\title{
The Synthesis and Evaluation of Heterocycles as Anti-Tuberculosis Agents
}

by

Kristiana Tika Santoso

A thesis

submitted to Victoria University of Wellington

in fulfilment of the requirements for the degree of

Doctor of Philosophy

Te Whare Wānanga o te Ūpoko o te Ika a Māui 


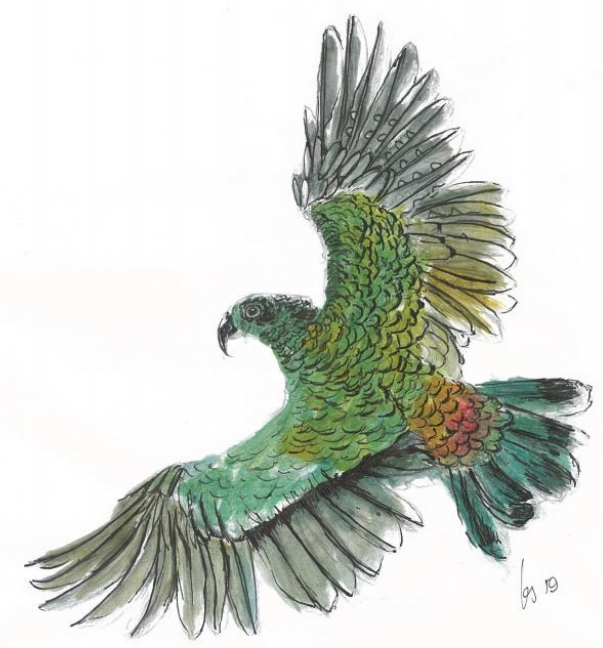

I hope that in this year to come, you make mistakes. Because if you are making mistakes, then you are making new things, trying new things, learning, living, pushing yourself, challenging yourself, changing your world. You're doing things you've never done before, and more importantly, you're doing something

Neil Gaiman 


\section{Abstract}

Tuberculosis (TB) is the leading cause of death from a single infectious agent, Mycobacterium tuberculosis $(M t b)$, worldwide. Currently, the efficacy of TB treatment regimens has declined due to the rise in antibacterial resistance and the shortage of new TB drugs. Thus, much effort has been spent in anti-tuberculosis drug development and in identifying new therapeutic targets against $M t b$. One such target is NADH dehydrogenase-II (NDH-II), an essential enzyme in the mycobacterial electron transport chain that is not present in mammalian cells. In this thesis, four classes of heterocyclic compounds that have the potential to target NDH-II and their evaluation as anti-tubercular agents, are described. An overview of TB drug development and NDH-II as a promising target for TB drugs are described in Chapter 1.

In Chapters 2 and 3, the potential of anti-tubercular drugs based on the quinolinequinone (QQ) scaffold is described. QQs have previously shown promise as TB drugs by activating NDH-II to overproduce harmful reactive oxygen species leading to bacterial cell death. Chapter 2 describes the total synthesis of the QQ natural products ascidiathiazone A and ascidiathiazone $\mathrm{B}$, and derivatives thereof, using a synthetic route that allows for high divergency and the efficient synthesis of the natural products and their intermediates. To this end, the first total synthesis of ascidiathiazone B is reported, as is the identification of ascidiathiazone $\mathrm{A}$ as a promising anti-tuberculosis drug with an $\mathrm{MIC}$ of $1.6 \mu \mathrm{M}$ against $\mathrm{Mtb}$. Insight into the ability of a representative quinone, 7-chloro-6-chloroethylamino-2-methylQQ, to increase NDH-II activity is also described. In Chapter 3, the syntheses of thirtytwo simplified QQs with different functional groups at the 6- and 7-positions of the QQ scaffold are described. These compounds were screened against $M t b$, with the lead compound from this library, 7-chloro-6-propargylamino-QQ, exhibiting an MIC of $8 \mu \mathrm{M}$ against $M t b$. Structure-activity data revealed diminished biological activity for QQs bearing tertiary amines, as compared to those with secondary amines, suggesting that the presence of a hydrogen bond donor at the 6- and 7-positions of QQs may play a critical role in antimycobacterial activity.

In Chapter 4, the synthesis and anti-mycobacterial activity of chromonyl-pyrimidines is presented. Chromonyl-pyrimidines have a structural resemblance to quinolinyl pyrimidines, a class of known NDH-II inhibitors and anti-TB agents. Chromones have shown promise as TB drugs, though they have not yet been reported to bind NDH-II. Despite this, 
chromonyl-pyrimidines contain a ketone functionality that may be able to bind the quinone binding site. For the first eleven-member library of chromonyl pyrimidines synthesised, all but two of the compounds exhibited inhibitory activity against $M t b$, however, the growth inhibition was modest $(\mathrm{MIC}=36-684 \mu \mathrm{M})$. Accordingly, a second generation of chromonyl pyrimidines was synthesised, which included six compounds with improved potency against $M t b$ - all with an MIC value of $12.5 \mu \mathrm{M}$. The activity of these chromonyl pyrimidines was attributed to the presence of aromatic rings both on the pyrimidine and the chromone scaffolds, though changes to the electronic properties of the aryl groups, i.e. the incorporations of electron-withdrawing and electron-donating groups, did not affect inhibitory activity.

Finally, in Chapter 5, a library of phthalazinones and pyrimidinyl-phthalazinones with antitubercular activity is described. While phthalazinones have not yet been extensively explored as anti-mycobacterial agents, the phthalazinone scaffold has the potential to act as an uncoupler. Uncouplers are typically weak acids or bases that act on the electron transport chain by dissipating the proton motive force and ultimately preventing the generation of ATP. In $M t b$, this uncoupling process is detrimental and leads to cell death. Phthalazinones are weakly basic and, due to their bicyclic ketone-bearing motif, has the potential to bind NDH-II at the proposed Q-site. Accordingly, a series of phthalazinones was synthesised to investigate their anti-tubercular activity and uncoupling activity. From the library of phthalazinones, $N$-tert-butyl- and nitro-substituted phthalazinones elicited high inhibitory activity, both with an MIC value of $3 \mu \mathrm{M}$. Of particular note among the pyrimidinylphthalazinones was the 4-fluorophenyl-pyrimidinyl-N-heptyl phthalazinone, which showed high potency against $M t b$ with an MIC of $1.6 \mu \mathrm{M}$. Further biological studies showed that some phthalazinones increased the rate of $\mathrm{NADH}$ oxidation in mycobacteria, which could be a result of uncoupling activity, while a number of pyrimidinyl-phthalazinones decreased NADH oxidation rates. These mechanistic results indicated that the two classes of compounds may have different modes of inhibition. 


\section{Acknowledgements}

"If I have seen further it is by standing on ye shoulders of Giants"

Isaac Newton

This $\mathrm{PhD}$ would not have existed if it weren't for the amazing giants whose shoulders I stood on. The first giants I'd like to thank are Mattie Timmer and Bridget Stocker, from whom I have learned almost everything I know about organic chemistry. Thank you for your dedication and patience in guiding me through this project. Your passion and enthusiasm for research are inspirational and I am grateful to have had the privilege to learn how to be a researcher from such accomplished scientists. Many thanks also to Greg Cook and his team for hosting me in their lab and helping me with the biology side of my project.

The second giants in my life are my beautiful whānau. Mum and dad, you've sacrificed so much to do the best by us and for that I'm eternally grateful. Yohanes, you're the wisest and most intelligent big brother a girl could have and Tania, thank you for being my best friend, my number one supporter, and my biggest inspiration. I'm so incredibly proud to be a Santoso.

Special thanks to Ian Vorster, to whom no request is unimportant, Patricia Stein, whose super power is to solve seemingly impossible problems, Martyn Coles and Mat Anker for their help with XRD experiments, Rohan Borah for his expertise in redox chemistry and all members of the immunoglycomics group, especially Stefan Munneke and Amy Foster for all their help. Special mention to Matthias Lein, who has cheered me on since my second-year, thank you for always having time for me no matter where you are in the world. Finally, thank you to my funding provider, the Maurice Wilkins Centre, for making this journey possible.

Huge hugs to those I'm honoured to call friends, who have been there for me at the beginning, and will now be there at the end: Stevie Jamieson, Claire Turner, Siobhan Bradley, Ethan Woolly, Lynne Gledstone-Brown. We can finally talk about something else now.

Finally, to my favourite Swiss family, the Lassueurs, thank you for the many care packages and kind words. In particular, to the most caring, beautiful and loyal person I could ever imagine to share my life with, Loïc Lassueur, thank you for coming along on this, at times, tumultuous journey without complaint. You've made the past three and a half years much easier by filling every spare moment with memorable adventures. Now let's celebrate with more adventures. 


\section{Table of Contents}

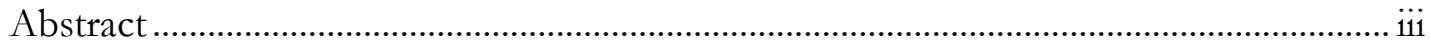

Acknowledgements ...........................................................................................................

List of abbreviations .................................................................................................... vii

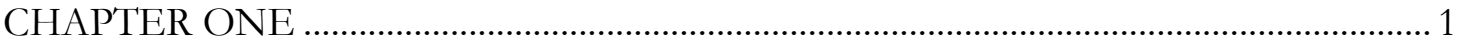

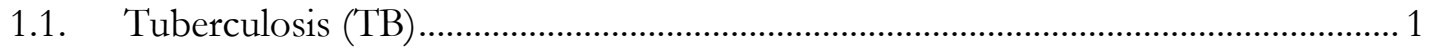

1.2. The electron transport chain as a new TB drug target ............................................. 10

1.3. NDH-II as an attractive TB drug target ................................................................... 16

1.4. NDH-II substrates have antimycobacterial activity ...............................................21

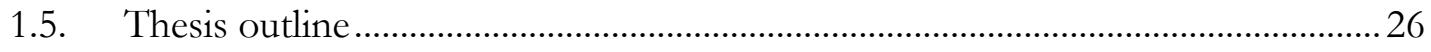

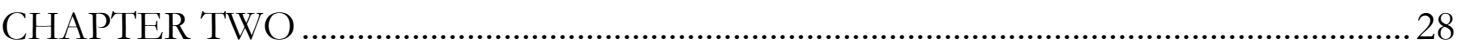

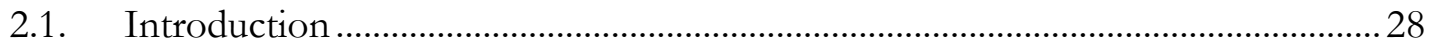

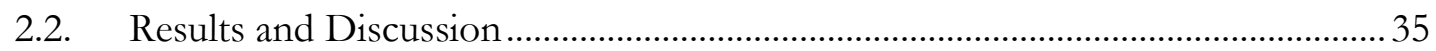

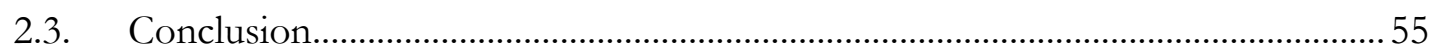

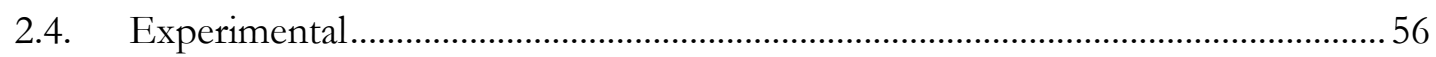

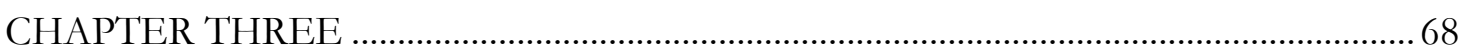

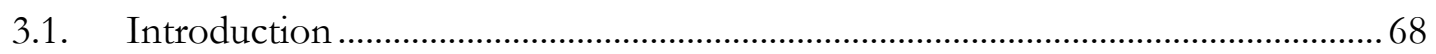

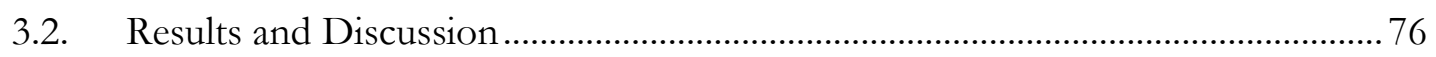

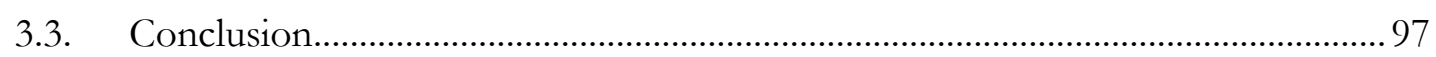

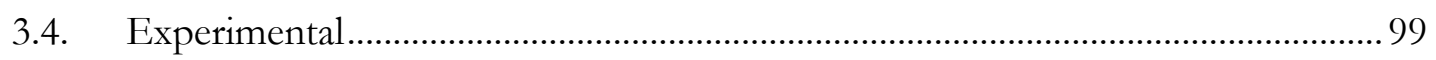

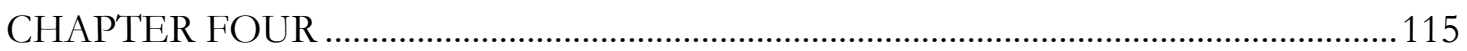

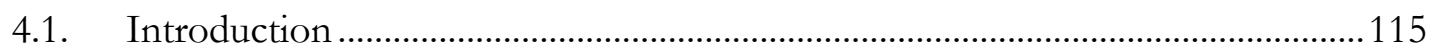

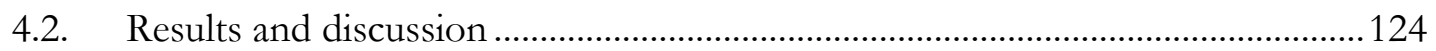

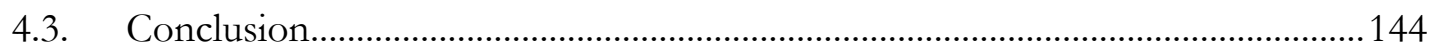

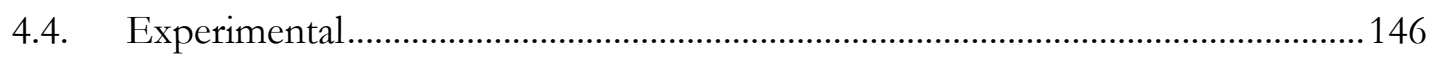

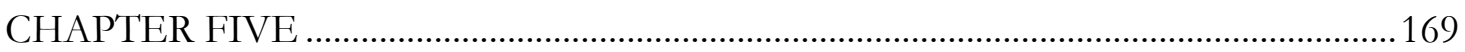

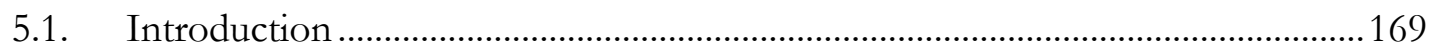

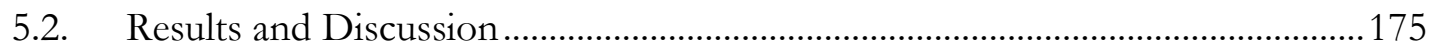

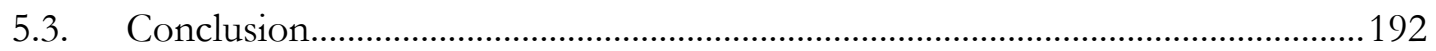

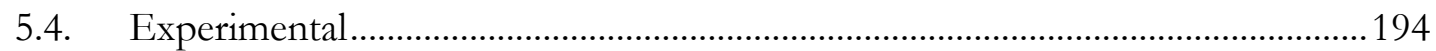

CHAPTER SIX

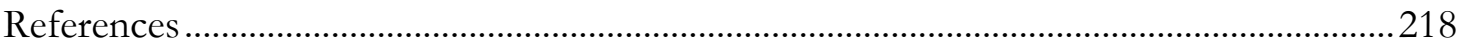


List of abbreviations

FAD

Ac

acetate

ADP adenosine diphosphate

AIBN

azobisisobutyronitrile

aq.

aqueous

ATP adenosine triphosphate

ATPase adenosinetriphosphatase

BDQ bedaquiline

Boc

tert-butyloxycarbonyl

br.

calcd.

broad

CCCP

COSY

CV

d

DC

DMAP

DMF

DMSO

DNA deoxyribonucleid acid

E. coli Escherischia coli

EDCI N-(3-

dimethylaminopropyl)-

$N$ 'ethylcarbodiimide-

hydrochloride

EMB ethambutol

ESI electrospray ionisation

Et ethyl

ETC electron transport chain
FCCP

flavin adenine

dinucleotide

carbonyl cyanide $p$ -

trifluoromethoxyphenyl

hydrazone

GC

HEPES (4-(2-hydroxyethyl)-1-

piperazineethanesulfonic acid)

HMBC heteronuclear multiple

bond correlation

HQNO 2-heptyl-4-

hydroxyquinoline- $N$ -

oxide

HRMS high resolution mass

spectrometry

HSQC heteronuclear single

quantum coherence

HTS high-throughput screen

IC $_{50}$ half maximal inhibitory concentration

IL interleukin

IMV inverted membrane

vesicle

INF- $\boldsymbol{\gamma}$ interferon- $\gamma$

INH isoniazid

IR infra-red

LAM lipoarabinomannan

$m / z \quad$ mass/charge

MBC minimum bactericidal

concentration

Me methyl

m-CPBA meta-

chloroperoxybenzoic acid

MIC minimum inhibition

concentration 


\begin{tabular}{|c|c|c|c|}
\hline MDR & multidrug-resistant & ROS & reactive oxygen species \\
\hline MHz & mega Hertz & $\mathbf{R R}$ & rifampicin-resistant \\
\hline MK & menaquinone & $\mathbf{s}$ & singlet \\
\hline$M t b$ & Mycobacterium tuberculosis & SAR & structure-activity \\
\hline \multirow{2}{*}{ NADH } & nicotinamide adenine & & relationship \\
\hline & dinucleotide & $\mathbf{t}$ & triplet \\
\hline NBS & N-bromosuccinimide & TB & tuberculosis \\
\hline NDH-I & type I NADH & THF & tetrahydrofuran \\
\hline \multirow{3}{*}{ NDH-II } & dehydrogenase I & TLC & thin layer \\
\hline & type II NADH & & chromatography \\
\hline & dehydrogenase & TLR & toll-like receptor \\
\hline NK & natural killer & TNF- $\alpha$ & tumour necrosis factor- $\alpha$ \\
\hline NMR & $\begin{array}{l}\text { nuclear magnetic } \\
\text { resonance }\end{array}$ & UV & ultra-violet \\
\hline obsd. & observed & XDR & extensively drug-resistant \\
\hline OD & optical density & XRD & X-ray diffraction \\
\hline PAS & para-amino salicylic acid & WHO & $\begin{array}{l}\text { World Health } \\
\text { Organisation }\end{array}$ \\
\hline PMF & proton motive force & & \\
\hline ppm & parts per million & & \\
\hline PRR & $\begin{array}{l}\text { pattern recognition } \\
\text { receptors }\end{array}$ & & \\
\hline PZA & pyrazinamide & & \\
\hline q & quartet & & \\
\hline $\mathbf{Q}$ & quinone & & \\
\hline $\mathrm{QH}_{2}$ & hydroquinone & & \\
\hline QQ & quinolinequinone & & \\
\hline Q-site & quinone binding site & & \\
\hline Q-TOF & quadrupole time of flight & & \\
\hline $\mathbf{R}_{f}$ & retention factor & & \\
\hline RIF & rifampicin & & \\
\hline RNAP & RNA polymerase & & \\
\hline RNI & $\begin{array}{l}\text { reactive nitrogen } \\
\text { intermediates }\end{array}$ & & \\
\hline
\end{tabular}




\section{CHAPTER ONE}

\section{Introduction}

\subsection{Tuberculosis (TB)}

Tuberculosis (TB) is an infectious disease caused by the bacterium complex Mycobacterium tuberculosis $(M t b)$, whereby M. tuberculosis, M. africanum, and M. bovis are human pathogens. ${ }^{1,2}$ Currently, an estimated 1.7 billion people, a quarter of the world's population, are infected with the bacteria. ${ }^{3}$ In the most recent global TB report, the World Health Organisation (WHO) recorded nearly 1.6 million TB deaths and 6.4 million new TB cases in $2017,{ }^{3}$ making TB the leading cause of death from a single infectious agent worldwide. Given the long history of TB, the fact that it remains a global threat today is alarming as almost all TB cases can be cured with timely diagnosis and prompt treatment. ${ }^{2}$ One reason for the continuous TB epidemic is 
the high rate of patient non-compliance due to the lengthy duration and financial burden of the treatment. ${ }^{4}$ As a consequence, countries with the highest incidence of TB are developing countries. In 2017, TB cases in just seven countries - India, Indonesia, China, the Philippines, Nigeria, Bangladesh and Pakistan - accounted for 63\% of the total global incidence of the disease. $^{3}$ Additionally, the rise of drug resistant $M t b$ strains continues to threaten the success of current treatment regimens and consequently, the need for new anti-tubercular agents has become an urgent global priority. However, the development of TB drugs is difficult due to the manner by which $M t b$ resides within the host. This is evidenced by the current treatment regime, which involves a combination therapy of four different antibiotics, and which has remained the same since it was introduced in the 1970s. ${ }^{2,5}$

\subsubsection{The immune response to TB}

The most common form of TB is pulmonary TB, where the bacteria reside in the lungs. Typical symptoms of this form of the disease include a chronic cough with blood-tinged sputum, fever and weight loss. These symptoms are present only in the active form of the disease. The bacteria are spread from the saliva of active pulmonary TB sufferers through aerosolised droplet nuclei of about 1-5 $\mu \mathrm{m}$ in diameter. ${ }^{6}$ The small size of the particles allows them to easily travel into the lungs where they are engulfed by alveolar macrophages. Mtb can also reside in other organs such as the brain and the kidneys (termed extrapulmonary TB). ${ }^{6-8}$ Most individuals infected by $M t b$ have a latent form of the disease, which is asymptomatic and noninfectious. Approximately $5-15 \%$ of latent TB cases will develop into active TB, and this typically occurs when the immune system becomes compromised. ${ }^{7}$

The immunological responses to the invasion of the lungs by $M t b$ are triggered by alveolar macrophages engulfing the bacteria. Here, the highly conserved mycobacterial cell wall structure allows pattern recognition receptors (PRRs) such as C-type lectins ${ }^{9}$ and toll-like receptors (TLRs) on the surface of macrophages to recognise specific cell wall components of the bacteria. ${ }^{10}$ As a defensive response, macrophages then induce the immediate killing of $M t b$ through cidal mechanisms such as the production of reactive nitrogen intermediates (RNI) and reactive oxygen species (ROS). ${ }^{11}$ However, the bacteria also have defence mechanisms that allow them to survive and replicate within the compartments of the macrophage. ${ }^{7}$ Although these defence mechanisms are not yet well understood, it has been reported that $M t b$ can prevent phagosome maturation and acidification, which inhibits fusion of the phagosome with the lysozome. ${ }^{12}$ Furthermore, $M t b$ can also reduce antigen expression by downregulating major 
histocompatibility complex (MHC) class II molecules on macrophages that are required for recognition of the internalised bacteria by $\mathrm{T}$ cells. ${ }^{11}$

The extraordinary ability of $M t b$ to remain viable within macrophages leads to the recruitment of other immune cells, such as dendritic cells (DCs), to the site of infection. The activation of macrophages and DCs then initiates further proliferation of immune cells, ultimately leading to granuloma formation (Figure 1.1). ${ }^{11}$ First, cytokines are produced, such as tumour necrosis factor- $\alpha(\mathrm{TNF}-\alpha)$ and the interleukins (ILs), including IL-6, IL-12, IL-1 $\alpha$, IL-1 $\beta$. Cytokines are an important part of the body's defence mechanisms as they can further activate macrophages (i.e. IL-12), control mycobacterial growth through granuloma formation (i.e. TNF- $\alpha$ ), or activate other immune cells such as T cells and natural killer $(\mathrm{NK})$ cells that can produce other pro-inflammatory cytokines such as interferon- $\gamma(\mathrm{INF}-\gamma) \cdot{ }^{13}$ The resulting aggregation of mononuclear immune cells marks the early stages of granuloma formation and the end of exponential bacterial growth. ${ }^{14}$ 


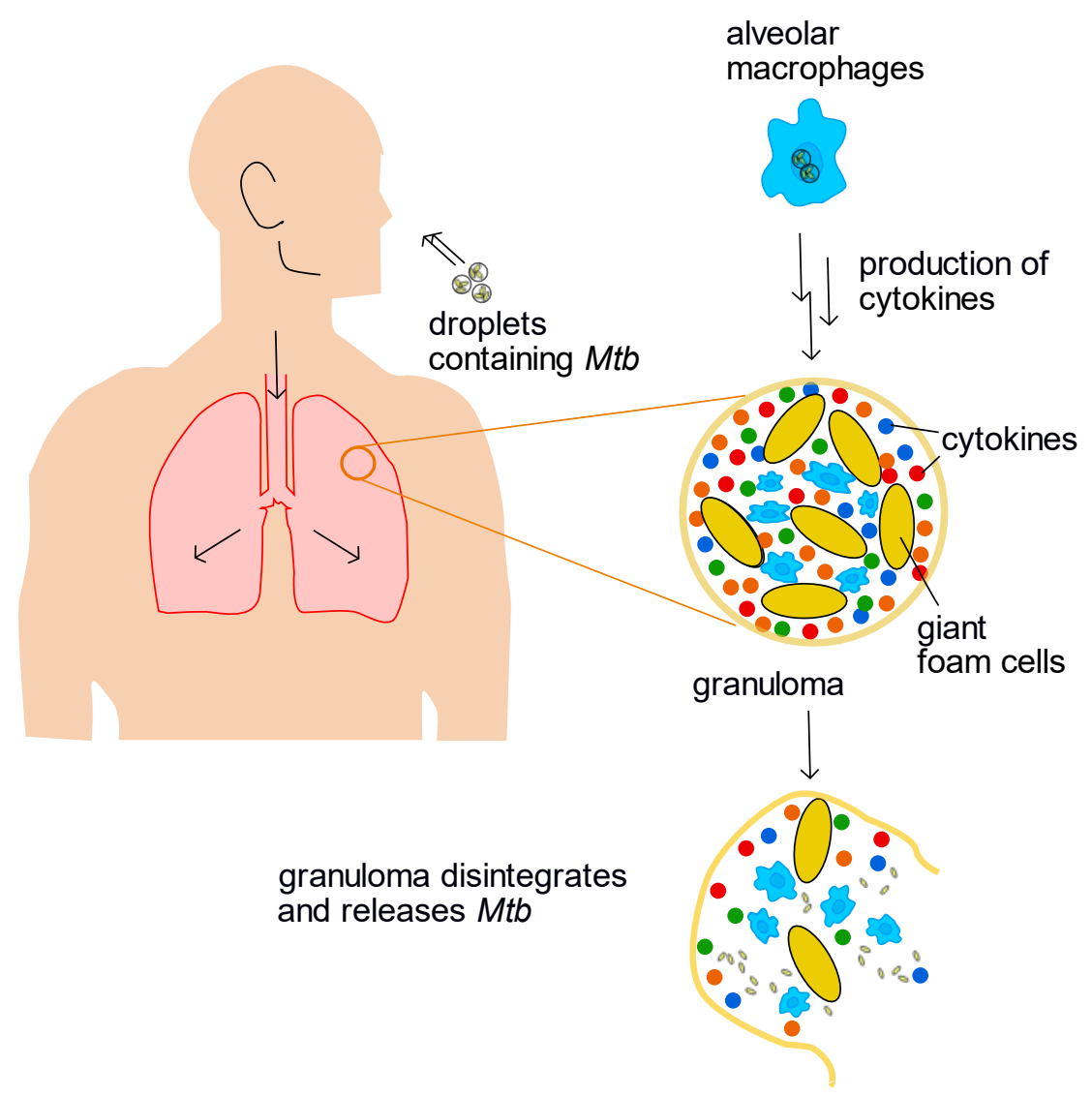

Figure 1.1: The immunological activities that occur during the invasion of Mtb. The mycobacteria is introduced into the body via small droplet nuclei before typically residing in the lungs, its primary site of infection. Here, the bacteria are recognised through their highly conserved cell wall structures by PRRs of macrophages and are engulfed. This activates a string of immunological events including the production of cytokines by macrophages, which can in turn activate $\mathrm{T}$ cells. The recruitment of immune cells leads to the formation of giant foam cells that continue to recruit more cytokines and chemokines until a granuloma is formed. Inside the granuloma, the mycobacteria are able to exist in their non-replicating, dormant form. This is the latent form of $\mathrm{TB}$, which can be reactivated when the immune system is weakened or compromised. The reactivation of the bacteria is marked by the disintegration of the granuloma and the release of $M t b$ into the body. ${ }^{13,14}$

Granuloma formation is an important immunological event resulting in the containment of the infection by preventing the mycobacteria from proliferating. This bacterial growth arrest is achieved as the granulomas mature, where fibrous capsules are formed surrounding the macrophages while more pro-inflammatory cytokines ${ }^{6}$ and chemokines ${ }^{15}$ are produced. Simultaneously, infected macrophages differentiate into foamy macrophages, macrophages laden with lipid droplets, which are collectively known as giant foam cells. The conversion of infected macrophages into foamy macrophages occurs due to the high lipid content of the TB bacterial cell wall ${ }^{14}$ and is hypothesised to assist in the bacilli's persistence by providing a potential nutrient source. ${ }^{16}$ Thus, granulomas provide a suitable environment for the 
mycobacteria to survive the bombardment of immune cells, allowing for infection localisation as the bacteria enter their dormant state. This dormant non-replicating form of mycobacteria can be reactivated when the immune system is compromised, ${ }^{11,13}$ for example during coinfection with HIV., ${ }^{6,17}$ With the immune system weakened, the granulomas can no longer contain the bacteria and the bacteria spread and infect other organs, which can lead to death of the host if the disease is left untreated. ${ }^{7}$

\subsubsection{Current treatment for TB}

The current therapeutic regimen for drug-susceptible TB has a high success rate $(>95 \%)^{2}$, however, it involves at least six months of directly observed therapy to ensure that patients remain compliant despite becoming asymptomatic before treatment is complete. ${ }^{2}$ The six months is comprised of two stages: the intensive phase, and the short-course chemotherapy phase. The intensive phase involves a combination therapy of all four first-line antibiotics, rifampicin (RIF, 1), isoniazid (INH, 2), pyrazinamide (PZA, 3), and ethambutol (EBM, 4) (Figure 1.2), for two months. The remaining four months are spent in the chemotherapy phase with INH and RIF., ${ }^{2,5}$ The lengthy treatment and requirement of DOT workers is often unachievable in areas with limited resources and this can lead to non-compliance on the patients' part, which can result in the exposure of the bacteria to sub-lethal concentrations of the antibiotics. This, in turn, can trigger the development of antibiotic resistance, ${ }^{18-20}$ which is evidenced by the rise of rifampicin-resistant (RR), multidrug-resistant (MDR), and extensively drug-resistant (XDR) strains of $M t b$. 


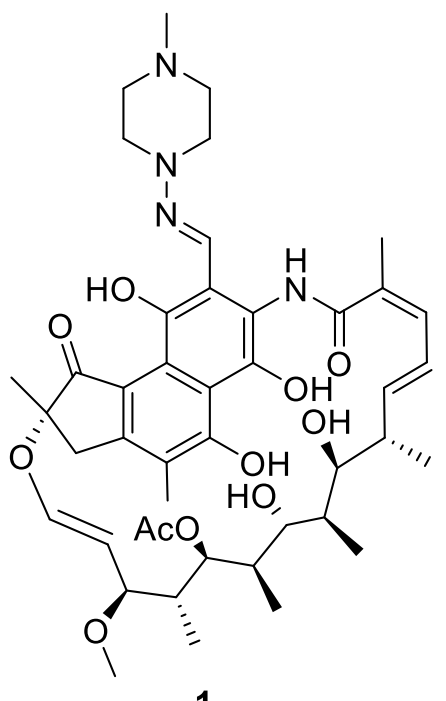

1

Rifampicin (RIF)<smiles>NNC(=O)c1ccncc1</smiles>

Isoniazid (INH)<smiles>NC(=O)c1cnccn1</smiles>

Pyrazinamide (PZA)<smiles>CCCCNC(CO)CNC(CC)CO</smiles>

Figure 1.2: Current first-line TB drugs RIF (1), INH (2), PZA (3), EMB (4). ${ }^{2}$

MDR-TB are defined as TB strains that are resistant to at least RIF (1) and INH (2), while, in addition to these drugs, XDR-TB are also resistant to any fluoroquinolones (broad-spectrum antibiotics) and at least one of the three injectable second-line drugs kanamycin (5), capreomycin (6), and amikacin (7) (Figure 1.3). ${ }^{2,18}$ In 2017, about 20\% of known TB cases were MDR-TB and RR-TB, a percentage that has been continuously increasing since $2009 .{ }^{3}$ Of these resistant strains, $8.5 \%$ were categorised as XDR-TB, an increase from $6.2 \%$ in $2016 .{ }^{3}$ To treat resistant strains of $M t b$, second-line drugs including para-amino salicylic acid (PAS) (8), ethionamide (9), ofloxacin (10), cycloserine (11) and streptomycin (12), are required. In contrast to first-line antibiotics, which provide the standard treatment for TB, second-line drugs have more unpleasant side effects. ${ }^{21}$ Patients with MDR-TB require a combination therapy of five or more drugs for at least 9 months. ${ }^{21}$ This therapy involves second-line injectable drugs during the intensive phase, where the duration of each phase varies among different individuals. Typically, the first round of treatment has a success rate of just $50 \%$ and patients are required to have repeat regimens, often with different drugs. XDR-TB cases require substantially longer treatment durations of up to three years, the involvement of third-line drugs, such as clofazimine $(\mathbf{1 3}),{ }^{2}$ and have a low $(26 \%)$ treatment success rate. ${ }^{21}$ 


\section{Second-line drugs}

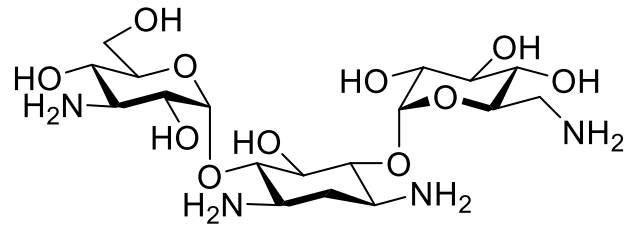

5

Kanamycin

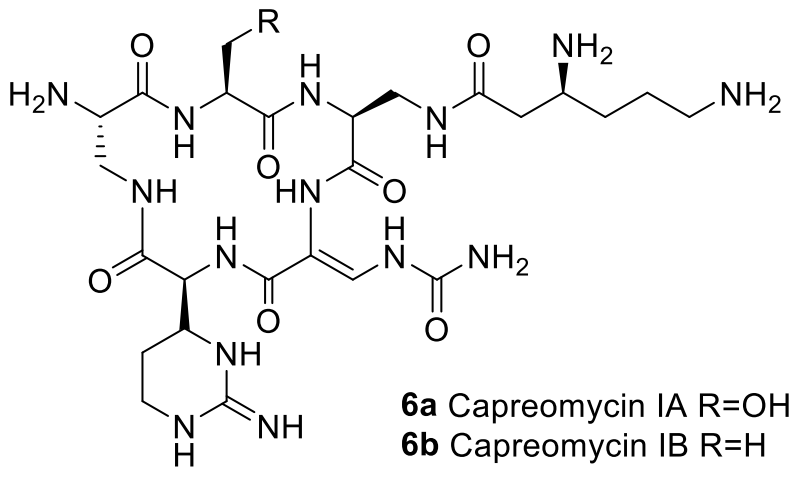

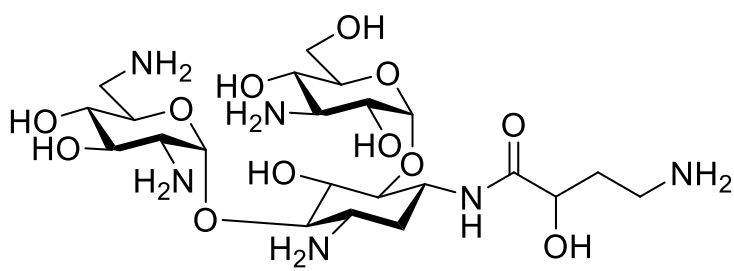

7

Amikacin<smiles>CC1COc2c(N3CCN(C)CC3)c(F)cc3c(=O)c(C(=O)O)cn1c23</smiles>

Ofloxacin<smiles>N[C@@H]1CONC1=O</smiles>

11<smiles>Nc1ccc(C(=O)O)c(O)c1</smiles>

Para-amino salicylic acid (PAS)<smiles>CCc1cc(C(N)=S)ccn1</smiles>

9

Third-line drug<smiles>CC(C)/N=c1\cc2n(-c3ccc(Cl)cc3)c3ccccc3nc-2cc1Nc1ccc(Cl)cc1</smiles>

Figure 1.3: Current second-line and third-line drugs for TB treatment. ${ }^{2}$ 


\subsubsection{The mechanisms of action of current TB drugs}

The first drug for TB, a protein synthesis inhibitor named streptomycin (12), was discovered in the mid-1940s by Albert Schatz and Selman Waksman. ${ }^{22}$ The immediate hope of eradication of TB, however, was short-lived when the first clinical trials saw patients relapsing and the bacteria quickly developing resistance to the drug. ${ }^{219}$ Fortunately, the revolutionary idea of treating TB with antibiotics soon led to the development of a second drug PAS (8) in $1944 .^{23}$ Although each antibiotic alone was unsuccessful in eradicating the disease from patients, together they were effective and resulted in little evidence of bacterial resistance. This marked the beginning of combination therapy for TB. ${ }^{19}$

The next few decades saw the emergence of a number of antibiotics that are still important for the control of the disease today; namely RIF (1) $,{ }^{24} \mathrm{INH}(\mathbf{2}),{ }^{25} \mathrm{PZA}(\mathbf{3}),{ }^{26}$ and EMB (4) ${ }^{27} \mathrm{RIF}$ (1) can effectively inhibit the DNA-dependent RNA polymerase (RNAP), an enzyme essential in the chain elongation process of transcription, ${ }^{18}$ while INH (2) and PZA (3) are fatty acid biosynthesis inhibitors. ${ }^{17}$ EMB (4) disrupts the integrity of the mycobacterial cell wall by inhibiting the arabinosyl transferase enzyme that is required for the synthesis of the arabinogalactans found in the outer cell wall. ${ }^{27}$

With the increasing occurrence of drug-resistant $M t b$ strains, much effort has been spent identifying the molecular mechanisms adopted by the bacteria to aid their survival. Although TB treatment involves many different antibiotics targeting different essential cell processes, each bacterial strain may only be exposed to one of these drugs at a time due to their compartmentalisation in the body. ${ }^{19}$ The compartmentalisation of the bacterial population in the body means $M t b$ can survive in different areas of the body; for example in pulmonary cavities, empyema pus (solid caseous material of dead tissue), or macrophages. This gives rise to different populations of bacteria that need to be targeted, each with their own unique environments. ${ }^{19}$

In pulmonary cavities, the bacteria have access to oxygen and are often in their replicating states. These bacteria typically exist in the early stages of acute TB and are readily targeted by INH (2), which is cidal to bacteria in aerobic environments. ${ }^{28}$ However, this is not the case for bacteria living in low $\mathrm{pH}$ environments such as empyema pus where antibiotics like INH (2) are not stable. In this case, PZA (3) is a more effective antibiotic as its active form, pyrazinoic acid, is stable under acidic conditions. ${ }^{20,29}$ In macrophages, bacteria exist in microaerophilic conditions, where they have occasional bursts of metabolic activity, but otherwise are in an ideal condition to resume their dormant state. Under these conditions, drugs are often 
tolerated by the bacteria due to their relatively low metabolic activities. This non-replicating form of bacteria are extremely difficult to target, with RIF (1) being one of the few antibiotics that is effective. ${ }^{18}$

As bacterial populations live in a variety of conditions, $M t b$ typically develop drug-resistance through chromosomal mutations rather than through transduction or transformation processes in which antibiotic-resistance is transferred through genetic materials. ${ }^{18-20}$ This type of acquired antibiotic resistance develops due to high survival pressures imposed by the drug the bacteria are exposed to. For example, EMB-resistance is established by chromosomal mutations in the gene encoding arabinosyl transferase, an enzyme inhibited by EMB, ${ }^{30}$ while RR-mutants induce reduced binding affinity of RIF (1) to RNAP. This was evident in the mutations found in 11 out of the 12 amino acids associated with RIF's binding pocket, usually into amino acids with longer side chains to prevent RIF binding. In addition, other RR-mutants also have a single nucleotide mutation involving a substitution in the $r p o B$ gene, which is the gene encoding the $\beta$-subunit of RNAP. ${ }^{18}$

PZA (3) is a prodrug that is activated by the bacterial enzyme pyrazinamidase to the active form, pyrazinoic acid. Accordingly, in mutants that are highly resistant to PZA (3), mutations associated with the $p n c A$ gene were found. PncA is the gene responsible for coding the pyrazinamidase enzyme and this mutation is observed in $70 \%$ of PZA-resistant strains. ${ }^{29-32}$ The development of PZA-resistance in strains without the $p n c A$ mutation is yet to be explained and this portrays the complexity of the molecular mechanisms that are modified as the bacteria evolve. ${ }^{19}$ The resistance to INH (1) is also complicated as it is associated with mutations in five different loci: kat $G$, $i n h A, a h p C$, kas $A$, and $n d h$. Some of the mutations are linked. For example, the loss of catalase activity due to kat $G$ mutations result in the overexpression of $a b p C$, the alkyl hydroperoxide reductase enzyme, though the reason for this association is unknown. ${ }^{19}$ Despite extensive research around Mtb's drug resistance, the mycobacteria's ability to quickly develop sophisticated resistance mechanisms, especially given their slow-replicating nature, remains poorly understood.

As well as acquired antibiotic resistance, $M t b$ also have intrinsic antibiotic resistance mechanisms, such as the highly complex cell wall (Figure 1.4). The cell wall is very thick, comprising of many layers of different components such as lipoarabinomannan glycolipids (LAM), arabinogalactans and peptidoglycans. LAM is a polymer made of phosphoryl-inositolmannose monomers on a polysaccharide backbone, whereas arabinogalactans are long polysaccharides comprised of linear galactan and branched arabinose chains, and 
peptidoglycans are polymers of amino acids and aminosugars. Arabinogalactans are also covalently bound to trehalose-dimycolates, which are highly hydrophobic long chain fatty acids, giving the outer bacterial membrane a waxy and non-fluid barrier. Accordingly, the highly lipophilic cell wall of $M t b$ makes it difficult for drugs to enter the cell, which in turn, imparts another level of complexity for effective anti-TB drug development. ${ }^{7,20}$
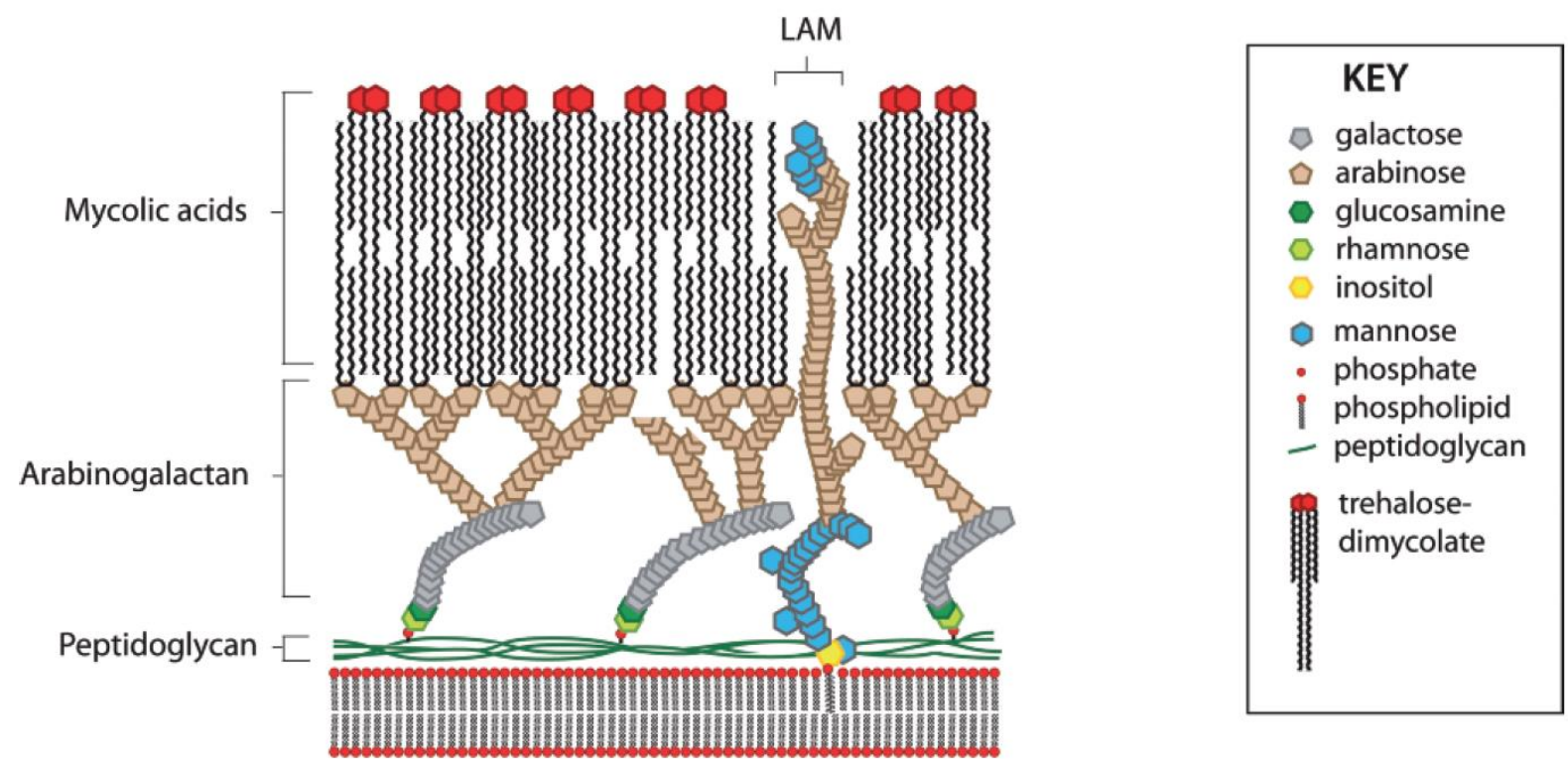

Figure 1.4: Cartoon depicting the complex structures making up the TB cell wall. The cell wall is comprised of structural layers of lipophilic mycolic acids, namely trehalose-dimycolates, and hydrophilic carbohydrates comprising of arabinogalactans and peptidoglycan. This makes the cell wall a difficult barrier for drugs to pass through and enter the cell. Image taken from Dangerfield E., et al., Chemistry in Australia, May 2010, with permission. ${ }^{7}$

\subsection{The electron transport chain as a new TB drug target}

\subsubsection{The electron transport chain (ETC) of $M t b$}

One way to develop new TB drugs is to target unexplored receptors in the bacteria, as evidenced by the recent development of bedaquiline (BDQ, 14, Figure 1.5), which targets the respiratory system of $M t b$ and is the first FDA-approved drug to treat TB in over forty years. ${ }^{33}$ Specifically, BDQ (14) inhibits $\mathrm{F}_{1} \mathrm{~F}_{0}$-ATP synthase, an enzyme found in the ETC that is responsible for the production of adenosine triphosphate (ATP) through oxidative phosphorylation. ${ }^{33}$ This exciting discovery has since triggered a number of studies into the essentiality of the ETC in Mtb and other ways in which the transport chain can be targeted. ${ }^{34}$ 38 


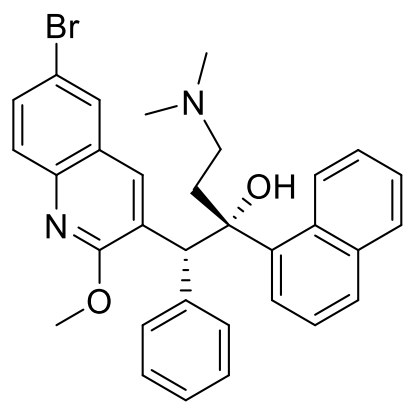

14

Figure 1.5: $\mathrm{F}_{1} \mathrm{~F}_{0}$-ATP synthase inhibitor bedaquiline (BDQ).

All living organisms generate energy in the form of ATP through phosphorylation reactions, which convert adenosine diphosphate (ADP) to ATP. ATP can be generated through substrate-level phosphorylation, where substrates are metabolised to generate ATP as seen in the citric acid cycle in human mitrochondria. ${ }^{39}$ This type of phosphorylation occurs within the bacterial cytoplasm and involves soluble enzymes. Alternatively, bacteria can synthesise ATP through oxidative phosphorylation. ${ }^{40}$ Oxidative phosphorylation couples oxygen intake with ATP synthesis and is a form of respiration used by obligate aerobes such as $M t b$. Specifically, the oxidative phosphorylation process generates ATP through the ETC, which involves a number of redox reactions ultimately leading to the reduction of $\mathrm{O}_{2}{ }^{34}$ This process has been shown to be crucial in both the replicating and non-replicating states of mycobacteria. ${ }^{41}$

The ETC is comprised of a group of membrane-bound enzymes that facilitate the downstream flow of electrons and the transport of protons out of the cell membrane. ${ }^{34}$ The protons remain outside the cell membrane because the membrane is impermeable to protons, resulting in the establishment of a transmembrane proton gradient that conserves energy. Consequently, two gradients, the electrical potential $(\Delta \varphi)$ and the chemical potential $(\Delta \mathrm{pH})$, are created, which together make up the proton motive force (PMF). ${ }^{40,41}$ The $\Delta \varphi$ is generated due to charge separation that results when protons are moved out of the cell. Similarly, $\Delta \mathrm{pH}$ occurs due to the decrease in $\mathrm{pH}$ outside the membrane when the protons move outside. The maintenance of the PMF is of paramount importance for bacteria as its aberration can lead to the loss of cell viability. ${ }^{41}$ For example, when the $\mathrm{pH}$ is neutral, the PMF will exist predominantly in the form of $\Delta \varphi$. As protons are removed from inside the bacterial membrane, the $\mathrm{pH}$ outside the 
membrane will decrease and $\Delta \mathrm{pH}$ will increase. ${ }^{41}$ This can be neutralised by the decrease in $\Delta \varphi$ through the influx of other cations such as $\mathrm{Mg}^{2+} \cdot{ }^{42}$

In $M t b$, the PMF is generated by the oxidation of electron donors, such as NADH and succinate, by $\mathrm{NADH}$ dehydrogenases (type 1: NDH-I, type 2: NDH-II), and succinate dehydrogenase $(\mathrm{SDH})$, respectively (Figure 1.6). These enzymatic processes release protons that are then translocated out of the cell membrane by proton-pumping enzymes (i.e. NDH-I and $\mathrm{SDH}$ ) to generate a transmembrane proton gradient. Such oxidation reactions are coupled to the reduction of menaquinone $(\mathrm{MK}, 15)$, an electron acceptor found in the ETC of $M t b$, to menaquinol $\left(\mathrm{MKH}_{2}, 16\right) .{ }^{34}$ The redox reactions then continue down the ETC, so that electrons flow from substrates with low reduction potentials, such as $\mathrm{NADH}\left(\mathrm{E}^{\circ}=-0.32 \mathrm{~V}\right)$, to those with higher reduction potentials, such as $\mathrm{O}_{2}\left(\mathrm{E}^{\circ}=+0.816 \mathrm{~V}\right) .{ }^{39}$ Hence, the direction of the electron flow can be determined by the position of each enzyme on the ETC. For example, $\mathrm{NDH}-\mathrm{I}$ and NDH-II, which bind and oxidise NADH to $\mathrm{NAD}^{+}$, are found at the beginning of the ETC due to the low reduction potential of NADH. On the other hand, oxygen, with a much more positive reduction potential, is the substrate of the cytochrome $b c$ complex, an enzyme found toward the end of the chain. Accordingly, substrates between these two enzymes have reduction potentials between the two extremes. ${ }^{39}$ After the reduction of $\mathrm{O}_{2}$ to $\mathrm{H}_{2} \mathrm{O}$, the next enzyme in the ETC is the $\mathrm{F}_{1} \mathrm{~F}_{0}$-ATP synthase. However, at this stage, the proton concentration is much higher outside the membrane than inside, and the ATP synthase catalyses proton translocation back into the membrane. Thus, protons move down the concentration gradient and generate energy that is captured in ATP through the phosphorylation of ADP. ${ }^{40}$ 


\section{NDH-I NDH-II SDH}

$$
\text { cytochrome } \mathrm{F}_{0} \mathrm{~F}_{1}
$$
bc complex ATPase
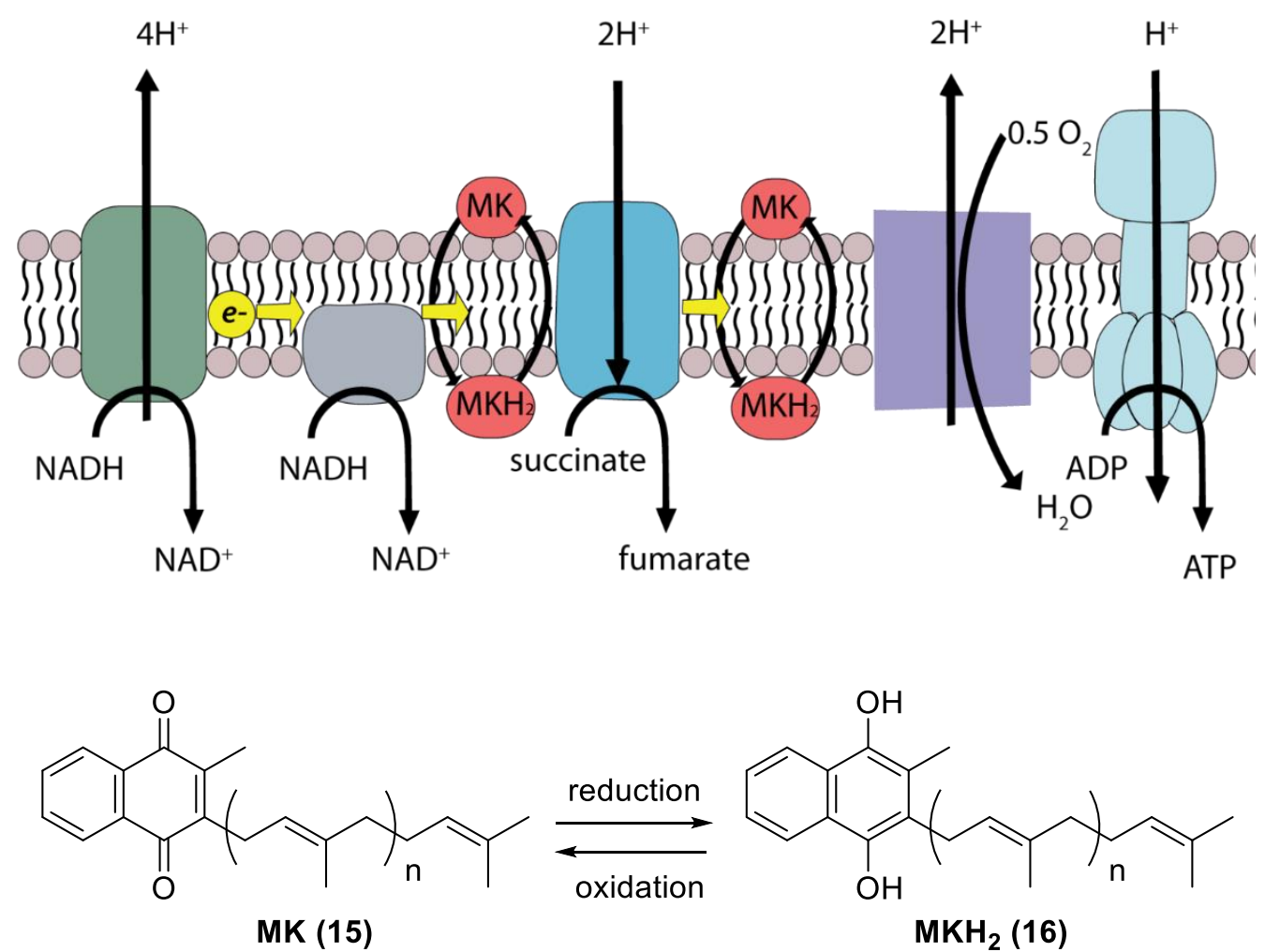

Figure 1.6: A schematic representation of the Mtb ETC showing the positions of oxidoreductase enzymes. The chain components are: NDH-I, NDH-II, SDH, cytochrome $b c$ complex, and $\mathrm{F}_{1} \mathrm{~F}_{\mathrm{o}}$ ATP synthase. Each enzyme catalyses redox reactions of different substrates, and the resulting protons and electrons are moved outside the membrane as well as down the ETC. The movement of electrons down the ETC is aided by the reduction of MK (15) to $\mathrm{MKH}_{2}$ (16), which is then reoxidised to form MK (15). ${ }^{41}$

\subsubsection{The oxidative phosphorylation process is essential to $M t b$}

Both types of ATP generation mechanisms, substrate-level phosphorylation and oxidative phosphorylation, are present in $M t b$, though oxidative phosphorylation produces a substantially greater amount of ATP. ${ }^{39}$ Although oxidative phosphorylation is much more efficient, it was hypothesised that the inhibition of $\mathrm{F}_{1} \mathrm{~F}_{0}$-ATP synthase would not be detrimental to $M t b$ given the bacteria's ability to produce ATP through substrate-level phosphorylation. In fact, this compensatory relationship between the two types of phosphorylation is seen in other aerobic bacteria such as Escherichia coli ${ }^{43}$ and Bacillus subtilis. ${ }^{44}$ However, the inhibition of ATP synthase by BDQ (14) was proven to be cidal to Mtb. ${ }^{33}$ This served as evidence that oxidative phosphorylation is a vital process for the survival of $M t b$. 
The $\mathrm{F}_{1} \mathrm{~F}_{0}$-ATP synthase facilitates the bacteria's ability to survive inside phagocytic vacuoles. ${ }^{42}$ Given the mildly acidic nature of these vacuoles with a $\mathrm{pH}$ range of $6.1-6.4^{45}$ and the sensitivity of all living cells to large deviations from the preferred neutral $\mathrm{pH}$ of $7, \mathrm{~F}_{1} \mathrm{~F}_{0}$-ATP synthase was found to have a role in the maintenance of a neutral internal $\mathrm{pH}$ despite the low external $\mathrm{pH}^{42}$ This was demonstrated in experiments where M. smegmatis and M. bovine maintained a near neutral internal $\mathrm{pH}$ in highly acidic environments $(\mathrm{pH}=4)$. However, cell viability and survival quickly deteriorated when a protonophore, carbonyl cyanide $m$-chlorophenylhydrazone (CCCP), was added to the growth medium, which allowed protons to enter the cells. The fact that cell viability was lost only when CCCP was added also portrayed the extraordinarily high impermeability of the cell wall to protons. ${ }^{45,46}$

As well as aiding in the survival of latent bacteria, $\mathrm{F}_{1} \mathrm{~F}_{0}$-ATP synthase is also reported to be essential in active forms of $M t b$, as evidenced by the loss of cell viability when the enzyme is inhibited. This was demonstrated in a 2005 study which showed that a lack of intact $\mathrm{F}_{1} \mathrm{~F}_{0}$-ATP synthase led to the inhibition of cell growth under both active and dormant stages of mycobacteria. Unlike E. coli and B. subtilis where the reduced ATP production is compensated by substrate-level phosphorylation, $\mathrm{F}_{1} \mathrm{~F}_{0}$-ATP synthase was required in mycobacteria. The speculative reasons for this essentiality are that the amount of ATP generated by glycolysis is not sufficient for the high demands required to build the complex cell wall of mycobacteria and/or that $\mathrm{F}_{1} \mathrm{~F}_{0}$-ATP synthase was needed as a translocation proton sink. ${ }^{47}$ Given the established impermeability of the mycobacterial cell wall, ${ }^{45} \mathrm{Mtb}$ may not support uncoupled respiration or allow uncontrolled proton entry through the adjustment of membrane permeability. ${ }^{47}$ To this end, the inhibition of oxidative phosphorylation in mycobacteria provides a new and exciting prospect for anti-tubercular drug design and has subsequently been the target of many recent drug developments.

\subsubsection{BDQ: The first TB drug to inhibit oxidative phosphorylation}

As mentioned above, BDQ (14) was the first drug that allowed researchers to determine the necessity of oxidative phosphorylation in mycobacteria. ${ }^{33,48}$ As an inhibitor of the $\mathrm{F}_{1} \mathrm{~F}_{0}$-ATP synthase, BDQ (14) binds to the $\mathrm{F}_{0} \mathrm{c}$-subunit of the enzyme ${ }^{49,50}$ and disconnects the mycobacterial respiration from ATP synthesis (Figure 1.7). Inhibitors with this mechanism of action are known as uncouplers. The uncoupling of respiration and ATP synthesis is detrimental to mycobacteria as they cannot use substrate-level phosphorylation as a compensatory mechanism. Moreover, the administration of BDQ (14) to Mtb leads to the 
downregulation of glycolysis, which occurs in the absence of fermentable carbon sources, such as succinate. Accordingly, the mycobacteria do not attempt to compensate for ATP depletion, suggesting that there is a more immediate threat introduced by the drug.

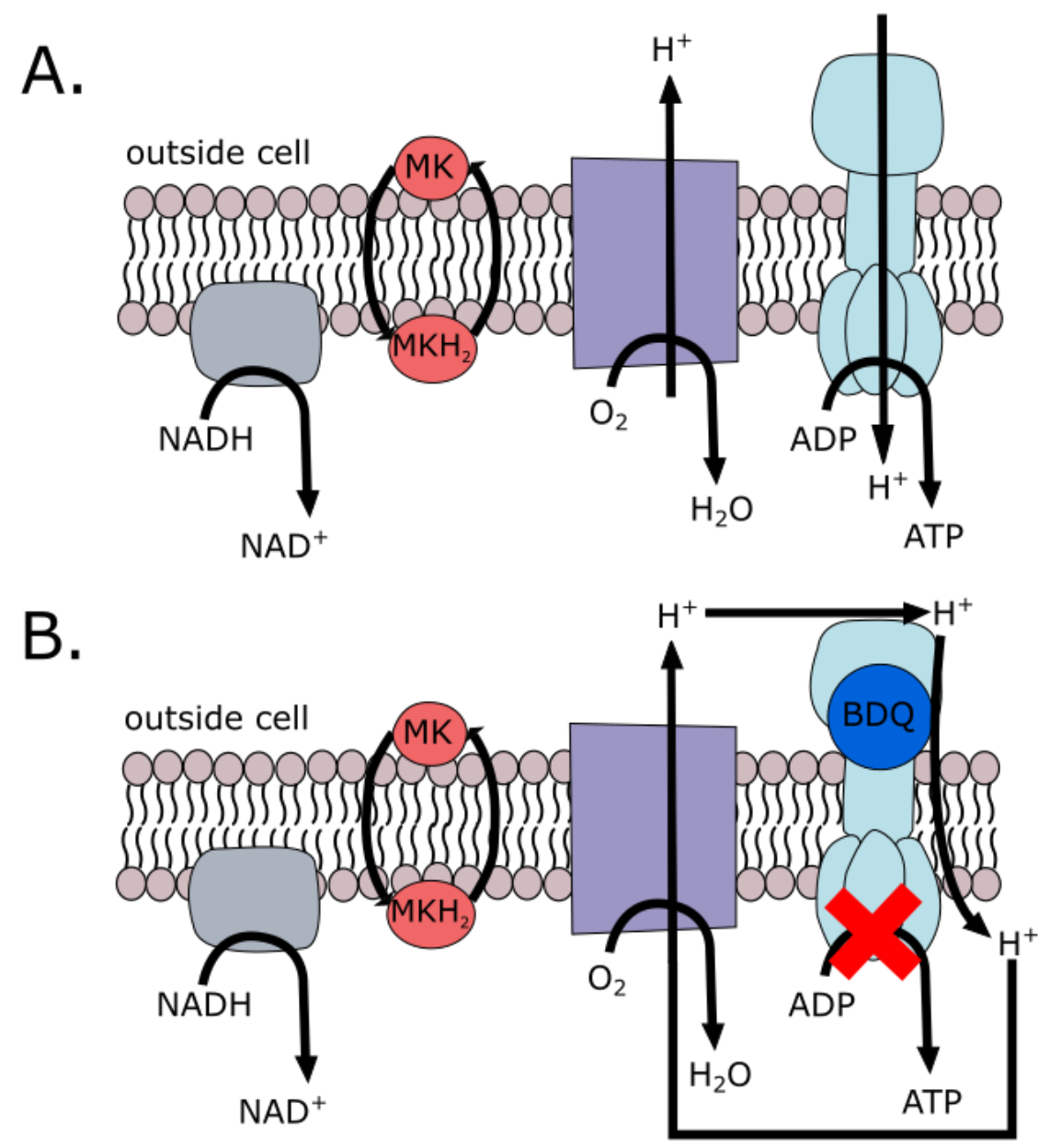

Figure 1.7: A cartoon representation of the uncoupler-like mechanism of BDQ (14). A). A simplified representation of the unimpaired ETC establishing a transmembrane proton gradient through successive redox reactions down the chain. The proton gradient is created through a number of membrane-bound enzymes catalysing redox reactions of different substrates such as $\mathrm{NADH}$, and $\mathrm{O}_{2}$. The protons and electrons produced from these reactions are either pumped out of the membrane or transferred down the chain, respectively. In addition, the redox reaction between $\mathrm{MK}(\mathbf{1 5})$ and $\mathrm{MKH}_{2}(\mathbf{1 6})$ aids in the downward movement of electrons. B). BDQ (14) binds to ATP synthase, thereby allowing protons to enter the cell freely. This results in a reduction of the $\mathrm{pH}$ gradient and, ultimately the dissipation of the PMF. ${ }^{33}$

Within the ETC, the uncoupling also results in the upregulation of non-proton pumps such as NDH-II and the downregulation of the majority of other proton translocating pumps. 
Additionally, processes that result in the production of reducing equivalents, such as NADH, are bypassed. Taken together, this suggests that the bacteria implements these modifications in order to avoid the hyperpolarisation of the PMF caused by the increased NADH/NAD ${ }^{+}$ ratio induced by BDQ (14). ${ }^{33}$ This is necessary because PMF homeostasis is important in all living things, as shown in a study where a hyperpolarised mitochondrial membrane resulted in ROS production and increased $\Delta \varphi$ in eukaryotes. ${ }^{51}$ Indeed, BDQ (14) elicits a transcriptional response indicative of ROS-production in $M t b$, though a change in $\Delta \varphi$ was not observed. Additionally, the binding of BDQ (14) to $\mathrm{F}_{1} \mathrm{~F}_{0}$-ATP synthase also resulted in increased $\mathrm{O}_{2}$ consumption and the reduction of $\Delta \mathrm{pH}$. On the whole, these responses suggest an uncouplerlike mode of action for BDQ (14), where the uncoupling of respiration from ATP synthesis is achieved via the free and uncontrolled movement of protons through the cell membrane. ${ }^{33}$ This leads to the dissipation of the PMF, which has been reported to be lethal in mycobacteria. ${ }^{52}$ The neutrality of $\Delta \varphi$ is likely to be maintained through the efflux of other neutral cations such as $\mathrm{K}^{+}{ }^{33}$

\subsection{NDH-II as an attractive TB drug target}

Of particular relevance to this thesis is the NDH-II enzyme in Mtb, which has shown promise as an antitubercular drug target. NDH-II is a small, monotopic membrane protein, weighing between $40-60 \mathrm{kDa} .{ }^{53}$ While it is absent in mammalian mitochondria, there are two copies of the enzyme in $M t b: n d h$ and its single subunit homologue $n d h A$. Along with NDH-I, NDH-II catalyses the oxidation of NADH that is fed into the ETC (Figure 1.6). ${ }^{54} \mathrm{NDH}-\mathrm{I}$ then translocates the released protons to the outside of the membrane, ${ }^{54}$ while $\mathrm{NDH}-\mathrm{II}$, being a non-proton pumping enzyme, uses the protons and electrons produced to drive the reduction of $\mathrm{MK}(\mathbf{1 5})$ to $\mathrm{MKH}_{2}(\mathbf{1 6})$ via a flavin co-factor (FAD). ${ }^{53,55}$

Mutagenesis studies have shown that NDH-I and NdhA are dispensable to $M t b$, however a lack of the $n d h$ gene leads to growth arrest and multiple amino acid auxotrophy which leads to the loss of cell viability. ${ }^{47}$ Furthermore, Ndh inhibition leads to a 25 -fold reduction in NADH oxidation, which indicates that NDH-II is the main enzyme responsible for this essential process. ${ }^{35,38,47}$ The preference of a non-proton pump to oxidise NADH over a proton pump is of interest, as NDH-II is less efficient in PMF and ATP generation than NDH-I. ${ }^{41}$ However, this may be beneficial under non-replicating conditions to avoid the accumulation of protons during low ATP synthase activity. ${ }^{56}$ Moreover, the use of NDH-II rather than NDH-I will allow for high metabolic flux, ${ }^{41}$ where $\mathrm{NADH}$ continues to get oxidised to $\mathrm{NAD}^{+}$and the 
PMF is maintained. PMF homeostasis is essential for mycobacterial persistence under hypoxic conditions. ${ }^{52}$ Furthermore, NDH-I activity in aerobes such as Mtb may lead to undesired production of ROS. ${ }^{41,57}$ Due to the essentiality of NDH-II for the growth and survival of $M t b$ and its absence in mammalian mitochondria, it is an attractive target for the development of new TB drugs. ${ }^{35,58}$

\subsubsection{Structure of NDH-II}

Crystal structures of NDH-II have been solved in the yeast strain Saccharomyces cerevisiae, ${ }^{59}$ as well as in bacterial species Caldalkealibacillus thermarum, ${ }^{53,58}$ Staphylococcus aureus, ${ }^{60}$ and Psendomonas falciparum. ${ }^{61}$ In all cases, the enzyme was crystallised as a homodimer comprising of three domains (Figure 1.8). The first and second domains are situated in the aqueous environment of the cytoplasm, binding NADH and FAD, respectively. The last domain is a hydrophobic membrane-anchoring domain in which the quinone binding site (Q-site) is found. ${ }^{58,62}$ The distinct binding sites are arranged such that both $\mathrm{NADH}$ and quinone are close in proximity to FAD, suggesting that the NADH oxidation and quinone reduction occurs via the reduction and oxidation of FAD, respectively (Scheme 1.1). ${ }^{53}$
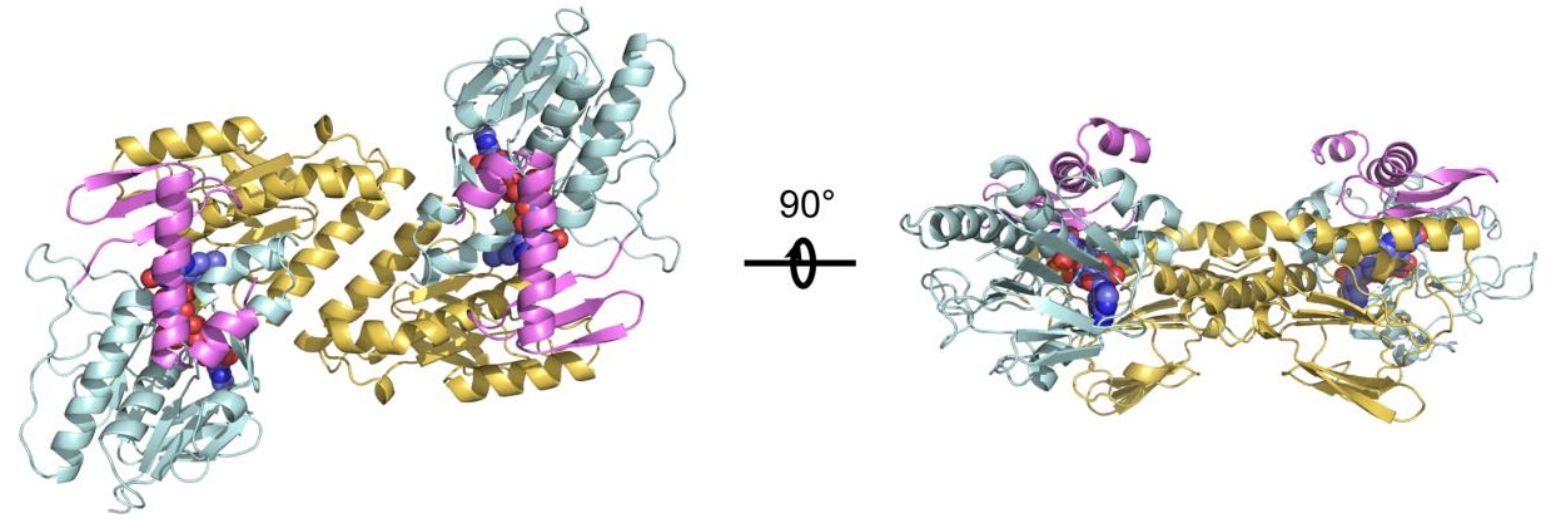

Figure 1.8: The homodimeric structure of bacterial NDH-II is comprised of three domains. The first domain binds NADH (blue), while the second domain binds the flavin co-factor FAD (gold) and the last domain is the membrane-anchoring domain (pink) where the quinone binding site (Q-site) is found. Image taken from Heikal, A., et al. Mol. Microbiol. (C) 2014 John Wiley \& Sons Ltd. with permission. ${ }^{53}$ 


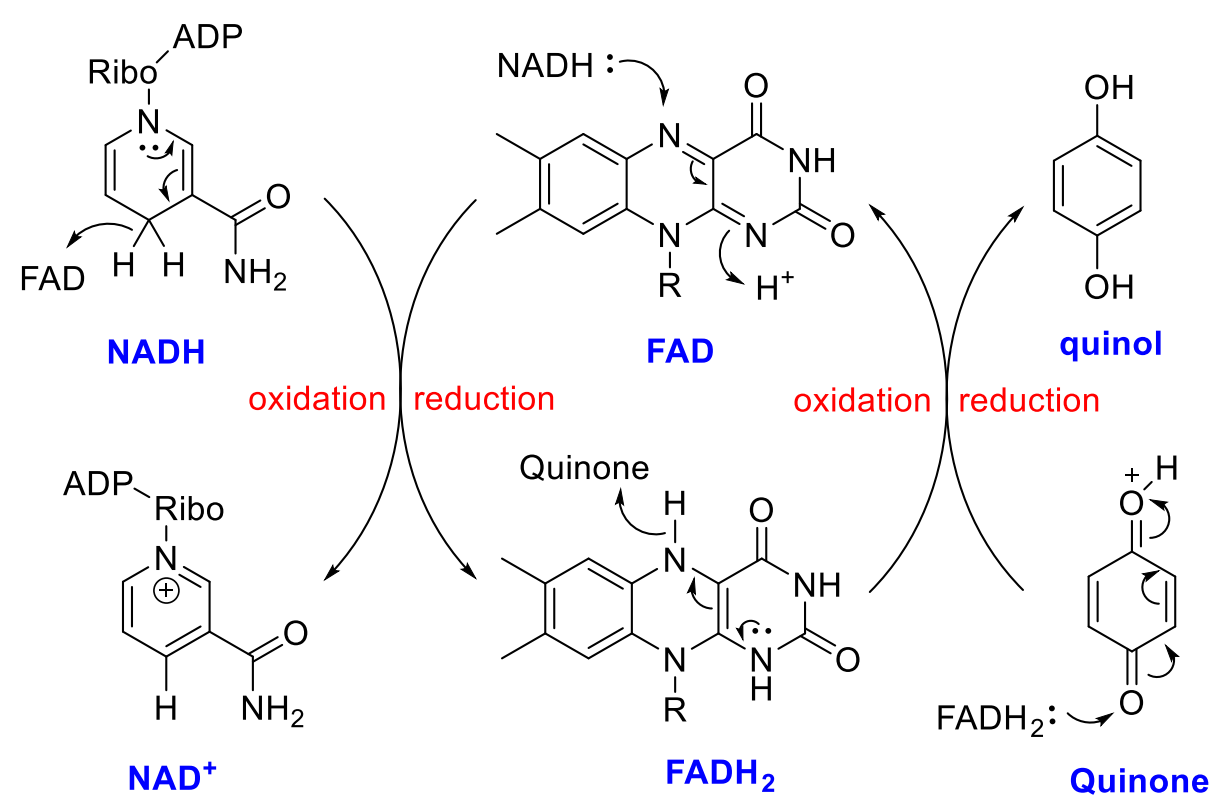

Scheme 1.1: The oxidation and reduction reactions involving NADH, FAD and a quinone species proposed to occur within the NDH-II enzyme. Here, NADH oxidation to $\mathrm{NAD}^{+}$is coupled to the reduction of the FAD co-factor to $\mathrm{FADH}_{2}$. The reoxidation of $\mathrm{FADH}_{2}$ to FAD in turn occurs as the quinone is reduced to the quinol species.

Unfortunately, the co-crystallisation of the enzyme with its natural quinone substrate is challenging and has only been achieved with the yeast equivalent of the enzyme, Ndi1, and its substrate ubiquinone (17) (Figure 1.9). ${ }^{63}$ To gain insight into the Q-site of the bacterial NDHII, Cook and co-workers conducted a docking study based on the crystal structure of the Ndi1ubiquinone complex using a high resolution $C$. thermarum NDH-II crystal structure. ${ }^{58}$ For this experiment, both physiologically irrelevant and relevant quinones, ubiquinone (17) and menadione (18), respectively, were chosen to be modelled at the Q-site. In addition, the cofactor FAD was included in the docking study. ${ }^{58}$<smiles>COC1=C(OC)C(=O)C(CC=CC(C)=O)=C(C)C1=O</smiles>

17<smiles>CC1=CC(=O)c2ccccc2C1=O</smiles>

18

Figure 1.9: Structures of ubiquinone (17) and menadione (18). 
The studies showed that both quinones 17 and 18 fit well into the Q-site, with the hydrophobic moieties of amino acids glutamine (Q317) and isoleucine (I379) accommodating the planar quinone ring (Figure 1.10). ${ }^{58,62}$ There is also a hydrogen bond between one of the carbonyl oxygens of quinones $\mathbf{1 7}$ and $\mathbf{1 8}$ and an amine proton on the FAD isoalloxazine ring. In the case of menadione (18), another hydrogen bond is observed between the second carbonyl oxygen of quinone $\mathbf{1 8}$ with a proton of an arginine group (R382) (Figure 1.10B), ${ }^{58}$ though it is unclear whether this interaction is physiologically plausible as this residue is suspected to be well within the membrane. ${ }^{53}$ In fact, it was speculated that this carbonyl would be interacting with a phosphate group in the phospholipid bilayer. ${ }^{58}$ Finally, a distant edge-to-face $\pi-\pi$ interaction was observed between menadione (18) with the FAD isoalloxazine ring. This interaction could contribute to the higher affinity of menadione (18) to NDH-II compared to ubiquinone (17) and, in turn, could explain why menadione (18) is the only quinone substrate found in C. thermarum. ${ }^{58}$ 

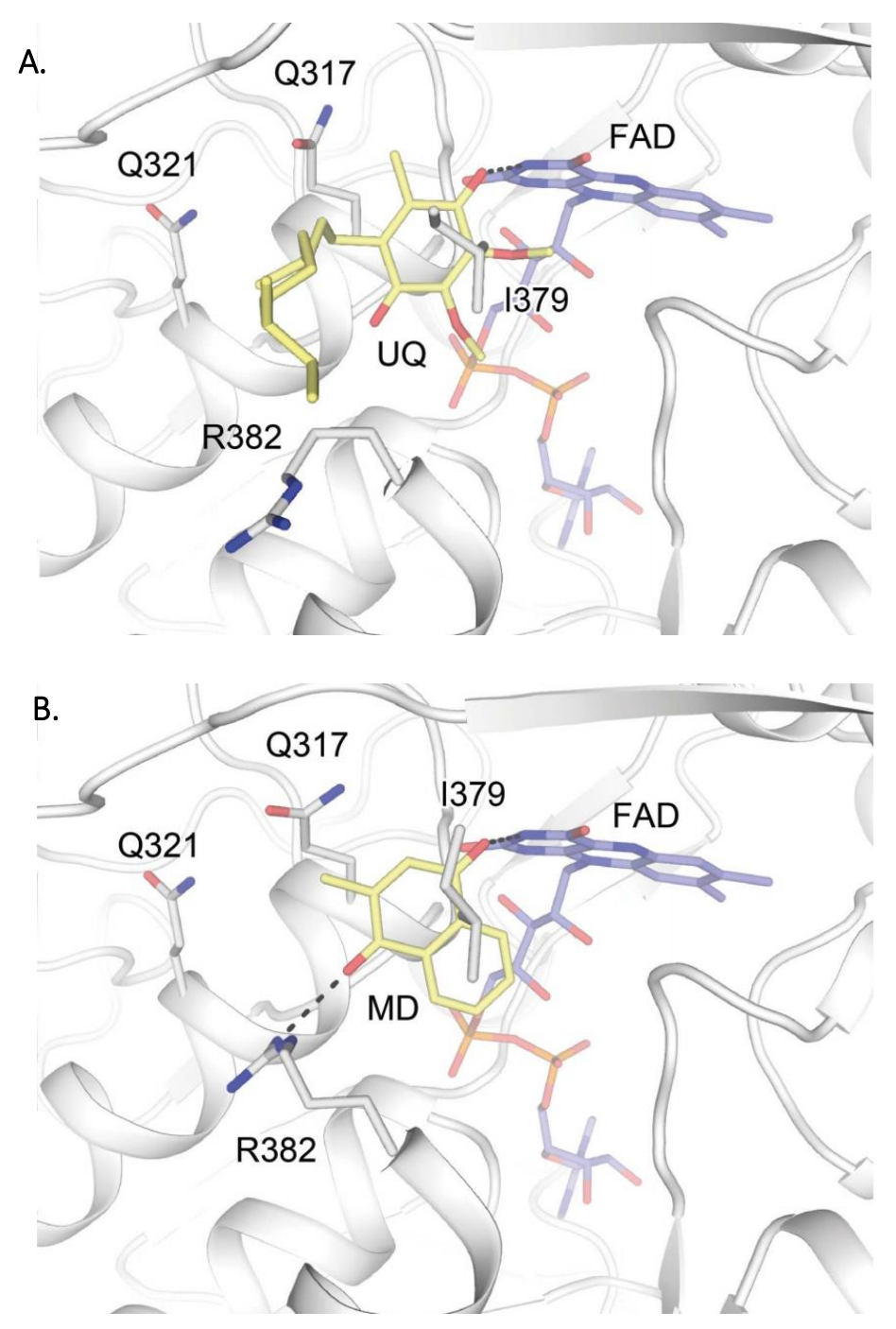

Figure 1.10: The docking of A). ubiquinone (17) and B). menadione (18) in the Q-site of NDH-II, showing the hydrophobic interactions between the quinone head group with Q317 and I379, as well as favourable interactions with the FAD isoalloxazine ring. ${ }^{58}$

Following the investigations into the predicted Q-site, the co-crystallisation of $C$. thermarum NDH-II with a known inhibitor 2-heptyl-4-hydroxyquinoline- $N$-oxide (HQNO) (19) was undertaken (Figure 1.11). ${ }^{62}$ Here, similar interactions were observed between NDH-II and HQNO (19) in the crystal structure as was also noted in the docking studies involving NDHII and menadione (18). ${ }^{58}$ Specifically, the pyridone group in HQNO (19) was sandwiched between hydrophobic side chains of Q317 and I379, while the ketone oxygen participates in hydrogen bonding with the FAD isoalloxazine ring. These interactions were previously observed in docking studies with menadione (18) in the NDH-II Q-site. ${ }^{58}$ Also, a lack of NDHII inhibition resulted when HQNO (19) was added to an I379-mutant, verifying the importance of this amino acid for binding. ${ }^{62}$ Further stabilisation of the compound was evident in the weak 
hydrophobic interactions to accommodate the alkyl chain in HQNO (19). Comparisons between this crystal structure and that of the yeast Ndi1-ubiquinone complex showed the same interactions with the quinone group, suggesting that these are conserved binding mechanisms between NDH-II and their substrates. ${ }^{62}$ Moreover, it also indicates that HQNO (19) inhibits NDH-II by a competitive inhibition mechanism.

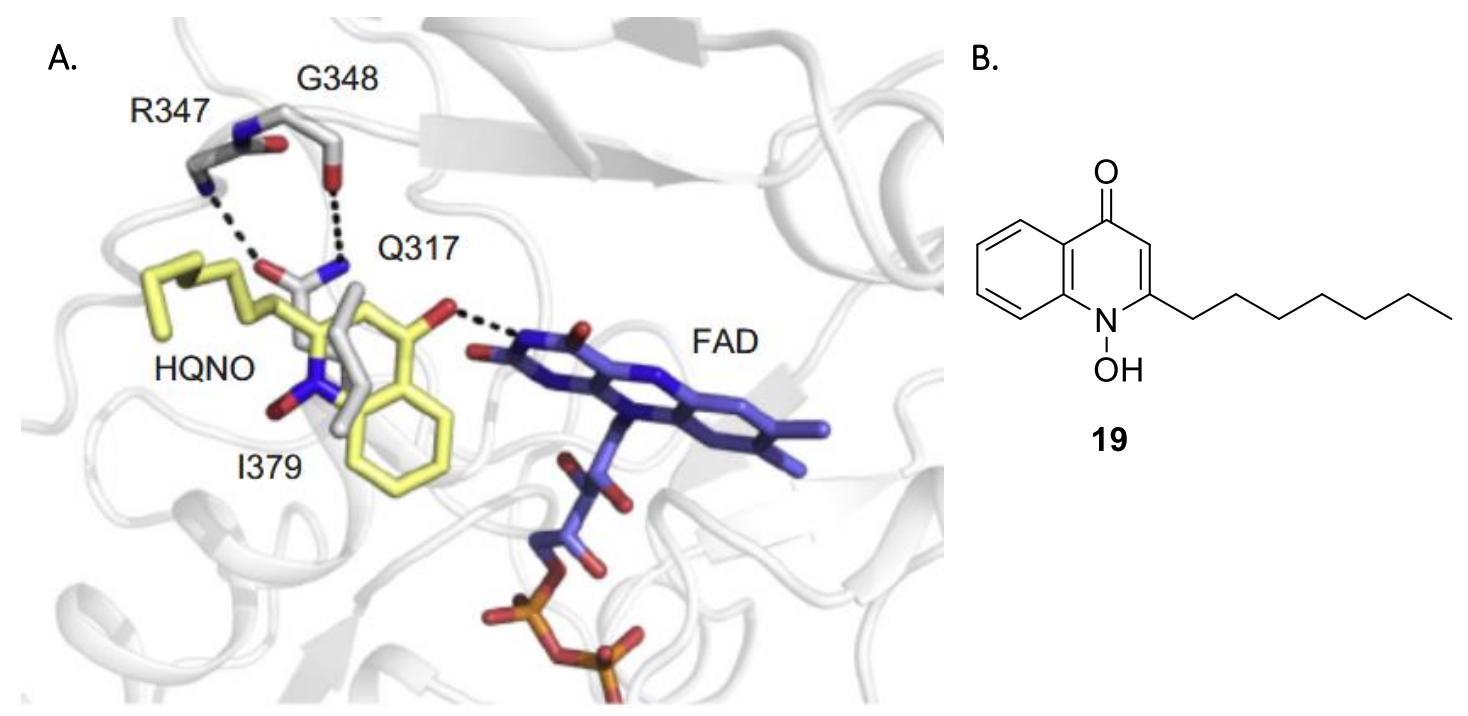

Figure 1.11: A). The crystal structure of NDH-II-HQNO complex showing interactions between the ketone head group with Q317, I379 and the FAD isoalloxazine ring. $\left.{ }^{62} \mathrm{~B}\right)$. The structure of HQNO (19).

\subsection{NDH-II substrates have antimycobacterial activity}

In the search for new TB drugs, NDH-II has become a prominent target for drug discovery and development. To this end, several classes of compounds have been reported to bind NDH-II and through enzyme activation ${ }^{64}$ or inhibition, ${ }^{56}$ and cause TB cell death. These classes of compounds will be discussed in the following overview.

\subsubsection{Quinolinequinones (QQs) activate NDH-II}

Quinones are nature's electron acceptors and, consequently, are vital for the biological redox processes that occur in the ETC. When quinolinequinones (QQs), a class of heterocyclic quinones, were reported to elicit anti-tubercular activity by Stocker and co-workers, ${ }^{65}$ it was hypothesised that NDH-II could be the target enzyme of these compounds. Accordingly, 
studies into the mode of action by which a representative QQ, 7-chloro-6-(2chloroethylamino)-5,8-QQ (QQ8c, 20, Figure 1.12), inhibited Mtb growth was undertaken. ${ }^{64}$<smiles>O=C1C(Cl)=C(NCCCl)C(=O)c2ncccc21</smiles>

Figure 1.12: The structure of QQ8c (20).

First, the minimum inhibition concentration (MIC) and minimum bactericidal concentration (MBC) against $M t b$ were determined for QQ8c (20) and found to both be $8.1 \mu \mathrm{M}$ (isoniazid = $0.72 \mu \mathrm{M}$ in the same assay). ${ }^{64}$ Here, the MIC is defined as the minimum concentration of the drug required to completely inhibit the growth of the bacteria, whereas the $\mathrm{MBC}$ is the minimum concentration required to kill the bacteria. When compared to the first-line TB drug INH (2), QQ8c (20) reduced Mtb cell viability to the same extent. Moreover, drug resistance, as is observed with INH (2), was not detected upon treatment of Mtb with QQ8c (20), which suggests a novel mode of action. ${ }^{64}$ Next, QQ8c (20) was shown to increase the rate of NADH oxidation both in aerobic and anaerobic environments. As NADH oxidation is coupled to quinone reduction, the concentration of the reduced quinol species was also measured. Here, the presence of the quinol was only observed in the absence of $\mathrm{O}_{2}$ and not under aerobic conditions. This hints to a redox cycling mechanism, whereby the quinol is re-oxidised by $\mathrm{O}_{2}$ to produce ROS in aerobic environments. Indeed, QQ8c (20)-induced $\mathrm{O}_{2}$ consumption was significantly reduced in the presence of ROS-scavenging enzymes. Moreover, in the presence of QQ8c (20), the rate of NADH oxidation was significantly increased in inverted membrane vesicles (IMVs) that overexpress NDH-II compared to wild type IMVs. Taken together, these results showed that QQ8c (20) specifically stimulates $M t b$ NDH-II to produce cidal levels of ROS. $^{64}$

\subsubsection{Phenothiazines are NDH-II inhibitors}


Phenothiazines are known to exhibit anti-mycobacterial activity, ${ }^{66}$ with some reported to be active against resistant strains of $M t b .{ }^{67-69}$ Although this anti-tubercular activity has been attributed to different drug targets within the bacteria, ${ }^{70,71}$ there is strong evidence supporting the hypothesis that phenothiazines specifically inhibit NDH-II. ${ }^{35}$ Trifluoperazine (21, Figure 1.13), with a reported MIC value of $10 \mu \mathrm{M}$ against $M t b,{ }^{68}$ exhibits a synergistic antitubercular acitivity with RIF (1) and INH (2). ${ }^{69}$ Mode of action studies have shown that trifluoperazine (21) inhibits $\mathrm{O}_{2}$ consumption in $\mathrm{M} t b$. In such cases, $\mathrm{O}_{2}$ consumption can be resumed by the addition of electron donors specific to cytochrome $c{ }^{35}$ which is found in the cytochrome $b c$ complex, indicating that inhibition occurs downstream from this complex in the ETC (Figure 1.6).$^{35}$ Indeed, the inhibition of purified $M t b$ NDH-II by trifluoperazine (21) was demonstrated in later experiments. ${ }^{38}$

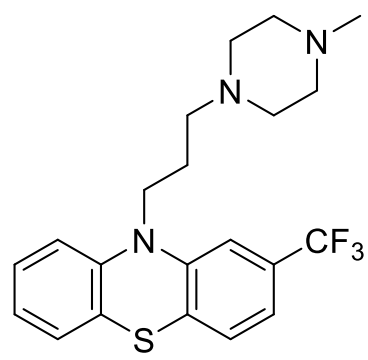

21

Trifluoperazine<smiles>CSc1ccc2c(c1)N(CC[C@@H]1CCCCN1C)c1cc(C(F)(F)F)ccc1S2</smiles>

22

Thioridazine

Figure 1.13: Structures of phenothiazines 21 and 22.

Also exhibiting synergistic effects with first-line TB drugs is thioridazine (22), ${ }^{72,73}$ an anti-psychotic drug that elicits anti-tubercular activity in vitro ${ }^{74,75}$ as well as in murine models of MDR-TB infections. ${ }^{73}$ Although the exact mechanism of this compound is unknown, thioridazine (22) causes a reduction in ATP synthesis through the dissipation of the mycobacterial $\Delta \varphi \cdot{ }^{56}$ Furthermore, phenothiazines were found to have a strong correlation between the inhibition of NDH-II and their antitubercular activities, suggesting NDH-II to be the target of these compounds. ${ }^{38,56}$ That said, other modes of action have also been described, whereby phenothiazines inhibit the bacterial efflux system and allow the lysosome to decrease its $\mathrm{pH}$ to a level lethal to $M t b \cdot{ }^{67,76,77}$ Unfortunately, phenothiazines are highly toxic ${ }^{78}$ and can induce mitochondrial toxicities in the human brain. ${ }^{79}$ 


\subsubsection{Thioquinazolinones disrupt NDH-II}

In 2013, thioquinazolinone 23 (Figure 1.14) was identified as an NDH-II inhibitor in a wholecell high throughput screen (HTS) against resistant $M t b$ strains ${ }^{80}$ Since then, two separate medicinal chemistry studies have been undertaken to obtain thioquinazolinones with low micromolar MIC values against $M t b .^{81,82}$<smiles>O=C(CSc1nc2ccccc2c(=O)[nH]1)NC1CCCCC1</smiles>
23

MIC: $0.3 \mu \mathrm{M}$<smiles>[Z4][X]c1nc2cccc(F)c2c(=O)[nH]1</smiles>

MIC: $0.09 \mu \mathrm{M}$

Figure 1.14: The structure of thioquinazolinone 23 and the optimised compound $24 .^{81}$

In the thioquinazolinones SAR studies, the replacement of the thioether group with other heteroatoms, such as oxygen and nitrogen, as well as a methylene group led to a loss of inhibitory activity. ${ }^{82}$ The substitution of the phenyl ring with saturated, smaller or heterocyclic rings also reduced antitubercular activity, giving the fluoro-phenyl analogue $\mathbf{2 3}$ as the most potent derivative. Similarly, the instalment of a gem-difluoro group on the cyclohexyl ring also increased the anti-tubercular properties of the drug. ${ }^{81}$ The efficacy of thioquinazolinones 23 and 24 against $M t b$ was attributed to NDH-II disruption as treatment of the bacteria with these compounds gave rise to mutations in the $N d h A$ gene, the non-essential gene for NDH-II. ${ }^{80}$ Although the mutations in the essential NDH-II gene, $N d h$, were not observed, quinazoline 24 was shown to elicit similar inhibitory activities against both $N d h$ and $N d h A .{ }^{81}$ This suggests that both isoforms of NDH-II were affected by thioquinazoline 24 and the lack of $\mathrm{Ndh}$ mutation may be due to deleterious effects that a single mutation could exert on the enzyme function. ${ }^{82}$ Mechanistic studies showed that quinazolinone $\mathbf{2 4}$ had very little effect on the rate of $\mathrm{O}_{2}$ consumption in $M t b$, indicating a different mode of inhibition to QQ8 (20). Kinetic studies also showed a noncompetitive mode of inhibition against $\mathrm{NADH}$ and a representative quinone, suggesting an allosteric inhibition mechanism. ${ }^{82}$ 


\subsubsection{Other anti-tubercular agents that bind NDH-II}

An important class of NDH-II inhibitors is the quinolinyl pyrimidines, of which the lead compound (25, Figure 1.15) exhibited a micromolar MIC value. ${ }^{79}$ These inhibitors were identified via a HTS against mycobacterial NDH-II. Through SAR studies, some information has been obtained regarding the binding pocket for this class of compounds, though the compounds' mode of action is yet to be determined. Here, primary amines on the quinoline and pyrimidine moieties of the quinolinyl pyrimidines were important for potential hydrogen bonding interactions within the NDH-II binding site. In addition, removing the aryl substituent on the quinoline ring also led to loss of antitubercular activity, while installing aromatic groups, rather than alkyl chains, on the pyrimidine ring increased the potency of the drug. ${ }^{79}$ Unfortunately, the mechanism by which quinolinyl pyrimidines inhibit Mtb growth has not been fully explored.<smiles>Nc1nc(Nc2ccc3nc(-c4ccc(F)cc4)cc(N)c3c2)cc(-c2ccc(Cl)cc2)n1</smiles>

Figure 1.15: The structure of the lead quinolinyl pyrimidine 25 against $M t b$.

The final class of NDH-II inhibitors are the quinolones. Quinolones are known anti-bacterial compounds and, in particular, fluoroquinolones are the current second-line TB drugs. ${ }^{83}$ Although quinolones are generally known to exert their anti-microbial activity by inhibiting DNA gyrase ${ }^{84}$ several compounds of this class, such as HQNO (19) ${ }^{62}$ have the potential to bind bacterial NDH-II. ${ }^{85,86}$ Recently, an anti-tubercular quinolone (26, Figure 1.16) with nanomolar $\mathrm{IC}_{50}$ values (concentration of drug required to inhibit bacterial growth by half) was discovered from a HTS against Mtb NDH-II and subsequent lead compound optimisation. ${ }^{87}$ SAR studies of this class of compounds showed that the ketone moiety was essential for antitubercular activity, as was the $\mathrm{C}$ ring. The instalment of fluoro-groups at C5 and C7 was important for inhibitory activity, though substitutions at other positions of the phenyl group 
led to a loss of growth inhibition. At the 3-position, different groups including $\mathrm{CH}_{3}, \mathrm{Br}$, and $\mathrm{Cl}$ were tolerated and the lack of subsitution at this position led to a loss in anti-tubercular activity. ${ }^{87}$ Despite their high potency against many bacterial species, quinolones have long been used as broad-spectrum antibiotics and, as a result, are becoming increasingly tolerated by many species of bacteria. ${ }^{84}$

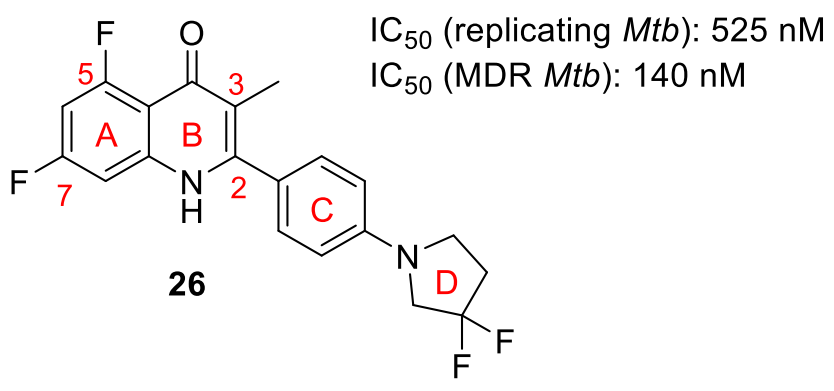

Figure 1.16: Lead anti-tubercular drug, quinolone 26.

\subsection{Thesis outline}

With insights into the structure of NDH-II and TB drugs that are reported to bind this enzyme, the overall objective of this thesis was to develop new heterocyclic compounds as TB drug candidates. To this end, the potential anti-tubercular activity of the natural products ascidiathiazone A (27) and ascidiathiazone B (28, Chapter 2), QQs (29, Chapter 3), chromonylpyrimidines (30, Chapter 4), and phthalazinones $(\mathbf{3 1}, \mathbf{3 2}$, Chapter 5) were investigated (Figure 1.17). Here, I conducted the biological screening of ascidiathiazones A (27) and B (28), and derivatives thereof, chromonyl-pyrimidines (30), and phthalazinones $(\mathbf{3 1}, \mathbf{3 2})$ under the supervision of Professor Gregory Cook, while the QQs (29) and several chromonylpyrimidines (30) were sent to the Cook laboratory at the University of Otago for testing. 
Chapter 2<smiles>O=C(O)c1ccc2c(n1)C(=O)C1=C(C2=O)S(=O)(=O)CCN1</smiles>

27

\section{Chapter 4}<smiles>Nc1nccc(Nc2ccc3occc(=O)c3c2)n1</smiles><smiles>O=C(O)c1ccc2c(n1)C(=O)C1=C(NC=CS1(=O)=O)C2=O</smiles>

28
Chapter 3<smiles>O=C1C=CC(=O)c2ncccc21</smiles>

29

Chapter 5<smiles>O=c1[nH]ncc2ccccc12</smiles><smiles>Nc1nccc(Nc2ccc3cn[nH]c(=O)c3c2)n1</smiles>

Figure 1.17: Synthetic target molecules 27 and 28 as well as general structures 29-32 to be explored for anti-tubercular activity. 


\section{CHAPTER TWO}

\section{Total synthesis and anti-tubercular}

\section{activity of Ascidiathiazones A and B}

\subsection{Introduction}

Ascidians, a class of sea squirts or tunicates ${ }^{88}$ are marine invertebrates that produce a vast array of bioactive secondary metabolites. ${ }^{89,90}$ Within ascidians, the polyclinidiae family is one of the most diverse families, of which the Aplidium genus is comprised of 320 distinct species. ${ }^{89}$ Metabolites from ascidians of this genus are of interest due to their high toxicity and favourable pharmacological properties. ${ }^{89-91}$ For example, aplidine (33, Figure 2.1), which is extracted from Aplidium albicans, is currently undergoing clinical trials for its anti-cancer potential, ${ }^{92,93}$ while the meridinianins (34), a group of brominated indoles isolated from Aplidium meridianum, act as protein kinase ${ }^{94}$ and tyrosine kinase inhibitors. ${ }^{95}$ The caelestines (35), a class of brominated 
quinolines that were isolated from the Australian ascidian Aplidium caelestis, exhibit modest antimicrobial activity against various bacterial strains including Staphylococcus aureus, Escherischia coli, and Pseudomonas aeruginosa. ${ }^{96}$

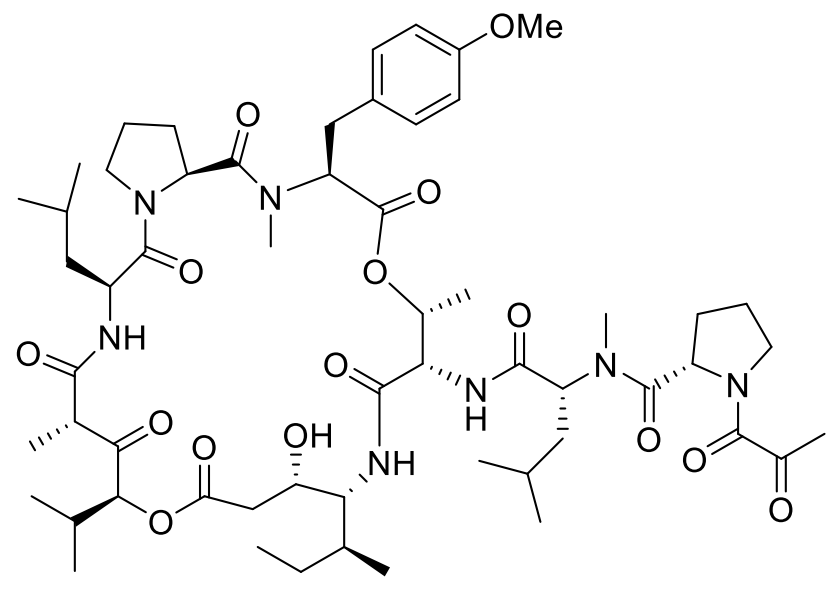

33<smiles>[R]c1c([R])c([R])c2c(-c3ccnc(N)n3)c[nH]c2c1[R]</smiles>

34, $\mathrm{R}=\mathrm{H}, \mathrm{OH}$, or $\mathrm{Br}$<smiles>[R]c1cc2c(=O)cc(C(=O)O)[nH]c2c([R])c1[R]</smiles>

35, $\mathrm{R}=\mathrm{H}, \mathrm{OMe}$, or $\mathrm{Br}$

Figure 2.1: Natural products aplidine (33), meridinianins (34) and caelestines (35)..$^{92,94,96}$

Ascidians of the Aplidium genus are a particularly rich source of meroterpenes that include prenylated quinones and hydroquinones. ${ }^{89,97}$ Meroterpenes exhibit significant biological activities that are often attributed to their ability to undergo redox reactions and produce ROS. Examples of bioactive prenylated hydroquinones from Aplidium tunicates include geranylhydroquinone (36) and rossinone A (37, Figure 2.2). ${ }^{97,98}$ Geranylhydroquinone (36) exhibited anti-proliferative activity against Rous sarcoma and mammary carcinoma, ${ }^{98-100}$ while rossinone A (37) was reported to suppress superoxide production in human peripheral blood neutrophils during respiratory burst activity and thus, shows promise as an anti-inflammatory agent. $^{97}$ 
<smiles>CC(C)=CCC/C(C)=C/Cc1cc(O)ccc1O</smiles>

36<smiles>CC(C)=C[C@@H](O)C(=O)/C(C)=C/CC/C(C)=C/Cc1cc(O)ccc1O</smiles>

37

Figure 2.2: Examples of prenylated hydroquinones: geranylhydroquinone (36) and rossinone A (37) ${ }^{97-99}$

Quinone meroterpenes have also been extracted from Aplidium ascidians, such as the Mediterranean Aplidium conicum, from which structurally unique quinones bearing a 1,1-dioxo1,4-thiazine ring were discovered. ${ }^{101}$ Four distinct families of dioxo-thiazinoquinones have been identified from this particular ascidian species: the conicaquinones $\left(\mathbf{3 8}, \mathbf{3 9}\right.$, Figure 2.3), ${ }^{102}$ the aplidinones (40-42), ${ }^{91}$ the thiaplidiaquinones $(\mathbf{4 3}, \mathbf{4 4}),{ }^{90}$ and more recently, the conithiaquinones $(\mathbf{4 5}, \mathbf{4 6}) .{ }^{103}$ These dioxo-thiaziquinones were found to exhibit anti-cancer properties, with conicaquinones A (38) and B (39) inhibiting the growth of rat glioma cells, ${ }^{102}$ aplidinone A (40) showing modest growth inhibition of a human leukaemia T cell line, ${ }^{101}$ thiaplidiaquinones A (43) and B (44) causing apoptosis of Jurkat cells, ${ }^{90}$ and the conithiaquinones A (45) and B (46) showing cytotoxicity against breast cancer cells. ${ }^{103}$ Despite the potential of this family of compounds as new pharmaceuticals, their modes of action are largely unexplored. Thiaplidiaquinones $\mathbf{4 3}$ and $\mathbf{4 4}$ however, have been shown to exhibit anticancer activity by the induction of ROS overproduction..$^{90}$ 
<smiles>CC(C)=CCCc1ccc2c(c1)C(=O)C1=C(NCCS1(=O)=O)C2=O</smiles>

38<smiles>[R]C1=C(C/C=C(\C)CCC=C(C)C)C(=O)C2=C(NCCS2(=O)=O)C1=O</smiles>

40, $\mathrm{R}=\mathrm{OCH}_{3}$

41, $\mathrm{R}=\mathrm{NH}_{2}$

42, $\mathrm{R}=\mathrm{NHCH}_{2} \mathrm{CH}_{2} \mathrm{SO}_{3}$<smiles>CC(C)=CCC/C(C)=C/Cc1cc2c(cc1O)C1=C(C(=O)C3=C(NCCS3(=O)=O)C1=O)C(/C=C(\C)CCC=C(C)C)O2</smiles><smiles>CC(C)=CCCc1ccc2c(c1)C(=O)C1=C(C2=O)S(=O)(=O)CCN1</smiles>

39<smiles>CC(C)=CCC/C(C)=C/Cc1cc(O)cc2c1OC(/C=C(\C)CCC=C(C)C)C1=C2C(=O)C2=C(NCCS2(=O)=O)C1=O</smiles><smiles>[R20][C@H]1C2=C(C(=O)C3=C(NCCS3(=O)=O)C2(C)C)[C@@H]2CC[C@](C)(OC)[C@@H]12</smiles>

45, $\mathrm{R}=\mathrm{H}$

46, $\mathrm{R}=\mathrm{CH}_{3}$

Figure 2.3: Conicaquinones A (38) and B (39), ${ }^{102}$ aplidinones A (40), B (41), and C (42), ${ }^{91}$ thiaplidiaquinones A (43), and B (44), ${ }^{91}$ and conithiaquinones $A(45)$ and B (46). ${ }^{103}$

\subsubsection{Ascidian natural products ascidiathiazones A and B}

The relatively unusual scaffold of a 1,1-dioxo-1,4-thiazine ring fused to a quinone ring has also been reported in ascidiathiazone A (27, Figure 2.4) and its structurally related analogue ascidiathiazone B (28). ${ }^{104}$ These ascidiathiazones were extracted from a New Zealand ascidian Aplidium sp. and have been reported to exhibit anti-inflammatory activity by inhibiting the production of superoxides by human neutrophils. ${ }^{104,105}$ 
<smiles>O=C1CCNC2=C1C(=O)c1ccc(C(=O)O)nc1C2=O</smiles>

27<smiles>O=C(O)c1ccc2c(n1)C(=O)C1=C(NC=CS1(=O)=O)C2=O</smiles>

28

Figure 2.4: Ascidiathiazone A (27) and ascidiathiazone B (28). ${ }^{104}$

In addition to the isolation and structural elucidation of natural products 27 and 28 , the total synthesis of ascidiathiazone A (27) was also achieved (Scheme 2.1). ${ }^{104}$ Here, quinolinic acid (47) was esterified to give methyl ester 48, which was then oxidised to the corresponding quinone (49) with Fremy's salt (potassium nitrosodisulfonate). Next, the thiazine ring was installed by treating QQ 49 with hypotaurine in the presence of cerium chloride to give tricyclic quinone 50, along with its regioisomer $\mathbf{5 1}$ as a minor product. Finally, thiazine $\mathbf{5 0}$ was hydrolysed to give ascidiathiazone A (27). Following the synthesis of ascidiathiazone A (27), a library of structural analogues were prepared, ${ }^{105}$ though the synthesis of ascidiathiazone B (28) has not been reported. 
<smiles>O=C(O)c1ccc2cccc(O)c2n1</smiles>

47

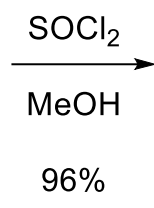

$96 \%$<smiles>COC(=O)c1ccc2cccc(O)c2n1</smiles>

48<smiles>COC(=O)c1ccc2c(n1)C(=O)C=CC2=O</smiles>

$90 \%$
49<smiles>O=C(O)c1ccc2c(n1)C(=O)C1=C(C2=O)S(=O)(=O)CCN1</smiles>

27<smiles>COC(=O)c1ccc2c(n1)C(=O)C1=C(C2=O)S(=O)(=O)CCN1</smiles>

50 (55\%)

$+$<smiles>COC(=O)c1ccc2c(n1)C(=O)C1=C(NCCS1(=O)=O)C2=O</smiles>

$51(7 \%)$

Scheme 2.1: Total synthesis of ascidiathiazone A (27) by Copp and co-workers. ${ }^{104}$

To explore the SAR between ascidiathiazones $\mathbf{2 7}$ and $\mathbf{2 8}$ and their associated anti-inflammatory activity, the natural products and their synthetic analogues were screened for their ability to suppress superoxide production. ${ }^{105}$ Here, it was noted that both the carboxylic acid moiety and the pyridinyl ring were required to retain the biological activity of the compounds. The absence of the thiazine ring however, resulted in good anti-inflammatory activity, though the inclusion of methylsulfone and methylamine substituents in place of the thiazine group led to a loss of anti-inflammatory activity. Furthermore, compounds with the $[2,3-g]$ regiochemistry (i.e. 27) exhibited greater anti-inflammatory activity compared to the corresponding [3,2-g] regioisomers (i.e. 28). ${ }^{105}$

\subsubsection{Project aim and retrosynthesis}

Natural products bearing the tricyclic thiazino-quinone scaffold exhibit a number of pharmacological properties, ${ }^{90,101,102}$ however their anti-tubercular activity has not been explored. Given the tuberculostatic activity of QQ ${ }^{65}$ as well as their ability to bind and activate 
mycobacterial NDH-II (Chapter 1, Section 1.4.1.), ${ }^{65}$ it was proposed that the dioxo-thiazinebearing QQs ascidiathiazone A (27) and ascidiathiazone B (28) may also show promise as TB drugs. Accordingly, methodology to allow for the efficient synthesis of ascidiathiazones A (27) and B (28), and derivatives thereof, was sought, with investigations into the anti-tubercular activity of these compounds subsequently being undertaken.

To this end, a retrosynthetic strategy was proposed whereby target ascidiathiazones $\mathbf{2 7}$ and $\mathbf{2 8}$ could be prepared through the treatment of primary chlorides $\mathbf{5 2 - 5 5}$ with sodium sulfide to give the desired thiazine ring (Scheme 2.2) ${ }^{65}$ Having obtained the tricyclic scaffold, oxidation of the sulfide moiety was then envisioned to give the corresponding sulfones (27 and 28 ). Here, direct oxidation of the $[2,3-g]$ sulfide will provide ascidiathiazone A (27), while an additional base-catalysed air oxidation of the $[3,2-g]$ sulfone derivative will give ascidiathiazone B (28). The primary chlorides 52-55 could, in turn, be synthesised from common intermediates dichloroquinone $\mathbf{5 6}$ or $\mathbf{5 7}$, which are oxidation products of the commercially available 8hydroxyquinaldine (58). Oxidation of methylpyridines to their corresponding carboxylic acids can be performed under the agency of $\mathrm{SeO}_{2}{ }^{106-108}$ and other oxidants such as $\mathrm{KMnO}_{4}{ }^{109}$ and $\mathrm{NaIO}_{4}{ }^{110}$ and, as such, it was proposed that this transformation could be conducted at any stage of the synthesis. Accordingly, both methyl-substituted (52, 54, and 56) and carboxylic acidsubstituted $(\mathbf{5 3}, \mathbf{5 5}$, and $\mathbf{5 7})$ quinones were included in the retrosynthesis. 
<smiles>CCS(=O)(=O)C1=C(NCC=C(C)C)C(=O)c2nc(C(=O)O)ccc2C1=O</smiles>

27<smiles>C=C/C=C\C=C1C=CC(=O)C2=C(N1)C(=O)c1ccc(C(=O)O)nc1C2=O</smiles>

28<smiles>[R]c1ccc2c(n1)C(=O)C(NCCCl)=C(Cl)C2=O</smiles>

52, $\mathrm{R}=\mathrm{Me}$ 53, $\mathrm{R}=\mathrm{CO}_{2} \mathrm{H}$<smiles>[R]c1ccc2c(n1)C(=O)C(Cl)=C(Cl)C2=O</smiles>

56, $\mathrm{R}=\mathrm{Me}$ 57, $\mathrm{R}=\mathrm{CO}_{2} \mathrm{H}$<smiles>C=CC=C</smiles><smiles>Cc1ccc2cccc(O)c2n1</smiles>

58

Scheme 2.2: Retrosynthesis of ascidiathiazones A (27) and ascidiathiazone B (28).

In contrast to the published synthesis of ascidiathiazone A (27) ${ }^{104}$ the described synthetic strategy has the advantage of accessing both the $[2,3-g]$ and $[3,2-g]$ regioisomers of thiazinoQQs from common intermediate dichloroquinones (i.e. 56 or 57). ${ }^{65}$ Moreover, through the early introduction of the sulfide moiety, which could subsequently be oxidised to the sulfoxide or the sulfone, ${ }^{65}$ investigations into the effect of the sulfur oxidation state on the antitubercular activity of ascidithiazone analogues could be explored. As such, studies on the biological activity of QQs have indicated that the oxidation state of the thiol moiety may influence the anti-tubercular and anti-proliferative activity of the QQs ${ }^{65}$ thus suggesting that SARs concerning the sulfur oxidation state in the ascidiathiazone scaffold is an important avenue to investigate.

\subsection{Results and Discussion}

\subsubsection{The synthesis of thiazinoquinones}

Having established a synthetic plan for the preparation of ascidiathiazone A (27) and ascidiathiazone $\mathrm{B}$ (28), 8-hydroxyquinaldine (58) was treated with $\mathrm{SeO}_{2}$ to give 8-hydroxyquinolinic acid 47 in a modest yield (Scheme 2.3). ${ }^{106}$ Isolation of acid 47 from the reaction 
mixture proceeded by extracting from the crude material through a Soxhlet apparatus using EtOAc, as described in literature. ${ }^{106} \mathrm{~A}$ second oxidation reaction under the agency of sodium chlorate in concentrated $\mathrm{HCl}^{65}$ was then performed in an attempt to convert carboxylic acid 47 to carboxy-dichloroquinone 57. Unfortunately, the reaction yielded a complex mixture of products, as observed by TLC analysis, and there was no evidence for quinone formation by NMR spectroscopy or HRMS analysis. Accordingly, an alternative route was explored whereby quinoline 58 was first oxidised to dichloroquinone $\mathbf{5 6}$ using sodium chlorate under acidic conditions. ${ }^{65}$ Although this reaction was low-yielding, it was readily performed on a multigram scale and required inexpensive reagents. Crystallisation of dichloride $\mathbf{5 6}$ from $\mathrm{MeOH}$ gave yellow crystals that were then subjected to a benzylic oxidation promoted by $\mathrm{SeO}_{2}$. The reaction progress was monitored by TLC analysis, which suggested that dichloroquinone $\mathbf{5 6}$ was fully consumed, but unfortunately, many products were formed during the course of the reaction. Despite formation of the desired dichloroquinone (57), as indicated by HRMS, (calcd. for $\left[\mathrm{C}_{10} \mathrm{H}_{3} \mathrm{Cl}_{2} \mathrm{NO}_{4}+\mathrm{H}\right]^{+}:$271.9512, obsd.: 271.9525; calcd. for $\left[\mathrm{C}_{10} \mathrm{H}_{3} \mathrm{Cl}_{2} \mathrm{NO}_{4}+\mathrm{Na}\right]^{+}$: 293.9331, obsd.: 293.9331; calcd. for $\left[\mathrm{C}_{10} \mathrm{H}_{3} \mathrm{Cl}_{2} \mathrm{NO}_{4}-\mathrm{H}\right]^{-}: 269.9366$, obsd.: 269.9366), attempts to isolate and purify carboxy-dichloroquinone $\mathbf{5 7}$ by filtration over Celite and various column chromatography methods (silica gel, reverse phase with $\mathrm{C}_{18}$, and ion exchange) proved futile, with futher product degradation being observed after each attempted purification.<smiles>Cc1ccc2cccc(O)c2n1</smiles>

58<smiles>O=C(O)c1ccc2cccc(O)c2n1</smiles>

47<smiles>N#CC1CCCCC1C(=O)O</smiles>

57<smiles>Cc1ccc2c(n1)C(=O)C(Cl)=C(Cl)C2=O</smiles><smiles>Cc1ccc(O)cc1</smiles>

Scheme 2.3: Synthetic strategies to form 2-carboxy-dichloroquinone 57. 
Due to the instability of quinone $\mathbf{5 7}$, it was proposed that the carboxylic acid moiety could be introduced during the final stages of the synthesis. Indeed, in literature procedures involving quinolinic acids, the carboxylic acid functionality is often protected as a methyl ester while synthetic manipulations take place, with hydrolysis occurring at the end of the synthesis. ${ }^{104,105,111-113}$ Given the selectivity of $\mathrm{SeO}_{2}$ for the oxidation of allylic and benzylic methyl groups, ${ }^{106,114}$ it was thus envisioned that this oxidation step could occur once the thiazine ring had been installed on the QQ scaffold. Accordingly, dichloroquinone $\mathbf{5 6}$ was subjected to a substitution reaction with 2-chloroethylamine hydrochloride to give regioisomers 59 and $\mathbf{6 0}$ in a 3:2 ratio, respectively (Scheme 2.4). Here, isomers $\mathbf{5 9}$ and $\mathbf{6 0}$ could be differentiated using 1D and 2D NMR spectroscopy. Specifically, carbons involved in tautomerisation are more broad and less visible ${ }^{65}$ and accordingly, peak broadening was observed in the ${ }^{13} \mathrm{C}$ NMR spectra for carbons 5, 6, and 7 of the 7-isomer (59), and carbons 6, 7, and 8 in the 6-isomer (60). Furthermore, an HMBC between $\mathrm{H} 4$ and $\mathrm{C} 5$ allowed for assignment of the regioisomers (Figure 2.5). Here, silica gel flash column chromatography was utilised to separate the two regioisomers, with the 7-isomer $\mathbf{5 9}$ being obtained as orange-red crystals, while the 6 -isomer $\mathbf{6 0}$ was isolated as dark red crystals. Both regioisomers were then treated with sodium sulfide ${ }^{65}$ to form highly reactive primary thiols that underwent subsequent intramolecular cyclisation in situ to give tricyclic quinones $\mathbf{6 1}$ and $\mathbf{6 2}$ as purple crystals.<smiles>Cc1ccc2c(n1)C(=O)C(Cl)=C(Cl)C2=O</smiles>

56<smiles>CCCNC1=C(Cl)C(=O)c2ccc(C)nc2C(=O)C1=O</smiles>

59<smiles>Cc1ccc2c(n1)C(=O)C(Cl)=C(NCCCl)C2=O</smiles>

60<smiles>Cc1ccc2c(n1)C(=O)C1=C(SCCN1)C2=O</smiles>

61

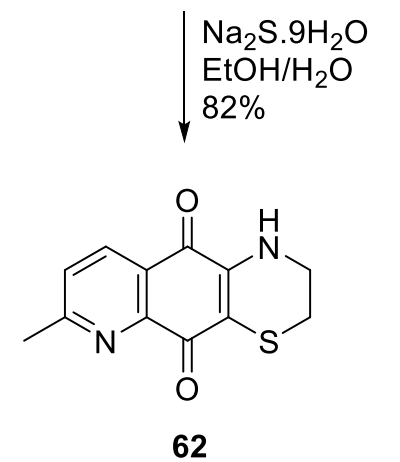

Scheme 2.4: The syntheses of tricyclic QQs 61 and 62 from dichloroquinone 56. 


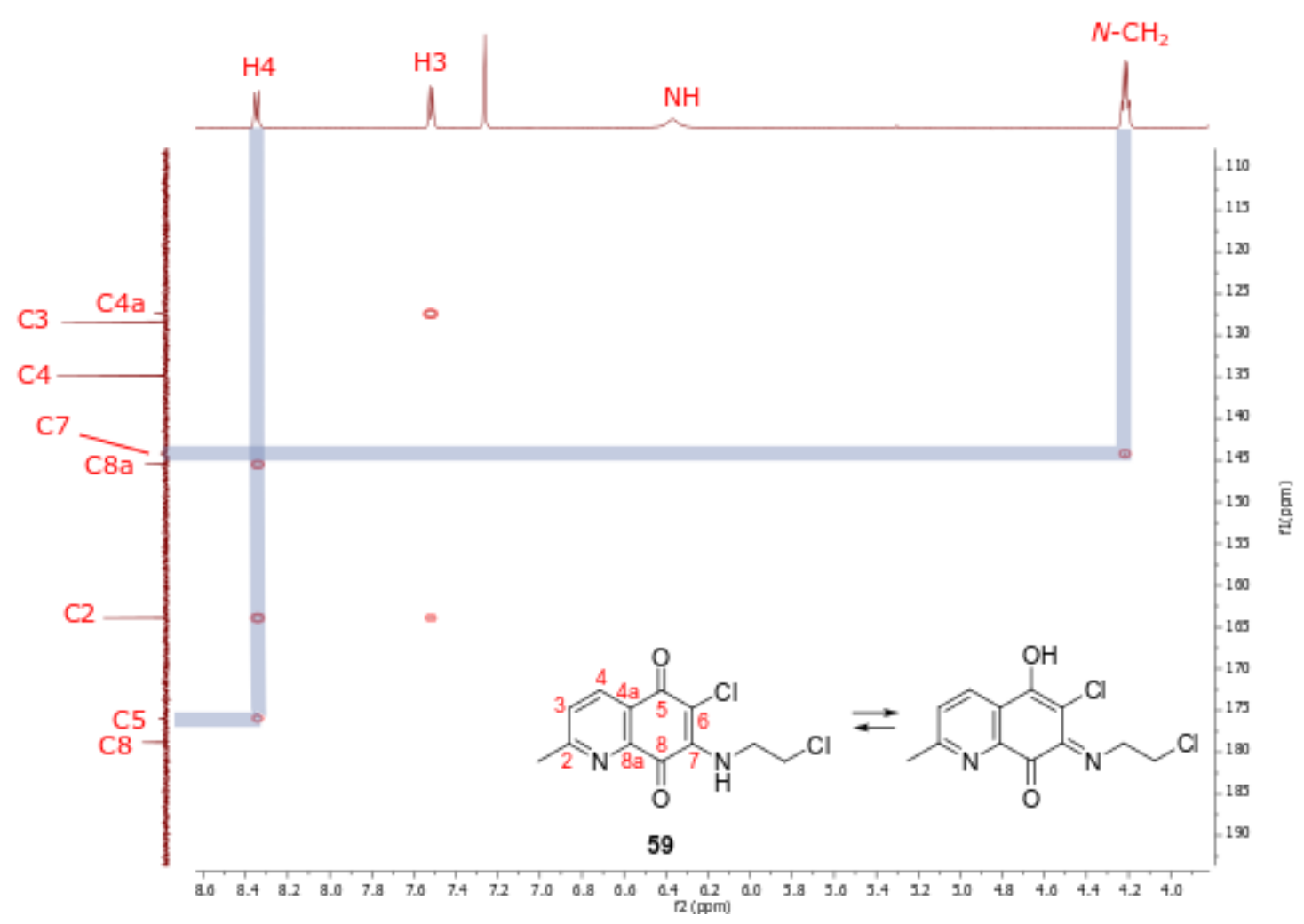

Figure 2.5: $\mathrm{HMBC}\left(500 \mathrm{MHz}, \mathrm{CDCl}_{3}\right)$ spectrum of chloroethylamino-QQ 59.

\subsubsection{The synthesis of ascidiathiazone $A$}

To prepare ascidiathiazone A (27), thiazine 61 was subjected to consecutive oxidation reactions of the sulfide and methyl groups to produce the desired sulfone and carboxylic acid moieties, respectively (Scheme 2.5). The oxidation of a sulfide to a sulfone is well-precedented and can be completed utilising either $m$ - $\mathrm{CPBA}^{65}$ or $\mathrm{H}_{2} \mathrm{O}_{2} \cdot{ }^{115,116}$ For compounds containing thiazine rings, the amine group is often protected, typically with a tert-butyloxycarbonyl (Boc) group, before the oxidation is undertaken, ${ }^{117-119}$ presumably to prevent oxidation of the nitrogen atom to a hydroxylamine. Notwithstanding, in other examples concerning thiazines, the desired sulfone was prepared without the need to first protect the amine moiety. ${ }^{120,121}$ As the oxidation of secondary amines to hydroxylamines is typically low-yielding ${ }^{122}$ and requires harsh conditions, ${ }^{123}$ the oxidation of thiazine $\mathbf{6 1}$ was thus performed without prior protection of the secondary amine. Here, $m$-CPBA was chosen as the oxidising agent as it allowed for the controlled oxidation of the sulfide moiety in a stepwise fashion via the sulfoxide intermediate, with $m$-CPBA being added stoichiometrically while $\mathrm{H}_{2} \mathrm{O}_{2}$ is typically added in excess. In this way, sulfoxide $\mathbf{6 3}$ was readily isolated from the chlorobenzoic acid by-product by an aqueous extraction and further oxidised using an additional equivalent of $m$-CPBA to give sulfone 64 . 
Alternatively, sulfone 64 could be prepared in one step and in higher yield $(92 \%)$ from thiazine 61 by adding 2 equivalents of $m$-CPBA.<smiles>Cc1ccc2c(n1)C(=O)C1=C(SCCN1)C2=O</smiles>

61

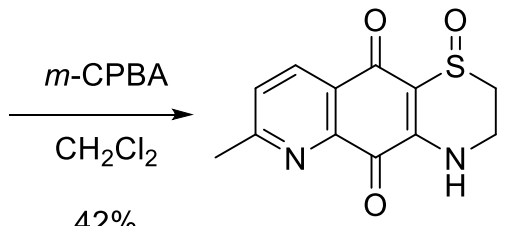

63 $92 \%$

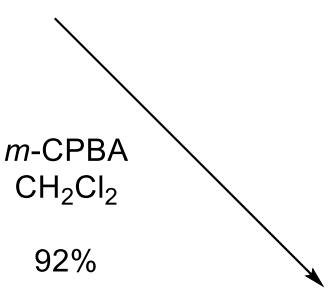<smiles>Cc1ccc2c(n1)C(=O)C1=C(C2=O)S(=O)(=O)CCN1</smiles>

64

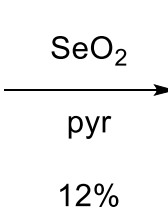

$12 \%$

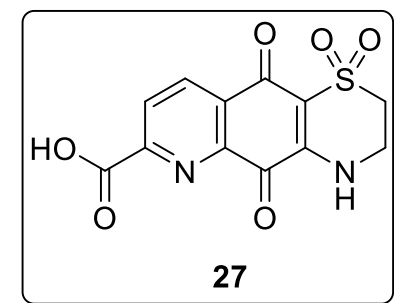

Scheme 2.5: The synthesis of ascidiathiazone A (27) via sulfone 64.

The formation of sulfoxide 63 was corroborated by ${ }^{1} \mathrm{H}$ NMR spectroscopy, whereby the four methylene protons at the 2- and 3-positions were observed at 2.52, 3.31, 4.00, and $4.13 \mathrm{ppm}$ (Figure 2.6A). This is due to the orientation of the thiazine ring in sulfoxide 63 , whereby the $\mathrm{S}=\mathrm{O}$ group is likely to adopt an axial conformation (I, Figure 2.7). The preferred axial position of a sulfoxide oxygen atom in a saturated thiazine ring is unconventional and has been a topic of controversy, however, the formation of a favoured pseudo-axial sulfoxide conformer has been realised in many experiments, ${ }^{121,124}$ with evidence to support this assignment including XRD data from crystal structures. ${ }^{125-129}$ The orientation of the sulfoxide substituent has been attributed to favourable axial SO/H interactions within the ring, ${ }^{121}$ which in turn results in the diastereopicity of the methylene protons at the 2-and 3-positions. Confirmation that sulfoxide 63 was formed was also obtained from HRMS data $\left(\left[\mathrm{C}_{12} \mathrm{H}_{10} \mathrm{~N}_{2} \mathrm{O}_{3} \mathrm{~S}+\mathrm{H}\right]^{+}\right.$calcd.: 293.0227, obsd.: 293.0198), as well as IR data showing a typical $S=O$ stretch at $1059 \mathrm{~cm}^{-1} .{ }^{130}$ In contrast, the ${ }^{1} \mathrm{H}$ NMR spectrum for the corresponding sulfone $\mathbf{6 4}$ revealed only two distinct resonances (3.72, and $4.27 \mathrm{ppm}$ ) for the 2- and 3-position as each pair of geminal protons occupies very similar proton environments (Figure 2.6B). 

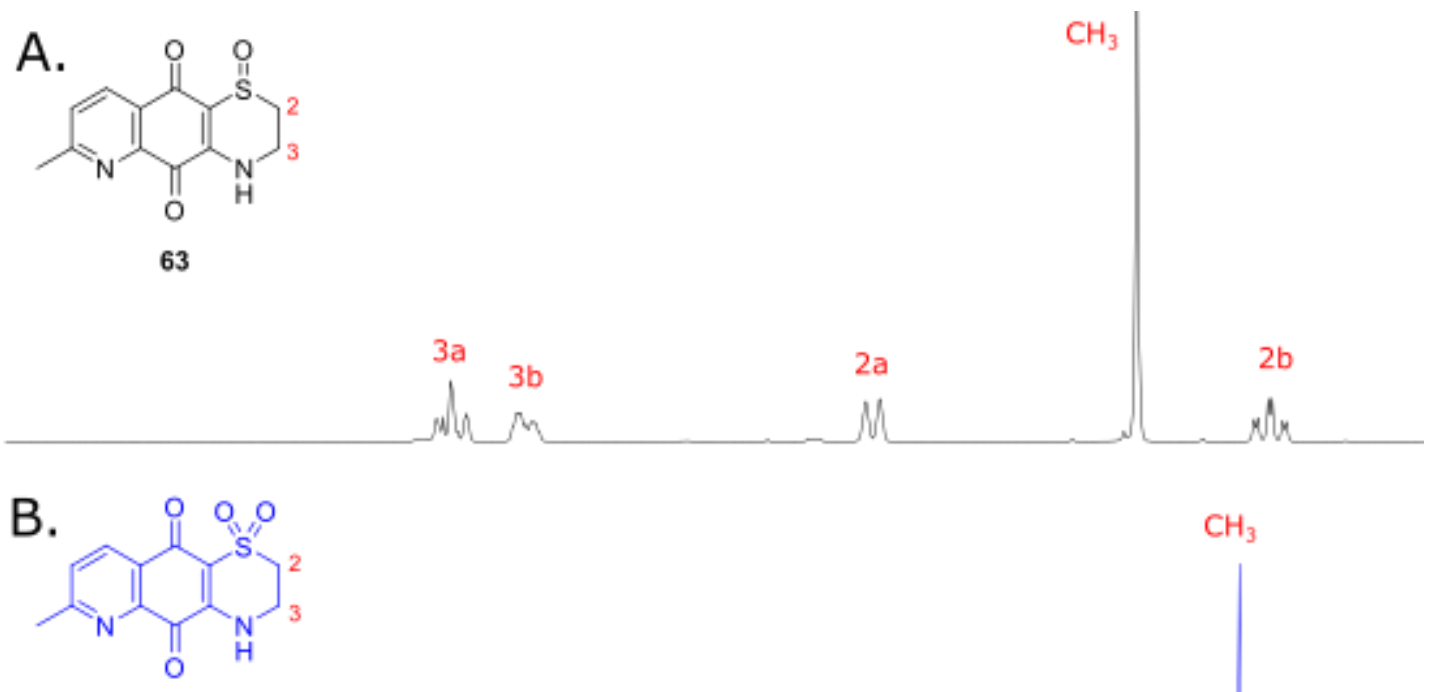

64
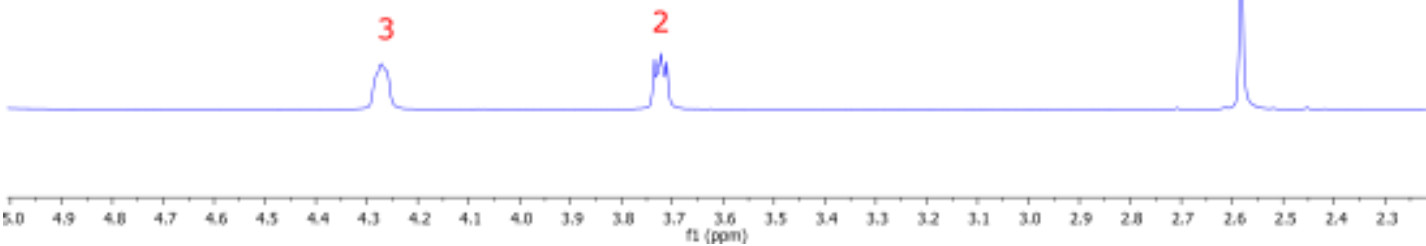

Figure 2.6: ${ }^{1} \mathrm{H}$ NMR spectra of A). sulfoxide $63\left(500 \mathrm{MHz}, \mathrm{CDCl}_{3}\right)$, and B). sulfone 64 (500 $\mathrm{MHz}$, pyridine- $\left.\mathrm{d}_{5}\right)$.

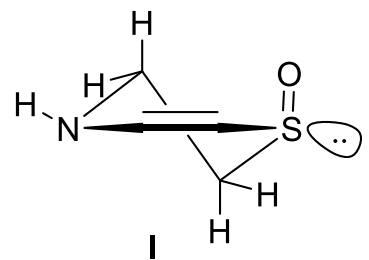

Figure 2.7: Structure of the thiazine ring, showing the $\mathrm{S}=\mathrm{O}$ substituent in the axial position.

With sulfone 64 in hand, a final oxidation step employing $\mathrm{SeO}_{2}$ was applied to synthesise ascidiathiazone A (27) (Scheme 2.6). The oxidation reaction was sluggish and required stirring at $90{ }^{\circ} \mathrm{C}$ for three days, as further increases in temperature led to quinone degradation. Nonetheless, the desired carboxylic acid $\mathbf{2 7}$ was isolated successfully as a bright yellow solid, albeit in a modest $(12 \%)$ yield, with the spectral data matching those reported in literature. ${ }^{104}$ 


\subsubsection{An alternative synthetic route to ascidiathiazone $A$}

In an attempt to increase the overall yield for the ascidiathiazone A (27) synthesis, an alternative approach involving the benzylic oxidation of thiazine 61 prior to sulfur oxidation was attempted (Scheme 2.6). Gratifyingly, the benzylic oxidation occurred selectively without any evidence of sulfur oxidation, giving carboxylic acid 65 in 83\% yield following filtration through Celite, acidification, and aqueous extraction.<smiles>Cc1ccc2c(n1)C(=O)C1=C(SCCN1)C2=O</smiles>

61<smiles>O=C(O)c1ccc2c(n1)C(=O)C1=C(SCCN1)C2=O</smiles>

65

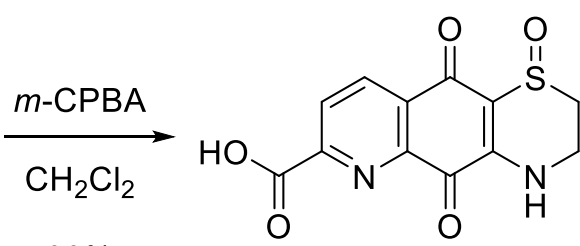

$93 \%$

66

1. $\mathrm{H}_{2} \mathrm{O}_{2}, \mathrm{H}_{2} \mathrm{O}$

2. $\mathrm{H}_{2} \mathrm{O}_{2}, \mathrm{AcOH}$

$52 \%$

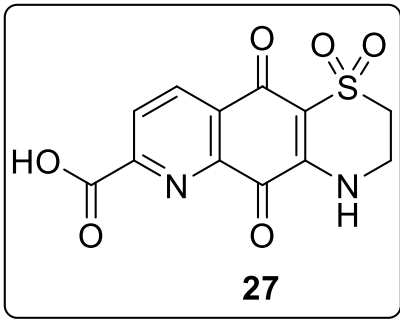

Scheme 2.6: The synthesis of ascidiathiazone A (27) through carboxylic acid 65.

Evidence for the formation of carboxylic acid 65 was obtained through ${ }^{1} \mathrm{H}$ and ${ }^{13} \mathrm{C}$ NMR analysis. The disappearance of the resonances attributed to the methyl group for methylpyrimidine 61 , which were noted at $\delta_{\mathrm{H}}=2.69 \mathrm{ppm}$ and $\delta_{\mathrm{C}}=25 \mathrm{ppm}$, in combination with the appearance of a resonance at $\delta_{\mathrm{C}}=165.7 \mathrm{ppm}$ in the ${ }^{13} \mathrm{C}$ NMR spectrum of $\mathbf{6 5}$ were indicative of the presence of a carboxylic acid carbonyl carbon. This carbon was also found to correlate to protons at the 8- and 9- positions, as determined by HMBC (Figure 2.8). Moreover, the mass of the desired compound was observed by HRMS (calcd. for $\left[\mathrm{C}_{12} \mathrm{H}_{8} \mathrm{~N}_{2} \mathrm{O}_{4} \mathrm{~S}+\mathrm{H}\right]^{+}$: 277.0278, obsd.: 277.0277; calcd. for $\left[\mathrm{C}_{12} \mathrm{H}_{8} \mathrm{~N}_{2} \mathrm{O}_{4} \mathrm{~S}-\mathrm{H}\right]^{-} .:$275.0132, obsd.: 275.0135), thereby corroborating the presence of acid $\mathbf{6 5}$. 


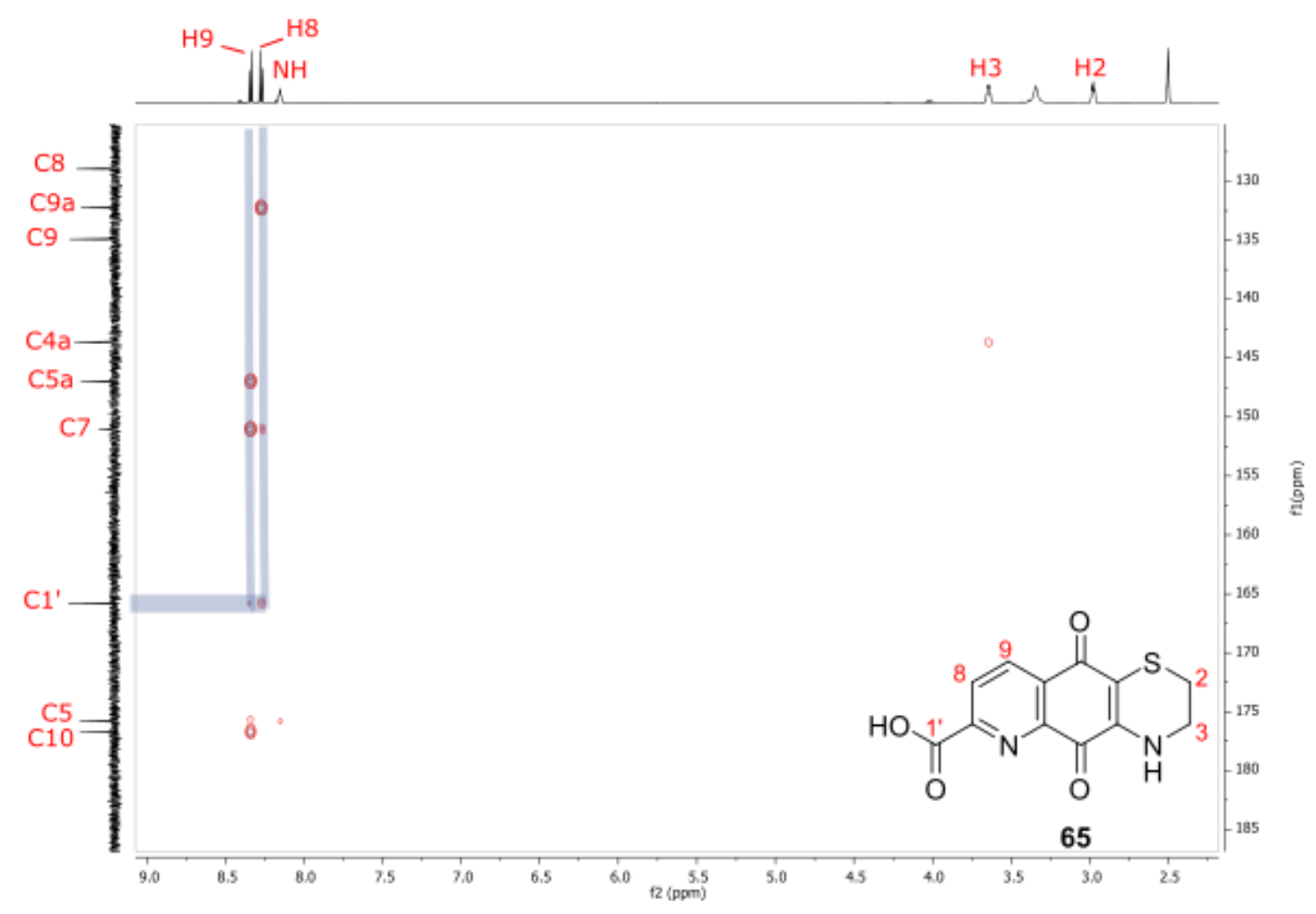

Figure 2.8: $\mathrm{HMBC}$ spectrum $\left(500 \mathrm{MHz}, \mathrm{DMSO}-\mathrm{d}_{6}\right)$ of thiazine-quinolinic acid 65.

With the carboxy-quinone $\mathbf{6 5}$ in hand, the final oxidation reactions were undertaken to install the sulfone functionality present in ascidiathiazone A (27). Again, an initial oxidation step was employed in order to isolate sulfoxide 66 in an excellent (93\%) yield. Due to its highly polar nature, sulfoxide $\mathbf{6 6}$ precipitated from the reaction mixture and was readily collected through filtration. However, when sulfoxide $\mathbf{6 6}$ was subjected to a second oxidation reaction, the reaction was sluggish and did not reach completion, which was presumably due to the low solubility of sulfoxide 66 in organic solvents $\left(\mathrm{CH}_{2} \mathrm{Cl}_{2}\right.$ and acetone). To circumvent this issue, a two-step oxidation reaction of $\mathbf{6 5}$ was conducted using two equivalents of $m$-CPBA in acetone. Although product formation was evidenced by TLC and HRMS analysis (calcd. for $\left[\mathrm{C}_{12} \mathrm{H}_{8} \mathrm{~N}_{2} \mathrm{O}_{6} \mathrm{~S}+\mathrm{H}\right]^{+}$: 309.0176, obsd.: 309.0175), the oxidation reaction did not reach completion and resulted in the recovery of sulfide $\mathbf{6 5}$ and sulfoxide $\mathbf{6 6}$. Additional equivalents of $m$-CPBA did not assist in the consumption of sulfide $\mathbf{6 5}$ and sulfoxide intermediate $\mathbf{6 6}$ and instead further complicated the purification procedure. Thus, in an alternative approach, sulfide 65 was treated with a $28 \% \mathrm{H}_{2} \mathrm{O}_{2}$ solution for two hours, followed by the addition of a second equivalent of $28 \% \mathrm{H}_{2} \mathrm{O}_{2}$, this time in $\mathrm{AcOH} .{ }^{115}$ Gratifyingly, the desired sulfone 27 precipitated from the solution after one hour of stirring at $70^{\circ} \mathrm{C}$ to give ascidiathiazone $\mathrm{A}(27)$ 
as a bright yellow solid in a moderate yield (52\%). In addition, crystallisation of ascidiathiazone A (27) from $\mathrm{H}_{2} \mathrm{O}$ resulted in small yellow crystals that were of sufficient quality for X-ray crystallography. The structure of ascidiathiazone A (27) was confirmed by XRD data, which revealed the sulfone and carboxylic acid functionalities (Figure 2.9).

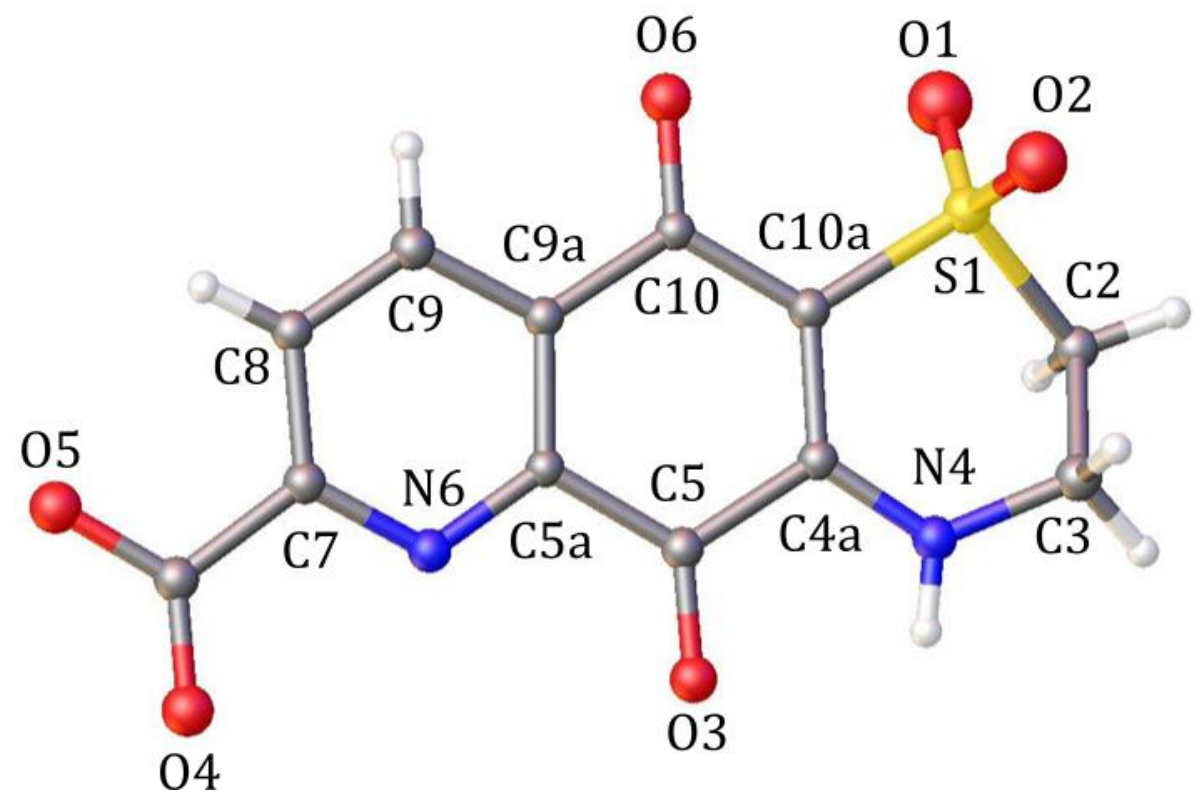

Figure 2.9: XRD crystal structure of ascidiathiazone A (27).

Compared to the literature synthesis of ascidiathiazone A (27), which was achieved in $32 \%$ yield over 4 steps, ${ }^{104}$ the presented synthetic route had a lower overall yield of $6 \%$ over 5 steps due to the low-yielding oxidation step of hydroxyquinoline $\mathbf{5 8}$ to afford dichloroquinone 56. That said, the presented synthetic routes allowed for a variety of ascidiathiazone A (27) analogues to be prepared for SAR studies of their anti-tubercular activity.

\subsubsection{The synthesis of ascidiathiazone $B$}

Having successfully prepared ascidiathiazone A (27), similar synthetic strategies were adopted to synthesise ascidiathiazone B (28) (Scheme 2.7). Accordingly, thiazine 62 was treated with $m$ - 
CPBA to form sulfoxide 67 and, subsequently, sulfone 68. Gratifyingly, improved yields were achieved upon the oxidation of thiazine $\mathbf{6 2}$ compared to the oxidation of its regioisomer $\mathbf{6 1}$, giving sulfone $\mathbf{6 8}$ in $98 \%$ yield or 30\% yield over two steps from the corresponding sulfide (62). Next, sulfone $\mathbf{6 8}$ was subjected to base-catalysed air oxidation with potassium hydroxide ${ }^{105,131}$ to give the unsaturated sulfone $\mathbf{6 9}$ in a high yield. The formation of the alkene moiety was evident by the loss of the resonances for the methyelene protons at 3.41 and 3.94 ppm in the ${ }^{1} \mathrm{H}$ NMR spectrum of sulfone 39 (Figure 2.10A), and the appearance of two doublets correlating to protons 2 and 3 at 7.24 and $6.45 \mathrm{ppm}$, respectively, in the ${ }^{1} \mathrm{H}$ NMR spectrum of thiazine 69 (Figure 2.10B). The observed $J_{\mathrm{H} 2, \mathrm{H} 3}$ value of $8.9 \mathrm{~Hz}$ is typical of $J_{\mathrm{H}, \mathrm{H}}$ values of cis alkene protons, ${ }^{130}$ while also matching literature data for proton resonances in unsaturated 1,1-dioxo-thiazino-quinones. ${ }^{105,131}$ Having prepared sulfone $\mathbf{6 8}$, oxidation of the methyl group to the corresponding carboxylic acid was then performed under the agency of $\mathrm{SeO}_{2}$. The oxidation was complete after 24 hours, whereby TLC analysis showed the appearance of a slower running product $\left[\mathrm{R}_{f}=0.09(\mathrm{EtOAc} / \mathrm{MeOH} / \mathrm{AcOH}, 4 / 1 / 0.1, \mathrm{v} / \mathrm{v} / \mathrm{v})\right]$. Due to the polar nature of ascidiathiazone B (28), purification was difficult and required both standard and reverse phase column chromatography, as well as size exclusion chromatography. Nonetheless, ascidiathiazone B (28) was successfully isolated in a 33\% yield, with data matching those reported for natural product 28. ${ }^{104}$ In this way, I confirmed the structural assignment of ascidiathiazone B (28) as this is the first reported total synthesis of this natural product. Here, ascidiathiazone B (28) was synthesised in 6 steps from commercially available starting materials (8-hydroxyquinoline $\mathbf{5 8}$ ) and in $2 \%$ overall yield. 


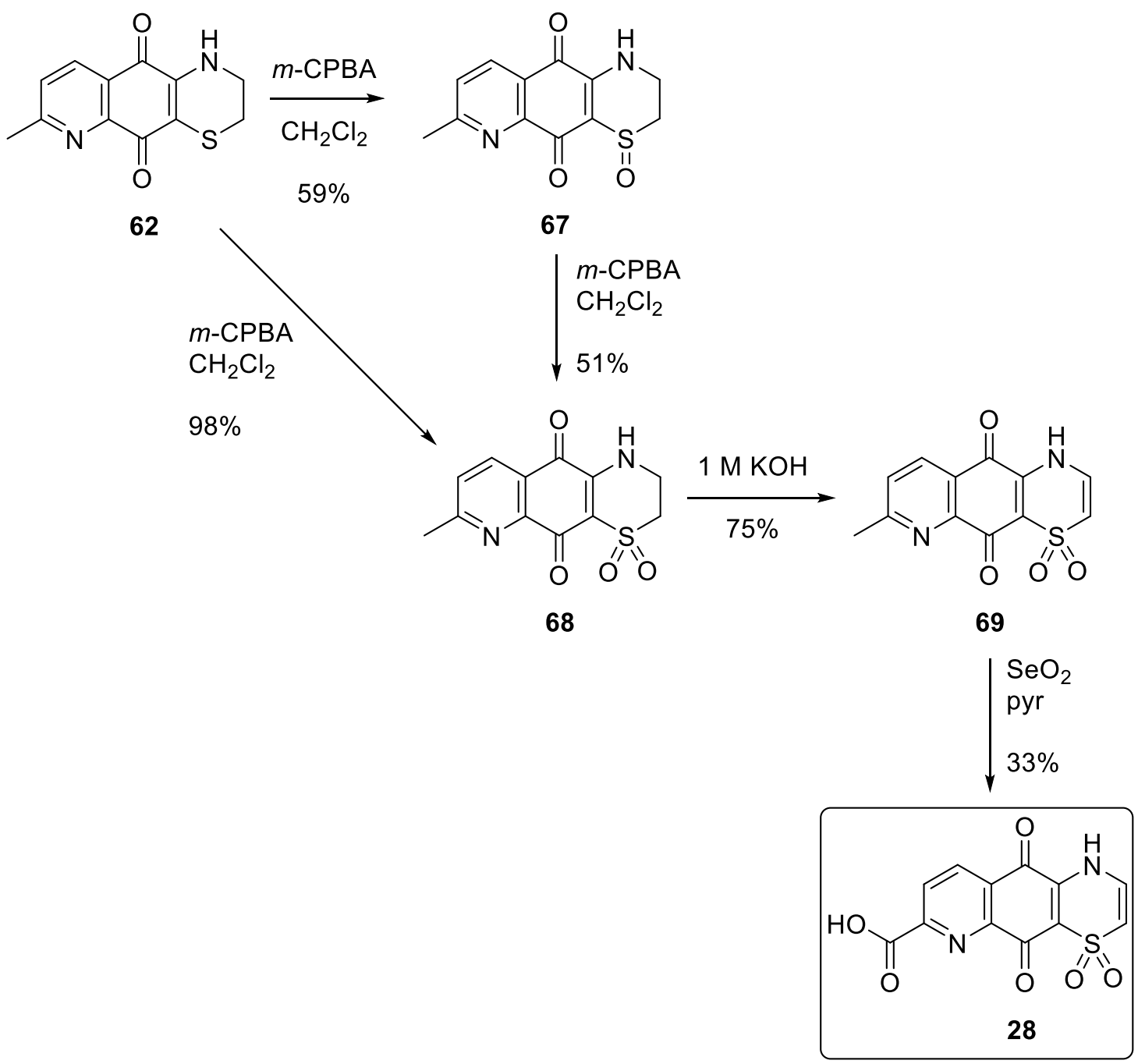

Scheme 2.7: The synthesis of ascidiathiazone B (28) from thiazine 62 in 3 steps. 


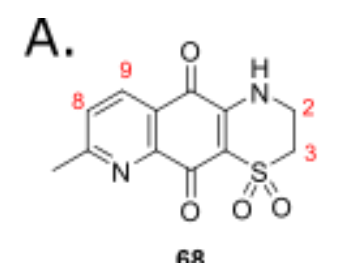

68
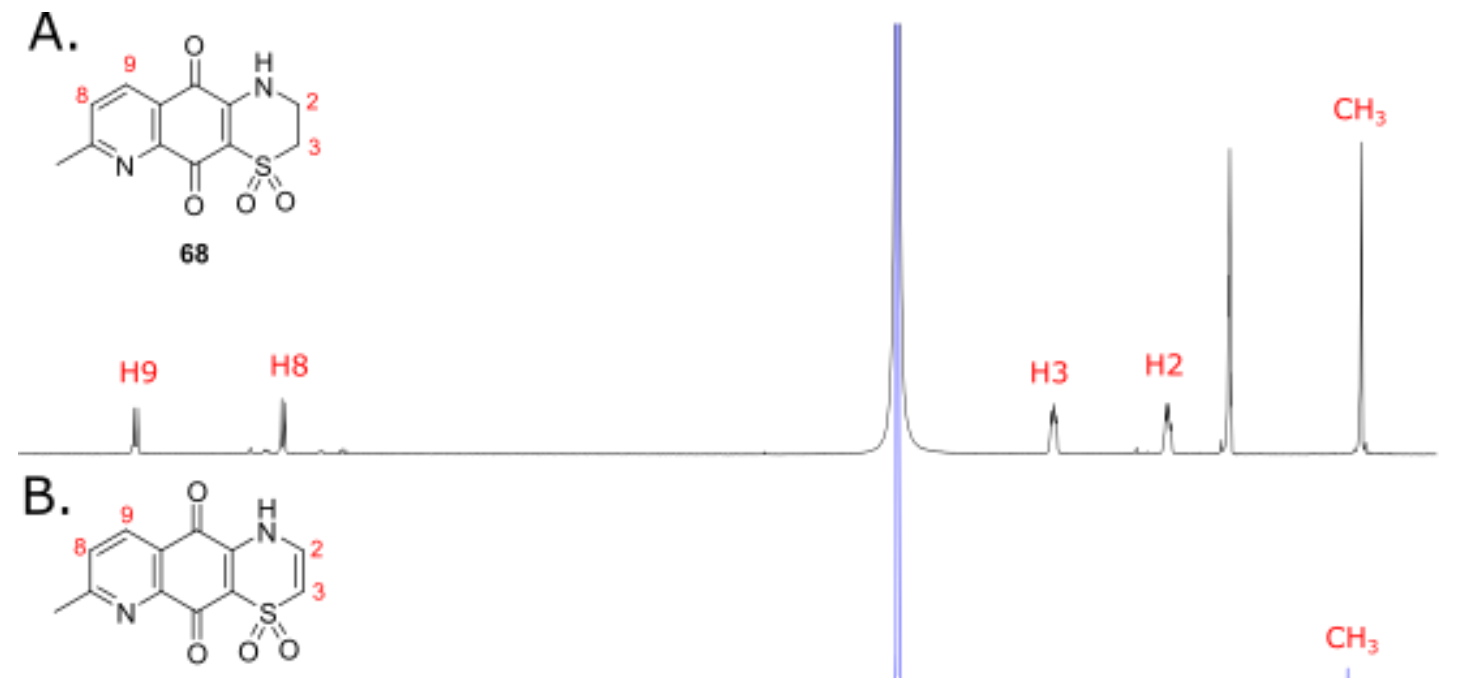

69

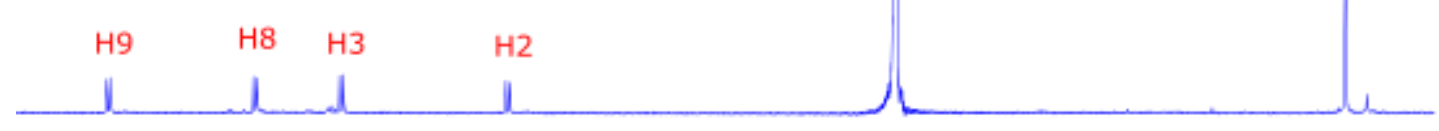

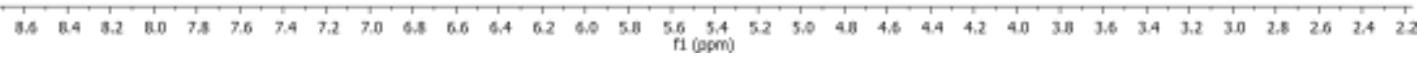

Figure 2.10: ${ }^{1} \mathrm{H}$ NMR spectrum $\left(500 \mathrm{MHz}, \mathrm{D}_{2} \mathrm{O}\right)$ of $\left.\mathrm{A}\right)$. saturated sulfone 68 and $\left.\mathrm{B}\right)$. unsaturated sulfone 69.

Next, the alternative synthetic route was applied to thiazine $\mathbf{6 2}$ in order to prepare additional derivatives of ascidiathiazone B (28) for assessment of their anti-tubercular activity. To this end, thiazine 62 was treated with $\mathrm{SeO}_{2}$ to give carboxy-quinone $\mathbf{7 0}$ in $72 \%$ yield (Scheme 2.8). Next, sulfide oxidation reactions with $m$-CPBA and $\mathrm{H}_{2} \mathrm{O}_{2}$ gave the corresponding sulfoxide 71 and sulfone $\mathbf{7 2}$, respectively, in moderate to good yields. Both sulfoxide $\mathbf{7 1}$ and sulfone $\mathbf{7 2}$ precipitated from $\mathrm{CH}_{2} \mathrm{Cl}_{2}$ and $\mathrm{H}_{2} \mathrm{O}$, respectively, and were collected by filtration. The formation of sulfone 72 was corroborated by HRMS (calcd. for $\left[\mathrm{C}_{12} \mathrm{H}_{8} \mathrm{~N}_{2} \mathrm{O}_{6} \mathrm{~S}+\mathrm{H}\right]^{+}$: 309.0176, obsd.: 309.0170, calcd. for $\left[\mathrm{C}_{12} \mathrm{H}_{8} \mathrm{~N}_{2} \mathrm{O}_{6} \mathrm{~S}-\mathrm{H}\right]: 307.0030$, obsd.: 307.0051 ), as well as by NMR analysis. NMR data for thiazine $\mathbf{7 2}$ was similar to its regioisomer, ascidiathiazone A (27), with the most notable difference being the slightly larger $\Delta \mathrm{H}$ 9-H8 value for sulfone 72 (8.48 ppm$8.27 \mathrm{ppm}=0.21 \mathrm{ppm})$, compared to that for ascidiathiazone A $(27,8.40 \mathrm{ppm}-8.22 \mathrm{ppm}=0.18$ ppm) (Figure 2.11). Finally, sulfone 72 was subjected to base-catalysed air oxidation to give ascidiathiazone B (28) in 80\% yield, giving ascidiathiazone B (28) in an improved overall yield of $2.5 \%$ over six steps, compared to the previous synthetic route. 


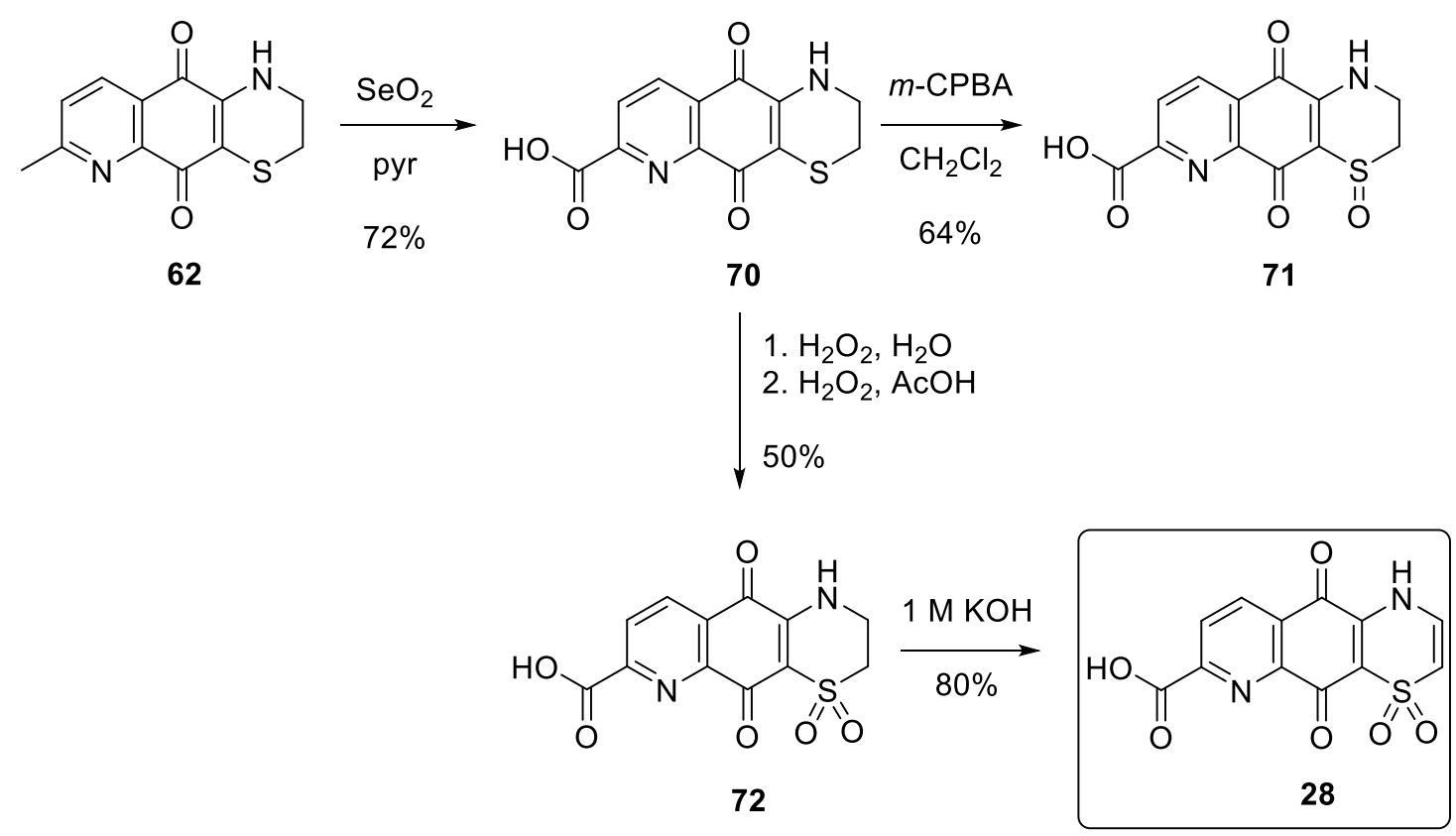

Scheme 2.8: Synthesis of ascidiathiazone B (28) via carboxyquinone $\mathbf{7 2 .}$

A.
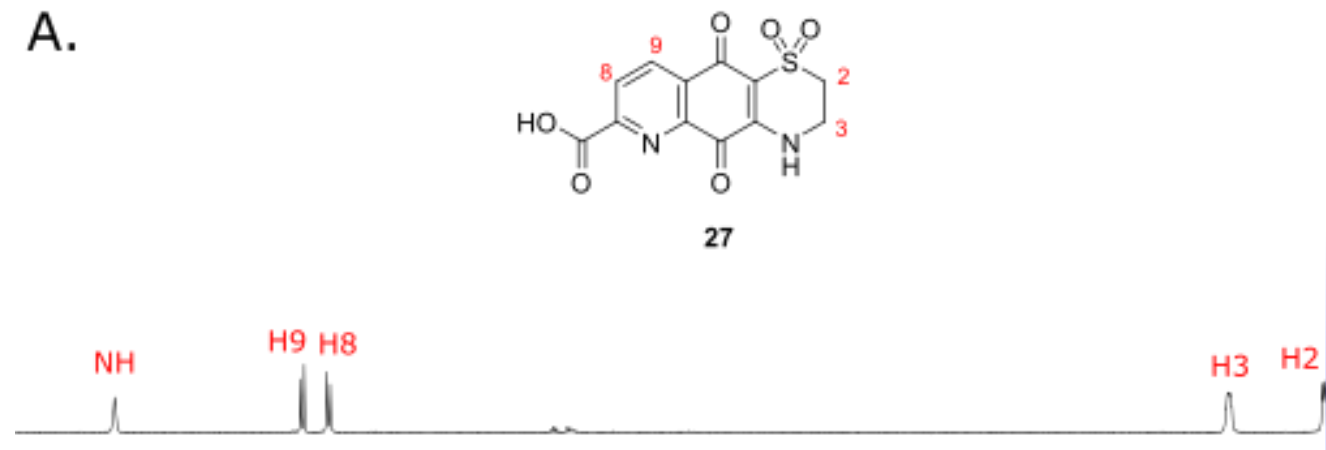

B.
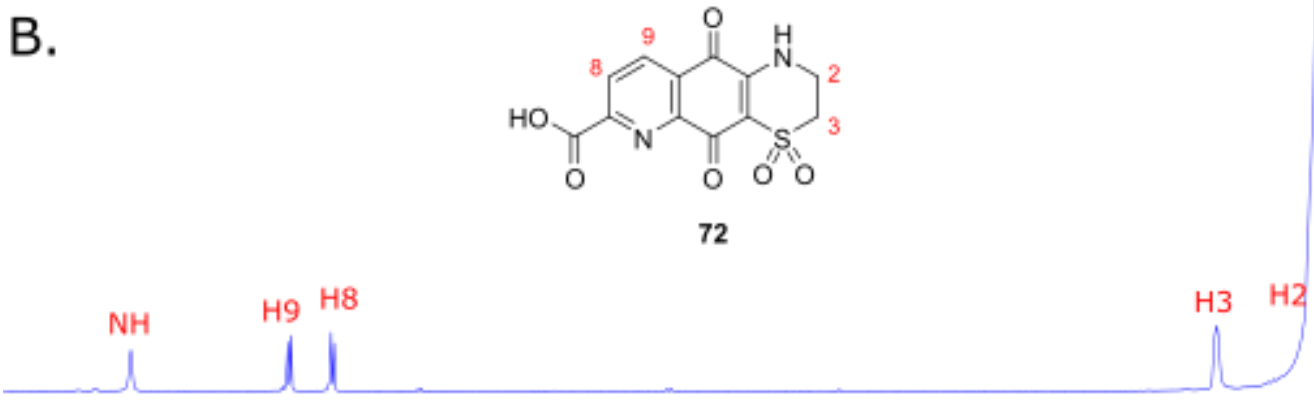

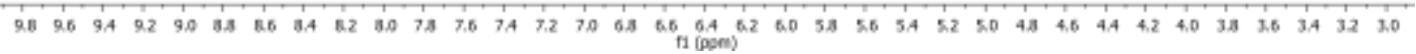

Figure 2.11: ${ }^{1} \mathrm{H}$ NMR spectra $\left(500 \mathrm{MHz}, \mathrm{DMSO}-\mathrm{d}_{6}\right)$ of $\left.\mathrm{A}\right)$. ascidiathiazone $\mathrm{A}(27)$, and its regioisomer B). carboxy-quinone $\mathbf{7 2}$. 


\subsubsection{MIC values}

To investigate the anti-tubercular activity of ascidiathiazone A (27) and ascidiathiazone B (28) along with synthetic derivatives 47, 59-72, two strains of mycobacteria, M. smegmatis mc $^{2} 155$ and $M t b \mathrm{mc}^{2} 6230$ were employed, as measured by the MIC (Table 2.1). Here, the MIC values were determined as the minimal concentration of the compound required to inhibit bacterial growth. Both $M$. smegmatis $\mathrm{mc}^{2} 155$ and $M t b \mathrm{mc}^{2} 6230$ are commonly used for mycobacterial studies as they are non-pathogenic, ${ }^{132}$ and, in the case of $M$. smegmatis, fast-growing. ${ }^{133,134}$ These studies were undertaken under the supervision of Professor Gregory Cook at the University of Otago.

Table 2.1: The MIC values $(\mu \mathrm{M})$ of ascidiathiazones $\mathbf{2 7}, \mathbf{2 8}$, and analogues $\mathbf{4 7}, \mathbf{5 9 - 7 2}$ against M. smegmatis and $M t b$

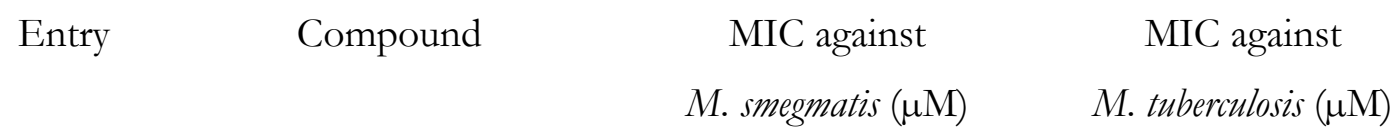<smiles>O=C(O)c1ccc2c(n1)C(=O)C1=C(SCCN1)C2=O</smiles>

2<smiles>O=C(O)c1ccc2c(n1)C(=O)C1=C(NCCS1)C2=O</smiles><smiles>O=C(O)c1ccc2c(n1)C(=O)C1=C(C2=O)S(=O)CCN1</smiles> 
4<smiles>O=C(O)c1ccc2c(n1)C(=O)C1=C(NCCS1=O)C2=O</smiles>

5<smiles>Cc1ccc2c(n1)C(=O)C1=C(C2=O)S(=O)CCN1</smiles>

63<smiles>Cc1ccc2c(n1)C(=O)C1=C(NCCS1=O)C2=O</smiles>

67<smiles>Cc1ccc2c(n1)C(=O)C1=C(C2=O)S(=O)(=O)CCN1</smiles>

64

8<smiles>Cc1ccc2c(n1)C(=O)C1=C(NCCS1(=O)=O)C2=O</smiles>

68

9<smiles>Cc1ccc2c(n1)C(=O)C1=C(SCCN1)C2=O</smiles>

61
$>100$

100 
10<smiles>Cc1ccc2c(n1)C(=O)C1=C(NCCS1)C2=O</smiles>

11<smiles>O=C(O)c1ccc2c(n1)C(=O)C1=C(NCCS1(=O)=O)C2=O</smiles>

72

12<smiles>O=C(O)c1ccc2c(n1)C(=O)C1=C(C2=O)S(=O)(=O)CCN1</smiles>

27

13<smiles>Cc1ccc2c(n1)C(=O)C(NCCCl)=C(Cl)C2=O</smiles>

59

14<smiles>Cc1ccc2c(n1)C(=O)C(Cl)=C(NCCCl)C2=O</smiles>

15<smiles>O=C(O)c1ccc2cccc(O)c2n1</smiles> 
16<smiles>Cc1ccc2c(n1)C(=O)C1=C(NC=CS1(=O)=O)C2=O</smiles>

69<smiles>O=C(O)c1ccc2c(n1)C(=O)C1=C(NC=CS1(=O)=O)C2=O</smiles>

From the MIC values, it was noted that the anti-mycobacterial activity of the ascidiathiazone analogues varied greatly between the two species of bacteria. Indeed, this is a common phenomenon, ${ }^{135}$ despite extensive literature precedence on the use of $M$. smegmatis as a bacterial model for $M t b$ in drug discovery. ${ }^{136,137}$ Herein, a lack of growth inhibition against M. smegmatis was observed for almost all of the compounds, with the exceptions of compounds 61, 62, 59, 47, and 69, which showed modest inhibitory activity $(50-100 \mu \mathrm{M}$, Entries 9, 10, 13, 15, 16). Notwithstanding, the anti-tubercular activity of thiazino-QQs 61, 62, 59, 47, 69 against $M$. smegmatis correlated to low MIC values against $M t b(3-12 \mu \mathrm{M})$.

In contrast, all compounds exhibited inhibitory activity against $M t b$, with MIC values ranging from $100 \mu \mathrm{M}$ to $1.6 \mu \mathrm{M}$. In most cases, $[2,3-g]$ and $[3,2-g]$ regioisomers similar potency. This can be seen in sulfides 65 and $70(100 \mu \mathrm{M})$, sulfoxides 66 and $71(100 \mu \mathrm{M})$ and 63 and $67(50$ $\mu \mathrm{M})$, and sulfones 64 and $68(25 \mu \mathrm{M})$ (Entries 1-8). When different MIC values were observed between regioisomers, the $[2,3-g]$ isomers tended to have higher inhibitory activity than the corresponding [3,2-g] isomers, as in thiazines 61 and $\mathbf{6 2}(12.5 \mu \mathrm{M}$ and $25 \mu \mathrm{M}$, Entries 9 and 10, respectively), and in sulfone $\mathbf{7 2}$, which was a considerably weaker inhibitor than its $[2,3-g]$ regioisomer, ascidiathiazone A (27) $(100 \mu \mathrm{M}$ and $1.6 \mu \mathrm{M}$, respectively, Entries 11 and 12). In chloroethylaminoQQs $\mathbf{5 9}$ and $\mathbf{6 0}$ however, the $[3,2-g]$ isomer gave better anti-tubercular activity (12.5 $\mu \mathrm{M}$ vs. $3 \mu \mathrm{M}$, Entries 13 and 14, respectively).

Previous studies on the biological activity of QQs have identified tuberculostatic QQs that bear sulfone and sulfoxide functionalities ${ }^{65}$ and, accordingly, the effect of different sulfur oxidation states on the anti-tubercular activity of QQs was analysed. Here, the sulfone moiety 
tended to increase growth inhibition compared to the corresponding sulfides and sulfoxides. For example, sulfones $\mathbf{6 4}$ and $\mathbf{6 8}$ had lower MIC values $(25 \mu \mathrm{M})$ than sulfoxides $\mathbf{6 3}$ and $\mathbf{6 7}$ (50 $\mu \mathrm{M}$, Entries 5-8), while ascidiathiazone A (27) was exceptionally potent against $M t b$ compared to the corresponding sulfide (61) and sulfoxide (66) (Entries 12, 1, and 3, respectively). In the case of the $[3,2-g]$ isomers, unsaturation at carbons 2 and 3 correlated to a remarkable decrease in MIC, with unsaturated thiazine $\mathbf{6 9}$ being much more potent than the saturated form $\mathbf{6 8}$ (6 $\mu \mathrm{M}$ compared to $25 \mu \mathrm{M}$, Entries 16 and 8, respectively), as well as ascidiathiazone B (28) with an MIC of $3 \mu \mathrm{M}$ compared to acid 72 at $100 \mu \mathrm{M}$ (Entries 17, 11, respectively).

As mentioned above, the carboxy-functionality was previously deemed important for the antiinflammatory activity of ascidiathiazone analogues ${ }^{105}$ and this structural feature was thus also explored for anti-tubercular activity. Here, compounds bearing a carboxylic acid group gave wide ranging MIC values. In particular, acids 65, 70, 66, 71, and 72 all have modest inhibitory activity at $100 \mu \mathrm{M}$ (Entries 1-4, 11), while acid 47, along with natural products 27 and 28 have the lowest MIC values of the library at 3, 1.6, and $3 \mu \mathrm{M}$, respectively (Entries 15, 12, and 17, respectively). These results suggest that the presence of both sulfone and carboxyfunctionalities are important for anti-tubercular activity of the tricylic ascidiathiazone analogues.

Overall, this in vitro assay demonstrated the potent anti-tubercular activity of natural products ascidiathiazone A (27) and ascidiathiazone B (28), with ascidiathiazone A (27) being twice as potent than ascidiathiazone B (28). Moreover, ascidiathiazone A (27) has previously been reported to exhibit low cytotoxicity in mammalian cell line L6 ( $\left.\mathrm{IC}_{50}: 170 \mu \mathrm{M}\right),{ }^{138}$ making it a promising lead compound as an anti-tubercular agent. That said, the mechanism by which ascidiathiazone A (27) exerts anti-mycobacterial activity is yet to be determined.

\subsubsection{NADH oxidation}

To investigate whether the ascidiathiazone analogues perturb NDH-II function, the effects of each compound on the rate of NADH oxidation in mycobacterial inverted membrane vesicles (IMVs) were measured. IMVs have intact ETCs that oxidise NADH, which provides an excellent model for mycobacterial NADH oxidation. As NADH absorbs light at $340 \mathrm{~nm},{ }^{33,64}$ NADH oxidation by IMVs was monitored by measuring the absorbance at $340 \mathrm{~nm}$ over time. The rate of NADH oxidation in the presence of each ascidiathiazone analogue, alongside the negative control, i.e. IMV only, and the basal rate of oxidation, i.e. IMV and NADH only, are 
shown in Figure 2.12. These experiments were undertaken under the supervision of Dr. Kiel Hards and Prof. Gregory Cook at the University of Otago.

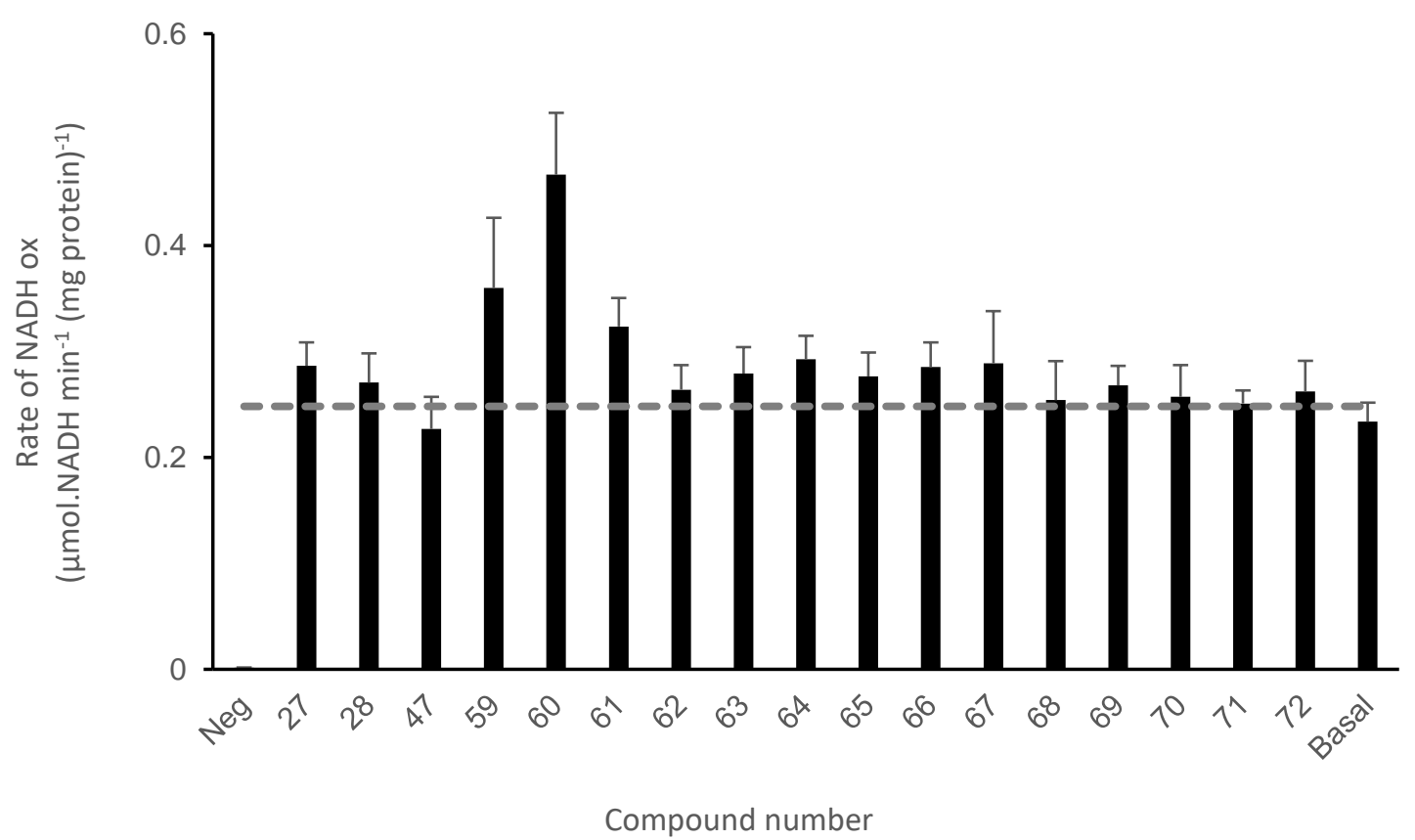

Figure 2.12: Rates of NADH oxidation after treating IMVs with compounds 27, 28, 47 and 59-74. The basal rate was determined by measuring the rate of NADH oxidation by IMVs without the addition of any drug, while the negative control contained only IMVs.

As shown in Figure 2.12, chloroethylamino-QQs $\mathbf{5 9}$ and $\mathbf{6 0}$ exerted an appreciable effect on NADH oxidation by increasing the reaction rate. This observation is consistent with previous studies whereby the closely related QQ8c (20, Figure 2.13) stimulated NADH oxidation and oxygen consumption in IMVs. ${ }^{64} \mathrm{Next}$, the $\mathrm{NADH}$ oxidation and $\mathrm{O}_{2}$ consumption induced by QQ 60 were determined by monitoring the fluorescence of the $\mathrm{pH}$-sensitive dye acridine orange (73) and the $\mathrm{O}_{2}$ concentration in the IMVs, respectively (Figure 2.14). Here, upon the addition of $\mathrm{NADH}(\mathrm{t}=5 \mathrm{~s})$, a reduction in $\mathrm{O}_{2}$ concentration and the fluorescence quenching of acridine orange (73) were observed as $\mathrm{NADH}$ undergoes oxidation and releases protons. When QQ 60 was added $(t=6 \mathrm{~s})$, the rate of $\mathrm{O}_{2}$ consumption increased drastically, which supported the hypothesis that QQ $\mathbf{6 0}$ stimulates NADH oxidation. However, the fluorescence of acridine orange (73) continued to decrease after QQ 60 was added, indicating that the IMV cell membrane remained impermeable to protons (the drop in fluorescence seen here is due to the inherent fluorescence of QQ 60). As uncouplers of respiration allow protons to move 
in and out of cell membranes, this observation indicated that QQ 60 does not uncouple respiration in $M t b$. This observation is consistent with the mode of action of QQ8c (20). ${ }^{64}$ As the oxygen concentration is depleted, ion diffusion through the membrane takes place and fluorescence increases as the $\mathrm{pH}$ increases (at $\mathrm{t}=7 \mathrm{~s}$ ).<smiles>O=C1C(Cl)=C(NCCCl)C(=O)c2ncccc21</smiles>

20<smiles>CN(C)c1ccc2cc3ccc(N(C)C)cc3nc2c1</smiles>

73

Figure 2.13: QQ8c (20) and acridine orange (73).

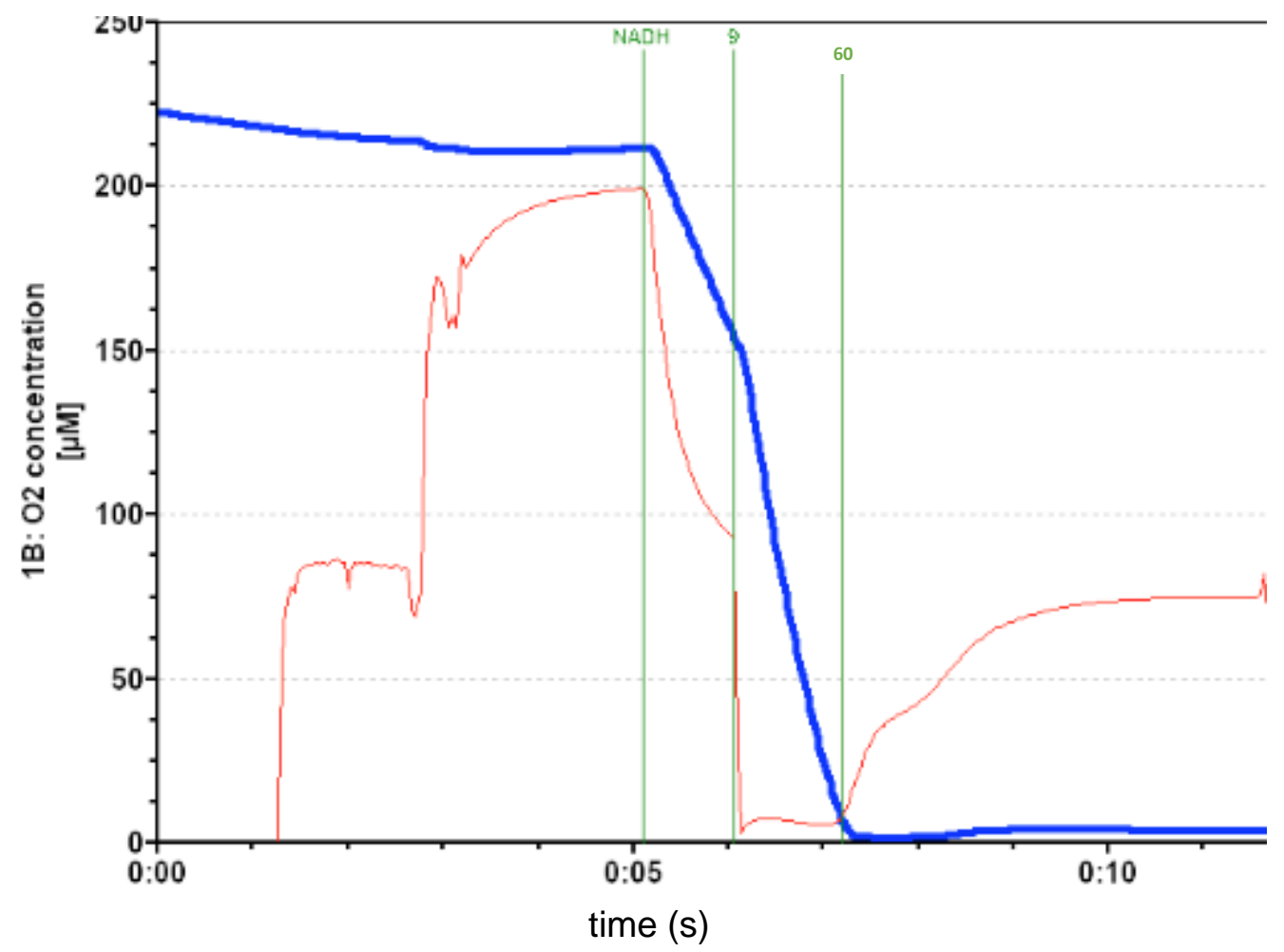

Figure 2.14: QQ 60 increased oxygen consumption in IMVs and did not act as an uncoupler. Oxygen concentration (blue line) decreases significantly faster after $\mathbf{6 0}$ was added to the solution (at $\mathrm{t}=6$ seconds), while the fluorescence of acridine orange (73) (red line) continued to decrease.

In the case of QQ8c (20), similar assays were conducted with IMVs that overexpress the NDH-2 enzyme, thereby confirming that QQ8c (20) activates NDH-2 specifically. ${ }^{64}$ Herein, 
wild type IMVs were used, though due to the structural resemblance of quinone 60 to QQ8c (20), as well as the widely accepted view that Mtb utilise NDH-II exclusively to oxidise $\mathrm{NADH},{ }^{41}$ it was postulated that compound $\mathbf{6 0}$ inhibits $M t b$ growth through NDH-II activation. Notwithstanding, studies of the modes of action of lead compounds ascidiathiazone A (27) and ascidiathiazone B (28) are underway.

\subsection{Conclusion}

In summary, the total synthesis of the natural product ascidiathiazone A (27) and, for the first time, the total synthesis of ascidiathiazone B (28) were achieved. Although the synthesis of ascidiathiazone A (27) has been previously reported (4 steps and 32\% overall yield from quinolinic acid 47), ${ }^{104}$ the present divergent synthetic strategy allowed for both natural products 27 and 28 to be synthesised from a common intermediate. Herein, ascidiathiazone A (27) was synthesised in $6 \%$ overall yield over five steps, while ascidiathiazone B (28) in $2.5 \%$ overall yield over six steps, both from the commercially available 8-hydroxyquinoline (58). In addition, this synthetic route allowed for the preparation of a number of related structures for SAR analysis. In paricular, the effect of a carboxylic acid functionality as well as different sulfur oxidation states on the anti-tubercular activity of the compounds was investigated.

Next, the library of ascidiathiazone analogues was screened against $M$. smegmatis and $M t b$, from which MIC values for each compound were obtained. While the compounds did not show inhibitory activity against $M$. smegmatis, they did inhibit $M t b$ growth at low concentrations. The lead compound from this library was ascidiathiazone A (27), with a remarkably low MIC of $1.6 \mu \mathrm{M}$, and, moreover, low toxicity against mammalian cell line L6. ${ }^{138}$ Other potent inhibitors identified were ascidiathiazone B (28), quinolinic acid 47, and quinone 60 - all with MIC values of $3 \mu \mathrm{M}$.

To gain some insight into the mode of action of the ascidiathiazone analogues, IMVs were treated with each compound, and the resulting NADH oxidation rates measured. In this assay, quinone 60 stimulated NADH oxidation, an observation that was further supported in assays that saw QQ 60 increase $\mathrm{O}_{2}$ consumption in IMVs without acting as an uncoupler. Taken together, compound $\mathbf{6 0}$ thus has a mode of action similar to the structurally related QQ8c (20), although QQ 60 is a more potent $M t b$ inhibitor than QQ8c (20) (MIC $=3 \mu \mathrm{M}$ vs. $8.1 \mu \mathrm{M},{ }^{64}$ respectively). 


\subsection{Experimental}

\subsubsection{Chemistry}

General procedure. Unless otherwise stated, all reactions were performed under atmospheric air. Prior to use, $\mathrm{CH}_{2} \mathrm{Cl}_{2}$ (Fisher) was distilled from $\mathrm{P}_{2} \mathrm{O}_{5}$, pyridine $(\mathrm{BDH})$ was distilled over $\mathrm{CaH}_{2}, \mathrm{SeO}_{2}$ (Aldrich) was sublimed, and EtOAc, petroleum ether, acetone, $\mathrm{MeOH}$ (Pure Science) and $\mathrm{H}_{2} \mathrm{O}$ were distilled. 8-hydroxy-2-methyl-quinoline (Aldrich), $\mathrm{NaOH}$ (Pure Science), 37\% HCl (Merck), sodium chlorate (Sigma Aldrich), EtOH (absolute, Pure Science), 2-chloroethylamine hydrochloride (Aldrich), $\mathrm{NaHCO}_{3}$ (Pure Science), $\mathrm{MgSO}_{4}$ (Pure Science), sodium sulfide (Pure Science), m-CPBA (Aldrich), AcOH (Panreac), 28\% $\mathrm{H}_{2} \mathrm{O}_{2}$ (Riedel-de Haen), KOH (Pure Science) were used as received.

All solvents were removed by evaporation under reduced pressure. Reactions were monitored by TLC-analysis on Macherey-Nagel silica gel coated plastic sheets $(0.20 \mathrm{~mm}$, with fluorescent indicator $\mathrm{UV}_{254}$ ) with detection by UV-absorption (short wave UV - $254 \mathrm{~nm}$; long wave UV $366 \mathrm{~nm}$ ) by dipping in $\mathrm{KMnO}_{4}$ in $\mathrm{H}_{2} \mathrm{O}$. Column chromatography was performed on Pure Science silica gel (40 - 63 micron). Infrared spectra were recorded as thin films using a Bruker Tensor 27 FTIR spectrometer equipped with an Attenuated Total Reflectance (ATR) sampling accessory and are reported in wavenumbers $\left(\mathrm{cm}^{-1}\right)$. Melting points (m.p.) were obtained on a DigiMelt MPA160 melting point appartus. UV data were obtained on an Agilent 8453 spectrometer. Nuclear magnetic resonance spectra were recorded at $20{ }^{\circ} \mathrm{C}$ in $\mathrm{D}_{2} \mathrm{O}, \mathrm{CDCl}_{3}$, pyridine- $\mathrm{d}_{5}$, or DMSO- $\mathrm{d}_{6}$ using either a Varian INOVA operating at $500 \mathrm{MHz}$ or Varian VNMRS operating at $600 \mathrm{MHz}$. Chemical shifts are given in $\mathrm{ppm}(\delta)$ relative to solvent residues. NMR peak assignments were made using COSY, HSQC, and HMBC 2D experiments. High-resolution (ESI) mass spectrometric data were obtained with an Agilent 6530 Accurate-Mass Q-TOF LC-MS equipped with a 1260 Infinity binary pump.<smiles>O=C(O)c1ccc2cccc(O)c2n1</smiles>

47

8-Hydroxy-2-quinolinic acid (47). In a round-bottomed flask was stirred 8-hydroxy-2-methyl-quinoline (225 mg, $1.4 \mathrm{mmol}$ ) and selenium dioxide (782 mg, $7.05 \mathrm{mmol}, 5$ equiv.) in pyridine $(10 \mathrm{~mL})$. The reaction was stirred at $90{ }^{\circ} \mathrm{C}$ for $20 \mathrm{~h}$, after which time the disappearance of the starting material was observed via TLC. The reaction was cooled and filtered over Celite and the solvent evaporated in vacuo. A solution of $\mathrm{NaOH}\left(2 \mathrm{M}\right.$ in $\left.\mathrm{H}_{2} \mathrm{O}\right)$ was then added to the brown residue, the resulting precipitate filtered, then the solution acidified with $2 \mathrm{M}$ aq. $\mathrm{HCl}$ 
to $\mathrm{pH}=4$. The product was then obtained by filtration as a brown solid $(51 \mathrm{mg}, 20 \%)$. The data obtained for this compound matched those reported in literature. ${ }^{106}$<smiles>Cc1ccc2c(n1)C(=O)C(Cl)=C(Cl)C2=O</smiles>

56

6,7-Dichloro-2-methyl-5,8-quinolinequinone (56). In a roundbottomed flask, 8-hydroxy-2-methyl-quinoline (14.5 g, $0.09 \mathrm{~mol}$ ) was dissolved in conc. $\mathrm{HCl}(600 \mathrm{~mL})$ and the reaction warmed to $40{ }^{\circ} \mathrm{C}$. Sodium chlorate ( $48.5 \mathrm{~g}, 0.45 \mathrm{~mol}, 5$ equiv.) was then slowly added to the reaction mixture over a period of $1 \mathrm{~h}$, after which time the reaction was stirred at room temperature for an additional $2 \mathrm{~h}$. The solution was then diluted to $1 \mathrm{~L}$ with $\mathrm{H}_{2} \mathrm{O}$ and the white precipitate filtered, the filtrate extracted with $\mathrm{CH}_{2} \mathrm{Cl}_{2}(6 \times 250 \mathrm{~mL})$ and the organic layers combined and concentrated in vacuo. The resulting bright orange solid was recrystallised in ethanol to provide the title compound as bright yellow crystals ( $6.20 \mathrm{~g}, 29 \%)$. The data obtained for this compound matched those reported in the literature. ${ }^{65}$<smiles>Cc1ccc2c(n1)C(=O)C(NCCCl)=C(Cl)C2=O</smiles>

59<smiles>Cc1ccc2c(n1)C(=O)C(Cl)=C(NCCCl)C2=O</smiles>

60
6-Chloro-7-(2-chloroethylamino)2-methyl quinolinequinone (59) and 7-chloro-6-(2chloroethylamino)-2-methyl quinolinequinone

Dichloroquinolinequinone $\mathbf{5 6}(2.5 \mathrm{~g}, 10 \mathrm{mmol})$ was dissolved in ethanol $(95 \mathrm{~mL})$ and water (48 $\mathrm{mL}$ ) before 2-chloroethylamine hydrochloride (1.7 g, $15 \mathrm{mmol}, 1.5$ equiv.) and $2 \mathrm{M}$ aq. $\mathrm{NaOH}$ (9 mL, $18 \mathrm{mmol}, 1.8$ equiv.) were added. The red solution was warmed to reflux and stirred for $1 \mathrm{~h}$, after which time TLC analysis indicated complete disappearance of the starting material. The solvent was removed in vacuo and the resulting red solid redissolved in $\mathrm{CH}_{2} \mathrm{Cl}_{2}$ $(100 \mathrm{~mL})$ and washed with sat. aq. $\mathrm{NaHCO}_{3}(100 \mathrm{~mL})$, followed by brine $(100 \mathrm{~mL})$ before being dried with anhydrous $\mathrm{MgSO}_{4}$, filtered and the filtrate concentrated in vacuo. Purification by silica gel column chromatography $\left(\mathrm{CH}_{2} \mathrm{Cl}_{2} / \mathrm{EtOAc}, 1 / 0 \rightarrow 3 / 1, \mathrm{v} / \mathrm{v}\right)$ allowed for the separatation of the two regioisomers giving $\mathbf{5 9}$ as red-orange crystals (1.6 g, 56\%) and $\mathbf{6 0}$ as dark red crystals $\left(1.1 \mathrm{~g}, 38 \%\right.$ ). Data for 59: $\mathrm{R}_{f}=0.43$ (EtOAc/petroleum ether, 1/1, v/v); m.p. $=230.9{ }^{\circ} \mathrm{C}$; UV-Vis $(\mathrm{MeOH}) \lambda_{\max }(\log \varepsilon): 465$ (3.51) nm; IR (U max $): 3310,1694,1598,1575$, $1557,1505,1450,1323,1248,1192,1147,1111,1092,850,818,737,566,466,423 \mathrm{~cm}^{-1} ;{ }^{1} \mathrm{H}$ NMR $\left(500 \mathrm{MHz}, \mathrm{CDCl}_{3}\right): \delta \mathrm{ppm} 8.35\left(\mathrm{~d}, J_{3,4}=8.0 \mathrm{~Hz}, 1 \mathrm{H}, \mathrm{H} 4\right), 7.52\left(\mathrm{~d}, J_{3,4}=8.0 \mathrm{~Hz}, 1 \mathrm{H}\right.$, 
H3), 6.36 (br. s, 1H, NH), 4.21 (q, $\left.J_{2^{\prime}, 3^{\prime}}=6.1 \mathrm{~Hz}, 2 \mathrm{H}, \mathrm{H} 2^{\prime}\right), 3.79$ (t, $\left.J_{2^{\prime}, 3^{\prime}}=5.7 \mathrm{~Hz}, 2 \mathrm{H}, \mathrm{H} 3^{\prime}\right), 2.74$ (s, 3H, $\left.\mathrm{CH}_{3}\right) ;{ }^{13} \mathrm{C}$ NMR (125 MHz, $\mathrm{CDCl}_{3}$ ) $\delta$ ppm 179.0 (C8), 176.1 (C5), 164.0 (C2), 145.6 (C8a), 144.3 (C7), 135.0 (C4), 128.6 (C3), 127.5 (C4a), 46.2 (C2'), 44.2 (C3'), $25.1\left(\mathrm{CH}_{3}\right)$; HRMSESI $(m / 2)$ calcd. for $\left[\mathrm{C}_{12} \mathrm{H}_{10} \mathrm{Cl}_{2} \mathrm{~N}_{2} \mathrm{O}_{2}+\mathrm{H}\right]^{+}: 285.0192$, obsd.: 285.0192. Data for 60: $\mathrm{R}_{f}=0.31$ $\left(1 / 1\right.$, EtOAc/petroleum ether, v/v); m.p. $=154.9^{\circ} \mathrm{C} ; \mathrm{IR}\left(\mathrm{u}_{\max }\right): 3337,1666,1600.2,1581,1518$, 1451, 1313, 1227, 1143, 1074, 931, 841, 752, 701, 643, 565, 471, $436 \mathrm{~cm}^{-1} ;{ }^{1} \mathrm{H}$ NMR $(500 \mathrm{MHz}$, $\left.\mathrm{CDCl}_{3}\right) \delta$ ppm 8.19 (d, J3,4 $\left.=8.2 \mathrm{~Hz}, 1 \mathrm{H}, \mathrm{H} 4\right), 7.42$ (d, J3,4 $\left.=7.9 \mathrm{~Hz}, 1 \mathrm{H}, \mathrm{H} 3\right), 6.31$ (br. s, $1 \mathrm{H}$, $\mathrm{NH}), 4.18\left(\mathrm{q}, J_{2^{\prime}, 3^{\prime}}=6.0 \mathrm{~Hz}, 2 \mathrm{H}, \mathrm{H} 2^{\prime}\right), 3.77\left(\mathrm{t}, J_{2^{\prime}, 3^{\prime}}=5.7 \mathrm{~Hz}, 2 \mathrm{H}, \mathrm{H} 3^{\prime}\right), 2.72\left(\mathrm{CH}_{3}\right) ;{ }^{13} \mathrm{C} \mathrm{NMR}$ (125 MHz, $\mathrm{CDCl}_{3}$ ) $\delta$ ppm 179.8 (C5), 175.6 (C8), 166.1 (C2), 147.8 (C8a), 143.3 (C6), 135.0 (C4), 126.7 (C3), 124.7 (C4a), $46.0\left(\mathrm{C}^{\prime}\right), 44.2\left(\mathrm{C}^{\prime}\right), 25.5\left(\mathrm{CH}_{3}\right)$ : HRMS-ESI ( $\left.\mathrm{m} / \mathrm{z}\right)$ calcd. for $\left[\mathrm{C}_{12} \mathrm{H}_{10} \mathrm{Cl}_{2} \mathrm{~N}_{2} \mathrm{O}_{2}+\mathrm{H}\right]^{+}:$285.0192, obsd.: 285.0191.

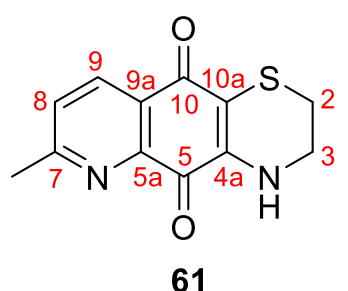

61

7-Methyl-2,3-dihydro-4H-[1,4] thiazino[2,3-g]quinoline-5,10dione (61). To a solution of QQ 59 (115 mg, $0.40 \mathrm{mmol})$ in EtOH (30 $\mathrm{mL}$ ) was added dropwise a solution of sodium sulfide (159 mg, 1.22 mmol, 3 equiv.) in $\mathrm{H}_{2} \mathrm{O}(15 \mathrm{~mL})$. The solution was stirred for $30 \mathrm{~min}$ at room temperature, after which time TLC analysis showed the disappearance of starting materials. The dark purple liquid was then diluted with water (100 $\mathrm{mL})$ and extracted with $\mathrm{CH}_{2} \mathrm{Cl}_{2}(2 \times 100 \mathrm{~mL})$ before being dried with anhydrous $\mathrm{MgSO}_{4}$, filtered and the filtrate concentrated in vacuo. Purification by silica gel flash column chromatography (petroleum ether/EtOAc, $3 / 1 \rightarrow 1 / 3$, v/v) yielded the title compound as purple crystals $(86 \mathrm{mg}, 86 \%) . \mathrm{R}_{f}=0.38(\mathrm{EtOAc}) ;$ m.p. $=259^{\circ} \mathrm{C}$; UV-vis $(\mathrm{MeOH}) \lambda_{\max }(\log \varepsilon)$ : $553 \mathrm{~nm}$ (3.42); IR (Umaz): 3358, 1676, 1620, 1592, 1574, 1547, 1488, 1204, 1153, 1119, 816, 733 , 538, 468, $413 \mathrm{~cm}^{-1}$; ${ }^{1} \mathrm{H}$ NMR $\left(500 \mathrm{MHz}, \mathrm{CDCl}_{3}\right) \delta \mathrm{ppm} 8.22$ (d, J8,9 $\left.=7.7 \mathrm{~Hz}, 1 \mathrm{H}, \mathrm{H} 9\right), 7.41$ (d, $J_{8,9}=7.9 \mathrm{~Hz}, 1 \mathrm{H}, \mathrm{H} 8$ ), 6.17 (br. s, 1H, NH), 3.79 (t, $J_{2,3}=4.3 \mathrm{~Hz}, 2 \mathrm{H}, \mathrm{H} 3$ ), 3.01 (q, $J_{2,3}=4.1$ $\mathrm{Hz}, 2 \mathrm{H}, \mathrm{H} 2), 2.69$ (s, 3H, $\left.\mathrm{CH}_{3}\right) ;{ }^{13} \mathrm{C} \mathrm{NMR}\left(125 \mathrm{MHz}, \mathrm{CDCl}_{3}\right) \delta \mathrm{ppm} 178.6$ (C5), 176.6 (C10), 163.1 (C7), 146.3 (C5a), 141.3 (C4a), 134.3 (C9), 128.4 (C9a), 127.8 (C8), 111.1 (C10a), 42.0 (C3), $25.0\left(\mathrm{CH}_{3}\right), 23.9$ (C2); HRMS-ESI (m/z) calcd. for $\left[\mathrm{C}_{12} \mathrm{H}_{10} \mathrm{~N}_{2} \mathrm{O}_{2} \mathrm{~S}+\mathrm{H}\right]^{+}$: 247.0536, obsd.: 247.0524 . 


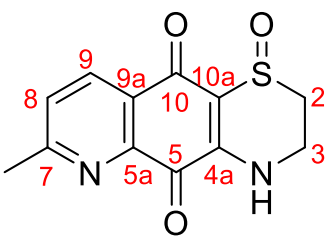

63
1-Oxo-7-methyl-2,3-dihydro-4H-[1,4] thiazino[2,3-g]quinoline5,10-dione (63). Thiazine 61 (95 $\mathrm{mg}, 0.39 \mathrm{mmol}$ ) was dissolved in freshly distilled $\mathrm{CH}_{2} \mathrm{Cl}_{2}(3.9 \mathrm{~mL})$ and cooled to $0{ }^{\circ} \mathrm{C}$ before $m$-CPBA (67 mg, $0.39 \mathrm{mmol}$ ) was added. The reaction was allowed to warm to room temperature and stirred for $30 \mathrm{~min}$ before TLC analysis indicated the consumption of starting materials and the presence of sulfoxide 63. The bright yellow solution was then concentrated in vacuo and purified by silica gel flash column chromatography $\left(\mathrm{CH}_{2} \mathrm{Cl}_{2} / \mathrm{MeOH}, 1 / 0 \rightarrow 4 / 1, \mathrm{v} / \mathrm{v}\right)$ to give the title compound as a yellow amorphous solid (45 $\mathrm{mg}, 44 \%) . \mathrm{R}_{f}=0.10(\mathrm{EtOAc} / \mathrm{MeOH}, 4 / 1, \mathrm{v} / \mathrm{v})$; IR (umax $): 2931,2830,1677,1603,1577,1508$, 1307, 1251, 1220, 1177, 1143, 1110, 1061, 1024, 842, 748, 701, 644, 554, $420 \mathrm{~cm}^{-1} ;{ }^{1} \mathrm{H}$ NMR $\left(500 \mathrm{MHz}, \mathrm{CDCl}_{3}\right) \delta$ ppm 8.45 (d, $\left.J_{8,9}=8.7 \mathrm{~Hz}, 1 \mathrm{H}, \mathrm{H} 9\right), 7.60$ (d, $\left.J_{8,9}=8.9 \mathrm{~Hz}, 1 \mathrm{H}, \mathrm{H} 8\right), 7.28$ (br. s, $1 \mathrm{H}, \mathrm{NH}$ ), 4.11 (t, $\left.J_{3 \mathrm{a}, 3 \mathrm{~b}}=15.3 \mathrm{~Hz}, 1 \mathrm{H}, \mathrm{H3a}\right), 3.97$ (d, J3a,3b $\left.=15.0 \mathrm{~Hz}, 1 \mathrm{H}, \mathrm{H} 3 \mathrm{~b}\right), 3.28$ (d, $\left.J_{2 \mathrm{a}, 2 \mathrm{~b}}=14.2 \mathrm{~Hz}, 1 \mathrm{H}, \mathrm{H} 2 \mathrm{a}\right), 2.76\left(\mathrm{~s}, 3 \mathrm{H}, \mathrm{CH}_{3}\right), 2.50\left(\mathrm{t}, J_{2 \mathrm{a}, 2 \mathrm{~b}}=14.5 \mathrm{~Hz}, 1 \mathrm{H}, \mathrm{H} 2 \mathrm{~b}\right) ;{ }^{13} \mathrm{C}$ NMR $(125$ MHz, $\mathrm{CDCl}_{3}$ ) $\delta$ ppm 178.7 (C5), 177.6 (C10), 164.4 (C7), 145.7 (C5a), 135.5 (C9), 129.5 (C8), 128.5 (C9a), 112.4 (C10a), 41.3 (C2), 31.2 (C3), $25.22\left(\mathrm{CH}_{3}\right)$; HRMS-ESI $(\mathrm{m} / \mathrm{z})$ calcd. for $\left[\mathrm{C}_{12} \mathrm{H}_{10} \mathrm{~N}_{2} \mathrm{O}_{3} \mathrm{~S}+\mathrm{H}\right]^{+}:$263.0485, obsd.: 263.0482 .

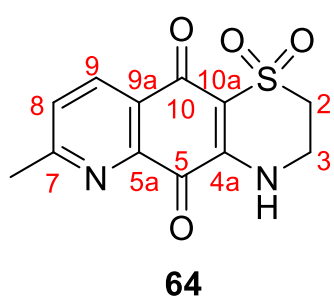

formation of sulfone 64 . The bright yellow solution was then concentrated in vacuo and purified by silica gel flash column chromatography $\left(\mathrm{CH}_{2} \mathrm{Cl}_{2} / \mathrm{MeOH}, 1 / 0 \rightarrow 4 / 1, \mathrm{v} / \mathrm{v}\right)$ to give the title compound as a yellow amorphous solid (50 mg, 86\%). $\mathrm{R}_{f}=0.20$ (EtOAc/MeOH, 4/1, v/v); IR (U $\left.u_{\max }\right): 2918,2853,1689,1635,1590,1578,1370,1329,1297,1242,1197,1130,1069,954$, 742, $\mathrm{cm}^{-1} ;{ }^{1} \mathrm{H}$ NMR $(500 \mathrm{MHz}$, pyridine-d 6 ) $\delta$ ppm 10.47 (br. s, $1 \mathrm{H}, \mathrm{NH}), 8.42$ (d, J8,9 $=8.1 \mathrm{~Hz}$, 1H, H9), 7.40 (d, $\left.J_{8,9}=8.1 \mathrm{~Hz}, 1 \mathrm{H}, \mathrm{H} 8\right), 4.27$ (m, 2H, H2), 3.73-3.71 (m, 2H, H3), 2.58 (s, 3H, $\left.\mathrm{CH}_{3}\right) ;{ }^{13} \mathrm{C}$ NMR (125 MHz, pyridine-d ${ }_{5}$ ) $\delta$ ppm 178.3 (C5), 175.5 (C10), 163.6 (C7), 148.(C4a), 146.7 (C5a), 135.0 (C9), 129.0 (C8), 112.6 (C10a), 49.8 (C2), 40.4 (C3), 24.9 ( CH $_{3}$ ); HRMS-ESI $(m / z)$ calcd. for $\left[\mathrm{C}_{12} \mathrm{H}_{10} \mathrm{~N}_{2} \mathrm{O}_{4} \mathrm{~S}+\mathrm{H}\right]^{+}: 279.0434$, obsd.: 279.0433 . 
<smiles>O=C(O)c1ccc2c(n1)C(=O)C1=C(SCCN1)C2=O</smiles>

65

2,3-Dihydro-4H-[1,4] thiazino[2,3-g] quinoline-5,10-dione-7carboxylic acid (65). Thiazine $\mathbf{6 1}(20 \mathrm{mg}, 0.080 \mathrm{mmol})$ was dissolved in freshly distilled pyridine $(0.76 \mathrm{~mL})$ to which $\mathrm{SeO}_{2}(40$ $\mathrm{mg}, 0.37 \mathrm{mmol}, 4.5$ equiv.) was then added. The reaction was warmed to $90{ }^{\circ} \mathrm{C}$ and stirred overnight before TLC analysis showed the disappearance of starting materials. The solution was then filtered through a pad of Celite and washed with EtOH $(5 \mathrm{~mL})$. The resulting filtrate was then concentrated in vacuo to give a dark purple residue that was re-dissolved in $\mathrm{H}_{2} \mathrm{O}$. The $\mathrm{pH}$ of the solution was then increased to 10 by the addition of $2 \mathrm{M}$ aq. $\mathrm{NaOH}$ and the reaction was washed with $\mathrm{CH}_{2} \mathrm{Cl}_{2}$ (3 $\mathrm{x} 10 \mathrm{~mL}$ ). The aqueous layer was then collected and acidified to $\mathrm{pH} 1$ with $2 \mathrm{M}$ aq. $\mathrm{HCl}$ before the product was extracted with EtOAc $(3 \times 10 \mathrm{~mL})$. The organic layers were collected, dried with anhydrous $\mathrm{MgSO}_{4}$, filtered, and the filtrate concentrated in vacuo to give the title compound as a dark purple amorphous solid (19 mg, 83\%). $\mathrm{R}_{f}=0.02$ (EtOAc); IR (U max): 3369, 2921, 1732, 1694, 1582, 1544, 1495, 1450, 1337, 1261, 1220, 1161, 982, 835, 721, 553, $440 \mathrm{~cm}^{-}$ '; ${ }^{1} \mathrm{H}$ NMR (500 MHz, DMSO-d 6 ) $\delta$ ppm 8.34 (d, $\left.J_{8,9}=8.0 \mathrm{~Hz}, 1 \mathrm{H}, \mathrm{H} 9\right), 8.27$ (d, J8,9 $=8.0 \mathrm{~Hz}$, 1H, H8), 8.15 (br. s, $1 \mathrm{H}, \mathrm{NH}), 3.66-3.63$ (m, 2H, H3), 2.99-2.97 (m, 2H, H2); ${ }^{13} \mathrm{C}$ NMR (125 MHz, DMSO-d 6 ) $\delta$ ppm 176.7 (C5), 175.8 (C10), 165.8 (CO $\left.{ }_{2} \mathrm{H}\right), 151.0$ (C7), 147.0 (C5a), 143.7 (C4a), 134.9 (C9), 132.3 (C9a), 129.0 (C8), 109.2 (C10a), 42.2 (C3), 23.1 (C2); HRMS-ESI (m/ z) calcd. for $\left[\mathrm{C}_{12} \mathrm{H}_{8} \mathrm{~N}_{2} \mathrm{O}_{4} \mathrm{~S}+\mathrm{H}\right]^{+}: 277.0278$, obsd.: 277.0277 , and for $\left[\mathrm{C}_{12} \mathrm{H}_{8} \mathrm{~N}_{2} \mathrm{O}_{4} \mathrm{~S}-\mathrm{H}\right]^{-}: 275.0132$, obsd.: 275.0135 .<smiles>O=C(O)c1ccc2c(n1)C(=O)C1=C(NCCS1=O)C2=O</smiles>

66

1-Oxo-2,3-dihydro-4H-[1,4] thiazino[2,3-g]quinoline-5,10dione-7-carboxylic acid (66). Acid 65 (9 mg, $0.03 \mathrm{mmol})$ was dissolved in acetone $(0.09 \mathrm{~mL})$ and cooled to $0{ }^{\circ} \mathrm{C}$ before $m$-CPBA (5.2 $\mathrm{mg}, 0.03 \mathrm{mmol})$ was added. The bright yellow solution was allowed to warm to room temperature and stirred for $30 \mathrm{~min}$ before TLC analysis showed the complete disappearance of starting materials. The solution was then concentrated in vacuo, re-dissolved in $\mathrm{H}_{2} \mathrm{O}(1 \mathrm{~mL})$ and washed with EtOAc $(3 \times 1 \mathrm{~mL})$. The aqueous solution was then concentrated to give the title compound as a yellow solid $(9.2$ mg, 94\% yield). $\mathrm{R}_{f}=0.18(\mathrm{MeOH} / \mathrm{EtOAc}, 4 / 1, \mathrm{v} / \mathrm{v}) ; \mathrm{IR}\left(\mathrm{u}_{\max }\right): 3375,2933,1603,1578,1379$, $1330,1252,1184,1110,1023,950,844,640,618,570 \mathrm{~cm}^{-1} ;{ }^{1} \mathrm{H}$ NMR $\left(600 \mathrm{MHz}, \mathrm{D}_{2} \mathrm{O}\right) \delta \mathrm{ppm}$ $8.65\left(\mathrm{~d}, J_{8,9}=8.1 \mathrm{~Hz}, 1 \mathrm{H}, \mathrm{H} 9\right), 8.31\left(\mathrm{~d}, J_{8,9}=8.1 \mathrm{~Hz}, 1 \mathrm{H}, \mathrm{H} 8\right), 4.17\left(\mathrm{dt}, J_{3 \mathrm{a}, 3 \mathrm{~b}}=15.1 \mathrm{~Hz}, J_{2 \mathrm{a}, 3 \mathrm{a}}=3.3\right.$ $\mathrm{Hz}, 1 \mathrm{H}, \mathrm{H} 3 \mathrm{a}), 3.81\left(\mathrm{td}, J_{3 \mathrm{a}, 3 \mathrm{~b}}=14.9 \mathrm{~Hz}, J_{2 \mathrm{~b}, 3 \mathrm{~b}}=2.9 \mathrm{~Hz}, 1 \mathrm{H}, 3 \mathrm{~b}\right), 3.47\left(\mathrm{dt}, J_{2 \mathrm{a}, 2 \mathrm{~b}}=14.7 \mathrm{~Hz}, J_{2 \mathrm{~b}, 3 \mathrm{~b}}\right.$ $=2.6 \mathrm{~Hz}, 1 \mathrm{H}, \mathrm{H} 2 \mathrm{~b}), 2.78\left(\mathrm{td}, J_{2 \mathrm{a}, 2 \mathrm{~b}}=14.6 \mathrm{~Hz}, J_{2 \mathrm{a}, 3 \mathrm{a}}=3.9 \mathrm{~Hz}, 1 \mathrm{H}, \mathrm{H} 2 \mathrm{a}\right) ;{ }^{13} \mathrm{C} \mathrm{NMR}(150 \mathrm{MHz}$, 
$\left.\mathrm{D}_{2} \mathrm{O}\right) \delta$ ppm 178.5 (C10), 177.8 (C5), $157.5\left(\mathrm{CO}_{2} \mathrm{H}\right), 147.2$ (C7 or C5a), 145.8 (C7 or C5a), 136.6 (C9), 130.1 (C9a), 128.6 (C8), 107.7 (C10a), 39.6 (C2), 30.7 (C3); HRMS-ESI (m/ g) calcd. for: $\left[\mathrm{C}_{12} \mathrm{H}_{8} \mathrm{~N}_{2} \mathrm{O}_{5} \mathrm{~S}-\mathrm{H}\right]: 291.0081$, found: 291.0092 .<smiles>O=C(O)c1ccc2c(n1)C(=O)C1=C(C2=O)S(=O)(=O)CCN1</smiles>

27

Ascidiathiazone A (27). Procedure A: To a solution of tricyclic acid $65(15 \mathrm{mg}, 0.05 \mathrm{mmol})$ in $\mathrm{H}_{2} \mathrm{O}(0.25 \mathrm{~mL})$ was added $\mathrm{H}_{2} \mathrm{O}_{2}$ $(28 \%, 0.025 \mathrm{~mL})$. The reaction was stirred for $2 \mathrm{~h}$ before $\mathrm{H}_{2} \mathrm{O}_{2}$ $(28 \%, 0.0125 \mathrm{~mL})$ and $\mathrm{AcOH}(0.25 \mathrm{~mL})$ were added. The yellow solution was then warmed to $70{ }^{\circ} \mathrm{C}$ for $3 \mathrm{~h}$. The resulting yellow solid was collected by filtration and dried in a dessicator to give the title compound $(8.0 \mathrm{mg}$, $52 \%$ ) as a yellow amorphous solid. Procedure B: Selenium dioxide (110 mg, $0.99 \mathrm{mmol}, 4.5$ equiv.) was added to a solution of sulfone $64(60 \mathrm{mg}, 0.22 \mathrm{mmol})$ pyridine $(2.1 \mathrm{~mL})$. The reaction was warmed to $90{ }^{\circ} \mathrm{C}$ and stirred for $24 \mathrm{~h}$, after which time TLC analysis indicated the consumption of the starting materials and the formation of the 27 . The yellow solution was filtered through Celite and concentrated in vacuo to give a yellow solid, which was then redissolved in $\mathrm{H}_{2} \mathrm{O}$ and carefully neutralised with glacial $\mathrm{AcOH}$ before being concentrated in vacuo. Purification by silica gel flash column chromatography $\left(\mathrm{CH}_{2} \mathrm{Cl}_{2} / \mathrm{MeOH}, 99 / 1 \rightarrow 4 / 1\right.$, $\mathrm{v} / \mathrm{v})$ yielded the title compound as a yellow amorphous solid $(8 \mathrm{mg}, 12 \%) . \mathrm{R}_{f}=0.62(\mathrm{MeOH})$; IR (thin film): 2997, 1728, 1595, 1557, 1349, 1301, 1270, 1210, 1168, 1106, 958, 827, 726, 640, 563, 478, $429 \mathrm{~cm}^{-1} ;{ }^{1} \mathrm{H}$ NMR (500 MHz, DMSO-d 6 ): $\delta$ ppm 9.44 (br. s, $1 \mathrm{H}, \mathrm{NH}$ ), 8.51 (d, J8, $=8.0 \mathrm{~Hz}, 1 \mathrm{H}, \mathrm{H} 9), 8.38$ (d, $\left.J_{8,9}=8.0 \mathrm{~Hz}, 1 \mathrm{H}, \mathrm{H} 9\right), 3.89-3.41$ (m, 2H, H3), 3.40-3.39 (m, 2H, H2); ${ }^{13} \mathrm{C}$ NMR (150 MHz, DMSO-d 6 ) $\delta$ ppm 176.4 (C5), 173.4 (C10), $165.3\left(\mathrm{CO}_{2} \mathrm{H}\right), 151.7$ (C7), 147.8 (C4a), 146.3 (C5a), 135.7 (C9), 131.5 (C9a), 129.0 (C8), 110.8 (C10a), 48.2 (C2), 40.1 (C3); HRMS-ESI ( $m / 2$ ): [ $\left.\mathrm{C}_{12} \mathrm{H}_{8} \mathrm{~N}_{2} \mathrm{O}_{6} \mathrm{~S}-\mathrm{H}\right]^{-}$calcd.: 307.0030, obsd.: 307.0034. The data obtained for this compound matched those reported in literature. ${ }^{104}$

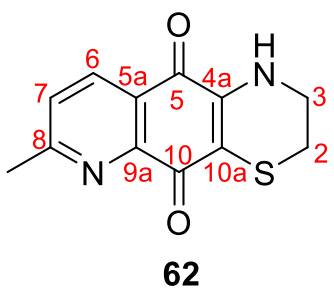

8-Methyl-2,3-dihydro-4H-[1,4] thiazino[3,2-g]quinoline-5,10dione (62). To a solution of QQ 60 (112.5 mg, $0.39 \mathrm{mmol})$ in EtOH $(28 \mathrm{~mL})$ was added a solution of sodium sulfide $(153 \mathrm{mg}, 1.17 \mathrm{mmol}, 3$ equiv.) in $\mathrm{H}_{2} \mathrm{O}(14 \mathrm{~mL})$ dropwise. The solution was stirred for $30 \mathrm{~min}$ at room temperature, after which time TLC analysis showed the disappearance of starting materials. The dark purple liquid was then diluted with water (100 
$\mathrm{mL})$ and extracted with $\mathrm{CH}_{2} \mathrm{Cl}_{2}(2 \times 100 \mathrm{~mL})$ before being dried with anhydrous $\mathrm{MgSO}_{4}$, filtered and the filtrate concentrated in vacuo. Purification by silica gel flash column chromatography (petroleum ether/EtOAc, $3 / 1 \rightarrow 1 / 3$, v/v) yielded the title compound as purple crystals $(81 \mathrm{mg}, 82 \%) . \mathrm{R}_{f}=0.38(\mathrm{EtOAc}) ; \mathrm{m} . \mathrm{p} .=268{ }^{\circ} \mathrm{C}$; UV-vis $(\mathrm{MeOH}) \lambda_{\max }(\log \varepsilon)$ : $550 \mathrm{~nm}$ (3.40); IR (Umaz): 1672, 1594, 1574, 1550, 1478, 1200, 1152, 1119, 812, 730, 538, 485 $\mathrm{cm}^{-1} ;{ }^{1} \mathrm{H}$ NMR $\left(500 \mathrm{MHz}, \mathrm{CDCl}_{3}\right) \delta$ ppm $8.22\left(\mathrm{~d}, J_{6,7}=7.7 \mathrm{~Hz}, 1 \mathrm{H}, \mathrm{H} 6\right), 7.41$ (d, J6,7 $=7.9 \mathrm{~Hz}$, 1H, H7), 6.17 (br. s, 1H, NH), 3.79 (t, $J_{2,3}=4.3 \mathrm{~Hz}, 2 \mathrm{H}, \mathrm{H} 3$ ), 3.01 (q, $J_{2,3}=4.1 \mathrm{~Hz}, 2 \mathrm{H}, \mathrm{H} 2$ ), 2.69 (s, 3H, $\left.\mathrm{CH}_{3}\right) ;{ }^{13} \mathrm{C}$ NMR (125 MHz, $\mathrm{CDCl}_{3}$ ) $\delta$ ppm 178.6 (C5), 176.6 (C10), 163.1 (C8), 146.3 (C9a), 141.3 (C4a), 134.3 (C6), 128.4 (C5a), 127.8 (C7), 111.1 (C10a), 42.0 (C3), 25.0 $\left(\mathrm{CH}_{3}\right)$, 23.9 (C2); HRMS-ESI (m/ z) calcd. for $\left[\mathrm{C}_{12} \mathrm{H}_{10} \mathrm{~N}_{2} \mathrm{O}_{2} \mathrm{~S}+\mathrm{H}\right]^{+}:$247.0536, obsd.: 247.0524.

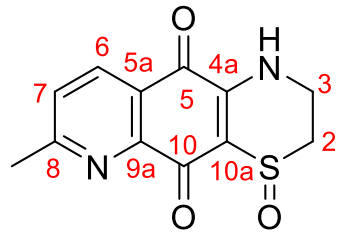

67

1-Oxo-8-methyl-2,3-dihydro- $4 H$-[1,4] thiazino[3,2-g]quinoline5,10-dione (67). Thiazine $62(60 \mathrm{mg}, 0.24 \mathrm{mmol})$ was dissolved in freshly distilled $\mathrm{CH}_{2} \mathrm{Cl}_{2}(2.4 \mathrm{~mL})$ and cooled to $0{ }^{\circ} \mathrm{C}$ in an ice-bath before $m$-CPBA (41 mg, $0.24 \mathrm{mmol}$ ) was added. The reaction was allowed to warm to room temperature and then stirred for $30 \mathrm{~min}$ before TLC analysis deemed the reaction complete. The bright yellow solution was then concentrated in vacuo, re-dissolved in $\mathrm{H}_{2} \mathrm{O}(5 \mathrm{~mL})$, and washed with EtOAc $(2 \times 10 \mathrm{~mL})$ to give the title compound as a yellow solid (38 mg, 60\%). $\mathrm{R}_{f}=0.4\left(\mathrm{CH}_{2} \mathrm{Cl}_{2} / \mathrm{MeOH}, 4 / 1, \mathrm{v} / \mathrm{v}\right)$; IR $\left(\nu_{\max }\right): 3060,2855,1654,1577,1328,1151,1078,965 \mathrm{~cm}^{-1} ;{ }^{1} \mathrm{H}$ NMR $\left(500 \mathrm{MHz}, \mathrm{D}_{2} \mathrm{O}\right) \delta \mathrm{ppm}$ $8.01\left(\mathrm{~d}, J_{6,7}=8.0 \mathrm{~Hz}, 1 \mathrm{H}, \mathrm{H} 6\right), 7.49$ (d, $\left.J_{6,7}=8.0 \mathrm{~Hz}, 1 \mathrm{H}, \mathrm{H} 7\right), 4.06\left(\mathrm{dt}, J_{3 \mathrm{a}, 3 \mathrm{~b}}=15.2 \mathrm{~Hz}, J_{2 \mathrm{a}, 3 \mathrm{a}}=\right.$ $3.2 \mathrm{~Hz}, 1 \mathrm{H}, \mathrm{H} 3 \mathrm{a}), 3.65$ (td, $\left.J_{3 \mathrm{a}, 3 \mathrm{~b}}=14.9 \mathrm{~Hz}, J_{2 \mathrm{~b}, 3 \mathrm{~b}}=2.5 \mathrm{~Hz}, 1 \mathrm{H}, \mathrm{H} 3 \mathrm{~b}\right), 3.40$ (dt, $J_{2 \mathrm{a}, 2 \mathrm{~b}}=14.7 \mathrm{~Hz}$, $\left.J_{2 \mathrm{~b}, 3 \mathrm{~b}}=2.6 \mathrm{~Hz}, 1 \mathrm{H}, \mathrm{H} 2 \mathrm{~b}\right), 2.74\left(\mathrm{td}, J_{2 \mathrm{a}, 2 \mathrm{~b}}=14.6 \mathrm{~Hz}, J_{2 \mathrm{a}, 3 \mathrm{a}}=3.9 \mathrm{~Hz}, 1 \mathrm{H}, \mathrm{H} 2 \mathrm{a}\right), 2.56$ (s, 3H, CH $\mathrm{CH}_{3}$; ${ }^{13} \mathrm{C}$ NMR (150 MHz, D $\left.2 \mathrm{O}\right): \delta$ ppm 178.2 (C5), 177.0 (C10), 166.6 (C8), 146.7 C9a), 145.9 (C4a), 135.4 (C6), 128.1 (C7), 125.0 (C5a), 108.3 (C10a), 39.7 (C2), 30.6 (C3), 23.8 ( CH $\left._{3}\right)$; HRMS-ESI $(m / z)$ calcd. for $\left[\mathrm{C}_{12} \mathrm{H}_{10} \mathrm{~N}_{2} \mathrm{O}_{3} \mathrm{~S}+\mathrm{H}\right]^{+}: 263.0485$, obsd.: 263.0486 .

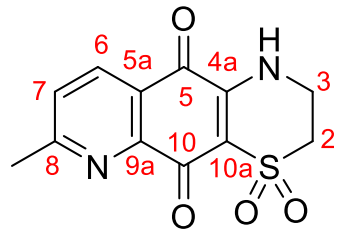

68

1,1-Dioxo-8-methyl-2,3-dihydro-4H-[1,4] thiazino[3,2g]quinoline-5,10-dione (68). To a solution of thiazine $62(128 \mathrm{mg}$, $0.52 \mathrm{mmol})$ in $\mathrm{CH}_{2} \mathrm{Cl}_{2}(7.8 \mathrm{~mL})$ was added $m$-CPBA (189 mg, 1.1 $\mathrm{mmol})$. The reaction was stirred for $20 \mathrm{~h}$ at room temperature after which time TLC analysis showed the disappearance of starting materials. The bright yellow solution was the concentrated in vacuo to give a yellow solid, which was subsequently purified by silica gel column chromatography $\left(\mathrm{CH}_{2} \mathrm{Cl}_{2} / \mathrm{MeOH}, 1 / 0 \rightarrow 19 / 1\right.$, 
$\mathrm{v} / \mathrm{v}$ ) to yield the title compound as a bright yellow amorphous solid (141 mg, 98\%). $\mathrm{R}_{f}=0.5$ $\left(\mathrm{CH}_{2} \mathrm{Cl}_{2} / \mathrm{MeOH}, 4 / 1, \mathrm{v} / \mathrm{v}\right)$; IR (U $\left.\mathrm{U}_{\max }\right): 2915,2850,1690,1635,1592,1565,1382,1329,1290$, 1240, 1195, 1136, 960, 752, $705 \mathrm{~cm}^{-1} ;{ }^{1} \mathrm{H}$ NMR (500 MHz, $\left.\mathrm{D}_{2} \mathrm{O} / \mathrm{CD}_{3} \mathrm{OD}\right): \delta \mathrm{ppm} 8.37$ (d, J6,7 $=8.1 \mathrm{~Hz}, 1 \mathrm{H}, \mathrm{H} 6), 7.64$ (d, $\left.J_{6,7}=8.2 \mathrm{~Hz}, 1 \mathrm{H}, \mathrm{H} 7\right), 4.08-4.05(\mathrm{~m}, 2 \mathrm{H}, \mathrm{H} 3), 3.56-3.52(\mathrm{~m}, 2 \mathrm{H}$, $\mathrm{H} 2), 2.64$ (s, $\left.3 \mathrm{H}, \mathrm{CH}_{3}\right) ;{ }^{13} \mathrm{C} \mathrm{NMR}\left(125 \mathrm{MHz}, \mathrm{D}_{2} \mathrm{O} / \mathrm{CD}_{3} \mathrm{OD}\right.$ ): $\delta$ ppm 177.8 (C5 or C10), 175.10 (C5 or C10), 166.4 (C8), 147.9 (C4a), 146.8 (C9a), 135.5 (C6), 127.9 (C7), 125.1 (C5a), 47.9 (C2), 39.1 (C3), $23.7\left(\mathrm{CH}_{3}\right)$; HRMS-ESI $(\mathrm{m} / \mathrm{z})$ calcd. for $\left[\mathrm{C}_{12} \mathrm{H}_{10} \mathrm{~N}_{2} \mathrm{O}_{4} \mathrm{~S}+\mathrm{H}\right]^{+}:$279.0434, obsd.: 279.0433.

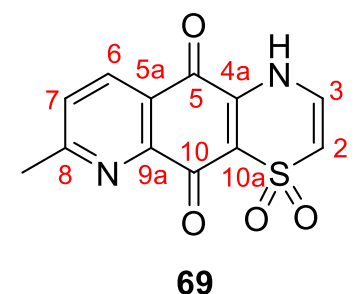

compound as a bright yellow amorphous solid (75 mg, 80\%). $\mathrm{R}_{f}=0.25\left(\mathrm{CH}_{2} \mathrm{Cl}_{2} / \mathrm{EtOAc}, 4 / 1\right.$, v/v); IR (Umax): 3464, 3054, 2853, 1689, 1664, 1636, 1594, 1570, 1508, 1437, 1374, 1329, 1285 , 1242, 1197, 1135, 1081, 964, 850, 751, 705, 603, $556 \mathrm{~cm}^{-1} ;{ }^{1} \mathrm{H}$ NMR (500 MHz, pyridine- $\left.\mathrm{d}_{5}\right): \delta$ $\operatorname{ppm} 8.36\left(\mathrm{~d}, J_{6,7}=8.0 \mathrm{~Hz}, 1 \mathrm{H}, \mathrm{H} 6\right), 7.51$ (d, $\left.J_{2,3}=8.9 \mathrm{~Hz}, 1 \mathrm{H}, \mathrm{H} 3\right), 7.43$ (d, $J_{6,7}=8.0 \mathrm{~Hz}, 1 \mathrm{H}$, $\mathrm{H} 7), 6.98\left(\mathrm{~d}, J_{2,3}=8.9 \mathrm{~Hz}, 1 \mathrm{H}, \mathrm{H} 2\right), 2.6\left(\mathrm{~s}, 3 \mathrm{H}, \mathrm{CH}_{3}\right) ;{ }^{13} \mathrm{C}$ NMR $\left(150 \mathrm{MHz}\right.$, pyridine- $\left.\mathrm{d}_{5}\right) \delta \mathrm{ppm}$ 179.5 (C5), 178.0 (C10), 166.8 (C8), 148.0 (C9a), 141.5 (C4a), 135.2 (C6), 131.2 (C3), 128.1 (C7), 126.5 (C5a), 119.1 (C10a), 113.5 (C2), $25.6\left(\mathrm{CH}_{3}\right)$; HRMS-ESI (m/z) calcd. for $\left[\mathrm{C}_{12} \mathrm{H}_{8} \mathrm{~N}_{2} \mathrm{O}_{4} \mathrm{~S}+\mathrm{H}\right]^{+}: 277.0278$, obsd.: 277.0277 .

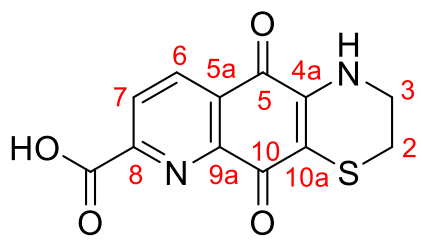

72
1,1-Dioxo-8-methyl-4H-[1,4] thiazino[3,2-g]quinoline-5,10-dione (69). Sulfone 68 (96 mg, $0.34 \mathrm{mmol}$ ) was dissolved in $1 \mathrm{M}$ aq. $\mathrm{KOH}$ $(10 \mathrm{~mL})$ and stirred for $3 \mathrm{~h}$ before being passed through a cationic ion exchange resin (BIO-REX 70, 200-400 mesh). The resulting yellow solution was concentrated in vacuo then freeze-dried to give the title 
and acidified to $\mathrm{pH} 1$ with $2 \mathrm{M} \mathrm{HCl}$ before the product was extracted with EtOAc $(3 \times 10$ $\mathrm{mL}$ ). The organic layers were collected and dried with anhydrous $\mathrm{MgSO}_{4}$, filtered, and the filtrate concentrated in vacuo to give the title compound as a dark purple amorphous solid (48 $\mathrm{mg}, 58 \%) \cdot \mathrm{R}_{f}=0.04$ (EtOAc); IR ( $\left.\nu_{\max }\right): 3369,2922,1732,1694,1582,1544,1495,1450,1337$, 1261, 1221, 1161, 982, 835, 721, 553, $440 \mathrm{~cm}^{-1}$; ${ }^{1} \mathrm{H}$ NMR (500 MHz, DMSO-d 6 ) $\delta$ ppm 8.39 (d, $\left.J_{6,7}=7.9 \mathrm{~Hz}, 1 \mathrm{H}, \mathrm{H} 6\right), 8.21$ (d, $\left.J_{6,7}=7.9 \mathrm{~Hz}, 1 \mathrm{H}, \mathrm{H} 7\right), 7.98$ (t, $J_{\mathrm{NH}, 3}=3.3 \mathrm{~Hz}, 1 \mathrm{H}, \mathrm{NH}$ ), 3.63-3.61 (m, 2H, H3), 3.00-2.98 (m, 2H, H2); ${ }^{13} \mathrm{C}$ NMR (125 MHz, DMSO-d 6 ) $\delta$ ppm 177.3 (C5 or C10), 176.3 (C5 or C10), $165.8\left(\mathrm{CO}_{2} \mathrm{H}\right), 152.2$ (C8), 148.8 (C9a), 142.3 (C4a), 135.8 (C6), 129.6 (C5a), 127.5 (C7), 111.8 (C10a), 41.8 (C3), 23.3 (C2); HRMS-ESI (m/z) calcd. for $\left[\mathrm{C}_{12} \mathrm{H}_{8} \mathrm{~N}_{2} \mathrm{O}_{4} \mathrm{~S}+\mathrm{H}\right]^{+}: 277.0278$, obsd.: 277.0277 , and for $\left[\mathrm{C}_{12} \mathrm{H}_{8} \mathrm{~N}_{2} \mathrm{O}_{4} \mathrm{~S}-\mathrm{H}\right]: 275.0132$, obsd.: 275.0135 .<smiles>O=C(O)c1ccc2c(n1)C(=O)C1=C(NCCS1=O)C2=O</smiles>

73
1-Oxo-2,3-dihydro-4H-[1,4] thiazino[3,2-g]quinoline-5,10dione-8-carboxylic acid (73). Acid $72(19 \mathrm{mg}, 0.07 \mathrm{mmol})$ was dissolved in acetone $(0.2 \mathrm{~mL})$ and cooled to $0{ }^{\circ} \mathrm{C}$ before $m$-CPBA (12 $\mathrm{mg}, 0.07 \mathrm{mmol}$ ) was added. The ice bath was then removed and the reaction was stirred for $30 \mathrm{~min}$ before TLC analysis showed the complete disappearance of starting materials. The solution was concentrated in vacuo, re-dissolved in $\mathrm{H}_{2} \mathrm{O}(1 \mathrm{~mL})$ and washed with EtOAc $(3 \times 1 \mathrm{~mL})$. The aqueous solution was then concentrated to give the title compound as a yellow amorphous solid (19 mg, 94\% yield). $\mathrm{R}_{f}=0.18(\mathrm{MeOH} /$ EtOAc, 4/1, v/v); IR (U $\max ): 3375,2923,1593,1560,1381,1333$, 1232, 1189, 1110, 1020, 953, 641, 618, $570 \mathrm{~cm}^{-1} ;{ }^{1} \mathrm{H}$ NMR (600 MHz, DMSO-d 6 ): $\delta$ ppm 9.45 (br. s, $1 \mathrm{H}, \mathrm{NH}), 8.51$ (d, $J_{6,7}=8.1 \mathrm{~Hz}, 1 \mathrm{H}, \mathrm{H6}$ ), 8.28 (d, $\left.J_{6,7}=8.2 \mathrm{~Hz}, 1 \mathrm{H}, \mathrm{H} 7\right), 3.86-3.83$ (m, 1H, H3a), 3.57 (t, $\left.J_{3 \mathrm{a}, 3 \mathrm{~b}}=14.5 \mathrm{~Hz}, 1 \mathrm{H}, \mathrm{H} 3 \mathrm{~b}\right), 3.15$ (t, $\left.J_{2 z, 3 \mathrm{~b}}=14.0 \mathrm{~Hz} .1 \mathrm{H}, \mathrm{H} 2 \mathrm{a}\right), 2.44$ (td, $J_{2 \mathrm{a}, 2 \mathrm{~b}}=$ $\left.14.1 \mathrm{~Hz}, J_{2 \mathrm{~b}, 3 \mathrm{~b}}=3.6 \mathrm{~Hz}, 1 \mathrm{H}, \mathrm{H} 2 \mathrm{~b}\right) ;{ }^{13} \mathrm{C}$ NMR (125 MHz, DMSO-d 6 ): $\delta$ ppm 179.7 (C5), 176.2 (C10), $165.8\left(\underline{\mathrm{CO}}_{2} \mathrm{H}\right), 153.5$ (C8), 148.4 (C9a), 145.9 (C4a), 136.4 (C6), 130.1 (C5a), 127.6 (C7), 113.2 (C10a), 40.9 (C2), 31.6 (C3); HRMS-ESI (m/ z) calcd. for: $\left[\mathrm{C}_{12} \mathrm{H}_{8} \mathrm{~N}_{2} \mathrm{O}_{5} \mathrm{~S}-\mathrm{H}\right]=291.0081$, found: 291.0092 . 
<smiles>O=C(O)c1ccc2c(n1)C(=O)C1=C(NCCS1(=O)=O)C2=O</smiles>

74

1,1-Dioxo-2,3-dihydro- $4 H$-[1,4] thiazino [3,2-g]quinoline5,10-dione-8-carboxylic acid (74). To a solution of acid $72(63$ $\mathrm{mg}, 0.23 \mathrm{mmol})$ in $\mathrm{H}_{2} \mathrm{O}(1.2 \mathrm{~mL})$ was added $\mathrm{H}_{2} \mathrm{O}_{2}(28 \%, 0.12 \mathrm{~mL})$. The solution was stirred at room temperature for $2 \mathrm{~h}$, after which time $\mathrm{H}_{2} \mathrm{O}_{2}(28 \%, 0.05 \mathrm{~mL})$ was added, followed by $\mathrm{AcOH}(1.3$ $\mathrm{mL}$ ). The yellow solution was then warmed to $70{ }^{\circ} \mathrm{C}$ and stirred for $3 \mathrm{~h}$. The resulting yellow solid was then collected by filtration and dried in a dessicator to give the title compound as a yellow solid (35.5 mg, 33\%). $\mathrm{R}_{f}=0.62(\mathrm{MeOH})$; IR (U $\left.\mathrm{u}_{\max }\right)$ : 3461, 1660, 1562, 1452, 1275, 1117 , 989, 950, 795, 708, 685, 549, 499, $459 \mathrm{~cm}^{-1} ;{ }^{1} \mathrm{H}$ NMR (500 MHz, DMSO-d 6 ): $\delta$ ppm 9.31 (br. s, $1 \mathrm{H}, \mathrm{NH}), 8.50$ (d, $\left.J_{6,7}=8.0 \mathrm{~Hz}, 1 \mathrm{H}, \mathrm{H} 6\right), 8.28$ (d, $\left.J_{6,7}=8.0 \mathrm{~Hz}, 1 \mathrm{H}, \mathrm{H} 7\right), 3.89-3.87$ (m, 2H, H3), 3.42-3.40 (m, 2H, H2); ${ }^{13} \mathrm{C}$ NMR (125 MHz, DMSO-d 6 ): $\delta$ ppm 178.8 (C5), $165.7\left(\mathrm{CO}_{2} \mathrm{H}\right)$, 153.9 (C8),148.1 (C9a), 147.3 (C4a), 136.4 (C6), 129.5 (C5a), 127.5 (C7),48.7 (C2), 39.9 (C3); HRMS-ESI $(m / z)$ calcd. for $\left[\mathrm{C}_{12} \mathrm{H}_{8} \mathrm{~N}_{2} \mathrm{O}_{6} \mathrm{~S}+\mathrm{H}\right]^{+}:$309.0176, obsd.: 309.0170, calcd. for $[\mathrm{M}-\mathrm{H}]$ : 307.0030, obsd.: 307.0034.<smiles>O=C(O)c1ccc2c(n1)C(=O)C1=C(NC=CS1(=O)=O)C2=O</smiles>

46

Ascidiathiazone B (28). Procedure A: Selenium dioxide (44 mg, $0.4 \mathrm{mmol}, 4.5$ equiv.) was added to a solution of thiazine $\mathbf{7 0}$ (14.6 $\mathrm{mg}, 0.09 \mathrm{mmol})$ in dry pyridine $(0.86 \mathrm{~mL})$. The reaction was stirred at $80{ }^{\circ} \mathrm{C}$ for $3 \mathrm{~d}$, after which time TLC analysis showed the disappearance of starting materials. The yellow solution was then filtered through a pad of celite, concentrated in vacuo, and re-dissolved in $\mathrm{H}_{2} \mathrm{O}(1 \mathrm{~mL})$ and 0.1 $\mathrm{mL}$ of $2 \mathrm{M}$ aq. $\mathrm{NaOH}$. After stirring at reflux for $10 \mathrm{~min}$, the reaction was allowed to cool to room temperature and neutralised with $2 \mathrm{M}$ aq. $\mathrm{HCl}$. The resulting orange mixture was then passed through a short C18 reverse chromatography column, concentrated in vacuo, and the residue purified by silica gel flash column chromatography $\left(\mathrm{CH}_{2} \mathrm{Cl}_{2} / \mathrm{MeOH}, 1 / 0 \rightarrow 1 / 1\right.$, v/v). The residue was then passed through a short reversed-phase C18 column and freeze dried to give the title compound as an orange-red amorphous solid $(9.0 \mathrm{mg}, 33 \%)$. Procedure B: Sulfone 74 (5 mg, $0.02 \mathrm{mmol})$ was dissolved in $1 \mathrm{M}$ aq. $\mathrm{KOH}(3 \mathrm{~mL})$ and stirred for $3 \mathrm{~h}$ before being passed through a cationic ion exchange resin (BIO-REX 70, 200-400 mesh). The resulting orange-red solution was concentrated in vacuo then freeze-dried to give the title compound as an orange-red amorphous solid (4.1 mg, 80\%). $\mathrm{R}_{f}=0.10$ (EtOAc/MeOH/AcOH, 4/1/0.1, v/v/v); IR (Umax): 3429, 1624, 1571, 1560, 1453, 1293, 1112 $\mathrm{cm}^{-1} ;{ }^{1} \mathrm{H}$ NMR $\left(600 \mathrm{MHz}, \mathrm{DMSO}_{-} \mathrm{d}_{6}\right): \delta \mathrm{ppm} 8.40$ (d, $\left.J_{6,7}=8.0 \mathrm{~Hz}, 1 \mathrm{H}, \mathrm{H} 6\right), 8.24$ (d, J6,7 $=8.0$ 
$\mathrm{Hz}, 1 \mathrm{H}, \mathrm{H} 7), 7.27$ (d, $\left.J_{2,3}=8.0 \mathrm{~Hz}, 1 \mathrm{H}, \mathrm{H} 3\right), 6.23$ (d, $\left.J_{2,3}=8.0 \mathrm{~Hz}, 1 \mathrm{H}, \mathrm{H} 2\right) ;{ }^{13} \mathrm{C}$ NMR $(125 \mathrm{MHz}$, DMSO-d $\left.{ }_{6}\right): \delta$ ppm $181.2(\mathrm{C} 5), 176.7$ (C10), $166.1\left(\underline{\mathrm{CO}}_{2} \mathrm{H}\right), 160.7$ (C8), 148.2 (C4a), 147.2 (C9a), 139.2 (C3), 136.0 (C7), 128.1 (C5a), 126.7 (C7), 115.5 (C10a), 113.3 (C2); HRMS-ESI (m/ z) calcd. for $\left[\mathrm{C}_{12} \mathrm{H}_{6} \mathrm{~N}_{2} \mathrm{O}_{6} \mathrm{~S}-\mathrm{H}\right]: 304.9874$, obsd.: 304.9875 . The data for this compound matched those reported in the literature. ${ }^{104}$

\subsubsection{Biology}

Samples. Each compound was dissolved in sterile DMSO to make $10 \mathrm{mM}$ stock solutions.

Obtaining MIC values. In clear-bottomed 96-well plates (Nunc), two-fold serial dilutions of each compound were added to volumes of $7 \mathrm{H} 9$ medium for $M t b$ and $\mathrm{HdB}$ minimal medium for M. smegmatis. The last column of each plate did not contain any compound and served as a negative control. Previously prepared M. smegmatis $\mathrm{mc}^{2} 6533$ and $M t b \mathrm{mc}^{2} 6230$ (Howard Hughes Medical Institute, Albert Einstein College of Medicine) inocula were diluted with their respective media to achieve $0.05 \mathrm{OD}_{600}$. The inocula were then added to each well and the plates incubated at $37^{\circ} \mathrm{C}$ for two and five days for those containing M. smegmatis and Mtb, respectively. After incubation, $20 \mu \mathrm{L}$ of resazurin was added to the wells, and the plates incubated at $37^{\circ} \mathrm{C}$ for one hour or 24 hours for M. smegmatis and Mtb, respectively. The wells that remained blue were deemed to contain non-replicating bacteria, while those that underwent a colour change to purple or pink were deemed to contain replicating bacteria. MIC values were ascertained by visual judgment of colour.

Measuring rates of NADH oxidation. $M$. smegmatis IMVs were prepared prior to testing as previously described. ${ }^{33}$ To make a stock solution of $M$. smegmatis IMVs, $1 \mu \mathrm{L}$ of IMV solution (20 $\mathrm{mg} / \mathrm{mL}$ protein) was added to $1 \mathrm{~mL}$ of HEPES-NaOH buffer ( $\mathrm{pH}$ 7.5). The HEPES$\mathrm{NaOH}$ was composed of $10 \mathrm{mM}$ HEPES-NaOH, $5 \mathrm{mM} \mathrm{MgCl}, 100 \mathrm{mM} \mathrm{KCl}$. In clearbottomed 96-well plates, $20 \mu \mathrm{L}$ of the IMV/buffer solution was added to each well, followed by $2 \mu \mathrm{L}$ of $10 \mathrm{mM}$ (in DMSO) solutions of each compound. NADH $(10 \mu \mathrm{L}, 400 \mu \mathrm{M})$ was then added to each well. The solution in the well was mixed with a pipette and put into a Thermo Scientific Varioskan Flash plate reader. Each plate had two wells without any drug, to serve as negative controls. The vehicle control contained IMV/buffer solution $(20 \mu \mathrm{L})$, while the second negative control consisted of IMV/buffer solution $(20 \mu \mathrm{L})$ and $\mathrm{NADH}(10 \mu \mathrm{L})$ to obtain the basal rate of NADH oxidation. Absorbance values were recorded every minute for twenty minutes at $340 \mathrm{~nm}$ to allow for calculations of the NADH consumption rate, using an 
extinction coefficient $\varepsilon_{340}=6.22 \mathrm{mM}^{-1} \mathrm{~cm}^{-1}$. The experiments were undertaken three times, and the average rate of reaction was taken for each compound.

Measuring $\mathrm{O}_{2}$ consumption and fluorescence of acridine orange. In a respiratory chamber, IMVs $(20 \mu \mathrm{L}, 20 \mathrm{mg} / \mathrm{mL})$ were incubated with $5 \mu \mathrm{M}$ acridine orange (75) in $1 \mathrm{~mL}$ HEPES-NaOH buffer ( $\mathrm{pH}$ 7.5) for 5 seconds before $1 \mathrm{mM}$ NADH was added. After one second, QQ 61 was added into the chamber until all of the $\mathrm{O}_{2}$ was consumed and the chamber enters anoxia. The change in $\mathrm{O}_{2}$ concentration and fluorescence over time was recorded. 


\section{CHAPTER THREE}

\section{The anti-tubercular and redox activity of}

quinolinequinones

\subsection{Introduction}

Quinones are vital to redox processes in living organisms due to their propensity to undergo reversible redox reactions. For example, ubiquinones (17, Figure 3.1) are electron acceptors that are essential for respiration in nearly all aerobic organisms. ${ }^{139-141}$ Within biological systems, the reduction of quinones occurs enzymatically as part of the oxidative phosphorylation process to generate ATP. ${ }^{142}$ Quinone species $(\mathrm{Q})$ are reduced to their respective hydroquinones $\left(\mathrm{QH}_{2}\right)$ through a carefully regulated two-electron reduction that occurs with the concomitant oxidation of $\mathrm{NADH}$ (equation 1). ${ }^{143,144}$ Subsequently, $\mathrm{QH}_{2}$ is re-oxidised to the original quinone by molecular oxygen by two one-electron steps (equations 2-4). ${ }^{145,146}$ Here, radicals 
$\mathrm{QH}^{*}$ and $\mathrm{HO}_{2}{ }^{\circ}$ are generated from the first one-electron redox reaction (equation 2). The hydroperoxy radical $\mathrm{HO}_{2}{ }^{*}$ can then undergo deprotonation reactions to form superoxide $\mathrm{O}_{2}{ }^{-}$ (equation 3), which triggers the formation of peroxides $\left(\mathrm{H}_{2} \mathrm{O}_{2}\right)$ and the regeneration of the quinone $\left(\mathrm{Q}\right.$, equation 4-5). Finally, peroxides $\left(\mathrm{H}_{2} \mathrm{O}_{2}\right)$ then react with superoxides $\left(\mathrm{O}_{2}{ }^{--}\right)$to produce hydroxyl radicals $\mathrm{HO}^{\bullet}$ (equation 6). ${ }^{147}$ While the formation of ROS is important for cell signalling, unregulated production of superoxides can be detrimental to the cell. ${ }^{146}$ Indeed, many quinone-based drugs exhibit their therapeutic activity by accepting electrons from $\mathrm{NADH}$ and generating harmful levels of ROS. ${ }^{144,148-151}$

$$
\begin{aligned}
& Q+N A D H+H^{+} \rightarrow Q H_{2}+N A D^{+} \\
& \mathrm{QH}_{2}+\mathrm{O}_{2} \rightarrow \mathrm{QH}^{\bullet}+\mathrm{HO}_{2}{ }^{\bullet} \\
& \mathrm{HO}_{2}{ }^{\bullet} \rightleftarrows \mathrm{O}_{2}^{\cdot-}+\mathrm{H}^{+} \\
& \mathrm{QH}^{\bullet}+\mathrm{O}_{2} \rightarrow \mathrm{Q}+\mathrm{HO}_{2}^{\bullet} \\
& 2 \mathrm{O}_{2}{ }^{--}+2 \mathrm{H}^{+} \rightarrow \mathrm{H}_{2} \mathrm{O}_{2}+\mathrm{O}_{2} \\
& \mathrm{O}_{2}^{\cdot-}+\mathrm{H}_{2} \mathrm{O}_{2} \rightarrow \mathrm{HO}^{\bullet}+\mathrm{HO}^{-}+\mathrm{O}_{2}
\end{aligned}
$$

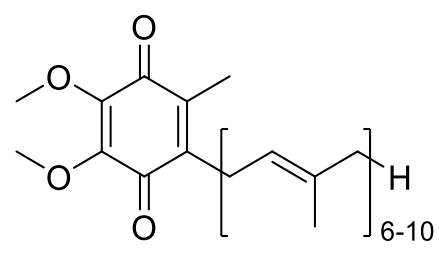

17

Figure 3.1: Ubiquinones (17), a class of quinones found in mammalian mitochondria. ${ }^{139-141}$

\subsubsection{Pharmacological properties of quinolinequinone-containing drugs}

Streptonigrin (74, Figure 3.2) is a QQ-bearing metabolite isolated from Streptomyces flocculus that exhibits anti-tumour properties. ${ }^{152,153}$ The QQ scaffold in streptonigrin (74) is essential for its anti-proliferative activity, which is attributed to the generation of radical species and peroxides during the redox cycling of quinone $74 .{ }^{144,151,153}$ These radical species can cleave DNA in tumour cells. The reduction of the quinone moiety was thought to be catalysed by the NQO1 enzyme, a two-electron reductase that is overexpressed in many cancer cells including lung, breast and colon. ${ }^{154,155}$ To improve the anti-cancer activity of streptonigrin (74), structurally refined QQs were synthesised and their rates of DNA cleavage measured. Here, methoxy-QQ $\mathbf{7 5}$ was as equally potent as streptonigrin (74) in its ability to cleave DNA, while QQs $\mathbf{7 6}$ and 
77 surpassed the activity induced by the natural product by almost two-fold. ${ }^{144}$ Another QQ with anti-tumour properties is LY83583 (78), ${ }^{156}$ a commercially available drug known to competitively inhibit guanylate cyclase. ${ }^{156,157}$ Again, the mode of action of LY83583 (78) was reportedly due to the formation of ROS and hydroxyl radical species ${ }^{156}$ through the redox cycling of the QQ motif. ${ }^{157}$<smiles>[Z4]Oc1ccc(-c2c(C)c(C(=O)O)nc(-c3ccc4c(n3)C(=O)C(N)=C(OC)C(=O)C4=O)c2N)c(O)c1OC</smiles>

74<smiles>COC1=C(N)C(=O)c2nc(-c3ccccc3N)ccc2C1=O</smiles>

75<smiles>COC1=CC(=O)c2nc(-c3ccccc3N)ccc2C1=O</smiles>

77<smiles>COC1=C(Cl)C(=O)c2nc(-c3ccccc3[N+](=O)[O-])ccc2C1=O</smiles><smiles>O=C1C(Nc2ccccc2)=CC(=O)c2ncccc21</smiles>

78

Figure 3.2: The structure of streptonigrin (74), a QQ-containing secondary metabolite, and biologically active QQ derivatives 75-78. ${ }^{144}$

In addition to their anti-cancer properties, QQs are also known to exhibit anti-bacterial activity. Recently, a library of aryl- and aniline-substituted QQs were tested for their ability to inhibit the growth of both the Gram positive and Gram negative bacteria, S. aureus and E. coli, respectively. ${ }^{150}$ For both bacterial species, aniline-substituted QQs exhibited higher inhibitory activity compared to QQs functionalised with aryl substituents. Of particular relevance to this project is the anti-mycobacterial activity of quinones. ${ }^{149,158-160}$ In seminal studies, 6-cyclo-octylQQ (79, Figure 3.3) was found to inhibit the growth of $M t b$ at low concentrations (MIC $=3.5$ $\mu \mathrm{M}) .{ }^{149,158}$ Since this work, others have demonstrated the anti-tubercular activity of isobenzoquinones, such as isoquinoline $\mathbf{8 0}\left(\mathrm{IC}_{50}=1.3 \mu \mathrm{M}\right),{ }^{159}$ while enamine-benzoquinone $\mathbf{8 1}$ was found to be potent against resistant strains of $M t b(\mathrm{MIC}=1.2 \mu \mathrm{M}) \cdot{ }^{160}$ In the latter study, SAR analysis of enamine-quinone analogues indicated that the quinone ring was essential for anti-tubercular activity as its removal led to reduced drug potency against $M t b .{ }^{160}$ 
<smiles>O=C1C(NC2CCCCCCC2)=CC(=O)c2ncccc21</smiles>

79<smiles>CC(C)n1c(=O)c2c3c(cnc2n(C(C)C)c1=O)C(=O)C1=C(C3=O)C2CCC1C2</smiles><smiles>CN(C)/C=C/c1c2c(nc3ccccc13)C(=O)c1ccccc1C2=O</smiles>

Figure 3.3: Examples of quinone-containing compounds 79-81 that exhibit anti-tubercular activity. ${ }^{149,158-160}$

In 2010, Stocker and co-workers synthesised a library of QQs that exhibited bacteriostatic activity against $M$. bovis BCG. ${ }^{65}$ Here, the most active compounds, QQs 82 and 83 (Figure 3.4), were found to possess anti-tubercular activity ( $\mathrm{MIC}=7.5-45 \mu \mathrm{M})$ that was comparable to the current first-line drug $\operatorname{EMB}(4, \mathrm{MIC}=15-30.9 \mu \mathrm{M})$ in the same assay. ${ }^{65}$ Following this discovery, mechanistic studies of a representative QQ, QQ8c (20), revealed that QQ8c (20) specifically targeted mycobacterial NDH-II, which led to the production of lethal levels of ROS (Chapter 1, Section 1.4.1.). ${ }^{64}$ Similarly, an analogue of QQ8c, methyl-QQ 60, increased $\mathrm{NADH}$ oxidation rates and $\mathrm{O}_{2}$ consumption in mycobacterial ETC (Chapter 2, Section 2.2.6).<smiles>NC1=C(Cl)C(=O)c2ncccc2C1=O</smiles>

82<smiles>O=C1C(Cl)=C(NCCCl)C(=O)c2ncccc21</smiles>

QQ8c (20)<smiles>CS(=O)C1=C(N)C(=O)c2cccnc2C1=O</smiles>

83<smiles>Cc1ccc2c(n1)C(=O)C(Cl)=C(NCCCl)C2=O</smiles>

Figure 3.4: Amino-QQs 82, 83, QQ8c (20) and QQ 60 are potent bacteriostatic agents. ${ }^{64,65}$

\subsubsection{Redox potential of quinones}

The ability of quinones to interact with oxidoreductases, both as natural substrates and as pharmaceutical agents, has been attributed to their propensity to undergo redox reactions. 
Redox reactions of organic materials typically involve the generation of highly reactive radical species that need to be adequately stabilised for the redox active compound to undergo redox cycling. ${ }^{161}$ If the radical species is too reactive, it can self-react, while a persistent radical may not readily undergo subsequent reduction or oxidation reactions. ${ }^{162}$ In quinones (II), a oneelectron reduction forms a semiquinone radical (III), which can then be reduced to form a quinol (IV, Scheme 3.1). Here, the negative charge of the semiquinone radical (III) is stabilised by resonance due to the extensive conjugation present in the benzene ring. Consequently, the two-step reduction of quinones (II) is reversible, thereby allowing for redox cycling. ${ }^{141,163}$ The tendency for a quinone to undergo reduction can be measured by its redox potential, whereby a high redox potential indicates that the quinone will readily accept electrons. ${ }^{163,164}$

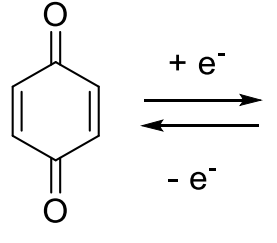

II

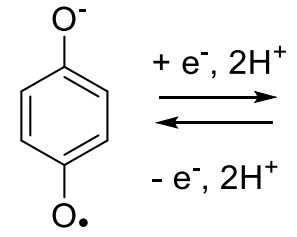

III<smiles>Oc1ccc(O)cc1</smiles>

IV

Scheme 3.1: The reversible reduction of quinones (II) to semiquinones (III) and quinols (IV).

The capacity of a given quinone to accept electrons can be fine-tuned through the installation of different functionalities on the ring. ${ }^{165}$ Generally, the addition of electron-donating groups on the quinone scaffold increases the electron density of the molecule, thereby reducing the ability of the quinone to accept electrons. Accordingly, quinones bearing electron-donating groups usually have lower redox potentials than those with electron-withdrawing groups. ${ }^{163,165}$ This trend was illustrated effectively in the study of substituted phenothiazines, whereby cyano- (84) and trifluoromethyl-substituted (85) phenothiazines had higher redox potentials compared to unsubstituted ethyl-phenothiazine $\mathbf{8 6}$, while dimethyl phenothiazine 87 had the lowest redox potential of the group (Figure 3.5). ${ }^{166}$ That said, exceptions to this trend have been noted, such as the lower reduction potential of sulfonic acid 88 compared to unsubstituted quinone 89. ${ }^{167}$ Moreover, as quinones behave differently in different media, ${ }^{168}$ their oxidising strength is likely to be affected by $\mathrm{pH}$, intermolecular interactions and entropy in physiological systems. ${ }^{169}$ 
<smiles>CCN1c2ccc(C#N)cc2Sc2cc(C#N)ccc21</smiles>

84

$E^{\circ}=0.73 \mathrm{~V}$

(vs. ferrocene)<smiles>CCN1c2ccccc2Sc2ccccc21</smiles>

86

$\mathrm{E}^{\circ}=0.27 \mathrm{~V}$

(vs. ferrocene)<smiles>O=C1C=C(S(=O)(=O)O)C(=O)c2ccccc21</smiles>

88

$E^{\circ}=-0.23 V$

(vs. $\mathrm{Hg} / \mathrm{Hg}^{+}$)<smiles>CCN1c2ccc(C(F)(F)F)cc2Sc2cc(C(F)(F)F)ccc21</smiles>

85

$\mathrm{E}^{\circ}=0.61 \mathrm{~V}$

(vs. ferrocene)<smiles>CCN1c2ccc(C)cc2Sc2cc(C)ccc21</smiles>

87

$E^{\circ}=0.13 \mathrm{~V}$

(vs. ferrocene)<smiles>O=C1C=CC(=O)C=C1</smiles>

89

$E^{\circ}=-0.11 \vee$

(vs. $\mathrm{Hg} / \mathrm{Hg}^{+}$)

Figure 3.5: Electron-withdrawing functional groups on the phenothiazine scaffold, as in phenothiazines 84 and $\mathbf{8 5}$, leads to an increase in reduction potential compared to unsubstituted phenothiazine $\mathbf{8 6}$. Conversely, methylphenothiazine $\mathbf{8 9}$ has a relatively low reduction potential. That said, quinone $\mathbf{8 8}$, which bears an electron withdrawing sulfonic acid functionality, has a lower reduction potential than unfunctionalised benzoquinone (89).

The redox potentials of quinones have been reported to correlate with their therapeutic effects. ${ }^{170,171}$ For example, Livertoux $e t$ al. described a linear relationship between the redox potentials of a range of quinones $\left(\mathrm{E}^{\circ}=-0.23 \mathrm{~V}\right.$ to $-0.72 \mathrm{~V}$ vs. $\left.\mathrm{Hg} / \mathrm{Hg}^{+}\right)$and the rate of superoxide production in rat microsomes. ${ }^{167}$ More recently, Ohayon et al. reported on the anticancer activity of an ortho-quinone-containing natural product, $\beta$-lapachone (90, Figure 3.6). $\beta$-Lapachone (90) inhibits deubiquitinases in tumour cells by generating ROS and irreversibly oxidising cysteine residues within the enzyme binding site. ${ }^{172} \mathrm{~A}$ study of several $\beta$-lapachone analogues demonstrated that the most potent deubiquitinase inhibitors had redox potentials between $-0.20 \mathrm{~V}$ and $-0.24 \mathrm{~V}$ (vs. $\left.\mathrm{Ag} / \mathrm{Ag}^{+}\right) .{ }^{171}$ Compounds that had redox potentials outside this optimal range exhibited minimal or no anti-cancer properties. ${ }^{171}$ However, the authors also reported that ortho-quinone $\mathbf{9 0}$ displayed a higher degree of enzyme inhibition than para- 
quinone $91(100 \%$ at $0.5 \mu \mathrm{M}$ vs. $11 \%$ inhibition at $1 \mu \mathrm{M}$, respectively), despite both having a reduction potential of $-0.24 \mathrm{~V}$ (vs. $\left.\mathrm{Ag} / \mathrm{Ag}^{+}\right)$. This difference in activity was attributed to the enhanced stability of ortho-quinol $(\mathbf{V})$ due to the formation of a hydrogen bond between the two quinol oxygens. ${ }^{171}$ This study simultaneously highlights both the value and the limitations of predicting the potency of redox active drugs through the measurement of redox potentials. While the reduction potentials of a compound can provide insight into the relative reactivities of different drug candidates, the ability of a compound to undergo redox reactions in a physiological setting cannot always be predicted. ${ }^{168,169}$

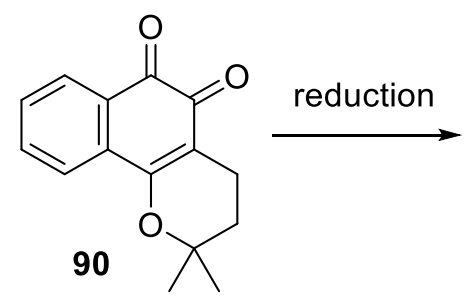<smiles></smiles><smiles>CC1(C)C=CC2=C(O1)C(=O)c1ccccc1C2=O</smiles>

Figure 3.6: The reduction of ortho-quinone $\beta$-lapachone $(\mathbf{9 0})$ to quinol $\mathbf{V}$, and the para-quinone analogue $\mathbf{9 1} .^{171}$

\subsubsection{Project aims and retrosynthetic analysis of QQs}

As discussed in Chapter 1 (Section 1.2), redox reactions facilitated by NDH-II are crucial for the viability of $M t b$. Given the capacity of QQs to activate $\mathrm{NDH}-\mathrm{II}^{64}$ and exhibit tuberculostatic activity, ${ }^{65}$ investigations into how changes to the QQ scaffold influence antitubercular activity could lead to the discovery of a more potent QQ TB drug. To this end, it was proposed that a library of QQs bearing different amine substituents would be synthesised and subsequently screened against $M t b$ for their bacteriostatic effects. Herein, particular focus was given to the installation of tertiary amines on the QQ scaffold, which will allow investigations into potential hydrogen bonding interactions involving the amine proton to be made. Similarly, the installation of amines with quaternary carbons, i.e. tert-butylamine and aniline, on the QQ scaffold may reduce the availability of the amine proton to participate in hydrogen bonding due to increased steric bulk. Next, QQs bearing aliphatic chains were included in the library as the NDH-II Q-site is reportedly surrounded by hydrophobic grooves $^{53,58,62}$ and, moreover, the natural substrate of NDH-II, MK (15), ${ }^{173}$ possesses a long hydrophobic tail. Finally, aryl-substituted QQs could enhance the binding affinity of the QQs 
to the target enzyme due to potential $\pi$-stacking interactions with the FAD isoalloxazine ring or other aromatic side chains in the NDH-II Q-site (i.e. Y13 and Y383). ${ }^{53}$

The synthesis of QQs is well-precedented and there are several synthetic routes available to access the QQ motif. Typically, the quinone moiety is prepared through employment of the Teuber reaction, ${ }^{174}$ whereby phenols or anilines are treated with Fremy's salt, ${ }^{105,139,175}$ though other oxidants such as ceric(IV) ammonium nitrate, ${ }^{176,177}$ (diacetoxyiodo)benzene, ${ }^{178}$ or potassium dichromate ${ }^{179}$ can also be used. Once the quinone ring is formed however, the installation of substituents on the quinone scaffold can be challenging and is often circumvented by the introduction of functional groups prior to quinone formation. ${ }^{139}$ Accordingly, to prepare a library of amine-substituted QQs, it was envisioned that the 6,7dichloroquinone backbone would first be prepared to allow for subsequent functionalisation. Here, the presence of chloride leaving groups would enable nucleophilic substitution reactions to take place, which would otherwise be sluggish and low-yielding. ${ }^{177}$ To this end, it was envisaged that the QQ analogues VI-IX could be synthesised by the substitution of dichloroQQs 92 and 56 by various alkyl- and aryl-amines (Scheme 3.2). For the purpose of this research, it was proposed that both the 6- (VI, VII) and 7-regioisomers (VIII, IX) would be prepared in the same synthetic step, though it should be noted that the 6-isomer (VI, VII) can be preferentially formed by the addition of a metal catalyst to the reaction mixture. ${ }^{65,180}$ DichloroQQs $\mathbf{9 2}$ and $\mathbf{5 6}$ could, in turn, be prepared by the oxidation of commercially available reagents, 8-hydroxyquinoline (93) and 8-hydroxyquinaldine (58), respectively. ${ }^{65}$ 
<smiles>[R]NC1=C(Cl)C(=O)c2nc([R])ccc2C1=O</smiles>

VI, $\mathrm{R}^{1}=\mathrm{H}, \mathrm{R}^{2}=$ alkyl, aryl

VII, $\mathrm{R}^{1}=\mathrm{CH}_{3}, \mathrm{R}^{2}=$ alkyl, aryl<smiles>[R]NC1=C(Cl)C(=O)c2ccc([R])nc2C1=O</smiles><smiles>[R]c1ccc2c(n1)C(=O)C(Cl)=C(Cl)C2=O</smiles>

92, $\mathrm{R}^{1}=\mathrm{H}$

93, $\mathrm{R}^{1}=\mathrm{H}$

56, $\mathrm{R}^{1}=\mathrm{CH}_{3}$

58, $\mathrm{R}^{1}=\mathrm{CH}_{3}$

VIII, $\mathrm{R}^{1}=\mathrm{H}, \mathrm{R}^{2}=$ alkyl, aryl

IX, $\mathrm{R}^{1}=\mathrm{CH}_{3}, \mathrm{R}^{2}=$ alkyl, aryl

Scheme 3.2: Retrosynthetic analysis of QQs VI-IX.

\subsection{Results and Discussion}

\subsubsection{The synthesis of $Q Q s$}

Having established a retrosynthetic plan for the synthesis of 6- and 7-amino-substituted QQs (VI-IX), 8-hydroxyquinoline (93) and 8-hydroxyquinaldine (58) were first oxidised with sodium chlorate to afford dichloroquinones 92 and 56, respectively (Scheme 3.3). ${ }^{65,181}$ After recrystallisation from ethanol, QQs $\mathbf{9 2}$ and $\mathbf{5 6}$ were then treated with a variety of amines to give the 6- and 7-isomers (94-111) in good combined yields and in an approximately 1:1 ratio. ${ }^{65}$ Derivatives prepared included those designed to probe the effect of bulky amines (i.e. QQs 9497, QQs 104-105), aliphatic chains (i.e. QQs 98-101, and 108-109) and aromatic groups (i.e. QQs 106-107 and 110-111) on anti-tubercular activity. 
93, $\mathrm{R}^{1}=\mathrm{H}$

93, $\mathrm{R}^{1}=\mathrm{H}$

58, $R^{1}=M e$ $\underset{28 \%}{\mathrm{NaClO}_{3}}$

92, $\mathrm{R}^{1}=\mathrm{H}$

56, $\mathrm{R}^{1}=\mathrm{Me}$
$\mathrm{HR}^{2}$

$\mathrm{NaOH}$<smiles>CCOCC</smiles>

$\sim 1: 1 \mathbf{a}: \mathbf{b}$

$R$

7-isomer (a)

6-isomer (b)

$$
\begin{aligned}
& 94 a, R^{1}=C H_{3}, 50 \% \\
& 94 b, R^{1}=C_{3}, 41 \% \\
& 95 a, R^{1}=\mathrm{H}, 40 \% \\
& 96 b, R^{1}=C_{3}, 33 \%
\end{aligned}
$$$$
\overbrace{\mathrm{H}}^{\mathrm{s}} \sim \mathrm{Ph}
$$$$
\text { 104a, } R^{1}=\mathrm{H}, 34 \%
$$$$
S^{5} \mathrm{~N}^{-\mathrm{Ph}} \quad 104 \mathrm{~b}, \mathrm{R}^{1}=\mathrm{H}, 36 \%
$$$$
\text { 105a, } \mathrm{R}^{1}=\mathrm{CH}_{3}, 24 \%
$$$$
\text { 105b, } \mathrm{R}^{1}=\mathrm{CH}_{3}, 66 \%
$$$$
\text { 106a, } R^{1}=H, 17 \%
$$$$
\text { 106b, } R^{1}=H, 55 \%
$$$$
\text { 107a, } \mathrm{R}^{1}=\mathrm{CH}_{3}, 28 \%
$$$$
\text { 107b, } \mathrm{R}^{1}=\mathrm{CH}_{3}, 37 \%
$$
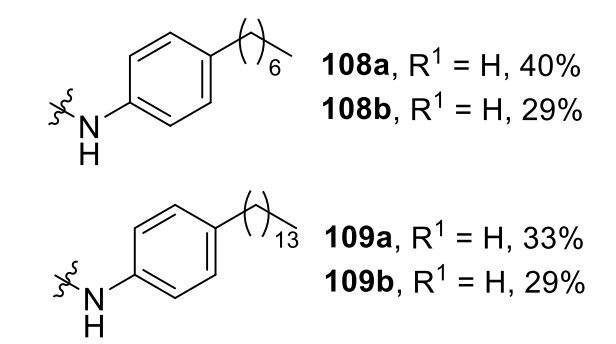

109a, $R^{1}=H, 33 \%$

109b, $R^{1}=H, 29 \%$<smiles>COc1ccc(CNC(C)(C)C)cc1</smiles>

110a, $R^{1}=H, 67 \%$

$110 b, R^{1}=H, 11 \%$

111a, $\mathrm{R}^{1}=\mathrm{CH}_{3}, 49 \%$

Scheme 3.3: Synthesis of QQs 94-111.

The amine-substitution conditions of dichloroQQs $\mathbf{9 2}$ and $\mathbf{5 6}$ were chosen to give a mixture of regioisomers as there is no suitable methodology available for the regioselective synthesis of both isomers. ${ }^{65}$ Notwithstanding, the two regioisomers were separable by silica gel flash column chromatography, with the 7-isomer being eluted first, which is consistent with literature precedent. ${ }^{178,182}$ Generally, the substitution of dichlorides $\mathbf{9 2}$ and $\mathbf{5 6}$ with different amines proceeded smoothly, giving close to a quantitative yield for the combined regioisomers. However, a number of QQ analogues required numerous purification steps to separate the regioisomers, which resulted in decreased yields. In particular, multiple attempts at purification by column chromatography were required for QQ analogues bearing long alkyl chains, such as octylamine-substituted QQs (102 and 103), heptylanilino-QQs 108 and tetradecylanilino- 
QQs 109. Here, the use of toluene as the mobile phase in silica gel column chromatography assisted in the separation.

As with the synthesis of 2-chloroethylamino-QQs 59 and $\mathbf{6 0}$ (Chapter 2, Section 2.2.1.), NMR spectroscopy was used to elucidate the structure of each isomer. ${ }^{65}$ Specifically, in the ${ }^{13} \mathrm{C}$ NMR spectrum, carbons involved in tautomerisation were observed as broad resonances that were less visible than typical quaternary carbon resonances. ${ }^{65}$ The carbonyl carbons involved in tautomerisation were then assigned via an HMBC between $\mathrm{H} 4$ and $\mathrm{C} 5$. In addition, the ${ }^{1} \mathrm{H}$ NMR revealed a smaller $\Delta \mathrm{H} 2-\mathrm{H} 4$ value for the 7-isomer compared to the 6-isomer in all QQs that were unfunctionalised at the 2-position, ${ }^{65}$ as illustrated in Figure 3.7 with QQs 98a and $98 b$.

A.<smiles>CCCNC1=C(Cl)C(=O)c2cccnc2C1=O</smiles>

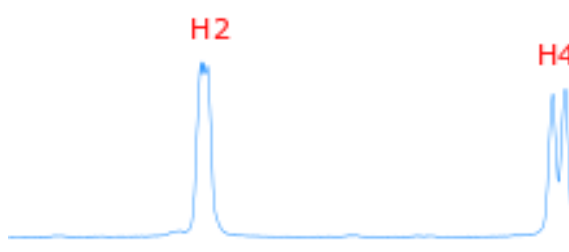

98a

$\mathrm{H3}$

B.
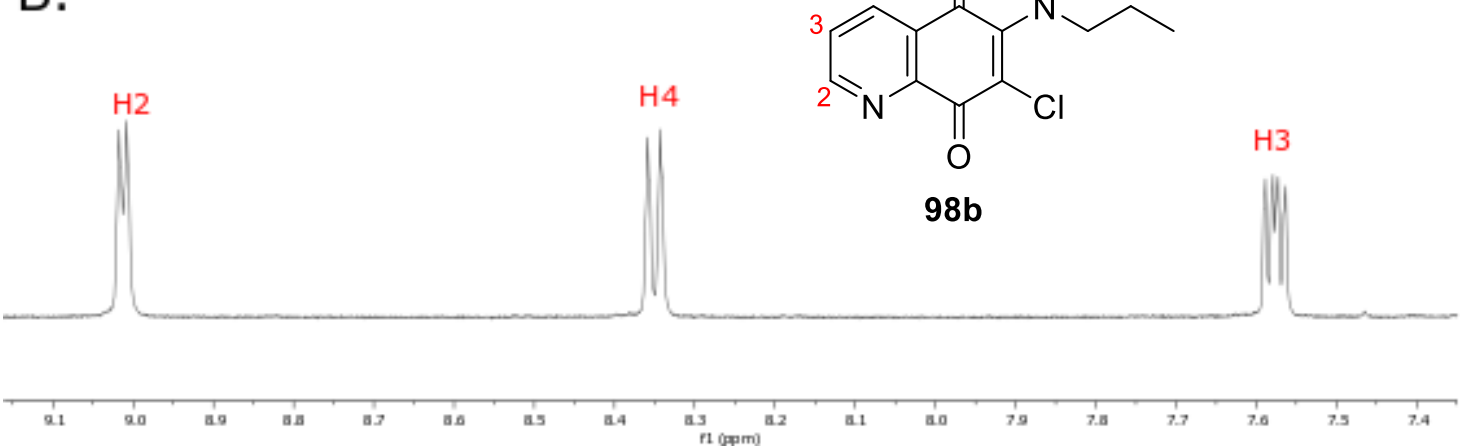

Figure 3.7: ${ }^{1} \mathrm{H}$ NMR (500 MHz, $\mathrm{CDCl}_{3}$ ) spectra of A). 6-chloro-7-propylaminoQQ 98a and B). its regioisomer $\mathbf{9 8 b}$.

In addition to NMR spectroscopy, the structural assignment of some analogues, such as the aniline-substituted QQ 104b, was confirmed by XRD (Figure 3.8). Here, the crystal structure confirmed the aromaticity of the pyridine ring, with an average $\mathrm{C}-\mathrm{C}$ bond length close to 1.40 $\AA$ (Appendix 3, Table A.3.1.), typical for aromatic C-C bond lengths, ${ }^{183}$ and N-C lengths of $1.34 \AA$, typical for aromatic C-N bonds. ${ }^{184}$ On the other hand, the quinone ring contained 
bonds of different length, whereby typical aromatic C-C bonds were observed, ${ }^{184}$ as well as longer bonds suggestive of single bond character $(1.46 \AA-1.50 \AA){ }^{183}$ The different bond lengths observed within the quinone ring indicated that the quinone moiety is not aromatic, despite its planar orientation. ${ }^{185}$

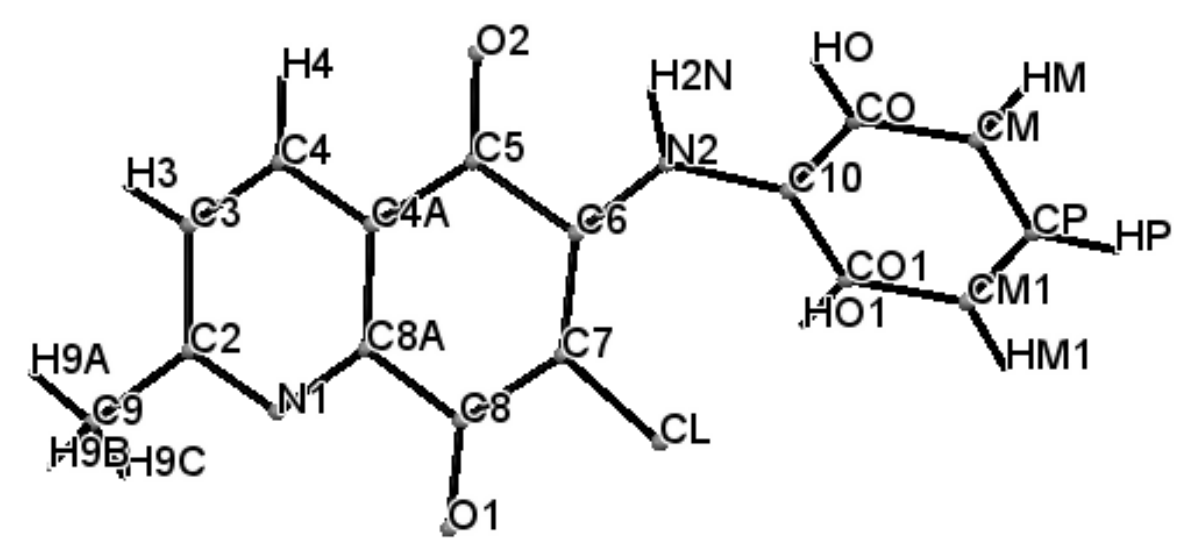

Figure 3.8: Crystal structure of 6-anilino-2-methylquinolinequinone (104b).

Intermolecular hydrogen bonds were also observed in the QQ 104b crystal structure, which showed the secondary amine as the hydrogen bond donor (H2n, Figure 3.9). Here, a bifurcated hydrogen bond was present between $\mathrm{H} 2 \mathrm{n}$ and the ring nitrogen $\mathrm{N} 1$, as well as the carbonyl O1 (for bond lengths, see Appendix 3, Table A.3.2.). Similar interactions have previously been reported in literature ${ }^{186,187}$ and provide the possibility for hydrogen bonding in a biological setting. 


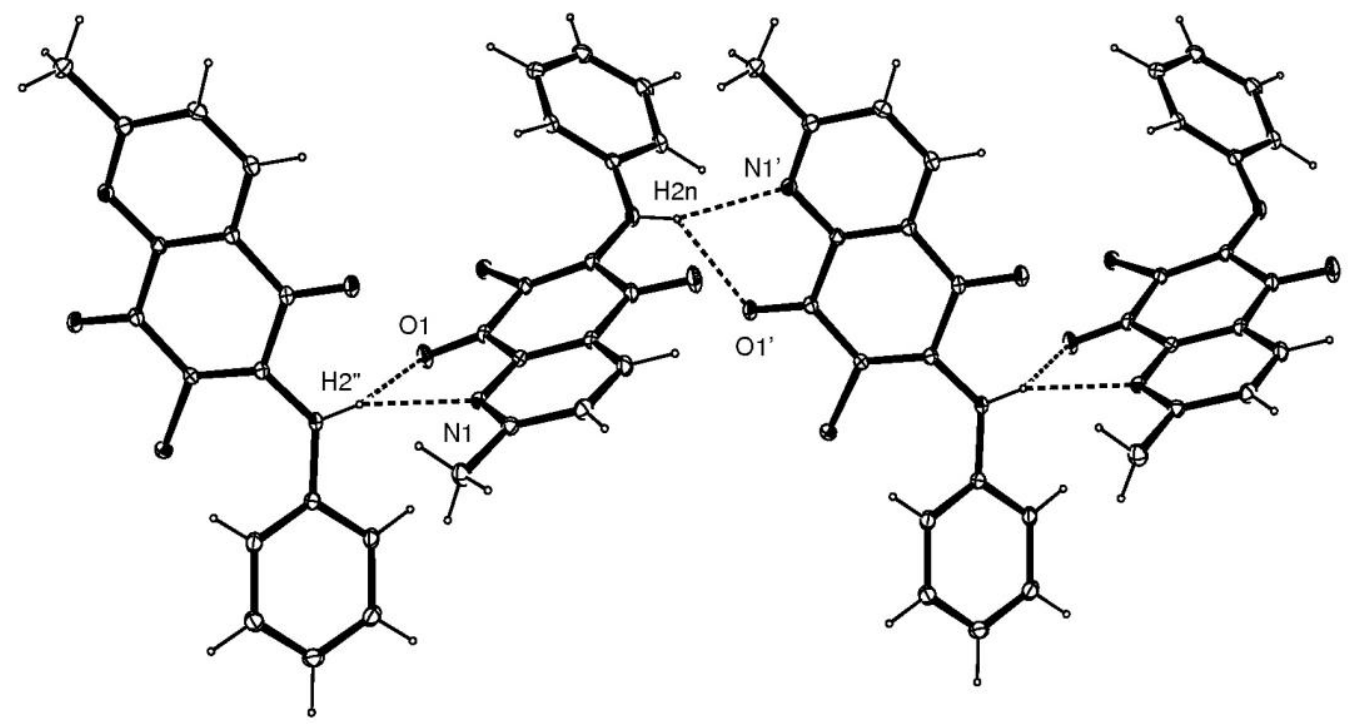

Figure 3.9: Intermolecular hydrogen bond interactions observed in anilino-QQ 104b.

\subsubsection{The anti-tubercular activity of QQs}

The QQs 94-111 were screened for their ability to inhibit Mtb growth by Professor Gregory Cook and co-workers at the University of Otago. Accordingly, the MIC value of each QQ was obtained (Table 3.1). Analysis of the QQ library highlighted the importance of several structural features for anti-tubercular activity. For example, QQs bearing tertiary amines, such as dimethylamino-QQs 94a and 94b (Entries 1 and 2, respectively) and piperidine-substituted QQs 95a and 96b (Entries 3 and 4), exhibited only low levels of Mtb growth inhibition. As tertiary amines are unable to act as hydrogen bond donors, this suggests that the ability of the QQ to form a hydrogen bond with the target enzyme may be important for anti-tubercular activity. 
Table 3.1: $\mathrm{MIC}$ values in $\mu \mathrm{M}$ and redox values $\left(\mathrm{E}_{1}\right.$ and $\mathrm{E}_{2}$ in acetonitrile and $\mathrm{E}^{\prime}$ in microemulsions) of synthesised QQ analogues 94-111, as well as literature MIC values of streptomycin (12) ${ }^{188}$ and QQ8c (20) ${ }^{64}$ and measured $E_{1}$ and $E_{2}$ values for QQ8c (20) in DMF.

$\begin{array}{lllll}\text { Entry Compound } & \mathrm{MIC} & \mathrm{E}_{1}(\mathrm{~V}) & \mathrm{E}_{2}(\mathrm{~V}) & \mathrm{E}^{\prime}(\mathrm{V})^{\mathrm{b}} \\ & (\mu \mathrm{M}) & & & \end{array}$<smiles>Cc1ccc2c(n1)C(=O)C(N(C)C)=C(Cl)C2=O</smiles>

2<smiles>Cc1ccc2c(n1)C(=O)C(Cl)=C(N(C)C)C2=O</smiles>

94b

3<smiles>O=C1C(Cl)=C(N2CCCCC2)C(=O)c2ncccc21</smiles>

95a

4<smiles>Cc1ccc2c(n1)C(=O)C(Cl)=C(N1CCCCC1)C2=O</smiles>

96b

5<smiles>O=C1C(Cl)=C(Nc2ccccc2)C(=O)c2ncccc21</smiles>

n.d.

$104 a$ 
6<smiles>O=C1C(Cl)=C(Nc2ccccc2)C(=O)c2ncccc21</smiles>

7<smiles>Cc1ccc2c(n1)C(=O)C(Nc1ccccc1)=C(Cl)C2=O</smiles>

$105 a$

8<smiles>Cc1ccc2c(n1)C(=O)C(Cl)=C(Nc1ccccc1)C2=O</smiles>

105b

9<smiles>CC1(c2ccc(NC3=C(Cl)C(=O)c4cccnc4C3=O)cc2)CC1</smiles>

108a

10<smiles>CC(C)(C)c1ccc(NC2=C(Cl)C(=O)c3cccnc3C2=O)cc1</smiles>

108b

11<smiles>CC(C)(C)c1ccc(NC2=C(Cl)C(=O)c3cccnc3C2=O)cc1</smiles>

109a

12<smiles>Cc1ccc(NC2=C(Cl)C(=O)c3ncccc3C2=O)cc1</smiles>

$$
\begin{array}{llll}
> & -12 & -0.46 & -1.06 \quad \text { n.d. }
\end{array}
$$

256

$\begin{array}{lll}-0.44 & -0.90 \quad \text { n.d. }\end{array}$

$512-0.47 \quad-1.05 \quad$ n.d.

32

$$
\begin{array}{lll}
-0.57 & -1.08 \quad-0.54
\end{array}
$$

$\begin{array}{llll}64 & -0.58 & -1.15 & -0.54\end{array}$

$>512 \quad-0.44^{\mathrm{a}} \quad-1.00^{\mathrm{a}} \quad$ n.d.

$>512 \quad-0.78^{\mathrm{a}} \quad-1.10^{\mathrm{a}} \quad$ n.d. 
13<smiles>COc1ccc(CNC2=C(Cl)C(=O)c3cccnc3C2=O)cc1</smiles>

110a

14<smiles>COc1ccc(CNC2=C(Cl)C(=O)c3ncccc3C2=O)cc1</smiles>

$110 \mathrm{~b}$

15<smiles>COc1ccc(CNC2=C(Cl)C(=O)c3ccc(C)nc3C2=O)cc1</smiles>

16<smiles>O=C1C(Cl)=C(NCCc2ccccc2)C(=O)c2ncccc21</smiles>

17<smiles>O=C1C(Cl)=C(NCCc2ccccc2)C(=O)c2ncccc21</smiles>

106b

18<smiles>Cc1ccc2c(n1)C(=O)C(NCCc1ccccc1)=C(Cl)C2=O</smiles>

107a $\begin{array}{llll}16 & -0.63 & -1.24 & \text { n.d. }\end{array}$

32

$-0.61$

$-1.18$

$-0.61$

16

-1.10 n.d.

16

$-0.64$

$-1.21$

$-0.59$

128

$-0.60$

-1.15 n.d.

16

$-0.70$

$-1.30$

$-0.60$ 
19<smiles>Cc1ccc2c(n1)C(=O)C(Cl)=C(NCCc1ccccc1)C2=O</smiles>

107b

20<smiles>CCCNC1=C(Cl)C(=O)c2cccnc2C1=O</smiles>

$98 a$

21<smiles>CCCNC1=C(Cl)C(=O)c2ncccc2C1=O</smiles>

98b

22<smiles>CCCNC1=C(Cl)C(=O)c2ccc(C)nc2C1=O</smiles>

99a

23<smiles>CCCNC1=C(Cl)C(=O)c2nc(C)ccc2C1=O</smiles>

99b

24<smiles>CC(C)NC1=C(Cl)C(=O)c2cccnc2C1=O</smiles>

102a $\begin{array}{llll}128 & -0.73 & -1.30 & \text { n.d. }\end{array}$

128

$$
-0.59 \quad-1.16 \quad \text { n.d. }
$$

$128-0.60 \quad-1.16 \quad$ n.d.

128

$$
\begin{array}{lll}
-0.56 & -0.97 \quad \text { n.d. }
\end{array}
$$

$\begin{array}{llll}512 & -0.60 & -1.17 & \text { n.d. }\end{array}$

$\begin{array}{llll}64 & -0.68 & -1.27 & -0.59\end{array}$ 
25<smiles>O=C1C(Cl)=C(NC(F)(F)F)C(=O)c2ncccc21</smiles>

102b

26<smiles>Cc1ccc2c(n1)C(=O)C(Cl)=C(NC(F)(F)F)C2=O</smiles>

103b

27<smiles>Cc1ccc2c(n1)C(=O)C(NC(C)(C)C)=C(Cl)C2=O</smiles>

97a

28<smiles>Cc1ccc2c(n1)C(=O)C(Cl)=C(NC(C)(C)C)C2=O</smiles>

97b

29<smiles>C#CCNC1=C(Cl)C(=O)c2cccnc2C1=O</smiles>

$100 a$

30<smiles>C#CCNC1=C(Cl)C(=O)c2ncccc2C1=O</smiles>

$100 b$ $\begin{array}{llll}16 & -0.67 & -1.26 & -0.62\end{array}$

$128 \quad-0.72 \quad-1.27 \quad$ n.d.

$\begin{array}{llll}>512 & -0.59 & -1.10 & -0.68\end{array}$

256

$-0.69$

$-1.25 \quad-0.68$

128

$\begin{array}{lll}-0.49 & -1.07 \quad \text { n.d. }\end{array}$

8

$-0.50 \quad-1.10 \quad$ n.d. 
31<smiles>C#CCNC1=C(Cl)C(=O)c2ccc(C)nc2C1=O</smiles>

$101 \mathrm{a}$

n.d.

32<smiles>C#CCNC1=C(Cl)C(=O)c2nc(C)ccc2C1=O</smiles>

64

n.d.

33

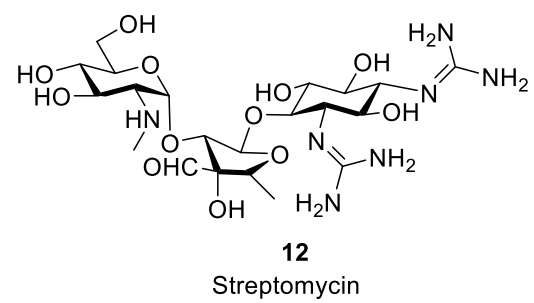

34<smiles>O=C1C(Cl)=C(NCCCl)C(=O)c2ncccc21</smiles>

QQ8c (20)
8.6 $6^{188}$ n.d. n.d. n.d

$8.1^{64}$

$-0.49^{c}$

$-0.95^{\mathrm{c}}$

n.d.

35<smiles>CC1=CC(=O)c2ccccc2C1=O</smiles>

n.d.

a $\mathrm{ACN} /$ toluene $(2 / 1, \mathrm{v} / \mathrm{v})$ was used; $\mathrm{b}$ in microemulsions; ${ }^{\mathrm{c}}$ in DMF; n.d. not determined

The effect of aniline-substituents on the Mtb growth inhibition of QQs was then analysed. Here, aniline-substituted QQs 104a, 105a, 105b were moderate to poor inhibitors of $M t b$ growth, with MIC values between 256 and $512 \mu \mathrm{M}$, while QQ 104b exerted no inhibitory activity (MIC $>512 \mu \mathrm{M}$, Entries 5-8). Next, the inhibitory activity of anilino-QQs bearing aliphatic chains of different length were investigated. In comparison to unsubstituted anilinoQQs 104-105, heptylanilino-QQs 108a and 108b resulted in lower MIC values of $32 \mu \mathrm{M}$ (Entry 
9) and $64 \mu \mathrm{M}$ (Entry 10), respectively. That said, the addition of a longer lipid chain, as in tetradecylanilino QQs 109a and 109b, led to a complete loss of anti-mycobacterial activity (Entries 11-12). These findings suggest that there could be an optimal carbon chain length on the QQ scaffold that confers higher inhibitory activity. Indeed, a similar observation was found by Bohne and co-workers, ${ }^{189}$ whereby quinolones with a 12 -carbon alkyl chain exerted higher inhibitory activity against Toxoplasma gandii NDH-II than a shorter 7-carbon chain. ${ }^{189}$ Moreover, when HQNO (19), a heptyl-substituted quinolone, was co-crystallised with $C$. thermarum NDH-II, the authors suggested a hydrophobic groove in the crystal structure comprising of V350, V362, L375 and A372 that could accommodate a longer alkyl chain. ${ }^{62}$

Next, the effect of the position of a phenyl group on the QQ scaffold was investigated by screening the Mtb growth inhibition of methoxybenzylamino- (110-111, Entries 13-15) and phenylethylamino-QQs 106-107 (Entries 16-19). Gratifyingly, these QQs, in particular the 7isomers 110a, 111a, 106a, 107a (Entries 13, 15, 16, 18, respectively), all with an MIC value of $16 \mu \mathrm{M}$, inhibited mycobacterial growth much more effectively than the anilino-QQs. The biological activity of QQs bearing aliphatic chains was then investigated (Entries 20-32). Here, propylamine-substituted QQs 98-99 displayed a modest growth inhibition of $M t b$, with MIC values between $128 \mu \mathrm{M}$ and $512 \mu \mathrm{M}$ (Entries 20-23). The longer alkyl chain lengths, as in octylamino-QQs 102a and 102b (Entries 24 and 25, respectively), led to increased antitubercular activity, though this increase was not observed in methyl-quinoline 103b (Entry 26). As with heptylanilino-QQs 108-109, the higher inhibition exerted by longer lipid tails in QQs 102a and 102b confirms the importance of enhanced hydrophobicity at this position. Finally, tert-butylamino-QQs 97a and 97b showed poor anti-mycobacterial activity (Entries 27 and 28, respectively), while propargylamino-QQs 100a, 100b, 101a, and 101b (Entries 29-32) showed a range of inhibitory activities (MIC $=8-256 \mu \mathrm{M}$ ), with 7-chloro-6-propargylamino QQ 100b having the lowest MIC value of the library $(8 \mu \mathrm{M}$, Entry 30$)$.

Another important SAR was the position at which the amine substituent was installed on the QQ scaffold. For QQs bearing aryl groups, the 7-isomers exhibited better anti-tubercular activity compared to the 6-isomer in all cases aside from tetradecylanilino-QQs 109a and 109b, which were both inactive against $M t b$ (Entries 11 and 12). This trend was evidenced by the difference in MIC values between regioisomers 104a and 104b (MIC $=512 \mu \mathrm{M}$ vs. $>512 \mu \mathrm{M}$, Entries 5 and 6, respectively), 105a and 105b (256 $\mu \mathrm{M}$ vs. $512 \mu \mathrm{M}$, Entries 7 and 8, respectively), 110 and $110 \mathrm{~b}$ (MIC $=16 \mu \mathrm{M}$ vs. $32 \mu \mathrm{M}$, Entries 13 and 14, respectively), 106a and 106b $(\mathrm{MIC}=16 \mu \mathrm{M}$ vs. $128 \mu \mathrm{M}$, Entries 16 and 17, respectively) and 107a/107b (16 $\mu \mathrm{M}$ vs. $128 \mu \mathrm{M}$, Entries 18 and 19, respectively). Conversely, the 6-isomers tended to exhibit higher 
inhibitory activity than the corresponding 7-isomers in QQs bearing aliphatic chains, with the exceptions of propylamino-QQs 98a/98b (MIC = $128 \mu \mathrm{M}$ for both, Entries 20 and 21), and 99a/99b (MIC $=128 \mu \mathrm{M}$ vs. $512 \mu \mathrm{M}$, Entries 22 and 23, respectively). Notable examples of this trend include the enhanced inhibitory activity of 6-isomers $97 \mathrm{~b}, 100 \mathrm{~b}, 101 \mathrm{~b}$ and $102 \mathrm{~b}$ (MIC $=256 \mu \mathrm{M}, 8 \mu \mathrm{M}, 64 \mu \mathrm{M}$ and $16 \mu \mathrm{M}$, Entries 28, 30, 32 and 25, respectively) compared to their corresponding 7-isomers 97a, 100a, 101a, 102a $(\mathrm{MIC}=>512 \mu \mathrm{M}, 128 \mu \mathrm{M}, 256 \mu \mathrm{M}$ and 64 $\mu \mathrm{M}$, Entries 27, 29, 32 and 24, respectively).

In summary, the QQ analogues exhibited good to modest anti-tubercular activity, with only five compounds (94a, 104b, 109a, 109b, 97a) failing to inhibit bacterial growth (Entries 1, 6, 11, 12, and 27). Propargylamine-substituted QQ 100b (MIC $=9 \mu \mathrm{M}$, Entry 30) was the most active compound of the series, exhibiting an MIC value comparable to first-line drug streptomycin $\left(\mathbf{1 2}, \mathrm{MIC}=8.6 \mu \mathrm{M}\right.$, Entry 33) ${ }^{188}$ and NDH-II activator QQ8c $(\mathbf{2 0}, \mathrm{MIC}=8.1$ $\mu \mathrm{M}$, Entry 34). ${ }^{64}$ QQs bearing aryl groups, i.e. heptylanilino-QQs 108, benzylamino-QQs 110, 111, and phenylethylamino-QQs 106, 107, were effective $M t b$ growth inhibitors. Here, the lack of inhibitory activity of QQs bearing longer lipid chains (QQs 108) suggests that an optimal alkyl chain length is important for anti-tubercular activity. Similarly, QQs bearing short aliphatic chains, i.e. propylamino-QQs $\mathbf{9 8 , 9 9}$ were less potent than those with longer alkyl groups, i.e. octylamino-QQs 102 and 103, though the addition of an acetylene group i.e. in propargylamines 100 and 101, led to increased growth inhibition. In addition, QQs bearing tertiary amines, i.e. dimethylamino-QQs 94 and piperidino-QQs 95, or amines adjacent to a quaternary carbon, i.e. tert-butylamino-QQs 97 and anilino-QQs 104-105, are poor growth inhibitors of $M t b$. Along with these new insights into the SAR between QQs and Mtb growth inhibition, trends from previous findings were also confirmed, ${ }^{65}$ such as the negligible effect of the methyl substituent on the 2-position of QQs on the biological activity of the compounds. Furthermore, this set of QQs allowed for the identification of a new lead antimycobacterial compound, propargylamino-QQ 100b. Given the stimulatory effects exerted by QQs $20^{64}$ and $\mathbf{6 0}$ (Chapter 2) on NADH oxidation, it's likely that QQ $100 \mathrm{~b}$ could have a similar inhibition mechanism, though this is yet to be confirmed.

\subsubsection{Reduction potentials of QQs}

Given the ability of quinones to undergo reduction and aid in ROS generation, ${ }^{64,149,150}$ redox potentials of QQs 94-111 were determined by cyclic voltammetry (CV). To serve as a reference, the redox potentials of menadione (18), a methyl analogue of the NDH-II substrate MK (15), 
was also measured. The CV experiments were conducted in acetonitrile, whereby two consecutive one-electron redox events occured, as reported for quinones in aprotic solvents (Scheme 3.1, page 81). ${ }^{168,190}$ For example, the voltammogram of QQ 111a (Figure 3.10) revealed two reduction or cathodic peaks as the voltage is scanned at negative potentials. When the switching potential of $-1.5 \mathrm{~V}$ was reached, the scan direction was reversed, resulting in the stepwise oxidation of the reduced species. ${ }^{191}$ The redox potentials for the first and second electron transfers, $\mathrm{E}_{1}$ and $\mathrm{E}_{2}$, respectively, are determined as the midpoint potential of the corresponding reduction and oxidation peaks. All potentials reported in this section are referenced against $\mathrm{Ag} / \mathrm{Ag}^{+}$.

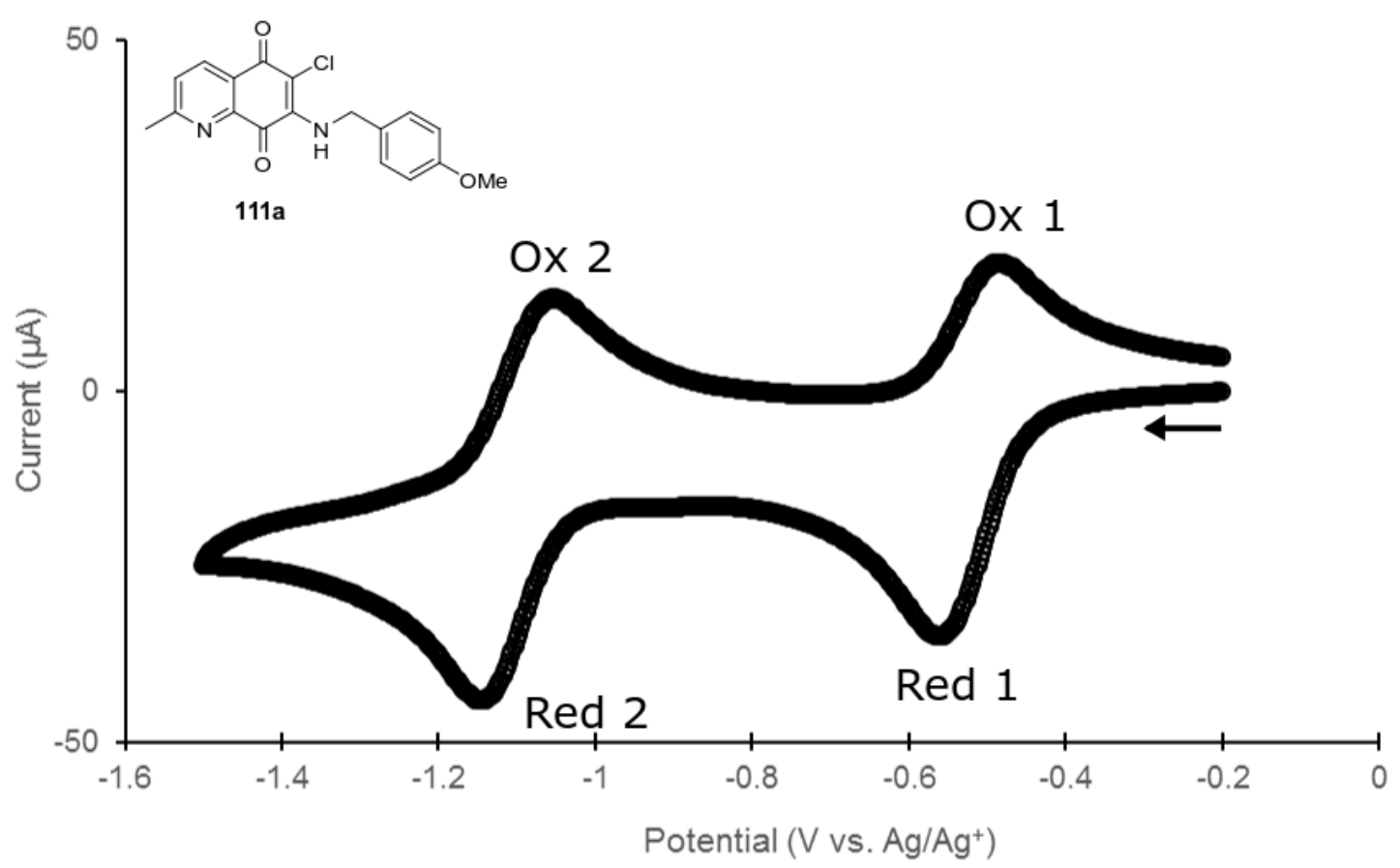

Figure 3.10: Voltammogram of $1.4 \mathrm{mM}$ QQ 111a in a $50 \mathrm{mM}\left(\mathrm{C}_{2} \mathrm{H}_{5}\right)_{4} \mathrm{~N}^{+}\left(\mathrm{PF}_{6}\right)^{-}$solution of acetonitrile at $100 \mathrm{mV} / \mathrm{s}$ on a glassy carbon (GC) electrode, in the presence of $\mathrm{N}_{2}$.

The redox potentials of QQs $\mathbf{9 4 - 1 1 1}$ were comparable to that of menadione $\left(\mathbf{1 8}, \mathrm{E}_{1}=-0.70 \mathrm{~V}\right.$, $\mathrm{E}_{2}=-1.27 \mathrm{~V}$, Entry 35, Table 3.1), whereby $\mathrm{E}_{1}$ ranged from $-0.42 \mathrm{~V}$ to $-0.78 \mathrm{~V}$ and $\mathrm{E}_{2}$ occurred between $-0.90 \mathrm{~V}$ and $-1.30 \mathrm{~V}$ (Entries 1-32). In addition, the difference in potential between the two redox events $\left(\Delta \mathrm{E}_{1}-\mathrm{E}_{2}\right)$ was consistent among the QQs, at approximately $0.55 \mathrm{~V}$, indicating that the QQ semiquinone radicals had a similar stability to menadione $\left(18, \Delta \mathrm{E}_{1}-\mathrm{E}_{2}\right.$ $=0.58 \mathrm{~V}$ ). The similarity in redox behaviour between synthesised QQs 94-111 and menadione (18) indicated that the QQs may be able to participate in redox reactions in NDH-II. Moreover, 
a $\mathrm{CV}$ conducted when the solution was not purged with $\mathrm{N}_{2}$ revealed additional reduction peaks at $-0.6 \mathrm{~V}$ and $-1.3 \mathrm{~V}$ and the absence of QQ dianion formation (Figure 3.11). The discrepancy between the two voltammograms (Figure 3.10 and Figure 3.11) is likely to be caused by the presence of $\mathrm{O}_{2}$ in the solvent that was not previously degassed prior to obtaining the CV. Here, $\mathrm{O}_{2}$ can undergo reduction at $-0.6 \mathrm{~V}$ to form superoxide anion radicals, which can react with the semiquinone radicals generated at $-0.85 \mathrm{~V}$. The by-product of the reaction between reduced oxygen species and semiquinone radicals can then undergo an irreversible reduction, observed at $-1.3 \mathrm{~V}$, while the remaining semiquinone species in the bulk solution are oxidised to the parent quinone at $-0.7 \mathrm{~V}$. The reaction between semiquinones and superoxide radicals is wellprecedented for quinones with low one-electron reduction potentials, ${ }^{163}$ and provide the possibility for QQs to undergo redox cycling to form ROS in Mtb.

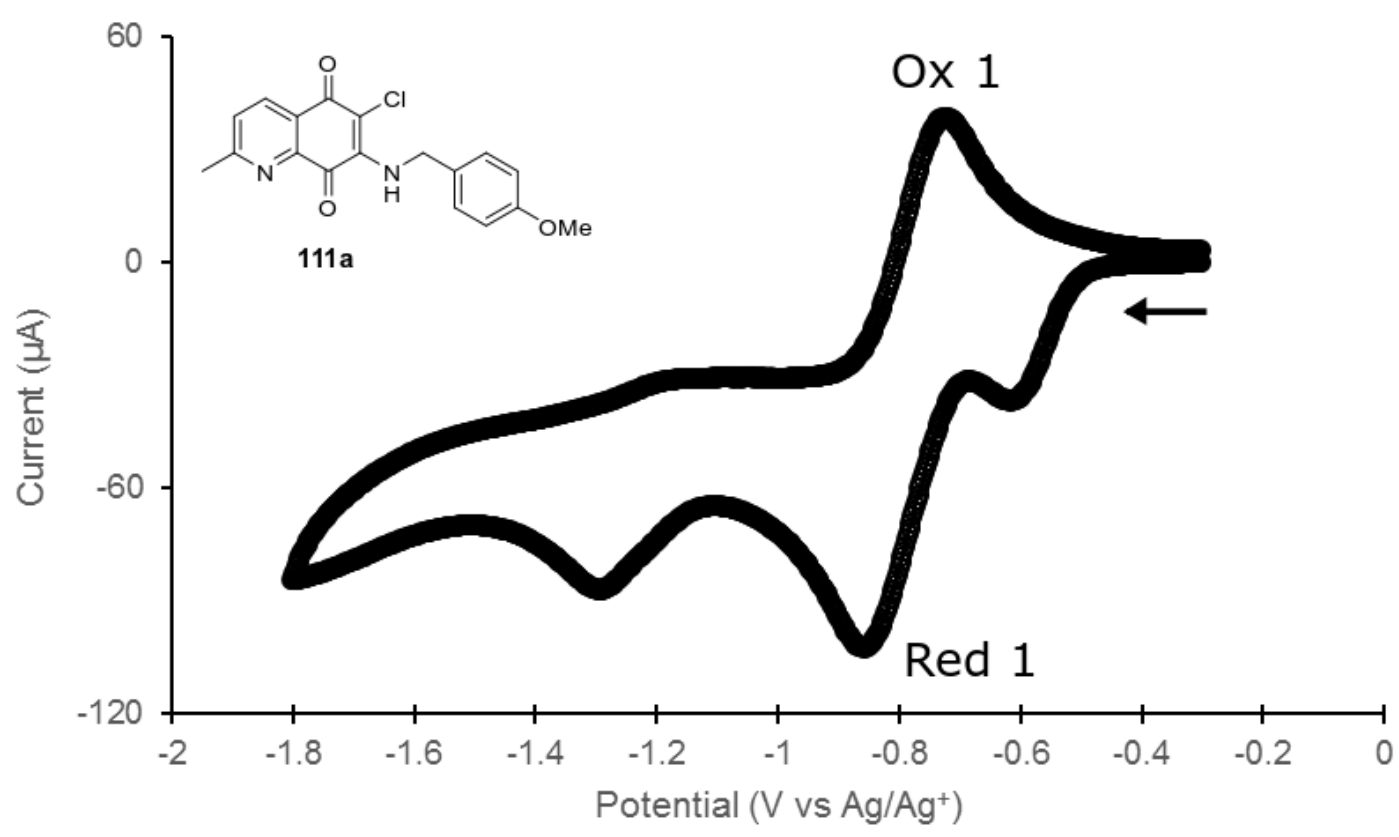

Figure 3.11: Cyclic voltammogram of $1.4 \mathrm{mM}$ QQ 111a in a $50 \mathrm{mM}\left(\mathrm{C}_{2} \mathrm{H}_{5}\right)_{4} \mathrm{~N}^{+}\left(\mathrm{PF}_{6}\right)^{-}$solution of acetonitrile at $100 \mathrm{mV} / \mathrm{s}$ on a GC electrode, without purging with $\mathrm{N}_{2}$.

The relationship between the different functionalities on the quinone scaffold and the redox potentials of QQs 94-111 was analysed. In general, QQs bearing anilines, in particular anilinesubstituted QQs 104-105 $\left(\mathrm{E}_{1}=-0.42 \mathrm{~V}\right.$ to $-0.47 \mathrm{~V}, \mathrm{E}_{2}=-1.00 \mathrm{~V}$ to $-1.06 \mathrm{~V}$, Entries 5-8) and tetradecylanilino-QQs $109\left(\mathrm{E}_{1}=-0.45 \mathrm{~V} /-0.53 \mathrm{~V}, \mathrm{E}_{2}=-1.02 \mathrm{~V}\right.$ for both, Entries 11-12), as well as propargylamine (QQs 100-101, $\mathrm{E}_{1}=-0.49 \mathrm{~V}$ to $-0.55 \mathrm{~V}, \mathrm{E}_{2}=-1.05 \mathrm{~V}$ to $-1.10 \mathrm{~V}$, Entries 
29-32) had the highest redox potentials of the library. Conversely, QQs bearing hydrocarbons, i.e. octylamino-QQs 102-103 $\left(\mathrm{E}_{1}=-0.67 \mathrm{~V}\right.$ to $-0.72 \mathrm{~V}, \mathrm{E}_{2}=-1.26 \mathrm{~V}$ to $-1.27 \mathrm{~V}$, Entries 24-26) and tert-butylamino-QQ $97\left(\mathrm{E}_{1}=-0.59 \mathrm{~V}\right.$ to $-0.68 \mathrm{~V}, \mathrm{E}_{2}=-1.10 \mathrm{~V}$ to $-1.25 \mathrm{~V}$, Entry 28), had relatively low reduction potentials. It was also noted that QQs with aromatic groups positioned further away from the QQ scaffold, i.e. phenylethylamino-QQs 106-107 $\left(\mathrm{E}_{1}=-0.60 \mathrm{~V}\right.$ to -0.73 $\mathrm{V}, \mathrm{E}_{2}=-1.15$ to -1.30 , Entries 16-19) and benzylamines $110\left(\mathrm{E}_{1}=-0.61 \mathrm{~V}\right.$ to $-0.63 \mathrm{~V}, \mathrm{E}_{2}=-$ 1.18 to -1.24 , Entries 13-14), were weaker oxidising agents compared to the anilino-QQs. This trend highlights the marked effect that functional groups have on the QQs' ability to accept electrons. In comparison to hydrocarbons, QQs bearing unsaturated substituents, i.e. phenyland propargyl groups, are higher in electronegativity, ${ }^{192-194}$ and thus result in weak electronwithdrawing properties. Hence, QQs bearing anilines and propargylamines underwent reduction more readily than those with hydrocarbon substituents, which is in agreement with literature. $^{165}$

In many cases, there was negligible difference in redox potentials between regioisomers (i.e. $\mathrm{E}_{1}$ $=-0.59 \mathrm{~V}$ and $-0.60 \mathrm{~V}, \mathrm{E}_{2}=-1.16 \mathrm{~V}$ and $-1.16 \mathrm{~V}$ for QQs $98 \mathrm{a}$ and $\mathbf{9 8 b}$, Entries 20 and 21, respectively, and $\mathrm{E}_{1}=-0.68 \mathrm{~V}$ and $-0.67 \mathrm{~V}, \mathrm{E}_{2}=-1.27 \mathrm{~V}$ and $-1.26 \mathrm{~V}$ for QQs 102a and 102b, Entries 24 and 25, respectively). However, when the regioisomers possessed different reduction potentials, the 7 -isomer tended to accept electrons more readily than the corresponding 6-isomer, particularly for the first electron transfer. For example, QQ 97a $\left(\mathrm{E}_{1}\right.$ $=-0.59 \mathrm{~V}$, Entry 27) had higher redox potentials than its regioisomer QQ 97b $\left(\mathrm{E}_{1}=-0.69 \mathrm{~V}\right.$, Entry 28), and, similarly, QQ 99a was more readily reduced than its regioisomer $99 \mathbf{b}\left(\mathrm{E}_{1}=\right.$ $0.56 \mathrm{~V} /-0.60 \mathrm{~V}$, Entries 22 and 23, respectively). The greater susceptibility for the 7-isomers (X) to undergo reduction compared to the 6-isomers (XI) could be reasoned by intramolecular hydrogen bonds within the QQ (Figure 3.12). As well as the hydrogen bond between the amine proton and the carbonyl oxygen, the ring nitrogen in the 7-isomer, when protonated, could form an additional hydrogen bond with the C8 oxygen. The hydrogen bonds in the 7-isomer may lead to enhanced electrophilicity of the compound, thereby increasing its propensity to undergo reduction reactions. ${ }^{163}$ 


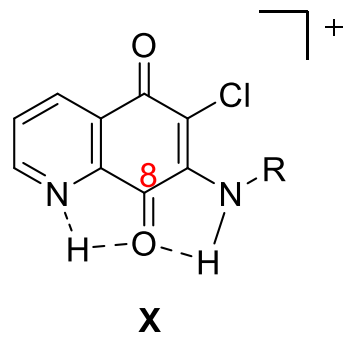

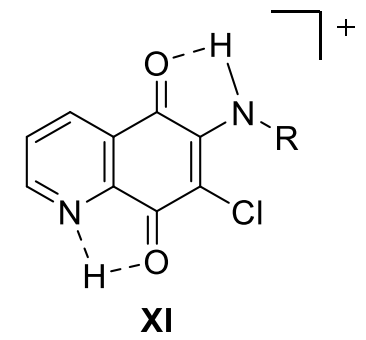

Figure 3.12: Intramolecular hydrogen bonding interactions present in QQs $\mathbf{X}$ and $\mathbf{X I}$.

In addition to the $\mathrm{CV}$ experiments in acetonitrile, experiments using $\mathrm{H}_{2} \mathrm{O}$ as the solvent were undertaken in order to better emulate physiological conditions. However, due to the poor solubility of QQs 94-111 in $\mathrm{H}_{2} \mathrm{O}$, a novel CV methodology involving microemulsions, as designed by Professor Thomas Nann and co-workers at Victoria University of Wellington, was applied (patent pending). ${ }^{195} \mathrm{~A}$ microemulsion is a thermodynamically stable mixture of immiscible liquids, typically oil and water, in combination with amphiphilic materials known as surfactants. This technique allows for the QQs to be solubilised in organic solvents in the microemulsion, while the redox reactions occur in an aqueous environment, whereby the quinones (II) undergo a two-electron step reaction to form quinols (IV) (Figure 3.13). ${ }^{190}$

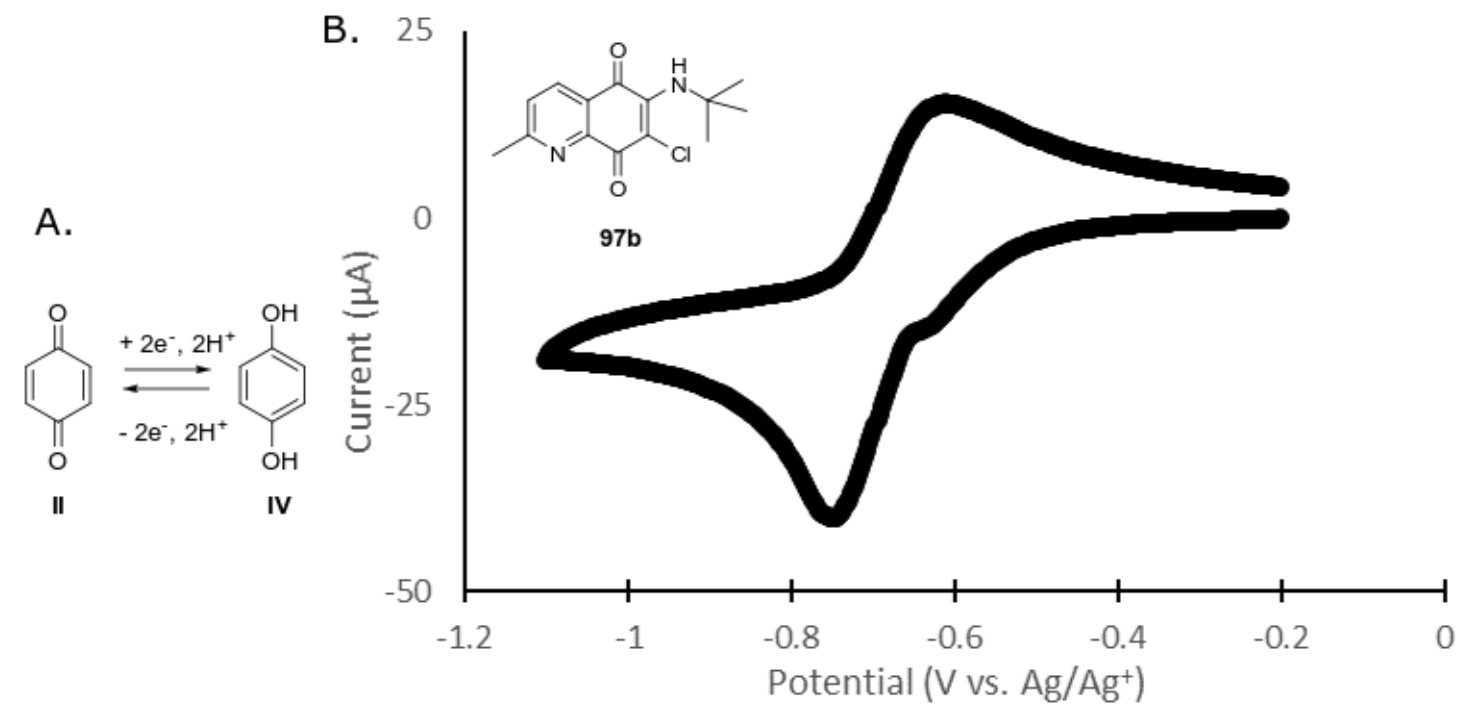

Figure 3.13: A). Two-electron reduction process from quinones (II) to quinols (IV) in aqueous solvents. B). Voltammogram of $50 \mathrm{mM}$ of QQ $\mathbf{9 7 b}$ in a microemulsion against GC electrode. 
CV experiments of QQs 94-111 were undertaken in water with the employment of microemulsions by Prof. Thomas Nann and co-workers. Here, the compounds were dissolved in toluene and mixed with $\mathrm{H}_{2} \mathrm{O}$ to generate microemulsions, which were stabilised with sodium dodecylsulfate and 1-butanol as surfactant and co-surfactant, respectively. Unfortunately, many of the QQs did not show reversible redox reactions and, accordingly, their reduction potentials, E', could not be determined. Notwithstanding, E' values were obtained for QQs 95-97, 102, 106a, 107a, 108, and 110b (Entries 3, 4, 9, 10, 14, 16, 18, 24, 25, 27 and 28, Table 3.1). Here, the redox potentials of the QQs obtained from the CVs involving microemulsions differed from those obtained from the CVs conducted in acetonitrile. For example, in the microemulsion, piperidino-QQs $\mathbf{9 5 a}$ and $\mathbf{9 6} \mathbf{b}$ gave high redox potentials at $-0.49 \mathrm{~V}$ and -0.47 $\mathrm{V}$, respectively (Entries 3 and 4), while in acetonitrile, QQs $\mathbf{9 5 a}$ and $\mathbf{9 6 \mathbf { b }}$ were weakly reducing relative to other QQs in the library. The inconsistency in redox activity of the QQs in the different solvents is perhaps not surprising, as the electrochemical behaviour of quinones has been reported to change in various media. ${ }^{168}$ Indeed, different redox values were also obtained when dimethylformamide (DMF) was used as solvent (see Appendix 3, Table A.3.3.)

\subsubsection{Relationship between redox potentials and anti-tubercular activity of QQs}

Next, the relationship between the tuberculostatic activity and redox potentials of QQs 94-111 was analysed. Unfortunately, no trend was observed between the redox activity of the QQs in acetonitrile $\left(\mathrm{E}_{1}\right.$ and $\left.\mathrm{E}_{2}\right)$ and their MIC values (Figure 3.14). Moreover, a lack of correlation was also observed with $E_{1} / E_{2}$ values obtained from $C V$ experiments conducted in DMF (Appendix 3, Figure A.3.1.). 


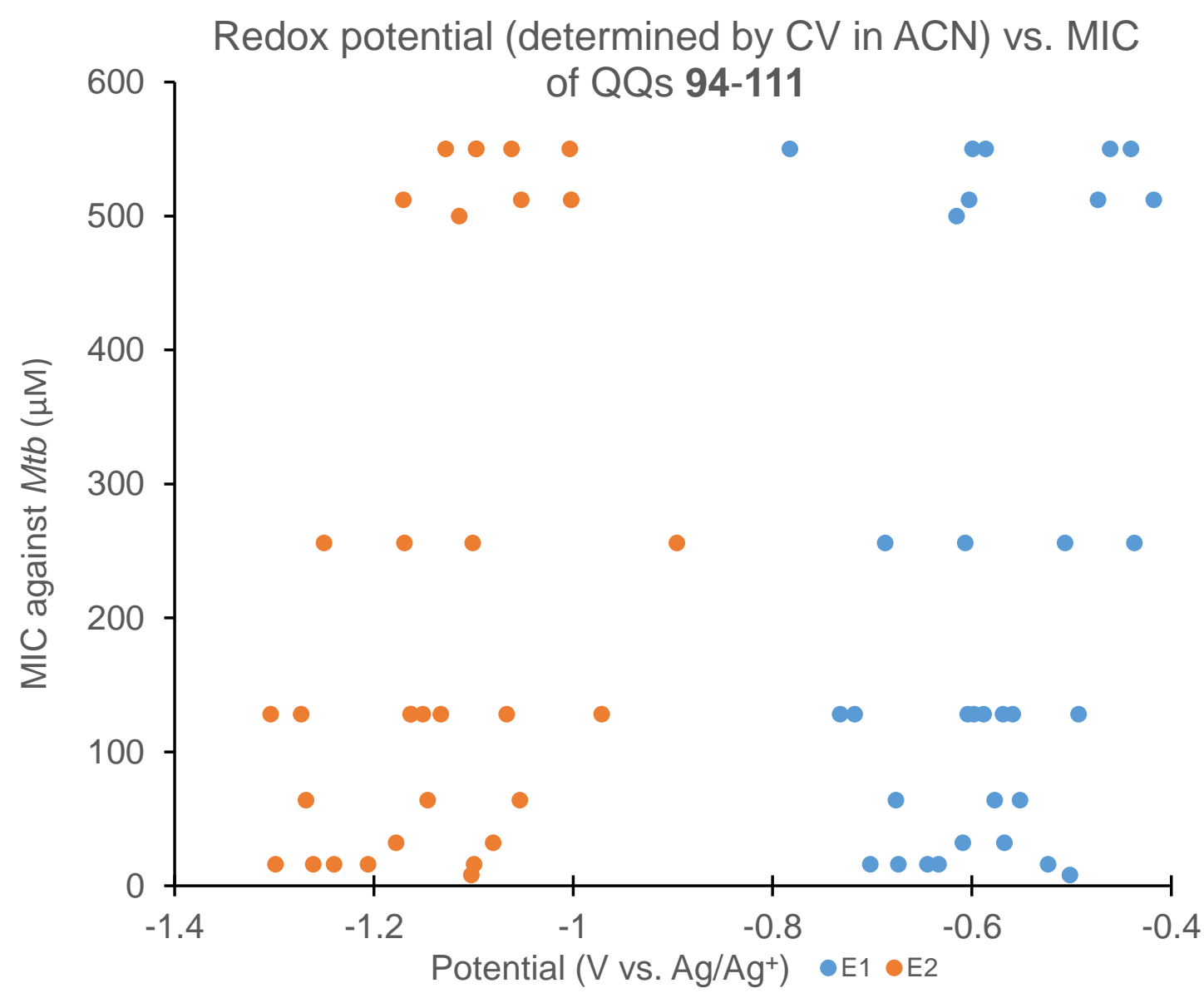

Figure 3.14: No relationship was found between the redox potential of QQs 94-111 determined by CV in acetonitrile and their anti-tubercular activity.

That said, when the MIC values were plotted against the redox potentials obtained from microemulsion CVs, an optimal redox potential window was observed, whereby QQs with redox potentials between $-0.50 \mathrm{~V}$ to $-0.62 \mathrm{~V}$ tended to exhibit the highest inhibitory activity (Figure 3.15). For QQs with redox potentials higher than $-0.62 \mathrm{~V}$, there was a near linear relationship between the redox potential and MIC values, suggesting that QQs with lower reduction potentials were more effective growth inhibitors of $M t b$. 


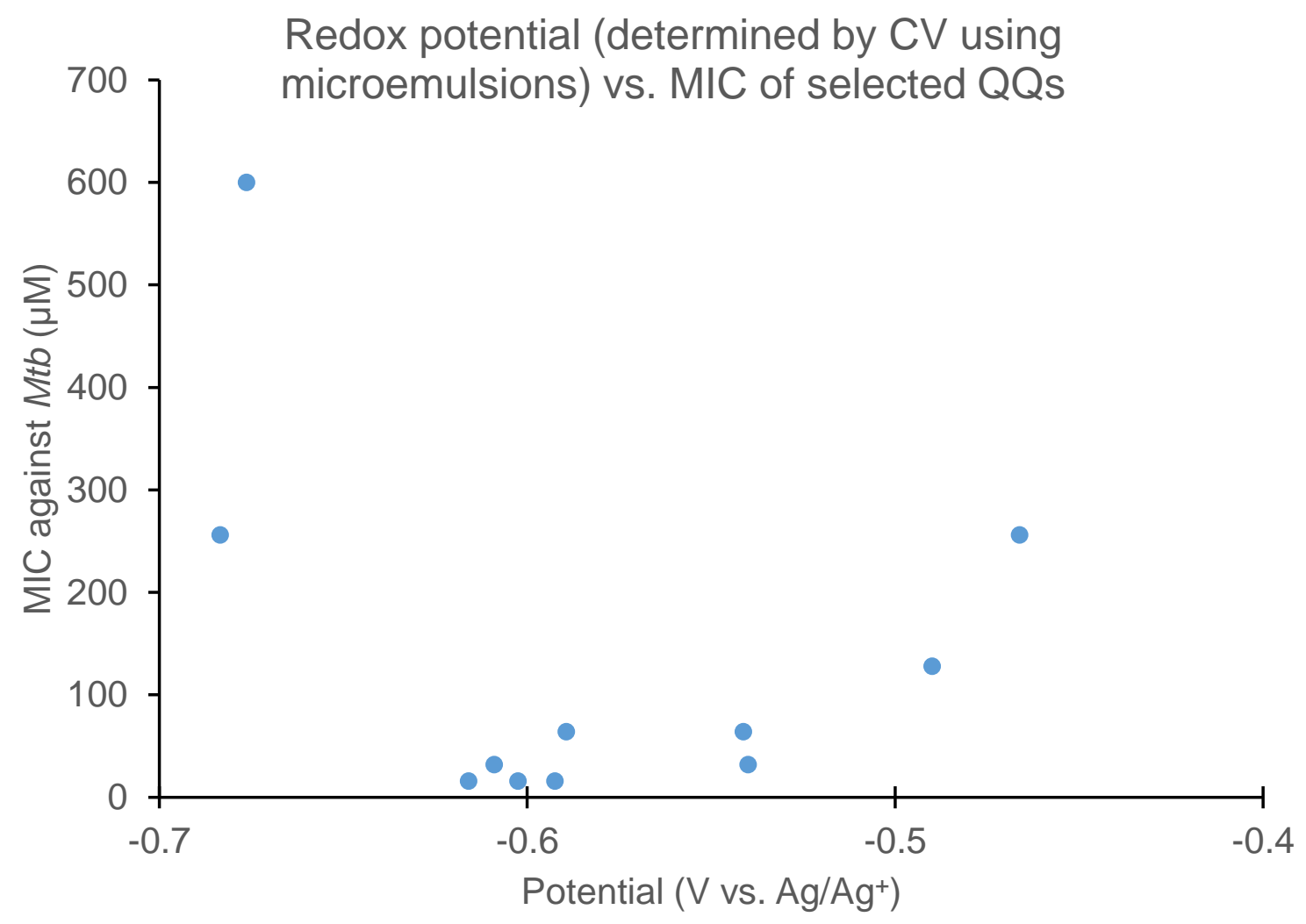

Figure 3.15: The relationship between the redox potential of selected QQs in microemulsions and their anti-tubercular activity as measured in MIC.

The optimal redox potential of $-0.6 \mathrm{~V}$ for the observed anti-tubercular activity of the QQs is comparable to that of Mtb NDH-II's natural substrate, MK (15), which has a redox potential of $-0.66 \mathrm{~V}$ in water (vs. $\mathrm{Fc} / \mathrm{Fc}^{+}$). ${ }^{196}$ Thus, it was hypothesised that QQs with redox potential much higher than $-0.66 \mathrm{~V}$ are likely to be reduced too quickly and are therefore less effective in interacting with NDH-II. Conversely, QQs with low redox potentials are less likely to undergo reduction and may not be involved in the enzymatic reduction by NDH-II. Taken together, these results suggest that an optimal redox potential of approximately $-0.6 \mathrm{~V}$, as determined when microemulsions of the QQs were measured appears to be required for the quinones to exert anti-tubercular activity. That said, the redox potentials of the remaining QQs need to be determined to ascertain the relationship between redox and anti-tubercular activity of the compounds. 


\subsubsection{Proposed mechanism of anti-tubercular activity of lead propargylamino-QQ}

The acetylene motif is a common moiety in many biologically active compounds, particularly in anti-cancer ${ }^{186,197,198}$ and anti-mycobacterial agents, ${ }^{199,200}$ and accordingly, a redox-independent mechanism of lead propargylamine-substituted QQ $100 \mathrm{~b}$ was postulated. Here, the acetylene group could covalently bind nucleophilic amino acid residues, resulting in irreversible inhibition (XII, Scheme 3.4A) ${ }^{201}$ Potential nucleophilic residues in the NDH-II Q-site that could form covalent bonds with an electrophilic group such as allene include two highly conserved glutamine residues, Q317 and Q321. ${ }^{53}$ Glutamines are nucleophilic amino acids ${ }^{202}$ that have previously been reported to be involved in covalent inhibition, i.e. with 2-deoxy-2fluoro- $\beta$-D-glucopyranoside. ${ }^{203,204}$ In addition, Q317 and Q321 play an important role in quinone binding, as evidenced by the decrease in quinone binding affinity when the glutamine residues were mutated to alanines. ${ }^{53}$

The above hypothesis could also explain the difference in biological activity between the structurally related QQs 20 (MIC $=8.1 \mu \mathrm{M})$ and $\mathbf{9 8 b}(\mathrm{MIC}=128 \mu \mathrm{M})$, where QQ8c 20 bears a chloroethylamine substituent on the 6-position and QQ $\mathbf{9 8 b}$ is substituted with a propylamine group (Figure 3.11). Thus, it is possible that nucleophilic residues, such as glutamine, can displace the chloride atom in QQ8c (20) to give the covalent adduct XIII (Scheme 3.4B). Alternatively, the chloride atom could be ejected via an intramolecular attack by the amine nitrogen to yield aziridinium XIV, which can then undergo ring-opening by a nucleophilic amino acid residue to give XIII (Scheme 3.4C). 
A.<smiles>C=C(N)CNC1=C(Cl)C(=O)c2ncccc2C(=O)C1=[W]</smiles>

\section{B.}

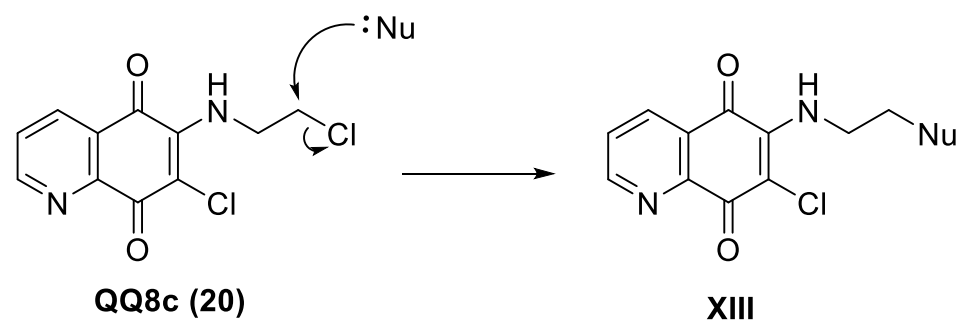

c.<smiles>CC(Cl)(Cl)CCNC1=C(Cl)C(=O)c2cccnc2C1=O</smiles><smiles>N#CC1CN1C1=CC(=O)c2cccnc2C1=O</smiles>

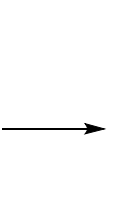<smiles>N#[N+]CCNC1=C(Cl)C(=O)c2ncccc2C1=O</smiles>

XIII

Scheme 3.4: Possible mechanisms of covalent bond formation between QQ 100b and QQ8c (20) with nucleophilic amino acid residues within the binding site. A). The acetylene group in QQ 100b is subject to nucleophilic attack to form the enol XII, while QQ8c (20) can undergo B). a substitution reaction with the nucleophile to afford covalent adduct XIII or C). an intramolecular substitution reaction to form aziridinium XIV before undergoing nucleophilic attack.

\subsection{Conclusion}

In this chapter a library of QQs containing different functional groups were synthesised to establish SAR for the growth inhibition of $M t b$. Herein, 32 compounds were successfully prepared, giving the desired QQs in two steps from commercially available materials in high yields (64-99\%). The synthesised QQs were then screened against Mtb to obtain MIC values for SAR analysis. The SAR study showed that QQs functionalised with phenylethylamine and benzylamine exhibited high anti-tubercular activity, while those with aniline substituents were poor $M t b$ growth inhibitors. QQ analogues with alkyl chains tended to have a mixed range of anti-tubercular activity, while those with tertiary amines did not exhibit growth inhibition. Notably, the most biologically active compound was propargylamino-substituted QQ 98b, with an MIC comparable to streptomycin (12) and QQ8c (20) (8 $\mu$ mol, $8.6 \mu \mathrm{mol},{ }^{188}$ and 8.1 $\mu \mathrm{mol},{ }^{64}$ respectively). In addition to MIC values, the redox potentials of a selection of QQs 
were also determined, and while no correlation between the redox potential and tuberculostatic activity in acetonitrile, an optimal redox potential of approximately $-0.60 \mathrm{~V}$ for biological activity against $M t b$ was observed for QQs compatible with the microemulsion system.

While the mode of action of the lead QQ analogue $\mathbf{9 8 b}$ is unknown, acetylene groups are known to enhance biological potential of a variety of anti-tumour agents. ${ }^{197}$ A potential explanation for this observation is that the acetylene moiety could undergo nucleophilic attack by amino acid residues in the binding site, such as glutamine, which could result in a covalent bond and irreversible binding. To confirm this hypothesis, further biological analyses are warranted and should include co-crystallisation of the quinone with NDH-II to examine the binding site more closely. Furthermore, future work could focus on the specificity of the QQs for bacterial NDH-II over other potential enzyme targets, such as NQO1, whereby the latter is overexpressed in many cancer cells. ${ }^{139}$ 


\subsection{Experimental}

\subsubsection{Chemistry - synthesis}

General procedure. Unless otherwise stated, all reactions were performed under atmospheric air. Prior to use, dioxane (Sigma Aldrich) was distilled over $\mathrm{CaCl}_{2}$, toluene (drum), EtOAc (drum), and petroleum ether (drum) were distilled. $\mathrm{CH}_{2} \mathrm{Cl}_{2}$ (LabServ), sodium chlorate (BDH), 8-hydroxyquinoline (BDH), 37\% $\mathrm{HCl}$ (Merck), EtOH (absolute, Pure Science), 8hydroxyquinaldine (Aldrich), $\mathrm{NaHCO}_{3}$ (Pure Science), $\mathrm{MgSO}_{4}$ (Pure Science), pyridine (Roth), dimethylamine $(\mathrm{BDH})$, piperidine $(\mathrm{BDH})$, tertbutylamine (Aldrich), propylamine (Aldrich), propargylamine (AKSci), octylamine (Aldrich), aniline (BDH), 4-heptylaniline (Aldrich), 4tetradecylaniline (Aldrich), methoxybenzylamine (Aldrich), and phenylethylamine (HW) were used as received.

All solvents were removed by evaporation under reduced pressure. Reactions were monitored by TLC-analysis on Macherey-Nagel silica gel coated plastic sheets $(0.20 \mathrm{~mm})$ with either visual detection, or detection by UV-absorption ( $254 \mathrm{~nm}$ ) and by dipping in $\mathrm{KMnO}_{4}$ in $\mathrm{H}_{2} \mathrm{O}$. Column chromatography was performed using Pure Science silica gel (40-63 micron) as the stationary phase and the solvent systems indicated. High-resolution (ESI) mass spectra were recorded on an Agilent 6530 Accurate-Mass Q-TOF LC-MS equipped with a 1260 Infinity binary pump. Infrared spectra were recorded as thin films using a Bruker Alpha II FTIR spectrometer equipped with an Attenuated Total Reflectance (ATR) sampling accessory, and are reported in wavenumbers $\left(\mathrm{cm}^{-1}\right)$. Melting points (m.p.) were obtained on a DigiMelt MPA160 melting point apparatus and UV data obtained on an Cary 150 spectrophotometer. Nuclear magnetic resonance (NMR) spectra were acquired at $20 \mathrm{C}$ in $\mathrm{CDCl}_{3}$, as indicated using a Varian UnityINOVA $500 \mathrm{MHz}$ spectrometer, where ${ }^{1} \mathrm{H}$ and ${ }^{13} \mathrm{C}$ were measured at 500 and $125 \mathrm{MHz}$, respectively. ${ }^{1} \mathrm{H}$ NMR spectral data are presented as illustrated: chemical shift $(\delta)$ in ppm, multiplicity [s (singlet), d (doublet), $\mathrm{t}$ (triplet), $\mathrm{m}$ (multiplet over the range specified)], number of protons, $(\mathrm{nH})$, coupling constants $(\mathrm{J})$ in Hertz, assignment of protons). ${ }^{1} \mathrm{H}$ NMR spectra are referenced to the $\mathrm{CHCl}_{3}(\delta 7.26 \mathrm{ppm})$, residual solvent peaks. ${ }^{13} \mathrm{C}$ NMR spectra were proton decoupled and referenced to TMS ( $\delta 0 \mathrm{ppm})$. NMR peak assignments were made using COSY, HSQC, and HMBC experiments. 
<smiles>O=C1C(Cl)=C(Cl)C(=O)c2ncccc21</smiles>

92

6,7-Dichloro-5,8-quinolinequinone (92). In a round-bottomed flask fitted with a condenser, 8-hydroxyquinoline ( $14.5 \mathrm{~g}, 0.1 \mathrm{~mol})$ was dissolved in conc. $\mathrm{HCl}(600 \mathrm{~mL})$. The reaction was then warmed to $40{ }^{\circ} \mathrm{C}$ before sodium chlorate ( $53 \mathrm{~g}, 0.5 \mathrm{~mol}, 5$ equiv.) was added in batches over a period of $1 \mathrm{~h}$, where each batch was added after the yellow gas produced had receded. The reaction was then left to stir for $2 \mathrm{~h}$ before it was diluted with $\mathrm{H}_{2} \mathrm{O}(1400 \mathrm{~mL})$. The white precipitate was filtered off, and the filtrate extracted with $\mathrm{CH}_{2} \mathrm{Cl}_{2}(6 \times 250 \mathrm{~mL})$ before the organic layer was collected and concentrated in vacuo. The bright orange solid was then recrystallised from ethanol to yield the dichloroquinolinequinone $\mathbf{9 2}$ as bright yellow crystals (6.6 g, 29\%). $\mathrm{R}_{f}=0.50$ (EtOAc); m.p.: $221.7^{\circ} \mathrm{C}$ (lit. ${ }^{65} 219.4-221.0^{\circ} \mathrm{C}$ ); IR (thin film): 2971, $1694,1677,1573,1558,1290,1275,1194,1137,895,824,724,693,636 \mathrm{~cm}^{-1}$; ${ }^{1} \mathrm{H}$ NMR $(500$ $\mathrm{MHz}_{\mathrm{CDCl}}$ ) $\delta$ ppm 9.10 (d, J2,3 $\left.=4.6 \mathrm{~Hz}, 1 \mathrm{H}, \mathrm{H} 2\right), 8.53$ (d, J3,4 $\left.=7.9 \mathrm{~Hz}, 1 \mathrm{H}, \mathrm{H} 4\right), 7.76$ (dd, $\left.J_{3,4}=7.8 \mathrm{~Hz}, J_{2,3}=4.6 \mathrm{~Hz}, 1 \mathrm{H}, \mathrm{H} 3\right) ;{ }^{13} \mathrm{C} \mathrm{NMR}\left(125 \mathrm{MHz}, \mathrm{CDCl}_{3}\right): \delta 175.6$ (C5), 174.4 (C8), 155.4 (C2), 146.8 (C8a), 144.4 (C6 or C7), 143.2 (C6 or C7), 135.7 (C4), 128.3 (C4a and C3); HRMS(ESI) $m /$ z calcd. for $\left[\mathrm{C}_{9} \mathrm{H}_{3} \mathrm{Cl}_{2} \mathrm{NO}_{2}+\mathrm{H}\right]^{+}:$227.9614, obsd.: 227.9618. The data obtained for this compound matched those reported in literature. ${ }^{65}$

General procedure for the synthesis of substituted QQs. To a solution of dichloroquinolinequinones 92 or $\mathbf{5 6}$ (1 equiv.) in dioxane $(5 \mathrm{~mL} / \mathrm{mmol}$ ) was added the desired amine (1.2 equiv.). The coloured solution was stirred at room temperature before pyridine (2.1 equiv.) was added and the reaction stirred for $4 \mathrm{~h}$, after which TLC analysis showed the disappearance of starting materials. The reaction was then concentrated in vacuo, redissolved in EtOAc and washed with sat. aq. $\mathrm{NaHCO}_{3}$, followed by brine. The organic layers were collected and dried using anhydrous $\mathrm{MgSO}_{4}$, which was filtered and the filtrate concentrated in vacuo. The resulting solid was then purified using silica gel flash column chromatography $\left(\mathrm{CH}_{2} \mathrm{Cl}_{2} / \mathrm{EtOAc}\right.$ or toluene/EtOAc) to give each regioisomer.<smiles>Cc1ccc2c(n1)C(=O)C(N(C)C)=C(Cl)C2=O</smiles>

94a<smiles>Cc1ccc2c(n1)C(=O)C(Cl)=C(N(C)C)C2=O</smiles>

94b

6-Chloro-7-dimethylamino-2-methyl-5,8quinolinequinone (94a) and 7-chloro-6dimethylamino-2-methyl-5,8-quinolinequinone (94b). By subjecting dichloroQQ 56 (227.3 mg, 1.0 mmol) and dimethylamine (33\% aq., $0.33 \mathrm{~mL}, 1.5$ $\mathrm{mmol})$ to the general procedure for the synthesis of substituted QQs, 94a (125.3 mg, 50\%) 
and 94b (100 mg, 41\%) were isolated as red crystals. Data for 94a: $\mathrm{R}_{f}=0.53\left(\mathrm{CH}_{2} \mathrm{Cl}_{2} / \mathrm{EtOAc}\right.$, 4/1, v/v); m.p.: $233.5^{\circ} \mathrm{C}$; IR (U $\left.\max \right): 2961,2922,2852,1690,1570,1508,1449,1412,1309$, 1259, 1090, 1067, 1015, 796, 775, 751, 706, 645, $567 \mathrm{~cm}^{-1}$; UV-vis (toluene) $\lambda_{\max }$ (log $\varepsilon$ ): 500 (3.32) nm; ${ }^{1} \mathrm{H}$ NMR $\left(500 \mathrm{MHz}, \mathrm{CDCl}_{3}\right) \delta \mathrm{ppm} 8.18\left(\mathrm{~d}, J_{3,4}=7.9 \mathrm{~Hz}, 1 \mathrm{H}, \mathrm{H} 4\right), 7.42\left(\mathrm{~d}, J_{3,4}=\right.$ $7.9 \mathrm{~Hz}, 1 \mathrm{H}, \mathrm{H} 3), 3.25$ (s, 6H, -N(CH$\left.)_{2}\right), 2.73$ (s, 3H, $\left.\mathrm{CH}_{3}\right) ;{ }^{13} \mathrm{C} \mathrm{NMR}\left(125 \mathrm{MHz}, \mathrm{CDCl}_{3}\right) \delta$ ppm 181.6 (C5), 176.6 (C8), 165.2 (C2), 150.1 (C7), 147.0 (C8a), 135.0 (C4), 126.8 (C3), 125.8 (C4a), 120.8 (C6), $44.5 \quad\left(-\mathrm{N}\left(\mathrm{CH}_{3}\right)_{2}\right), \quad 25.3 \quad\left(\mathrm{CH}_{3}\right) ; \quad \mathrm{HRMS}(\mathrm{ESI}) \mathrm{m} / \mathrm{z}$ calcd. for $\left[\mathrm{C}_{12} \mathrm{H}_{11} \mathrm{ClN}_{2} \mathrm{O}_{2}+\mathrm{H}\right]^{+}:$251.0582, obsd.: 251.0587. Data for 94b: $\mathrm{R}_{f}=0.46\left(\mathrm{CH}_{2} \mathrm{Cl}_{2} / \mathrm{EtOAc}, 4 / 1\right.$, v/v); m.p.: $152.9^{\circ} \mathrm{C}$; UV-vis (toluene) $\lambda_{\max }(\log \varepsilon): 485$ (2.32) nm; IR (U $\left.\nu_{\max }\right): 2945,1671,1639$, 1553, 1435, 1372, 1286, 1202, 1158, 1138, 1098, 1064, 1010, 933, 869, 859, 830, 809, 772, 746, 700, 650, $565 \mathrm{~cm}^{-1} ;{ }^{1} \mathrm{H}$ NMR (500 MHz, CDCl $) \delta$ ppm 8.20 (d, J3,4 $\left.=8.0 \mathrm{~Hz}, 1 \mathrm{H}, \mathrm{H} 4\right), 7.43$ (d, $\left.J_{3,4}=8.0 \mathrm{~Hz}, 1 \mathrm{H}, \mathrm{H} 3\right), 3.26\left(\mathrm{~s}, 6 \mathrm{H},-\mathrm{N}\left(\mathrm{CH}_{3}\right)_{2}\right), 2.75$ (s, 3H, $\left.\mathrm{CH}_{3}\right) ;{ }^{13} \mathrm{C} \mathrm{NMR}\left(125 \mathrm{MHz}, \mathrm{CDCl}_{3}\right)$

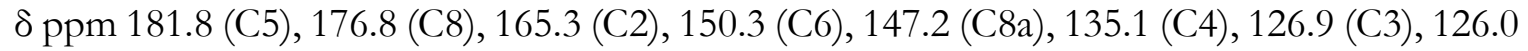
(C4a), 120.9 (C7), $44.7 \quad\left(\mathrm{~N}_{\left.\left(\mathrm{CH}_{3}\right)_{2}\right),} 25.44 \quad\left(\mathrm{CH}_{3}\right) ; \quad \mathrm{HRMS}(\mathrm{ESI}) \mathrm{m} / \mathrm{z}\right.$ calcd. for $\left[\mathrm{C}_{12} \mathrm{H}_{11} \mathrm{ClN}_{2} \mathrm{O}_{2}+\mathrm{H}\right]^{+}:$251.0582, obsd.: 251.0587.

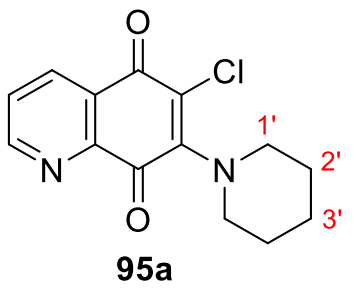

6-Chloro-7-piperidinyl-5,8-quinolinequinone (95a). By subjecting dichloroQQ $92(139.1 \mathrm{mg}, 0.61 \mathrm{mmol})$ and piperidine $(0.07 \mathrm{~mL}, 0.73$ $\mathrm{mmol}$ ) to the general procedure for the synthesis of substituted QQs, the title compound was isolated as purple crystals (67.5 mg, 40\%); $\mathrm{R}_{f}$ $=0.59$ (4/1, $\left.\mathrm{CH}_{2} \mathrm{Cl}_{2} / \mathrm{EtOAc}, \mathrm{v} / \mathrm{v}\right)$; m.p.: $95.2^{\circ} \mathrm{C} ; \mathrm{IR}\left(\mathrm{u}_{\max }\right)$ : 2934, 2849, 1689, 1641, 1587, 1555, 1450, 1397, 1314, 1293, 1244, 1218, 1187, 1141, 1118, 1073, 1035, $987,852,834,809,784,722,644,509,562,444,420 \mathrm{~cm}^{-1}$; UV-vis (toluene) $\lambda_{\max }$ (log ع): 465 (2.32) nm; ${ }^{1} \mathrm{H}$ NMR (500 MHz, $\left.\mathrm{CDCl}_{3}\right) \delta \mathrm{ppm} 8.90\left(\mathrm{dd}, J_{2,3}=4.6 \mathrm{~Hz}, J_{2,4}=1.7 \mathrm{~Hz}, 1 \mathrm{H}, \mathrm{H} 2\right)$, $8.40\left(\mathrm{dd}, J_{3,4}=7.9 \mathrm{~Hz}, J_{2,4}=1.7 \mathrm{~Hz}, 1 \mathrm{H}, \mathrm{H} 4\right), 7.61\left(\mathrm{dd}, J_{3,4}=7.9 \mathrm{~Hz}, J_{2,3}=4.7 \mathrm{~Hz}, 1 \mathrm{H}, \mathrm{H} 3\right)$, 3.58-3.56 (m, 4H, H1'), 3.45, 1.79-1.75 (m, 4H, H2'), 1.72-1.68 (m, 2H, H3'); ${ }^{13} \mathrm{C}$ NMR (125 $\mathrm{MHz}, \mathrm{CDCl}_{3}$ ) $\delta$ ppm 180.3 (C8), 176.9 (C5), 153.8 (C2), 151.20 (C7), 147.2 (C8a), 134.5 (C4), 128.5 (C4a), 127.8 (C3), 120.8 (C6), 53.2 (C1'), 26.9 (C2'), 24.0 (C3'); HRMS(ESI) m/z calcd. for $\left[\mathrm{C}_{14} \mathrm{H}_{13} \mathrm{ClN}_{2} \mathrm{O}_{2}+\mathrm{H}\right]^{+}: 277.0738$, obsd.: 277.0736. 
<smiles>Cc1ccc2c(n1)C(=O)C(Cl)=C(N1CCCCC1)C2=O</smiles>

7-Chloro-6-piperidinyl-2-methyl-5,8-quinolinequinone (96b).

By subjecting dichloroQQ 56 (202.5 mg, $0.84 \mathrm{mmol})$ and piperidine $(0.1 \mathrm{~mL}, 1.0 \mathrm{mmol})$ to the general procedure for the synthesis of substituted QQs, the title compound was isolated as purple crystals

(80.6 mg, 33\%); $\mathrm{R}_{f}=0.32\left(\mathrm{CH}_{2} \mathrm{Cl}_{2} /\right.$ EtOAc, 24/1, v/v); m.p.: 153.2 ${ }^{\circ} \mathrm{C}$; IR (U $\left.u_{\max }\right):$ 2922, 1672, 1648, 1548, 1443, 1293, 1242, 1206, 1101, 985, 859, 745, 702, 568, 448, $420 \mathrm{~cm}^{-1}$; UV-vis (toluene) $\lambda_{\max }(\log \varepsilon): 515$ (2.90) nm; ${ }^{1} \mathrm{H}$ NMR $\left(500 \mathrm{MHz}, \mathrm{CDCl}_{3}\right) \delta \mathrm{ppm}$ $8.19\left(\mathrm{~d}, J_{3,4}=8.0 \mathrm{~Hz}, 1 \mathrm{H}, \mathrm{H} 4\right), 7.43$ (d, $\left.J_{3,4}=8.0 \mathrm{~Hz}, 1 \mathrm{H}, \mathrm{H} 3\right), 3.54$ (m, 4H, H1'), 2.74 (s, 3H, $\mathrm{CH}_{3}$ ), 1.77 (m, 4H, H2'), 1.71 (m, 2H, H3'); ${ }^{13} \mathrm{C}$ NMR (125 MHz, $\left.\mathrm{CDCl}_{3}\right) \delta$ ppm 181.6 (C5), 176.8 (C8), 165.2 (C2), 150.1 (C6), 147.1 (C8a), 135.1 (C4), 127.0 (C3), 126.2 (C4a), 123.0 (C7), $53.2\left(\mathrm{C}^{\prime}\right), 27.0\left(\mathrm{C}^{\prime}\right), 25.3\left(\mathrm{CH}_{3}\right), 24.2\left(\mathrm{C}^{\prime}\right)$; HRMS(ESI) $m / z$ calcd. for $\left[\mathrm{C}_{15} \mathrm{H}_{15} \mathrm{ClN}_{2} \mathrm{O}_{2}+\mathrm{H}\right]^{+}$: 291.0895, obsd.: 291.0899 .<smiles>Cc1ccc2c(n1)C(=O)C(NC(C)(C)C)=C(Cl)C2=O</smiles>

97a<smiles>Cc1ccc2c(n1)C(=O)C(Cl)=C(NC(C)(C)C)C2=O</smiles>

97b

6-Chloro-2-methyl-7-tertbutylamino5,8-quinolinequinone (97a) and 7chloro-2-methyl-7-tertbutylamino-5,8quinolinequinone (97b). By subjecting dichloroQQ 56 (195 mg, $0.81 \mathrm{mmol})$ and tert-butylamine $(0.13 \mathrm{~mL}, 1.2 \mathrm{mmol})$ to the general procedure for the synthesis of substituted QQs, 97a (88.1 mg, 39\%) and 97b (56.4 mg, 25\%) were isolated as red crystals. Data for 97a: $\mathrm{R}_{f}=0.70\left(\mathrm{CH}_{2} \mathrm{Cl}_{2} /\right.$ EtOAc, 9/1, v/v); m.p.: $113.2^{\circ} \mathrm{C}$; IR (umax): 3371, 1670, 1594, 1560, 1534, 1369, 1314, 1201, 1163, 1092, 848, 678, $422 \mathrm{~cm}^{-1}$; UV-vis (toluene) $\lambda_{\max }(\log \varepsilon): 485$ (2.39) nm; ${ }^{1} \mathrm{H}$ NMR $\left(500 \mathrm{MHz}, \mathrm{CDCl}_{3}\right) \delta \mathrm{ppm} 8.31\left(\mathrm{~d}, J_{3,4}=8.0 \mathrm{~Hz}, 1 \mathrm{H}, \mathrm{H} 4\right), 7.48\left(\mathrm{~d}, J_{3,4}=8.0 \mathrm{~Hz}, 1 \mathrm{H}\right.$, H3), 6.04 (br. s, $1 \mathrm{H}, \mathrm{NH}), 2.73$ (s, 3H, $\mathrm{CH}_{3}$ ), 1.57 (s, 9H, $\left.{ }^{\circ} \mathrm{Bu}-\mathrm{CH}_{3}\right) ;{ }^{13} \mathrm{C}$ NMR (125 MHz, $\mathrm{CDCl}_{3}$ ): $\delta$ ppm 179.4 (C8), 175.8 (C5), 163.7 (C2), 146.6 (C7), 146.1 (C8a), 134.8 (C4), 128.2 (C3), 127.2 (C4a), $55.8\left({ }^{(} \mathrm{Bu}-\mathrm{C}_{\mathrm{q}}\right), 31.6\left({ }^{\mathrm{Bu}}-\mathrm{CH}_{3}\right), 25.1\left(\mathrm{CH}_{3}\right)$; HRMS-ESI ( $\left.\mathrm{m} / \mathrm{q}\right)$ calcd. for $\left[\mathrm{C}_{14} \mathrm{H}_{15} \mathrm{ClN}_{2} \mathrm{O}_{2}+\mathrm{H}\right]^{+}: 279.0895$, obsd.: 279.0896. Data for 97b: $\mathrm{R}_{f}=0.35\left(\mathrm{CH}_{2} \mathrm{Cl}_{2} / \mathrm{EtOAc}, 9 / 1\right.$, v/v); m.p.: $177.2^{\circ} \mathrm{C}$; IR (U $\left.\max \right): 3376,1670,1594,1535,1369,1314,1201,1163,1092,849,749$, 678, $414 \mathrm{~cm}^{-1}$; UV-vis (toluene) $\lambda_{\max }(\log \varepsilon): 485(1.48) \mathrm{nm} ;{ }^{1} \mathrm{H}$ NMR $\left(500 \mathrm{MHz}, \mathrm{CDCl}_{3}\right) \delta \mathrm{ppm}$ $8.22\left(\mathrm{~d}, J_{3,4}=8.0 \mathrm{~Hz}, 1 \mathrm{H}, \mathrm{H} 4\right), 7.42$ (d, $\left.J_{3,4}=8.0 \mathrm{~Hz}, 1 \mathrm{H}, \mathrm{H} 3\right), 6.04$ (br s., 1H, NH), 2.76 (s, $3 \mathrm{H}, \mathrm{CH}_{3}$ ), 1.57 (s, 9H, $\left.{ }^{\mathrm{Bu}}-\mathrm{CH}_{3}\right) ;{ }^{13} \mathrm{C}$ NMR (125 MHz, $\mathrm{CDCl}_{3}$ ) $\delta$ ppm 180.3 (C5), 175.5 (C8), 165.6 (C2), 147.6 (C8a), 145.4 (C6), 135.1 (C4), 126.6 (C3), 125.2 (C4a), 55.4 ('Bu-C) 31.6 $\left({ }^{\circ} \mathrm{Bu}-\mathrm{CH}_{3}\right), 25.4\left(\mathrm{CH}_{3}\right)$; HRMS-ESI $(\mathrm{m} / \mathrm{z})$ calcd. for $\left[\mathrm{C}_{14} \mathrm{H}_{15} \mathrm{ClN}_{2} \mathrm{O}_{2}+\mathrm{H}\right]^{+}$: 279.0895, obsd.: 
<smiles>CCCNC1=C(Cl)C(=O)c2cccnc2C1=O</smiles>

98a<smiles>CCCNC1=C(Cl)C(=O)c2ncccc2C1=O</smiles>

$98 b$

6-Chloro-7-propylamino-5,8quinolinequinone (98a) and 7-chloro6-propylamino-5,8-quinolinequinone (98b). By subjecting dichloroQQ 92 (44.2 $\mathrm{mg}, 0.5 \mathrm{mmol})$ and propylamine $(0.05$ $\mathrm{mL}, 0.6 \mathrm{mmol}$ ) to the general procedure for the synthesis of substituted QQs, $98 \mathbf{a}(75.2 \mathrm{mg}$, $60 \%$ ) and $\mathbf{9 8 b}(47.6 \mathrm{mg}, 39 \%)$ were isolated as red crystals. Data for 98a: $\mathrm{R}_{f}=0.43$ $\left(\mathrm{CH}_{2} \mathrm{Cl}_{2} /\right.$ EtOAc, 4/1, v/v); m.p.: $145.7^{\circ} \mathrm{C}$ (lit. $\left.{ }^{187} 135-136{ }^{\circ} \mathrm{C}\right)$; IR (umax): 3284, 2929, 1693 , 1576, 1553, 1506, 1464, 1431, 1375, 1284, 1259, 1236, 1195, 1165, 1151, 1137, 1093, 1036, 1026, 1006, 984, 871, 834, 818, 726, 611, 551, 493, 455, 439, $418 \mathrm{~cm}^{-1}$; UV-vis (toluene) $\lambda_{\max }$ (log $\varepsilon): 480(2.64) \mathrm{nm} ;{ }^{1} \mathrm{H}$ NMR (500 MHz, $\left.\mathrm{CDCl}_{3}\right): \delta \mathrm{ppm} 8.92\left(\mathrm{~d}, J_{2,3}=4.7 \mathrm{~Hz}, 1 \mathrm{H}, \mathrm{H} 2\right), 8.47$ $\left(\mathrm{d}, J_{3,4}=7.9 \mathrm{~Hz}, 1 \mathrm{H}, \mathrm{H} 4\right), 7.65$ (dd, $\left.J_{3,4}=7.9 \mathrm{~Hz}, J_{2,3}=4.7 \mathrm{~Hz}, 1 \mathrm{H}, \mathrm{H} 3\right), 6.28$ (br. s, 1H, NH), 3.85 (q, $\left.J_{1^{\prime}, 2^{\prime}}=6.9 \mathrm{~Hz}, 2 \mathrm{H}, \mathrm{H} 1^{\prime}\right), 1.75$ (sext., $\left.J_{1^{\prime}, 2^{\prime}}=J_{2^{\prime}, 3^{\prime}}=7.2 \mathrm{~Hz}, 2 \mathrm{H}, \mathrm{H} 2^{\prime}\right), 1.03$ (t, $J_{2^{\prime}, 3^{\prime}}=7.3 \mathrm{~Hz}$, 2H, H3'); ${ }^{13} \mathrm{C}$ NMR (125 MHz, $\mathrm{CDCl}_{3}$ ): $\delta 179.0$ (C8), 175.7 (C5), 153.4 (C2), 146.0 (C8a), 134.8 (C4), 130.1 (C4a), 128.5 (C3), 123.8 (C6), 46.9 (C1'), 24.4 (C2'), 11.2 (C3'); HRMS-ESI (m/ z) calcd. for $\left[\mathrm{C}_{12} \mathrm{H}_{11} \mathrm{ClN}_{2} \mathrm{O}_{2}+\mathrm{H}\right]^{+}: 251.0582$, obsd.: 251.0588. The spectral data obtained for this compound were consistent with those reported in literature. ${ }^{187}$ Data for $\mathbf{9 8 b}: \mathrm{R}_{f}=0.26$ $\left(\mathrm{CH}_{2} \mathrm{Cl}_{2}\right.$ /EtOAc, 4/1, v/v); m.p.: $154.4^{\circ} \mathrm{C}$ (lit. ${ }^{187} 134-135{ }^{\circ} \mathrm{C}$ ); IR (umax): 3227, 2956, 2919 , 1676, 1560, 1551, 1458, 1330, 1304, 1266, 1207, 1134, 1110, 1071, 1040, 1023, 831, 821, 803, 746, 732, 710, 677, 656, 649, 563, $492 \mathrm{~cm}^{-1}$; UV-vis (toluene) $\lambda_{\max }(\log \varepsilon): 480(3.48) \mathrm{nm} ;{ }^{1} \mathrm{H}$ $\operatorname{NMR}\left(500 \mathrm{MHz}, \mathrm{CDCl}_{3}\right) \delta \mathrm{ppm} 9.02\left(\mathrm{dd}, J_{2,3}=4.7 \mathrm{~Hz}, J_{2,4}=1.4 \mathrm{~Hz}, 1 \mathrm{H}, \mathrm{H} 2\right), 8.35\left(\mathrm{dd}, J_{3,4}=\right.$ $\left.8.1 \mathrm{~Hz}, J_{2,4}=1.4 \mathrm{~Hz}, 1 \mathrm{H}, \mathrm{H} 4\right), 7.59$ (dd, $\left.J_{3,4}=7.9 \mathrm{~Hz}, J_{2,3}=4.7 \mathrm{~Hz}, 1 \mathrm{H}, \mathrm{H} 3\right), 6.06$ (br. s, $1 \mathrm{H}$, $\mathrm{NH}), 3.85$ (q, $\left.J_{1^{\prime}, 2^{\prime}}=7.0 \mathrm{~Hz}, 2 \mathrm{H}, \mathrm{H} 1^{\prime}\right), 1.74$ (sext., $\left.J_{1^{\prime}, 2^{\prime}}=J_{2^{\prime}, 3^{\prime}}=7.0 \mathrm{~Hz}, 2 \mathrm{H}, \mathrm{H} 2^{\prime}\right), 1.03$ (t, $J_{2^{\prime}, 3^{\prime}}=$ $\left.7.5 \mathrm{~Hz}, 2 \mathrm{H}, \mathrm{H} 3{ }^{\prime}\right) ;{ }^{13} \mathrm{C}$ NMR (125 MHz, $\mathrm{CDCl}_{3}$ ) $\delta$ ppm 180.3 (C5), 155.4 (C2), 148.7 (C8a), 134.8 (C4), 126.8 (C4a), 126.6 (C3), 46.9 (C1'), 24.4 (C2'), 11.2 (C3'); HRMS-ESI (m/ z) calcd. for $\left[\mathrm{C}_{12} \mathrm{H}_{11} \mathrm{ClN}_{2} \mathrm{O}_{2}+\mathrm{H}\right]^{+}:$251.0582, obsd.: 251.0588. The spectral data obtained for this compound were consistent with those reported in literature. ${ }^{187}$ 
<smiles>CCCNC1=C(Cl)C(=O)c2ccc(C)nc2C1=O</smiles>

6-Chloro-2-methyl-6-

propylamino-5,8-

quinolinequinone $(99 a)$ and $7-$

chloro-2-methyl-6-

propylamino-5,8-

quinolinequinone (99b). By subjecting dichloroQQ 56 (49.0 mg, $0.20 \mathrm{mmol}$ ) and propylamine $(0.02 \mathrm{~mL}, 0.24 \mathrm{mmol})$ to the general procedure for the synthesis of substituted QQs, 99a (27 mg, 51\%) and 99b (24.9 mg, 47\%) were isolated as red crystals. Data for 99a: $\mathrm{R}_{f}$ $=0.40\left(\mathrm{CH}_{2} \mathrm{Cl}_{2} /\right.$ EtOAc, $\left.9 / 1, \mathrm{v} / \mathrm{v}\right) ;$ m.p.: $150.7{ }^{\circ} \mathrm{C} ; \mathrm{IR}\left(\mathrm{U}_{\max }\right): 3296,1696,1600,1577,1513$, $1460,1443,1320,1258,1200,1165,1140,1105,1089,851,819,738,707,595,565,525,501$, 474, $418 \mathrm{~cm}^{-1}$; UV-vis $\left(\mathrm{CH}_{2} \mathrm{Cl}_{2}\right) \lambda_{\max }(\log \varepsilon): 480$ (2.28), 310 (2.89) nm; ${ }^{1} \mathrm{H}$ NMR $(500 \mathrm{MHz}$, $\left.\mathrm{CDCl}_{3}\right) \delta$ ppm 8.35 (d, $\left.J_{3,4}=7.9 \mathrm{~Hz}, 1 \mathrm{H}, \mathrm{H} 4\right), 7.49$ (d, $\left.J_{3,4}=8.0 \mathrm{~Hz}, 1 \mathrm{H}, \mathrm{H} 3\right), 6.18$ (br. s, $1 \mathrm{H}$, $\mathrm{NH}), 3.83\left(\mathrm{q}, J_{1^{\prime}, 2^{\prime}}=J_{2^{\prime}, 3^{\prime}}=7.3 \mathrm{~Hz}, 2 \mathrm{H}, \mathrm{H} 1^{\prime}\right), 2.73\left(\mathrm{~s}, 3 \mathrm{H}, \mathrm{CH}_{3}\right), 1.73$ (sext., $J_{1^{\prime}, 2^{\prime}}=J_{2^{\prime}, 3^{\prime}}=7.1 \mathrm{~Hz}$, 2H, H2'), 1.01 (t, $\left.J_{2^{\prime}, 3^{\prime}}=7.4 \mathrm{~Hz}, 2 \mathrm{H}, \mathrm{H} 3^{\prime}\right) ;{ }^{13} \mathrm{C}$ NMR (125 MHz, CDCl $) \delta$ ppm $179.2(\mathrm{C} 8), 176.0$ (C5), 163.5 (C2), 145.5 (C8a), 144.4 (C7), 134.0 (C4), 128.4 (C3), 127.9 (C4a), 46.8 (C1'), 25.0 $\left(\mathrm{CH}_{3}\right), 24.3\left(\mathrm{C}^{\prime}\right), 11.06\left(\mathrm{C}^{\prime}\right)$; HRMS-ESI $(\mathrm{m} / \mathrm{q})$ calcd. for $\left[\mathrm{C}_{13} \mathrm{H}_{13} \mathrm{ClN}_{2} \mathrm{O}_{2}+\mathrm{H}\right]^{+}: 265.0738$, obsd.: 265.0740. Data for 99b: $\mathrm{R}_{f}=0.3\left(\mathrm{CH}_{2} \mathrm{Cl}_{2} / \mathrm{EtOAc}, 9 / 1, \mathrm{v} / \mathrm{v}\right)$, m.p.: $114.1{ }^{\circ} \mathrm{C}$; IR (U max $)$ : 2958, 1673, 1571, 1509, 1453, 1310, 1225, 1111, 1067, 839, 776, 748, 701, 649, 574, $419 \mathrm{~cm}^{-1}$; UV-vis $\left(\mathrm{CH}_{2} \mathrm{Cl}_{2}\right) \lambda_{\max }(\log \varepsilon): 475(2.30) \mathrm{nm} ;{ }^{1} \mathrm{H}$ NMR $\left(500 \mathrm{MHz}, \mathrm{CDCl}_{3}\right) \delta \mathrm{ppm} 8.21\left(\mathrm{~d}, J_{3,4}=\right.$ $8.0 \mathrm{~Hz}, 1 \mathrm{H}, \mathrm{H} 4), 7.41$ (d, $\left.J_{3,4}=8.0 \mathrm{~Hz}, 1 \mathrm{H}, \mathrm{H} 3\right), 6.04$ (br. s, $\left.1 \mathrm{H}, \mathrm{NH}\right), 3.82$ (q, $J_{1^{\prime}, 2^{\prime}}=J_{2^{\prime}, 3^{\prime}}=6.9$ $\left.\mathrm{Hz}, 2 \mathrm{H}, \mathrm{H} 1^{\prime}\right), 2.75$ (s, 3H, CH 3 ), 1.72 (sext., $J_{1^{\prime}, 2^{\prime}}=J_{2^{\prime}, 3^{\prime}}=7.2 \mathrm{~Hz}, 2 \mathrm{H}, \mathrm{H} 2$ '), 1.01 (t, $J_{2^{\prime}, 3^{\prime}}=7.4$ $\left.\mathrm{Hz}, 2 \mathrm{H}, \mathrm{H}^{\prime}\right) ;{ }^{13} \mathrm{C}$ NMR (125 MHz, CDCl $)$ \& ppm 180.2 (C5), 175.5 (C8), 166.0 (C2), 148.3 (C8a), 143.6 (C7), 134.9 (C4), 126.5 (C3), 124.6 (C4a), 46.8 (C1'), $25.6\left(\mathrm{CH}_{3}\right), 24.4(\mathrm{C} 2 '), 11.2$ (C3'); HRMS-ESI $(m / z)$ calcd. for $\left[\mathrm{C}_{13} \mathrm{H}_{13} \mathrm{ClN}_{2} \mathrm{O}_{2}+\mathrm{H}\right]^{+}:$265.0738, obsd.: 265.0740.<smiles>C#CCNC1=C(Cl)C(=O)c2cccnc2C1=O</smiles>

$100 \mathrm{a}$<smiles>C#CCNC1=C(Cl)C(=O)c2cccnc2C1=O</smiles>
quinolinequinone (100b). By subjecting dichloroQQ 92 (189.0 mg,

$0.83 \mathrm{mmol})$ and propargylamine $(0.06 \mathrm{~mL}, 1.0 \mathrm{mmol})$ to the general procedure for the synthesis of substituted QQs, 100a (63.5 mg, 31\%) and (57.3 mg, 28\%) were isolated as red crystals. Data for 100a: $\mathrm{R}_{f}=0.27\left(\mathrm{CH}_{2} \mathrm{Cl}_{2} /\right.$ EtOAc, 4/1, v/v); m.p.: $199.8^{\circ} \mathrm{C}$ (lit. $\left.{ }^{186} 140-142{ }^{\circ} \mathrm{C}\right)$; IR $\left(U_{\max }\right): 3189,1697,1599,1565,1507,1422,1348,1322,1196,1152,1093,817,724,575 \mathrm{~cm}^{-1}$; 
UV-vis $\left(\mathrm{CH}_{2} \mathrm{Cl}_{2}\right) \lambda_{\max }(\log \varepsilon): 455(1.71) \mathrm{nm} ;{ }^{1} \mathrm{H}$ NMR $\left(500 \mathrm{MHz}, \mathrm{CDCl}_{3}\right) \delta \mathrm{ppm} 8.96\left(\mathrm{dd}, J_{2,3}\right.$ $\left.=4.6 \mathrm{~Hz}, J_{2,4}=1.5 \mathrm{~Hz}, 1 \mathrm{H}, \mathrm{H} 2\right), 8.49\left(\mathrm{dd}, J_{3,4}=7.9 \mathrm{~Hz}, J_{2,4}=1.5 \mathrm{~Hz}, 1 \mathrm{H}, \mathrm{H} 4\right), 7.69$ (dd, $J_{3,4}=$ $\left.7.9 \mathrm{~Hz}, J_{2,3}=4.6 \mathrm{~Hz}, 1 \mathrm{H}, \mathrm{H} 3\right), 6.21$ (br. s, $1 \mathrm{H}, \mathrm{NH}$ ), 4.69 (dd, $J_{1^{\prime}, \mathrm{NH}}=6.0 \mathrm{~Hz}, J_{1^{\prime}, 3^{\prime}}=2.5 \mathrm{~Hz}$, 2H, H1'), $2.43\left(\mathrm{t}, J_{1^{\prime}, 3^{\prime}}=2.5 \mathrm{~Hz}, 1 \mathrm{H}, \mathrm{H} 3^{\prime}\right) ;{ }^{13} \mathrm{C} \mathrm{NMR}\left(125 \mathrm{MHz}, \mathrm{CDCl}_{3}\right) \delta \mathrm{ppm} 178.6(\mathrm{C} 8), 176.0$ (C5), 153.9 (C2), 146.1 (C8a), 144.1 (C7), 134.9 (C4), 129.7 (C4a), 128.6 (C3), 79.0 (C2'), 74.0 $\left(\mathrm{C}^{\prime}\right)$, $35.3\left(\mathrm{C}^{\prime}\right)$; HRMS-ESI $(\mathrm{m} / \mathrm{z})$ calcd. for $\left[\mathrm{C}_{12} \mathrm{H}_{7} \mathrm{ClN}_{2} \mathrm{O}_{2}+\mathrm{H}^{+}\right.$: 247.0269, obsd.: 247.0260. The spectral data obtained for this compound were consistent with those reported in literature. ${ }^{186}$ Data for 100b: $\mathrm{R}_{f}=0.16\left(\mathrm{CH}_{2} \mathrm{Cl}_{2} /\right.$ EtOAc, 4/1, v/v); m.p.: $137.1{ }^{\circ} \mathrm{C}$ (lit. ${ }^{186} 140$ $\left.142{ }^{\circ} \mathrm{C}\right)$; IR (Umax $): 3248,1691,1595,1570,1513,1345,1308,1267,1207,1147,1117,1075$, 832, 806, 759, 731, 680, 656, 595, 451, $411 \mathrm{~cm}^{-1}$; UV-vis (toluene) $\lambda_{\max }(\log \varepsilon): 460$ (3.72), 300 (1.23) nm; ${ }^{1} \mathrm{H}$ NMR (500 MHz, $\mathrm{CDCl}_{3}$ ): $\delta$ ppm 9.03 (dd, $\left.J_{2,3}=4.7 \mathrm{~Hz}, J_{2,4}=1.7 \mathrm{~Hz}, 1 \mathrm{H}, \mathrm{H} 2\right)$, $8.38\left(\mathrm{dd}, J_{3,4}=7.9 \mathrm{~Hz}, J_{2,4}=1.8 \mathrm{~Hz}, 1 \mathrm{H}, \mathrm{H} 4\right), 7.62\left(\mathrm{dd}, J_{3,4}=7.8 \mathrm{~Hz}, J_{2,3}=4.7 \mathrm{~Hz}, 1 \mathrm{H}, \mathrm{H} 3\right)$, 6.02 (br. s, $1 \mathrm{H}, \mathrm{NH}), 4.66\left(\mathrm{dd}, J_{1^{\prime}, \mathrm{NH}}=6.0 \mathrm{~Hz}, J_{1^{\prime}, 3^{\prime}}=2.6 \mathrm{~Hz}, 2 \mathrm{H}, \mathrm{H} 1^{\prime}\right), 2.42\left(\mathrm{t}, J_{1^{\prime}, 3^{\prime}}=2.5 \mathrm{~Hz}\right.$, 1H, H3); ${ }^{13} \mathrm{C}$ NMR (125 MHz, $\mathrm{CDCl}_{3}$ ) $\delta$ ppm 179.7 (C5), 155.3 (C2), 148.1 (C8a), 143.1 (C6), 134.8 (C4), 126.8 (C3), 78.9 (C2'), 73.7 (C3'), 35.2 (C1'); HRMS-ESI (m/ z) calcd. for $\left[\mathrm{C}_{12} \mathrm{H}_{7} \mathrm{ClN}_{2} \mathrm{O}_{2}+\mathrm{H}\right]^{+}: 247.0269$, obsd.: 247.0260 . The data obtained for this compound were consistent with those reported in literature. ${ }^{186}$
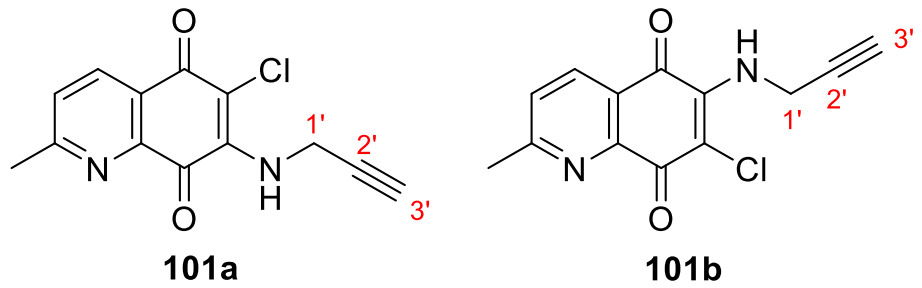

6-Chloro-2-methyl-7propargylamino-5,8quinolinequinone (101a) and 7chloro-2-methyl-6propargylamino-5,8-

quinolinequinone (101b). By subjecting dichloroQQ 56 (77 $\mathrm{mg}, 0.32 \mathrm{mmol}$ ) and propargylamine $(0.03 \mathrm{~mL}, 0.48 \mathrm{mmol})$ to the general procedure for the synthesis of substituted QQs, 101a (37.5 mg, 45\%) was isolated as red crystals and 101b (33.4 mg, 40\%) was isolated as orange crystals. Data for 101a: $\mathrm{R}_{f}=0.32\left(\mathrm{CH}_{2} \mathrm{Cl}_{2} / \mathrm{EtOAc}, 4 / 1, \mathrm{v} / \mathrm{v}\right) ;$ m.p.: $213.7{ }^{\circ} \mathrm{C}$; IR $\left(U_{\max }\right): 3310,3216,1691,1603,1580,1497,1352,1320,1279,1250,1202,1154,1114,830,740$, 706, 683, 600, 558, 473, 435, $421 \mathrm{~cm}^{-1}$; UV-vis $\left(\mathrm{CH}_{2} \mathrm{Cl}_{2}\right) \lambda_{\max }(\log \varepsilon): 475$ (2.22) nm; ${ }^{1} \mathrm{H}$ NMR $\left(500 \mathrm{MHz}, \mathrm{CDCl}_{3}\right): \delta$ ppm 8.34 (d, $\left.J_{3,4}=8.0 \mathrm{~Hz}, 1 \mathrm{H}, \mathrm{H} 4\right), 7.51$ (d, $\left.J_{3,4}=8.0 \mathrm{~Hz}, 1 \mathrm{H}, \mathrm{H} 3\right), 6.12$ (br. s, $1 \mathrm{H}, \mathrm{NH}), 4.66\left(\mathrm{dd}, J_{1^{\prime}, \mathrm{NH}}=4.7 \mathrm{~Hz}, J_{1^{\prime}, 3^{\prime}}=2.5 \mathrm{~Hz}, 2 \mathrm{H}, \mathrm{H} 1^{\prime}\right), 2.74\left(\mathrm{~s}, 3 \mathrm{H}, \mathrm{CH}_{3}\right), 2.41\left(\mathrm{t}, J_{1^{\prime}, 3^{\prime}}\right.$ $\left.=2.5 \mathrm{~Hz}, 1 \mathrm{H}, \mathrm{H}^{\prime}\right) ;{ }^{13} \mathrm{C}$ NMR (125 MHz, CDCl $): \delta$ ppm 178.9 (C8), 176.1 (C5), 164.1 (C2), 145.6 (C8a), 143.9 (C7), 135.1 (C4), 128.6 (C3), 127.5 (C6), 79.1 (C2'), 73.8 (C3'), 35.2 (C1'), $25.2\left(\mathrm{CH}_{3}\right)$; HRMS-ESI $(\mathrm{m} / \mathrm{q})$ calcd. for $\left[\mathrm{C}_{13} \mathrm{H}_{9} \mathrm{ClN}_{2} \mathrm{O}_{2}+\mathrm{H}\right]^{+}:$261.0425, obsd.: 261.0426. Data 
for 101b: $\mathrm{R}_{f}=0.19\left(\mathrm{CH}_{2} \mathrm{Cl}_{2} /\right.$ EtOAc, 4/1, v/v); m.p.: $163.2^{\circ} \mathrm{C}$; IR (U $\left.\mathrm{U}_{\max }\right): 3180,2924,1611$, 1574, 1558, 1504, 1454, 1357, 1312, 1294, 1257, 1145, 1068, 1025, 930, 750, 734, 702, 612, 553, $509 \mathrm{~cm}^{-1}$; UV-vis $\left(\mathrm{CH}_{2} \mathrm{Cl}_{2}\right) \lambda_{\max }(\log \varepsilon): 455(2.17) \mathrm{nm} ;{ }^{1} \mathrm{H} \mathrm{NMR}\left(500 \mathrm{MHz}, \mathrm{CDCl}_{3}\right) \delta \mathrm{ppm}$ $8.25\left(\mathrm{~d}, J_{3,4}=8.0 \mathrm{~Hz}, 1 \mathrm{H}, \mathrm{H} 4\right), 7.45$ (d, J3,4 $\left.=8.0 \mathrm{~Hz}, 1 \mathrm{H}, \mathrm{H} 3\right), 6.00$ (br. s, $\left.1 \mathrm{H}, \mathrm{NH}\right), 4.65$ (dd, $\left.J_{1^{\prime}, \mathrm{NH}}=4.7 \mathrm{~Hz}, J_{1^{\prime}, 3^{\prime}}=2.5 \mathrm{~Hz}, 2 \mathrm{H}, \mathrm{H} 1^{\prime}\right), 2.77\left(\mathrm{~s}, 3 \mathrm{H}, \mathrm{CH}_{3}\right), 2.41\left(\mathrm{t}, J_{1^{\prime}, 3^{\prime}}=2.5 \mathrm{~Hz}, 1 \mathrm{H}, \mathrm{H} 3^{\prime}\right) ;{ }^{13} \mathrm{C}$ NMR (125 MHz, $\mathrm{CDCl}_{3}$ ) $\delta$ ppm 179.8 (C8), 175.9 (C5), 166.2 (C2), 147.8 (C8a), 143.1 (C6), 135.1 (C4), 126.8 (C3), $79.2\left(\mathrm{C}^{\prime}\right), 73.8$ (C3'), $35.2\left(\mathrm{C}^{\prime}\right)$, $25.6\left(\mathrm{CH}_{3}\right)$; HRMS-ESI (m/z) calcd. for $\left[\mathrm{C}_{13} \mathrm{H}_{9} \mathrm{ClN}_{2} \mathrm{O}_{2}+\mathrm{H}\right]^{+}: 261.0425$, obsd.: 261.0426 .
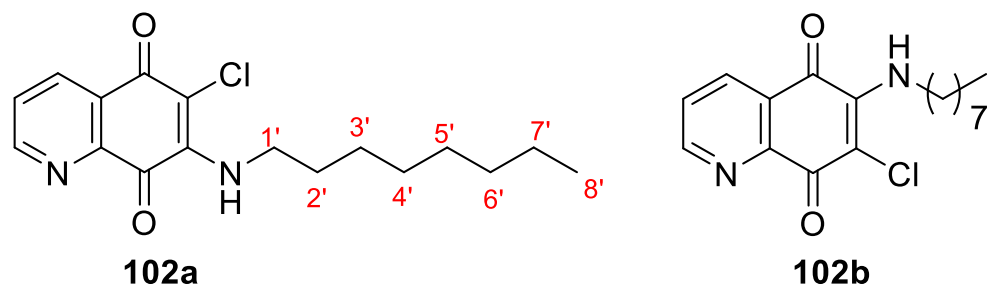

6-Chloro-7-octylamino-5,8quinolinequinone (102a) and 7-chloro-6-octylamino5,8-quinolinequinone

(102b). By subjecting dichloroQQ $92(176 \mathrm{mg}, 0.77 \mathrm{mmol})$ and octylamine $(0.15 \mathrm{~mL}, 0.93 \mathrm{mmol})$ to the general procedure for the synthesis of substituted QQs, 102a (69.2 mg, 28\%) and 102b (88.9 mg, 36\%) were isolated as red crystals. Data for 102a: $\mathrm{R}_{f}=0.69$ (EtOAc); m.p.: $148.9{ }^{\circ} \mathrm{C}$; IR $\left(\mathrm{U}_{\max }\right): 2922$, 2851, 1691, 1592, 1559, 1508, 1428, 1325, 1306, 1252, 1196, 1138, 1098, 820, $728 \mathrm{~cm}^{-1}$; UVvis (toluene) $\lambda_{\max }(\log \varepsilon): 485(2.46) \mathrm{nm} ;{ }^{1} \mathrm{H}$ NMR $\left(500 \mathrm{MHz}, \mathrm{CDCl}_{3}\right) \delta \mathrm{ppm} 8.88\left(\mathrm{~d}, J_{2,4}=4.6\right.$ $\mathrm{Hz}, 1 \mathrm{H}, \mathrm{H} 2), 8.44$ (d, $\left.J_{3,4}=7.8 \mathrm{~Hz}, 1 \mathrm{H}, \mathrm{H} 4\right), 7.63$ (dd, $J_{3,4}=7.8 \mathrm{~Hz}, J_{2,3}=4.5 \mathrm{~Hz}, 1 \mathrm{H}, \mathrm{H} 3$ ), 6.24 (br. s, $1 \mathrm{H}, \mathrm{NH}$ ), 3.84 (q, $J_{1^{\prime}, 2^{\prime}}=J_{1^{\prime}, \mathrm{NH}}=7.2 \mathrm{~Hz}, 2 \mathrm{H}, \mathrm{H} 1^{\prime}$ ), 1.68 (pent., $J_{1^{\prime}, 2^{\prime}}=J_{2^{\prime}, 3^{\prime}}=7.6 \mathrm{~Hz}$, 2H, H2'), 1.39- 1.25 (m, 8H, H3'-H7'), 0.85 (t, $\left.J_{7^{\prime}, 8^{\prime}}=7.0 \mathrm{~Hz}, 2 \mathrm{H}, \mathrm{H} 8^{\prime}\right) ;{ }^{13} \mathrm{C}$ NMR (125 MHz, $\mathrm{CDCl}_{3}$ ) $\delta$ ppm 179.0 (C8), 175.7 (C5), 153.4 (C2), 144.6 (C7), 134.8 (C4), 130.1 (C4a), 128.5 (C3), 45.2 (C1'), 31.8 (C6'), 31.1 (C2'), 29.3 (C4'), 29.2 (C3'), 26.7 (C5'), 22.7 (C7'), 14.15 (C8'); HRMS-ESI $(m / z)$ calcd. for $\left[\mathrm{C}_{17} \mathrm{H}_{21} \mathrm{ClN}_{2} \mathrm{O}_{2}+\mathrm{H}\right]^{+}:$321.1364, obsd.: 321.1364. Data for 102b: $\mathrm{R}_{f}$ $=0.33$ (EtOAc); m.p.: $116.2^{\circ} \mathrm{C}$; IR (U max $): 3237,2921,1675,1589,1560,1513,1443,1373$, $1305,1264,1206,1113,1073,827,803,748,714,654,572,411 \mathrm{~cm}^{-1}$; UV-vis (toluene) $\lambda_{\max }$ $(\log \varepsilon): 485(2.67) \mathrm{nm} ;{ }^{1} \mathrm{H}$ NMR $\left(500 \mathrm{MHz}, \mathrm{CDCl}_{3}\right) \delta \mathrm{ppm} 9.00$ (d, $\left.J_{2,4}=4.5 \mathrm{~Hz}, 1 \mathrm{H}, \mathrm{H} 2\right), 8.34$ (d, $\left.J_{3,4}=7.9 \mathrm{~Hz}, 1 \mathrm{H}, \mathrm{H} 4\right), 7.57$ (dd, $\left.J_{3,4}=7.8 \mathrm{~Hz}, J_{2,3}=4.7 \mathrm{~Hz}, 1 \mathrm{H}, \mathrm{H} 3\right), 6.05$ (br. s, $1 \mathrm{H}, \mathrm{NH}$ ), 3.85 (q, $\left.J_{1^{\prime}, 2^{\prime}}=J_{1^{\prime}, \mathrm{NH}}=7.2 \mathrm{~Hz}, 2 \mathrm{H}, \mathrm{H} 11^{\prime}\right), 1.69$ (pent., $J_{1^{\prime}, 2^{\prime}}=J_{2^{\prime}, 3^{\prime}}=7.2 \mathrm{~Hz}, 2 \mathrm{H}, \mathrm{H} 2^{\prime}$ ), 1.39-1.24 (m, 8H, H3'- H7'), 0.87 (t, $\left.J_{7^{\prime}, 8^{\prime}}=7.0 \mathrm{~Hz}, 2 \mathrm{H}, \mathrm{H} 8^{\prime}\right) ;{ }^{13} \mathrm{C}$ NMR $\left(125 \mathrm{MHz}, \mathrm{CDCl}_{3}\right) \delta \mathrm{ppm}$ 180.1(C8), 175.2(C5), 155.4 (C2), 148.5 (C8a), 143.8 (C6), 134.8 (C4), 134.7 (C4a), 126.7 (C3), 45.2 (C1'), 31.8 (C6'), 31.0 (C2'), 29.20 (C4'), 29.16 (C3'), 26.6 (C5'), 22.6 (C7'), 14.05 (C8'); calcd. for $\left[\mathrm{C}_{17} \mathrm{H}_{21} \mathrm{ClN}_{2} \mathrm{O}_{2}+\mathrm{H}\right]^{+}$: 321.1364, obsd.: 321.1361 . 


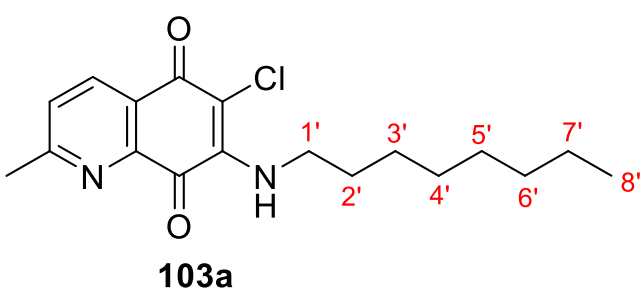

6-Chloro-2-methyl-7octylamino-5,8quinolinequinone (103a) and 7-chloro-2methyl-6-octylamino-

5,8-quinolinequinone (103b). By subjecting dichloroQQ 56 (113.9 mg, $0.47 \mathrm{mmol}$ ) and octylamine $(0.09 \mathrm{~mL}, 0.56 \mathrm{mmol})$ to the general procedure for the synthesis of substituted QQs, 103a (58.2 mg, 37\%) and 103b (53.5 mg, 34\%) were isolated as red crystals. Data for 103a: $\mathrm{R}_{f}=0.52\left(\mathrm{CH}_{2} \mathrm{Cl}_{2} /\right.$ EtOAc, 9/1, v/v); m.p.: $118^{\circ} \mathrm{C}$; IR (U $\left.\mathrm{u}_{\max }\right): 3304,2923,1694,1598$, 1574, 1553, 1509, 1443, 1318, 1258, 1202, 1162, 1143, 1109, 1090, 819, 739, 707, 587, 537, 479, 448, $417 \mathrm{~cm}^{-1}$; UV-vis (toluene) $\lambda_{\max }(\log \varepsilon): 485$ (2.84) nm; ${ }^{1} \mathrm{H}$ NMR $\left(500 \mathrm{MHz}, \mathrm{CDCl}_{3}\right)$ : $\delta$ ppm 8.32 (d, J3,4 $=7.9 \mathrm{~Hz}, 1 \mathrm{H}, \mathrm{H} 4), 7.48$ (d, $\left.J_{3,4}=8.0 \mathrm{~Hz}, 1 \mathrm{H}, \mathrm{H} 3\right), 6.19$ (br. s, 1H, NH), $3.84\left(\mathrm{q}, J_{1^{\prime}, 2^{\prime}}=6.8 \mathrm{~Hz}, 2 \mathrm{H}, \mathrm{H} 1^{\prime}\right), 2.71\left(\mathrm{~s}, 3 \mathrm{H}, \mathrm{CH}_{3}\right), 1.71-1.66\left(\mathrm{~m}, 2 \mathrm{H}, \mathrm{H} 2^{\prime}\right), 1.41-1.25(\mathrm{~m}, 10 \mathrm{H}$, $\left.\mathrm{H} 2^{\prime}-\mathrm{H} 7^{\prime}\right), 0.86\left(\mathrm{t}, J_{7^{\prime}, 8^{\prime}}=6.4 \mathrm{~Hz}, 2 \mathrm{H}, \mathrm{H} 8^{\prime}\right) ;{ }^{13} \mathrm{C} \mathrm{NMR}\left(125 \mathrm{MHz}, \mathrm{CDCl}_{3}\right): \delta$ ppm $179.2(\mathrm{C} 8)$, 174.2 (C5), 163.5 (C2), 145.4 (C8a), 134.9 (C4), 128.4 (C3), 127.8 (C4a), 45.2 (C1'), 31.7 (C6'), 31.1 (C2'), 29.2 (C4'), 29.28 (C3'), 26.6 (C5'), 22.6 (C7'), 14.1 (C8'); HRMS-ESI ( $m / z)$ calcd. for $\left[\mathrm{C}_{18} \mathrm{H}_{23} \mathrm{ClN}_{2} \mathrm{O}_{2}+\mathrm{H}\right]^{+}$: 335.1521, obsd.: 335.1525. Data for 103b: $\mathrm{R}_{f}=0.49\left(\mathrm{CH}_{2} \mathrm{Cl}_{2} / \mathrm{EtOAc}\right.$, 9/1, v/v); m.p.: $111.7^{\circ} \mathrm{C}$; IR (U $\left.\max \right): 2918,2853,1675,1563,1519,1313,1286,1224,1115,839$, 776, 750, 704, 645, $572 \mathrm{~cm}^{-1}$; UV-vis (toluene) $\lambda_{\max }(\log \varepsilon): 485(2.19) \mathrm{nm} ;{ }^{1} \mathrm{H}$ NMR $(500 \mathrm{MHz}$, $\mathrm{CDCl}_{3}$ ): $\delta$ ppm 8.21 (d, $\left.J_{3,4}=8.0 \mathrm{~Hz}, 1 \mathrm{H}, \mathrm{H} 4\right), 7.41$ (d, $\left.J_{3,4}=8.0 \mathrm{~Hz}, 1 \mathrm{H}, \mathrm{H} 3\right), 6.03$ (br. s, $1 \mathrm{H}$,

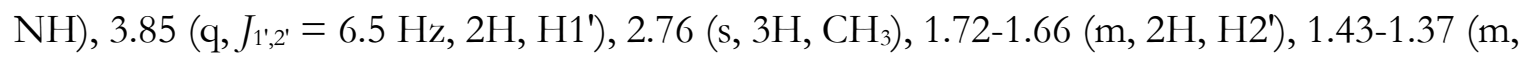
2H, H2'), 1.35-1.25 (m, 8H, H3'- H7'), 0.88 (t, $\left.J_{7^{\prime}, 8^{\prime}}=6.8 \mathrm{~Hz}, 2 \mathrm{H}, \mathrm{H} 8^{\prime}\right) ;{ }^{13} \mathrm{C}$ NMR $(125 \mathrm{MHz}$, $\mathrm{CDCl}_{3}$ ): $\delta$ ppm 180.2 (C8), 166.1 (C2), 148.1 (C8a), 135.0 (C4), 126.5 (C3), 124.6 (C4a), 45.2 (C1'), 31.9 (C6'), 31.1 (C2'), 29.33 (C4'), 29.28 (C3'), 25.6 (C5'), 22.8 (C7'), 14.22 (C8'); HRMSESI $(m / z)$ calcd. for $\left[\mathrm{C}_{18} \mathrm{H}_{23} \mathrm{ClN}_{2} \mathrm{O}_{2}+\mathrm{H}\right]^{+}: 335.1521$, obsd.: 335.1525 .<smiles>O=C1C(Cl)=C(Nc2ccccc2)C(=O)c2ncccc21</smiles><smiles>O=C1C(Cl)=C(Nc2ccccc2)C(=O)c2ncccc21</smiles>

6-Chloro-7-phenylamino-5,8quinolinequinone (104a) and 7-chloro6-phenylamino-5,8-quinolinequinone (104b). By subjecting dichloroQQ 92 (120.9 mg, $0.53 \mathrm{mmol}$ ) and aniline (0.06 $\mathrm{mL}, 0.62 \mathrm{mmol})$ to the general procedure for the synthesis of substituted QQs, 104a $(51.3 \mathrm{mg}$, 
$24 \%$ ) and 104b (54.3 mg, 36\%) were isolated as red crystals. Data for 104a: $\mathrm{R}_{f}=0.33$ $\left(\mathrm{CH}_{2} \mathrm{Cl}_{2} /\right.$ EtOAc, 24/1, v/v); m.p.: $235.7^{\circ} \mathrm{C}$; IR (U $\left.\mathrm{u}_{\max }\right): 2955,2922,2852,1692,1558,1462$, 1447, 1378, 1315, 1245, 1152, 1066, 852, 758, 723, 691, 629, 579, 550, 540, 452, $418 \mathrm{~cm}^{-1}$; UVvis $\left(\mathrm{CH}_{2} \mathrm{Cl}_{2}\right) \lambda_{\max }(\log \varepsilon): 490(2.48) \mathrm{nm} ;{ }^{1} \mathrm{H} \mathrm{NMR}\left(500 \mathrm{MHz}, \mathrm{CDCl}_{3}\right): \delta \mathrm{ppm} 9.00\left(\mathrm{~d}, J_{2,4}=4.5\right.$ $\mathrm{Hz}, 1 \mathrm{H}, \mathrm{H} 2$ ), 8.52 (d, $\left.J_{3,4}=7.2 \mathrm{~Hz}, 1 \mathrm{H}, \mathrm{H} 4\right), 7.86$ (br. s, $1 \mathrm{H}, \mathrm{NH}$ ), 7.71 (dd, $J_{3,4}=7.8 \mathrm{~Hz}, J_{2,3}$ $=4.7 \mathrm{~Hz}, 1 \mathrm{H}, \mathrm{H} 3), 7.37\left(\mathrm{t}, J_{0-\mathrm{CH} 2, m-\mathrm{CH} 2}=J_{m-\mathrm{CH} 2, p-\mathrm{CH} 2}=7.6 \mathrm{~Hz}, 2 \mathrm{H}, m-\mathrm{PhCH}\right), 7.25\left(\mathrm{t}, J_{m-\mathrm{CH} 2, p-\mathrm{CH} 2}\right.$ $=7.3 \mathrm{~Hz}, 2 \mathrm{H}, p-\mathrm{PhCH}), 7.12\left(\mathrm{~d}, J_{o-\mathrm{CH} 2, m-\mathrm{CH} 2}=7.8 \mathrm{~Hz}, 2 \mathrm{H}, o-\mathrm{PhCH}\right) ;{ }^{13} \mathrm{C}$ NMR $(125 \mathrm{MHz}$, CDCl ${ }_{3}$ ): $\delta$ ppm 179.0 (C8), 176.5 (C5), 153.9 (C2), 146.1 (C8a), 141.9 (C7), 136.9 (Ph-Cq), 135.0 (C4), 130.0 (C4a), 128.53 (C3), 128.5 (m-PhCH), 126.1 (p-PhCH), 124.6 (o-PhCH); HRMS-ESI $(m / z)$ calcd. for $\left[\mathrm{C}_{15} \mathrm{H}_{9} \mathrm{ClN}_{2} \mathrm{O}_{2}+\mathrm{H}\right]^{+}: 285.0245$, obsd.: 285.0426. Data for 104b: $\mathrm{R}_{f}$ $=0.26\left(\mathrm{CH}_{2} \mathrm{Cl}_{2} /\right.$ EtOAc, 24/1, v/v); m.p. $205.8^{\circ} \mathrm{C}$; IR (U $\left.\max \right): 2923,1674,1646,1588,1559$, 1484, 1444, 926, 858, 800, 757, 731, 685, 577, 549, 510, 449, $415 \mathrm{~cm}^{-1}$; UV-vis $\left(\mathrm{CH}_{2} \mathrm{Cl}_{2}\right) \lambda_{\max }$ (log ع): 485 (2.54) nm; ${ }^{1} \mathrm{H}$ NMR (500 MHz, $\left.\mathrm{CDCl}_{3}\right): \delta \mathrm{ppm} 9.06\left(\mathrm{~d}, J_{2,4}=4.8 \mathrm{~Hz}, 1 \mathrm{H}, \mathrm{H} 2\right), 8.44$ $\left(\mathrm{dd}, J_{3,4}=8.0 \mathrm{~Hz}, J_{2,4}=1.6 \mathrm{~Hz}, 1 \mathrm{H}, \mathrm{H} 4\right), 7.71$ (br. s, $\left.1 \mathrm{H}, \mathrm{NH}\right), 7.68$ (dd, $J_{3,4}=8.0 \mathrm{~Hz}, J_{2,3}=4.7$ $\mathrm{Hz}, 1 \mathrm{H}, \mathrm{H} 3), 7.37$ (t, $\left.J_{o-\mathrm{CH} 2, m-\mathrm{CH} 2}=J_{m-\mathrm{CH} 2, p-\mathrm{CH} 2}=7.6 \mathrm{~Hz}, 2 \mathrm{H}, m-\mathrm{PhCH}\right), 7.25\left(\mathrm{t}, J_{m-\mathrm{CH} 2, p-\mathrm{CH} 2}=7.3\right.$ $\mathrm{Hz}, 2 \mathrm{H}, p-\mathrm{PhCH}), 7.10\left(\mathrm{~d}, J_{0-\mathrm{CH} 2, m-\mathrm{CH} 2}=7.8 \mathrm{~Hz}, 2 \mathrm{H}, o_{-} \mathrm{PhCH}\right) ;{ }^{13} \mathrm{C} \mathrm{NMR}\left(125 \mathrm{MHz}, \mathrm{CDCl}_{3}\right) \delta$ ppm 180.3 (C5), 176.0 (C8), 155.6 (C2), 148.5 (C8a), 141.2 (C6), 137.2 (Ph-Cq ), 135.0 (C4), 128.69 (C3), $128.64(m-\mathrm{PhCH}), 127.1$ (C4a), 126.2 (p-PhCH), 124.7 (o-PhCH); HRMS-ESI $(m / z)$ calcd. for $\left[\mathrm{C}_{15} \mathrm{H}_{9} \mathrm{ClN}_{2} \mathrm{O}_{2}+\mathrm{H}\right]^{+}: 285.0245$, obsd.: 285.0426 .<smiles>Cc1ccc2c(n1)C(=O)C(Nc1ccccc1)=C(Cl)C2=O</smiles>

$105 a$

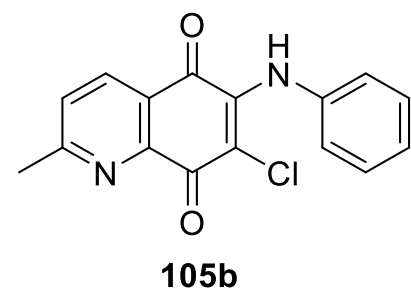

6-Chloro-2-methyl-7phenylamino-5,8quinolinequinone (105a) and 7chloro-2-methyl-6-phenylamino5,8-quinolinequinone (105b). By

subjecting dichloroQQ $56(115 \mathrm{mg}, 0.48 \mathrm{mmol})$ and aniline $(0.05 \mathrm{~mL}, 0.57 \mathrm{mmol})$ to the general procedure for the synthesis of substituted QQs, 105a (34.4 mg, 24\%) and 105b (94.6 $\mathrm{mg}, 24 \%)$ were isolated as red crystals. Data for 105a: $\mathrm{R}_{f}=0.32\left(\mathrm{CH}_{2} \mathrm{Cl}_{2} / \mathrm{EtOAc}, 24 / 1, \mathrm{v} / \mathrm{v}\right)$; m.p.: $226.2^{\circ} \mathrm{C}$; IR (U $\left.\mathrm{U}_{\max }\right): 3236,1688,1634,1552,1485,1443,1284,1225,1151,1073,986,910$, $872,848,815,768,736,693,574,562,501,472,448,419 \mathrm{~cm}^{-1}$; UV-vis $\left(\mathrm{CH}_{2} \mathrm{Cl}_{2}\right) \lambda_{\max }(\log \varepsilon)$ : 490 (2.68) nm; ${ }^{1} \mathrm{H}$ NMR $\left(500 \mathrm{MHz}, \mathrm{CDCl}_{3}\right) \delta \mathrm{ppm} 8.38$ (d, J3,4 $\left.=8.0 \mathrm{~Hz}, 1 \mathrm{H}, \mathrm{H} 4\right), 7.81$ (br. s, $1 \mathrm{H}, \mathrm{NH}), 7.54\left(\mathrm{~d}, J_{3,4}=8.0 \mathrm{~Hz}, 1 \mathrm{H}, \mathrm{H} 3\right), 7.36\left(\mathrm{t}, J_{0-\mathrm{CH}, m-\mathrm{CH}}=J_{m-\mathrm{CH}, p-\mathrm{CH} 3}=7.9 \mathrm{~Hz}, 2 \mathrm{H}, m-\mathrm{PhCH}\right)$, $7.24\left(\mathrm{t}, J_{m-\mathrm{CH}, p-\mathrm{CH} 3}=7.6 \mathrm{~Hz}, 1 \mathrm{H}, p-\mathrm{PhCH}\right), 7.11\left(\mathrm{~d}, J_{0-\mathrm{CH}, m-\mathrm{CH}}=7.8 \mathrm{~Hz}, 2 \mathrm{H}, o-\mathrm{PhCH}\right), 2.77(\mathrm{~s}, 3 \mathrm{H}$, $\left.\mathrm{CH}_{3}\right) ;{ }^{13} \mathrm{C}$ NMR $\left(125 \mathrm{MHz}, \mathrm{CDCl}_{3}\right.$ ) $\delta$ ppm 179.4 (C8), 176.9 (C5), 164.3 (C2), 145.8 (C8a), 
141.9 (C6 or C7), $137.2\left(\mathrm{Ph}-\mathrm{C}_{\mathrm{q}}\right), 135.3$ (C4), 128.64 (C3), $128.62(m-\mathrm{PhCH}), 128.0(\mathrm{C} 4 \mathrm{a}), 126.1$ $(\not-\mathrm{PhCH}), 124.6(0-\mathrm{PhCH}), 25.2\left(\mathrm{CH}_{3}\right)$; HRMS-ESI $(\mathrm{m} / \mathrm{z})$ calcd. for $\left[\mathrm{C}_{16} \mathrm{H}_{11} \mathrm{ClN}_{2} \mathrm{O}_{2}+\mathrm{H}\right]^{+}$: 299.0582, obsd.: 299.0586. Data for 105b: $\mathrm{R}_{f}=0.22\left(\mathrm{CH}_{2} \mathrm{Cl}_{2} / \mathrm{EtOAc}, 24 / 1\right.$, v/v); m.p: 226.7 ${ }^{\circ} \mathrm{C}$; IR (U $\left.U_{\max }\right): 2955,2918,1678,1646,1546,1420,1375,1287,1222,1199,1144,1033,905$, 888, 849, 707, 707, 698, 586, 560, $509 \mathrm{~cm}^{-1}$; UV-vis $\left(\mathrm{CH}_{2} \mathrm{Cl}_{2}\right) \lambda_{\max }(\log \varepsilon): 485(2.18) \mathrm{nm} ;{ }^{1} \mathrm{H}$ $\operatorname{NMR}\left(500 \mathrm{MHz}, \mathrm{CDCl}_{3}\right) \delta \mathrm{ppm} 8.31$ (d, $\left.J_{3,4}=8.0 \mathrm{~Hz}, 1 \mathrm{H}, \mathrm{H} 4\right), 7.66$ (br. s, 1H, NH), 7.48 (d, $\left.J_{3,4}=8.0 \mathrm{~Hz}, 1 \mathrm{H}, \mathrm{H} 3\right), 7.37\left(\mathrm{t}, J_{0 \cdot \mathrm{CH}, m-\mathrm{CH}},=J_{m-\mathrm{CH}, p-\mathrm{CH} 3}=7.8 \mathrm{~Hz}, 2 \mathrm{H}, m-\mathrm{PhCH}\right), 7.24\left(\mathrm{t}, J_{m-\mathrm{CH}, p-\mathrm{CH} 3}\right.$ $=7.8 \mathrm{~Hz}, 1 \mathrm{H}, p-\mathrm{PhCH}), 7.09\left(\mathrm{~d}, J_{0-\mathrm{CH}, m-\mathrm{CH}}=7.8 \mathrm{~Hz}, 2 \mathrm{H}, o_{-} \mathrm{PhCH}\right), 2.79\left(\mathrm{~s}, 3 \mathrm{H}, \mathrm{CH}_{3}\right) ;{ }^{13} \mathrm{C} \mathrm{NMR}$

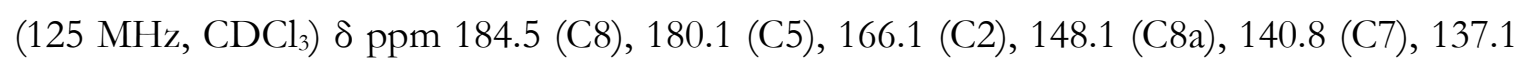
$\left(\mathrm{Ph}-\mathrm{C}_{\mathrm{q}}\right), 135.0$ (C4), 128.5 (m-PhCH), 126.8 (C3), 125.9 (p-PhCH), 124.7 (C4a), 124.4 (o$\mathrm{PhCH}), 25.5\left(\mathrm{CH}_{3}\right)$; HRMS-ESI $(m / z)$ calcd. for $\left[\mathrm{C}_{16} \mathrm{H}_{11} \mathrm{ClN}_{2} \mathrm{O}_{2}+\mathrm{H}\right]^{+}:$299.0582, obsd.: 299.0586.
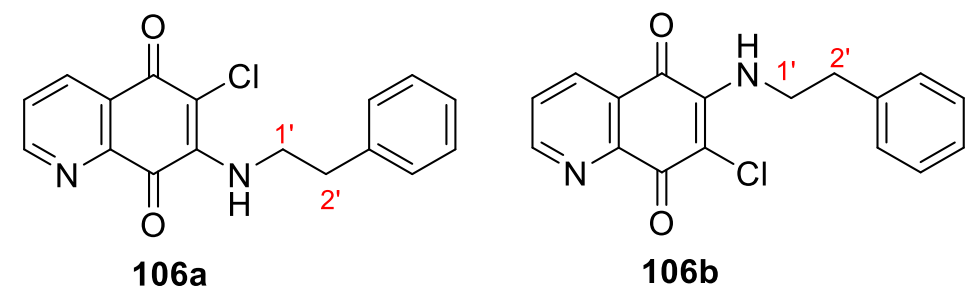

6-Chloro-7phenylethylamino-5,8quinolinequinone (106a) and 7-chloro-6phenylethylamino-5,8-

quinolinequinone (106b). By subjecting dichloroQQ 92 (168.5 mg, $0.74 \mathrm{mmol}$ ) and 2phenylethylamine $(0.11 \mathrm{~mL}, 0.89 \mathrm{mmol})$ to the general procedure for the synthesis of substituted QQs, 106a (39.3 mg, 17\%) and 106b (127.3 mg, 55\%) were isolated as red crystals. Data for 106a: $\mathrm{R}_{f}=0.31\left(\right.$ EtOAc/petroleum ether, 2/1, v/v); m.p.: $170.7^{\circ} \mathrm{C}$; IR (Umax): 3286, 2164, 1638, 1639, 1591, 1583, 1520, 1356, 1307, 1253, 1189, 1142, 1096, 830, 817, 756, 732, 724, 699, 667, 647, 613, 530, 498, 482, $419 \mathrm{~cm}^{-1}$; UV-vis (toluene) $\lambda_{\max }(\log \varepsilon): 485$ (3.69) nm; ${ }^{1} \mathrm{H}$ NMR $\left(500 \mathrm{MHz}, \mathrm{CDCl}_{3}\right) \delta \mathrm{ppm} 8.92\left(\mathrm{~d}, J_{2,3}=4.5 \mathrm{~Hz}, 1 \mathrm{H}, \mathrm{H} 2\right), 8.47\left(\mathrm{~d}, J_{3,4}=7.8 \mathrm{~Hz}, 1 \mathrm{H}\right.$, H4), $7.65\left(\mathrm{dd}, J_{3,4}=7.7 \mathrm{~Hz}, J_{2,3}=4.7 \mathrm{~Hz}, 1 \mathrm{H}, \mathrm{H} 3\right), 7.34-7.31(\mathrm{~m}, 2 \mathrm{H}, m-\mathrm{PhCH}), 7.26-7.24(\mathrm{~m}$, $3 \mathrm{H}, o-\mathrm{PhCH}, p-\mathrm{PhCH}$ ), 6.25 (br. s, 1H, NH), 4.14 (q, $\left.J_{1^{\prime}, 2^{\prime}}=J_{1^{\prime}, \mathrm{NH}}=6.8 \mathrm{~Hz}, 2 \mathrm{H}, \mathrm{H} 1^{\prime}\right), 3.01$ (t, $\left.J_{1^{\prime}, 2^{\prime}}=6.9 \mathrm{~Hz}, 2 \mathrm{H}, \mathrm{H} 2^{\prime}\right), 3.00 ;{ }^{13} \mathrm{C} \mathrm{NMR}\left(125 \mathrm{MHz}, \mathrm{CDCl}_{3}\right) \delta \mathrm{ppm} 178.8$ (C8), 175.6 (C5), 153.4 (C2), 146.0 (C8a), 137.5 (Ph-Cq), 134.7 (C4), 128.9 (m-PhCH), 128.8 (o-PhCH), 128.4 (C3), $127.0(p-\mathrm{PhCH}), 46.2\left(\mathrm{C}^{\prime}\right), 37.3\left(\mathrm{C}^{\prime}\right)$; HRMS-ESI $(\mathrm{m} / \mathrm{z})$ calcd. for $\left[\mathrm{C}_{17} \mathrm{H}_{13} \mathrm{ClN}_{2} \mathrm{O}_{2}+\mathrm{H}\right]^{+}$: 313.0738, obsd.: 313.0738. Data for 106b: $\mathrm{R}_{f}=0.18$ (EtOAc/petroleum ether, 2/1, v/v); m.p.: $165.2^{\circ} \mathrm{C}$; IR (U $\left.U_{\max }\right): 3183,2953,2922,2853,1688,1596,1563,1497,1356,1327,1260,1215$, 1111, 1075, 744, 731, 680, 554, 498, 463, $410 \mathrm{~cm}^{-1}$; UV-vis (toluene) $\lambda_{\max }(\log \varepsilon): 485(2.72) \mathrm{nm}$; ${ }^{1} \mathrm{H}$ NMR $\left(500 \mathrm{MHz}, \mathrm{CDCl}_{3}\right): \delta \mathrm{ppm} 9.01$ (d, $\left.J_{2,3}=5.1 \mathrm{~Hz}, 1 \mathrm{H}, \mathrm{H} 2\right), 8.32\left(\mathrm{~d}, J_{3,4}=7.8 \mathrm{~Hz}, 1 \mathrm{H}\right.$, 
H4), $7.57\left(\mathrm{dd}, J_{3,4}=7.9 \mathrm{~Hz}, J_{2,3}=4.6 \mathrm{~Hz}, 1 \mathrm{H}, \mathrm{H} 3\right), 7.35-7.32(\mathrm{~m}, 2 \mathrm{H}, m-\mathrm{PhCH}), 7.27-7.23(\mathrm{~m}$, 3H, o- $\mathrm{PhCH}, p-\mathrm{PhCH}$ ), 6.07 (br. s, 1H, NH), 4.14 (q, $\left.J_{1^{\prime}, 2^{2}}=J_{1^{\prime}, \mathrm{NH}}=6.8 \mathrm{~Hz}, 2 \mathrm{H}, \mathrm{H} 1^{\prime}\right), 3.00$ (t, $\left.J_{1^{\prime}, 2^{\prime}}=7.1 \mathrm{~Hz}, 2 \mathrm{H}, \mathrm{H} 2\right) ;{ }^{\prime 3} \mathrm{C}$ NMR (125 MHz, $\left.\mathrm{CDCl}_{3}\right): \delta$ ppm 180.0 (C5), 155.2 (C2), 148.4 (C8), 143.6 (C6), 137.4 (Ph-Cq), 134.7 (C4), 128.9 (m-PhCH), 128.81 (o-PhCH), 127.7 (C4a), 127.0 (p-PhCH), 126.5 (C3), 46.1 (C1'), $37.2\left(\mathrm{C}^{\prime}\right)$; HRMS-ESI ( $\left.m / 2\right)$ calcd. for $\left[\mathrm{C}_{17} \mathrm{H}_{13} \mathrm{ClN}_{2} \mathrm{O}_{2}+\mathrm{H}\right]^{+}$: 313.0738, obsd.: 313.0738 .
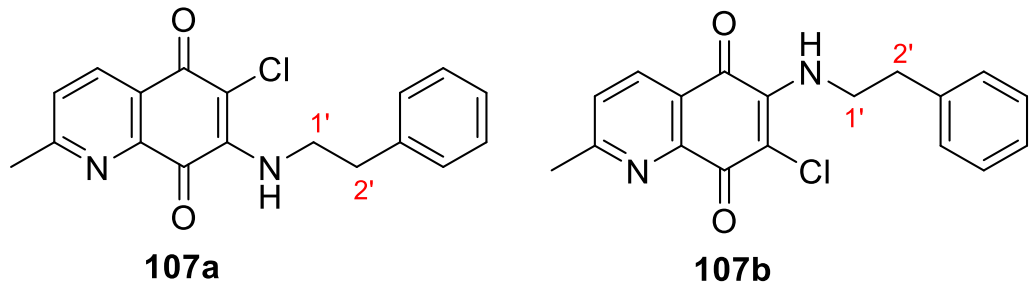

6-Chloro-2-methyl-7phenylethylamino-5,8quinolinequinone (107a) and 7-chloro-2-methyl-6phenylethylamino-5,8-

quinolinequinone (107b). By subjecting dichloroQQ 56 (185.6 $\mathrm{mg}, 0.77 \mathrm{mmol}$ ) and 2phenylethylamine $(0.12 \mathrm{~mL}, 0.92 \mathrm{mmol})$ to the general procedure for the synthesis of substituted QQs, 107a (70.5 mg, 28\%) and 107b (93.1 mg, 37\%) were isolated as red crystals. Data for 107a: $\mathrm{R}_{f}=0.42\left(\right.$ EtOAc/petroleum ether, 2/1, v/v); m.p.: $165.9^{\circ} \mathrm{C}$; IR (U $\left.\mathrm{U}_{\max }\right): 3302$, 1695, 1633, 1571, 1493, 1464, 1305, 1230, 1147, 1107, 851, 817, 735, 694, 665, 595, 579, 517, 492, 476, 449, 419, $404 \mathrm{~cm}^{-1}$; UV-vis (toluene) $\lambda_{\max }(\log \varepsilon): 490$ (2.83) nm; ${ }^{1} \mathrm{H}$ NMR $(500 \mathrm{MHz}$, $\left.\mathrm{CDCl}_{3}\right) \delta \mathrm{ppm} 8.32\left(\mathrm{~d}, J_{3,4}=8.0 \mathrm{~Hz}, 1 \mathrm{H}, \mathrm{H} 4\right), 7.48\left(\mathrm{~d}, J_{3,4}=8.0 \mathrm{~Hz}, 1 \mathrm{H}, \mathrm{H} 3\right), 7.33-7.30(\mathrm{~m}$, $2 \mathrm{H}, m-\mathrm{PhCH}), 7.25-7.22(\mathrm{~m}, 3 \mathrm{H}, o-\mathrm{PhCH}, p-\mathrm{PhCH}), 6.21$ (br. s, $1 \mathrm{H}, \mathrm{NH}), 4.12$ (q, $J_{\mathrm{H} 1^{\prime}, \mathrm{H} 2^{\prime}}=$ $\left.J_{\mathrm{H}^{\prime}, \mathrm{NH}}=6.9 \mathrm{~Hz}, 2 \mathrm{H}, \mathrm{H} 1^{\prime}\right), 2.99$ (t, $\left.J_{1^{\prime}, 2^{\prime}}=7.2 \mathrm{~Hz}, 2 \mathrm{H}, \mathrm{H} 2^{\prime}\right), 2.72\left(\mathrm{~s}, 3 \mathrm{H}, \mathrm{CH}_{3}\right) ;{ }^{13} \mathrm{C} \mathrm{NMR}(125$ $\mathrm{MHz}, \mathrm{CDCl}_{3}$ ) $\delta$ ppm 179.1 (C8), 175.9 (C5), 163.6 (C2), 145.5 (C8a), 144.4 (C7). 137.7 (PhCq), 134.9 (C4), 129.0 (m-PhCH), 128.9 (o-PhCH), 128.5 (C3), 128.3 (C4a), 127.0 (p-PhCH), 46.1 (C1'), 37.4 (C2'), $25.1\left(\mathrm{CH}_{3}\right)$; HRMS-ESI $(m / z)$ calcd. for $\left[\mathrm{C}_{18} \mathrm{H}_{15} \mathrm{ClN}_{2} \mathrm{O}_{2}+\mathrm{H}\right]^{+}: 327.0895$, obsd.: 327.0895. Data for 107b: $\mathrm{R}_{f}=0.27$ (EtOAc/petroleum ether, 2/1, v/v); m.p.: $143.0^{\circ} \mathrm{C}$; IR (U $\left.U_{\max }\right): 3210,1682,1567,1507,1440,1362,1306,1230,1114,1069,844,770,749,697,645$, 566, 506, 489, 460, $421 \mathrm{~cm}^{-1}$; UV-vis (toluene) $\lambda_{\max }(\log \varepsilon): 475$ (2.78) nm; ${ }^{1} \mathrm{H}$ NMR $(500 \mathrm{MHz}$, $\left.\mathrm{CDCl}_{3}\right) \delta \mathrm{ppm} 8.20\left(\mathrm{~d}, J_{3,4}=8.0 \mathrm{~Hz}, 1 \mathrm{H}, \mathrm{H} 4\right), 7.41$ (d, $\left.J_{3,4}=8.0 \mathrm{~Hz}, 1 \mathrm{H}, \mathrm{H} 3\right), 7.33-7.32(\mathrm{~m}$, $2 \mathrm{H}, m-\mathrm{PhCH}$ ), 7.26-7.24 (m, 3H, o- $\mathrm{PhCH}, p-\mathrm{PhCH}), 6.06$ (br. s, $1 \mathrm{H}, \mathrm{NH}), 4.12$ (q, J $\mathrm{H}_{1^{\prime}, \mathrm{H} 2^{\prime}}=$ $\left.J_{\mathrm{H}^{\prime}, \mathrm{NH}}=6.9 \mathrm{~Hz}, 2 \mathrm{H}, \mathrm{H} 1^{\prime}\right), 2.99$ (t, $\left.J_{1^{\prime}, 2^{\prime}}=7.2 \mathrm{~Hz}, 2 \mathrm{H}, \mathrm{H} 2^{\prime}\right), 2.75$ (s, 3H, CH $) ;{ }^{13} \mathrm{C} \mathrm{NMR}^{(125}$ $\mathrm{MHz}, \mathrm{CDCl}_{3}$ ) $\delta$ ppm 179.9 (C5), 175.5 (C8), 165.9 (C2), 148.0 (C8a), 143.5 (C6), 137.5 (PhCq), 134.8 (C4), 128.9 (o-PhCH), 128.8 (m-PhCH), 127.0 (p-PhCH), 126.4 (C3), 124.5 (C4a), $46.1\left(\mathrm{C} 1^{\prime}\right), 37.2\left(\mathrm{C}^{\prime}\right), 25.5\left(\mathrm{CH}_{3}\right)$; HRMS-ESI $(\mathrm{m} / \mathrm{z})$ calcd. for $\left[\mathrm{C}_{18} \mathrm{H}_{15} \mathrm{ClN}_{2} \mathrm{O}_{2}+\mathrm{H}\right]^{+}$: 327.0895 , obsd.: 327.0895 . 


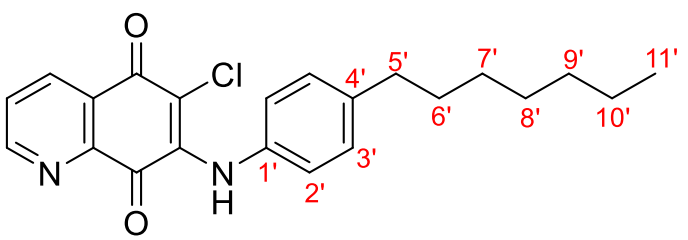

$108 a$<smiles>CC(C)(C)c1ccc(NC2=C(Cl)C(=O)c3ncccc3C2=O)cc1</smiles>

108b
6-Chloro-7-(4heptyl)anilino-

5,8-quinolinequinone (108a) and 7-chloro-6-(4-heptyl)anilino-5,8-quinolinequinone (108b). By subjecting dichloroQQ $92(136.3 \mathrm{mg}, 0.60 \mathrm{mmol})$ and 4-heptylaniline $(0.15 \mathrm{~mL}$, $0.72 \mathrm{mmol})$ to the general procedure for the synthesis of substituted QQs, 108a $(91.9 \mathrm{mg}$, $40 \%$ ) and 108b (66.6 mg, 29\%) were isolated as red crystals. Data for 108a: $R_{f}=0.23$ (petroleum ether/EtOAc, 1/1, v/v); m.p.: $150.5^{\circ} \mathrm{C}$; IR (U $\left.\max \right): 2915,2849,1690,1636,1589$, 1577, 1559, 1555, 1513, 1481, 1470, 1452, 1322, 1311, 1302, 1277, 1248, 1217, 1180, 1152, 1067, 856, 844, 725, 623, $567 \mathrm{~cm}^{-1}$; UV-vis (toluene) $\lambda_{\max }$ (log ع): 505 (2.69) nm; ${ }^{1} \mathrm{H}$ NMR (500 $\mathrm{MHz}_{\mathrm{CDCl}}$ ) $\delta \mathrm{ppm} 8.98$ (d, J2,3 $\left.=4.7 \mathrm{~Hz}, 1 \mathrm{H}, \mathrm{H} 2\right), 8.51$ (d, $\left.J_{3,4}=7.9 \mathrm{~Hz}, 1 \mathrm{H}, \mathrm{H} 4\right), 7.84$ (br. s, $1 \mathrm{H}, \mathrm{NH}), 7.70\left(\mathrm{dd}, J_{3,4}=7.7 \mathrm{~Hz}, J_{2,3}=4.7 \mathrm{~Hz}, 1 \mathrm{H}, \mathrm{H} 3\right), 7.16$, (d, $\left.J_{2^{\prime}, 3^{\prime}}=7.6 \mathrm{~Hz}, 2 \mathrm{H}, \mathrm{H} 3^{\prime}\right)$, $7.02\left(\mathrm{~d}, J_{2^{\prime}, 3^{\prime}}=7.6 \mathrm{~Hz}, 2 \mathrm{H}, \mathrm{H} 2^{\prime}\right), 2.62\left(\mathrm{t}, J_{5^{\prime}, 6^{\prime}}=7.7 \mathrm{~Hz}, 2 \mathrm{H}, \mathrm{H} 5^{\prime}\right), 1.65-1.59$ (m, 2H, H6'), 1.32$1.27\left(\mathrm{~m}, 8 \mathrm{H}, \mathrm{H} 7^{\prime}-\mathrm{H} 10^{\prime}\right), 0.88$ (t, $\left.J_{10^{\prime}, 1^{\prime}}=6.1 \mathrm{~Hz}, 3 \mathrm{H}, \mathrm{H} 11^{\prime}\right) ;{ }^{13} \mathrm{C} \mathrm{NMR}\left(125 \mathrm{MHz}, \mathrm{CDCl}_{3}\right) \delta$ ppm 179.2 (C8), 176.5 (C5), 153.9 (C2), 146.3 (C8a), 142.2 (C6), 141.4 (C4'), 135.1 (C4), 134.6 (C1'), 130.2 (C4a), 128.6 (C3), 128.5 (C3'), 124.7 (C2'), 113.5 (C7), 35.6 (C5'), 31.9 (C9'), 31.5 (C6'), 29.3 (C7'), 29.3 (C8'), 22.8 (C10'), 14.2 (C11'); HRMS(ESI) $m / z$ calcd. for $\left[\mathrm{C}_{22} \mathrm{H}_{23} \mathrm{ClN}_{2} \mathrm{O}_{2}+\mathrm{H}\right]^{+}$: 383.1521, obsd.: 358.1520. Data for 108b: $\mathrm{R}_{f}=0.11$ (petroleum ether/EtOAc, 1/1, v/v); m.p: $174.4^{\circ} \mathrm{C}$; IR (Umax): 3340, 2920, 1670, 1606, 1578, 1518, 1492 , 1309, 1213, 1144, 863, 839, 813, 746, 687, 638. 525, $458 \mathrm{~cm}^{-1}$; UV-vis (toluene) $\lambda_{\max }(\log \varepsilon):$ 495 (2.72) nm; ${ }^{1} \mathrm{H}$ NMR (500 MHz, CDCl $) \delta$ ppm $9.04\left(\mathrm{dd}, J_{3,4}=4.7 \mathrm{~Hz}, J_{2,4}=1.4 \mathrm{~Hz}, 1 \mathrm{H}\right.$, H4), $8.42\left(\mathrm{dd}, J_{2,3}=7.8 \mathrm{~Hz}, J_{2,4}=1.3 \mathrm{~Hz}, 1 \mathrm{H}, \mathrm{H} 2\right), 7.66$ (br. s, $\left.1 \mathrm{H}, \mathrm{NH}\right), 7.63$ (dd, $J_{3,4}=4.7 \mathrm{~Hz}$, $\left.J_{2,3}=7.8 \mathrm{~Hz}, 1 \mathrm{H}, \mathrm{H} 3\right), 7.16$, (d, $\left.J_{2^{\prime}, 3^{\prime}}=8.3 \mathrm{~Hz}, 2 \mathrm{H}, \mathrm{H} 3^{\prime}\right), 7.01$ (d, $\left.J_{2^{\prime}, 3^{\prime}}=8.2 \mathrm{~Hz}, 2 \mathrm{H}, \mathrm{H} 2^{\prime}\right), 2.61$ $\left(\mathrm{t}, J_{5^{\prime}, 6^{\prime}}=7.9 \mathrm{~Hz}, 2 \mathrm{H}, \mathrm{H} 5^{\prime}\right), 1.61$ (pent., $\left.J_{5^{\prime}, 6^{\prime}, 7^{\prime}}=7.6 \mathrm{~Hz}, 2 \mathrm{H}, \mathrm{H} 6^{\prime}\right), 1.32-1.27$ (m, 8H, H7'- H10'), $0.87\left(\mathrm{t}, J_{10^{\prime}, 11^{\prime}}=6.9 \mathrm{~Hz}, 3 \mathrm{H}, \mathrm{H} 11^{\prime}\right) ;{ }^{13} \mathrm{C}$ NMR $\left(125 \mathrm{MHz}, \mathrm{CDCl}_{3}\right) \delta \mathrm{ppm} 180.2$ (C5), 176.0 (C8), 155.5 (C2), 148.4 (C8a), 141.1 (C4'), 134.5 (C1'), 134.9 (C4), 128.6 (C3'), 127.0 (C3), 124.6 (C2'), 115.6 (C6), 35.6 (C5'), 31.9 (C9'), 31.5 (C6'), 29.3 (C7'), 29.3 (C8'), 22.8 (C10'), 14.2 (C11'); HRMS-ESI $(m / z)$ calcd. for $\left[\mathrm{C}_{22} \mathrm{H}_{23} \mathrm{ClN}_{2} \mathrm{O}_{2}+\mathrm{H}\right]^{+}: 383.1521$, obsd.: 358.1520 . 


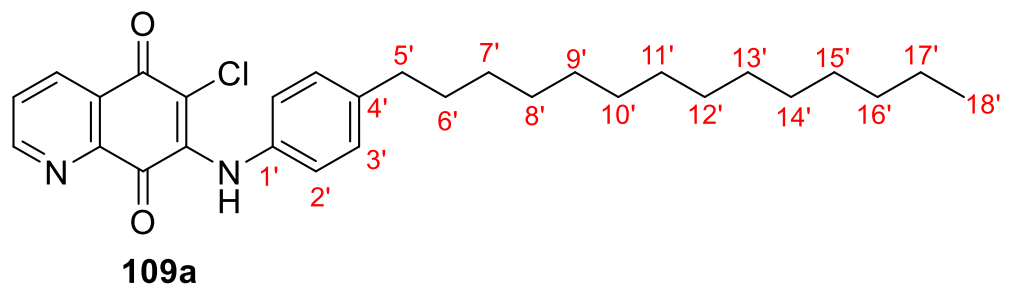

6-Chloro-7-(4-tetradecyl)anilino-5,8-quinolinequinone<smiles>CC(C)c1ccc(NC2=C(Cl)C(=O)c3ncccc3C2=O)cc1</smiles>

109b

tetradecyl)anilino-5,8-quinolinequinone (109b). By subjecting dichloroQQ 92 (129.7 mg, $0.57 \mathrm{mmol})$ and 4-tetradecylaniline $(196.2 \mathrm{mg}, 0.68 \mathrm{mmol})$ to the general procedure for the synthesis of substituted QQs, 109a (90.5 mg, 33\%) and 109b (79.5 mg, 29\%) were isolated as red crystals. Data for 109a: $\mathrm{R}_{f}=0.67\left(\mathrm{CH}_{2} \mathrm{Cl}_{2} / \mathrm{EtOAc}, 5 / 1, \mathrm{v} / \mathrm{v}\right)$; m.p.: $152.3{ }^{\circ} \mathrm{C}$; IR (U $\left.\max \right)$ : 3236, 2913, 2848, 1691, 1637, 1588, 1556, 1513, 1467, 1322, 1303, 1217, 1151, 1067, 1018, $857,834,817,725,623,571,551,825,510,469,450,417 \mathrm{~cm}^{-1}$; UV-vis $\left(\mathrm{CH}_{2} \mathrm{Cl}_{2}\right) \lambda_{\max }(\log \varepsilon)$ : 495 (2.39) nm; ${ }^{1} \mathrm{H}$ NMR $\left(500 \mathrm{MHz}, \mathrm{CDCl}_{3}\right) \delta \mathrm{ppm} 8.99$ (d, $\left.J_{2,3}=4.7 \mathrm{~Hz}, 1 \mathrm{H}, \mathrm{H} 2\right), 8.52$ (d, J3,4 $=7.9 \mathrm{~Hz}, 1 \mathrm{H}, \mathrm{H} 4), 7.83$ (br. s, $1 \mathrm{H}, \mathrm{NH}), 7.71$ (dd, $J_{3,4}=7.8 \mathrm{~Hz}, J_{2,3}=4.7 \mathrm{~Hz}, 1 \mathrm{H}, \mathrm{H} 3$ ), 7.17 $\left(\mathrm{d}, J_{2^{\prime}, 3^{\prime}}=8.2 \mathrm{~Hz}, 2 \mathrm{H}, \mathrm{H} 3^{\prime}\right), 7.02\left(\mathrm{~d}, J_{2^{\prime}, 3^{\prime}}=8.2 \mathrm{~Hz}, 2 \mathrm{H}, \mathrm{H} 2{ }^{\prime}\right), 2.62\left(\mathrm{t}, J_{5^{\prime}, 6^{\prime}}=7.4 \mathrm{~Hz}, 2 \mathrm{H}, \mathrm{H} 5^{\prime}\right)$, 1.62-1.55 (m, H6'), 1.31-1.26 (m, 22H, H7'-H17'), 0.88 (t, $\left.J_{17^{\prime}, 18^{\prime}}=7.0 \mathrm{~Hz}, 2 \mathrm{H}, \mathrm{H} 18^{\prime}\right) ;{ }^{13} \mathrm{C}$ NMR (125 MHz, CDCl $)$ \& ppm 179.1 (C8), 176.4 (C5), 153.8 (C2), 146.1 (C8a), 141.3 (C1'), 134.9 (C4), 134.5 (C4'), 130.0 (C4a), 128.5 (C3), 128.4 (C3'), 124.6 (C2'), 113.5 (C6), 35.5 (C5'), 31.9 (C6'), 31.4, 29.69, 29.68, 29.67, 29.65, 29.6, 29.5, 29.4, 29.3, 22.7 (C7'-C17'), 14.12 (C18'); HRMS-ESI ( $m / z)$ calcd. for $\left[\mathrm{C}_{29} \mathrm{H}_{37} \mathrm{ClN}_{2} \mathrm{O}_{2}+\mathrm{H}\right]^{+}:$481.2616, obsd.: 481.2618. Data for 109b: $\mathrm{R}_{f}$ $=0.5\left(\mathrm{CH}_{2} \mathrm{Cl}_{2} /\right.$ EtOAc, 5/1, v/v); m.p.: $160.7^{\circ} \mathrm{C} ; \mathrm{IR}\left(\mathrm{u}_{\max }\right): 3339,2916,2848,1670,1616,1608$, 1578, 1471, 1313, 1215, 1144, 863, 837, 818, 623, $453 \mathrm{~cm}^{-1}$; UV-vis $\left(\mathrm{CH}_{2} \mathrm{Cl}_{2}\right) \lambda_{\max }(\log \varepsilon): 495$ (2.29) nm; ${ }^{1} \mathrm{H}$ NMR $\left(500 \mathrm{MHz}, \mathrm{CDCl}_{3}\right) \delta \mathrm{ppm} 9.05$ (d, $\left.J_{2,3}=4.8 \mathrm{~Hz}, 1 \mathrm{H}, \mathrm{H} 2\right), 8.43\left(\mathrm{~d}, J_{3,4}=\right.$

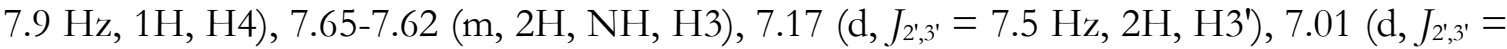

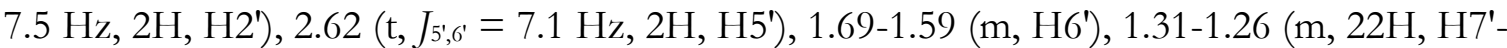
H17'), 0.88 (t, $\left.J_{17^{\prime}, 18^{\prime}}=7.2 \mathrm{~Hz}, 2 \mathrm{H}, \mathrm{H} 18^{\prime}\right) ;{ }^{13} \mathrm{C}$ NMR $\left(125 \mathrm{MHz}, \mathrm{CDCl}_{3}\right) \delta$ ppm $180.3(\mathrm{C} 5), 176.0$ (C8), 155.5 (C2), 148.6 (C8a), 141.4 (C4'), 134.9 (C4), 134.7 (C1'), 128.6 (C3'), 127.0 (C3), 126.9 (C4a), 124.6 (C2'), 35.6 (C5'), 32.1 (C6'), 31.5, 29.9, 29.8, 29.8, 29.8, 29.7, 29.6, 29.5, 22.8 (C7'C17'), 14.3 (C18'); HRMS-ESI ( $m /$ z) calcd. for $\left[\mathrm{C}_{29} \mathrm{H}_{37} \mathrm{ClN}_{2} \mathrm{O}_{2}+\mathrm{H}\right]^{+}$: 481.2616, obsd.: 481.2616. 
<smiles>COc1ccc(CNC2=C(Cl)C(=O)c3cccnc3C2=O)cc1</smiles><smiles>COc1ccc(CNC2=C(Cl)C(=O)c3ncccc3C2=O)cc1</smiles>

6-Chloro-7-(4methoxylbenzyl)amino-

5,8-quinolinequinone (110a) and 7-chloro-6-(4methoxylbenzyl)amino-

5,8-quinolinequinone (110b). By subjecting dichloroQQ 92 (143.6 mg, $0.63 \mathrm{mmol}$ ) and methoxybenzylamine $(0.10 \mathrm{~mL}, 0.76 \mathrm{mmol})$ to the general procedure for the synthesis of substituted QQs, $110 \mathrm{a}(167.4 \mathrm{mg}, 67 \%)$ and $\mathbf{1 1 0 b}(52.5 \mathrm{mg}, 21 \%)$ were isolated as red crystals. Data for 110a: $\mathrm{R}_{f}=0.29\left(\mathrm{CH}_{2} \mathrm{Cl}_{2} /\right.$ EtOAc, 5/1, v/v); m.p.: $179.3{ }^{\circ} \mathrm{C}$; IR (U max $): 3308,1698$, 1595, 1560, 1509, 1447, 1359, 1302, 1246, 1181, 1149, 1089, 1036, 809, 724, 580, 545, 517, 445, $410 \mathrm{~cm}^{-1}$; UV-vis (DMF) $\lambda_{\max }(\log \varepsilon): 465(3.53) \mathrm{nm} ;{ }^{1} \mathrm{H}$ NMR $\left(500 \mathrm{MHz}, \mathrm{CDCl}_{3}\right) \delta \mathrm{ppm}$ $8.91\left(\mathrm{~d}, J_{2,3}=4.8 \mathrm{~Hz}, 1 \mathrm{H}, \mathrm{H} 2\right), 8.46\left(\mathrm{~d}, J_{3,4}=7.8 \mathrm{~Hz}, 1 \mathrm{H}, \mathrm{H} 4\right), 7.64$ (dd, $J_{3,4}=7.9 \mathrm{~Hz} J_{2,3}=4.6$ $\mathrm{Hz}, 1 \mathrm{H}, \mathrm{H} 3), 7.25\left(\mathrm{~d}, J_{0-\mathrm{CH}, m-\mathrm{CH}}=8.1 \mathrm{~Hz}, 2 \mathrm{H}, o_{-} \mathrm{PhCH}\right), 6.90\left(\mathrm{~d}, J_{0-\mathrm{CH}, m-\mathrm{CH}}=8.1 \mathrm{~Hz}, 2 \mathrm{H}, m-\right.$ $\mathrm{PhCH}), 6.30$ (br s, $1 \mathrm{H}, \mathrm{NH}), 5.00\left(\mathrm{~d}, J_{\mathrm{CH} 2 \mathrm{NH}}=5.1 \mathrm{~Hz}, 2 \mathrm{H}, \mathrm{CH}_{2}\right) 3.79\left(\mathrm{~s}, 3 \mathrm{H}, \mathrm{O}-\mathrm{CH}_{3}\right) ;{ }^{13} \mathrm{C} \mathrm{NMR}$ (125 MHz, $\left.\mathrm{CDCl}_{3}\right) \delta$ ppm 179.0 (C8), 175.6 (C5), $159.7\left(\mathrm{C}_{\mathrm{q}}-\mathrm{OCH}_{3}\right), 153.6(\mathrm{C} 2), 146.1$ (C8a), 144.4 (C7), 134.9 (C4), 129.9 (C4a), 128.6 (C3), 129.3 (o-PhCH), 114.5 (m-PhCH), $55.5\left(\mathrm{OCH}_{3}\right)$; HRMS-ESI $(m / z)$ calcd. for $\left[\mathrm{C}_{17} \mathrm{H}_{13} \mathrm{ClN}_{2} \mathrm{O}_{2}+\mathrm{H}\right]^{+}:$329.0887, obsd.: 328.0688. Data for 110b: $\mathrm{R}_{f}$ $=0.20\left(\mathrm{CH}_{2} \mathrm{Cl}_{2} / \mathrm{EtOAc}, 5 / 1, \mathrm{v} / \mathrm{v}\right) ; \mathrm{IR}\left(\mathrm{u}_{\max }\right): 3310,1699,1603,1576,1516,1350,1235,1100$, 1024, 800, 725, 678, $511 \mathrm{~cm}^{-1}$; UV-vis (DMF) $\lambda_{\max }(\log \varepsilon): 460(2.03) \mathrm{nm} ;{ }^{1} \mathrm{H}$ NMR $(500 \mathrm{MHz}$, $\left.\mathrm{CDCl}_{3}\right) \delta$ ppm $8.92\left(\mathrm{~d}, J_{2,3}=4.6 \mathrm{~Hz}, 1 \mathrm{H}, \mathrm{H} 2\right), 8.43\left(\mathrm{~d}, J_{3,4}=7.8 \mathrm{~Hz}, 1 \mathrm{H}, \mathrm{H} 4\right), 7.68-7.62(\mathrm{~m}$, $1 \mathrm{H}, \mathrm{H} 3), 7.24\left(\mathrm{~d}, J_{0-\mathrm{CH}, m-\mathrm{CH}}=8.7 \mathrm{~Hz}, 2 \mathrm{H}, o-\mathrm{PhCH}\right), 6.91\left(\mathrm{~d}, J_{0-\mathrm{CH}, m-\mathrm{CH}}=8.1 \mathrm{~Hz}, 2 \mathrm{H}, m-\mathrm{PhCH}\right)$, $5.86(\mathrm{br} \mathrm{s}, 1 \mathrm{H}, \mathrm{NH}), 4.33\left(\mathrm{~d}, J_{\mathrm{CH} 2 \mathrm{NH}}=5.5 \mathrm{~Hz}, 2 \mathrm{H}, \mathrm{CH}_{2}\right) 3.81\left(\mathrm{~s}, 3 \mathrm{H}, \mathrm{O}_{-} \mathrm{CH}_{3}\right) ;{ }^{13} \mathrm{C} \mathrm{NMR}^{(125}$ $\left.\mathrm{MHz}, \mathrm{CDCl}_{3}\right) \delta$ ppm $181.6(\mathrm{C} 8), 175.5$ (C5), $159.5\left(\mathrm{C}_{\mathrm{q}}-\mathrm{OCH}_{3}\right), 153.4$ (C2), $146.0(\mathrm{C} 8 \mathrm{a}), 144.5$ (C6), 134.7 (C4), 129.9 (C4a), 128.4 (C3), 129.3 (o-PhCH), 114.4 (m-PhCH), $55.3\left(\mathrm{OCH}_{3}\right)$; HRMS-ESI $(m / z)$ calcd. for $\left[\mathrm{C}_{17} \mathrm{H}_{13} \mathrm{ClN}_{2} \mathrm{O}_{2}+\mathrm{H}\right]^{+}: 329.0887$, obsd.: 328.0688 .

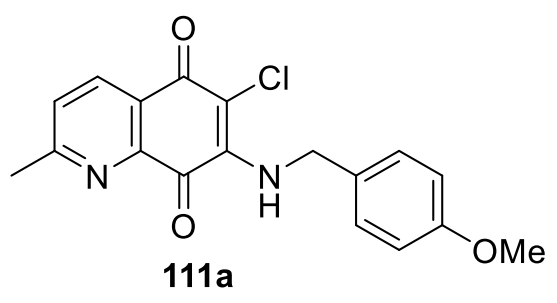
orange crystals $(147.8 \mathrm{mg}, 49 \%) ; \mathrm{R}_{f}=0.39\left(\mathrm{CH}_{2} \mathrm{Cl}_{2} /\right.$ EtOAc, 9/1, v/v); m.p.: $169.3{ }^{\circ} \mathrm{C}$; IR $\left(U_{\max }\right): 3315,1699,1600,1576,1557,1506,1436,1357,1323,1239,1177,1153,1106,1094$, $1034,818,801,738,678,599,562,518,472,417 \mathrm{~cm}^{-1}$; UV-vis (toluene) $\lambda_{\max }(\log \varepsilon): 485$ (2.20),

6-Chloro-7-(4-methoxybenzyl)amino-2-methyl-5,8quinolinequinone (111a). By subjecting dichloroQQ 56 (211.8 mg, $0.88 \mathrm{mmol})$ and methoxybenzylamine (0.14 $\mathrm{mL}, 1.05 \mathrm{mmol}$ ) to the general procedure for the synthesis of substituted QQs, the title compound was isolated as 
$320(2.51) \mathrm{nm} ;{ }^{1} \mathrm{H}$ NMR $\left(500 \mathrm{MHz}, \mathrm{CDCl}_{3}\right): \delta \mathrm{ppm} 8.29$ (d, $\left.J_{3,4}=8.1 \mathrm{~Hz}, 1 \mathrm{H}, \mathrm{H} 4\right), 7.47$ (d, J3,4 $=8.0 \mathrm{~Hz}, 1 \mathrm{H}, \mathrm{H} 3), 7.24\left(\mathrm{~d}, J_{o-\mathrm{CH}, m_{-} \mathrm{CH}}=8.1 \mathrm{~Hz}, 2 \mathrm{H}, o_{-} \mathrm{PhCH}\right), 6.87\left(\mathrm{~d}, J_{o-\mathrm{CH}, m_{-} \mathrm{CH}}=8.1 \mathrm{~Hz}, 2 \mathrm{H}\right.$, $m-\mathrm{PhCH}$ ), 6.23 (br s, 1H, NH), 4.97 (d, J $\mathrm{NH}_{\mathrm{CH} 2}=6.0 \mathrm{~Hz}, 2 \mathrm{H}, \mathrm{CH}_{2}-\mathrm{Ph}$ ), 3.77 (s, 3H, O-CH 3 ), 2.69 (s, 3H, $\left.\mathrm{CH}_{3}\right) ;{ }^{13} \mathrm{C}$ NMR (125 MHz, $\mathrm{CDCl}_{3}$ ): $\delta$ ppm 179.1 (C8), 176.0 (C5), 163.6 (C2), $159.5\left(\mathrm{C}_{\mathrm{q}}-\mathrm{OCH}_{3}\right), 145.5(\mathrm{C} 8 \mathrm{a}), 144.3(\mathrm{C} 7), 134.9(\mathrm{C} 4), 129.6\left(\mathrm{C}_{\mathrm{q}}-\mathrm{CH}_{2}\right), 129.2(o-\mathrm{PhCH}), 128.4$ (C3), 127.7 (C4a), $114.4(m-\mathrm{PhCH}), 55.3\left(\mathrm{O}_{-} \mathrm{CH}_{3}\right)$, 50.53, $48.7\left(\mathrm{CH}_{2}\right), 25.0\left(\mathrm{CH}_{3}\right)$; HRMS-ESI $(m / z)$ calcd. for $\left[\mathrm{C}_{18} \mathrm{H}_{15} \mathrm{ClN}_{2} \mathrm{O}_{2}+\mathrm{H}\right]^{+}: 343.0844$, obsd.: 343.0846 .

\subsubsection{Cyclic voltammetry (acetonitrile)}

The electrochemical measurements were conducted at ambient room temperature $\left(\sim 20^{\circ} \mathrm{C}\right)$ using a PGSTAT128N potentiostat-galvanostat (Metrohm Autolab Instruments, Switzerland), equipped with a three-electrode cell. Glassy carbon electrode and platinum wire were used for the working and counter electrodes, respectively, and a silver wire was used for a reference electrode. For all samples the QQ concentration was approximately $1 \mathrm{mM}$ and the electrolyte concentration $\left(\mathrm{C}_{2} \mathrm{H}_{5}\right)_{4} \mathrm{~N}\left(\mathrm{PF}_{6}\right)$ was $50 \mathrm{mM}$ in a $30 \mathrm{~mL}$ solution of acetonitrile. All the CVs were recorded at $100 \mathrm{mV} / \mathrm{s}$. 


\section{CHAPTER FOUR}

\section{The synthesis of chromonyl-}

\section{pyrimidines and their potential as anti-}

\section{tubercular agents}

\subsection{Introduction}

Recently, a high-throughput screen of 100,000 compounds identified amino-quinolinyl pyrimidines (i.e. XV, Figure 4.1) as a promising class of $M t b \mathrm{NDH}-\mathrm{II}$ inhibitor. ${ }^{79}$ In particular, the incorporation of primary amines at the 2'- and 4-positions on the quinolinyl pyrimidine scaffold were found to be essential for enzyme inhibition and were thus postulated to participate in hydrogen bonding interactions within the enzyme binding site. Quinolinyl pyrimidines $(\mathbf{X V})$ bearing monosubstituted phenyl substituents at the 2- and 6'-positions were excellent NDH-II inhibitors, with lead compound 25 exhibiting an $\mathrm{IC}_{50}$ value of $0.043 \mu \mathrm{M} .^{79}$ SAR studies of monosubstituted phenyl quinolinyl pyrimidines revealed that alterations to the 
phenyl substituents did not greatly affect the anti-tubercular activity of the compounds. For example, the potency of para-chlorophenyl quinolinyl pyrimidine 25 against NDH-II (measured in $\mathrm{IC}_{50}$ ) and $\mathrm{Mtb}$ (measured in MIC) was comparable to derivatives 112-114, despite differences in substituents on the phenyl ring. Here, quinolinyl pyrimidine $\mathbf{1 1 2}$ featured a chloride at the meta-position of the phenyl group on the pyrimidine ring, while this chloride was absent in quinolinyl pyrimidine 113. In quinolinyl pyrimidine 114, the strongly electronwithdrawing fluoro-substituent on the phenyl substituent in quinolinyl pyrimidine 25 was replaced with an electron-donating methoxy group. These observations suggest that small structural changes to aryl-substituted quinolinyl pyrimidines $\mathbf{X V}$ may be well-tolerated and, moreover, the correlation between NDH-II inhibition and MIC values against $M t b$ indicated that NDH-II may be the target of growth inhibition by quinolinyl pyrimidines $(\mathbf{X V}) .{ }^{79}$ Since this study, little work has been reported on the development of quinolinyl pyrimidines as antitubercular agents and their mode of action is yet to be confirmed. ${ }^{205}$

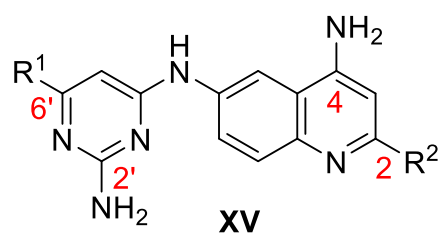

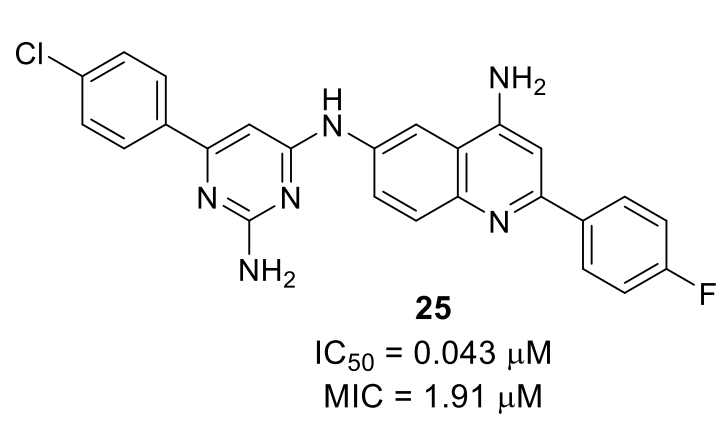

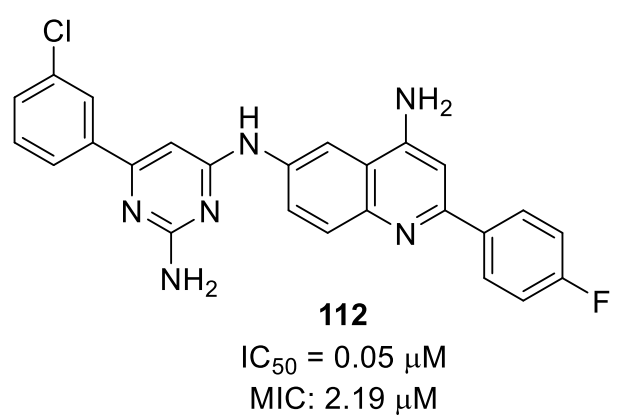<smiles>Nc1nc(Nc2ccc3nc(-c4ccc(F)cc4)cc(N)c3c2)cc(-c2ccccc2)n1</smiles>

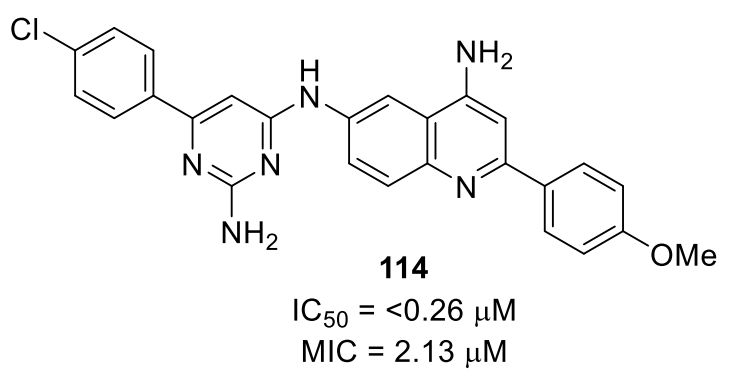

Figure 4.1: Quinolinyl pyrimidine general structure (XV) and derivatives 25, 112-114, with enzyme inhibition activity $\left(\mathrm{IC}_{50}\right)$ and anti-tubercular activity (MIC). ${ }^{79}$ 
Given the anti-tubercular activity of quinolinyl pyrimidines $(\mathbf{X V})$, it was envisioned that a structurally related class of compounds, chromonyl-pyrimidines (XVI, Figure 4.2A), could be promising new TB drug candidates. The bicylic 1,4-chromone scaffold in chromonylpyrimidines (XVI) mimics the quinone motif and is anticipated to bind NDH-II at the proposed Q-site. Moreover, the replacement of the quinoline group with a less polar group, such as a chromone, might enhance interactions with the hydrophobic NDH-II active site. Here, the primary amine in the quinoline moiety of quinolinyl pyrimidines is replaced by a ketone, which could act as a hydrogen acceptor, for example with the amine proton of the FAD isoalloxazine ring. In addition, both quinolinyl pyrimidines $\mathbf{X V}$ and chromonylpyrimidines XVI can stabilise a positive charge through resonance (Figure 4.2B), allowing them to remain stable under acidic environments, such as empysema pus, in which $M t b$ can reside (Chapter 1, Section 1.1.3). ${ }^{206}$

chromone

A.

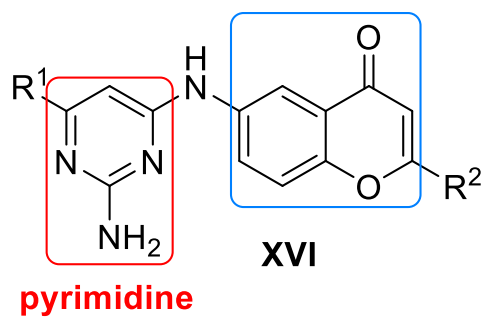

B.<smiles></smiles><smiles>[R]C1=CC([O-])c2cc(Nc3cc([R])nc(N)n3)ccc2O1</smiles>

Figure 4.2: A).Proposed anti-tubercular drug scaffold, chromonyl-pyrimidines (XVI). B). Both quinolinyl pyrimidines $\mathbf{X V}$ and chromonyl-pyrimidines XVI can stabilise a positive charge through resonance.

\subsubsection{The role of chromones in drug discovery}

Chromones are oxygen-containing heterocyclic compounds known for their wide range of pharmacological properties. ${ }^{207}$ In particular, the therapeutic potential of chromone-based drugs for the treatment of cancer, ${ }^{208-213}$ neurodegenerative diseases, ${ }^{214}$ and infections ${ }^{210,215,216}$ have been extensively investigated. Accordingly, many chromone-containing drugs have advanced to clinical trials, ${ }^{217-220}$ though none hitherto as anti-mycobacterial agents. That said, the anti- 
tubercular activity of chromones has been demonstrated with tricyclic chromones $\mathbf{1 1 5}$ and $\mathbf{1 1 6}$ (Figure 4.3), ${ }^{221,222}$ which inhibited $M t b$ cell growth by $99 \%$ at $62.5 \mu \mathrm{g} / \mathrm{mL} .{ }^{222}$ In addition, pyridinyl tricyclic chromones $\mathbf{1 1 7}$ and $\mathbf{1 1 8}$ showed promise as anti-tubercular agents against MDR-TB, with MIC values of $0.07 \mu \mathrm{M}$ and $0.61 \mu \mathrm{M}$, respectively. ${ }^{223}$<smiles>NC(=S)Nc1nc(=S)[nH]c2oc3c(Br)cc(Br)cc3c(=O)c12</smiles><smiles>NC(=S)Nc1nc(=S)[nH]c2oc3ccc(Cl)cc3c(=O)c12</smiles><smiles>CCc1ccc(C(=O)NCC2=CC3Oc4ccccc4C(=O)C3=CN2c2ncccc2C)cc1</smiles><smiles>Cc1ccc2ccc(C(=O)NCC3=CC4Oc5ccccc5C(=O)C4=CN3c3ncc(Br)cc3Br)cc2c1</smiles>

Figure 4.3: Examples of anti-tubercular chromones 115-118. ${ }^{222,223}$

The biological activity of chromones has been attributed to their redox properties and their role as antioxidants. ${ }^{224,225}$ For example, flavone (119, Figure 4.4) inhibited the NADH : oxidoreductase enzyme in Saccharomyces cerevisiae, ${ }^{226}$ while glycosyl-chromones 120 and 121 showed radical scavenging activity against ROS 1,1-diphenyl 2-picryl hydrazyl (DPPH), ${ }^{227,228}$ and 2-(cis-1,2-dihydroxy-4-oxo-cyclohex-5-enyl)-5,7-dihydroxy-chromone (DEDC, 122) elevated ROS generation in human neuroblastoma SH-SY5Y cells. ${ }^{229}$ Chromones are also involved in kinase $\mathrm{e}^{230}$ and tubulin polymerisation inhibition ${ }^{231}$ in tumour cells, adenylyl cyclase type 1 inhibition for chronic pain, ${ }^{232}$ DNA polymerase inhibition in HIV-1, ${ }^{233}$ and cyclooxygenase inhibition in inflammatory diseases. ${ }^{234}$ Hence, the diverse pharmaceutical properties and low cytotoxicity against mammalian cells ${ }^{235}$ of chromones make them an attractive scaffold in the search for new NDH-II inhibitors. 
<smiles>O=c1cc(-c2ccccc2)oc2ccccc12</smiles>

119<smiles>O=C(/C=C/c1coc2cc(OC3OC(CO)C(O)C(O)C3O)ccc2c1=O)c1ccc([N+](=O)[O-])cc1</smiles><smiles></smiles>

120

Figure 4.4: Chromone-based drugs, flavone (119), ${ }^{226}$ glycosylated chromones $\mathbf{1 2 0}^{227}$ and $\mathbf{1 2 1},{ }^{228}$ and DEDC (122), ${ }^{228}$ that exert their pharmacological properties through their redox and antioxidant activities.

\subsubsection{The synthesis of chromones}

Typically, 2-substituted 1,4-chromones are prepared through the esterification of 2'hydroxyacetophenone (123) to give ester $124,25,236$ which can undergo a Baker-Venkatamaran rearrangement $(\rightarrow \mathbf{1 2 5})^{237,238}$ and subsequent acid-catalysed annulation to yield 2-substituted chromones, i.e. 126 (Scheme 4.1). ${ }^{207,239,240}$ Other methodologies for preparing chromones include the Simonis reaction of phenols (127), ${ }^{241,242}$ a Kostanecki reaction to afford cayboxychromones (128), ${ }^{243,244}$ and an intramolecular Wittig reaction of silylated ester 129, which can be prepared from salicylic acid (130). ${ }^{245}$ 
<smiles>CC(=O)c1ccccc1O</smiles>

123<smiles>[R2]OC(=O)C(=O)O[R20]</smiles><smiles>[R]OC(=O)c1cc(=O)c2ccccc2o1</smiles><smiles>[R]C(=O)Oc1ccccc1C(C)=O</smiles>

Baker-Venkatamaran rearrangement<smiles>CC1CC1</smiles>

124<smiles>Oc1ccccc1</smiles>

127<smiles>[R]C(=O)CC(=O)c1ccccc1O</smiles>

125<smiles>[R]c1cc(=O)c2ccccc2o1</smiles><smiles>CS(=O)(=C[As])P(c1ccccc1)c1ccccc1</smiles>

Wittig reaction<smiles>O=C(O)c1ccccc1O</smiles>

130<smiles>[R16][OH+]C(=O)c1ccccc1OC([R15])=O</smiles>

129

Scheme 4.1: Common strategies for the synthesis of chromones 126 and carboxy-chromones 128. ${ }^{207,225,236-245}$

\subsubsection{The role of pyrimidines in drug discovery}

The pyrimidine scaffold is a well-cited structural motif in drug discovery ${ }^{246}$ and is one of the most common nitrogen-containing heterocycles present in the current repertoire of FDAapproved dugs. ${ }^{247}$ Pyrimidine-containing pharmaceuticals have primarily been used to treat cancer, ${ }^{248-250}$ cardiovascular, ${ }^{251,252}$ and infectious diseases. ${ }^{253-256}$ Of particular interest for this project is a study on the anti-tubercular activity of a series of aniline-substituted-pyrimidines, which resulted in the identification of a lead pyrimidine candidate, derivative 131 (MIC $=8.9$ $\mu \mathrm{M}$ against $M t b$, Figure 4.5). ${ }^{257}$ In other studies, 2-hydroxypyrimidine 132 demonstrated $96 \%$ inhibition of $M t b$ growth at $6.25 \mu \mathrm{g} / \mathrm{mL},{ }^{258}$ and thiophene-substituted pyrimidine $\mathbf{1 3 3}$ gave a low MIC value of $0.4 \mu \mathrm{M}$ against $M t b$, comparable to that of INH (2). ${ }^{259}$ 


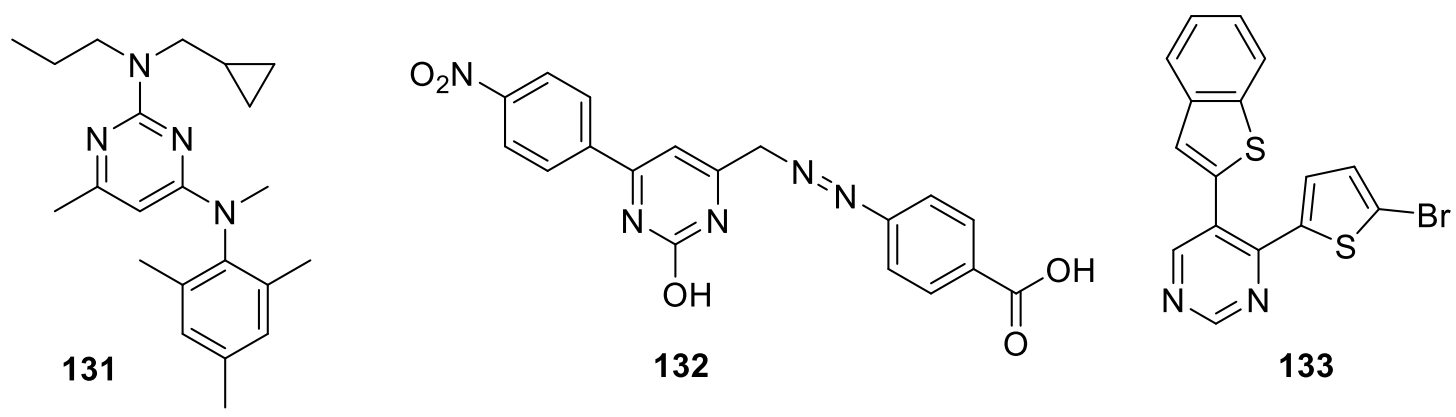

Figure 4.5: Examples of pyrimidine-containing anti-tubercular agents 131-133. ${ }^{257-259}$

Although many pyrimidine-containing anti-tubercular agents have been identified, ${ }^{258,260-266}$ the mechanisms by which the heterocycles inhibit mycobacterial growth have only been investigated in a few cases. For example, some pyrimidines are known to inhibit bacterial dihydrofolate reductases, an enzyme responsible for the reduction of folates to tetrahydrofolate, which in turn is important for nucleic and amino acid biosynthesis. ${ }^{267}$ Accordingly, their anti-mycobacterial activity is typically attributed to their antifolate activity, as in epiroprim (134, Figure 4.6) and azapteridine 135, which exhibited antifolate activity against $M$. leprae and $M$. avium, respectively. ${ }^{268-270}$ Unfortunately, however, pyrimidines 134 and 135 were less efficaciouis against $M t b$. Another notable enzyme target of pyrimidines is the acetohydroxacid synthase, which is inhibited by pyrimidine 136 in RR-TB and MDR-TB. ${ }^{260}$ Acetohydroxyacid synthase is involved in the biosynthesis of amino acids leucine, isoleucine and valine.<smiles>CCOc1cc(Cc2cnc(N)nc2N)cc(OCC)c1-n1cccc1</smiles><smiles>CCOc1ccc(OCC)c(N(C)Cc2cnc3nc(N)ncc3c2C)c1</smiles><smiles>COC(=O)c1ccccc1S(=O)(=O)NC(=O)Nc1nc(C)cc(C)n1</smiles>

Figure 4.6: Epiroprim $(134)^{268}$ and azapteridine $135^{270}$ inhibit dihydrofolate reductase in bacteria, while sulfonamide 136 inhibits acetohydroxacid synthase. ${ }^{260}$ 


\subsubsection{Pyrimidine synthesis}

To synthesise 2-amino-substituted pyrimidines (XVII) guanidinium salts (i.e. 137) are often used (Scheme 4.2). Key in the pyrimidine synthesis is the carbon-nitrogen bond formation to provide the 6-membered heterocycle, and accordingly, several methodologies involving different substrates have been developed. For example, treatment of guanidines 137 with malonitrile (138) or 1,3-diesters 139 affords tri-aminopyrimidines $\mathbf{1 4 0}^{271}$ or dihydroxypyrimidines $141,{ }^{272-274}$ respectively. To synthesise alkyl- or aryl-substituted pyrimidines, guanidines 137 can be reacted with 1,3-diketones 142 to give pyrimidines $143,^{275-277}$ or chlorovinyl aldehydes 144 , which can be prepared by applying Vilsmeier-Haack conditions to ketones 145 , to give dialkyl pyrimidines $146 .{ }^{278}$

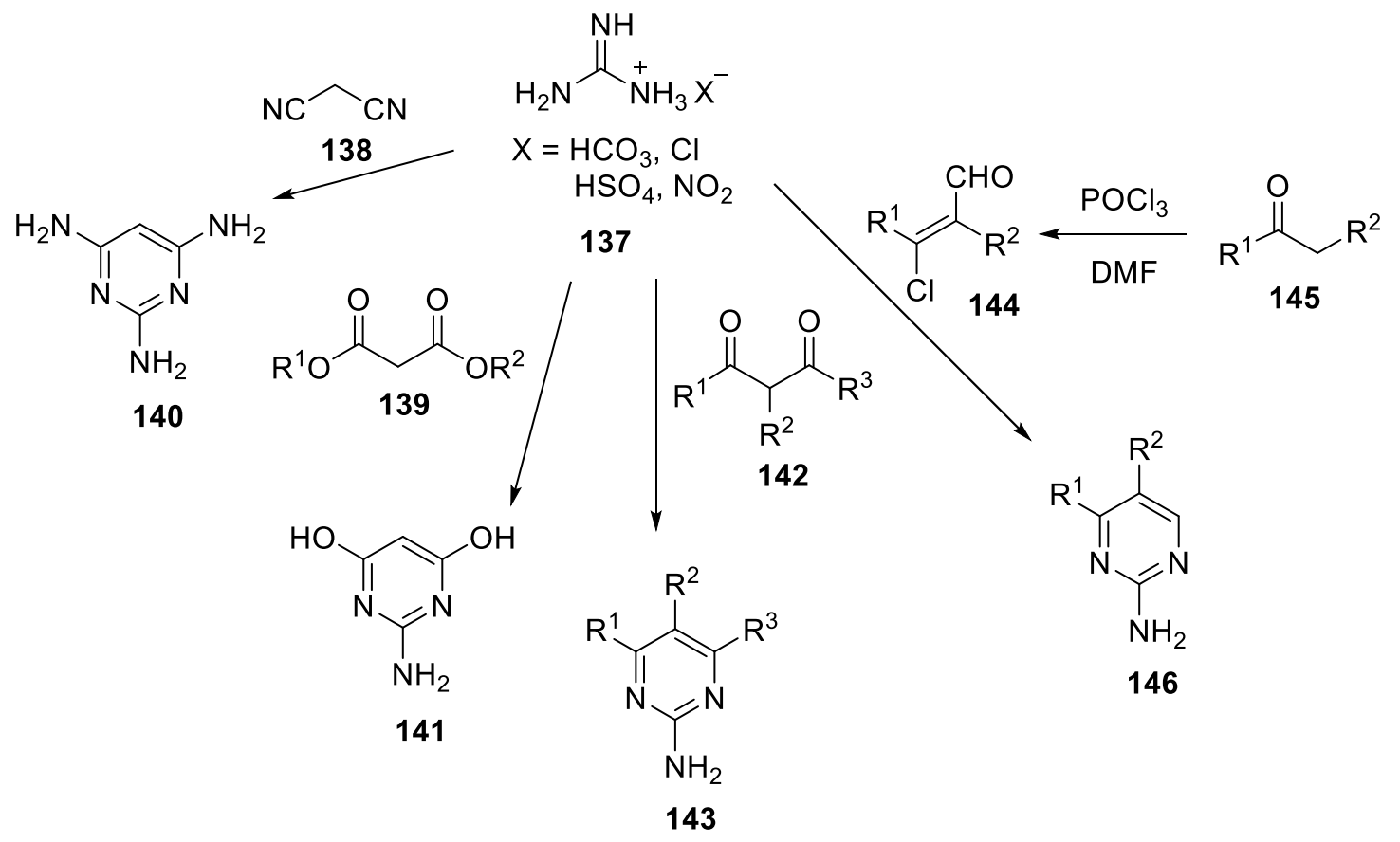

Scheme 4.2: Synthetic approaches to form functionalised pyrimidines from guanidinium salts 137..$^{271-274}$

\subsubsection{Project aims and retrosynthesis}

The aim of the research in this chapter is to investigate the potential of chromonyl-pyrimidines (XVI) as anti-tubercular agents. Accordingly, the preparation of a library of chromonylpyrimidines (XVI) with various functionalisations at the 6'- and 2-positions was proposed (Scheme 4.3). Here, the synthesis of pyrimidines installed with methyl, chloromethyl, and 
methoxymethyl groups was proposed so as to provide insight into how the electronic properties of the pyrimidine ring influences the anti-tubercular activity of the compounds. Similarly, substituents at the 2-position were chosen to probe the pharmacophore of chromonyl-pyrimidines, whereby an ester and cyclopropyl group provide an electrophilic centre for the chromone to interact with nucleophilic residues in NDH-II. In addition, cyclopropyl and phenyl groups are apolar and are thus anticipated to enhance the binding affinity of chromonyl-pyrimidines to the hydrophobic Q-site.

Retrosynthetically, it was envisioned that chromonyl-pyrimidines XVI would be synthesised by a nucleophilic substitution of chloropyrimidines XVII by amino-chromones XVIII. Chloropyrimidines XVII could, in turn, be prepared by the condensation of functionalised beta-ketoesters 147 with guanidine, followed by a chlorination reaction. ${ }^{279}$ Substituted chromones XVIII could be constructed from the condensation of 2'-hydroxyacetophenone (123) with functionalised acid chlorides or carboxylic acids to form an ester, which would then undergo an acid-catalysed cyclisation reaction. ${ }^{280}$ The library of functionalised chromonylpyrimidines (XVI) will then be screened for their ability to inhibit $M t b$.<smiles>[R1]c1cc(Nc2ccc3oc([R])cc(=O)c3c2)nc(N)n1</smiles><smiles>C=CC</smiles><smiles>[R]c1cc(Cl)nc(N)n1</smiles><smiles>[R]c1cc(=O)c2cc(N)ccc2o1</smiles>

$\mathrm{R}^{1}=\mathrm{CH}_{3}, \mathrm{CH}_{2} \mathrm{Cl}, \mathrm{CH}_{2} \mathrm{OCH}_{3}$ $\mathrm{R}^{2}=\mathrm{COCH}_{2} \mathrm{CH}_{3}, \mathrm{C}_{3} \mathrm{H}_{5}$, Ph<smiles>[R17]C(=O)CC(=O)OCC</smiles>

Scheme 4.3: The retrosynthetic analysis of chromonyl-pyrimidines (XVI). 


\subsection{Results and discussion}

\subsubsection{Docking studies}

To understand how chromonyl-pyrimidines (XVI) could bind NDH-II, ${ }^{281}$ docking studies of 6-((2-amino-6-methylpyrimidin-4-yl)amino)-2-methyl-4H-chromen-4-one (148, Figure 4.7A) in the C. thermarum NDH-II crystal structure (PDB: 6BDO) ${ }^{62}$ were undertaken using the UCSF Chimera/DOCK6 programme suite. ${ }^{282,283}$ In the docking studies, chromonyl-pyrimidine 148 was well-placed in the proposed Q-site of NDH-II (Figure 4.7B-D). Specifically, chromonylpyrimidine 148 is sandwiched between hydrophobic residues Q317 and I379, with the pyrimidine moiety occupying a site surrounded by more polar groups, R382 and E324 (Figure 4.7B). The methyl substituent of the pyrimidine ring, however, interacts with hydrophobic side chains I320 and Q321, indicating that less polar substituents at this position may be wellaccommodated. The hydrophobic interactions of the bicyclic chromone moiety with Q317 and 1379 , as shown in Figure 4.7C, are consistent with those of quinone and quinolone scaffolds in menadione $(\mathbf{1 8})^{58}$ and HQNO (19), ${ }^{62}$ respectively (Chapter 1, Section 1.3.1). Unfortunately, the ketone oxygen of chromone 148, which was anticipated to form a hydrogen bond with the isoalloxazine ring nitrogen is not well-positioned to interact with FAD. Notwithstanding, a hydrogen bond measured at $2.09 \AA$ was observed between an amine proton of the pyrimidine ring and the oxygen in glutamic acid E324 (Figure 4.7D). Taken together, these results demonstrated that chromonyl-pyrimidines XVII could be accommodated in the putative NDH-II Q-site in a similar manner to other substrates, i.e. menadione $(\mathbf{1 8})^{58}$ and HQNO (19). ${ }^{62}$ 
A.<smiles>Cc1cc(Nc2ccc3oc(C)cc(=O)c3c2)nc(N)n1</smiles>
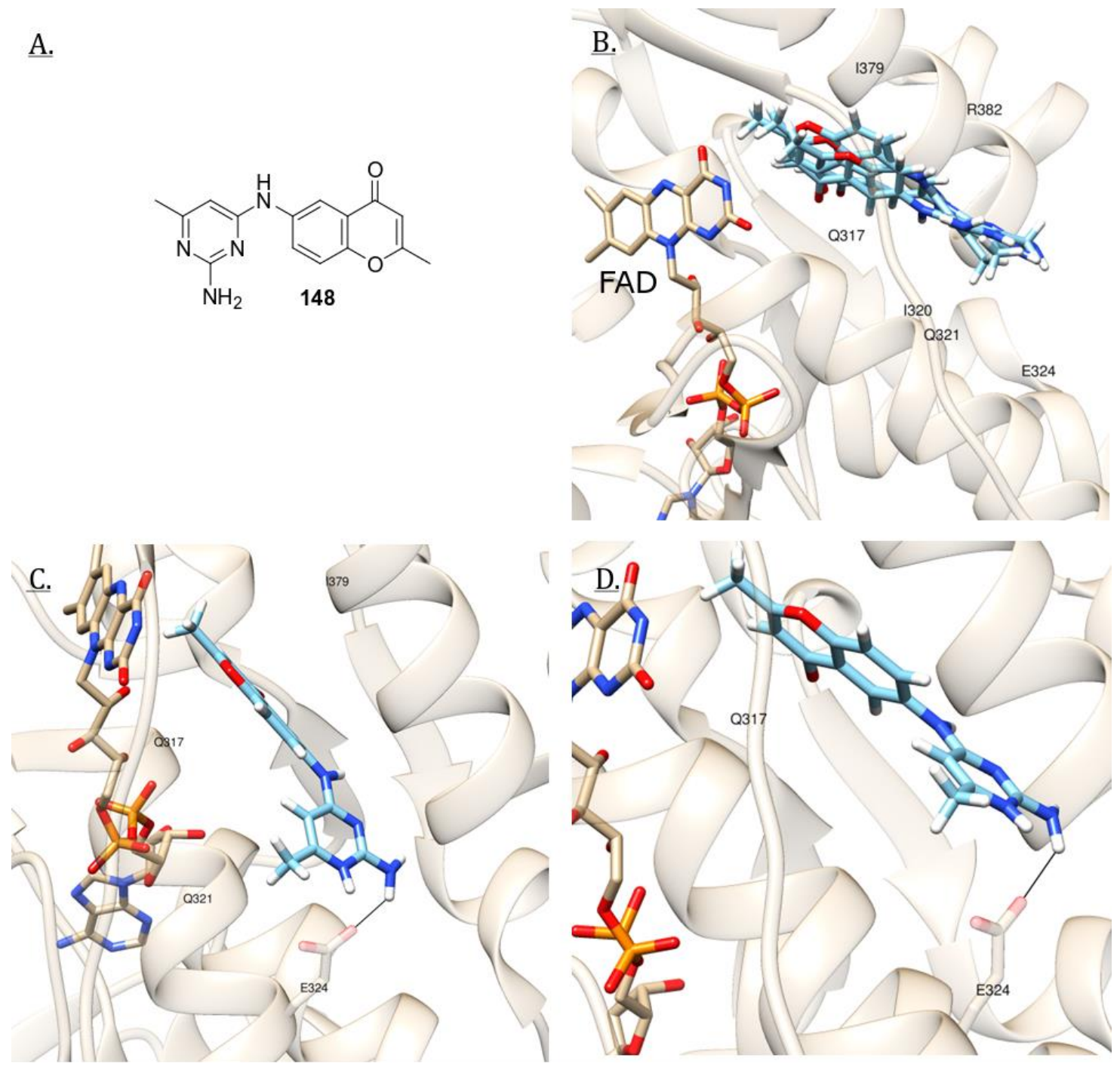

Figure 4.7: Docking studies of chromonyl-pyrimidine 148 in C. thermarum NDH-II (PDB: $\left.6 \mathrm{BDO}){ }^{62} \mathrm{~A}\right)$. Structure of 6-((2-amino-6-methylpyrimidin-4-yl)amino)-2-methyl-4Hchromen-4-one (148). B). Several binding modes of chromonyl-pyrimidine 148 (light blue) in the putative NDH-II Q-site are in close proximity to FAD (gold). C). The bicyclic chromone is sandwiched between amino acids Q317 and I379, which are important residues for quinone binding. D). There is a hydrogen bond (black line) between an amine proton of the pyrimidine ring and the oxygen of glutamic acid E324. Molecular graphics and analyses were performed with USCF Chimera, developed by the Resource for Biocomputing, Visualization, and Informatics at the University of California, San Francisco.

\subsubsection{The synthesis of functionalised pyrimidines}

The synthesis of functionalised chloropyrimidines commenced with the condensation of guanidine hydrochloride (137) and ethyl acetoacetate in the presence of sodium hydride to give

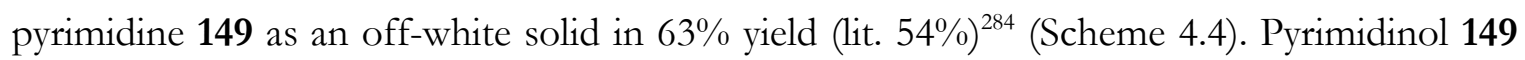
was then chlorinated under the agency of $\mathrm{POCl}_{3}$ to give 4-chloro-6-methylpyrimidine (150). Similarly, guanidine 137 was treated with ethyl 4-chloroacetoacetate to give both the 
hydroxymethyl- as well as the methoxymethyl-substituted pyrimidines $\mathbf{1 5 1}^{285}$ and $\mathbf{1 5 2}$, respectively, in an approximately 2:1 ratio and a high combined yield of $83 \%$ (53\% for 151, lit.: $\left.46 \%{ }^{285}\right)$. Hydroxypyrimidines 151 and 152 were readily separated by silica gel flash chromatography $\left(\mathrm{R}_{f}=0.37\right.$ and $0.53, \mathrm{CH}_{2} \mathrm{Cl}_{2} / \mathrm{MeOH} / \mathrm{EtOH} / \mathrm{NH}_{3}(25 \%), 5 / 2 / 2 / 1 \mathrm{v} / \mathrm{v} / \mathrm{v} / \mathrm{v}$, respectively) before they were treated with $\mathrm{POCl}_{3}$ to give the desired chloropyrimidines, $\mathbf{1 5 3}$ and $\mathbf{1 5 4}$, respectively. The spectral data obtained for pyrimidines $\mathbf{1 5 0}^{279}$ and $\mathbf{1 5 3}^{285}$ matched those reported in the literature, while the formation of chloride 154 was evidenced by NMR spectroscopy and HRMS data (calcd. for $\left[\mathrm{C}_{6} \mathrm{H}_{8} \mathrm{ClN}_{3} \mathrm{O}+\mathrm{H}\right]^{+}: 174.0429$, obsd.: 174.0429).<smiles>CCOC(=O)CC(C)=O</smiles>

137

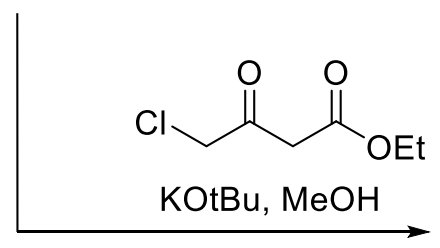

$83 \%, 2: 1,151: 152$<smiles>Cc1cc(O)nc(N)n1</smiles>

149<smiles>Nc1nc(O)cc(CO)n1</smiles>

151<smiles>Cc1cc(Cl)nc(N)n1</smiles>

150<smiles>COCc1cc(Cl)nc(N)n1</smiles>

Scheme 4.4: The synthesis of chloropyrimidines $150,^{279} \mathbf{1 5 3}^{285}$ and 154 .

\subsubsection{The synthesis of functionalised chromones}

To synthesise 2-substituted amino-chromones, 2'-hydroxyacetophenone (123) was first subjected to Kostanecki conditions to give ethyl ester 155, ${ }^{243,244}$ which was then nitrated to give 6-nitro chromone 156 in 90\% yield over the two steps (Scheme 4.5). ${ }^{286}$ Next, a Claisen 
condensation between acetophenone 123 and ethyl cyclopropanecarboxylate afforded chromone $157,{ }^{287}$ which was isolated in $74 \%$ yield. A subsequent nitration reaction then successfully gave cyclopropyl-substituted nitrochromone $\mathbf{1 5 8}$ in high yield.

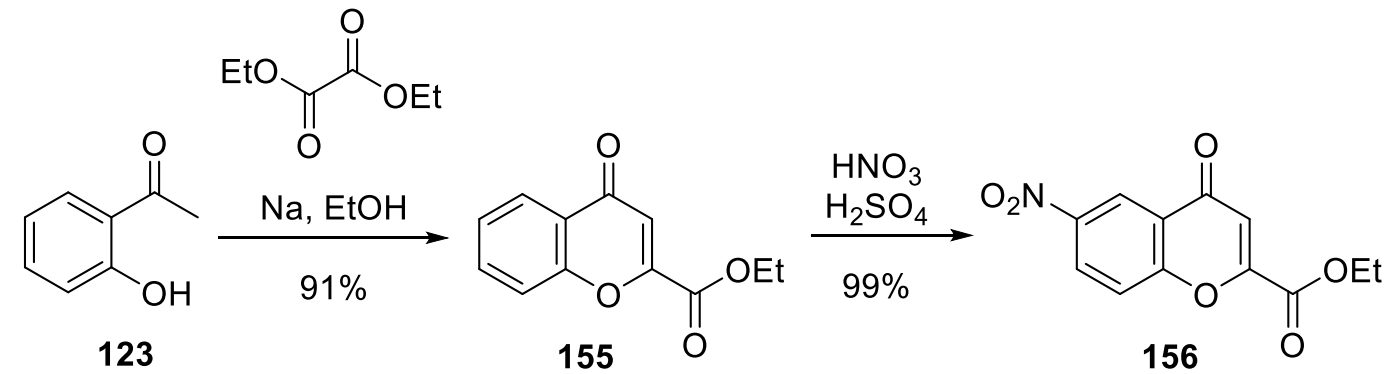

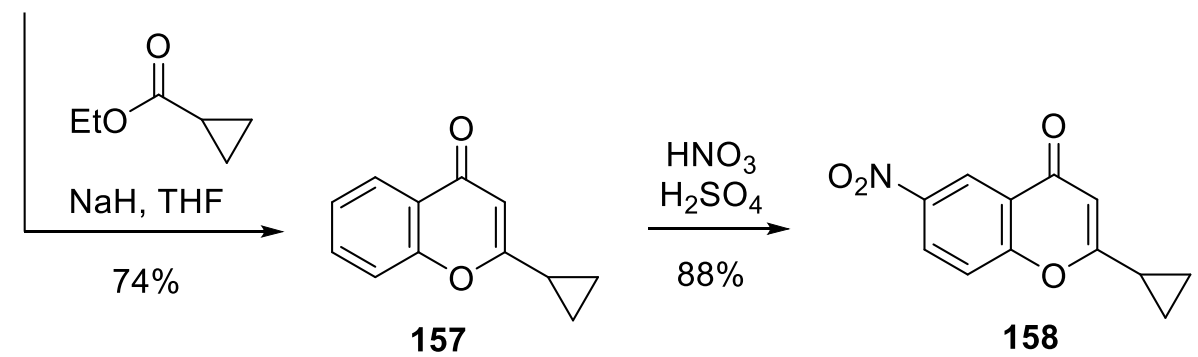

Scheme 4.5: The synthesis of nitro-chromones 156 and $158 .{ }^{286,287}$

Finally, in an attempt to synthesise 2-phenyl-chromone (159), 2'-hydroxyacetophenone (123) was first reacted with ethyl benzoate under basic conditions (Scheme 4.6). However, this reaction was unsuccessful, as evidenced by the recovery of starting material 123 and the isolation of benzoic acid as a by-product. Accordingly, it was decided that the ester carbonyl carbon was not sufficiently electrophilic to undergo nucleophilic attack with acetophenone $\mathbf{1 2 3}$ and, instead, benzaldehyde would react more readily with phenol 123 as the carbonyl carbon in an aldehyde group is more electron-deficient than that of an ester. Gratifyingly, the condensation of acetophenone $\mathbf{1 2 3}$ with benzaldehyde proceeded smoothly to yield chalcone 160, which underwent iodine-promoted oxidation to provide the desired phenylchromone 159 in good yield. ${ }^{288}$ Next, chromone 159 was subjected to nitration conditions. Unfortunately, mono-nitration was not possible as an inseparable 1:1 mixture of di-nitrochromones 161 and 162 was obtained even when milder conditions, such as lower temperatures and the use of weaker acids, were applied. 
<smiles>CC(=O)c1ccccc1O</smiles>

123

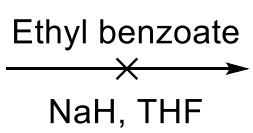

$\mathrm{NaH}, \mathrm{THF}$<smiles>C1CCCC1</smiles><smiles>O=c1cc(-c2ccccc2)oc2ccccc12</smiles>

Benzaldehyde $\mathrm{NaOH}, \mathrm{EtOH}$<smiles>O=C1CC(c2ccccc2)Oc2ccccc21</smiles>

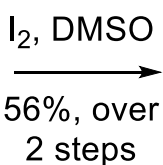<smiles>O=c1cc(-c2ccccc2)oc2ccccc12</smiles>

$\mathrm{HNO}_{3}$ $\mathrm{H}_{2} \mathrm{SO}_{4}$ or $\mathrm{AcOH}$ $65 \%, 1: 1,161 ; 162$<smiles>O=c1cc(-c2ccc([N+](=O)[O-])cc2)oc2ccc([N+](=O)[O-])cc12</smiles><smiles>O=c1cc(-c2cccc([N+](=O)[O-])c2)oc2ccc([N+](=O)[O-])cc12</smiles>

Scheme 4.6: The synthesis of phenylchromone 159 from acetophenone 123 and the subsequent nitration reaction to give structural isomers 161 and 162.

Structural isomers 161 and 162 were characterised using NMR spectroscopy, whereby the ${ }^{1} \mathrm{H}$ NMR spectrum showed multiciplity patterns corresponding to both meta- and para-substituted nitrophenyl groups (Figure 4.8). Specifically for $p$-nitrophenyl 161, proton resonances at 8.45 ppm and $8.15 \mathrm{ppm}\left(\mathrm{d}, J_{1^{\prime}, 2^{\prime}}=8.5 \mathrm{~Hz}\right)$ correlated to protons at ortho- $\left(\mathrm{H} 11^{\prime}\right)$ and meta- $\left(\mathrm{H} 2^{\prime}\right)$ positions with respect to the chromone scaffold, respectively. The proton resonances correlating to $m$-phenylchromone 162 were more complicated as each nitrophenyl proton occupied different chemical environments. Here, the proton resonances corresponding to $\mathrm{H}^{\prime}$ and $\mathrm{H} 4^{\prime}$ revealed ${ }^{4} J_{3^{\prime}, 4^{\prime}}$ and ${ }^{4} J_{3^{\prime}, 6^{\prime}}$ coupling constants of $2.1 \mathrm{~Hz}$ and $2.0 \mathrm{~Hz}$, respectively, which are typical of a long range $\mathrm{W}$ coupling. ${ }^{130}$ Moreover, coupling constants $3^{3} 4^{4}, 5^{\prime}$ and ${ }^{3} 5_{5^{\prime}, 6^{\prime}}$ for geminal protons $\mathrm{H} 4^{\prime} / \mathrm{H}^{\prime}$ and $\mathrm{H} 5^{\prime} / \mathrm{H}^{\prime}$ ' were observed at $8.2 \mathrm{~Hz}$ and $7.9 \mathrm{~Hz}$, respectively. ${ }^{130}$ Further evidence of the presence of the di-nitro chromones was observed by HRMS data (calcd. for $\left[\mathrm{C}_{15} \mathrm{H}_{8} \mathrm{~N}_{2} \mathrm{O}_{6}+\mathrm{H}\right]^{+}: 313.0455$, obsd.: 313.0453). 
<smiles>O=c1cc(-c2ccc([N+](=O)[O-])cc2)oc2ccc([N+](=O)[O-])cc12</smiles><smiles>O=c1cc(-c2cccc([N+](=O)[O-])c2)oc2ccc([N+](=O)[O-])cc12</smiles>

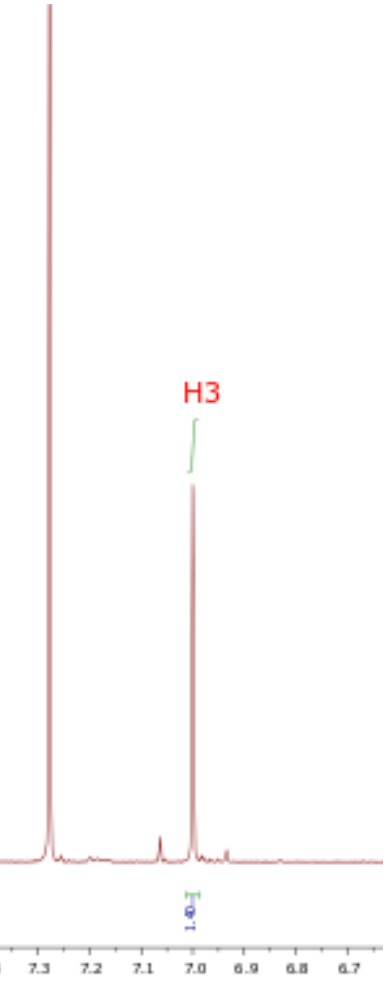

Figure 4.8: ${ }^{1} \mathrm{H}$ NMR $\left(500 \mathrm{MHz}, \mathrm{CDCl}_{3}\right)$ of a 1:1 mixture of structural isomers 161 and 162.

Consequently, it was decided that 2-phenyl-6-nitro chromone could be synthesised by first installing the nitro-substituent on 2'-hydroxyacetophenone (123, Scheme 4.7). Here, acetophenone 123 was treated with $\mathrm{HNO}_{3}$ and $\mathrm{AcOH}$ to give nitro-acetophenone 163 in 25\% yield (lit. 30\%), ${ }^{289}$ with 3'-nitro-acetophenone (164) as a minor (5\% yield) by-product. The structures of acetophenones 163 and 164 were confirmed through XRD data (Figure 4.9A and Figure 4.9B, respectively). The formation of structural isomers 163 and 164 occurred due to the meta-directing properties of the carbonyl functionality combined with the ortho- and paradirecting hydroxyl group, giving $\mathrm{C}^{\prime}$ and $\mathrm{C}^{\prime}$ as the most favoured positions for electrophilic aromatic substitution. ${ }^{290}$ However, the lack of steric hindrance at the 5 -position relative to the 3'-position led to 5'-nitro acetophenone 163 being the major product. Fortuitously, the regioisomers were readily separated using silica gel flash column chromatography, with the desired nitro-acetophenone $\mathbf{1 6 3}$ being eluted first $\left(\mathrm{R}_{f}\right.$ for $\mathbf{1 6 3}=0.54$ in toluene/EtOAc, 95/5, $\mathrm{v} / \mathrm{v} ; \mathrm{R}_{f}$ for $\mathbf{1 6 4}=0.24$ in toluene/EtOAc, 95/5, v/v). Next, nitro-acetophenone 163 was reacted with benzoyl chloride in the presence of $\mathrm{KOH}$ and then treated with acid under reflux to give phenylchromone 165 as the major product, ${ }^{291}$ along with diketone $\mathbf{1 6 6}$ as a minor byproduct. Both compounds were isolated and included in the final library of chromones. The 
spectral data obtained for phenylchromone 165 matched those reported in literature, ${ }^{291}$ while evidence for the formation of diketone $\mathbf{1 6 6}$ included the presence of two carbonyl carbon resonances at 194 and $175 \mathrm{ppm}$ in the ${ }^{13} \mathrm{C}$ NMR spectra, as well as HRMS data (calcd. for $\left[\mathrm{C}_{22} \mathrm{H}_{13} \mathrm{NO}_{5}+\mathrm{H}\right]^{+}:$372.0867, obsd.: 372.0865).<smiles>CC(=O)c1ccccc1O</smiles>

123

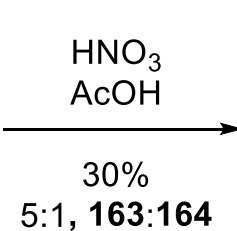

$5: 1,163: 164$<smiles>CC(=O)c1cc([N+](=O)[O-])ccc1O</smiles>
163<smiles>CC(=O)c1cccc([N+](=O)[O-])c1O</smiles>

164<smiles>O=c1cc(-c2ccccc2)oc2ccc([N+](=O)[O-])cc12</smiles>

165

1. Benzoyl chloride $\mathrm{KOH}, \mathrm{pyr}$

2. $\mathrm{H}_{2} \mathrm{SO}_{4}, \mathrm{AcOH}$

$\downarrow 75 \%, 15: 1,165: 166$

Scheme 4.7: The synthesis of phenyl chromone 165 
A.

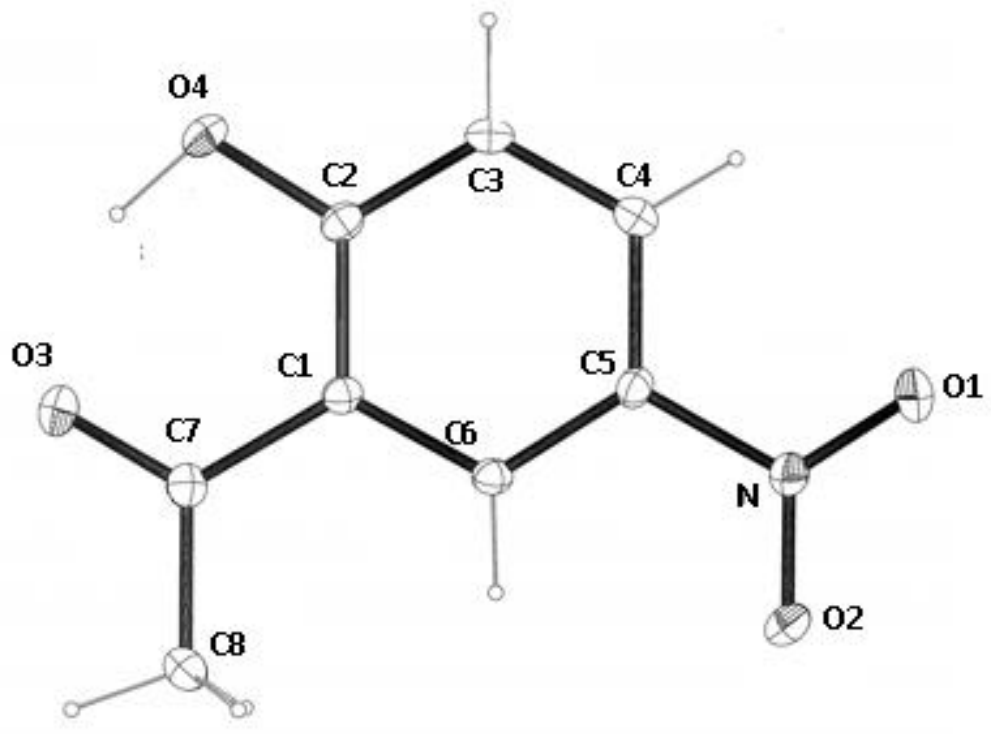

B.

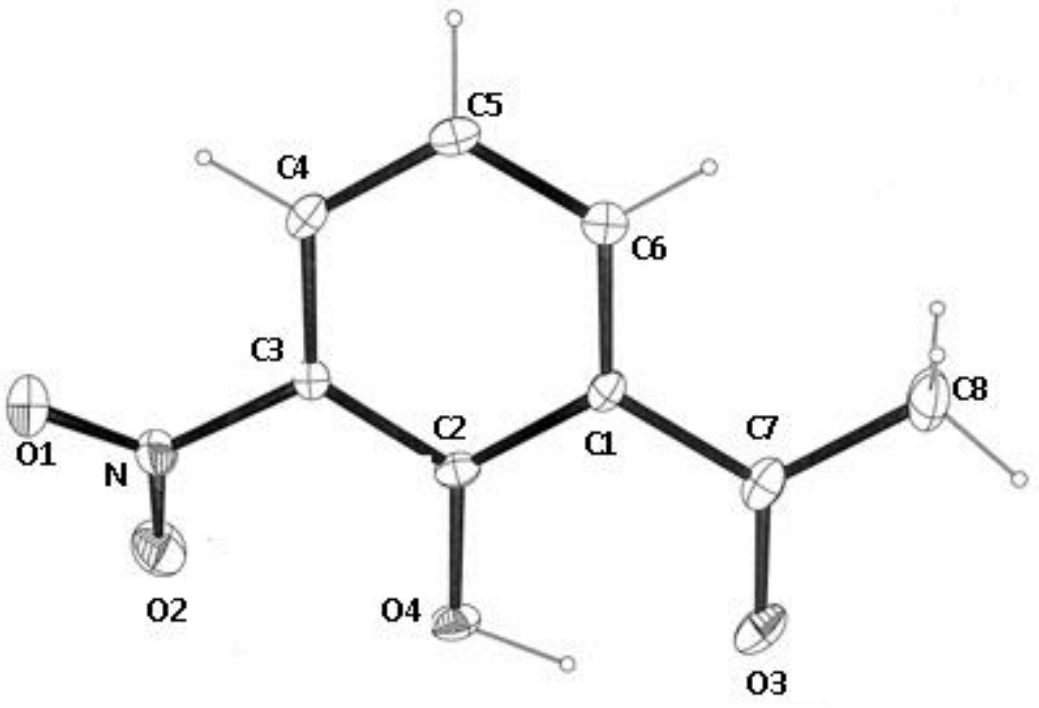

Figure 4.9: XRD crystal structures of nitro-acetophenones 163 (A) and 164 (B).

Initially, it was speculated that the formation of the diketone by-product 166 occurred due to the presence of excess benzoyl chloride from the previous step. Accordingly, ester 167 (Scheme 4.8) was purified using silica gel flash column chromatography prior to acid treatment. Notwithstanding, the formation of by-product 166 occurred even in the absence of benzoyl chloride, albeit in a lower yield, along with the recovery of acetophenone 163 . Thus, it was postulated that upon the treatment of ester 167 with $\mathrm{H}_{2} \mathrm{SO}_{4}$ and $\mathrm{AcOH}$, some ester hydrolysis occurred in situ and this allowed for the addition of the phenyl carbonyl group at the $\alpha$-carbon of enolate XIX (Scheme 4.8). Next, elimination of water from ketone $\mathbf{X X}$ leading to the formation of diketone XXI and, subsequently, enol XXII was proposed, with enol XXII undergoing an intramolecular aldol-Claisen to give chalcone XXIII. E1 reaction would then 
yield by-product 166. To corroborate this mechanism, the second step of this reaction was undertaken in the absence of $\mathrm{H}_{2} \mathrm{O}$ to yield phenyl-chromone 165 selectively in 65\% yield.

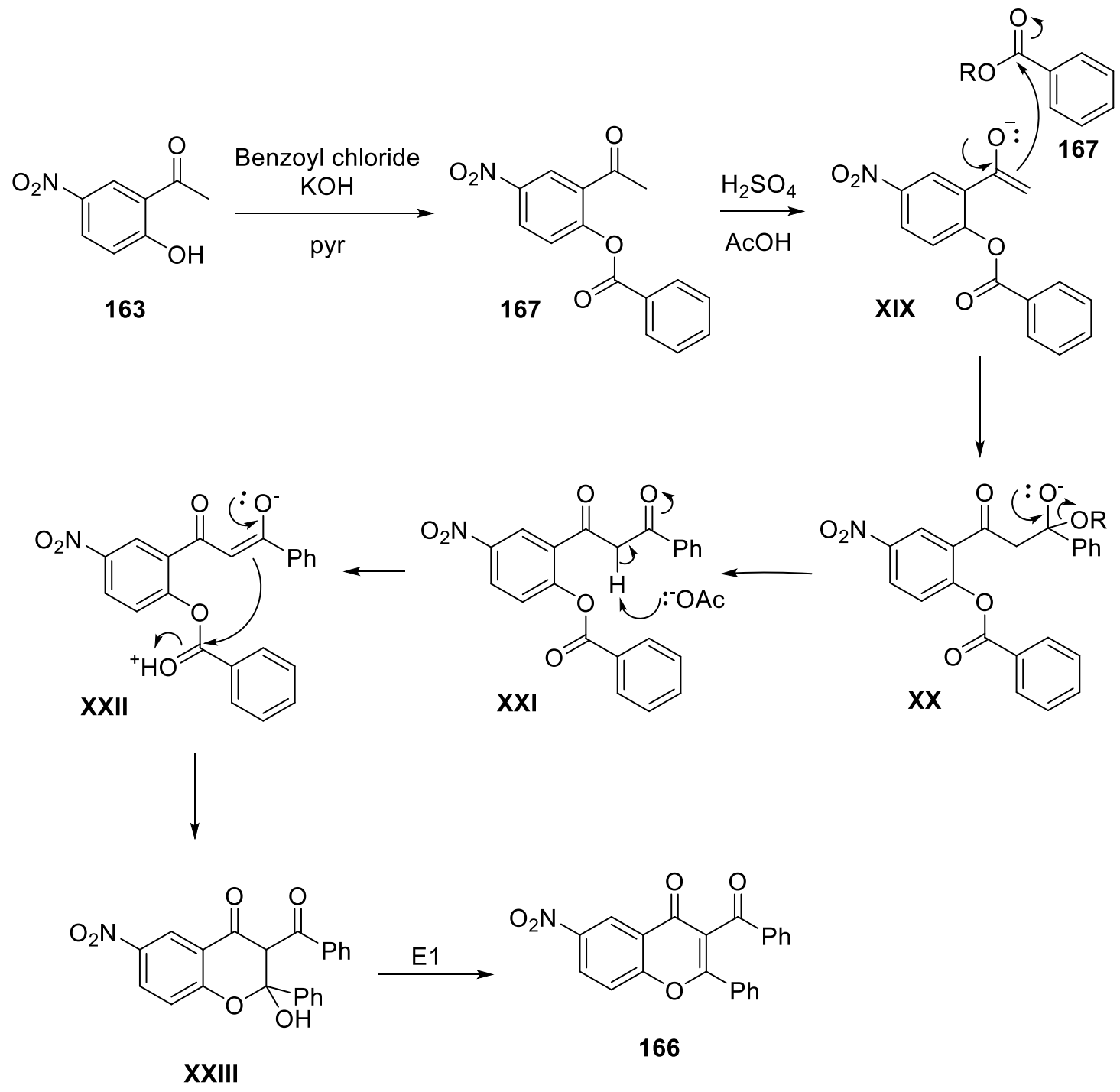

Scheme 4.8: The mechanism of by-product 166 formation.

Finally, the iron-mediated reduction of nitrochromones 156, 158, 165, and 166 was undertaken $^{292}$ to give amines $\mathbf{1 6 8 - 1 7 1}$ in good yields (49\% - 93\%) from their respective nitrosubstituted precursors (Scheme 4.9). Evidence for the successful reduction of the nitro group was obtained by IR spectroscopy, whereby N-H stretching frequencies at approximately 3400 $\mathrm{cm}^{-1}{ }^{130}$ and the loss of $\mathrm{NO}_{2}$ stretches (asymmetric stretches: $1550-1500 \mathrm{~cm}^{-1}$ and symmetric stretches: $1360-1290 \mathrm{~cm}^{-1}$ ) were observed. ${ }^{130}$ Moreover, broad singlets at appoximately $4.0 \mathrm{ppm}$ in $\mathrm{CDCl}_{3}$ that were associated with amine protons were observed by ${ }^{1} \mathrm{H}$ NMR spectroscopy. 
<smiles>[R]c1oc2ccc([N+](=O)[O-])cc2c(=O)c1[R]</smiles>

156, $\mathrm{R}^{1}=\mathrm{H}, \mathrm{R}^{2}=\mathrm{CO}_{2} \mathrm{Et}$

158, $\mathrm{R}^{1}=\mathrm{H}, \mathrm{R}^{2}=\mathrm{C}_{3} \mathrm{H}_{5}$

165, $R^{1}=H, R^{2}=P h$

166, $R^{1}=$ COPh, $R^{2}=P h$<smiles>[R]c1oc2ccc(N)cc2c(=O)c1[R]</smiles>

168, $\mathrm{R}^{1}=\mathrm{H}, \mathrm{R}^{2}=\mathrm{CO}_{2} \mathrm{Et}, 73 \%$

169, $\mathrm{R}^{1}=\mathrm{H}, \mathrm{R}^{2}=\mathrm{C}_{3} \mathrm{H}_{5}, 51 \%$

170, $R^{1}=H, R^{2}=P h, 49 \%$

$171, R^{1}=C O P h, R^{2}=P h, 93 \%$

Scheme 4.9: Reduction of nitrochromones $156,158,165$ and 166 with iron to give amines 168 171.

\subsubsection{The synthesis of chromonyl-pyrimidines}

Having prepared functionalised chloropyrimidines 150, 153, and 154 and aminochromones 168-171, the assembly of the chromonyl-pyrimidines was undertaken. Accordingly, chloropyrimidines 150, 153 and 154 were subjected to nucleophilic substitution by aminochromones 168-171 under acidic conditions to give chromonyl-pyrimidines 172-181 in good yields (Scheme 4.10). Here, the substitution reactions were performed in anhydrous conditions under an argon atmosphere to avoid acid-catalysed hydrolysis of chloropyrimidines 150,153 , and 154 to their respective pyrimidinols $(149,151,152$, respectively). Subsequently, ethyl ester 179 was subjected to hydrolysis to afford carboxylic acid $\mathbf{1 8 2}$ in $90 \%$ yield (Scheme 4.11). 
<smiles>[R]c1cc(Cl)nc(N)n1</smiles>

150, $\mathrm{R}^{1}=\mathrm{CH}_{3}$

153, $\mathrm{R}^{1}=\mathrm{CH}_{2} \mathrm{Cl}$

154, $\mathrm{R}^{1}=\mathrm{CH}_{2} \mathrm{OCH}_{3}$<smiles>[R]c1oc2ccc(N)cc2c(=O)c1[R]</smiles>

168, $\mathrm{R}^{2}=\mathrm{H}, \mathrm{R}^{3}=\mathrm{CO}_{2} \mathrm{Et}$

169, $\mathrm{R}^{2}=\mathrm{H}, \mathrm{R}^{3}=\mathrm{C}_{3} \mathrm{H}_{5}$

$170, R^{2}=H, R^{3}=P h$

$171, R^{2}=$ COPh, $R^{3}=P h$

$\mathrm{Cl}$ or $\mathrm{HBF}_{4}$

1,4-dioxane<smiles>[R]c1cc(Nc2ccc3oc([R])c([R])c(=O)c3c2)nc(N)n1</smiles>

172, $\mathrm{R}^{1}=\mathrm{CH}_{3}, \mathrm{R}^{2}=\mathrm{H}, \mathrm{R}^{3}=\mathrm{CO}_{2} \mathrm{Et}, 87 \%$

173, $\mathrm{R}^{1}=\mathrm{CH}_{3}, \mathrm{R}^{2}=\mathrm{H}, \mathrm{R}^{3}=\mathrm{C}_{3} \mathrm{H}_{5}, 39 \%$

174, $\mathrm{R}^{1}=\mathrm{CH}_{3}, \mathrm{R}^{2}=\mathrm{H}, \mathrm{R}^{3}=\mathrm{Ph}, 44 \%$

175, $\mathrm{R}^{1}=\mathrm{CH}_{3}, \mathrm{R}^{2}=\mathrm{COPh}, \mathrm{R}^{3}=\mathrm{Ph}, 20 \%$

176, $\mathrm{R}^{1}=\mathrm{CH}_{2} \mathrm{Cl}, \mathrm{R}^{2}=\mathrm{H}, \mathrm{R}^{3}=\mathrm{CO}_{2} \mathrm{Et}, 61 \%$

177, $\mathrm{R}^{1}=\mathrm{CH}_{2} \mathrm{Cl}, \mathrm{R}^{2}=\mathrm{H}, \mathrm{R}^{3}=\mathrm{C}_{3} \mathrm{H}_{5}, 40 \%$

178, $\mathrm{R}^{1}=\mathrm{CH}_{2} \mathrm{Cl}, \mathrm{R}^{2}=\mathrm{H}, \mathrm{R}^{3}=\mathrm{Ph}, 71 \%$

179, $\mathrm{R}^{1}=\mathrm{CH}_{2} \mathrm{OCH}_{3}, \mathrm{R}^{2}=\mathrm{H}, \mathrm{R}^{3}=\mathrm{CO}_{2} \mathrm{Et}, 54 \%$

180, $\mathrm{R}^{1}=\mathrm{CH}_{2} \mathrm{OCH}_{3}, \mathrm{R}^{2}=\mathrm{H}, \mathrm{R}^{3}=\mathrm{C}_{3} \mathrm{H}_{5}, 70 \%$

181, $\mathrm{R}^{1}=\mathrm{CH}_{2} \mathrm{OCH}_{3}, \mathrm{R}^{2}=\mathrm{H}, \mathrm{R}^{3}=\mathrm{Ph}, 58 \%$

Scheme 4.10: Synthesis of chromonyl-pyrimidines 172-181<smiles>CCOC(=O)c1cc(=O)c2cc(Nc3cc(COC)nc(N)n3)ccc2o1</smiles>

179

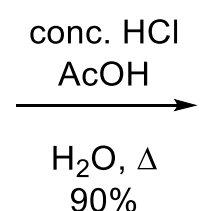<smiles>COCc1cc(Nc2ccc3oc(C(=O)O)cc(=O)c3c2)nc(N)n1</smiles>

Scheme 4.11: The hydrolysis of $\mathbf{1 7 9}$ to give acid $\mathbf{1 8 2 .}$

The successful synthesis of chromonyl-pyrimidines $172-182$ was demonstrated by NMR analysis, whereby the ${ }^{1} \mathrm{H}$ NMR spectra revealed a broad singlet at approximately $10 \mathrm{ppm}$, corresponding to a secondary amine proton. Moreover, HMBCs between the secondary amine 
proton and carbons at the 4"-, 5- and 7-positions were observed, as illustrated in Figure 4.10 with chromonyl-pyrimidine 172, thereby demonstrating that successful coupling between the chloropyrimidine and the amino-chromone has taken place.

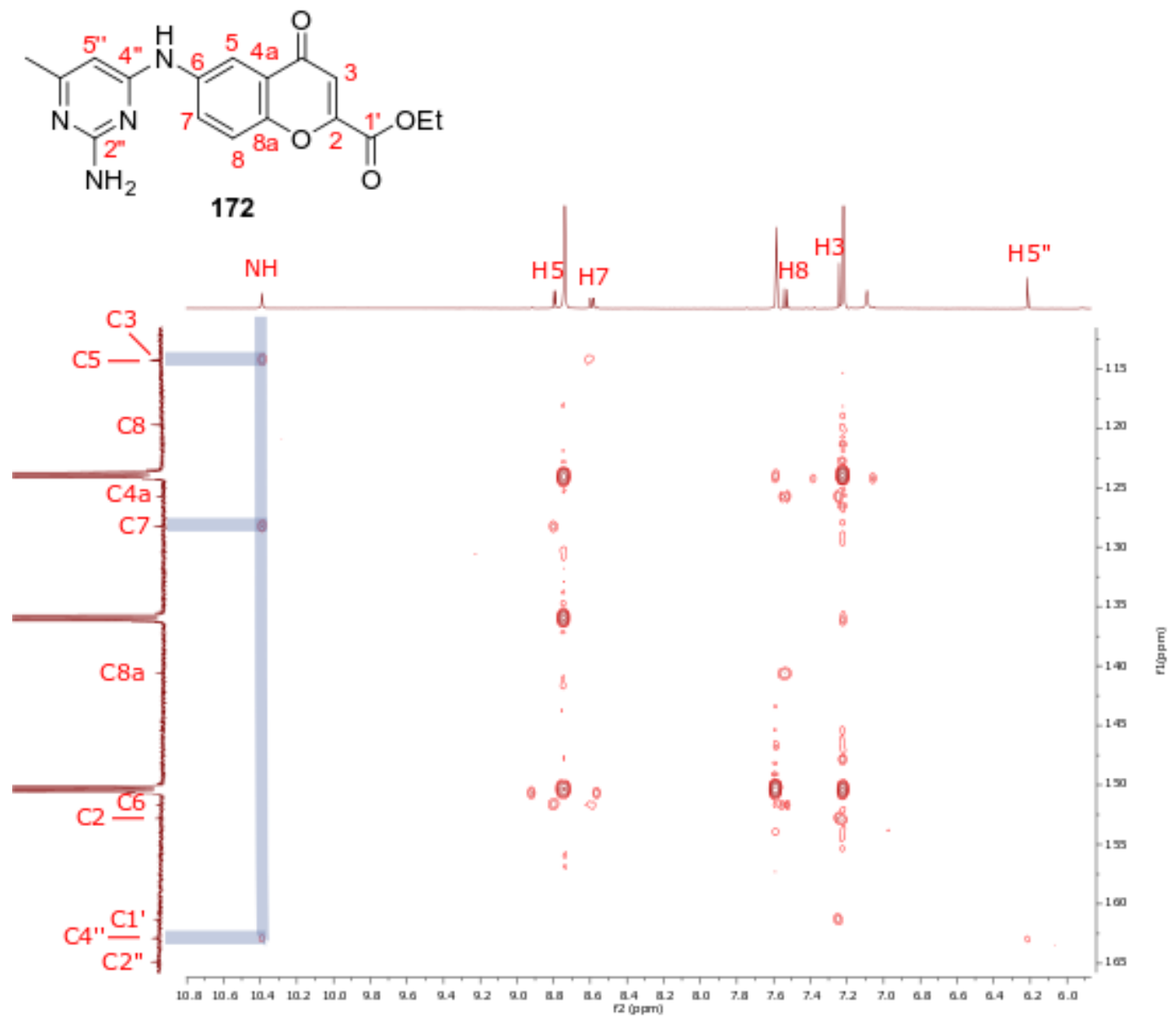

Figure 4.10: HMBC (500 MHz, pyridine- $\mathrm{d}_{5}$ ) of chromonyl pyrimidine 172.

In the synthesis of cyclopropyl-substituted chromonyl-pyrimidines $173,177,180, \mathrm{HBF}_{4}$ was used instead of $\mathrm{HCl}$ to avoid nucleophilic cyclopropyl ring-opening by chloride ions. This side reaction was observed during the $\mathrm{HCl}$-mediated synthesis of methyl-substituted chromonyl pyrimidine 173, which resulted in the isolation of chloropropylchromone 183 (Scheme 4.12) and the observation of the coupled product between chromone $\mathbf{1 8 3}$ and methylpyrimidine $\mathbf{1 5 0}$ by HRMS ( $m /$ z calcd. for $\left[\mathrm{C}_{17} \mathrm{H}_{17} \mathrm{ClN}_{4} \mathrm{O}_{2}+\mathrm{H}\right]^{+}: 345.1113$, obsd.: 345.1112$)$. Here, different regioisomers of chloropropyl 183 could be produced by nucleophilic attack at either the 1'- or 2'-positions, however, attack at the 2'-position was more likely to occur as C2' is less sterically hindered compared to $\mathrm{C} 1$ '. Moreover, conjugate addition of the chloride anion was favoured due to the stabilisation of the resulting negative charge through resonance structures of the 
$\alpha, \beta$-unsaturated ketone (i.e. XXV), giving primary chloride $\mathbf{1 8 3}$ as the addition product. Evidence for the formation of primary chloride 183 was observed in the ${ }^{1} \mathrm{H}$ NMR and COSY spectra, whereby three methylene protons at $3.63 \mathrm{ppm}\left(\mathrm{t}, J_{1^{\prime}, 2^{\prime}}=6.3 \mathrm{~Hz}, \mathrm{H} 1^{\prime}\right), 2.80 \mathrm{ppm}\left(\mathrm{t}, J_{2^{\prime}, 3^{\prime}}\right.$ $\left.=7.7 \mathrm{~Hz}, \mathrm{H} 3^{\prime}\right)$, and $2.22 \mathrm{ppm}\left(\mathrm{m}, \mathrm{H} 2^{\prime}\right)$ were shown to correlate to each other.

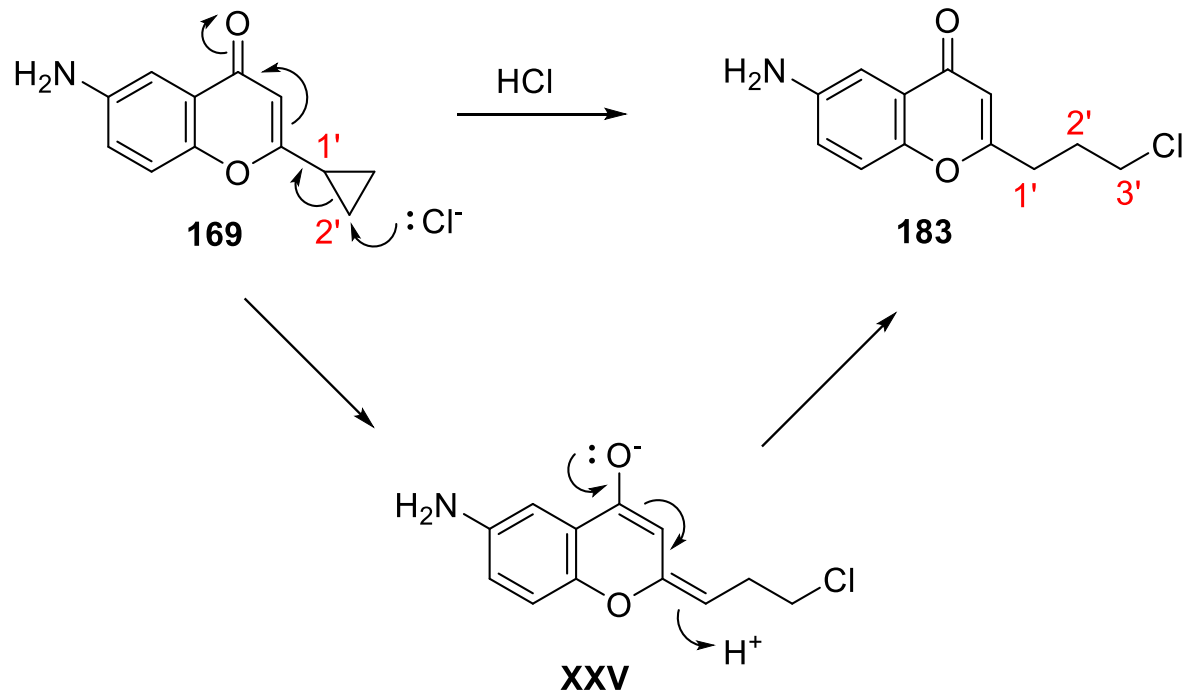

Scheme 4.12: The side-reaction of amino-chromone 169 with $\mathrm{HCl}$ to give by-product 183 via intermediate $\mathbf{X X V}$.

\subsubsection{Anti-tubercular activity of chromonyl-pyrimidines}

To investigate the anti-tubercular activity of chromonyl-pyrimidines 172-182, the compounds were screened for their ability to inhibit $M t b$ by the Cook laboratory at the University of Otago (Table 4.1). Here, all chromonyl-pyrimidines apart from methoxy-methyl-substituted compounds 179 and 182 (Entries 1 and 2) inhibited Mtb growth. Notably, chromonylpyrimidines bearing a methoxymethyl group at the 6"-position had relatively high MIC values (Entries 1-3), though cyclopropyl 180 exhibited better inhibitory activity (MIC $=95 \mu \mathrm{M}$, Entry 4). The instalment of a chloromethyl group, as in chromonyl-pyrimidines 176 and $\mathbf{1 7 8}$, generally resulted in greater inhibition of $\mathrm{Mtb}(\mathrm{MIC}=171 \mu \mathrm{M}$ and $337 \mu \mathrm{M}$, Entries 5 and 6 , respectively) compared to their corresponding methoxymethyl-substituted chromonylpyrimidines 179, 181 (MIC >1382 $\mu \mathrm{M}$ and $684 \mu \mathrm{M}$, Entries 1 and 3, respectively), though this trend was not observed with the cyclopropyl derivatives (MIC for $\mathbf{1 7 7}=186 \mu \mathrm{M}$, Entry 7, vs. MIC for $\mathbf{1 8 0}=95 \mu \mathrm{M}$, Entry 4). Overall however, the methyl-substituted pyrimidines 172-175 were the most effective inhibitors of mycobacterial growth (Entries 8-11), with 
phenylchromones 174 and 175 giving the lowest MIC values of the library (MIC $=46 \mu \mathrm{M}$ and $36 \mu \mathrm{M}$, Entries 10 and 11, respectively).

Table 4.1: MIC values $(\mu \mathrm{g} / \mathrm{mL}$ and $\mu \mathrm{M})$ of chromonylpyrimidines 172-182 against $M t b$.
Entry
Compound
$\operatorname{MIC}(\mu \mathrm{g} / \mathrm{mL})$
$\operatorname{MIC}(\mu \mathrm{M})$

2<smiles>CCOC(=O)c1cc(=O)c2cc(Nc3cc(COC)nc(N)n3)ccc2o1</smiles>

3<smiles>COCc1cc(Nc2ccc3oc(C(=O)O)cc(=O)c3c2)nc(N)n1</smiles>

4<smiles>COCc1cc(Nc2ccc3oc(-c4ccccc4)cc(=O)c3c2)nc(N)n1</smiles><smiles>COCc1cc(Nc2ccc3oc(C4CC4)cc(=O)c3c2)nc(N)n1</smiles><smiles>CCOC(=O)c1cc(=O)c2cc(Nc3cc(CCl)nc(N)n3)ccc2o1</smiles>

6<smiles>Nc1nc(CCl)cc(Nc2ccc3oc(-c4ccccc4)cc(=O)c3c2)n1</smiles><smiles>Nc1nc(CCl)cc(Nc2ccc3oc(C4CC4)cc(=O)c3c2)n1</smiles> 
8<smiles>[Y7]C(F)(F)c1cc(=O)c2cc(Nc3cc(C)nc(N)n3)ccc2o1</smiles>

9<smiles>Cc1cc(Nc2ccc3oc(C4CC4)cc(=O)c3c2)nc(N)n1</smiles>

10<smiles>Cc1cc(Nc2ccc3oc(-c4ccccc4)cc(=O)c3c2)nc(N)n1</smiles>

11

Next, the effect of different substituents at the 2-position of chromonyl-pyrimidines on their ability to inhibit $M t b$ growth was analysed. Here, the addition of carbonyl groups, as in esters 179, 176, 172 and acid 182 (Entries 1, 5, 8 and 2, respectively), resulted in reduced potency against $M t b$ compared to the corresponding cyclopropyl- and phenyl-substituted compounds across all derivatives. It was postulated that the esters may undergo hydrolysis to form carboxylic acids. Indeed, both ester $\mathbf{1 7 9}$ and its hydrolysed derivative 182, were inactive against Mtb (Entries 1 and 2). Although only speculation, a carboxylic acid group could perhaps participate in acid-base interactions away from the putative Q-site, i.e. Q321 in NDH-II.

Compared to the respective ester- and phenyl-substituted derivatives, chromonyl-pyrimidines bearing a cyclopropyl group $(\mathbf{1 8 0}, \mathbf{1 7 7}, \mathbf{1 7 3}$, Entries 4, 7, and 9, respectively) elicited good inhibitory activity against $M t b$, with methoxymethyl-pyrimidine $\mathbf{1 8 0}$ leading to a particularly low MIC of $95 \mu \mathrm{M}$. Finally, the instalment of phenyl substituents at the 2-position led to contrasting results, where reduced potency was exhibited by chloromethyl- (181) and methoxymethyl-substituted (178) analogues $(\mathrm{MIC}=684 \mu \mathrm{M}$ and $337 \mu \mathrm{M}$, Entries 3 and 7, respectively), while methyl-substituted chromonyl-pyrimidines 174 and 175 were the lead compounds of the library. 
Taken together, chromonyl-pyrimidines with a methyl substituent at the 6"-position led to the lowest MIC values compared to those bearing a chloromethyl or methoxymethyl group at this position. This anti-tubercular activity could potentially be attributed to the hydrophobic methyl substituent, which, in docking studies of methyl-pyrimidine 148, could be accommodated in a hydrophobic groove in the NDH-II Q-site. In addition, installing a phenyl group at the 2position of chromonyl-pyrimidines resulted in high anti-tubercular activity. The presence of an aromatic substituent at this position could lead to favourable $\pi-\pi$ interactions with the FAD isoalloxazine ring within NDH-II. These results provided information for the development of a second generation of chromonyl pyrimidines.

\subsubsection{The synthesis of a second generation of chromonyl pyrimidines}

Having established the SAR of chromonyl-pyrimidines 172-182, a second library of chromonyl-pyrimidines was prepared to further explore the pharmacophore of this class of compound. In light of the high anti-tubercular activity of methyl-pyrimidinyl phenylchromones 174 and 175, it was proposed that methyl, phenyl and $p$-fluorophenyl groups would be installed at the $6 "$ "-position so as to probe potential $\pi-\pi$ interactions within the target enzyme active site. Moreover, the electron-withdrawing chloromethyl group in the initial library of chromonyl pyrimidines showed modest anti-tubercular activity and the instalment of a fluorophenyl substituent on the pyrimidine ring could be used to investigate the effect of a less polar electron-withdrawing group on anti-tubercular activity. The effect of a phenyl substituent at the 2-position will be explored by the introduction of strong electron-withdrawing and electron-donating groups at the para-position of the phenyl substituent.

To this end, chromonyl pyrimidines bearing a methyl, phenyl, and $p$-fluorophenyl groups at the 6"-position and a nitrophenyl, aminophenyl and phenyl substituents at the 2-position were synthesised. First, guanidine carbonate (137) was treated with ethyl benzoylacetate $(\mathbf{1 8 3})^{293}$ and ethyl 4-fluorobenzoylacetate (184) to give hydroxypyrimidines 185 and 186, respectively, which were readily converted to chloropyrimidines $187^{294}$ and 188 , respectively (Scheme 4.13). 
<smiles>N=C(N)N</smiles>

137<smiles>[R]c1cc(Cl)nc(N)n1</smiles>

187, $\mathrm{R}=\mathrm{Ph}, 55 \%$

$188, \mathrm{R}=4-\mathrm{F}-\mathrm{Ph}, 62 \%$<smiles>COC(C)C</smiles><smiles>[R]C(=O)CC(=O)OCC</smiles>

183, $\mathrm{R}=\mathrm{Ph}$

184, $\mathrm{R}=4-\mathrm{F}-\mathrm{Ph}$<smiles>CC(O)O</smiles><smiles>[R]c1cc(O)nc(N)n1</smiles>

185, $\mathrm{R}=\mathrm{Ph}, 75 \%$

186, $R=4-F-P h, 84 \%$

Scheme 4.13: The synthesis of phenyl- (187 $)^{294}$ and fluorophenyl pyrimidines (188).

With chloropyrimidines 187 and 188 in hand, the synthesis of the aryl chromone 192 was undertaken starting with nitro-acetophenone 163 (Scheme 4.14). As target chromone 192 bears a nitrophenyl group, it was envisaged that the amine functionality would be introduced at the beginning of the synthesis. In addition, the electron-donating properties of the amine substituent in acetophenone $\mathbf{1 8 9}$ would increase the nucleophilicity of the phenolic oxygen and expedite subsequent esterification reactions. Accordingly, nitro-acetophenone 163 was reduced under the agency of iron powder to form amino-acetophenone 189, which was then $\mathrm{N}$-protected as Boc-amidoacetophenone 190 in 35\% yield over two steps. Next, acetophenone 190 was esterified with 4-nitro benzoic acid in the presence of coupling reagents EDCI and DMAP $^{295}$ to afford ester 191, which readily underwent a Baker-Venkataraman rearrangement ${ }^{295-297}$ to give the desired chromone 192. 
<smiles>CC(=O)c1cc([N+](=O)[O-])ccc1O</smiles>

163

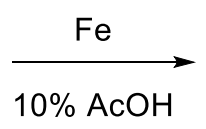

$39 \%$<smiles>CC(=O)c1cc(N)ccc1O</smiles>

189

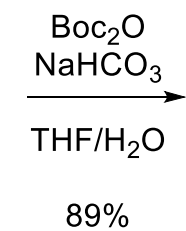<smiles>CC(=O)c1cc(NC(=O)OCc2ccccc2)ccc1O</smiles><smiles>O=C(O)c1ccc([N+](=O)[O-])cc1</smiles><smiles>CC(=O)c1cc(NC(=O)OCc2ccc([N+](=O)[O-])cc2)ccc1OC(=O)c1ccc(-c2cc(=O)c3cc(N)ccc3o2)cc1</smiles>

Scheme 4.14: The synthesis of nitro-phenyl chromone 192 from acetophenone 163.

Having synthesised the necessary building blocks, the assembly of the second library of chromonyl-pyrimidines was undertaken. As with the first library of chromonyl pyrimidines, the substitution reactions between chloropyrimidines 150,188 , and 189 and aminochromones 170 and 192 were performed under acidic conditions to give the final compounds in good yields (43\%-73\%, Scheme 4.15). Again, evidence for the successful synthesis of chromonylpyrimidines was provided by NMR spectroscopy, including an HMBC of the bridging amine proton with protons at the 5"-, 5- and 7-positions. In addition to these chromonyl-pyrimidines, nitro-phenylchromones 193 and 198 were reduced to give aminophenyl-substituted chromonyl-pyrimidines 194 and 199 as yellow solids. Here, the presence of an amine group was confirmed by IR spectroscopy $\left(3349 \mathrm{~cm}^{-1}\right.$ and $3310 \mathrm{~cm}^{-1}$ for chromonyl-pyrimidines 194 and 199, respectively) and HRMS (calcd. for $\left[\mathrm{C}_{20} \mathrm{H}_{17} \mathrm{~N}_{5} \mathrm{O}_{2}+\mathrm{H}\right]^{+}: 360.1455$, obsd.: 360.1455 , and calcd. for $\left[\mathrm{C}_{25} \mathrm{H}_{18} \mathrm{FN}_{5} \mathrm{O}_{2}+\mathrm{H}\right]^{+}: 440.1517$, obsd.: 440.1519). 
<smiles>[R]c1cc(Cl)nc(N)n1</smiles>

150, $\mathrm{R}^{1}=\mathrm{CH}_{3}$

188, $R^{1}=P h$

$189, R^{1}=4-F-P h$<smiles>[R]c1cc(=O)c2cc(N)ccc2o1</smiles>

$170, \mathrm{R}^{2}=\mathrm{Ph}$

192, $\mathrm{R}^{2}=4-\mathrm{NO}_{2}-\mathrm{Ph}$

conc. $\mathrm{HCl}$

2-ethoxy-ethanol<smiles>[R]c1cc(Nc2ccc3oc([R])cc(=O)c3c2)nc(N)n1</smiles>

$$
\begin{aligned}
\mathrm{Fe}-193, \mathrm{R}^{1}=\mathrm{CH}_{3}, \mathrm{R}^{2}=4-\mathrm{NO}_{2}-\mathrm{Ph}, 43 \% \\
10 \% \text { AcOH } \rightarrow 194, \mathrm{R}^{1}=\mathrm{CH}_{3}, \mathrm{R}^{2}=4-\mathrm{NH}_{2}-\mathrm{Ph}, 33 \% \\
195, \mathrm{R}^{1}=\mathrm{Ph}, \mathrm{R}^{2}=\mathrm{Ph}, 58 \% \\
196, \mathrm{R}^{1}=\mathrm{Ph}, \mathrm{R}^{2}=4-\mathrm{NO}_{2}-\mathrm{Ph}, 54 \% \\
197, \mathrm{R}^{1}=4-\mathrm{F}-\mathrm{Ph}, \mathrm{R}^{2}=\mathrm{Ph}, 63 \% \\
\mathrm{Fe} \longrightarrow 198, \mathrm{R}^{1}=4-\mathrm{F}-\mathrm{Ph}, \mathrm{R}^{2}=4-\mathrm{NO}_{2}-\mathrm{Ph}, 73 \% \\
10 \% \text { AcOH } \rightarrow 199, \mathrm{R}^{1}=4-\mathrm{F}-\mathrm{Ph}, \mathrm{R}^{2}=4-\mathrm{NH}_{2}-\mathrm{Ph}, 42 \%
\end{aligned}
$$

Scheme 4.15: The synthesis of chromonyl pyrimidines 193-199.

\subsubsection{Anti-tubercular activity of a second library of chromonyl-pyrimidines}

Having successfully prepared chromonyl-pyrimidines 193-199, biological screening of the synthesised compounds for inhibitory activity against $M t b$ was undertaken under the supervision of Professor Greg Cook at the University of Otago. The MIC values of each chromonyl-pyrimidine against $M t b$ are reported in Table 4.2.

Table 4.2: MIC values $(\mu \mathrm{M})$ of chromonyl-pyrimidines 193-199.

Entry

Compound

$\operatorname{MIC}(\mu \mathrm{M})$

1<smiles>Cc1cc(Nc2ccc3oc(-c4ccc([N+](=O)[O-])cc4)cc(=O)c3c2)nc(N)n1</smiles> 
<smiles>Cc1cc(Nc2ccc3oc(-c4ccc(N)cc4)cc(=O)c3c2)nc(N)n1</smiles><smiles>Nc1nc(Nc2ccc3oc(-c4ccccc4)cc(=O)c3c2)cc(-c2ccccc2)n1</smiles>

4<smiles>Nc1cc([N+](=O)[O-])cc(Nc2ccc3oc(-c4ccccc4)cc(=O)c3c2)n1</smiles>

5<smiles>Nc1nc(Nc2ccc3oc(-c4ccccc4)cc(=O)c3c2)cc(-c2ccc(F)cc2)n1</smiles>

6<smiles>Nc1nc(Nc2ccc3oc(-c4ccc(F)cc4)cc(=O)c3c2)ccc1[N+](=O)[O-]</smiles><smiles>Nc1ccc(-c2cc(=O)c3cc(Nc4cc(-c5ccc(F)cc5)nc(N)n4)ccc3o2)cc1</smiles>

In comparison to the first library of chromonyl pyrimidines (172-182, Table 4.1), the second generation of chromonyl pyrimidines (193-199, Table 4.2) generally exhibited higher antitubercular activity. While methyl-substituted nitrophenyl 193 (MIC $=100 \mu \mathrm{M}$, Entry 1) was less potent against $M t b$ compared to the previous lead compound $174(\mathrm{MIC}=46 \mu \mathrm{M})$, the aminophenyl-substituted chromonyl-pyrimidine 194 elicited higher anti-tubercular activity at $\mathrm{MIC}=12.5 \mu \mathrm{M}$ (Entry 2). Surprisingly, chromonyl-pyrimidines 195-199 with aromatic 
substituents at the 6"-position inhibited Mtb growth to the same extent (MIC $=12.5 \mu \mathrm{M}$, Entries 3-7) as aminophenyl-substituted chromonyl-pyrimidine 194, despite the varied electronic properties among the compounds. The lack of SAR among the chromonylpyrimidines with different phenyl substituents was unexpected, though a similar observation was noted with phenyl-substituted quinolinyl pyrimidines, as discussed in Section 4.1. ${ }^{79}$ In light of these results, diphenyl-substituted chromonyl-pyrimidine 196 and the corresponding diphenyl quinolinyl-pyrimidine were docked into the NDH-II Q-site, which showed distant edge-to-face $\pi-\pi$ interactions with the FAD isoalloxazine ring (Appendix 4, Figure A.4.1). In both cases, no hydrogen bonds were observed between the residues in the Q-site and the arylsubstituted scaffolds, which provides a possibility that these class of compounds could bind $\mathrm{NDH}-\mathrm{II}$ at a different site or the two scaffolds have different target proteins. Notwithstanding, the incorporation of aromatic substituents to the chromonyl-pyrimidine scaffold improved their anti-tubercular activity compared to the first generation of chromonyl-pyrimidines.

\subsection{Conclusion}

In summary, this chapter describes the investigation of chromonyl-pyrimidines as potential anti-tubercular agents. Here, a convergent synthesis was utilised, whereby functionalised amino-chromones and chloro-pyrimidines were first prepared prior to the chromonylpyrimidine assembly. Screening of the first library of chromonyl-pyrimidines resulted in the identification of two lead chromonyl pyrimidines, 174 and 175, with MIC values of $46 \mu \mathrm{M}$ and $36 \mu \mathrm{M}$, respectively. The SAR from this set of compounds facilitated the design and synthesis of a second generation of chromonyl pyrimidines. Specifically, nitro-phenyl, phenyl, and aminophenyl groups were installed at the 2-position of the chromonyl scaffold while methyl, phenyl, and fluoro-phenyl substituents were incorporated at the 6"-position. Gratifyingly, the second library of chromonyl-pyrimidines elicited higher anti-tubercular activity against $M t b$, with chromonyl-pyrimidines 194-199 all exhibiting the same MIC of $12.5 \mu \mathrm{M}$, despite the wide range of electronic properties among the compounds. From these studies, it is postulated that chromonyl pyrimidines bind NDH-II at a site that tolerates minor structural variations to the aromatic substituents of the compounds.

Key pharmacophores were obtained from the two libraries of chromonyl-pyrimidines, whereby chromonyl-pyrimidines with aromatic substituents at both 6"- and 2-positions were the most potent anti-tubercular agents (Figure 4.11). At the 6"-position, electrophilic carbons 
gave good anti-tubercular activity, while aliphatic groups on the 2-position elicited higher antimycobacterial activity than electron-withdrawing groups.

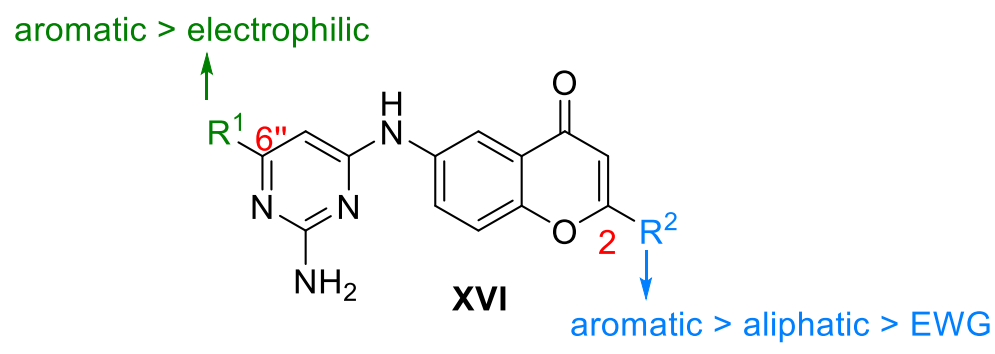

Figure 4.11: Overall SAR of chromonyl-pyrimidines from this study. 


\subsection{Experimental}

\subsubsection{Docking}

Chromonyl-pyrimidine 148 was prepared for docking using the DockPrep tool in USCF Chimera (version 13.1). ${ }^{282}$ The quinone binding site of chain B of the C. thermarum NDH-II crystal structure (PDB: 6BDO) ${ }^{62}$ was used for docking. The NDH-II crystal structure was prepared by removing the co-crystallised ligand HQNO (19) and applying the DockPrep tool in USCF Chimera (version 13.1). A $5 \AA$ grid was generated whereby the centre was defined as residues 46, 47, 316, 317, 318, 347, 348, 349, 376, 379, 380, and 383. Chromonyl-pyrimidine 148 was then modelled into the NDH-II quinone binding site using the anchor and grow algorithm in DOCK 6 (version 6.9). ${ }^{298}$ The conformers with the top 50 binding scores were analysed.

\subsubsection{Chemistry}

General procedure. Unless otherwise stated, all reactions were performed under atmospheric air. Prior to use, DMF (Acros) was distilled from $\mathrm{BaO}$, THF (Pancreac) was distilled over sodium wire and benzophenone, toluene (Roth) was dried with sodium wire, pyridine (BDH) was distilled over $\mathrm{CaH}_{2}, \mathrm{CH}_{2} \mathrm{Cl}_{2}$ (Fisher) was distilled from $\mathrm{P}_{2} \mathrm{O}_{5}$, and EtOAc (drum), petroleum ether (drum), acetone (drum), $\mathrm{MeOH}$ (Pure Science) and $\mathrm{H}_{2} \mathrm{O}$ was distilled. AcOH (Panreac), diethyl oxalate (BDH), 1,4-dioxane (Aldrich), EtOH (absolute, Pure Science), 2 ethoxyethanol (Unilab), ethyl cyclopropane carboxylate (Alfa Aesar), guanidine carbonate (Sigma Aldrich), guanidine hydrochloride (Sigma Aldrich), 37\% $\mathrm{HCl}$ (Merck), $\mathrm{HNO}_{3}$ (Labserv), $\mathrm{H}_{2} \mathrm{SO}_{4}$ (Panreac), 2'-hydroxyacetophenone (Merck), iron (Scientific Supplies), KOH (Pure Science), $\mathrm{MgSO}_{4}$ (Pure Science), 60\% NaH in oil (Sigma Aldrich), $\mathrm{NaHCO}_{3}$ (Pure Science), 28\% $\mathrm{NH}_{3}$ (aq) (UNIVAR), phosphoryl chloride (PS), silver triflate (Sigma Aldrich), sodium (Aldrich) were used as received.

All solvents were removed by evaporation under reduced pressure. Reactions were monitored by TLC-analysis on Macherey-Nagel silica gel coated plastic sheets $(0.20 \mathrm{~mm}$, with fluorescent indicator $\mathrm{UV}_{254}$ ) with detection by UV-absorption (short wave UV - $254 \mathrm{~nm}$; long wave UV $366 \mathrm{~nm}$ ) and by dipping in $\mathrm{KMnO}_{4}$ in $\mathrm{H}_{2} \mathrm{O}$. Column chromatography was performed on Pure Science silica gel (40 - 63 micron). Infrared spectra were recorded as thin films using a Bruker Alpha II FTIR spectrometer equipped with an Attenuated Total Reflectance (ATR) sampling accessory and are reported in wavenumbers $\left(\mathrm{cm}^{-1}\right)$. Melting points (m.p.) were obtained on a DigiMelt MPA160 melting point apparatus. UV data were obtained on an Agilent 8453 
spectrometer. Nuclear magnetic resonance spectra were recorded at $20{ }^{\circ} \mathrm{C}$ in $\mathrm{D}_{2} \mathrm{O}, \mathrm{CDCl}_{3}$, pyridine- $\mathrm{d}_{5}$, or DMSO- $\mathrm{d}_{6}$ using either a Varian INOVA operating at $500 \mathrm{MHz}$ or Varian VNMRS operating at $600 \mathrm{MHz}$. Chemical shifts are given in $\mathrm{ppm}(\delta)$ relative to solvent residues. NMR peak assignments were made using COSY, HSQC, and HMBC 2D experiments. High-resolution (ESI) mass spectrometric data were obtained with an Agilent 6530 Accurate-Mass Q-TOF LC-MS equipped with a 1260 Infinity binary pump.<smiles>Cc1cc(O)nc(N)n1</smiles>

149

2-Amino-6-methyl-pyrimidin-4-ol (149). In a two-necked roundbottomed flask was added $4 \AA$ mol sieves, followed by dry DMF $(50 \mathrm{~mL})$ and $\mathrm{NaH}$ (60\% in oil, $4.0 \mathrm{~g}, 0.1 \mathrm{~mol}, 2$ equiv.). The solution was stirred under argon at room temperature for $30 \mathrm{~min}$ before a solution of guanidine hydrochloride $(5 \mathrm{~g}, 52.3 \mathrm{mmol})$ in dry DMF $(50 \mathrm{~mL})$ was added dropwise into the solution over $30 \mathrm{~min}$. The white suspension was then stirred at room temperature for a further $30 \mathrm{~min}$ before a solution of ethylacetoacetate $(6.6 \mathrm{~mL}, 52.3 \mathrm{mmol}, 1$ equiv.) in dry DMF $(50 \mathrm{~mL})$ was added dropwise into the reaction flask, after which time the orange solution was heated to reflux. After $15 \mathrm{~h}$, TLC analysis showed the disappearance of starting materials and the reaction was filtered, washed with hot dioxane and concentrated in vacuo. The resulting brown solid was then purified by silica gel flash column chromatography $\left[\mathrm{CH}_{2} \mathrm{Cl}_{2} / \mathrm{MeOH} / \mathrm{EtOH} / \mathrm{NH}_{3}(25 \%), 35 / 2 / 2 / 1 \rightarrow 10 / 2 / 2 / 1, \mathrm{v} / \mathrm{v} / \mathrm{v} / \mathrm{v}\right]$ to yield the title compound as a white solid (4.1 g, 63\% yield). $\mathrm{R}_{f}=0.24$ (EtOAc); IR ( $\left.\mathrm{U}_{\max }\right): 3145,1660$, 1454, 846, $557 \mathrm{~cm}^{-1} ;{ }^{1} \mathrm{H}$ NMR (500 MHz, $\mathrm{D}_{2} \mathrm{O}$ ): $\delta$ ppm 5.73 (s, 1H, H5), 2.17 (s, 3H, CH 3 ); ${ }^{13} \mathrm{C}$ NMR (125 MHz, D $\left.2 \mathrm{O}\right): \delta$ ppm 155.7 (C6), 101.3 (C5), $19.9\left(\mathrm{CH}_{3}\right)$; HRMS-ESI (m/z) calcd. for $\left[\mathrm{C}_{5} \mathrm{H}_{7} \mathrm{~N}_{3} \mathrm{O}+\mathrm{H}\right]^{+}:$126.0662, obsd.: 126.0664. The data obtained for this compound were consistent with those reported in literature. ${ }^{299}$<smiles>Nc1nc(O)cc(CO)n1</smiles>

151<smiles>COCc1cc(O)nc(N)n1</smiles>

152
2-Amino-6-hydroxymethylpyrimidin-4-ol $\quad(151)^{285}$ and 2-amino-6-methoxymethylpyrimidin-4-ol (152). Guanidine hydrochloride (5 g, $52.3 \mathrm{mmol}, 1.2$ equiv.) and $\mathrm{KO}{ }^{`} \mathrm{Bu}(26.3 \mathrm{~g}, 0.23 \mathrm{~mol}, 5.4$ equiv.) were dissolved in $\mathrm{MeOH}(78 \mathrm{~mL})$ and the solution was stirred at room

temperature before ethyl 4-chloroacetoacetate $(6 \mathrm{~mL}, 43.4 \mathrm{mmol})$ was added dropwise over $1 \mathrm{~h}$. The reaction was warmed to $60^{\circ} \mathrm{C}$ and stirred for $2 \mathrm{~h}$ before it was cooled to room temperature. After $12 \mathrm{~h}$ of stirring, TLC analysis showed the disappearance of starting 
materials and the reaction was quenched by the addition of $\mathrm{HCl}$ until $\mathrm{pH} 5$ was reached. The resulting brown precipitate was filtered and separated by silica gel flash column chromatography $\left[\mathrm{CH}_{2} \mathrm{Cl}_{2} / \mathrm{MeOH} / \mathrm{EtOH} / \mathrm{NH}_{3}(25 \%), 95 / 2 / 2 / 1 \rightarrow 5 / 2 / 2 / 1, \mathrm{v} / \mathrm{v} / \mathrm{v} / \mathrm{v}\right]$ to give hydroxymethylpyrimidine $151(4.2 \mathrm{~g}, 57 \%)$ and methoxymethylpyrimidine 152 (2 g, 24\%) both as white solids. Data for 151: $\mathrm{R}_{f}=0.37\left[\mathrm{CH}_{2} \mathrm{Cl}_{2} / \mathrm{MeOH} / \mathrm{EtOH} / \mathrm{NH}_{3}(25 \%)\right.$, 5/2/2/1, v/v/v/v]; IR (U $\left.\max _{\max }\right): 3097,3010,2924,2806,2272,1645.1613,1389,1337,1243$, 1193, 1135, 959, 697, $597 \mathrm{~cm}^{-1} ;{ }^{1} \mathrm{H}$ NMR (500 MHz, D $\left.2 \mathrm{O}\right): \delta$ ppm 5.91 (s, 1H, H-5), 4.37 (s, 2H, CH $\mathrm{CH}_{2}$; ${ }^{13} \mathrm{C}$ NMR (150 MHz, $\left.\mathrm{D}_{2} \mathrm{O}\right): \delta$ ppm 97.92 (C5), $61.7\left(\mathrm{CH}_{2}\right) ; \operatorname{HRMS}-\mathrm{ESI}(\mathrm{m} / \mathrm{z})$ calcd. for $\left[\mathrm{C}_{5} \mathrm{H}_{7} \mathrm{~N}_{3} \mathrm{O}_{2}+\mathrm{H}\right]^{+}$: 142.0611, obsd.: 142.0618. Data for 152: $\mathrm{R}_{f}=0.53$ $\left(\mathrm{CH}_{2} \mathrm{Cl}_{2} / \mathrm{MeOH} / \mathrm{EtOH} / \mathrm{NH}_{3}\right.$ (25\%), 5/2/2/1, v/v/v/v); IR (U $\left.\max \right): 3325,3118,2995$, 2878, 2826, 2753, 1601, 1380, 1349, 1120, 958, 902, $589 \mathrm{~cm}^{-1} ;{ }^{1} \mathrm{H}$ NMR $\left(500 \mathrm{MHz}, \mathrm{D}_{2} \mathrm{O}\right)$ : $\delta$ ppm 5.84 (s, 1H, H5), 4.22 (s, 2H, $\left.\mathrm{CH}_{2}\right), 3.40$ (s, 3H, $\left.\mathrm{CH}_{3}\right) ;{ }^{13} \mathrm{C} \mathrm{NMR}\left(150 \mathrm{MHz}, \mathrm{D}_{2} \mathrm{O}\right): \delta$ ppm 157.0 (C4), 100.5 (C5), $73.3\left(\mathrm{CH}_{2}\right), 59.4\left(\mathrm{CH}_{3}\right)$; HRMS-ESI $(\mathrm{m} / \mathrm{q})$ calcd. for $\left[\mathrm{C}_{6} \mathrm{H}_{9} \mathrm{~N}_{3} \mathrm{O}_{2}+\mathrm{H}\right]^{+}:$156.0768, obsd.: 156.0768 .

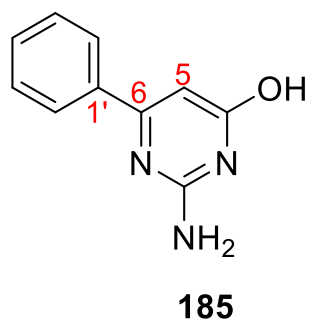

2-Amino-6-phenyl-pyrimidin-4-ol (185). Ethyl benzoylacetate $(1 \mathrm{~mL}$, $5.8 \mathrm{mmol})$ and guanidine carbonate $(1.05 \mathrm{~g}, 5.8 \mathrm{mmol}, 1$ equiv.) were dissolved in $\mathrm{EtOH}(10 \mathrm{~mL})$ and warmed to $100{ }^{\circ} \mathrm{C}$. After $24 \mathrm{~h}$ of stirring, the precipitate was collected by filtration to give the title compound as a white solid (912 mg, 84\%). $\mathrm{R}_{f}=0.3$ (EtOAc); IR (Umax): 3165, 1561, 1517, 1396, 1232, 979, 888, 773, $669 \mathrm{~cm}^{-1} ;{ }^{1} \mathrm{H}$ NMR (500 MHz, pyridine- $\mathrm{d}_{5}$ ) $\delta$ ppm 8.89 (br. s, $1 \mathrm{H}$, $\mathrm{OH}), 8.45\left(\mathrm{~d}, J_{2^{\prime}, 3^{\prime}}=7.1 \mathrm{~Hz}, 2 \mathrm{H}, \mathrm{H} 2^{\prime}\right), 7.55\left(\mathrm{t}, J_{2^{\prime}, 3^{\prime}}=J_{3^{\prime}, 4^{\prime}}=7.3 \mathrm{~Hz}, 2 \mathrm{H}, \mathrm{H} 3^{\prime}\right), 7.49$ (t, $J_{3^{\prime}, 4^{\prime}}=7.2$ $\left.\mathrm{Hz}, 1 \mathrm{H}, \mathrm{H} 4{ }^{\prime}\right), 6.94$ (s, 1H, H5); HRMS-ESI $(m / z)$ calcd. for $\left[\mathrm{C}_{10} \mathrm{H}_{9} \mathrm{~N}_{3} \mathrm{O}+\mathrm{H}\right]^{+}:$188.0818, obsd.: 188.0819. The data obtained for this compound were consistent with those reported in literature. ${ }^{300}$

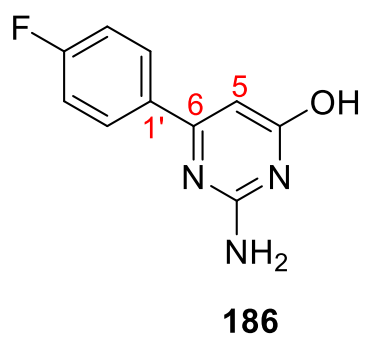

2-Amino-6-(4'-fluorophenyl)-pyrimidin-4-ol (186). Ethyl 3-(4fluorophenyl)-3-oxopropanoate $(0.5 \mathrm{~mL}, 2.78 \mathrm{mmol})$ and guanidine carbonate (500 mg, $2.78 \mathrm{mmol}, 1$ equiv.) were dissolved in EtOH (5 $\mathrm{mL}$ ) and warmed to $100{ }^{\circ} \mathrm{C}$. After $24 \mathrm{~h}$ of stirring, the precipitate was collected by filtration to give the title compound as a white solid (427 $\mathrm{mg}, 75 \%) . \mathrm{R}_{f}=0.36$ (EtOAc); IR (Umax): 3070, 1654, 1600, 1566, 1506, 1465, 1406, 1388, 1225, 1155, $826 \mathrm{~cm}^{-1} ;{ }^{1} \mathrm{H}$ NMR (500 MHz, pyridine-d $\left.\mathrm{d}_{5}\right): \delta$ ppm $8.26-8.23$ (m, 2H, H2'), 7.73 (br. s, 
2H, $\mathrm{NH}_{2}$ ), 7.19 (m, 2H, H3'), 6.74 (s, 1H, H5); ${ }^{13} \mathrm{C}$ NMR (125 MHz, pyridine-d $\mathrm{d}_{5}$ ): $\delta$ ppm 165.4 $\left(\mathrm{d}, J_{4^{\prime}, \mathrm{F}}=280 \mathrm{~Hz}, \mathrm{C}^{\prime}\right), 163.9(\mathrm{C} 6), 161.4(\mathrm{C} 4), 130.7$ (C2'), 116.8 (d, J $\left.J_{3^{\prime}, \mathrm{F}}=22 \mathrm{~Hz}, \mathrm{C}^{\prime}\right), 100.2$ (C5); HRMS-ESI $(m / z)$ calcd. for $\left[\mathrm{C}_{10} \mathrm{H}_{8} \mathrm{FN}_{3} \mathrm{O}+\mathrm{H}\right]^{+}:$206.0724, obsd.: 206.0725. The data obtained for this compound were consistent with those reported in literature. ${ }^{79}$

General procedure for the synthesis of chloropyrimidines. 4-Hydroxypyrimidine (1 equiv.) was evaporated three times in dry toluene $(0.5 \mathrm{~mL} / \mathrm{mmol})$ under argon before it was dissolved in $\mathrm{POCl}_{3}(0.5 \mathrm{~mL} / \mathrm{mmol})$. The reaction was heated to reflux for $2 \mathrm{~h}$, after which time the $\mathrm{POCl}_{3}$ was removed in vacuo. The solid was dissolved in a mixture of aqueous $\mathrm{NH}_{3}$ (28\%) and ice, and the crude product extracted with $\mathrm{CH}_{2} \mathrm{Cl}_{2}$ three times. The organic layers were then dried using anhydrous $\mathrm{MgSO}_{4}$, filtered and the filtrate concentrated to give the crude product, which could then be recrystallised from hot ethanol.<smiles>Cc1cc(C)nc(N)n1</smiles>

150

2-Amino-4-chloro-6-methyl-pyrimidine (150). By subjecting pyrimidinol $149(3 \mathrm{~g}, 26.3 \mathrm{mmol})$ and $\mathrm{POCl}_{3}(15 \mathrm{~mL})$ to the general procedure for the synthesis of chloropyrimidines, the title compound was isolated as white crystals $\left(2.0\right.$ g, 53\%); $\mathrm{R}_{f}=0.6$ (EtOAc); m.p.: $182{ }^{\circ} \mathrm{C}$ (lit. $\left.{ }^{279} 182-183{ }^{\circ} \mathrm{C}\right) ; \mathrm{IR}$ $\left(U_{\max }\right): 3309,3192,2925,1637,1548,1476,1288,872,782,490 \mathrm{~cm}^{-1} ;{ }^{1} \mathrm{H}$ NMR (500 MHz, $\mathrm{CDCl}_{3}$ ): $\delta$ ppm 6.55 (s, $\left.1 \mathrm{H}, \mathrm{H}-5\right), 5.15$ (br. s, $2 \mathrm{H}, \mathrm{NH}_{2}$ ), 2.32 (s, $\left.3 \mathrm{H}, \mathrm{CH}_{3}\right) ;{ }^{13} \mathrm{C}$ NMR (125 MHz, CDCl $)$ : $\delta$ ppm 170.2 (C6), 162.7 (C2), 161.5 (C4), 110.6 (C5), $23.9\left(\mathrm{CH}_{3}\right)$; HRMS-ESI $(m / z)$ calcd. for $\left[\mathrm{C}_{5} \mathrm{H}_{6}{ }^{35} \mathrm{ClN}_{3}+\mathrm{H}\right]^{+}:$144.0323, obsd.: 144.0324 .<smiles></smiles>

153
2-Amino-4-chloro-6-chloromethyl-pyrimidine $\quad$ (153). ${ }^{285} \quad$ By subjecting pyrimidinol $151(235.6 \mathrm{mg}, 1.67 \mathrm{mmol})$ and $\mathrm{POCl}_{3}(2 \mathrm{~mL})$ to the general procedure for the synthesis of chloropyrimidines, the title compound was isolated as a white amorphous solid (184.3 mg, 62\%); $\mathrm{R}_{f}$ $=0.48($ EtOAc); IR (U $\max ): 2948,2855,1625,1550,1466,1413,1377$, 1313, 1292, 1222, 1164, 1010, 925, 887, 805, 779, 769, 629, $505 \mathrm{~cm}^{-1} ;{ }^{1} \mathrm{H}$ NMR $(500 \mathrm{MHz}$, $\left.\mathrm{CDCl}_{3}\right) \delta$ ppm 6.83 (C5), 5.60 (br. s, 2H, NH ), 4.37 (s, 2H, $\left.\mathrm{CH}_{2}\right) ;{ }^{13} \mathrm{C}$ NMR (125 MHz, $\left.\mathrm{CDCl}_{3}\right) \delta$ ppm 167.7 (C6), 162.8 (C4), 162.7 (C2), 109.2 (C5), $44.8\left(\mathrm{CH}_{2}\right) ;$ HRMS-ESI (m/ z) calcd. for $\left[\mathrm{C}_{5} \mathrm{H}_{5}{ }^{35} \mathrm{Cl}_{2} \mathrm{~N}_{3}+\mathrm{H}\right]^{+}: 177.9933$, obsd.: 177.9933 . 
<smiles>COCc1cc(Cl)nc(N)n1</smiles>

2-Amino-4-chloro-6-methoxymethyl-pyrimidine (154). Вy subjecting pyrimidinol $152(48 \mathrm{mg}, 0.3 \mathrm{mmol})$ and $\mathrm{POCl}_{3}(0.18 \mathrm{~mL})$ to the general procedure for the synthesis of chloropyrimidines, the title compound was isolated as a white amorphous solid ( $25 \mathrm{mg}, 48 \%) ; \mathrm{R}_{f}=$ 0.35 (EtOAc); IR (U $\left.\mathrm{U}_{\max }\right): 3398,3316,1637,1553,1475,1421,1291$, 1125, 889, 781, $524 \mathrm{~cm}^{-1} ;{ }^{1} \mathrm{H}$ NMR $\left(500 \mathrm{MHz}, \mathrm{CDCl}_{3}\right) \delta$ ppm 6.76 (s, 1H, H5), 5.57 (br. s., $\left.2 \mathrm{H}, \mathrm{NH}_{2}\right), 4.32$ (s, 2H, CH ), 3.45 (s, $\left.1 \mathrm{H}, \mathrm{CH}_{3}\right) ;{ }^{13} \mathrm{C} \mathrm{NMR}\left(125 \mathrm{MHz}, \mathrm{CDCl}_{3}\right) \delta$ ppm 170.5 (C6), 162.7 (C4), 162.3 (C3), 107.8 (C5), $73.9\left(\mathrm{CH}_{2}\right), 59.2\left(\mathrm{CH}_{3}\right)$; HRMS-ESI (m/ z) calcd. for $\left[\mathrm{C}_{6} \mathrm{H}_{8} \mathrm{ClN}_{3} \mathrm{O}+\mathrm{H}\right]^{+}$: 174.0429, obsd.: 174.0429 .

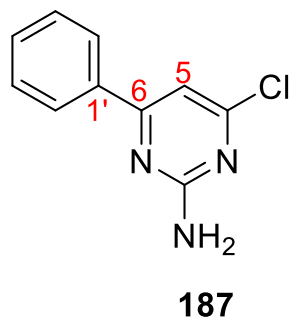

2-Amino-4-chloro-6-phenyl-pyrimidine (187). By subjecting pyrimidinol $185(238.5 \mathrm{mg}, 1.31 \mathrm{mmol})$ and $\mathrm{POCl}_{3}(4.89 \mathrm{~mL})$ to the general procedure for the synthesis of chloropyrimidines, the title compound was isolated as a white amorphous solid (148.2 mg, 55\%); $\mathrm{R}_{f}$ $=0.32\left(\mathrm{CH}_{2} \mathrm{Cl}_{2} / \mathrm{EtOAc}, 1 / 1, \mathrm{v} / \mathrm{v}\right) ; \mathrm{IR}\left(\mathrm{U}_{\max }\right): 3173,1651,1625,1589,1557$, 1538, 1496, 1480, 1462, 1447, 1418, 1319, 1295, 1228, 1193, 1171, 995, 947, 818, 813, 761, 687, 651, $562 \mathrm{~cm}^{-1} ;{ }^{1} \mathrm{H}$ NMR $\left(500 \mathrm{MHz}, \mathrm{CDCl}_{3}\right) \delta \mathrm{ppm} 7.92-7.90(\mathrm{~m}, 2 \mathrm{H}, \mathrm{H} 2$ '), $7.44-7.40$ (m, 3H, H3', H4'), 7.00 (s, 1H, H5); HRMS-ESI (m/z) calcd. for $\left[\mathrm{C}_{10} \mathrm{H}_{8}{ }^{35} \mathrm{ClN}_{3}+\mathrm{H}\right]^{+}: 246.0480$, obsd.: 246.0481. The data obtained for this compound were consistent with those reported in literature. ${ }^{294}$

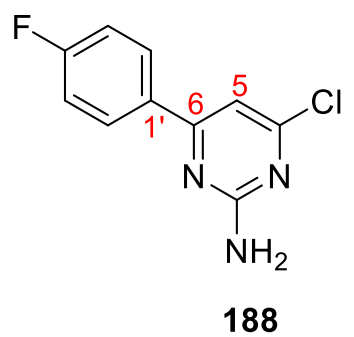

2-Amino-4-chloro-6-(4-fluorophenyl)-pyrimidine (188). Ву subjecting pyrimidinol $186(183.5 \mathrm{mg}, 0.90 \mathrm{mmol})$ and $\mathrm{POCl}_{3}(3.34$ $\mathrm{mL}$ ) to the general procedure for the synthesis of chloropyrimidines, the title compound was isolated as a white amorphous solid (124.8 mg, 62\%); $\mathrm{R}_{f}=0.29\left(\mathrm{CH}_{2} \mathrm{Cl}_{2} /\right.$ EtOAc, 1/1, v/v); IR (U $\left.u_{\max }\right): 2747,1664,1606,1570,1541,1510,1468,1234,1161,995,942,827,550$, $482 \mathrm{~cm}^{-1} ;{ }^{1} \mathrm{H}$ NMR (500 MHz, $\left.\mathrm{CDCl}_{3}\right): \delta$ ppm 8.00 - 7.97 (m, 2H, H2'), 7.18 - $7.14(\mathrm{~m}, 2 \mathrm{H}$, H3'), 7.03 (s, 1H, H5); ${ }^{13} \mathrm{C}$ NMR (500 MHz, $\mathrm{CDCl}_{3}$ ): $\delta$ ppm 166.6 (C6), 162.1 (C4), 164.8 $\left(\mathrm{d},{ }_{J^{\prime}, \mathrm{F}}=266.9 \mathrm{~Hz}, \mathrm{C} 4^{\prime}\right), 129.3\left(\mathrm{~d}, J_{2^{\prime}, \mathrm{F}}=9.0 \mathrm{~Hz}, \mathrm{C} 2^{\prime}\right), 115.9$ (d, $\left.J_{3^{\prime}, \mathrm{F}}=21.6 \mathrm{~Hz}, \mathrm{C} 3^{\prime}\right), 106.8$ (C5); HRMS-ESI $(m / z)$ calcd. for $\left[\mathrm{C}_{10} \mathrm{H}_{7}^{35} \mathrm{ClFN}_{3}+\mathrm{H}\right]^{+}:$224.0385, obsd.: 224.0386. 
<smiles></smiles>

Ethyl 4-oxo-4H-chromene-2-carboxylate (155). To a solution of sodium ( $2 \mathrm{~g}, 87 \mathrm{mmol}, 5$ equiv.) in $\mathrm{EtOH}(120 \mathrm{~mL})$, was added dropwise a solution of 2'-hydroxyacetophenone $(2.1 \mathrm{~mL}, 17.4 \mathrm{mmol})$ and diethyl oxalate $(6 \mathrm{~mL}, 43.5 \mathrm{mmol}, 2.5$ equiv. $)$ in EtOH $(10 \mathrm{~mL})$. by $1 \mathrm{~h}$ at reflux, at which time TLC analysis showed the disappearance of starting materials. The reaction was quenched by the addition of $2 \mathrm{M} \mathrm{HCl}$ until $\mathrm{pH} 1$ was reached. The resulting precipitate was filtered and the filtrate concentrated to get the crude product, which was purified by silica gel flash column chromatography (petroleum ether/EtOAc, $1 / 0 \rightarrow 3 / 1, \mathrm{v} / \mathrm{v}$ ) to give the title compound as a white solid ( $3.4 \mathrm{~g}, 91 \%$ yield). $\mathrm{R}_{f}=0.41$ (EtOAc/petroleum ether, 1/1, v/v); IR (U $\max ): 1638,1609,1564,1362,1249,1073,1023$, 999, 829, 768, 653, 550, 481, $464 \mathrm{~cm}^{-1} ;{ }^{1} \mathrm{H}$ NMR (500 MHz, $\left.\mathrm{CDCl}_{3}\right): \delta \mathrm{ppm} 8.06\left(\mathrm{~d}, J_{5,6}=\right.$ $8.1 \mathrm{~Hz}, 1 \mathrm{H}, \mathrm{H} 5), 7.63$ (t, $\left.J_{6,7}=J_{7,8}=7.8 \mathrm{~Hz}, 1 \mathrm{H}, \mathrm{H} 7\right), 7.48$ (d, $\left.J_{7,8}=8.4 \mathrm{~Hz}, 1 \mathrm{H}, \mathrm{H} 8\right), 7.33$ (t, $\left.J_{5,6}=J_{6,7}=7.5 \mathrm{~Hz}, 1 \mathrm{H}, \mathrm{H} 6\right), 6.99$ (s, 1H, H3), 4.64 (q, $\left.J_{\mathrm{CH} 2-\mathrm{CH} 3}=7.1 \mathrm{~Hz}, 2 \mathrm{H}, \mathrm{CH}_{2}\right), 1.62$ (t, $\left.J_{\mathrm{CH} 2-\mathrm{CH} 3}=7.1 \mathrm{~Hz}, 3 \mathrm{H}, \mathrm{CH}_{3}\right) ;{ }^{13} \mathrm{C} \mathrm{NMR}\left(125 \mathrm{MHz}, \mathrm{CDCl}_{3}\right): \delta \mathrm{ppm} 178.2(\mathrm{C} 4), 160.4\left(\mathrm{C}^{\prime}\right)$, 155.8 (8a), 152.1 (C2), 134.6 (C7), 125.8 (C6), 125.6 (C5), 124.3 (C4a), 118.7 (C8), 114.6 (C3), $62.9\left(\mathrm{CH}_{2}\right), 14.0\left(\mathrm{CH}_{3}\right)$; HRMS-ESI $(\mathrm{m} / \mathrm{z})$ calcd. for $\left[\mathrm{C}_{12} \mathrm{H}_{10} \mathrm{O}_{4}+\mathrm{H}\right]^{+}$: 219.0652, obsd.: 219.0656. The data obtained for this compound were consistent with those reported in literature. $^{280}$<smiles>CCOC(=O)c1cc(=O)c2cc([N+](=O)[O-])ccc2o1</smiles>

156

Ethyl 6-nitro-4-oxo-4H-chromene-2-carboxylate (156). Concentrated $\mathrm{HNO}_{3}(70 \%, 0.1 \mathrm{~mL})$ was added to chromone 155 (200 mg, $0.92 \mathrm{mmol}$ ) and cooled to $0{ }^{\circ} \mathrm{C}$ before concentrated $\mathrm{H}_{2} \mathrm{SO}_{4}(1.5 \mathrm{~mL})$ was added to the reaction flask.

The solution was stirred at room temperature for $8 \mathrm{~h}$, after which time the reaction was poured into an ice-water mixture. The resulting white precipitate was filtered and dried to obtain the crude compound, which was recrystallised in $\mathrm{MeOH} / \mathrm{CH}_{2} \mathrm{Cl}_{2}(3 / 1, \mathrm{v} / \mathrm{v})$ to yield white crystals ( $240 \mathrm{mg}, 99 \%$ yield). $\mathrm{R}_{f}=0.54$ (petroleum ether $\left./ \mathrm{EtOAc}, 1 / 1, \mathrm{v} / \mathrm{v}\right) ; \mathrm{IR}\left(\mathrm{u}_{\max }\right)$ : 2954, 2925, 2853, 1737, 1667, 1530, 1494, 1363, 1346, 1271, 1081, $967 \mathrm{~cm}^{-1} ;{ }^{1} \mathrm{H}$ NMR $(500$ $\left.\mathrm{MHz}, \mathrm{CDCl}_{3}\right): \delta \mathrm{ppm} 9.07\left(\mathrm{~d}, J_{5,7}=2.6 \mathrm{~Hz}, 1 \mathrm{H}, \mathrm{H} 5\right), 8.58\left(\mathrm{dd}, J_{5,7}=9.2, J_{6,7}=2.8 \mathrm{~Hz}, 1 \mathrm{H}\right.$, H7), 7.78 (d, $\left.J_{7,8}=9.2 \mathrm{~Hz}, 1 \mathrm{H}, \mathrm{H} 8\right), 7.19$ (s, 1H, H3), 4.50 (q, Jل $\mathrm{CH}_{2-\mathrm{CH} 3}=7.1 \mathrm{~Hz}, 2 \mathrm{H}, \mathrm{CH}_{2}$ ), $1.46\left(\mathrm{t}, J_{\mathrm{CH} 2-\mathrm{CH} 3}=7.1 \mathrm{~Hz}, 3 \mathrm{H}, \mathrm{CH}_{3}\right) ;{ }^{13} \mathrm{C} \mathrm{NMR}\left(125 \mathrm{MHz}, \mathrm{CDCl}_{3}\right): \delta$ ppm 176.97 (C4), 159.90 (C1), 158.8 (C8a), 152.9 (C2), 145.0 (C6), 129.1 (C7), 124.6 (C4a), 122.7 (C5), 120.78 
(C8), $115.2(\mathrm{C} 3), 63.6\left(\mathrm{CH}_{2}\right), 14.3\left(\mathrm{CH}_{3}\right)$; HRMS-ESI $(\mathrm{m} / \mathrm{q})$ calcd. for $\left[\mathrm{C}_{12} \mathrm{H}_{9} \mathrm{NO}_{6}+\mathrm{H}\right]^{+}$: 264.0503, obsd.: 264.0502. The data obtained for this compound matched those reported in literature. ${ }^{286}$

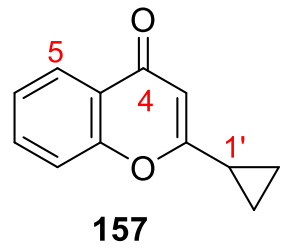

equiv.) in THF ( $1 \mathrm{~mL}$ ) was then added into the $\mathrm{NaH}$ suspension dropwise. The reaction was stirred under reflux for $15 \mathrm{~min}$ before it was poured into an ice-water mixture and acidified to $\mathrm{pH} 6$ with $2 \mathrm{M} \mathrm{HCl}$. The product was then extracted using EtOAc, washed with brine, and dried with anhydrous $\mathrm{MgSO}_{4}$. After filtration, the filtrate was then concentrated in vacuo into a yellow-orange oil, to which $\mathrm{MeOH}(20 \mathrm{~mL})$ and conc. $\mathrm{HCl}(37 \%, 1 \mathrm{~mL})$ were added. The resulting orange solution was stirred at room temperature for $5 \mathrm{~h}$, after which time TLC analysis showed the appearance of the product and the disappearance of starting materials. The reaction was concentrated in vacuo and the product extracted with EtOAc. After washing with $\mathrm{NaHCO}_{3}$ and brine, the organic solution was dried with anhydrous $\mathrm{MgSO}_{4}$, filtered, and the resulting filtrate concentrated. Purification by silica gel flash column chromatography (petroleum ether/EtOAc, 10/1 $\rightarrow 3 / 1, \mathrm{v} / \mathrm{v}$ ) gave the title compound as a white solid (1.03 g, 74\% yield). $\mathrm{R}_{f}=0.29$ (petroleum ether/EtOAc, 3/1, v/v); IR (U $\left.U_{\max }\right): 1640,1570,1461,1384,1124,1035,956,842,775 \mathrm{~cm}^{-1} ;{ }^{1} \mathrm{H}$ NMR $(500 \mathrm{MHz}$, $\left.\mathrm{CDCl}_{3}\right): \delta \mathrm{ppm} 8.17$ (d, $\left.J_{5,6}=8.2 \mathrm{~Hz}, 1 \mathrm{H}, \mathrm{H} 5\right), 7.61$ (t, $\left.J_{6,7}=J_{7,8}=7.8 \mathrm{~Hz}, 1 \mathrm{H}, \mathrm{H} 7\right), 7.37-7.34$ (m, 2H, H8, H6), 6.21 (s, 1H, H3), 1.90 (m, 1H, C1'), 1.19-1.14 (m, 2H, C2'a), 1.13-1.08 (m, 2H, C2'b); ${ }^{13} \mathrm{C}$ NMR (125 MHz, $\mathrm{CDCl}_{3}$ ): $\delta$ ppm 177.9 (C4), 170.4 (C2), 156.2 (C8a), 133.3 (C7), 125.8 (C5), 125.0 (C8), 124.1 (C4a), 117.7 (C6), 108.0 (C3), 14.7 (C1'), 8.70 (C2'); HRMS-ESI $(m / 2)$ calcd. for $\left[\mathrm{C}_{12} \mathrm{H}_{10} \mathrm{O}_{2}+\mathrm{H}\right]^{+}: 187.0754$, obsd.: 187.0755. The data obtained for this compound were consistent with those reported in literature. ${ }^{301}$

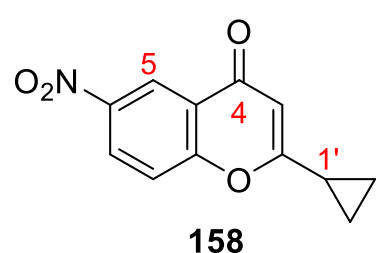

2-Cyclopropyl-6-nitro-4H-chromen-4-one (158). $\mathrm{HNO}_{3}$

(70\%, $0.16 \mathrm{~mL})$ was added to cyclopropyl chromene 157 (294 mg, $1.58 \mathrm{mmol}$ ) and the mixture was cooled to $0{ }^{\circ} \mathrm{C}$ before conc. $\mathrm{H}_{2} \mathrm{SO}_{4}(2.4 \mathrm{~mL})$ was added. The reaction was allowed to warm to room temperature over $30 \mathrm{~min}$ before it was poured into an ice-water mixture and extracted 
using EtOAc. The organic solution was collected, dried with anhydrous $\mathrm{MgSO}_{4}$ and filtered. The filtrate was concentrated in vacuo to give the crude product as a yellow oil. Purification via silica gel flash column chromatography (petroleum ether/EtOAc, 20/1 $\rightarrow$ 10/1, v/v) gave the title compound as a white solid (322 mg, 88\% yield). $\mathrm{R}_{f}=0.40\left(\mathrm{CH}_{2} \mathrm{Cl}_{2} / \mathrm{EtOAc}\right.$, 9/1, v/v); IR (Umax $): 2921,2851,1649$, 1641, 1606, 1528, 1465, 1443, 1368, 1340, 1275, 1206 , 1056, 1026, 953, 838, 743, $677 \mathrm{~cm}^{-1} ;{ }^{1} \mathrm{H}$ NMR (500 MHz, $\left.\mathrm{CDCl}_{3}\right): \delta \mathrm{ppm} 8.98$ (d, $J_{5,7}=2.4$ $\mathrm{Hz}, 1 \mathrm{H}, \mathrm{H} 5), 8.42$ (dd, $\left.J_{7,8}=9.1 \mathrm{~Hz}, J_{5,7}=2.6 \mathrm{~Hz}, 1 \mathrm{H}, \mathrm{H} 7\right), 7.50$ (d, $\left.J_{7,8}=9.1 \mathrm{~Hz}, 1 \mathrm{H}, \mathrm{H} 8\right), 6.25$ (s, 1H, H3), 1.96-1.91 (m, 1H, C1'), 1.23-1.15 (m, 4H, C2'); ${ }^{13} \mathrm{C}$ NMR (125 MHz, $\left.\mathrm{CDCl}_{3}\right): \delta$ 175.7 (C4), 171.3 (C2), 158.9 (C8a), 144.5 (C6), 127.6 (C7), 124.0 (C4a), 122.2 (C5), 119.4 (C8), 108.1 (C3), 9.49 (C1'), 9.19 (C2'); HRMS-ESI (m/z) calcd. for $\left[\mathrm{C}_{12} \mathrm{H}_{9} \mathrm{NO}_{4}+\mathrm{H}\right]^{+}$: 232.0604, obsd.: 232.0603.<smiles>CC(=O)c1cc([N+](=O)[O-])ccc1O</smiles>

163

2'-Hydroxy-5'-nitro-acetophenone (163). A solution of 2'hydroxyacetophenone $(10 \mathrm{~mL}, 83 \mathrm{mmol})$ in $\mathrm{AcOH}(20 \mathrm{~mL})$ was cooled to $0{ }^{\circ} \mathrm{C}$ before concentrated $\mathrm{HNO}_{3}(70 \%, 20 \mathrm{~mL})$ was added. The flask was fitted with a condenser and warmed to room temperature. After 1 $h$, the reaction was poured into an ice- $\mathrm{H}_{2} \mathrm{O}$ mixture, and was stirred for a further $30 \mathrm{~min}$ at room temperature. The resulting white precipitate was collected and recrystallised in hot $\mathrm{MeOH}$ to give the title compound as white crystals $(4.5 \mathrm{~g}, 30 \%) . \mathrm{R}_{f}=0.54$ (toluene/EtOAc, 95/5, v/v); m.p: $105.5{ }^{\circ} \mathrm{C}$ (lit. $\left.{ }^{302} 102{ }^{\circ} \mathrm{C}\right)$; IR (Umax $): 3089,1644,1519,1337,1295,1248$, 1212, 1116, 964, 903, 807, 746, 729, $644 \mathrm{~cm}^{-1} ;{ }^{1} \mathrm{H}$ NMR (500 MHz, $\left.\mathrm{CDCl}_{3}\right): \delta \mathrm{ppm} 12.87$ $(\mathrm{s}, 1 \mathrm{H}, \mathrm{OH}), 8.71\left(\mathrm{~d}, J_{4^{\prime}, 6^{\prime}}=2.6 \mathrm{~Hz}, 1 \mathrm{H}, \mathrm{H} 6^{\prime}\right), 8.35\left(\mathrm{dd}, J_{3^{\prime}, 4^{\prime}}=9.2 \mathrm{~Hz} J_{4^{\prime}, 6^{\prime}}=2.7 \mathrm{~Hz}, \mathrm{H} 4^{\prime}\right)$, 7.09 (d, $\left.J_{3^{\prime}, 4^{\prime}}=9.2 \mathrm{~Hz}, 1 \mathrm{H}, \mathrm{H} 3^{\prime}\right), 2.75$ (s, 3H, H2); ${ }^{13} \mathrm{C}$ NMR (125 MHz, $\left.\mathrm{CDCl}_{3}\right): \delta \mathrm{ppm}$ 204.1 (C1), 167.2 (C2'), 139.6 (C5'), 131.2 (C4'), 127.3 (C6'), $119.6\left(\mathrm{C}^{\prime}\right), 118.6\left(\mathrm{C}^{\prime}\right), 26.8$ (C2); HRMS-ESI (m/2) calcd. for $\left[\mathrm{C}_{8} \mathrm{H}_{7} \mathrm{NO}_{4}-\mathrm{H}\right]^{-}:$180.0302, obsd.: 180.0306. The data obtained for this compound matched those reported in literature. ${ }^{302}$

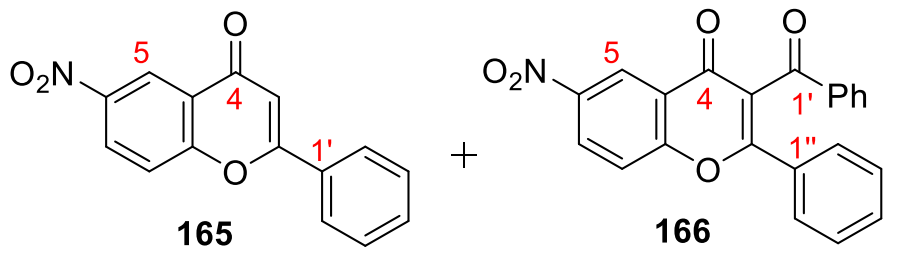

2-Phenyl-6-nitro-4Hchromen-4-one (165) and 3benzoyl-6-nitro-2-phenyl-4Hchromen-4-one (166). A

solution of benzoyl chloride ( $0.4 \mathrm{~mL}, 3.36 \mathrm{mmol}, 12$ equiv.) in freshly distilled pyridine (6 $\mathrm{mL})$ was cooled to $0{ }^{\circ} \mathrm{C}$ before acetophenone $163(50 \mathrm{mg}, 0.28 \mathrm{mmol})$ was added. The ice 
bath was then removed and the solution was allowed to warm to room temperature and stirred for $12 \mathrm{~h}$, after which time TLC analysis showed the disappearance of starting materials and the appearance of new products. The reaction was then poured into an ice$\mathrm{HCl}(2 \mathrm{M})$ mixture and extracted with EtOAc. The organic layers were collected, washed with brine, dried with anhydrous $\mathrm{MgSO}_{4}$ and filtered. The filtrate was then concentrated in vacuo before it was re-dissolved in pyridine ( $4.5 \mathrm{~mL}) . \mathrm{KOH}(424 \mathrm{mg}, 7.5 \mathrm{mmol}, 2.25$ equiv.) was added to the solution before it was warmed to reflux. After $2 \mathrm{~h}$, the reaction was diluted with $\mathrm{H}_{2} \mathrm{O}$ and extracted with EtOAc $(3 \times 5 \mathrm{~mL})$. The organic layers were then collected, washed with $0.1 \mathrm{M} \mathrm{HCl}$ and brine before it was dried with anhydrous $\mathrm{MgSO}_{4}$ and filtered. After concentration in vacuo, the resulting solid was re-dissolved in $\mathrm{AcOH}(4 \mathrm{~mL})$ and conc. $\mathrm{H}_{2} \mathrm{SO}_{4}(0.2 \mathrm{~mL})$ and the solution warmed to reflux for $2 \mathrm{~h}$. The reaction was then quenched by the addition of $0.1 \mathrm{M} \mathrm{HCl}$, extracted with EtOAc, and washed with aqueous $\mathrm{NaHCO}_{3}$ and brine. The organic layer was then dried with anhydrous $\mathrm{MgSO}_{4}$, filtered, and the filtrate concentrated in vacuo to give the crude product as a brown solid. Purification by silica gel column chromatography (petroleum ether/toluene, $1 / 0 \rightarrow 1 / 1, \mathrm{v} / \mathrm{v}$ ) gave the title compound 165 as a white solid (52 mg, 70\%) with diketone 166 as a minor by-product (5 mg, 5\%). Data for 165: $\mathrm{R}_{f}=0.40$ (toluene/EtOAc, 95/5, v/v); IR ( $\mathrm{u}_{\max }$ ): 3329, 3067, 1656, 1493, 1387, 1176, 768, 663, $560 \mathrm{~cm}^{-1} ;{ }^{1} \mathrm{H}$ NMR (500 MHz, $\left.\mathrm{CDCl}_{3}\right): \delta$ ppm 9.08-9.07 (m, 1H, H5), 8.53 (dd, $\left.J_{5,7}=2.8 \mathrm{~Hz}, J_{7,8}=9.0 \mathrm{~Hz}, 1 \mathrm{H}, \mathrm{H} 7\right), 7.93-7.91$ (m, 2H, H2'), 7.73 (d, J7, $=9.3 \mathrm{~Hz}, 1 \mathrm{H}, \mathrm{H} 8), 7.61-7.53$ (m, 3H, H3', H4'), 6.87 (s, 1H, H3); ${ }^{13} \mathrm{C}$ NMR (125 MHz, CDCl $\mathrm{CD}_{3}$ : $\delta$ ppm 176.8 (C4), 164.2 (C2), 159.1 (C), 144.9 (C8a), 132.5 (C4'), 130.8 (C1'), 129.4 (C2'), 128.2 (C7), 126.5 (C3'), 124.1 (C4a), 122.6 (C5), 120.0 (C8), 107.9 (C3); HRMSESI $(m / z)$ calcd. for $\left[\mathrm{C}_{15} \mathrm{H}_{9} \mathrm{NO}_{4}+\mathrm{H}\right]^{+}:$268.0604, obsd.: 268.0606: The data obtained for this compound were consistent with that reported in literature. ${ }^{303}$ Data for $166: \mathrm{R}_{f}=0.46$ (toluene/EtOAc, 95/5, v/v); IR (U max): 3068, 1658, 1645, 1493, 1387, 1177, 769, 561, 458, $442 \mathrm{~cm}^{-1} ;{ }^{1} \mathrm{H}$ NMR $\left(500 \mathrm{MHz}, \mathrm{CDCl}_{3}\right): \delta \mathrm{ppm} 9.11$ (d, $\left.J_{5,7}=2.7 \mathrm{~Hz}, 1 \mathrm{H}, \mathrm{H} 7\right), 8.60$ (dd, $J_{7,8}$ $\left.=9.2 \mathrm{~Hz}, J_{5,7}=2.7 \mathrm{~Hz}, 1 \mathrm{H}, \mathrm{H} 7\right), 7.91\left(\mathrm{~d}, J_{3^{\prime}, 4^{\prime}}=8.0 \mathrm{~Hz}, 2 \mathrm{H}, \mathrm{H} 3^{\prime}\right), 7.76\left(\mathrm{~d}, J_{7,8}=9.2 \mathrm{~Hz}, 1 \mathrm{H}\right.$, H8), 7.66 (d, $\left.J_{2^{\prime \prime}, 3^{\prime \prime}}=8.4 \mathrm{~Hz}, 2 \mathrm{H}, \mathrm{H} 2^{\prime \prime}\right), 7.57$ (t, $\left.J_{4^{\prime}, 5^{\prime}}=7.4 \mathrm{~Hz}, 1 \mathrm{H}, \mathrm{H} 5^{\prime}\right), 7.50-7.36(\mathrm{~m}, 5 \mathrm{H}$, H4', H3", H4"); ${ }^{13} \mathrm{C}$ NMR (125 MHz, CDCl $): \delta$ ppm 192.4 (C1'), 175.2 (C4), 163.2 (C2), 160.0 (C6), 145.2 (C8a), 136.6 (C2'), 134.3 (C5'), 132.3 (C4"), 130.9 (C1"), 129.5 (C3'), 129.13 (C3"), 129.05 (C4'), 128.7 (C7), 126.6 (C2'), 123.6 (C4a), 123.0 (C5), 120.1 (C8); HRMS-ESI $(\mathrm{m} / \mathrm{g})$ calcd. for $\left[\mathrm{C}_{22} \mathrm{H}_{13} \mathrm{NO}_{5}+\mathrm{H}\right]^{+}: 372.0867$, obsd.: 372.0865 . 
General procedure for the reduction of nitroaryl groups. Nitro-chromone or nitrophenyl chromone (1 equiv.) was dissolved in $\mathrm{AcOH}\left(10 \%\right.$ in $\left.\mathrm{H}_{2} \mathrm{O}, 5 \mathrm{~mL} / \mathrm{mmol}\right)$ and stirred under argon. Fe $(1 / 1, w t / w t)$ was then added into the reaction flask before the reaction was heated to reflux for $12 \mathrm{~h}$, after which TLC analysis showed the disappearance of starting materials. The reaction was filtered and rinsed with hot $\mathrm{EtOH}$, and the filtrate was concentrated in vacuo to give a brown solid, which was purified using silica gel column chromatography (petroleum ether/EtOAc, $9 / 1 \rightarrow 1 / 1, \mathrm{v} / \mathrm{v}$ ) to yield the desired aminochromones.<smiles>CCOC(=O)c1cc(=O)c2cc(N)ccc2o1</smiles>

Ethyl-6-amino-4-oxo- $4 \mathrm{H}$-chromene-2-carboxylate (168). By subjecting nitrochromone 156 (962 mg, $3.65 \mathrm{mmol})$, iron powder $(962 \mathrm{mg})$ and $\mathrm{AcOH}\left(10 \%\right.$ in $\left.\mathrm{H}_{2} \mathrm{O}, 15 \mathrm{~mL}\right)$ to the general procedure for the reduction of nitroaryl groups, the title compound was isolated as a white amorphous solid (621 $\mathrm{mg}, 73 \%) ; \mathrm{R}_{f}=0.23$ $\left(\mathrm{CH}_{2} \mathrm{Cl}_{2} / \mathrm{EtOAc}, 2 / 1, \mathrm{v} / \mathrm{v}\right)$; IR (U $\left.\max \right): 3453,3367,3248,1728,1633,1605,1489,1473,1276$, 1252, $1208 \mathrm{~cm}^{-1} ;{ }^{1} \mathrm{H}$ NMR (500 MHz, $\mathrm{CDCl}_{3}$ ): $\delta$ ppm 7.44 (d, $\left.J_{7,8}=8.8 \mathrm{~Hz}, 1 \mathrm{H}, \mathrm{H} 8\right), 7.34$ $\left(\mathrm{d}, J_{5,7}=1.3 \mathrm{~Hz}, 1 \mathrm{H}, \mathrm{H} 5\right), 7.08$ (d, $\left.J_{7,8}=8.9 \mathrm{~Hz}, 1 \mathrm{H}, \mathrm{H} 7\right), 7.04$ (s, 1H, H3), 4.44 (q, $J_{\mathrm{CH} 2, \mathrm{CH} 3}$ $=7.1 \mathrm{~Hz}, 2 \mathrm{H}, \mathrm{CH}_{2}$ ), 3.98 (br. s, $\left.2 \mathrm{H}, \mathrm{NH}_{2}\right), 1.42$ (t, $\left.J_{\mathrm{CH} 2, \mathrm{CH} 3}=7.2 \mathrm{~Hz}, 3 \mathrm{H}, \mathrm{CH}_{3}\right) ;{ }^{13} \mathrm{C} \mathrm{NMR}$ (125 MHz, $\left.\mathrm{CDCl}_{3}\right)$ : $\delta$ ppm 178.6 (C4), 160.9 (C1'), 151.9 (C2), 149.7 (C8a), 144.8 (C6), 125.5 (C4a), 123.3 (C7), 120.0 (C8), 113.7 (C3), 107.4 (C5), $63.0\left(\mathrm{CH}_{2}\right), 14.2\left(\mathrm{CH}_{3}\right)$; HRMS-ESI $(m / z)$ calcd. for $\left[\mathrm{C}_{12} \mathrm{H}_{11} \mathrm{NO}_{4}+\mathrm{H}\right]^{+}: 234.0761$, obsd.: 234.0762 . The data obtained for this compound matched those reported literature. ${ }^{286}$<smiles>Nc1ccc2oc(C3CC3)cc(=O)c2c1</smiles>

2-Cyclopropyl-6-amino-4H-chromen-4-one (169). Ву subjecting nitrochromone $158(83.5 \mathrm{mg}, 0.361 \mathrm{mmol})$, iron powder $(83.5 \mathrm{mg})$ and $\mathrm{AcOH}(10 \%, 2 \mathrm{~mL})$ to the general procedure for the reduction of nitroaryl groups, the title compound was isolated as a white amorphous solid (37.0 $\mathrm{mg}, 51 \%) ; \mathrm{R}_{f}=0.3$ $\left(\mathrm{CH}_{2} \mathrm{Cl}_{2} / \mathrm{EtOAc}, 4 / 1, \mathrm{v} / \mathrm{v}\right)$; IR (umax): 3333, 3221, 1622, 1574, 1488, 1459, 1422, 1395, 1305, $1201,1148,1072,1053,1023,998,927,838,797,748,731,711,635,586,533,441 \mathrm{~cm}^{-1} ;{ }^{1} \mathrm{H}$ $\operatorname{NMR}\left(500 \mathrm{MHz}, \mathrm{CDCl}_{3}\right): \delta \mathrm{ppm} 7.35$ (d, $\left.J_{5,7}=2.8 \mathrm{~Hz}, 1 \mathrm{H}, \mathrm{H} 5\right), 7.18\left(\mathrm{~d}, J_{7,8}=8.9 \mathrm{~Hz}, 1 \mathrm{H}\right.$, H8), $6.96\left(\mathrm{dd}, J_{7,8}=8.9 \mathrm{~Hz}, J_{5,7}=2.8 \mathrm{~Hz}, 1 \mathrm{H}, \mathrm{H} 7\right), 6.14$ (s, 1H, H3), 1.88-1.83 (m, 1H, C1'), 1.14-1.11 (m, 2H, C2'a), 1.07-1.04 (m, 2H, C2'b); $\left.{ }^{13} \mathrm{C} \mathrm{NMR} \mathrm{(125} \mathrm{MHz,} \mathrm{CDCl}_{3}\right): \delta$ ppm 177.9 (C4), 170.0 (C2), 149.9 (C8a), 143.8 (C6), 124.7 (C4a), 121.7 (C7), 118.6 (C8), 108.3 (C5), 
107.1 (C3), 14.7 (C1'), 8.6 (C2'); HRMS-ESI ( $m / z)$ calcd. for $\left[\mathrm{C}_{12} \mathrm{H}_{11} \mathrm{NO}_{2}+\mathrm{H}\right]^{+}$: 202.0863, obsd.: 202.0864.<smiles>Nc1ccc2oc(-c3ccccc3)c(C(=O)c3ccccc3)c(=O)c2c1</smiles>

6-Amino-3-benzoyl-2-phenyl-4H-chromen-4-one (171). Ву subjecting nitrochromone 166 (20 mg, $0.05 \mathrm{mmol}$ ), iron powder $(20 \mathrm{mg})$ and $\mathrm{AcOH}(10 \%, 0.26 \mathrm{~mL})$ to the general procedure for the reduction of nitroaryl groups, the title compound was isolated as a white amorphous solid (15.9 mg, 93\%); $\mathrm{R}_{f}=0.21\left(\mathrm{CH}_{2} \mathrm{Cl}_{2} / \mathrm{EtOAc}, 4 / 1, \mathrm{v} / \mathrm{v}\right)$;

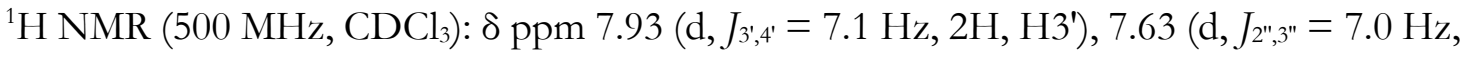

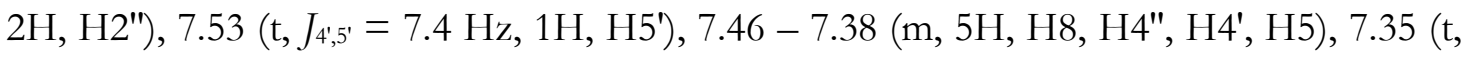
$\left.J_{2^{\prime \prime}, 3^{\prime \prime}}=J_{3^{\prime \prime}, 4^{\prime \prime}}=7.5 \mathrm{~Hz}, 2 \mathrm{H}, \mathrm{H} 3 "\right), 7.11$ (dd, J = 8.9 Hz, 2.9 Hz, 1H, H7); ${ }^{13} \mathrm{C}$ NMR (125 MHz, $\mathrm{CDCl}_{3}$ ): $\delta$ ppm 194.1 (C1'), 176.4 (C4), 149.8 (C6), 137.19 (C8a), 137.16 (C2') 133.6 (C5'), 132.1 (C1"), 131.2 (C4"), 129.5 (C3'), 128.7 (C4'), 128.5 (C2", C3"), 124.14 (C4a), 122.7 (C7), 119.1 (C8), 108.1 (C5); HRMS-ESI (m/z) calcd. for $\left[\mathrm{C}_{22} \mathrm{H}_{5} \mathrm{NO}_{3}+\mathrm{H}\right]^{+}: 342.1125$, obsd.: 342.1121.

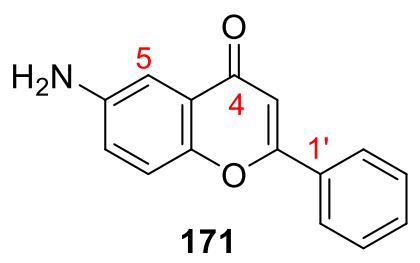

2-Phenyl-6-amino-4H-chromen-one (171). By subjecting nitrochromone 165 (100.4 mg, $0.38 \mathrm{mmol}$ ), iron powder (100.4 $\mathrm{mg})$ and $\mathrm{AcOH}(10 \%, 2 \mathrm{~mL})$ to the general procedure for the reduction of nitroaryl groups, the title compound was isolated as a white amorphous solid (44.2 mg, 49\%); $\mathrm{R}_{f}=0.1\left(\mathrm{CH}_{2} \mathrm{Cl}_{2} / \mathrm{EtOAc}, 4 / 1, \mathrm{v} / \mathrm{v}\right) ; \mathrm{IR}\left(\mathrm{U}_{\max }\right)$ : 3319, 3212, 2917, 2849, 1614, 1566, 1484, 1450, 1361, 1119, 772, $687 \mathrm{~cm}^{-1}$; ${ }^{1} \mathrm{H}$ NMR (500 $\left.\mathrm{MHz}, \mathrm{CDCl}_{3}\right): \delta$ ppm 7.90-7.88 (m, 2H, H2'), 7.51-7.49 (m, 3H, H2', H4'), 7.42 (d, J5,7 = 2.9 $\mathrm{Hz}, 1 \mathrm{H}, \mathrm{H} 5), 7.40$ (d, $\left.J_{7,8}=8.7 \mathrm{~Hz}, 1 \mathrm{H}, \mathrm{H} 8\right), 7.05$ (dd, $\left.J_{5,7}=2.9 \mathrm{~Hz}, J_{7,8}=8.9 \mathrm{~Hz}, 1 \mathrm{H}, \mathrm{H} 7\right)$, 6.77 (s, 1H, H3), 3.93 (br. s, 2H, NH ); ${ }^{13} \mathrm{C}$ NMR (125 MHz, $\mathrm{CDCl}_{3}$ ): $\delta$ ppm 178.6 (C4), 163.1 (C2), 150.1 (C6), 144.2 (C8a), 132.2 (C1'), 131.4 (C4'), 129.0 (C2'), 126.3 (C3'), 124.8 (C4a), 122.4 (C7), 119.2 (C8), 108.0 (C5), 106.8 (C3); HRMS-ESI (m/ v) calcd. for $\left[\mathrm{C}_{15} \mathrm{H}_{11} \mathrm{NO}_{2}+\mathrm{H}\right]^{+}:$238.0863, obsd.: 238.0868. The data obtained for this compound were consistent with those reported in literature. ${ }^{304}$ 
<smiles>CC(=O)c1[c-]c(N)ccc1O</smiles>

189

5'-Amino-2'-hydroxy-acetophenone (189). By subjecting acetophenone $163(650 \mathrm{mg}, 3.59 \mathrm{mmol})$, iron powder $(650 \mathrm{mg})$ and $\mathrm{AcOH}(10 \%, 18 \mathrm{~mL})$ to the general procedure for the reduction of nitroaryl groups, the title compound was isolated as a white amorphous solid (211.6 mg, 39\%); $\mathrm{R}_{f}=$ 0.20 (EtOAc/petroleum ether, 1/1, v/v); m.p.: $104.6^{\circ} \mathrm{C}$; IR (U $\left.\max \right): 3437,2953,2853,1637$, 1608, 1487, 1369, 1329, 1264, 1134, 967, 788, 725, 639, $521 \mathrm{~cm}^{-1} ;{ }^{1} \mathrm{H}$ NMR $\left(300 \mathrm{MHz}, \mathrm{CDCl}_{3}\right)$ : $\delta$ ppm 11.72 (br. s, $1 \mathrm{H}, \mathrm{OH}), 7.06\left(\mathrm{~d}, J_{4^{\prime}, 6^{\prime}}=2.5 \mathrm{~Hz}, 1 \mathrm{H}, \mathrm{H} 6^{\prime}\right), 6.90\left(\mathrm{dd}, J_{3^{\prime}, 4^{\prime}}=8.8 \mathrm{~Hz}, J_{4^{\prime}, 6^{\prime}}=2.5\right.$ $\left.\mathrm{Hz}, 1 \mathrm{H}, \mathrm{H} 4^{\prime}\right), 6.82$ (d, $\left.J_{3^{\prime}, 4^{\prime}}=8.6 \mathrm{~Hz}, 1 \mathrm{H}, \mathrm{H} 3^{\prime}\right), 2.61$ (s, 3H, H2); HRMS-ESI $(m / 2)$ calcd. for $\left[\mathrm{C}_{8} \mathrm{H}_{9} \mathrm{NO}_{2}+\mathrm{H}\right]^{+}:$152.0706, obsd.: 152.0700 .

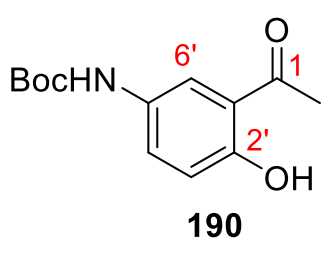

2'-Hydroxy-5'-(tert-butyloxycarbonyl)amino-acetophenone (190). A solution of $\mathrm{NaHCO}_{3}\left(485 \mathrm{mg}, 5.8 \mathrm{mmol}, 2.5\right.$ equiv.) in $\mathrm{H}_{2} \mathrm{O}(8.1 \mathrm{~mL})$ was added to a solution of acetophenone $189(350 \mathrm{mg}, 2.3 \mathrm{mmol})$ in THF (3.5 mL). Di-tert-butyl dicarbonate ( $0.72 \mathrm{~mL}, 3.5 \mathrm{mmol}$, 1.5 equiv.) was then added to the reaction and the reaction was left to stir for $12 \mathrm{~h}$, after which time TLC analysis showed the disappearance of starting materials. The solution was then concentrated in vacuo before it was dissolved in EtOAc and washed with $\mathrm{H}_{2} \mathrm{O}$. The organic layers were then dried with anhydrous $\mathrm{MgSO}_{4}$, filtered, and the filtrate concentrated in vacuo. Purification by silica gel flash column chromatography (petroleum ether/EtOAc, $1 / 0 \rightarrow 4 / 1$, v/v) gave the title compound as a white solid (514 mg, 89\%). $\mathrm{R}_{f}=0.24$ (petroleum ether/EtOAc, 9/1, v/v); IR (Umax $): 3354,2920,1710,1635,1589 ; 1437,1347,1301,1213,1025,827,749,615,508 \mathrm{~cm}^{-1}$;

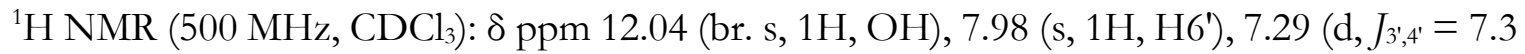

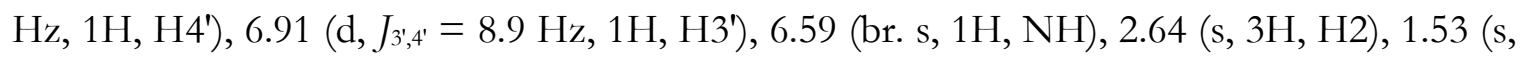
9H, $\left.{ }^{\mathrm{Bu}}-\mathrm{CH}_{3}\right) ;{ }^{13} \mathrm{C} \mathrm{NMR}\left(125 \mathrm{MHz}, \mathrm{CDCl}_{3}\right)$ : $\delta$ ppm 204.5 (C1), 158.4 (C2'), 153.19, $129.8\left(\mathrm{C}^{\prime}\right)$, 128.2 (C4'), 120.7 (C6'), 119.2 (1'), 118.7 (C3'), 80.8 ('Bu-Cq), 28.4 ('Bu-CH3), 26.8 (C2); HRMSESI $(m / q)$ calcd. for $\left[\mathrm{C}_{13} \mathrm{H}_{17} \mathrm{NO}_{4}-\mathrm{H}\right]: 250.1085$, obsd.: 250.1085 .

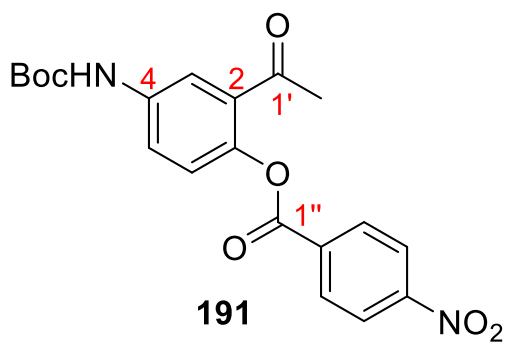

2-Acetyl-4-((tert-butoxycarbonyl)amino)phenyl-4nitrobenzoate (191). Acetophenone 190 (55 mg, 0.22 mmol) and 4-nitrobenzoic acid $(73.5 \mathrm{mg}, 0.44 \mathrm{mmol}, 2$ equiv.) were dissolved in $\mathrm{CH}_{2} \mathrm{Cl}_{2}(1.1 \mathrm{~mL})$ under argon before EDCI (105.4 mg, $0.55 \mathrm{mmol}, 2.5$ equiv.) and DMAP ( $27 \mathrm{mg}, 0.22$, 1 equiv.) were added. The mixture was stirred at room temperature for $12 \mathrm{~h}$, after 
which time TLC analysis showed the disappearance of the starting material. The reaction was then quenched by the addition of $\mathrm{NaHCO}_{3}$, followed by dilution with $\mathrm{CH}_{2} \mathrm{Cl}_{2}$. The resulting solution was then stirred for 10 minutes before the product was extracted using EtOAc and washed with $\mathrm{H}_{2} \mathrm{O}$ and brine. The organic layers were then dried with anhydrous $\mathrm{MgSO}_{4}$, which was filtered and the filtrate concentrated in vacuo. Purification by silica gel flash column chromatography (petroleum ether/EtOAc, $1 / 0 \rightarrow 4 / 1, \mathrm{v} / \mathrm{v}$ ) gave the title compound as white crystals (69 mg, 78\%). $\mathrm{R}_{f}=0.29$ (petroleum ether/EtOAc, 4/1, v/v); m.p: $149.8^{\circ} \mathrm{C}$; IR ( $\mathrm{u}_{\max }$ ): 3370, 1737, 1721, 1688, 1591, 1522, 1404, 1350, 1280, 1199, 1150, 1052, 1012, 852, 825, 775, 712, 622, $498 \mathrm{~cm}^{-1}$; ${ }^{1} \mathrm{H}$ NMR (500 MHz, $\mathrm{CDCl}_{3}$ ): $\delta$ ppm 8.39 (s, 4H, H3", H4"), 8.04 (s, $1 \mathrm{H}$, H3), 7.56 (d, $\left.J_{5,6}=8.0 \mathrm{~Hz}, 1 \mathrm{H}, \mathrm{H} 5\right), 7.19$ (d, $J_{5,6}=8.3 \mathrm{~Hz}, 1 \mathrm{H}, \mathrm{H} 6$ ), 6.69 (br. s, 1H, NH), 2.57 (s, 3H, H2'), 1.56 (s, 9H, $\left.{ }^{\mathrm{B} u}-\mathrm{CH}_{3}\right) ;{ }^{13} \mathrm{C} \mathrm{NMR}\left(125 \mathrm{MHz}, \mathrm{CDCl}_{3}\right): \delta$ ppm $197.2(\mathrm{C} 1), 163.8$ (C1"), 150.9 (C5"), 143.8 (C1), 137.0 (C4), 134.9 (C2"), 131.4 (C3"/C4"), 130.4 (C2), 124.3 (C6), 123.8 (C5), 120.2 (C3), 81.3 ('Bu Cq), 29.2 (C2'), 28.3 ('Bu CH3); HRMS-ESI ( $m /$ z) calcd. for $\left[\mathrm{C}_{20} \mathrm{H}_{20} \mathrm{~N}_{2} \mathrm{O}_{7}+\mathrm{NH}_{4}\right]^{+}$: 418.1609, obsd.: 418.1609 .

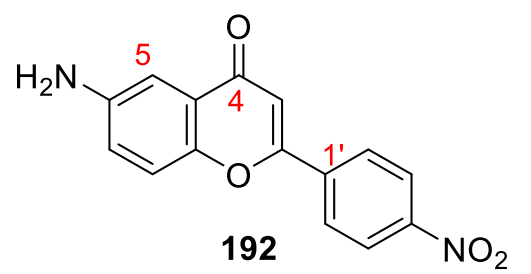

6-Amino-2-(4'-nitrophenyl)-4H-chromen-4-one (192). Ester 191 (86.5 mg, $0.22 \mathrm{mmol})$ was dissolved in pyridine (0.74 mL) and $\mathrm{KOH}(18.5 \mathrm{mg}, 0.33 \mathrm{mmol}, 1.5$ equiv.) under argon was added before the solution was warmed at $60^{\circ} \mathrm{C}$. After two hours, the reaction was neutralised by the addition of $2 \mathrm{M}$ aq. $\mathrm{HCl}$. The product was then extracted with EtOAc and dried with anhydrous $\mathrm{MgSO}_{4}$, which was filtered off and the filtrate concentrated in vacuo. The crude product was then dissolved in $\mathrm{AcOH}(0.74 \mathrm{~mL})$ before $\mathrm{H}_{2} \mathrm{SO}_{4}(0.01 \mathrm{~mL})$ was added and the solution warmed to reflux. After $1 \mathrm{~h}$, the reaction was cooled to room temperature, diluted with EtOAc and washed with sat. aq. $\mathrm{NaHCO}_{3}$. The organic layers were then dried with anhydrous $\mathrm{MgSO}_{4}$, which was filtered off and the filtrate concentrated in vacuo. Purification with silica gel flash column chromatography (petroleum ether/EtOAc, $1 / 0 \rightarrow 1 / 1, \mathrm{v} / \mathrm{v}$ ) gave the title compound as white crystals (46.6 mg, 75\%). $\mathrm{R}_{f}$ $=0.20$ (petroleum ether/EtOAc, 1/1, v/v); m.p.: $222.4^{\circ} \mathrm{C}$; IR $\left(\mathrm{u}_{\max }\right): 3338,3230,2918,2849$, 1620, 1606, 1571, 1540, 1518, 1494, 1485, 1466, 1413, 1372, 1341, 1321, 1297, 1256, 1209, 1137, 1107, 1078, 1041, 1012, 911, 846, 813, 768, 760, 752, 691, 651, 608, $550 \mathrm{~cm}^{-1}$; ${ }^{1} \mathrm{H}$ NMR $\left(500 \mathrm{MHz}, \mathrm{CDCl}_{3}\right): \delta \mathrm{ppm} 8.40\left(\mathrm{~d}, J_{2^{\prime}, 3^{\prime}}=8.7 \mathrm{~Hz}, 2 \mathrm{H}, \mathrm{H} 2^{\prime}\right), 8.11\left(\mathrm{~d}, J_{2^{\prime}, 3^{\prime}}=8.7 \mathrm{~Hz}, 2 \mathrm{H}, \mathrm{H} 3^{\prime}\right)$, $7.46\left(\mathrm{~d}, J_{7,8}=8.8 \mathrm{~Hz}, 1 \mathrm{H}, \mathrm{H} 8\right), 7.42\left(\mathrm{~d}, J_{5,7}=2.7 \mathrm{~Hz}, 1 \mathrm{H}, \mathrm{H} 5\right), 7.11$ (d, $J_{7,8}=8.8 \mathrm{~Hz}, J_{5,7}=2.8$ $\mathrm{Hz}, 1 \mathrm{H}, \mathrm{H} 7), 6.87$ (s, 1H, H3), 3.95 (s, 2H, NH 2$) ;{ }^{13} \mathrm{C}$ NMR (125 MHz, $\mathrm{CDCl}_{3}$ ): $\delta$ ppm 178.1 (C4), 160.1 (C2), 149.9 (C6), 149.3 (C4'), 144.5 (C8a), 138.0 (C1'), 127.1 (C3'), 124.8 (C4a), 
124.2 (C2'), 122.7 (C7), 119.2 (C8), 108.6 (C3), 107.8 (C5); HRMS-ESI (m/g) calcd. for $\left[\mathrm{C}_{15} \mathrm{H}_{10} \mathrm{~N}_{2} \mathrm{O}_{4}+\mathrm{H}\right]^{+}:$283.0713, obsd.: 283.0713.

General procedure for the synthesis of chromonyl-pyrimidines. Chloropyrimidine (1 equiv.) and aminochromone (1 equiv.) were co-evaporated three times in dry toluene to remove traces of water before being dissolved in dry 1,4-dioxane or 2-ethoxyethanol (5 $\mathrm{mL} / \mathrm{mmol}$ ). Concentrated $\mathrm{HCl}$ or $\mathrm{HBF}_{4}$ (0.5 equiv.) was then added, and the reaction was stirred under reflux for 22 hours. The precipitate was filtered and washed with $\mathrm{Et}_{2} \mathrm{O}$ to give the title compound as a yellow solid.<smiles></smiles>

Ethyl 6-((2"-amino-6"--methylpyrimidin-4"yl)amino)-4-oxo-4H-chromene-2-carboxylate) (172). By subjecting chloropyrimidine $150(10 \mathrm{mg}$, $0.07 \mathrm{mmol})$ and aminochromone $168(16 \mathrm{mg}, 0.07$ $\mathrm{mmol})$ in conc. $\mathrm{HCl}(0.1 \mu \mathrm{L})$ and dioxane $(0.7 \mathrm{~mL})$ to the general procedure for the synthesis of chromonyl-pyrimidines, the title compound was isolated as a yellow amorphous solid (20.7 mg, 87\%); $\mathrm{R}_{f}=0.14(\mathrm{EtOAc} / \mathrm{EtOH}, 4 / 1, \mathrm{v} / \mathrm{v}) ; \mathrm{IR}\left(\mathrm{U}_{\max }\right): 3325,1682,1638,1604$, 1530, 1484, 1392, 1250, 1013, 959, 866, 805, 778, 616, 531, $450 \mathrm{~cm}^{-1} ;{ }^{1} \mathrm{H}$ NMR $(500 \mathrm{MHz}$, pyridine-d $\mathrm{d}_{5}$ ): $\delta$ ppm 10.39 (br. s, $\left.1 \mathrm{H}, \mathrm{NH}\right), 8.79$ (d, $\left.J_{5,7}=2.7 \mathrm{~Hz}, 1 \mathrm{H}, \mathrm{H} 5\right), 8.59$ (dd, $J_{7,8}=9.2$, $\left.J_{5,7}=2.7 \mathrm{~Hz}, 1 \mathrm{H}, \mathrm{H} 7\right), 7.53$ (d, $\left.J_{7,8}=9.1 \mathrm{~Hz}, 1 \mathrm{H}, \mathrm{H} 8\right), 7.24$ (s, 1H, H3), 6.21 (s, 1H, H5"), $4.36\left(\mathrm{q}, J_{\mathrm{CH} 2-\mathrm{CH} 3}=7.1 \mathrm{~Hz}, 2 \mathrm{H}, \mathrm{CH}_{2}\right), 2.25\left(\mathrm{~s}, 3 \mathrm{H}, 1^{\prime \prime \prime}\right), 1.24\left(\mathrm{t}, J_{\mathrm{CH} 2-\mathrm{CH} 3}=7.1 \mathrm{~Hz}, 3 \mathrm{H}, \mathrm{CH}_{3}\right)$; ${ }^{13} \mathrm{C}$ NMR (125 MHz, pyridine-d $\mathrm{d}_{5}$ ): $\delta$ ppm 176.3 (C4), 165.4 (C6"), 162.7 (C2"), 160.7 (C4"), 159.1 (C1'), 150.7 (C2), 149.5 (C8a), 138.5 (C6), 126.1 (C7), 123.6 (C4a), 117.6 (C8), 112.2 (C3), 112.1 (C5), 94.2 (C5"), $61.2\left(\mathrm{CH}_{2}\right), 22.4$ (C1"'), $12.3\left(\mathrm{CH}_{3}\right)$; HRMS-ESI (m/z) calcd. for $\left[\mathrm{C}_{17} \mathrm{H}_{16} \mathrm{~N}_{4} \mathrm{O}_{4}+\mathrm{H}\right]^{+}$: 341.1244, obsd.: 341.1242 .

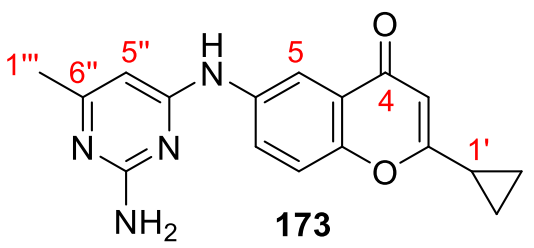

\section{6-((2"'Amino-6"'-methylpyrimidin-4"'-yl)amino)-} 2-cyclopropyl-4H-chromen-4-one (173). Ву subjecting chloropyrimidine $150(21.5 \mathrm{mg}, 0.15 \mathrm{mmol})$ and aminochromone 169 (20.1 $\mathrm{mg}, 0.07 \mathrm{mmol})$ in conc. $\mathrm{HBF}_{4}(0.1 \mu \mathrm{L})$ and dioxane $(0.7 \mathrm{~mL})$ to the general procedure for the synthesis of chromonyl-pyrimidines, the title compound was isolated as a yellow amorphous solid (8.4 
mg, 39\%); $\mathrm{R}_{f}=0.10$ (EtOAc/MeOH, 4/1, v/v); IR (Umax): 3316, 1567, 1481, 1435, 1365, 1205, 1057, 961, $813 \mathrm{~cm}^{-1} ;{ }^{1} \mathrm{H}$ NMR (500 MHz, CD $\left.3 \mathrm{OD}\right): \delta$ ppm 8.70 (d, $J_{5,7}=2.7 \mathrm{~Hz}, 1 \mathrm{H}$, H5), 7.79 (dd, $\left.J_{7,8}=9.0 \mathrm{~Hz}, J_{5,7}=2.8 \mathrm{~Hz}, 1 \mathrm{H}, \mathrm{H} 7\right), 7.42$ (d, $\left.J_{7,8}=9.0 \mathrm{~Hz}, 1 \mathrm{H}, \mathrm{H} 8\right), 6.28$ (s, 1H, H3), 5.97 (s, 1H, H5"), 2.21 (s, 3H, H1'"), 2.07-2.03 (m, 1H, C1'), 1.22-1.17 (m, 4H, $\left.\mathrm{C}^{\prime}\right) ;{ }^{13} \mathrm{C}$ NMR (125 MHz, CD $\left.{ }_{3} \mathrm{OD}\right): \delta$ ppm 178.7 (C4), 172.0 (C2), 163.0 (C6"), 161.7 (C4"), 151.7 (C8a), 137.7 (C6), 126.2 (C7), 123.1 (C4a), 117.9 (C8), 113.5 (C5), 106.3 (C3), 95.8 (C5"), 21.1 (C1"'), $14.0\left(\mathrm{C}^{\prime}\right), 8.12$ (C2'); HRMS-ESI ( $\left.\mathrm{m} / \mathrm{z}\right)$ calcd. for $\left[\mathrm{C}_{17} \mathrm{H}_{16} \mathrm{~N}_{4} \mathrm{O}_{2}+\mathrm{H}\right]$ : 309.1346, obsd.: 309.1349 .

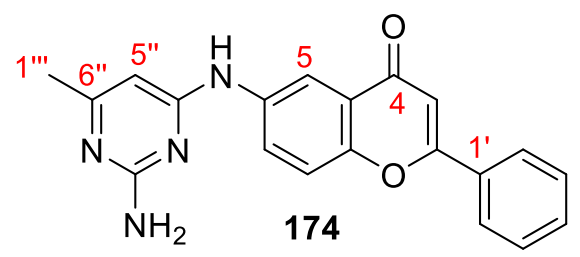

6-((2"-Amino-6"'-methylpyrimidin-4"'yl)amino)-2-phenyl-4H-chromen-4-one (70). By subjecting chloropyrimidine $150(13.6 \mathrm{mg}, 0.10$ $\mathrm{mmol}$ ) and aminochromone 170 (22.5 $\mathrm{mg}, 0.10$ $\mathrm{mmol})$ in conc. $\mathrm{HCl}(0.2 \mu \mathrm{L})$ and dioxane $(0.5 \mathrm{~mL})$ to the general procedure for the synthesis of chromonyl-pyrimidines, the title compound was isolated as a yellow amorphous solid (15.2 mg, 44\%); $\mathrm{R}_{f}=0.79$ (1/1, $\mathrm{CH}_{2} \mathrm{Cl}_{2} /$ EtOAc, v/v); IR (U $\left.\max \right): 1738,1591,1484,1452$, 1363, 995, 774, $601 \mathrm{~cm}^{-1}$; ${ }^{1} \mathrm{H}$ NMR (500 MHz, pyridine-d $)_{5}$ : $\delta$ ppm 10.35 (br. s, $1 \mathrm{H}, \mathrm{NH}$ ), $8.86\left(\mathrm{~d}, J_{5,7}=2.7 \mathrm{~Hz}, 1 \mathrm{H}, \mathrm{H} 5\right), 8.59\left(\mathrm{dd}, J_{7,8}=9.0 \mathrm{~Hz}, J_{5,7}=2.7 \mathrm{~Hz}, 1 \mathrm{H}, \mathrm{H} 7\right), 8.00-7.98(\mathrm{~m}$, 2H, H3'), 7.60 (d, J7,8 = 9.0 H, 1H, H8), 7.51-7.48 (m, 3H, H4', H2'), 7.15 (s, 1H, H3), 7.06 (br. s, 2H, NH 2 ), 6.25 (s, 1H, H5"), 2.26 (s, 3H, H1"'); ${ }^{13} \mathrm{C}$ NMR (125 MHz, pyridine-d $\mathrm{d}_{5}$ ): $\delta$ ppm 177.6 (C4), 166.8 (C4"), 164.3 (C2"), 162.7 (C2), 162.4 (C6"), 151.5 (C6), 139.3 (C8a), 132.2 (C1'), 131.4 (C4'), 129.1 (C2'), 127.0 (C7), 126.4 (C3'), 124.7 (C4a), 118.7 (C8), 114.3 (C5), 107.2 (C3), 95.5 (C5"), 23.9 (C1"'); HRMS-ESI (m/z) calcd. for $\left[\mathrm{C}_{20} \mathrm{H}_{16} \mathrm{~N}_{4} \mathrm{O}_{2}+\mathrm{H}\right]^{+}$: 345.1346, obsd.: 345.1378 .

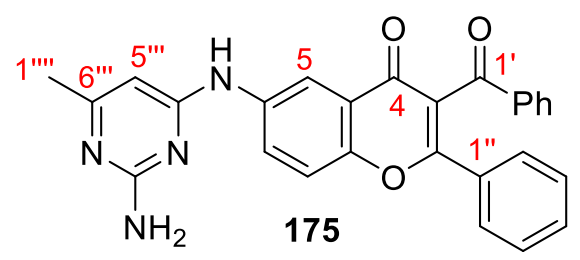

6-((2"-Amino-6"-methylpyrimidin-4"'yl)amino)-3-benzoyl-2-phenyl-4H-chromen-4one (175). By subjecting chloropyrimidine 150 (7.0 $\mathrm{mg}, 0.05 \mathrm{mmol})$ and aminochromone $171(17.3 \mathrm{mg}$, $0.05 \mathrm{mmol})$ in conc. $\mathrm{HCl}(0.2 \mu \mathrm{L})$ and dioxane $(0.25 \mathrm{~mL})$ to the general procedure for the synthesis of chromonyl-pyrimidines, the title compound was isolated as a yellow amorphous solid (4.9 mg, 22\%); $\mathrm{R}_{f}=0.54(\mathrm{EtOAc} / \mathrm{MeOH}, 9 / 1, \mathrm{v} / \mathrm{v}) ; \mathrm{IR}\left(\mathrm{U}_{\max }\right): 3321,1619$, 
$1578,1554,1516,1484,1446,1384,1357,1311,1257,1234,1203,1176,892,769,689 \mathrm{~cm}$

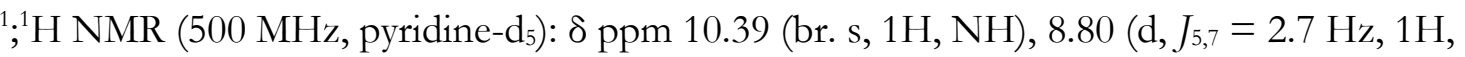
H5), 8.67 (dd, $\left.J_{7,8}=9.2 \mathrm{~Hz}, 2.8 \mathrm{~Hz}, 1 \mathrm{H}, \mathrm{H} 7\right), 8.21$ (d, $\left.J_{3^{\prime}, 4^{\prime}}=8.1 \mathrm{~Hz}, 2 \mathrm{H}, \mathrm{H} 3^{\prime}\right), 7.82$ (d, J2",3"

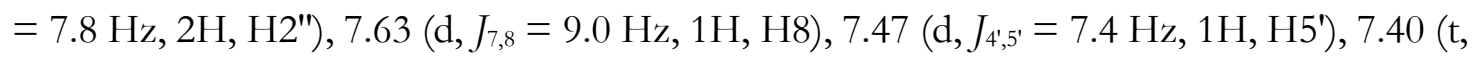
$\left.J_{3^{\prime}, 4^{\prime}}=J_{4^{\prime}, 5^{\prime}}=7.4 \mathrm{~Hz}, 2 \mathrm{H}, \mathrm{H} 4^{\prime}\right), 7.36-7.29$ (m, 3H, H3", H4"), 6.24 (s, 1H, H5'"), 2.26 (s, 3H, H1'"'); ${ }^{13} \mathrm{C}$ NMR (125 MHz, pyridine-d $\left.\mathrm{d}_{5}\right): \delta$ ppm 194.1 (C1'), 166.8 (C6"'), 163.0 (C2), 151.4 (C6), 139.7 (C8a), 137.8 (C2'), 133.8 (C5'), 132.5 (C1"), 131.3 (C4"), 129.6 (C3'), 129.0 (C4'), 128.9 (C3"), 128.7 (C2"), 127.4 (C7), 123.9 (C4a), 118.8 (C8), 114.1 (C5), 95.7 (C5"'), 23.8 (C1'"'); HRMS-ESI (m/z) calcd. for $\left[\mathrm{C}_{27} \mathrm{H}_{20} \mathrm{~N}_{4} \mathrm{O}_{3}+\mathrm{H}\right]^{+}$: 449.1608, obsd.: 449.1611.<smiles>[3H]C(Cl)[14c]1cc(Nc2ccc3oc(C(=O)OCC)cc(=O)c3c2)nc(N)n1</smiles>

Ethyl chloromethylpyrimidin-4"'-yl)-amino)-4-oxo4H-chromene-2-carboxylate (176). By subjecting chloropyrimidine 153 (32.1 $\mathrm{mg}, 0.18$ $\mathrm{mmol})$ and aminochromone $168(42 \mathrm{mg}, 0.18 \mathrm{mmol})$ in conc. $\mathrm{HCl}(0.2 \mu \mathrm{L})$ and dioxane $(3.6$ $\mathrm{mL}$ ) to the general procedure for the synthesis of chromonyl-pyrimidines, the title compound was isolated as a yellow amorphous solid (41.2 mg, 61\%); $\mathrm{R}_{f}=0.37$ (EtOAc); IR (U $\left.U_{\max }\right): 3334,1734,1638,1597,1485,1390,1303,1231,1100,956,871,842,777 \mathrm{~cm}^{-1} ;{ }^{1} \mathrm{H}$ NMR (500 MHz, pyridine- $\mathrm{d}_{5}$ ): $\delta$ ppm 10.65 (s, 1H, NH), 8.80 (d, J5,7 = 2.6 Hz, 1H, H5), $8.56\left(\mathrm{dd}, J_{7,8}=9.2 \mathrm{~Hz}, J_{5,7}=2.6 \mathrm{~Hz}, 1 \mathrm{H}, \mathrm{H} 7\right), 7.53$ (d, $\left.J_{7,8}=9.2 \mathrm{~Hz}, 1 \mathrm{H}, \mathrm{H} 8\right), 7.39$ (br. s,

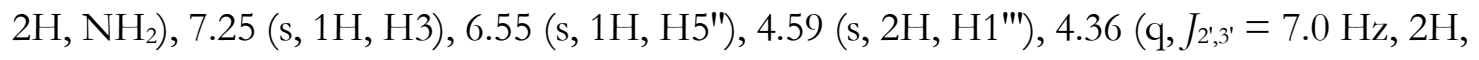
C2'), $1.24\left(\mathrm{t}, J_{2^{\prime}, 3^{\prime}}=7.1 \mathrm{~Hz}, 3 \mathrm{H}, \mathrm{C} 3^{\prime}\right) ;{ }^{13} \mathrm{C}$ NMR (125 MHz, pyridine-d $\left.\mathrm{d}_{5}\right): \delta$ ppm $177.8(\mathrm{C} 4)$, 164.6 (C6"), 162.6 (C4"), 160.4 (C1'), 152.2 (C2), 151.2 (C6), 139.6 (C8a), 127.7 (C7), 124.9 C4a), 119.1 (C8), 114.0 (C5), 113.7 (C3), 95.1 (C5"), 62.7 (C2'), 46.4 (C1"'), 13.6 (C3'); HRMS (ESI) calcd. for $\left[\mathrm{C}_{17} \mathrm{H}_{15} \mathrm{ClN}_{4} \mathrm{O}_{4}+\mathrm{H}\right]^{+}: 375.0855$, obsd.: 375.0859 .

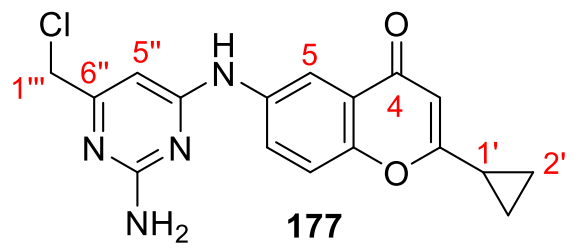

6-((2"-Amino-6"'-chloromethylpyrimidin-4"'yl)amino)-2-cyclopropyl-4H-chromen-4-one (177). By subjecting chloropyrimidine $153(30.7 \mathrm{mg}$, $0.17 \mathrm{mmol})$ and aminochromone $169(23.2 \mathrm{mg}, 0.12$ mmol) in conc. $\mathrm{HBF}_{4}(0.2 \mu \mathrm{L})$ and dioxane $(0.58 \mathrm{~mL})$ to the general procedure for the synthesis of chromonyl-pyrimidines, the title compound was isolated as a yellow amorphous solid (15.4 mg, 39\%); $\mathrm{R}_{f}=0.63$ (EtOAc); IR ( $\left.\cup_{\max }\right): 3388,1592,1486,1441$, 
$1375 \mathrm{~cm}^{-1} ;{ }^{1} \mathrm{H}$ NMR (500 MHz, $\left.\mathrm{CDCl}_{3} / \mathrm{CD}_{3} \mathrm{OD}\right): \delta \mathrm{ppm} 8.42$ (d, $\left.J_{5,7}=2.6 \mathrm{~Hz}, 1 \mathrm{H}, \mathrm{H} 5\right)$, $7.69\left(\mathrm{dd}, J_{7,8}=9.0 \mathrm{~Hz}, J_{5,7}=2.7 \mathrm{~Hz}, 1 \mathrm{H}, \mathrm{H} 7\right), 7.23$ (d, $\left.J_{7,8}=9.0 \mathrm{~Hz}, 1 \mathrm{H}, \mathrm{H} 8\right), 6.11(\mathrm{~s}, 1 \mathrm{H}$, H3), 6.06 (s, 1H, H5"), 4.15 (s, 2H, H1"'), 1.84-1.79 (m, 1H, C1'), 1.09-1.06 (m, 2H, C2'a), 1.03-0.99 (m, 2H, C2'b); ${ }^{13} \mathrm{C}$ NMR (125 MHz, $\left.\mathrm{CDCl}_{3} / \mathrm{CD}_{3} \mathrm{OD}\right): \delta$ ppm 178.8 (C4), 171.6 (C2), 163.0 (C6"), 151.8 (C6), 149.31 (C4"), 137.3 (C8a), 126.4 (C7), 123.4 (C4a), 118.1 (C8), 114.3 (C5), 106.8 (C3), 95.6 (C5"), 44.7 (C1'"), 14.6 (C1'), 8.8 (C2'); HRMS-ESI (m/ z) calcd. for $\left[\mathrm{C}_{17} \mathrm{H}_{15} \mathrm{ClN}_{4} \mathrm{O}_{2}+\mathrm{H}\right]^{+}$: 343.0956, obsd.: 343.0956.

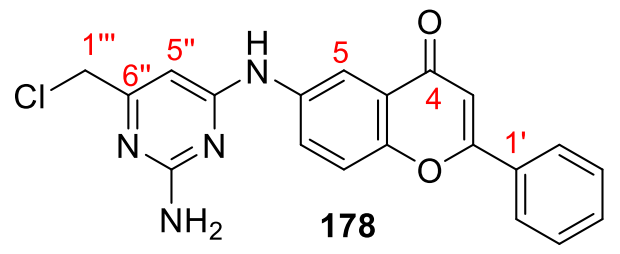

6-((2"'-Amino-6"'-chloromethylpyrimidin-4"'yl)amino)-2-phenyl-4H-chromen-4-one (178).

By subjecting chloropyrimidine $153(15.0 \mathrm{mg}, 0.09$ mmol) and aminochromone 170 (20.0 $\mathrm{mg}, 0.09$ $\mathrm{mmol})$ in conc. $\mathrm{HCl}(0.2 \mu \mathrm{L})$ and dioxane $(0.5 \mathrm{~mL})$ to the general procedure for the synthesis of chromonyl-pyrimidines, the title compound was isolated as a yellow amorphous solid (22.8 mg, 71\%); $\mathrm{R}_{f}=0.13\left(\mathrm{CH}_{2} \mathrm{Cl}_{2} / \mathrm{MeOH}, 1 / 1, \mathrm{v} / \mathrm{v}\right)$; IR (Umax): 1738, 1652, 1552, 1473 , 1427, 1263, 1155, 992, 775, 686, 470, $429 \mathrm{~cm}^{-1} ;{ }^{1} \mathrm{H}$ NMR (500 MHz, $\left.\mathrm{CDCl}_{3} / \mathrm{CD}_{3} \mathrm{OD}\right): \delta$ ppm 8.48 (s, 1H, H5), 7.95 - 7.85 (m, 3H, H7, H2'), 7.57 - 7.43 (m, 4H, H8, H3', H4'), 6.80 (s, 1H, H3), 6.17 (s, 1H, H5"), 4.25 (s, 2H, H1"'); ${ }^{13} \mathrm{C} \mathrm{NMR} \mathrm{(500} \mathrm{MHz,} \mathrm{CDCl}_{3} / \mathrm{CD}_{3} \mathrm{OD}$ ): $\delta$ ppm 179.5 (C4), 164.1 (C2), 163.4 (C4"), 152.0 (C6), 137.6 (C8a), 131.9 (C4'), 131.2 (C1'), 129.1 (C3'), 127.1 (C7), 126.4 (C2'), 123.6 (C4a), 118.7 (C8), 114.4 (C5), 106.5 (C3), 95.6 (C5"), 44.8 (C1"'); HRMS-ESI $(\mathrm{m} / \mathrm{q})$ calcd. for $\left[\mathrm{C}_{20} \mathrm{H}_{15} \mathrm{ClN}_{4} \mathrm{O}_{2}+\mathrm{H}\right]^{+}: 379.0956$, obsd.: 379.0959 .<smiles></smiles>

\section{Ethyl}

6-((2"'-amino-6"'methoxymethylpyrimidin-4"'-yl)-amino)-4oxo-4H-chromene-2-carboxylate (179). Вy subjecting chloropyrimidine $154(17.2 \mathrm{mg}, 0.10$ mmol) and aminochromone $168(23.3 \mathrm{mg}, 0.10 \mathrm{mmol})$ in conc. $\mathrm{HCl}(0.2 \mu \mathrm{L})$ and dioxane $(0.5 \mathrm{~mL})$ to the general procedure for the synthesis of chromonyl-pyrimidines, the title compound was isolated as a yellow amorphous solid (20 mg, 54\%); $\mathrm{R}_{f}=0.26$ (EtOAc/EtOH, 4/1, v/v); IR (U $\max ): 3326,1732,1606,1534,1483,1396,1250,1203,1126$, 1007, 950, 877, 826, 777, 648, $529 \mathrm{~cm}^{-1} ;{ }^{1} \mathrm{H}$ NMR (500 MHz, pyridine-d $\mathrm{d}_{5}$ ): $\delta$ ppm 10.54 (br. 
s, $1 \mathrm{H}, \mathrm{NH}), 8.82\left(\mathrm{~d}, J_{5,7}=2.7 \mathrm{~Hz}, 1 \mathrm{H}, \mathrm{H} 5\right), 8.60\left(\mathrm{dd}, J_{7,8}=9.2 \mathrm{~Hz}, J_{5,7}=2.7 \mathrm{~Hz}, 1 \mathrm{H}, \mathrm{H} 7\right)$, 7.53 (d, J7,8 $=9.2 \mathrm{~Hz}, 1 \mathrm{H}, \mathrm{H} 8), 7.24$ (s, 1H, H3), 6.60 (s, 1H, H5"), 4.46 (s, 2H, C1'"), 4.36 $\left(\mathrm{q}, J_{2^{\prime}, 3^{\prime}}=7.1 \mathrm{~Hz}, 2 \mathrm{H}, \mathrm{C} 2^{\prime}\right), 3.31$ (s, 3H, H2'"), $1.24\left(\mathrm{t}, J_{2^{\prime}, 3^{\prime}}=7.1 \mathrm{~Hz}, 3 \mathrm{H}, \mathrm{C} 3^{\prime}\right) ;{ }^{13} \mathrm{C}$ NMR $(125$ MHz, pyridine-d $\mathrm{d}_{5}$ ): $\delta$ ppm 178.4 (C4), 168.5 (C6"), 164.8 (C2"), 163.2 (C4"), 161.3 (C1'), 152.8 (C2), 151.8 (C6), 140.5 (C8a), 128.3 (C7), 125.8 (C4a), 119.8 (C8), 114.42 (C3), 114.39 (C5), 94.0 (C5"), 75.4 (C1'"), 63.4 (C2'), 23.9 (C2"'), 13.8 (C3'); HRMS-ESI (m/z) calcd. for $\left[\mathrm{C}_{18} \mathrm{H}_{18} \mathrm{~N}_{4} \mathrm{O}_{5}+\mathrm{H}\right]^{+}:$371.1350, obsd.: 371.1346 .

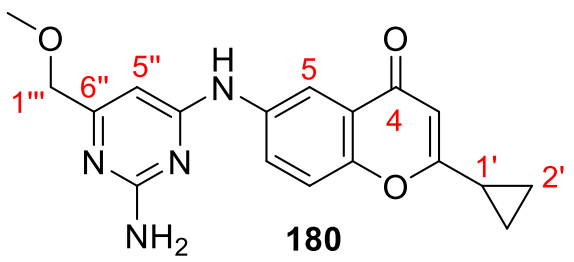

6-((2"-Amino-6"'-methoxymethylpyrimidin-4"yl)amino)-2-cyclopropyl-4H-chromen-4-one (180). By subjecting chloropyrimidine $154(31.3 \mathrm{mg}$, $0.18 \mathrm{mmol})$ and aminochromone $169(24.0 \mathrm{mg}, 0.12$ mmol) in conc. $\mathrm{HBF}_{4}(0.2 \mu \mathrm{L})$ and dioxane $(0.60 \mathrm{~mL})$ to the general procedure for the synthesis of chromonyl-pyrimidines, the title compound was isolated as a yellow amorphous solid (28.4 mg, 70\%); $\mathrm{R}_{f}=0.25(\mathrm{EtOAc} / \mathrm{MeOH}, 9 / 1, \mathrm{v} / \mathrm{v}) ; \mathrm{IR}\left(\mathrm{U}_{\max }\right): 1629$, 1588, 1542, 1522, 1477, 1449, 1340, 1329, 1300, 1195, 1116, 1055, 958, $820 \mathrm{~cm}^{-1} ;{ }^{1} \mathrm{H}$ NMR $\left(500 \mathrm{MHz}, \mathrm{CDCl}_{3} / \mathrm{CD}_{3} \mathrm{OD}\right): \delta \mathrm{ppm} 8.55$ (d, $\left.J_{5,7}=2.7 \mathrm{~Hz}, 1 \mathrm{H}, \mathrm{H} 5\right), 7.72\left(\mathrm{dd}, J_{7,8}=9.1 \mathrm{~Hz}\right.$, $\left.J_{5,7}=2.7 \mathrm{~Hz}, 1 \mathrm{H}, \mathrm{H} 7\right), 7.29$ (d, $\left.J_{7,8}=9.1 \mathrm{~Hz}, 1 \mathrm{H}, \mathrm{H} 8\right), 6.17$ (s, 1H, H3), 6.12 (s, 1H, H5"), 4.22 (s, 2H, H1'"), 3.38 (s, 3H, H2"'), 1.89-1.84 (m, 1H, H1'), 1.15-1.12 (m, 2H, H2'-a), 1.101.05 (m, 2H, H2'-b); ${ }^{13} \mathrm{C}$ NMR (125 MHz, $\mathrm{CDCl}_{3} / \mathrm{CD}_{3} \mathrm{OD}$ ): $\delta$ ppm 178.7 (C4), 171.8 (C2), 161.62 (C4"), 158.3 (C6"), 152.4 (C6), 136.4 (C8a), 126.7 (C7), 123.4 (C4a), 118.4 (C8), 115.1 (C5), 107.0 (C3), 95.3 (C-5'), 71.2 (C1"'), 58.9 (C2"'), 14.6 (C1'), 9.1 (C2'); HRMS-ESI ( $/$ / z) calcd. for $\left[\mathrm{C}_{18} \mathrm{H}_{18} \mathrm{~N}_{4} \mathrm{O}_{3}+\mathrm{H}\right]^{+}$: 339.1452, obsd.: 339.1452 .

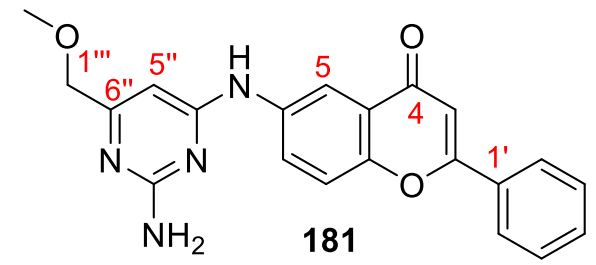

\section{6-((2"'-Amino-6"'-methoxymethylpyrimidin-4"'-} yl)amino)-2-phenyl-4H-chromen-4-one (181). By subjecting chloropyrimidine 154 (25.7 mg, 0.15 mmol) and aminochromone 170 (35.1 $\mathrm{mg}, 0.15$ $\mathrm{mmol})$ in conc. $\mathrm{HCl}(0.2 \mu \mathrm{L})$ and dioxane $(0.75 \mathrm{~mL})$ to the general procedure for the synthesis of chromonyl-pyrimidines, the title compound was isolated as a yellow amorphous solid (35.6 mg, 58\%); $\mathrm{R}_{f}=0.31(\mathrm{EtOAc} / \mathrm{MeOH}, 4 / 1, \mathrm{v} / \mathrm{v}) ; \mathrm{IR}\left(\mathrm{U}_{\max }\right): 3323$, 1638, 1568, 1519, 1479, 1452, 1356, 1340, 1242, 989, 908, 855, 799, 760, $650 \mathrm{~cm}^{-1} ;{ }^{1} \mathrm{H}$ NMR 
(500 MHz, pyridine- $\left.\mathrm{d}_{5}\right): \delta$ ppm $10.50(\mathrm{~s}, 1 \mathrm{H}, \mathrm{NH}), 8.87$ (d, $\left.J_{5,7}=2.7 \mathrm{~Hz}, 1 \mathrm{H}, \mathrm{H} 5\right), 8.58$ (dd, $\left.J_{7,8}=9.1 \mathrm{~Hz}, J_{5,7}=2.7 \mathrm{~Hz}, 1 \mathrm{H}, \mathrm{H} 7\right), 8.00-7.98$ (m, 2H, H3'), 7.59 (m, 1H, H8), $7.51-7.49$ (m, 3H, H2', H4'), 7.16 (s, 1H, H3), 6.63 (s, 1H, H5"), 4.48 (s, 2H, H1"'), 3.32 (s, 3H, H2"'); ${ }^{13} \mathrm{C}$ NMR (125 MHz, pyridine-d $\mathrm{d}_{5}$ ): $\delta$ ppm 133.0 (C4'), 130.6 (C2'), 128.5 (C7), 127.9 (C3'), 120.3 (C8), 116.1 (C5), 108.8 (C3), 94.9 (C5"), 76.11 (C1"'), 59.9 (C2"'); HRMS-ESI (m/ q) calcd. for $\left[\mathrm{C}_{21} \mathrm{H}_{18} \mathrm{~N}_{4} \mathrm{O}_{3}+\mathrm{H}\right]^{+}$: 375.1452 , obsd.: 375.1452 .

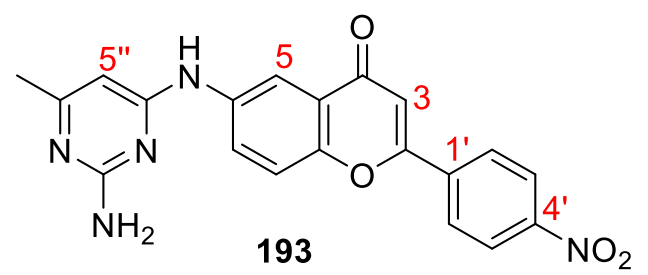

6-((2"-Amino-6"'-methylpyrimidin-4"'yl)amino)-2-(4'-nitrophenyl)-4H-chromen-4one (193). By subjecting chloropyrimidine 150 (15.7 $\mathrm{mg}, 0.2 \mathrm{mmol}$ ) and aminochromone $192(30.0 \mathrm{mg}$, $0.10 \mathrm{mmol})$ in conc. $\mathrm{HCl}(0.2 \mu \mathrm{L})$ and dioxane $(0.5 \mathrm{~mL})$ to the general procedure for the synthesis of chromonyl-pyrimidines, the title compound was isolated as a yellow amorphous solid (16.7 mg, 43\%); $\mathrm{R}_{f}=0.55$ (petroleum ether/EtOAc, 1/1, v/v); Fluorescence $(\mathrm{MeOH})$ : Ex 386 nm, Em 484 nm; IR (Umax): 3323, 1634, 1567, 1519, 1479, 1454, 1343, 1300, 1202, 989, 908, 853, 799, 760, 689, $468 \mathrm{~cm}^{-1} ;{ }^{1} \mathrm{H}$ NMR (600 MHz, pyridine- $\mathrm{d}_{5}$ ): $\delta$ ppm 11.45 (br. s, $1 \mathrm{H}$, $\mathrm{NH}), 8.82\left(\mathrm{~d}, J_{5,7}=2.6 \mathrm{~Hz}, 1 \mathrm{H}, \mathrm{H} 5\right), 8.49\left(\mathrm{dd}, J_{7,8}=9.0 \mathrm{~Hz}, J_{5,7}=3.0 \mathrm{~Hz}, 1 \mathrm{H}, \mathrm{H} 7\right), 8.39$ (d, $J_{2^{\prime}, 3^{\prime}}$

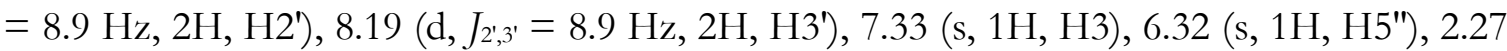
(s, 3H, $\mathrm{CH}_{3}$ ); ${ }^{13} \mathrm{C}$ NMR (150 MHz, pyridine-d $\mathrm{d}_{5}$ ): $\delta$ ppm 178.8 (C4), 164.0 (C4"), 161.7 (C2), 153.6 (C6), 150.5 (C4'), 139.3 (C1'), 137.9 (C8a), 129.5 (C4a), 129.0 (C3'), 128.8 (C7), 125.6 (C2'), 120.4 (C8), 116.6 (C5), 110.8 (C3), 97.7 (C5'), $24.2\left(\mathrm{CH}_{3}\right)$; HRMS-ESI (m/z) calcd. for $\left[\mathrm{C}_{20} \mathrm{H}_{15} \mathrm{~N}_{5} \mathrm{O}_{4}+\mathrm{H}\right]^{+}$: 390.1197, obsd.: 390.1193 .

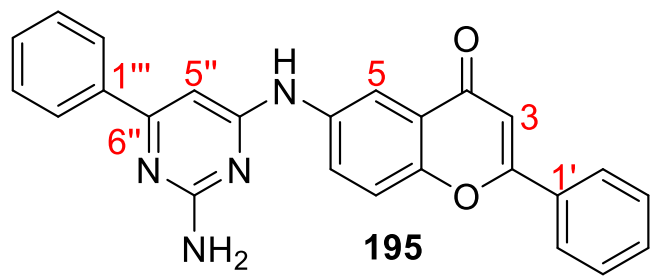

6-((2"-Amino-6"'-phenylpyrimidin-4"'yl)amino)-2-phenyl-4H-chromen-4-one (195). By subjecting chloropyrimidine 188 (49 $\mathrm{mg}, 0.24$ $\mathrm{mmol})$ and aminochromone $170(37.8 \mathrm{mg}, 0.16$ $\mathrm{mmol})$ in conc. $\mathrm{HCl}(7 \mu \mathrm{L})$ and dioxane $(0.7 \mathrm{~mL})$ to the general procedure for the synthesis of chromonyl-pyrimidines, the title compound was isolated as a yellow amorphous solid (37.5 mg, $58 \%) ; \mathrm{R}_{f}=0.54(\mathrm{EtOAc} / \mathrm{MeOH}, 4 / 1, \mathrm{v} / \mathrm{v}) ; \mathrm{IR}\left(\mathrm{u}_{\max }\right): 1636,1564,1521,1485,1450,1377$, 1203, 912, 822, 764, $677 \mathrm{~cm}^{-1}$; Fluorescence (MeOH): Ex 384 nm, Em 485 nm; ${ }^{1}$ H NMR (500 MHz, pyridine- $\mathrm{d}_{5}$ ): $\delta$ ppm 10.67 (br. s, $1 \mathrm{H}, \mathrm{NH}$ ), 8.88 (d, $\left.J_{5,7}=2.8 \mathrm{~Hz}, 1 \mathrm{H}, \mathrm{H} 5\right), 8.61$ (dd, $J_{7,8}$ $=9.1 \mathrm{~Hz}, 2.8 \mathrm{~Hz}, 1 \mathrm{H}, \mathrm{H} 7), 8.33-8.24$ (m, 2H, H2"'), $8.04-7.95$ (m, 2H, H2'), $7.52-7.47$ (m, 
3H, H3', H4'), 7.47 - 7.40 (m, 3H, H3'", H4"'), 7.17 (s, 1H, H3), 6.89 (s, 1H, H5"); ${ }^{13} \mathrm{C}$ NMR (150 MHz, pyridine-d $\mathrm{d}_{5}$ ): $\delta$ ppm 178.6 (C4), 165.1 (C4"), 163.7 (C2), 152.4 (C6), 139.9 (C8a), 139.3 (C1"'), 133.3 (C1'), 133.16, 132.9 (C4'), 132.3 (C4"'), 130.7 (C3'), 130.68, 129.9 (C3"'), 129.8 (C7), 129.4 (C2"'), 127.8 (C2'), 127.22 (C4a), 119.5 (C8), 115.2 (C5), 107.9 (C3), 94.0 (C5"); HRMS-ESI $(m / z)$ calcd. for $\left[\mathrm{C}_{25} \mathrm{H}_{18} \mathrm{~N}_{4} \mathrm{O}_{2}+\mathrm{H}\right]^{+}:$407.1503, obsd.: 407.1503.<smiles></smiles>

\section{6-((2"-Amino-6"'-phenylpyrimidin-4"'- yl)amino)-2-(4'-nitrophenyl)-4H-} chromen-4-one (196). By subjecting chloropyrimidine 188 (53.0 mg, $0.26 \mathrm{mmol}$ ) and aminochromone $192(72.6 \mathrm{mg}, 0.26$

mmol in conc. $\mathrm{HCl}(0.1 \mathrm{~mL})$ and dioxane $(1.1 \mathrm{~mL})$ to the general procedure for the synthesis of chromonyl-pyrimidines, the title compound was isolated as a yellow amorphous solid (63.4 mg, 54\%); $\mathrm{R}_{f}=0.42(\mathrm{EtOAc} / \mathrm{MeOH}, 4 / 1, \mathrm{v} / \mathrm{v}) ; \mathrm{IR}$ (Umax): 1739, 1558, 1457, 1344, 1217, 773, 560, 584, 555, 502, 476, 456, 431, $416 \mathrm{~cm}^{-1}$; Fluorescence (MeOH): Ex 385 nm, Em 489 nm; ${ }^{1} \mathrm{H}$ NMR (500 MHz, pyridine-d $\left.\mathrm{d}_{5}\right): \delta$ ppm 10.59 (br. s, $\left.1 \mathrm{H}, \mathrm{NH}\right), 8.95$ (d, $\left.J_{5,7}=2.7 \mathrm{~Hz}, 1 \mathrm{H}, \mathrm{H} 5\right)$, $8.66\left(\mathrm{dd}, J_{7,8}=9.1 \mathrm{~Hz}, J_{5,7}=2.8 \mathrm{~Hz}, 1 \mathrm{H}, \mathrm{H} 7\right), 8.38$ (d, $J_{2^{\prime}, 3^{\prime}}=8.9 \mathrm{~Hz}, 2 \mathrm{H}, \mathrm{H} 3$ ) $), 8.29-8.23$ (m, 2H, H2'"), 8.17 (d, $\left.J_{2^{\prime} 3^{\prime}}=8.7 \mathrm{~Hz}, 2 \mathrm{H}, \mathrm{H} 2^{\prime}\right), 7.63$ (d, $\left.J_{7,8}=9.0 \mathrm{~Hz}, 1 \mathrm{H}, \mathrm{H} 8\right), 7.47-7.39$ (m, 3H, H3"', H4"'), 7.32 (m, 3H, H3, NH $), 6.91$ (s, 1H, H5"); ${ }^{13} \mathrm{C}$ NMR (150 MHz, pyridine- $\left.\mathrm{d}_{5}\right): \delta$ ppm 177.5 (C4), 164.63, 164.2 (C4"), 162.9 (C6"), 160.1 (C2), 151.5 (C6), 149.3 (C4'), 139.6 (C8a), 138.9 (C1"'), 137.9 (C1'), 129.9 (C4"'), 128.7 (C3"'), 127.4 (C2'), 127.2 (C6), 127.0 (C2"'), 124.7 (C4a), 124.1 (C3), 118.8 (C8), 114.1 (C5), 109.2 (C3), 93.0 (C5"); HRMS-ESI ( $m /$ z) calcd. for $\left[\mathrm{C}_{25} \mathrm{H}_{17} \mathrm{~N}_{5} \mathrm{O}_{4}+\mathrm{H}\right]^{+}:$452.1353, obsd.: 452.1351.<smiles></smiles>

$\mathrm{mmol})$ in conc. $\mathrm{HCl}(9.5 \mu \mathrm{L})$ and dioxane $(0.955 \mathrm{~mL})$ to the general procedure for the synthesis of chromonyl-pyrimidines, the title compound was isolated as a yellow amorphous solid (50.8 $\mathrm{mg}, 63 \%) ; \mathrm{R}_{f}=0.16\left[\mathrm{CH}_{2} \mathrm{Cl}_{2} / \mathrm{EtOH} / \mathrm{MeOH} / \mathrm{NH}_{3}\right.$ (aq) (25\%), 25/2/2/1, v/v/v/v]; Fluorescence (MeOH): Ex 380 nm, Em 492 nm; IR (U $\max ):$ 1640, 1062, 1568, 1520, 1496, 1484, 
1454, 1378, 1364, 1082, 1069, $696 \mathrm{~cm}^{-1} ;{ }^{1} \mathrm{H}$ NMR (500 MHz, pyridine-d $\left.\mathrm{d}_{5}\right): \delta \mathrm{ppm} 10.55$ (br. s, $1 \mathrm{H}, \mathrm{NH}), 8.93$ (d, $\left.J_{5,7}=2.8 \mathrm{~Hz}, 1 \mathrm{H}, \mathrm{H} 5\right), 8.61$ (dd, $\left.J_{7,8}=9.0 \mathrm{~Hz}, J_{5,7}=2.7 \mathrm{~Hz}, 1 \mathrm{H}, \mathrm{H} 7\right), 8.23$ (m, 2H, C2"'), 8.00 (dt, $\left.J_{2^{\prime}, 3^{\prime}}=7.6, J_{2^{\prime}, 4^{\prime}}=1.7 \mathrm{~Hz}, 2 \mathrm{H}, \mathrm{H} 2^{\prime}\right), 7.62$ (d, $\left.J_{7,8}=9.0 \mathrm{~Hz}, 1 \mathrm{H}, \mathrm{H} 8\right), 7.53$ - 7.49 (m, 3H, H3', H4'), 7.20 - 7.13 (m, 3H, H3"', H3), 6.84 (s, 1H, H5"'); ${ }^{13} \mathrm{C}$ NMR (150 MHz, pyridine- $\mathrm{d}_{5}$ ): $\delta$ ppm $178.3(\mathrm{C} 4), 164.6$ (d, $\left.J_{4^{\prime \prime \prime}, \mathrm{F}}=248.4 \mathrm{~Hz}, \mathrm{C} 4{ }^{\prime \prime \prime}\right), 165.3$ (C4"), 163.7 (C6"), 163.6 (C2), 163.5 (C2"), 152.4 (C6), 139.8 (C8a), 132.9 (C1'), 132.2 (C4'), 129.8 (C3'), 129.74, 129.7

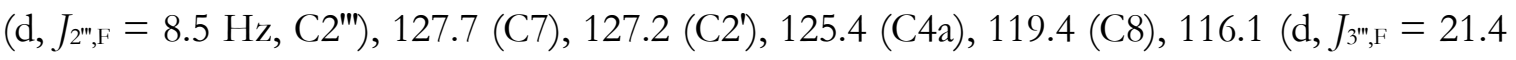
$\mathrm{Hz}, \mathrm{C}^{\prime \prime \prime}$ ), 115.2 (C5), 107.9 (C3), 93.3 (C5"); ${ }^{19} \mathrm{~F}$ NMR (282 MHz, pyridine-d 5 ) $\delta$ ppm -111.90; HRMS-ESI $(m / 2)$ calcd. for $\left[\mathrm{C}_{25} \mathrm{H}_{17} \mathrm{FN}_{4} \mathrm{O}_{2}+\mathrm{H}\right]^{+}:$425.1408, obsd.: 425.1408.

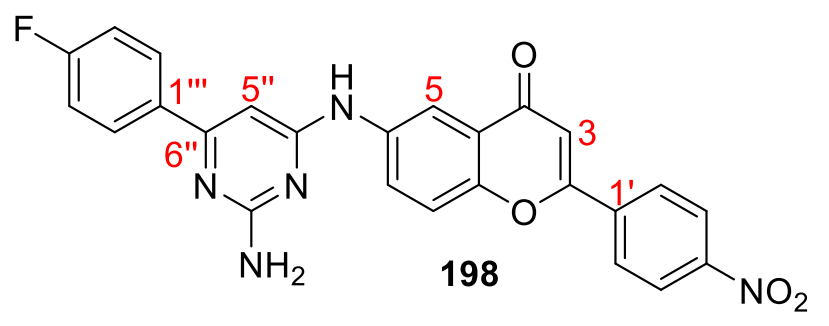

\section{6-((2"-Amino-6"'-(4"'"- fluorophenyl)pyrimidin-4"'- yl)amino)-2-(4'-nitrophenyl)-4H-} chromen-4-one (198). By subjecting

chloropyrimidine $189(14.5 \mathrm{mg}, 0.07$ mmol) and aminochromone $192(11.0 \mathrm{mg}, 0.05 \mathrm{mmol})$ in conc. $\mathrm{HCl}(0.2 \mu \mathrm{L})$ and dioxane $(0.5$ $\mathrm{mL}$ ) to the general procedure for the synthesis of chromonyl-pyrimidines, the title compound was isolated as a yellow amorphous solid (17.1 mg, 73\%); $\mathrm{R}_{f}=0.20$ (EtOAc); Fluorescence (MeOH): Ex 384 nm, Em 488 nm; IR (Umax): 1683, 1640, 1606, 1520, 1487, 1455, 1345, 1222, 1169, 914, 852, 823, 755, 693, $562 \mathrm{~cm}^{-1} ;{ }^{1} \mathrm{H}$ NMR (500 MHz, pyridine-d $\left.\mathrm{d}_{5}\right): \delta \mathrm{ppm} 11.29(\mathrm{~s}, 1 \mathrm{H}$, $\mathrm{NH}), 8.98(\mathrm{t}, J=2.7 \mathrm{~Hz}, 1 \mathrm{H}), 8.66(\mathrm{dt}, J=8.8,2.8 \mathrm{~Hz}, 1 \mathrm{H}), 8.39(\mathrm{dd}, J=8.9,2.6 \mathrm{~Hz}, 2 \mathrm{H})$, $8.29(\mathrm{ddd}, J=8.7,5.5,2.6 \mathrm{~Hz}, 2 \mathrm{H}), 8.25-8.15(\mathrm{~m}, 2 \mathrm{H}), 7.64-7.60(\mathrm{~m}, 2 \mathrm{H}), 7.33(\mathrm{~d}, J=2.7$ $\mathrm{Hz}, 1 \mathrm{H}), 7.20-7.14(\mathrm{~m}, 1 \mathrm{H}), 7.00(\mathrm{~d}, J=2.7 \mathrm{~Hz}, 1 \mathrm{H}) ;{ }^{13} \mathrm{C}$ NMR (150 MHz, pyridine- $\left.\mathrm{d}_{5}\right): \delta$ ppm 178.9 (C4), 165.3 (d, J4",F $=239.8$ Hz, C4"'), 160.5 (C4"), 158.96, 153.5 (C6), 150.9 (C2), 139.9 (C8a), 139.3 (C1'), 134.0 (C1"'), 130.6 (d, J2",F = 8.5 Hz, C2'"'), 129.0 (C7), 128.8 (C2'), 126.2 (C4a), 125.6 (C3'), 120.3 (C8), 117.0 (d, J3",F $=21.7$ Hz, C3"'), 115.8 (C5), 110.8 (C3), 94.2 $(\mathrm{C} 5 ") ;{ }^{19} \mathrm{~F}$ NMR (282 MHz, pyridine-d $\left.\mathrm{d}_{5}\right): \delta \mathrm{ppm}-111.48$; HRMS-ESI $(\mathrm{m} / \mathrm{q})$ calcd. for $\left[\mathrm{C}_{25} \mathrm{H}_{16} \mathrm{FN}_{5} \mathrm{O}_{4}+\mathrm{H}\right]^{+}: 470.1259$, obsd.: 470.1256 .<smiles></smiles>

2"-Amino-6"'-methoxymethylpyrimidin-4"-yl(6-amino-4-oxo-4H-chromene-2-carboxylic acid) (182). Chromonyl-pyrimidine $179(7.2 \mathrm{mg}$, $0.02 \mathrm{mmol}$ ) was dissolved in $\mathrm{AcOH} / \mathrm{H}_{2} \mathrm{O}(3 \mathrm{~mL}$, 
2/1, v/v) before conc. $\mathrm{HCl}(0.01 \mathrm{~mL})$ was added. The reaction was stirred under reflux for 1 $\mathrm{h}$, after which time TLC analysis showed the disappearance of the starting materials and the appearance of carboxylic acid 182. Purification by a short C18 reverse column chromatography $\left(1 / 0 \rightarrow 1 / 1, \mathrm{H}_{2} \mathrm{O} /\right.$ acetone, $\left.\mathrm{v} / \mathrm{v}\right)$ gave the title compound as a yellow solid (6.2 $\left.\mathrm{mg}, 90 \%\right) . \mathrm{R}_{f}$ $=0.02\left(1 / 1, \mathrm{CH}_{2} \mathrm{Cl}_{2} / \mathrm{MeOH}, \mathrm{v} / \mathrm{v}\right) ; \mathrm{IR}\left(\mathrm{U}_{\max }\right): 1639,1578,1597,1499,1414,1324,1371,1276,1037$, 892, 860, 725, 753, 710, 623, 668, 511, 531, 561, $407 \mathrm{~cm}^{-1} ;{ }^{1} \mathrm{H}$ NMR (500 MHz, pyridine- $\left.d_{5}\right): \delta$ ppm $7.89\left(\mathrm{~d}, J_{5,7}=2.8 \mathrm{~Hz}, 1 \mathrm{H}, \mathrm{H} 5\right), 7.54\left(\mathrm{~d}, J_{7,8}=9.0 \mathrm{~Hz}, 1 \mathrm{H}, \mathrm{H} 8\right), 7.53(\mathrm{~s}, 1 \mathrm{H}, \mathrm{H} 3), 7.33$ (dd, $J_{7,8}=$ $\left.9.0 \mathrm{~Hz}, J_{5,7}=2.8 \mathrm{~Hz}, 1 \mathrm{H}, \mathrm{H} 5\right), 6.35$ (s, 1H, H5"), 4.35 (s, 2H, H1"'), 3.33 (s, 3H, CH 3 ); ${ }^{13} \mathrm{C}$ NMR (125 MHz, pyridine): $\delta$ ppm 180.2 (C4), 166.9 (C4"), 165.8 (C1'), 158.9 (C2"), 156.9 (C2), 150.5 (C6 or C8a), 149.0 (C6 or C8a), 127.5 (C4a), 124.3 (C7), 121.3 (C3), 114.1 (C8), 107.7 (C5), 100.8 (C5"), 75.4 (C1"'), $59.9\left(\mathrm{CH}_{3}\right)$; HRMS-ESI (m/v) calcd. for $\left[\mathrm{C}_{16} \mathrm{H}_{14} \mathrm{~N}_{4} \mathrm{O}_{5}-\mathrm{H}\right]^{-}$: 341.0891, obsd.: 341.0890 .

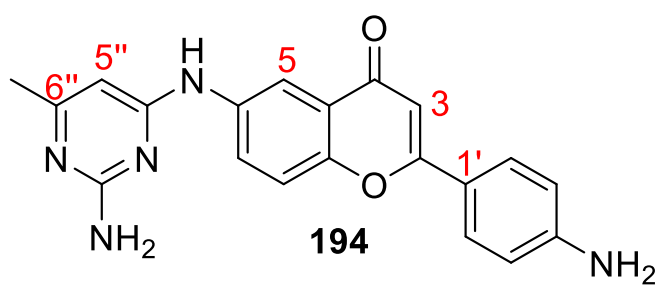

6-((2"'-Amino-6"'-methylpyrimidin-4"'yl)amino)-2-(4'-aminophenyl)-4H-chromen-4one (194). To a solution of nitrochromone 193 $(11.8 \mathrm{mg}, 0.03 \mathrm{mmol})$ in $\mathrm{EtOH} / \mathrm{H}_{2} \mathrm{O}(2 / 1, \mathrm{v} / \mathrm{v}$, $0.225 \mathrm{~mL})$ was added iron powder $(6.7 \mathrm{mg}, 0.12$ mmol, 4 equiv.) and conc. $\mathrm{HCl}(3 \mu \mathrm{L})$. The reaction was warmed to reflux and stirred for 12 $\mathrm{h}$, after which time TLC analysis showed complete disappearance of starting materials. The reaction mixture was then cooled and filtered through Celite before it was concentrated in vacuo to give the title compound as a yellow amorphous solid (3.63 $\mathrm{mg}, 33 \%) ; \mathrm{R}_{f}=0.08$ (EtOAc/MeOH, 4/1, v/v); IR (Umax): 3349, 2921, 1590, 1420, 1346, 1310, 1176, 1124, 962, 826, 798, 718, 558, $536 \mathrm{~cm}^{-1}$; Fluorescence (MeOH): Ex 386 nm, Em 495 nm; ${ }^{1} \mathrm{H}$ NMR (500 $\mathrm{MHz}_{\text {, pyridine- }}$ ) ): $\delta$ ppm 10.56 (br. s, $\left.1 \mathrm{H}, \mathrm{NH}\right), 8.82$ (d, $\left.J_{5,7}=2.7 \mathrm{~Hz}, 1 \mathrm{H}, \mathrm{H} 5\right), 8.46$ (dd, $J_{7,8}$ $\left.=9.0 \mathrm{~Hz}, J_{5,7}=2.7 \mathrm{~Hz}, 1 \mathrm{H}, \mathrm{H} 7\right), 7.89\left(\mathrm{~d}, J_{2^{\prime}, 3^{\prime}}=8.9 \mathrm{~Hz}, 2 \mathrm{H}, \mathrm{H} 2^{\prime}\right), 7.55\left(\mathrm{~d}, J_{7,8}=9.0 \mathrm{~Hz}, 1 \mathrm{H}\right.$, H8), 7.04 (s, 1H, H3), 7.02 (d, $\left.J_{2^{\prime}, 3^{\prime}}=8.7 \mathrm{~Hz}, 2 \mathrm{H}, \mathrm{H} 3^{\prime}\right), 6.23$ (s, 1H, H5"), 2.24 (s, 3H, CH 3 ); ${ }^{13} \mathrm{C}$ NMR (125 MHz, pyridine-d $\left.\mathrm{d}_{5}\right): \delta$ ppm 177.3 (C4), 164.4 (C4"), 164.1 (C2), 163.1 (C2"), 162.5 (C6"), 159.10, 153.0 (C1'), 151.8 (C6), 138.3 (C8a), 128.3 (C2'), 126.9 (C7), 124.8 (C4a), 119.0 (C4'), 118.4 (C7), 115.3 (C5), 114.3 (C3'), 103.8 (C3), 95.6 (C5"), $23.0\left(\mathrm{CH}_{3}\right)$; HRMS-ESI (m/ z) calcd. for $\left[\mathrm{C}_{20} \mathrm{H}_{17} \mathrm{~N}_{5} \mathrm{O}_{2}+\mathrm{H}\right]^{+}: 360.1455$, obsd.: 360.1455 . 
<smiles></smiles>

6-((2"'-Amino-6"'-(4"'-fluorophenyl)pyrimidin-4''-yl)amino)2-(4'-aminophenyl)-4H-chromen-4one) (199). To a solution of nitrochromone 198 (22.1 $\mathrm{mg}, 0.05 \mathrm{mmol})$

in $\mathrm{MeOH}(2.9 \mathrm{~mL})$ was added iron powder $(11.17 \mathrm{mg}, 0.2 \mathrm{mmol}, 4$ equiv.) and conc. $\mathrm{HCl}(5$ $\mu \mathrm{L})$. The reaction was warmed to reflux and stirred for $12 \mathrm{~h}$, after which time TLC analysis showed complete disappearance of starting materials. The reaction mixture was then cooled and filtered through Celite before it was concentrated in vacuo to vie the title compound as a yellow amorphous solid (9.2 mg, 42\%); $\mathrm{R}_{f}=0.63(\mathrm{EtOAc} / \mathrm{MeOH}, 4 / 1, \mathrm{v} / \mathrm{v})$; IR (U $\left.\max \right): 3310$, 2949, 1931, 1699, 1652, 1593, 1575, 1515, 1398, 1362, 1258, 1185, 1082, 984, 958, 802, 481, 455, 436, $423 \mathrm{~cm}^{-1}$; ${ }^{1} \mathrm{H}$ NMR (500 MHz, pyridine- $\mathrm{d}_{5}$ ): $\delta$ ppm 10.82 (br. s, $1 \mathrm{H}, \mathrm{NH}$ ), 8.88 (s, 1H, H5), 8.52 (d, J7,8 = $8.5 \mathrm{~Hz}, 1 \mathrm{H}, \mathrm{H} 7) 8.27-8.24$ (m, 2H, H3'"), 7.91 (d, $J_{2^{\prime}, 3^{\prime}}=8.4 \mathrm{~Hz}, 2 \mathrm{H}$,

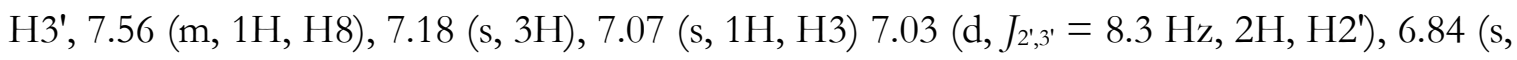
1H, H5"); ${ }^{13} \mathrm{C}$ NMR (150 MHz, pyridine-d $\left.\mathrm{d}_{5}\right): \delta$ ppm 178.0 (C4), 165.3 (d, $J_{4^{\mathrm{m}}, \mathrm{F}}=195.8 \mathrm{~Hz}, 1 \mathrm{H}$, H4"'), 164.8 (C1'), 163.7 (C6"), 153.7 (C2), 152.6 (C6), 138.9 (C8a), 130.7 (d, J2"',F $=7.9$ Hz, 2H,

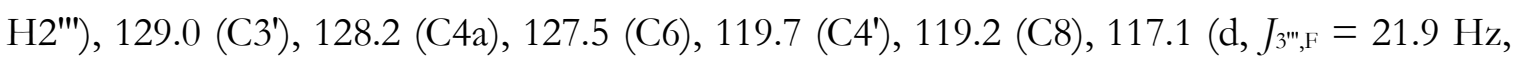
2H, H3"'), 117.0 (C5), 115.0 (C2'), 104.5 (C3), 92.5 (C5"); ${ }^{19} \mathrm{~F}$ NMR (282 MHz, pyridine-d $\left.\mathrm{d}_{5}\right) \delta$ ppm -109.70; HRMS-ESI (m/z) calcd. for $\left[\mathrm{C}_{25} \mathrm{H}_{18} \mathrm{FN}_{5} \mathrm{O}_{2}+\mathrm{H}\right]^{+}: 440.1517$, obsd.: 440.1519.

\subsubsection{Biology - testing of chromonyl-pyrimidines 193-199}

Samples. Each compound was dissolved in sterile DMSO to make $10 \mathrm{mM}$ stock solutions.

Obtaining MIC values. In clear-bottomed 96-well plates (Nunc), two-fold serial dilutions of each compound were added to volumes of $100 \mu \mathrm{L}$ of $\mathrm{HdB}$ minimal medium. The last column of each plate did not contain any compound and served as a negative control. Previously prepared $M t b \mathrm{mc}^{2} 6230$ inocula were diluted to $5 \%$ with $\mathrm{HdB}$ medium to achieve $0.05 \mathrm{OD}_{600}$. The inocula were then added to each well and the plates incubated at $37^{\circ} \mathrm{C}$ for five days. After incubation, $20 \mu \mathrm{L}$ of resazurin was added to the wells, and the plates incubated at $37^{\circ} \mathrm{C}$ for 24 $\mathrm{h}$. The wells that remained blue were deemed to contain non-replicating bacteria, while those that underwent a colour change to purple or pink were deemed to contain replicating bacteria. MIC values were ascertained by visual judgment of colour. 


\section{CHAPTER FIVE}

\section{The synthesis and anti-tubercular properties of phthalazinones}

\subsection{Introduction}

BDQ (14) is the first FDA-approved TB drug to inhibit oxidative phosphorylation in Mtb. To exert its inhibitory activity, BDQ (14) acts as an uncoupler by dissociating the transmembrane proton transfer from phosphorylation reactions responsible for ATP generation (Chapter 1, Section 1.2.3). ${ }^{33}$ This uncoupling mechanism leads to the dissipation of the mycobacterial PMF, a process that has been reported to be detrimental to $M t b .{ }^{33,52}$ The PMF is comprised of $\Delta \varphi$ and $\Delta \mathrm{pH}$ gradients, whereby $\Delta \varphi$ measures the charge separation across the membrane (positive $_{\text {outside }}$ : negative $\mathrm{inside}_{\text {) }}$ and $\Delta \mathrm{pH}$ is the transmembrane $\mathrm{pH}$ gradient (acidic outside : alkaline inside). $^{41,52}$ Typically, uncouplers are protonophores or ionophores that translocate 
protons across proton-impermeable membranes, perturbing the $\Delta \varphi$ or $\Delta \mathrm{pH}$ and ultimately collapsing the PMF. ${ }^{305}$ Examples of potent uncouplers are weak acids with ionisable protons, such as carbonyl cyanide $p$-trifluoromethoxyphenyl hydrazone (FCCP, 200, Figure 5.1) and CCCP (201). ${ }^{306}$ When neutral, FCCP (200) and CCCP (201) are apolar and can reside in the hydrophobic environment of cell membranes. However, as FCCP (200) and CCCP (201) move through the membrane, they dissociate into their respective anions and release a proton into the aqueous environment, which may be either outside the cell or within the cytosol. This mechanism allows for uncontrolled movement of protons in and out of the cell, which leads to the loss of the transmembrane proton gradient. ${ }^{305}$<smiles>N#CC(C#N)=NNc1cccc(Cl)c1</smiles>

Figure 5.1: Potent uncouplers FCCP (200) and CCCP (201)..$^{306}$

\subsubsection{Enzyme-specific uncouplers}

Although FCCP (200) and CCCP (200) are strong uncouplers, they are non-site specific and exert their ionophoric activity in both eukaryotic and prokaryotic cells, which causes toxicity in humans. ${ }^{41}$ Notwithstanding, uncouplers that target specific enzymes have been reported, such as BDQ (14), which binds to mycobacterial ATPase, and anthraquinone derivatives emodin (202, Figure 5.2) and emodinanthrone (203) that target cytochrome $c$ in E. coli. ${ }^{307}$ Of particular interest are phenothiazines that inhibit NDH-II by uncoupling oxidative phosphorylation in bacteria. ${ }^{308}$ Mechanistic studies have shown that thioridazine (22), a weak base with a $\mathrm{pKa}$ of $8.82,{ }^{309,310}$ induced a dose-dependent collapse in membrane potentials, and consequently, ATP depletion in $M t b^{52}$ and S. aureus. ${ }^{308}$ Here, the authors postulated that when protonated, thioridazine (22) carries charge across the membrane by accepting a proton from the periplasm and releasing it inside the cell, thereby reducing the PMF. ${ }^{308}$ Unfortunately, treatment with thioridazine (22) exhibits significant adverse side effects and has been reported to uncouple oxidative phosphorylation in mammalian mitochondria. ${ }^{311}$ 
<smiles>Cc1cc(O)c2c(c1)C(=O)c1cc(O)cc(O)c1C2=O</smiles>

202<smiles>Cc1cc(O)c2c(c1)Cc1cc(O)cc(O)c1C2=O</smiles>

203<smiles>CSc1ccc2c(c1)N(CCC1CCCCN1C)c1cc(C(F)(F)F)ccc1S2</smiles>

22

Figure 5.2: Emodin (202) and emodinanthrone (203) are uncouplers of cytochrome $c$ in E. coli; ${ }^{307}$ while phenothiazine 22 uncouples oxidative phosphorylation in $M t b$ and $S$. aureus. ${ }^{308}$

In 2015, Oldfield et al. conducted a study demonstrating the uncoupling activity of antitubercular agents. ${ }^{312}$ Here, a correlation was found between the degree of PMF dissipation and $M t b$ cell growth inhibition. Particularly potent uncouplers included lipophilic cationic species, such as SQ109 (204, Figure 5.3) and clofazimine (13), though AU1235 (205) was also active as a neutral uncoupler. ${ }^{312}$ SQ109 (204) was found to inhibit cell growth in Mtb by acting as an uncoupler. In addition SQ109 (204) also inhibited essential enzymes, such as MenA and MenG, which are enzymes required for MK biosynthesis, and transporter MmpL3, which is involved in cell wall biosynthesis. ${ }^{34,313,314}$ SAR studies of SQ109 (204) analogues showed that at least one protonable amine group was essential for anti-tubercular activity, indicating that SQ109 (204) had activity as a cation. ${ }^{314}$ Indeed, SQ109 (204) was found to be protonated at physiological pHs, allowing it to carry charge across the bacterial cell membrane and collapse the transmembrane proton gradient. ${ }^{312}$ Another effective uncoupler is clofazimine (13), a current third-line TB drug that produces $\operatorname{ROS}^{315}$ by binding to and undergoing reduction by NDH-II. ${ }^{36}$ In Oldfield's study, clofazimine (13) also induced PMF dissipation to the same extent as SQ109 (204) and BDQ (14). ${ }^{312}$ Finally, urea 205 is a neutral uncoupler of tubercular PMF that targets the MmpL3 enzyme. ${ }^{316}$ Unlike BDQ (14), which exerts its uncoupling activity by binding to its target enzyme ATPase, TB drugs 204, 205 and 13 are uncouplers of respiration that also target essential Mtb enzymes. Compounds with multiple modes of action are promising TB drug candidates as they are less susceptible to the development of bacterial resistance. Indeed, the rise in mycobacterial resistance to BDQ (14) has been reported, ${ }^{317}$ while SQ109 (204) has a strikingly low frequency of persister $M t b$ populations. ${ }^{318}$ 
<smiles>CC(C)=CCC/C(C)=C/CNCCNC1C2CC3CC(C2)CC1C3</smiles><smiles>O=C(Nc1ccc(F)c(F)c1F)NC1C2CC3CC(C2)CC1C3</smiles>

205<smiles>CC(C)/N=c1\cc2n(-c3ccc(Cl)cc3)c3ccccc3nc-2cc1Nc1ccc(Cl)cc1</smiles>

Figure 5.3: Anti-tubercular drugs 204, 205, and 13 induce PMF dissipation in bacteria.

\subsubsection{The phthalazinone scaffold in drug discovery}

Phthalazinones are becoming increasingly important in the field of drug discovery due to their diverse range of pharmaceutical properties, such as anti-cancer, ${ }^{319-321}$ anti-inflammatory, ${ }^{322-324}$ anti-bacterial, ${ }^{319,325}$ antioxidant, ${ }^{319}$ and anti-neurodegenerative ${ }^{326}$ properties. In particular, antibacterial phthalazinones include inosine-5'-monophosphate dehydrogenase inhibitor 206 (Figure 5.4), which has an MBC of $0.94 \mu \mathrm{M}$ against Francisella tularensis, ${ }^{327}$ as well as oxadiazole 207, which inhibit the growth of E. coli and Salmonella typhi. ${ }^{328}$ In addition, many clinically available phthalazinone-containing drugs are known, all with specific mechanisms of action. For example, oliparib (208) inhibits the poly(ADP-ribose)polymerase, an enzyme family responsible for repairing DNA damage, in tumour cells, ${ }^{329}$ while azelastine (209) is an effective histamine inhibitor that is preferentially taken up by the lung and alveolar macrophages. ${ }^{330}$ Others are known phosphodiesterase inhibitors (i.e. EG626, 210) (311 $^{33}$ and aldose reductase inhibitors in tumour cells (i.e. zopolrestate, 211). ${ }^{332}$ 
<smiles>COc1cc(NC(=O)Cc2nn(C)c(=O)c3c(C)cccc23)ccc1Cl</smiles>
206<smiles>Cc1cccc2c(Cc3nnc(-c4ccsc4)o3)nn(Cc3ccc(Cl)nc3)c(=O)c12</smiles>

207<smiles>O=C(c1cc(Cc2n[nH]c(=O)c3ccccc23)ccc1F)N1CCN(C(=O)C2CC2)CC1</smiles>

208<smiles>O=C(O)Cc1nn(Cc2nc3cc(C(F)(F)F)ccc3s2)c(=O)c2ccccc12</smiles>

211

Figure 5.4: Anti-bacterial phthalazinones 206 and 207, 327,328 and FDA-approved phthalazinone-containing drugs oliparib (208), azelastine (209), EG626 (210), and zopolrestat (211).

\subsubsection{The phthalazinone scaffold as potential uncouplers}

Effective uncouplers are commonly characterised as weak acids ${ }^{333}$ or bases, ${ }^{312}$ such as thioridazine (22) and BDQ (14), both of which have a pKa of 8.89. ${ }^{334,312}$ Electron-withdrawing and lipophilic groups have also been reported to enhance the uncoupling capabilities of weakly acidic or basic compounds. ${ }^{305}$ In this chapter, the phthalazinone scaffold (31, Figure 5.4) will be investigated for their anti-tubercular activity, as well as their capacity to uncouple oxidative phosphorylation in $M t b$. The capability of phthalazinones to act as an uncoupler has yet to be investigated, however phthalazinones are weak bases that can be functionalised with lipophilic and electron-withdrawing groups. The basic $\mathrm{pKa}$ of unfunctionalised phthalazinones is calculated to be $8.88,{ }^{335}$ while functionalised phthalazinones, i.e. azelastine (209) has a pKa of 9.5. ${ }^{336}$ Here, unfunctionalised phthalazinone $\mathbf{3 1}$ has an ionisable proton akin to FCCP (200) and CCCP (201), which can assist in charge transport across cell membranes, ${ }^{305}$ while the hydrazone moiety in $N$-substituted phthalazinones can be protonated, thereby enabling the cationic species to act as a charge carrier in a comparable manner to phenothiazines. ${ }^{308}$ 


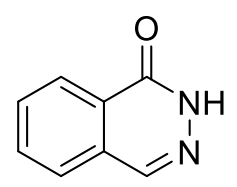

31

Figure 5.5: Phthalazinones (31) are weakly basic and could act as an uncoupler.

\subsubsection{Project aims and retrosynthesis}

In light of the uncoupling mechanisms of effective anti-tubercular drugs BDQ (14), SQ109 (204) and clofazimine (13), it was proposed that phthalazinones could also act as an uncoupler. In addition, it was envisioned that the ketone-containing bicyclic phthalazinone scaffold would fulfill the structural requirements for the NDH-II Q-site. Specifically, the planar heterocyclic moiety could be accommodated between hydrophobic residues Q317 and I379, while the ketone oxygen could participate in hydrogen bonding interactions with the ring nitrogen of the FAD isoalloxazine ring, as observed with menadione (18) and HQNO (19) in the NDH-II crystal structure (Chapter 1, Section 1.3.1). ${ }^{58,62}$ Given the ability of quinolinyl pyrimidines to inhibit NDH-II, ${ }^{79}$ it was envisaged that the addition of a pyrimidine to the phthalazinone scaffold could enhance their specificity for NDH-II. Moreover, docking studies of chromonylpyrimidines (Chapter 4) have shown possible hydrogen bonds between the pyrimidine amine protons and a glutamic acid residue (E324) in the NDH-II Q-site.

To this end, a retrosynthetic route was devised, whereby pyrimidinyl-phthalazinones $\mathbf{X X V}$ functionalised at the 2- and 6"-positions could be assembled from functionalised aminophthalazinones XXVI (where $\mathrm{R}^{3}=\mathrm{NH}_{2}$ ) and chloropyrimidines XXVII (Scheme 5.1). Here, the synthesis of phthalazinones bearing apolar aryl and alkyl groups was proposed in order to increase the lipophilicity of the phthalazinone scaffold and assist with binding to the hydrophobic NDH-II Q-site. ${ }^{58,62}$ Moreover, the addition of electron-withdrawing groups, such as fluorophenyl and sulfonamide, was envisioned to enhance the ability of the phthalazinones to disrupt the mycobacterial ETC. ${ }^{305}$ For this series of compounds, previously synthesised methyl-, phenyl- and fluorophenyl-substituted chloropyrimidines were to be used (Chapter 4). These pyrimidines were chosen as they contributed to the high anti-tubercular activity of chromonyl-pyrimidines 194-199. Functionalised phthalazinones XXVI could be prepared from the condensation of 3-bromo-6-nitro-isobenzofuranone (XXVIII, $\mathrm{R}^{3}=\mathrm{NO}_{2}$ ) with various hydrazines, followed by reduction of the nitro group, while the chloropyrimidines XXVII were 
to be available from the condensation of functionalised beta-ketoesters (XXIX) with guanidine, as previously described in Chapter 4. Furanone XXVIII could in turn be prepared from the reduction of phthalic anhydride, followed by nitration and subsequent benzylic bromination. Using this same synthetic route, a library of phthalazinones XXVI (where $\mathrm{R}^{3}=$ H) could also be synthesised from phthalic anhydride.

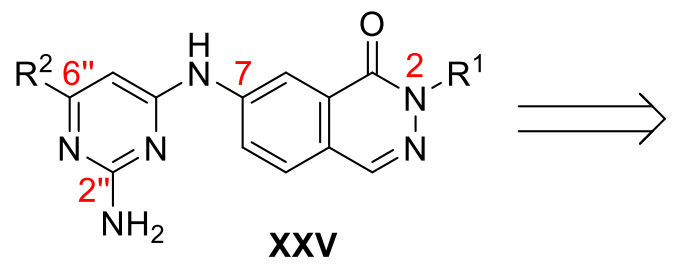<smiles>[R]c1ccc2cnn([R])c(=O)c2c1</smiles><smiles>[R]c1cc(Cl)nc(N)n1</smiles>

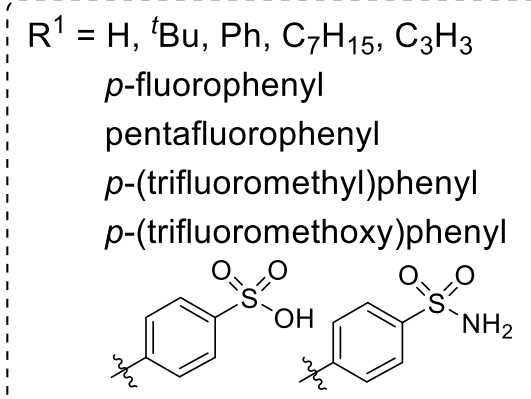<smiles>C=CC=C</smiles><smiles>[R]C(=O)OC1OC(=O)c2cc([R])ccc21</smiles>
XXVII

$$
\mathrm{R}^{2}=\mathrm{Me}, \mathrm{Ph}, 4-\mathrm{F}-\mathrm{Ph}
$$

$\mathrm{R}^{3}=\mathrm{H}, \mathrm{NH}_{2}, \mathrm{NO}_{2}$<smiles>C=CC=C</smiles>

phthalic anhydride

Scheme 5.1: Retrosynthetic analysis of pyrimidinyl-phthalazinones XXV and phthalazinones XXVI.

\subsection{Results and Discussion}

\subsubsection{Docking studies of phthalazinones and pyrimidinyl phthalazinones}

To investigate whether phthalazinones could be accommodated in the NDH-II Q-site, molecular docking of 2-methylphthalazin-1(2H)-one 212 (Figure 5.6A) was undertaken using USCF Chimera/DOCK6. ${ }^{282}$ Here, different molecular orientations with similar binding scores were adopted by the phthalazinone scaffold (Figure 5.6B). In all instances, the docked phthalazinone molecules were placed between the hydrophobic residues Q317 and I379, providing a similar binding orientation reported of menadione (18) and HQNO (19). ${ }^{58,62}$ 
Moreover, hydrogen bonding was also evident between phthalazinone 212 and R382 side chains. A similar interaction was also observed for the docked structure of menadione (18) in the NDH-II Q-site, ${ }^{58}$ whereby R382 interacts with one of the ketone oxygen atoms. That said, the authors queried the plausibility of this interaction, as crystal structures have shown this fragment of NDH-II to be embedded in the cell membrane and R382 was likely to interact with phosopholipids in the cell wall..$^{58}$ Notwithstanding, a hydrogen bond interaction between phthalazinone 212 and the ring nitrogen in FAD was observed for certain binding modes (Figure 5.6C).

A.<smiles>Cn1ncc2ccccc2c1=O</smiles>

212
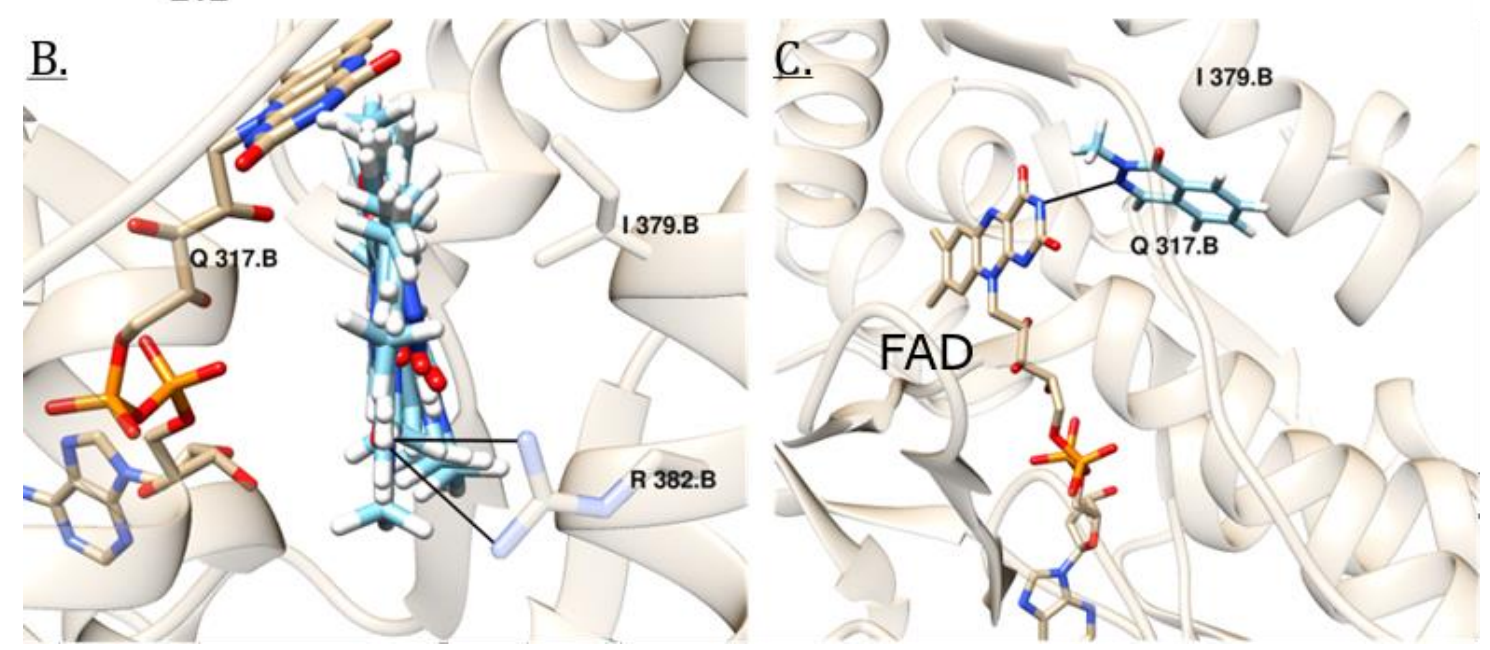

Figure 5.6: Docking studies of phthalazinone 212 in C. thermarum NDH-II (PDB: 6BDO), ${ }^{62}$ calculated using USCF Chimera/DOCK 6. A). Structure of 2-methylphthalazin-1(2H)-one (212). B). Phthalazinone 212 (light blue) can adopt many different conformations in the binding tunnel, sandwiched between Q317 and I379. C). Positioning of a high ranked conformer of 212 with a hydrogen bond to FAD (gold).

Next, docking studies of 7-((2-amino-6-methylpyrimidin-4-yl)-2-methylphthalazin-1(2H)-one (213, Figure 5.7A) in NDH-II were also conducted. Again, many different orientations of the pyrimidinyl-phthalazinone 213 were placed into the putative binding tunnel between hydrophobic residues Q317 and I379 (Figure 5.7B-D). Here, hydrogen bonds involving the pyrimidine amine group and Q317, Q321, E324, and R382 (Figure 5.7B), as well as that between the hydrazone nitrogen and the FAD isoalloxazine ring (Figure 5.7C) were observed. 
A well-positioned pyrimidinyl-phthalazinone 213 is shown in Figure 5.7D, which shows phthalazinone 213 occupying the putative binding tunnel, while the bridging and primary amine protons form a hydrogen bond with R382 and Q321, respectively. As previously noted however, the binding of R382 is physiologically unlikely as this portion of the enzyme is hypothesised to be well within the cell membrane. Taken together, these interactions suggest that compared to the phthalazinone ring on its own, pyrimidinyl-phthalazinones could bind the NDH-II Q-site more specifically. Further analysis of the NDH-II Q-site also indicated that the binding pocket could accommodate substituents at the 2- and 6"-positions. 
A.<smiles></smiles>
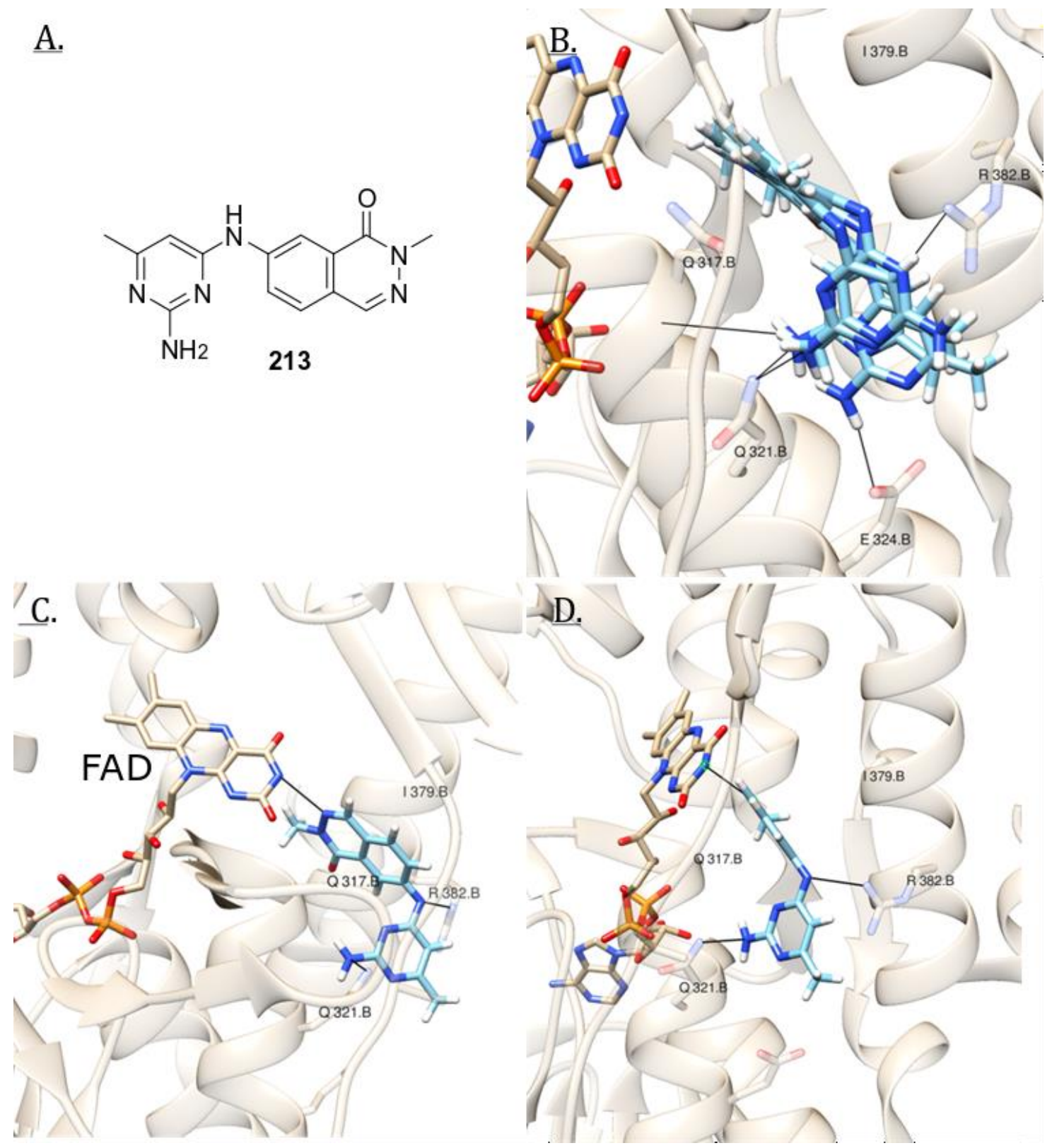

Figure 5.7: Docking studies of pyrimidinyl phthalazinone 213 in C. thermarum NDH-II (PDB: 6BDO). ${ }^{62}$ A). Structure of 7-((2-amino-6-methylpyrimidin-4-yl)amino)-2-methylphthalazin1(2H)-one (213). B). Possible binding modes of pyrimidinyl-phthalazinone 213 within the putative Q-site of NDH-II. C). Hydrogen bond interactions between phthalazinone 213 and the FAD isoalloxazine ring. D). Pyrimidinyl phthalazinone 213 is bound between Q317 and I379, while forming hydrogen bonds with surrounding amino acids R382 and Q321. Molecular graphics and analyses were performed with USCF Chimera, developed by the Resource for Biocomputing, Visualization, and Informatics at the Universtiy of California, San Fransisco

\subsubsection{The synthesis of phthalazinones}

The synthesis of $N$-substituted phthalazinones commenced with the reduction of phthalic anhydride with sodium borohydride to give isobenzofuranone 214 in good yield (Scheme 
5.2). ${ }^{337}$ A Wohl-Ziegler reaction was then performed on isobenzofuranone $214^{338}$ to give bromo-ester $215,{ }^{337}$ which was then treated with various hydrazines to afford phthalazinones 31, 216-223 in good yields (35-92\%).<smiles>O=C1CC(=O)c2ccccc21</smiles><smiles>O=C1OCc2ccccc21</smiles>

$65 \%$<smiles>[R]n1ncc2ccccc2c1=O</smiles>

1. $5 \% \mathrm{HCl}$

2. $\mathrm{H}_{2} \mathrm{NNHR}$

31, $\mathrm{R}^{1}=\mathrm{H}, 47 \%$<smiles>O=C1OC(Br)c2ccccc21</smiles>

215

216, $R^{1}=\mathrm{tBu}, 92 \%$

217, $\mathrm{R}^{1}=\mathrm{Ph}, 61 \%$

218, $\mathrm{R}^{1}=p$-fluorophenyl, $80 \%$

219, $R^{1}=$ pentafluorophenyl, $35 \%$

220, $\mathrm{R}^{1}=p$-(trifluoromethyl)phenyl, $58 \%$

221, $\mathrm{R}^{1}=p$-(trifluoromethoxy)phenyl, $65 \%$

222, $\mathrm{R}^{1}=$

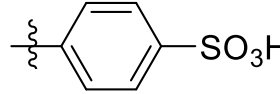

$76 \%$

223, $R^{1}=$

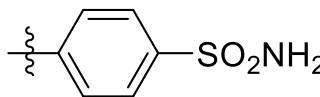

$38 \%$

Scheme 5.2: Synthesis of phthalazinones 31, 216-223.

The successful formation of phthalazinones 31 and $216-223$ were confirmed by ${ }^{1} \mathrm{H}$ and ${ }^{13} \mathrm{C}$ NMR spectroscopy, which showed the loss of resonances corresponding to H3 and C3 of bromide 215 (7.41 ppm and $74.8 \mathrm{ppm}$, respectively, in $\left.\mathrm{CDCl}_{3}\right)$ and the appearance of resonances corresponding to the 4-position of the phthalazinones $(8.00-8.30 \mathrm{ppm}, 136.0-$ 139.0 ppm, respectively, in $\mathrm{CDCl}_{3}$ ). In addition, IR spectra of phthalazinones showed bands between $1102-1120 \mathrm{~cm}^{-1}$, typical of N-N stretches, ${ }^{339}$ while spectral data of phthalazinones $\mathbf{3 1},{ }^{340} \mathbf{2 1 6},,^{341} \mathbf{2 1 7},{ }^{341} \mathbf{2 1 8},{ }^{342} \mathbf{2 2 0},{ }^{343}$ and $\mathbf{2 2 1 ^ { 3 4 2 }}$ matched those reported in literature. In addition to these compounds, phthalazinone $\mathbf{3 1}$ was alkylated using heptylbromide and 
propargylbromide under basic conditions to give alkyl phthalazinones 224 and 225, respectively (Scheme 5.3). Here, the connectivity of the alkyl group to the ring nitrogen was confirmed by $\mathrm{HMBC}$, in which correlations between the $\mathrm{N}-\mathrm{CH}_{2}$ and the carbonyl carbon were observed (i.e. Figure 5.8 for phthalazinone 224).
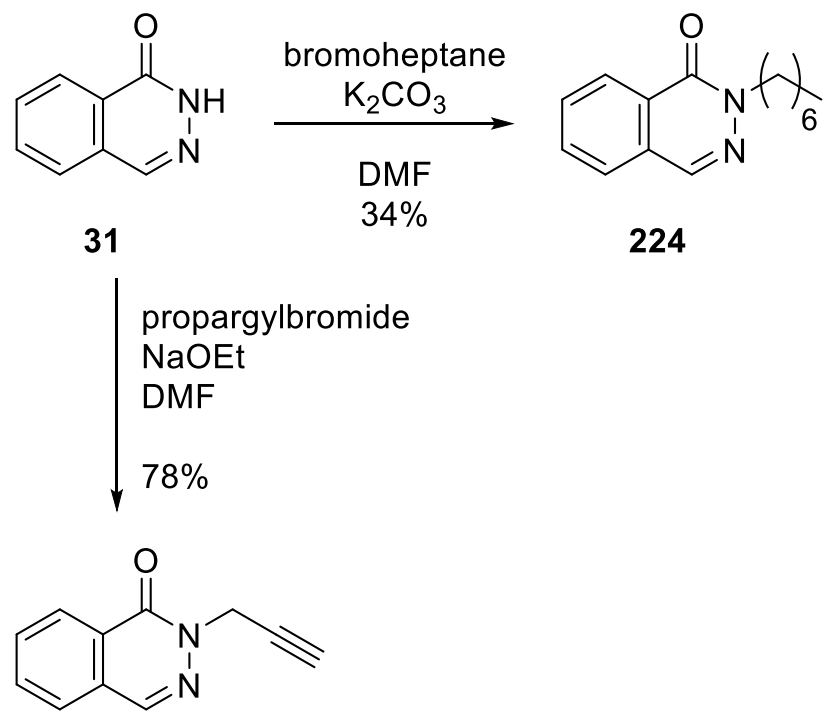

225

Scheme 5.3: The synthesis of heptylphthalazinone 224 and propargylphthalazinone 225 . 


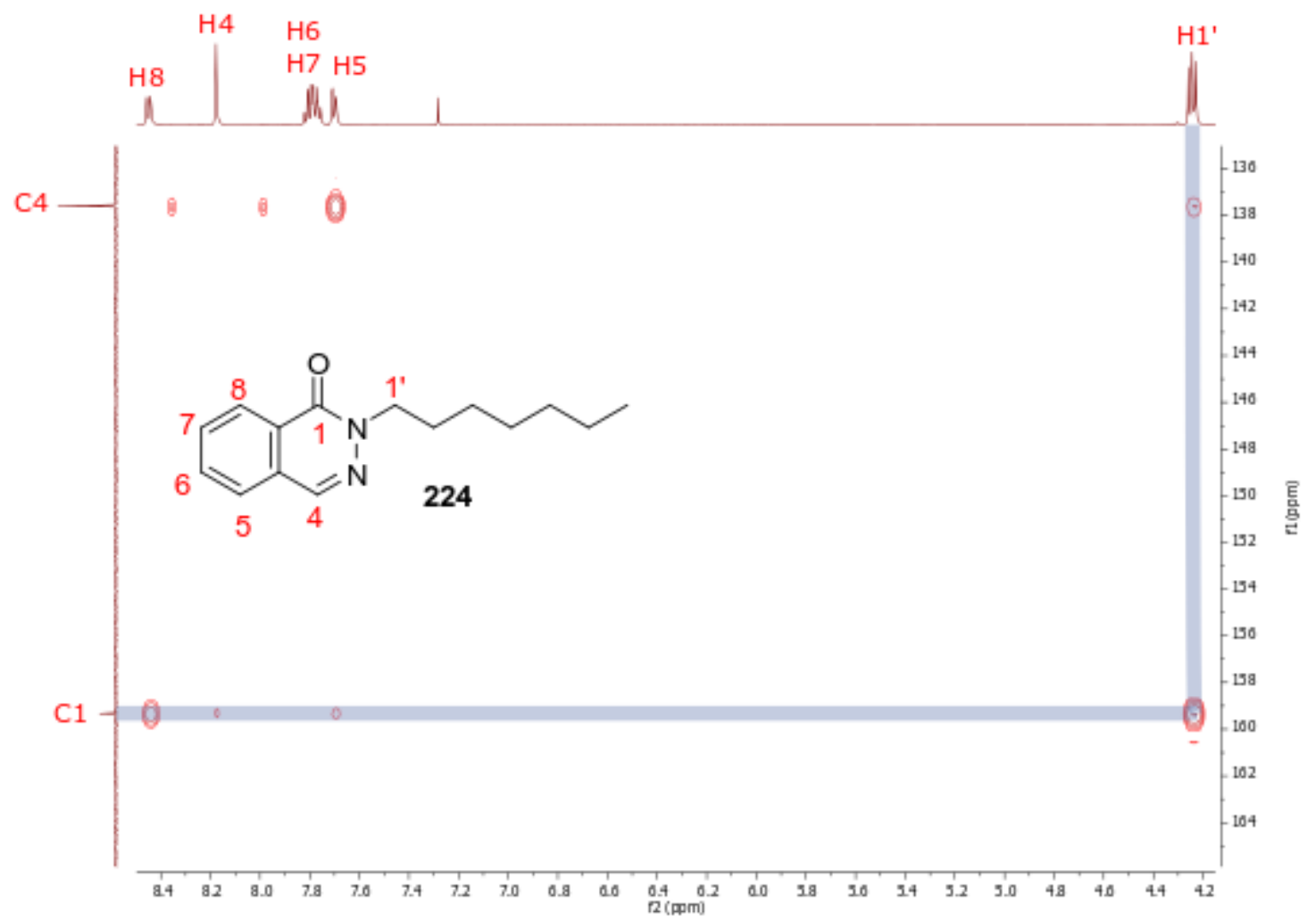

Figure 5.8: $\mathrm{HMBC}\left(500 \mathrm{MHz}, \mathrm{CDCl}_{3}\right)$ of heptyl phthalazinone 224 .

Having completed the synthesis of phthalazinones 31 and 216-225, 7-substituted phthalazinones were prepared (Scheme 5.4). Initially, it was envisaged that nitro-phthalide 226 could be synthesised by subjecting bromo-benzofuranone 215 to nitric acid. Unfortunately however, benzofuranone 215 was unstable under highly acidic conditions, due to the lability of the bromide group. Accordingly, nitration of isobenzofuran-1(3H)-one (214) was first undertaken to give nitro-phthalide 227, which was then subjected to Wohl Ziegler conditions ${ }^{338}$ to yield the desired phthalide 226 . $^{344}$ 
<smiles>[Z15]C1OC(=O)c2ccccc21</smiles>

215<smiles>CC(C)(C)O[Na]</smiles>

$\mathrm{H}_{2} \mathrm{SO}_{4}$<smiles>O=C1OC(Br)c2ccc([N+](=O)[O-])cc21</smiles>

NBS, AIBN

$\mathrm{CCl}_{4}$

$82 \%$<smiles>O=C1OCc2ccccc21</smiles>

214<smiles>O=C1OCc2ccc([N+](=O)[O-])cc21</smiles>

227

Scheme 5.4: Synthesis of nitro phthalide $226 .{ }^{344}$

Next, bromo-phthalide 226 was reacted with phenylhydrazinium chloride to give phenylphthalazinone 228 (Scheme 5.5), ${ }^{345}$ the structure of which was unequivocally confirmed by XRD data (Figure 5.9). Next, an iron-mediated reduction of nitro-phthalazinone 228 was then undertaken to afford 7-amino-2-phenyl phthalazinone (229) in 99\% yield. ${ }^{345}$ Similarly, treatment of nitrophthalide $\mathbf{2 2 6}$ with hydrazine hydrate gave nitro-phthalazinone $\mathbf{2 3 0}$, which was alkylated with propargylbromide and bromoheptane to give 7-nitro-2-propargylphthalazinone 231 and 7-nitro-2-heptyl-phthalazinone 233, respectively. Finally, nitrophthalazinones 231 and 233 were reduced using iron powder to give amino-propargyl phthalazinone 232 and amino-heptyl-phthalazinone 234, respectively, in excellent yields from their respective nitro-functionalised precursors. Confirmation of successful synthesis of amino-phthalazinones 229, 232, and 234 was observed by ${ }^{1} \mathrm{H}$ NMR spectroscopy, whereby a broad singlet at approximately $7 \mathrm{ppm}$ in $\mathrm{CDCl}_{3}$ corresponded to primary amine protons and IR spectroscopy revealed stretching frequencies between $3400-3300 \mathrm{~cm}^{-1}$, typical of $\mathrm{N}-\mathrm{H}$ stretches. ${ }^{130}$ 
<smiles>O=C1OC(Br)c2ccc([N+](=O)[O-])cc21</smiles>

1. $5 \% \mathrm{HCl}$

2. Hydrazine $\underset{73 \%}{\stackrel{\text { hydrate }}{\longrightarrow}}$

226

1. $5 \% \mathrm{HCl}$

2. Phenylhydrazinium chloride $73 \%$<smiles>O=c1c2cc([N+](=O)[O-])ccc2cnn1-c1ccccc1</smiles>

228

$\mathrm{Fe}, \mathrm{NH}_{4} \mathrm{HCO}_{2}$ $\mathrm{EtOH} / \mathrm{H}_{2} \mathrm{O}$ $99 \%$<smiles>Nc1ccc2cnn(-c3ccccc3)c(=O)c2c1</smiles>

229<smiles>O=c1[nH]ncc2ccc([N+](=O)[O-])cc12</smiles>

230<smiles></smiles>

233

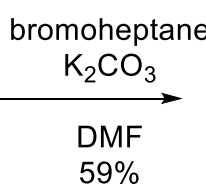

$59 \%$ $\mathrm{Fe}, \mathrm{NH}_{4} \mathrm{HCO}_{2}$ $\mathrm{EtOH} / \mathrm{H}_{2} \mathrm{O}$ $98 \%$<smiles>CC(C)(C)n1ncc2ccc(N)cc2c1=O</smiles>

234

231 $\mathrm{Fe}, \mathrm{NH}_{4} \mathrm{HCO}_{2}$ $\mathrm{EtOH} / \mathrm{H}_{2} \mathrm{O}$ $88 \%$<smiles>C#CCn1ncc2ccc(N)cc2c1=O</smiles>

232

Scheme 5.5: Synthesis of amino- and nitro-phthalazinones 228-234.

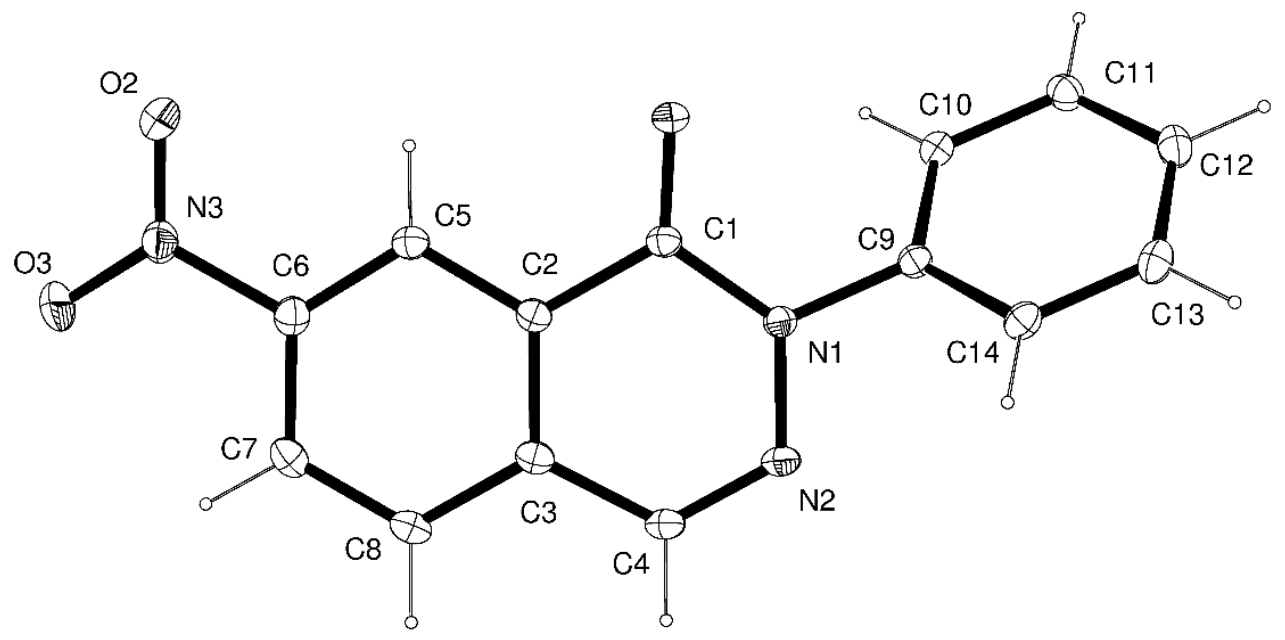

Figure 5.9: Crystal structure of nitrophthalazinone 228 . 


\subsubsection{The synthesis of pyrimidinyl-phthalazinones}

With amino phthalazinones 229, 232, and 234 in hand, the assembly of pyrimidinyl phthalazinones was undertaken. Accordingly, the condensation of amino-phthalazinones 229, 232, and 234 and chloropyrimidines 150, 187, and 188 under acidic conditions gave pyrimidinyl-phthalazinones 235-243 in moderate to good (24\%-83\%) yields (Scheme 5.6). Successful formation of pyrimidinyl-phthalazinones 235-243 was confirmed by NMR spectroscopy, which included an HMBC between the bridging amine and protons at the 5"-, 8-, and 6- positions, as illustrated in Figure 5.10 for phthalazinone 242.<smiles>[R]c1cc(Cl)nc(N)n1</smiles><smiles>[R]n1ncc2ccc(N)cc2c1=O</smiles>

$$
\begin{aligned}
150, \mathrm{R}^{1} & =\mathrm{CH}_{3} \\
187, \mathrm{R}^{1} & =\mathrm{Ph}
\end{aligned}
$$$$
\text { 229, } \mathrm{R}^{2}=\mathrm{Ph}
$$$$
\text { 232, } \mathrm{R}^{2}=\mathrm{C}_{3} \mathrm{H}_{3}
$$

188, $\mathrm{R}^{1}=4-\mathrm{F}-\mathrm{Ph}$

234, $\mathrm{R}^{2}=\mathrm{C}_{7} \mathrm{H}_{15}$<smiles>[R][Y12]1ncc2ccc(Nc3cc([R1])nc(N)n3)cc2c1=O</smiles>

$$
\begin{aligned}
& \text { 235, } R^{1}=\mathrm{CH}_{3}, \mathrm{R}^{2}=\mathrm{Ph}, 60 \% \\
& \text { 236, } \mathrm{R}^{1}=\mathrm{CH}_{3}, \mathrm{R}^{2}=\mathrm{C}_{7} \mathrm{H}_{15}, 79 \% \\
& \text { 237, } \mathrm{R}^{1}=\mathrm{CH}_{3}, \mathrm{R}^{2}=\mathrm{C}_{3} \mathrm{H}_{3}, 29 \% \\
& \text { 238, } \mathrm{R}^{1}=\mathrm{Ph}, \mathrm{R}^{2}=\mathrm{Ph}, 24 \% \\
& \text { 239, } \mathrm{R}^{1}=\mathrm{Ph}, \mathrm{R}^{2}=\mathrm{C}_{7} \mathrm{H}_{15}, 45 \% \\
& \text { 240, } \mathrm{R}^{1}=\mathrm{Ph}, \mathrm{R}^{2}=\mathrm{C}_{3} \mathrm{H}_{3}, 83 \% \\
& \text { 241, } \mathrm{R}^{1}=4-\mathrm{F}-\mathrm{Ph}, \mathrm{R}^{2}=\mathrm{Ph}, 38 \% \\
& \text { 242, } \mathrm{R}^{1}=4-\mathrm{F}-\mathrm{Ph}, \mathrm{R}^{2}=\mathrm{C}_{7} \mathrm{H}_{15}, 27 \% \\
& \text { 243, } \mathrm{R}^{1}=4-\mathrm{F}-\mathrm{Ph}, \mathrm{R}^{2}=\mathrm{C}_{3} \mathrm{H}_{3}, 53 \%
\end{aligned}
$$

Scheme 5.6: The synthesis of pyrimidinyl phthalazinones 235-243. 


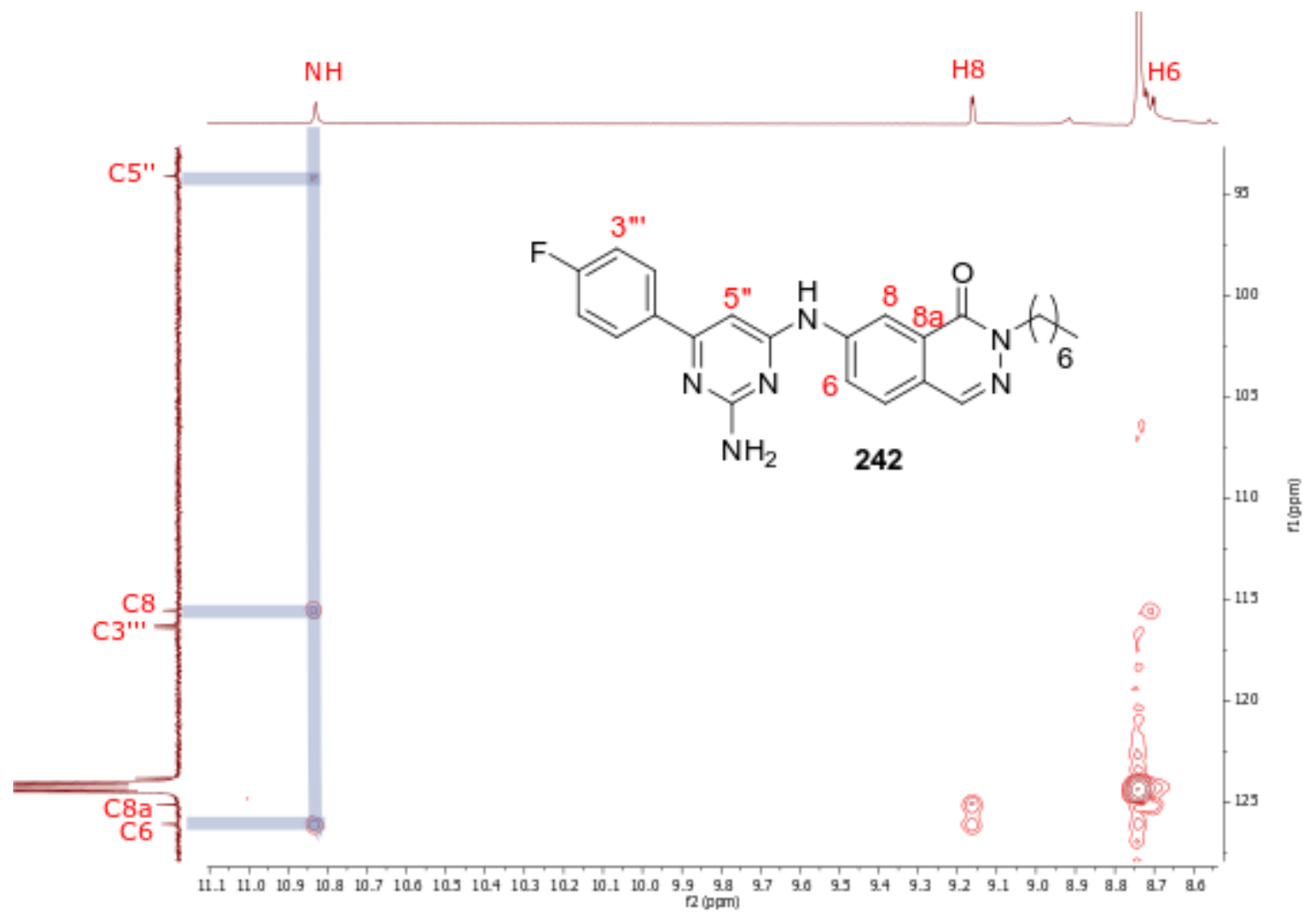

Figure 5.10: HMBC (500 MHz, pyridine- $\mathrm{d}_{5}$ ) of pyrimidinyl phthalazinone 242 .

\subsubsection{The anti-tubercular activity of phthalazinones and pyrimidinyl-phthalazinones}

Having completed the synthesis of phthalazinones and pyrimidinyl-phthalazinones, the compounds were then screened for their ability to inhibit the growth of Mtb (Table 5.1). The biological testing was conducted at the University of Otago under the supervision of Professor Gregory Cook. First, the anti-tubercular activity of unfunctionalised phthalazinone 31 (MIC = $50 \mu \mathrm{M}$, Entry 1) was compared with phthalazinones 212-225, which bear various lipophilic and electron-withdrawing substituents at the 2-position (Entries 2-11). Here, the incorporation of an alkyl chain at the 2-position led to an increase in inhibitory activity of the phthalazinones, with tert-butyl- (216), heptyl- (224) and propargyl- (225) phthalazinones all exhibiting low micromolar MIC values (3 $\mu \mathrm{M}, 6 \mu \mathrm{M}, 12.5 \mu \mathrm{M}$, respectively, Entries 2-4). However, phenylsubstituted phthalazinone 217 was a weaker inhibitor (MIC $=100 \mu \mathrm{M}$, Entry 5) compared to phthalazinone 31, though when an electron-withdrawing group was incorporated onto the phenyl ring (i.e. phenylphthalazinones 218-223, Entries 6-11), lower MIC values were obtained (MIC $=50 \mu \mathrm{M}$ for 218, 219, 221, 222, 223, MIC $=12.5 \mu \mathrm{M}$ for 220). These results supported the hypothesis that increasing lipophilicity and electron-withdrawing nature of the phthalazinones can increase the inhibitory activity of the compounds against $M t b$. 
Table 5.1: Inhibition of $M t b$ by phthalazinones and pyrimidinyl-phthalazinones.

Entry

224

4<smiles>C#CCn1ncc2ccccc2c1=O</smiles>

225

5<smiles>O=c1c2ccccc2cnn1-c1ccccc1</smiles>

100

217

6<smiles>O=c1c2ccccc2cnn1-c1ccc(F)cc1</smiles>

50

218

7<smiles>O=c1c2ccccc2cnn1-c1c(F)c(F)c(F)c(F)c1F</smiles>

219 
8<smiles>O=c1c2ccccc2cnn1-c1ccc(C(F)(F)F)cc1</smiles>

220

9<smiles>O=c1c2ccccc2cnn1-c1ccc(OC(F)(F)F)cc1</smiles>

221<smiles>O=c1c2ccccc2cnn1-c1ccc(S(=O)(=O)O)cc1</smiles>

222

11<smiles>NS(=O)(=O)c1ccc(-n2ncc3ccccc3c2=O)cc1</smiles>

223

12<smiles>O=c1[nH]ncc2ccc([N+](=O)[O-])cc12</smiles>

230

13<smiles>C#CCn1ncc2ccc([N+](=O)[O-])cc2c1=O</smiles>

231

14<smiles>CC(C)(C)n1ncc2ccc([N+](=O)[O-])cc2c1=O</smiles>

233 
<smiles>O=c1c2cc([N+](=O)[O-])ccc2cnn1-c1ccccc1</smiles>

228

16<smiles>CC1(n2ncc3ccc(N)cc3c2=O)CC1</smiles>

234

17<smiles>C#CCn1ncc2ccc(N)cc2c1=O</smiles>

232

18<smiles>Nc1ccc2cnn(-c3ccccc3)c(=O)c2c1</smiles>

229

19<smiles>Cc1cc(Nc2ccc3cnn(C4CC4)c(=O)c3c2)nc(N)n1</smiles>

20<smiles>C#CCn1ncc2ccc(Nc3cc(C)nc(N)n3)cc2c1=O</smiles>

21<smiles>Cc1cc(Nc2ccc3cnn(-c4ccccc4)c(=O)c3c2)nc(N)n1</smiles>

22<smiles>Nc1nc(Nc2ccc3cnn(C4CC4)c(=O)c3c2)cc(-c2ccccc2)n1</smiles>

100 
23<smiles></smiles>

24<smiles>[R4]OCC#C</smiles>

25<smiles>C#CCn1ncc2ccc(Nc3cc(-c4ccc(F)cc4)nc(N)n3)cc2c1=O</smiles>

27<smiles></smiles>

26<smiles></smiles>

100

12.5

50

12.5

1.6

Next, the effects of including electron-withdrawing and electron-donating groups at the 7 position of the phthalazinones on their anti-tubercular activity were analysed. Here, the incorporation of a nitro group at the 6-position led to a remarkable increase in anti-tubercular activity for phthalazinone 230 (MIC $=3 \mu \mathrm{M}$, Entry 12) as compared to the unfunctionalised phthalazinone 31 (MIC = $50 \mu \mathrm{M}$, Entry 1) and a two-fold improvement in activity for nitrophthalazinone 231 (MIC $=6 \mu \mathrm{M}$, Entry 13) when compared to the corresponding propargyl $225(\mathrm{MIC}=12.5 \mu \mathrm{M}$, Entry 4). For heptylphthalazinone 233 however, the 6-nitro group led to a loss in anti-tubercular activity $(\mathrm{MIC}=100 \mu \mathrm{M}$, Entry 14 vs. $\mathrm{MIC}=6 \mu \mathrm{M}$ for 224, Entry 3), while no change in inhibitory activity was observed between phenyl-phthalazinones 228 and 217 (Entries 15 and 5, respectively). The addition of electron-donating amine substituents 
at the 7-position (i.e. phthalazinones 234, 232 and 229, Entries 16-18) resulted in a loss of Mtb growth inhibition when compared to phthalazinones 224, 225, and 217, respectively (Entries 3-5). These results demonstrated that the instalment of an electron-donating group at the 7 position appears to reduce the inhibitory action of the drugs, while the incorporation of an electron-withdrawing group led to variable results with respect to their ability to inhibit mycobacterial growth. The decreased inhibition exhibited by aminophthalazinones 229, 232 and 234 could be attributed to the ability of amine groups to form hydrogen bonds with residues away from the putative NDH-II Q-site, i.e. E324. Moreover, the relatively high polarity of aminophthalazinones 229, 232 and 234 could also lower their binding affinity to the hydrophobic NDH Q-site.

Finally, the ability of pyrimidinyl-phthalazinones 235-243 to inhibit Mtb growth were determined. In general, compounds bearing a 6-methyl-pyrimidine moiety were poor $M t b$ inhibitors, with phthalazinones 235-237 having high MIC values (50 - $100 \mu \mathrm{M}$, Entries 19-21). Similarly, 6-phenyl-pyrimidines $\mathbf{2 3 9}$ and $\mathbf{2 2 8}$ had low potency against $M t b$ with an MIC value of $100 \mu \mathrm{M}$ (Entries 22 and 23), while phenyl-pyrimidinyl-alkyne 240 elicited good antitubercular activity (MIC = $12.5 \mu \mathrm{M}$, Entry 24). Aside from propargyl phthalazinone 243 (Entry 25), the incorporation of a fluoro-phenyl pyrimidine led to an increase of anti-tubercular activity of the pyrimidinyl-phthalazinones. Evidence for this trend include the low MIC values of fluorophenyl phthalazinones 241 (MIC $=12.5 \mu \mathrm{M}$, Entry 26) and $242(\mathrm{MIC}=1.6 \mu \mathrm{M}$, Entry 27), with phthalazinone 242 exhibiting the highest anti-tubercular activity of the series. Thus, while incoporating a pyrimidine group did not significantly increase the anti-tubercular activity of all phthalazinones, the lead $M t b$ inhibitor did contain this functional group, with fluorophenyl-pyrimidines contributing to higher anti-tubercular activity than the methyl- and phenylpyrimidines.

Taken together, these studies demonstrated that the inclusion of lipophilic and electronwithdrawing groups of the phthalazinones increased the anti-tubercular activity of the compounds. That said, the size and position of such functionalities also influence the inhibitory activity of the phthalazinones. To better explain the anti-tubercular activity of the compounds, investigations into the ability of the phthalazinones to affect NADH oxidation were undertaken. 


\subsubsection{The effect of NADH oxidation exerted by phthalazinones}

To investigate whether phthalazinones could affect mycobacterial respiration, the rates of $\mathrm{NADH}$ oxidation in the presence and absence of phthalazinones were measured using mycobacterial IMVs at the University of Otago under the supervision of Prof. Gregory Cook. Here, the results suggested that the addition of several phthalazinones to IMVs led to higher rates of NADH oxidation as compared to the basal rate (Figure 5.11). In particular, pyrimidinyl phthalazinones 238, 241 and 243 caused a higher rate of oxidation, suggesting that these compounds activate NDH-II, possibly via a redox-cycling mechanism. In contrast, many phthalazinones induced a lower rate of NADH oxidation, notably phenyl phthalazinone 217, heptyl phthalazinone $\mathbf{2 2 4}$, and nitrophthalazinone $\mathbf{2 3 3}$. The lower rates of NADH oxidation promoted by phthalazinones 217, 224, and 233 could be a result of NDH-II inhibition or uncoupling of oxidative phosphorylation, as observed with thioridazine (22). ${ }^{52,308}$ In the latter, NADH oxidation could slow down or halt when the $\Delta \varphi$ has collapsed, due to the release of charged species as a result of NADH oxidation. Taken together, it is likely that phthalazinones and pyrimidinyl phthalazinones could inhibit $M t b$ growth through different modes of action, whereby representative pyrimidinyl phthalazinones activate NDH-II, which could lead to the formation of lethal levels of ROS. ${ }^{37}$ On the other hand, several phthalazinones may exert their anti-tubercular activity through the inhibition of NDH-II and/or acting as an uncoupler of oxidative phosphorylation in mycobacteria. ${ }^{64}$ Notwithstanding, the mode of action of lead phthalazinones 216 and 230 and pyrimidinyl-phthalazinone 242 appears to be independent of NDH-II. 


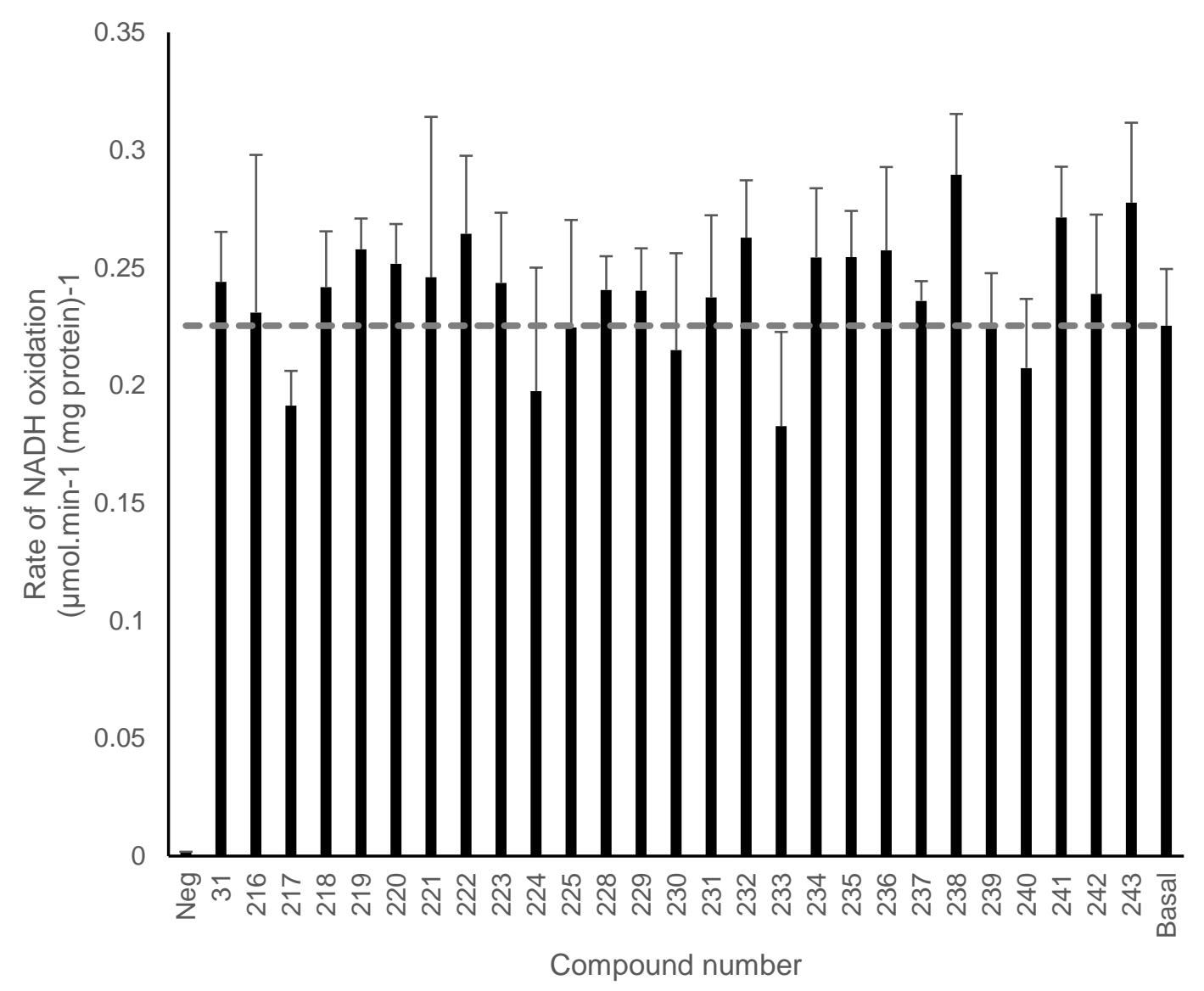

Figure 5.11: Rates of NADH oxidation when IMVs are treated with phthalazinones 31, 216225, 228-243, and pyrimidinyl phthalazinones 237-245. Negative control is the vehicle control, whereby IMV was not treated with any compound, nor was NADH added. The basal rate is the rate of NADH oxidation by IMVs without the addition of any drug. Data is shown as the average \pm standard deviation of three independent replicates.

\subsection{Conclusion}

In summary, a new class of anti-tubercular heterocyclic compounds, phthalazinones, was investigated. The bicyclic ketone-containing structure of phthalazinones make them a potential NDH-II substrate. As a weakly basic and apolar scaffold, phthalazinones also showed promise as an uncoupler. In the present study, a library of phthalazinones functionalised at the 2- and 7-positions, including pyrimidinyl-phthalazinones, were synthesised and tested against $M t b$. Docking studies of phthalazinones and pyrimidinyl-phthalazinones undertaken using the $C$. thermarum NDH-II protein showed that the phthalazinone scaffold could be accomodated in the putative NDH-II Q-site. To this end, a library of phthalazinones functionalised at the 2and 6'-positions were synthesised and screened for their ability to inhibit the growth of $M t b$. 
In general, phthalazinones bearing alkyl chains (phthalazinones 216, 224, 225) and electronwithdrawing substituents (i.e. phthalazinones 218-223) elicited good anti-tubercular activity. Moreover, the inclusion of amine groups at the 7-position led to reduced inhibitory activity of the phthalazinones, while the effect of a nitro group at this position gave varying inhibitory results. The pyrimidinyl-phthalazinones also exerted good inhibitory activity against $M t b$, identifying a lead compound, fluorophenyl-pyrimidinyl-heptyl-phthalazinone 244, with an MIC of $1.6 \mu \mathrm{M}$. The compounds were then screened for their ability to affect NADH oxidation IMVs. Here, increased rates of NADH oxidation were induced by pyrimidinyl phthalazinones 238, 241, and 243, while decreased rates were observed when the IMVs were treated with phthalazinones 217, 224, and 233. Other anti-tubercular phthalazinones, such as heptylphthalazinone 242 , did not greatly affect NADH oxidation, suggesting that the mechanism by which these compounds inhibit $M t b$ growth is independent of mycobacterial respiration. Taken together, the work presented in this chapter is the first to demonstrate the potential for phthalazinones to act as anti-tubercular agents. Further studies into optimising the antitubercular activity of these compounds and to better understand their mode of action are underway. 


\subsection{Experimental}

\subsubsection{Docking studies}

Phthalazinone 212 and pyrimidinyl-phthalazinone 213 were prepared for docking using the DockPrep tool in USCF Chimera (version 13.1). ${ }^{282}$ The quinone binding site of chain B of the C. thermarum NDH-II crystal structure (PDB: 6BDO) ${ }^{62}$ was used for docking. The NDH-II crystal structure was prepared by removing the co-crystallised ligand HQNO (19) and applying the DockPrep tool in USCF Chimera (version 13.1). A $5 \AA$ grid was generated whereby the centre was defined as residues 46, 47, 316, 317, 318, 347, 348, 349, 376, 379, 380, and 383. Ligands 212 and 213 were then modelled into the NDH-II quinone binding site using the anchor and grow algorithm in DOCK 6 (version 6.9). ${ }^{298}$ The conformers with the top 50 binding scores were analysed.

\subsubsection{Chemistry}

General procedure. Unless otherwise stated, all reactions were performed under atmospheric air. Prior to use, DMF (Acros) was distilled over $\mathrm{BaO}$, THF (Panreac) was distilled over sodium wire and benzophenone, $\mathrm{CH}_{2} \mathrm{Cl}_{2}$ (Fisher) was distilled from $\mathrm{P}_{2} \mathrm{O}_{5}$, pyridine $(\mathrm{BDH})$ was distilled over $\mathrm{CaH}_{2}$, and EtOAc (drum), petroleum ether (drum), acetone (drum), $\mathrm{MeOH}$ (Pure Science) and $\mathrm{H}_{2} \mathrm{O}$ were distilled. $12 \%$ AIBN in acetone (Aldrich), ammonium formate (AnalaR), $\mathrm{CCl}_{4}$ (AnalaR), diethyl ether (Aldrich), 2-ethoxyethanol (Unilab), 4-fluorophenyl hydrazine hydrochloride (Alfa Aesar), Fe (Scientific supplies), 37\% $\mathrm{HCl}$ (Merck), 4-hydrazine benzene sulfonic acid (Alfa Aesar), hydrazine hydrate (BDH), $\mathrm{MgSO}_{4}$ (Pure Science), NBS (Unilab), nitric acid (Aldrich), pentafluorophenyl hydrazine (Alfa Aesar), phenylhydrazinium chloride $(\mathrm{BDH})$, phthalic anhydride (Aldrich), potassium carbonate (Panreac), propargyl bromide (Acros), sodium (Aldrich), sodium borohydride (Aldrich), 4-sulfonamide hydrazine hydrochloride (Alfa Aesar), sulfuric acid (Panreac), tert-butyl hydrazine hydrochloride (Alfa Aesar), 4-trifluoromethoxyphenyl hydrazine hydrochloride (Alfa Aesar), 4(trifluoromethyl)phenyl hydrazine (Alfa Aesar), were used as received.

All solvents were removed by evaporation under reduced pressure. Reactions were monitored by TLC-analysis on Macherey-Nagel silica gel coated plastic sheets $(0.20 \mathrm{~mm}$, with fluorescent indicator $\mathrm{UV}_{254}$ ) with detection by UV-absorption (short wave UV - $254 \mathrm{~nm}$; long wave UV $366 \mathrm{~nm}$ ) by dipping in $\mathrm{KMnO}_{4}$ in $\mathrm{H}_{2} \mathrm{O}$. Column chromatography was performed on Pure Science silica gel (40 - 63 micron). Infrared spectra were recorded as thin films using a Bruker Alpha II FTIR spectrometer equipped with an Attenuated Total Reflectance (ATR) sampling 
accessory and are reported in wavenumbers $\left(\mathrm{cm}^{-1}\right)$. Melting points (m.p.) were obtained on a DigiMelt MPA160 melting point apparatus. Fluorescence data were obtained on a Shimadzu RF-5301PC spectrophotometer. Nuclear magnetic resonance spectra were recorded at $20{ }^{\circ} \mathrm{C}$ in $\mathrm{D}_{2} \mathrm{O}, \mathrm{CDCl}_{3}$, pyridine- $\mathrm{d}_{5}$, or DMSO- $\mathrm{d}_{6}$ using either a Varian INOVA operating at $500 \mathrm{MHz}$ $\left(500 \mathrm{MHz}\right.$ for ${ }^{1} \mathrm{H}$ nuclei and $125 \mathrm{MHz}$ for ${ }^{13} \mathrm{C}$ nuclei) or Varian Direct Drive operating at 600 $\mathrm{MHz}\left(600 \mathrm{MHz}\right.$ for ${ }^{1} \mathrm{H}$ nuclei, $150 \mathrm{MHz}$ for ${ }^{13} \mathrm{C}$ nuclei). Chemical shifts are given in ppm $(\delta)$ relative to solvent residues. NMR peak assignments were made using COSY, HSQC, and HMBC 2D experiments. High-resolution (ESI) mass spectrometric data were obtained with an Agilent 6530 Accurate-Mass Q-TOF LC-MS equipped with a 1260 Infinity binary pump.

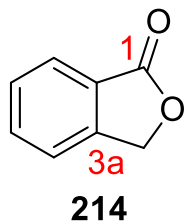

Isobenzofuran-1(3H)-one (214). To a solution of phthalic anhydride (13 g, 87 mmol) in freshly distilled THF $(152 \mathrm{~mL})$ at $0{ }^{\circ} \mathrm{C}$ was added $\mathrm{NaBH}_{4}(3.8 \mathrm{~g}, 87$ mmol, 1 equiv.) portionwise. The thick white suspension was then stirred for 12 $\mathrm{h}$ at room temperature, after which time the reaction was acidified with $2 \mathrm{M}$ aq. $\mathrm{HCl}$ until $\mathrm{pH} 1$ was reached. The resulting mixture was again stirred for $12 \mathrm{~h}$ at room temperature, after which time the reaction was concentrated in vacuo to give a brown solid. The crude product was then extracted with ethyl acetate $(50 \mathrm{~mL} \times 3)$ and washed with sat. aq. $\mathrm{NaHCO}_{3}\left(50 \mathrm{~mL}\right.$ x 2), followed by brine $(50 \mathrm{~mL})$, dried with anhydrous $\mathrm{MgSO}_{4}$, filtered, and concentrated in vacuo. Recrystallisation from $\mathrm{Et}_{2} \mathrm{O}$ gave the title compound as white crystals (9.2 g, 79\%). $\mathrm{R}_{f}=0.31$ (petroleum ether/EtOAc, 2/1, v/v); m.p.: $79.8^{\circ} \mathrm{C}\left(\right.$ lit. $^{337} 72-73{ }^{\circ} \mathrm{C}$ ); IR (Umax): 1745, 1712, 1615, 1595, 1466, 1438, 1367, 1318, 1286, 1217, 1199, 1110, 1048, 1017 , 999, 959, 737, 700, 680, 563, $473 \mathrm{~cm}^{-1} ;{ }^{1} \mathrm{H}$ NMR (500 MHz, $\left.\mathrm{CDCl}_{3}\right): \delta$ ppm $7.91\left(\mathrm{~d}, J_{6,7}=7.2\right.$ $\mathrm{Hz}, 1 \mathrm{H}, \mathrm{H} 7), 7.68$ (t, $\left.J_{4,5}=J_{5,6}=7.3 \mathrm{~Hz}, 1 \mathrm{H}, \mathrm{H} 5\right), 7.54-7.51$ (m, 2H, H4, H6), 5.33 (s, 2H, H3); ${ }^{13} \mathrm{C}$ NMR (125 MHz, $\mathrm{CDCl}_{3}$ ): $\delta$ ppm 171.24 (C1), 146.6 (C7a), 134.3 (C5), 129.2 (C6), 125.8 (C7), 125.7 (C3a), 122.2 (C4), 69.7 (C3); HRMS-ESI (m/s) calcd. for $\left[\mathrm{C}_{8} \mathrm{H}_{6} \mathrm{O}_{2}+\mathrm{H}\right]^{+}$: 135.0441, obsd.: 135.0444 The data obtained for this compound matched those reported in literature. $^{337}$

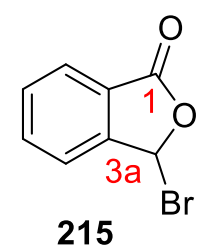

3-Bromoisobenzofuran-1(3H)-one (215). ${ }^{337}$ To a solution of phthalide 214 ( $2.6 \mathrm{~g}, 19.3 \mathrm{mmol})$ in $\mathrm{CCl}_{4}(77 \mathrm{~mL})$ was added NBS (10.3 g, $58 \mathrm{mmol}, 3$ equiv.) and AIBN (12\% in acetone, $2.2 \mathrm{~mL}, 0.97 \mathrm{mmol}, 0.1$ equiv.). The reaction was stirred at $75^{\circ} \mathrm{C}$ for $12 \mathrm{~h}$, after which time TLC analysis showed the disappearance of starting materials and the appearance of bromide 213. The precipitate was filtered and 
discarded, and the filtrate concentrated in vacuo. Purification via silica gel flash column chromatography (petroleum ether/EtOAc, $1 / 0 \rightarrow 3 / 1, \mathrm{v} / \mathrm{v}$ ) gave the title compound as colourless crystals $(2.6 \mathrm{~g}, 63 \%) . \mathrm{R}_{f}=0.44$ (petroleum ether/EtOAc, 1/1, v/v); m.p.: $87.3{ }^{\circ} \mathrm{C}$ (lit. $\left.{ }^{337} 79-82{ }^{\circ} \mathrm{C}\right)$; IR (U $\left.\mathrm{v}_{\max }\right): 1774,1601,1463,1341,1297,1277,1214,1172,1095,1034,959$, 772, 691, 650, 598, $459 \mathrm{~cm}^{-1} ;{ }^{1} \mathrm{H}$ NMR $\left(500 \mathrm{MHz}, \mathrm{CDCl}_{3}\right) \delta \mathrm{ppm} 7.93\left(\mathrm{~d}, J_{6,7}=7.5 \mathrm{~Hz}, 1 \mathrm{H}\right.$, $\mathrm{H} 7), 7.79\left(\mathrm{t}, J_{5,6}=J_{4,5}=7.6,1.0 \mathrm{~Hz}, 1 \mathrm{H}, \mathrm{H} 5\right), 7.64-7.61$ (m, 2H, H4, H6), 7.41 (s, H3); ${ }^{13} \mathrm{C}$ NMR (125 MHz, CDCl ${ }_{3}$ ) $\delta$ ppm 167.5 (C1), 149.0 (C3a), 135.4 (C5), 131.1 (C6), 126.1 (C7), 124.2 (C7a), 123.4 (C4), 74.7 (C3); HRMS-ESI ( $/$ / 2 ) calcd. for $\left[\mathrm{C}_{8} \mathrm{H}_{5}{ }^{79} \mathrm{BrO}_{2}+\mathrm{NH}_{4}\right]^{+}$: 229.9811, obsd.: 229.9812.<smiles>O=C1OC(Br)c2cc([N+](=O)[O-])ccc21</smiles>

after which time TLC analysis showed the disappearance of starting materials and the appearance of bromide 226. The precipitate was filtered and discarded, and the filtrate concentrated in vacuo. Purification via silica gel flash column chromatography (petroleum ether/EtOAc, $1 / 0 \rightarrow 3 / 1, \mathrm{v} / \mathrm{v}$ ) then gave the title compound as a yellow solid (554 mg, 82\%). $\mathrm{R}_{f}=0.6$ (petroleum ether/EtOAc, 4/1, v/v); IR (Umax): 1648, 1589, 1483, 1454, 1344, 1304, 1236, 1181, 1084, 948, 895, 890, 796, 761, 687, 602, 568, $487 \mathrm{~cm}^{-1} ;{ }^{1} \mathrm{H}$ NMR (500 MHz, $\left.\mathrm{CDCl}_{3}\right)$ : $\delta$ ppm 8.78 (s, 1H, H7), 8.67 (d, J4,5 = 8.4, 1H H5), 7.88 (d, J4,5 = 8.4 Hz, 1H, H4), 7.51 (s, 1H, $\mathrm{H} 3) ;{ }^{13} \mathrm{C}$ NMR (125 MHz, $\mathrm{CDCl}_{3}$ ): $\delta$ ppm 165.0 (C1), 153.6 (C7a), 149.8 (C6), 130.2 (C5), 125.8 (C3a), 125.1 (C4), 121.6 (C7), 73.1 (C3).

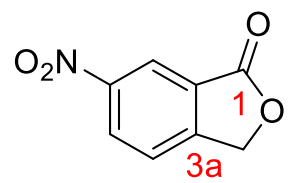

227

6-Nitrobenzofuran-1(3H)-one (227). ${ }^{344}$ Benzofuranone 214 (878 mg, 6.5 mmol) was dissolved in nitric acid $(0.91 \mathrm{~mL})$ and sulfuric acid $(1.1 \mathrm{~mL})$ at $0{ }^{\circ} \mathrm{C}$. The ice bath was then removed, allowing it to warm to room temperature, and stirred for $12 \mathrm{~h}$, after which time TLC analysis showed the disappearance of starting materials and the appearance of phthalide 229. The reaction was then poured into an ice- $\mathrm{H}_{2} \mathrm{O}$ bath and collected via filtration to give the crude product as a white solid. Recrystallisation from $\mathrm{Et}_{2} \mathrm{O}$ then gave the title compound as white crystals (919 mg, 79\%); m.p.: $176.2^{\circ} \mathrm{C} ; \mathrm{R}_{f}=0.21$ (petroleum ether/EtOAc, 2/1, v/v); IR ( $\left.\mathrm{u}_{\max }\right): 3056,1654$, 1560, 1522, 1457, 1309, 1143, 927, 848, 826, 782, 744, 681, 618, 571, 497, $418 \mathrm{~cm}^{-1} ;{ }^{1} \mathrm{H}$ NMR $\left(500 \mathrm{MHz}, \mathrm{CDCl}_{3}\right): \delta \mathrm{ppm} 8.76\left(\mathrm{~d}, J_{5,7}=1.8 \mathrm{~Hz}, 1 \mathrm{H}, \mathrm{H} 7\right), 8.58\left(\mathrm{dd}, J_{4,5}=8.3 \mathrm{~Hz}, J_{5,7}=2.0 \mathrm{~Hz}\right.$, 
1H, H5), $7.72\left(\mathrm{~d}, J_{4,5}=8.4 \mathrm{~Hz}, 1 \mathrm{H}, \mathrm{H} 4\right), 5.46(\mathrm{~s}, 2 \mathrm{H}, \mathrm{H} 3) ;{ }^{13} \mathrm{C} \mathrm{NMR}\left(125 \mathrm{MHz}, \mathrm{CDCl}_{3}\right) \delta \mathrm{ppm}$ 168.7 (C1), 151.9 (C3a), 149.2 (C6), 129.0 (C5), 127.7 (C7a), 123.7 (C4), 121.6 (C7), 69.7 (C3).

General procedure for the formation of phthalazinones. Bromophthalide 215 or 226 (1 equiv.) was dissolved in $5 \% \mathrm{HCl}$ in $\mathrm{H}_{2} \mathrm{O}(5 \mathrm{~mL} / \mathrm{mmol})$ and stirred at reflux for $2 \mathrm{~h}$, after which time the reaction was allowed to cool to room temperature. Once cooled, a hydrazinium chloride (1.2 equiv.) was added to the solution and the reaction stirred at room temperature for a further $2 \mathrm{~h}$. The resulting precipitate was then collected and recrystallised from hot ethanol to afford the title compounds.

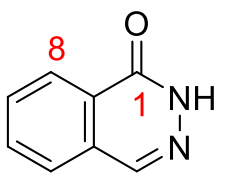

31

Phthalazin-1(2H)-one (31). ${ }^{340}$ By subjecting bromophthalide 215 (125.7 mg, $0.59 \mathrm{mmol})$ and hydrazine hydrate $(0.03 \mathrm{~mL}, 0.71 \mathrm{mmol})$ to the general procedure for the formation of phthalazinones to obtain the title compound as a white amorphous solid (40.5 $\mathrm{mg}, 47 \%$ ); $\mathrm{R}_{f}=0.27$ (petroleum ether/EtOAc, 1/1, v/v); IR (Umax): 2922, 1651, 1348, 1152, 1102, 1053, 877, 797, 769, 681, 591, 592, $490 \mathrm{~cm}^{-1} ;{ }^{1} \mathrm{H}$ NMR (500 MHz, $\left.\mathrm{CDCl}_{3}\right) \delta$ ppm 10.87 (br. s, $\left.1 \mathrm{H}, \mathrm{NH}\right), 8.46$ (d, J7,8 $=7.8 \mathrm{~Hz}$, 1H, H8), 8.20 (s, 1H, H4), $7.92-7.79$ (m, 2H, H6, H7), 7.76 (d, J5,6 = 7.9 Hz, 1H, H5); ${ }^{13} \mathrm{C}$ NMR (125 MHz, $\mathrm{CDCl}_{3}$ ) $\delta$ ppm 160.8 (C1), 139.2 (C4), 133.9 (C6), 132.0 (C7), 130.4 (C4a), 128.2 (C8a), 126.6 (C8), 126.4 (C5); HRMS-ESI (m/z) calcd. for $\left[\mathrm{C}_{8} \mathrm{H}_{6} \mathrm{~N}_{2} \mathrm{O}+\mathrm{H}\right]^{+}:$147.0553, obsd.: 147.0549 .

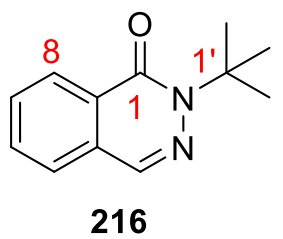

2-Tert-butylphthalazin-1(2H)-one (216). By subjecting bromophthalide $215(52 \mathrm{mg}, 0.24 \mathrm{mmol}$ ) and tert-butylhydrazinium hydrochloride $(35.9 \mathrm{mg}$, $0.29 \mathrm{mmol}$ ) to the general procedure for the formation of phthalazinones to obtain the title compound as a white amorphous solid (44.7 $\mathrm{mg}, 92 \%)$; $\mathrm{R}_{f}=0.74$ (EtOAc/petroleum ether, 1/1, v/v); IR (Umax): 1650, 1594, 1365, 1198, 1151, 1103, 1086, 761, 690, $602 \mathrm{~cm}^{-1} ;{ }^{1} \mathrm{H}$ NMR (500 MHz, $\left.\mathrm{CDCl}_{3}\right) \delta \mathrm{ppm} 8.44$ (d, $J_{7,8}=7.8 \mathrm{~Hz}, 1 \mathrm{H}, \mathrm{H} 8$ ), 8.13 (s, 1H, H4), 7.81 - 7.71 (m, 2H, H6, H7), 7.67 (d, J6,7 = 7.6 Hz, 1H, H5), 1.74 (s, 9H, H2); ${ }^{13} \mathrm{C}$ NMR (125 MHz, CDCl 3 ) $\delta$ ppm 160.0 (C1); 135.6 (C4), 132.7 (C7), 131.1 (C6), 129.4 (C4a), 129.3 (C8a), 126.6 (C8), 125.3 (C5), 64.2 (C1'), 28.3 (C2'); HRMS-ESI (m/z) calcd. for $\left[\mathrm{C}_{12} \mathrm{H}_{14} \mathrm{~N}_{2} \mathrm{O}+\mathrm{H}\right]^{+}:$203.1179, obsd.: 203.1178. The data obtained for this compound matched those reported in literature. ${ }^{341}$ 
<smiles>O=c1c2ccccc2cnn1-c1ccccc1</smiles>

217

2-Phenylphthalazin-1(2H)-one (217). By subjecting bromophthalide $215(50.2 \mathrm{mg}, 0.24 \mathrm{mmol})$ and phenylhydrazinium hydrochloride (41.6 $\mathrm{mg}, 0.29 \mathrm{mmol}$ ) to the general procedure for the formation of phthalazinones to obtain the title compound as a colourless crystals $(32.5$ $\mathrm{mg}, 61 \%) ; \mathrm{R}_{f}=0.45\left(\right.$ EtOAc/petroleum ether, 1/1, v/v); m.p.: $106.4^{\circ} \mathrm{C}$ (lit. ${ }^{341} 104-106{ }^{\circ} \mathrm{C}$ ); IR (U $\left.U_{\max }\right): 1654,1584,1488,1452,1330,1136,1060,904,862,798,764,735,706,685,617,599$, 582, 538, $483 \mathrm{~cm}^{-1} ;{ }^{1} \mathrm{H}$ NMR (500 MHz, $\left.\mathrm{CDCl}_{3}\right) \delta \mathrm{ppm} 8.52$ (d, J7,8 $\left.=7.7 \mathrm{~Hz}, 1 \mathrm{H}, \mathrm{H} 8\right), 8.30$ (s, 1H, H4), $7.87-7.80$ (m, 2H, H6, H7), 7.76 (d, $\left.J_{5,6}=7.8 \mathrm{~Hz}, 1 \mathrm{H}, \mathrm{H} 5\right), 7.66$ (d, $J_{2^{\prime}, 3^{\prime}}=8.0 \mathrm{~Hz}$, $\left.2 \mathrm{H}, \mathrm{H} 2^{\prime}\right), 7.50\left(\mathrm{t}, J_{2^{\prime}, 3^{\prime}}=J_{3^{\prime}, 4^{\prime}}=7.9 \mathrm{~Hz}, 2 \mathrm{H}, \mathrm{H} 3^{\prime}\right), 7.39$ (t, $\left.J_{3^{\prime}, 4^{\prime}}=7.7 \mathrm{~Hz}, 1 \mathrm{H}, \mathrm{H} 4^{\prime}\right) ;{ }^{13} \mathrm{C}$ NMR $(125$ MHz, CDCl ${ }_{3}$ ) $\delta$ ppm 159.2 (C1), 141.9 (C1'), 138.5 (C4), 133.5 (C6), 132.0 (C7), 129.5 (C4a), 128.8 (C8), 128.6 (C8a), 127.8 (C3'), 127.3 (C4'), 126.2 (C5), 125.8 (C2'); HRMS-ESI (m/z) calcd. for $\left[\mathrm{C}_{14} \mathrm{H}_{1} \mathrm{~N}_{2} \mathrm{O}+\mathrm{H}\right]^{+}: 223.0866$, obsd.: 223.0864. The data obtained for this compound matched those reported in literature. ${ }^{341}$<smiles>O=c1c2ccccc2cnn1-c1ccc(F)cc1</smiles>

218

2-(4-Fluorophenyl)phthalazin-1(2H)-one (218). By subjecting bromophthalide 215 (28.0 $\mathrm{mg}, 0.13 \mathrm{mmol}$ ) and 4-fluorophenyl hydrazine hydrochloride $(25.4 \mathrm{mg}, 0.16 \mathrm{mmol})$ to the general procedure for the formation of phthalazinones to obtain the title compound as white crystals $(25.0 \mathrm{mg}, 80 \%) ; \mathrm{R}_{f}=0.57$ (EtOAc/petroleum ether, 1/1, v/v);

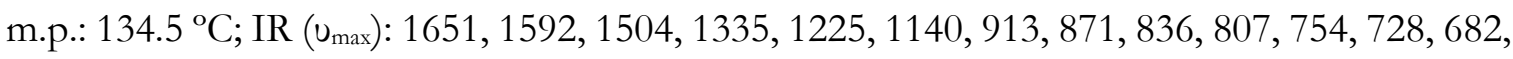
611, 596, 451, 518, $477 \mathrm{~cm}^{-1} ;{ }^{1} \mathrm{H}$ NMR (500 MHz, $\left.\mathrm{CDCl}_{3}\right) \delta \mathrm{ppm} 8.53\left(\mathrm{~d}, J_{7,8}=7.5 \mathrm{~Hz}, 1 \mathrm{H}\right.$, H8), 8.31 (s, 1H, H4), $7.93-7.81$ (m, 2H, H6, H7), 7.78 (d, J5,6 = 7.4 Hz, 1H, H5), $7.70-7.63$ (m, 2H, H2'), $7.23-7.15$ (m, 2H, H3'); ${ }^{13} \mathrm{C}$ NMR (125 MHz, CDCl $) \delta$ ppm $161.6\left(\mathrm{~d}, J_{4^{\prime}, \mathrm{F}}=\right.$ 245.4 Hz, C4'), 159.2 (C1), 137.9 (C1'), 138.6 (C4), 133.6 (C6), 132.1 (C7), 129.5 (C8a), 128.5

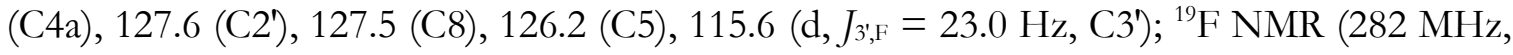
$\left.\mathrm{CDCl}_{3}\right) \delta \mathrm{ppm}$-113.90; HRMS-ESI $(\mathrm{m} / \mathrm{z})$ calcd. for $\left[\mathrm{C}_{14} \mathrm{H}_{9} \mathrm{FN}_{2} \mathrm{O}+\mathrm{H}\right]^{+}: 241.0772$, obsd.: 241.0769. The data for this compound matched that in literature. ${ }^{342}$<smiles>O=c1c2ccccc2cnn1-c1c(F)c(F)c(F)c(F)c1F</smiles>

219

2-(Pentafluorophenyl)phthalazin-1(2H)-one (219). By subjecting bromophthalide $215(51.0 \mathrm{mg}, 0.24 \mathrm{mmol})$ and pentafluorophenyl hydrazine $(57.0 \mathrm{mg}, 0.29 \mathrm{mmol})$ to the general procedure for the formation of phthalazinones to obtain the title compound as white crystals (25.5 mg, 34\%); $\mathrm{R}_{f}=0.23$ (petroleum ether/EtOAc, 4/1, v/v); m.p.: $136.8^{\circ} \mathrm{C}$; IR (Umax): 1678, 1509, 1301, 1136, 1070, 992, 958, 899, 801, 764, 734, 682, 
616, 598, $489 \mathrm{~cm}^{-1} ;{ }^{1} \mathrm{H}$ NMR (500 MHz, $\left.\mathrm{CDCl}_{3}\right) \delta \mathrm{ppm} 8.51$ (d, J7,8 $\left.=7.8 \mathrm{~Hz}, 1 \mathrm{H}, \mathrm{H} 8\right), 8.32$ (s, 1H, H4), $7.95\left(\operatorname{td}, J_{6,7}=J_{5,6}=7.6 \mathrm{~Hz}, J_{6,8}=1.4 \mathrm{~Hz}, 1 \mathrm{H}, \mathrm{H} 6\right), 7.89$ (dd, $J_{7,8}=8.6 \mathrm{~Hz}, J_{5,7} 1.3 \mathrm{~Hz}$, $1 \mathrm{H}, \mathrm{H} 7), 7.82\left(\mathrm{dd}, J_{4,5}=7.8 \mathrm{~Hz}, J_{5,7}=1.3 \mathrm{~Hz}, 1 \mathrm{H}, \mathrm{H} 5\right) ;{ }^{13} \mathrm{C} \mathrm{NMR}\left(125 \mathrm{MHz}, \mathrm{CDCl}_{3}\right) \delta \mathrm{ppm}$ 162.3 (C1'), 158.9 (C1), 145.2 (CF), 143.1 (CF), 140.9 (CF), 140.4 (C4), 139.2 (CF), 137.0 (CF), 134.6 (C6), 132.8 (C7), 129.8 (C8a), 127.8 (C4a), 127.5 (C8), 126.9 (C5); ${ }^{19}$ F NMR (282 MHz, $\left.\mathrm{CDCl}_{3}\right) \delta \mathrm{ppm}-144.49\left(\mathrm{~d}, J_{\mathrm{o}, \mathrm{m}}=17.8 \mathrm{~Hz}, 2 \mathrm{~F}, o-\mathrm{F}\right),-152.85\left(\mathrm{t}, J_{\mathrm{m}, \mathrm{p}}=21.1 \mathrm{~Hz}, 1 \mathrm{~F}, p-\mathrm{F}\right),-161.74$ $\left(\mathrm{t}, J_{\mathrm{o}, \mathrm{m}}=J_{\mathrm{m}, \mathrm{p}}=21.89 \mathrm{~Hz}, 2 \mathrm{~F}, m-\mathrm{F}\right)$; HRMS-ESI $(m / z)$ calcd. for $\left[\mathrm{C}_{14} \mathrm{H}_{5} \mathrm{~F}_{5} \mathrm{~N}_{2} \mathrm{O}+\mathrm{H}\right]^{+}: 313.0395$, obsd.: 313.0398 .

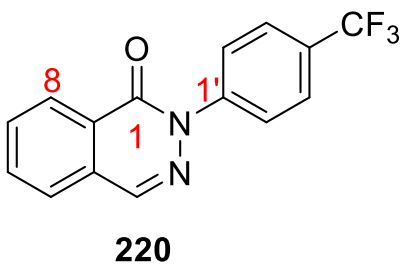

2-(4-Trifluoromethyl)phenyl)phthalazin-1(2H)-one (220). By subjecting bromophthalide 215 (45.9 $\mathrm{mg}, 0.22 \mathrm{mmol}$ ) and 4(trifluoromethyl)phenyl hydrazine $(46.5 \mathrm{mg}, 0.26 \mathrm{mmol})$ to the general procedure for the formation of phthalazinones to obtain the title compound as white crystals $(37.0 \mathrm{mg}, 58 \%) ; \mathrm{R}_{f}=0.52($ EtOAc/petroleum ether, $1 / 1$, v/v); m.p.: $164.9^{\circ} \mathrm{C}$; IR ( $\left.\mathrm{u}_{\max }\right): 2173,1671,1327,1105,1073,1057,870,834,7674,682,626$, 602, 536, 503, 435, 424, $407 \mathrm{~cm}^{-1} ;{ }^{1} \mathrm{H}$ NMR $\left(500 \mathrm{MHz}, \mathrm{CDCl}_{3}\right) \delta \mathrm{ppm} 8.51$ (d, J7,8 $=7.9 \mathrm{~Hz}$, 1H, H8), 8.31 (s, 1H, H4), $7.91-7.80$ (m, 4H, H6, H7, H2'), $7.80-7.71$ (m, 3H, H5, H3) ; ${ }^{13} \mathrm{C}$ NMR (125 MHz, $\mathrm{CDCl}_{3}$ ) $\delta$ ppm 159.3 (C1), 144.8 (C1), 139.2 (C4), 134.0 (C6), 132.4 (C7), $129.5\left(\mathrm{q}, J_{4^{\prime}, \mathrm{F}}=32.6 \mathrm{~Hz}, \mathrm{C} 4\right), 129.4(\mathrm{C} 8 \mathrm{a}), 128.5(\mathrm{C} 4 \mathrm{a}), 127.3(\mathrm{C} 8), 126.4(\mathrm{C} 5), 125.9$ (q, J $J_{3^{\prime}, \mathrm{F}}=$ $\left.3.7 \mathrm{~Hz}, \mathrm{C}^{\prime}\right), 125.9$ (C2'), 124.0 (q, $\left.J_{\mathrm{CF} 3, \mathrm{~F}}=272.9 \mathrm{~Hz}, \mathrm{CF}_{3}\right) ;{ }^{19} \mathrm{~F}$ NMR $\left(282 \mathrm{MHz}, \mathrm{CDCl}_{3}\right) \delta \mathrm{ppm}$ -62.46; HRMS-ESI $(\mathrm{m} / \mathrm{z})$ calcd. for $\left[\mathrm{C}_{15} \mathrm{H}_{9} \mathrm{~F}_{3} \mathrm{~N}_{2} \mathrm{O}+\mathrm{H}\right]^{+}:$291.0740, obsd.: 291.0740. The data obtained for this compound matched those reported in literature. ${ }^{343}$

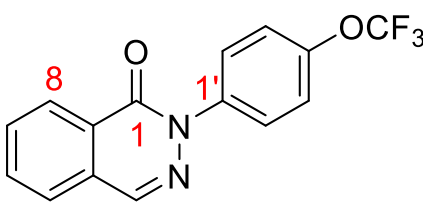

221

\section{2-(4-(Trifluoromethoxy)phenyl)phthalazin-1(2H)-one}

(221). By subjecting bromophthalide 215 (21.4 mg, $0.10 \mathrm{mmol})$ and 4-(trifluoromethoxy)phenyl hydrazine $(27.4 \mathrm{mg}, 0.12 \mathrm{mmol})$ to the general procedure for the formation of phthalazinones to

obtain the title compound as white crystals (19.9 mg, 65\%); $\mathrm{R}_{f}=0.6$ (EtOAc/petroleum ether, 1/1, v/v); m.p.: $115^{\circ} \mathrm{C}$; IR (U $\max$ ): 1660. 1590. 1504. 1137. 902. 842. 766. 685. 592. 504. 481 $\mathrm{cm}^{-1} ;{ }^{1} \mathrm{H}$ NMR $\left(500 \mathrm{MHz}, \mathrm{CDCl}_{3}\right) \delta$ ppm 8.51 (d, J7,8 $\left.=7.8 \mathrm{~Hz}, 1 \mathrm{H}, \mathrm{H} 8\right), 8.30$ (s, 1H, H4), 7.90

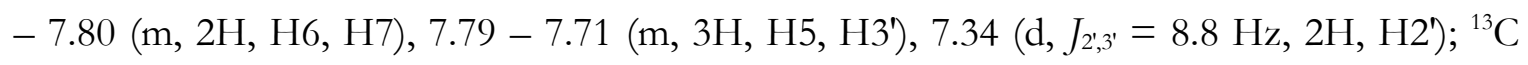
NMR (125 MHz, $\mathrm{CDCl}_{3}$ ) $\delta$ ppm 159.3 (C1), 148.2 (C4'), 140.4 (C1'), 139.0 (C4), 133.9 (C6), 132.4 (C7), 129.6 (C8a), 128.6 (C4a), 127.5 (C8), 127.2 (C3'), 126.4 (C5), 121.4 (C2'), 120.6 (q, 
$\left.J_{\mathrm{CF} 3, \mathrm{~F}}=269.7 \mathrm{~Hz}, \mathrm{CF}_{3}\right) ;{ }^{19} \mathrm{~F}$ NMR $\left(282 \mathrm{MHz}, \mathrm{CDCl}_{3}\right) \delta \mathrm{ppm}-57.85$; HRMS-ESI $(m / z)$ calcd. for $\left[\mathrm{C}_{15} \mathrm{H}_{9} \mathrm{~F}_{3} \mathrm{~N}_{2} \mathrm{O}_{2}+\mathrm{H}\right]^{+}:$207.0689, obsd.: 307.0691 .

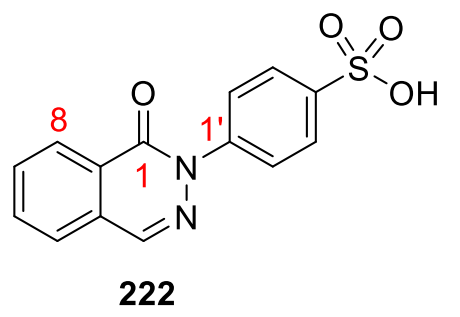

2-(1-Oxophthalazin-1(2H)-yl)benzenesulfonic acid (222).

By subjecting bromophthalide $215(49.3 \mathrm{mg}, 0.23 \mathrm{mmol})$ and $4-$ hydrazine benzene sulfonic acid $(51.9 \mathrm{mg}, 0.28 \mathrm{mmol})$ to the general procedure for the formation of phthalazinones to obtain the title compound as colourless crystals $(52.8 \mathrm{mg}, 76 \%) ; \mathrm{R}_{f}=$ 0.19 (EtOAc/MeOH, 4/1, v/v); m.p.: $214.8^{\circ} \mathrm{C}$; IR (umax): 3032, 1645, 1591, 1428, 1332, 1225 , 1177, 1120, 1029, 987, 912, 8334, 762, 684, 666, 618, 593, $572 \mathrm{~cm}^{-1} ;{ }^{1} \mathrm{H}$ NMR (500 MHz, DMSO-d $)_{6} \delta$ ppm 8.58 (s, 1H, H4), $8.32\left(\mathrm{dd}, J_{7,8}=7.9 \mathrm{~Hz}, J_{4,6}=1.1 \mathrm{~Hz}, 1 \mathrm{H}, \mathrm{H} 8\right), 8.04-7.95$ (m, 2H, H5, H7), 7.91 (t, J5,6 = 7.3 Hz, 1H, H6), 7.78 - 7.72 (m, 2H, H2'), 7.63 - 7.57 (m, 2H, $\left.\mathrm{H}^{3}\right)$; ${ }^{13} \mathrm{C}$ NMR (125 MHz, dmso-d 6 ) $\delta$ ppm 158.8 (C1), 142.2 (C4'), 139.4 (C1'), 134.5 (C7), 133.0 (C6), 129.7 (C4a), 128.1 (C8a), 127.6 (C5), 126.7 (C8), 126.3 (C2'), 125.8 (C3'); HRMS$\operatorname{ESI}(m / 2)$ calcd. for $\left[\mathrm{C}_{14} \mathrm{H}_{10} \mathrm{~N}_{2} \mathrm{O}_{4} \mathrm{~S}+\mathrm{H}\right]^{+}: 303.0434$, obsd.: 303.0434 .

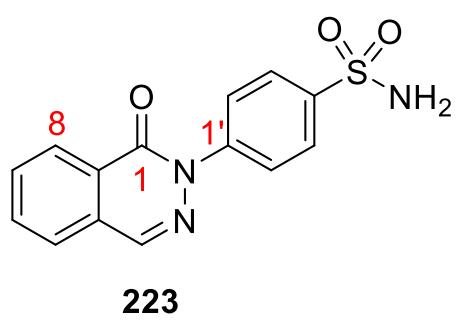

2-(1-Oxophthalazin-1(2H)-yl)benzenesulfonamide (223). By subjecting bromophthalide 215 (35.1 $\mathrm{mg}, 0.17 \mathrm{mmol}$ ) and 4sulfonamide phenyl hydrazine hydrochloride $(44.3 \mathrm{mg}, 0.20$ $\mathrm{mmol}$ ) to the general procedure for the formation of phthalazinones to obtain the title compound as white crystals (19.5 mg, 38\%); $\mathrm{R}_{f}=0.52$ (EtOAc); IR (U $\left.\max \right): 3290,1643,1580,1491,1331,1156,1091,903$, 831, 758, 685, 612, 584, 549, $524 \mathrm{~cm}^{-1} ;{ }^{1} \mathrm{H}$ NMR (500 MHz, pyridine-d $\left.{ }_{5}\right) \delta \mathrm{ppm} 9.19$ (s, 2H, $\mathrm{NH}_{2}$ ), 8.56 (d, J7,8 $\left.=7.9 \mathrm{~Hz}, 1 \mathrm{H}, \mathrm{H} 8\right), 8.45-8.38$ (m, 3H, H4, H2'), 8.18 - 8.07 (m, 2H, H3'), 7.81 - 7.69 (m, 3H, H6, H7, H7); ${ }^{13} \mathrm{C}$ NMR (125 MHz, pyridine-d $\left.\mathrm{d}_{5}\right) \delta$ ppm 160.39 (C1), 146.5 (C4'), 145.3 (C1), 140.7 (C4), 135.3 (C6), 133.7 (C7), 131.1 (C4a), 130.0 (C8a), 128.5 (C8), 128.26 (C2'), 128.23 (C5), 127.7 (C3'); HRMS-ESI ( $m / z)$ calcd. for $\left[\mathrm{C}_{14} \mathrm{H}_{11} \mathrm{~N}_{3} \mathrm{O}_{3} \mathrm{~S}+\mathrm{H}\right]^{+}$: 302.0594, obsd.: 302.0592 . 
<smiles>O=c1c2ccc([N+](=O)[O-])cc2cnn1-c1ccccc1</smiles>

228

7-Nitro-2-phenylphthalazin-1(2H)-one (228). By subjecting bromophthalide 226 (326 mg, $1.26 \mathrm{mmol}$ ) and phenylhydrazinium chloride (219 mg, $1.51 \mathrm{mmol})$ to the general procedure for the formation of phthalazinones to obtain the title compound as yellow amorphous solid (252.5 mg, 75\%); $\mathrm{R}_{f}=0.21$ (petroleum ether/EtOAc, 1/1, v/v); m.p.: $149{ }^{\circ} \mathrm{C}$; IR ( $\left.U_{\max }\right): 1656,1591,1518,1348,1142,924,847,823,753,680,618,588 \mathrm{~cm}^{-1} ;{ }^{1} \mathrm{H}$ NMR $\left(500 \mathrm{MHz}, \mathrm{CDCl}_{3}\right) \delta$ ppm $9.34\left(\mathrm{~d}, J_{6,8}=2.2 \mathrm{~Hz}, 1 \mathrm{H}, \mathrm{H} 8\right), 8.65$ (dd, $J_{5,6}=8.5, J_{6,8}=2.3$ $\mathrm{Hz}, 1 \mathrm{H}, \mathrm{H6}$ ), 8.41 (s, 1H, H4), 7.95 (d, J5,6 = $8.7 \mathrm{~Hz}, 1 \mathrm{H}, \mathrm{H} 5), 7.66$ (d, $J_{2^{\prime} 3^{\prime}}=7.4 \mathrm{~Hz}, 2 \mathrm{H}, \mathrm{H} 2^{\prime}$ ), $7.53\left(\mathrm{t}, J_{2^{\prime}, 3^{\prime}}=J_{3^{\prime}, 4^{\prime}}=7.9 \mathrm{~Hz}, 2 \mathrm{H}, \mathrm{H} 3^{\prime}\right), 7.44\left(\mathrm{t}, J_{3^{\prime}, 4^{\prime}}=7.4 \mathrm{~Hz}, 1 \mathrm{H}, \mathrm{H} 4^{\prime}\right) ;{ }^{13} \mathrm{C}$ NMR $(125 \mathrm{MHz}$, $\mathrm{CDCl}_{3}$ ) $\delta$ ppm 157.9 (C1), 149.5 (C7), 141.2 (C1'), 136.8 (C4), 133.0 (C8a), 129.4 (C4a), 129.0 (C3'), 128.4 (C4'), 128.0 (C5), 127.7 (C6), 125.5 (C2'), 123.5 (C8); HRMS-ESI ( $m / 2)$ calcd. for $\left[\mathrm{C}_{14} \mathrm{H}_{9} \mathrm{~N}_{3} \mathrm{O}_{3}+\mathrm{H}\right]^{+}:$268.0717, obsd.: 268.0716. The data obtained for this compound were consistent with those reported in literature. ${ }^{345}$<smiles>O=c1[nH]ncc2ccc([N+](=O)[O-])cc12</smiles>

230

7-Nitro-phthalazin-1(2H)-one (230). By subjecting bromophthalide 226 (184 mg, $0.71 \mathrm{mmol})$ and hydrazine hydrate $(0.05 \mathrm{~mL}, 1.07 \mathrm{mmol})$ to the general procedure for the formation of phthalazinones to obtain the title compound as yellow amorphous solid (99.1 mg, 73\%); $\mathrm{R}_{f}=0.35$ (EtOAc/petroleum ether, 1/1, v/v); IR ( $\left.U_{\max }\right): 3036,2919,2870,1685,1670,1617,1513,1334$, 1225, 1154, 1091, 926, 897, 800, 745, 677, 622, 562, $529 \mathrm{~cm}^{-1}$; ${ }^{1} \mathrm{H}$ NMR $(500 \mathrm{MHz}$, pyridine$\left.\mathrm{d}_{5}\right) \delta \mathrm{ppm} 9.33\left(\mathrm{~d}, J_{6,8}=2.3 \mathrm{~Hz}, 1 \mathrm{H}, \mathrm{H} 8\right), 8.57\left(\mathrm{dd}, J_{5,6}=8.6 \mathrm{~Hz}, J_{6,8}=2.4 \mathrm{~Hz}, 1 \mathrm{H}, \mathrm{H} 6\right), 8.48$ (s, 1H, H4), 7.95 (d, $\left.J_{5,6}=8.6 \mathrm{~Hz}, 1 \mathrm{H}, \mathrm{H} 5\right) ;{ }^{13} \mathrm{C}$ NMR (125 MHz, pyridine-d 5 ) $\delta$ ppm 161.1 (C1), 150.5 (C7), 138.5 (C4), 135.6 (C4a), 130.6 (C8a), 130.0 (C5), 128.8 (C6), 123.5 (C8); HRMS-ESI $(m / z)$ calcd. for $\left[\mathrm{C}_{8} \mathrm{H}_{5} \mathrm{~N}_{3} \mathrm{O}_{3}+\mathrm{H}\right]^{+}:$192.0404, obsd.: 192.0408.

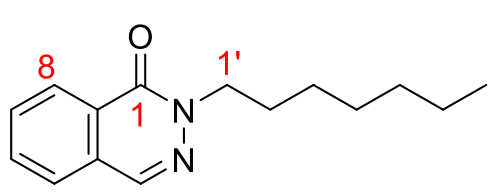

224

2-Heptylphthalazin-1(2H)-one (224). Phthalazinone 31 (34.4 mg, $0.24 \mathrm{mmol}$ ) was dissolved in DMF $(1.18 \mathrm{~mL}$ ) before potassium carbonate $(132.7 \mathrm{mg}, 0.96 \mathrm{mmol})$ and bromoheptane $(0.11 \mathrm{~mL}, 0.72 \mathrm{mmol})$ were added. The reaction was warmed to $50{ }^{\circ} \mathrm{C}$ and stirred for $12 \mathrm{~h}$, after which time TLC analysis showed complete disappearance of the starting material. Purification by silica gel flash column chromatography (petroleum ether/EtOAc, $1 / 0 \rightarrow 4 / 1, \mathrm{v} / \mathrm{v}$ ) gave the title compound as a white amorphous solid (19.9 mg, 34\%); $\mathrm{R}_{f}=0.47$ (EtOAc/petroleum ether, 1/1, v/v); ${ }^{1} \mathrm{H}$ NMR (500 MHz, $\left.\mathrm{CDCl}_{3}\right) \delta$ ppm 8.43 (d, J7,8 = $\left.7.7 \mathrm{~Hz}, 1 \mathrm{H}, \mathrm{H} 8\right), 8.16$ (s, 1H, H4), $7.82-7.72$ 
(m, 2H, H7, H6), 7.68 (d, J6,7 $=7.6 \mathrm{~Hz}, 1 \mathrm{H}, \mathrm{H} 5), 4.22$ (t, $\left.J_{1^{\prime}, 2^{\prime}}=7.5 \mathrm{~Hz}, 2 \mathrm{H}, \mathrm{H} 1^{\prime}\right), 1.89-1.80$

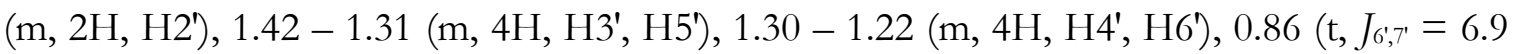
$\mathrm{Hz}, 3 \mathrm{H}, \mathrm{H} 7$ ) $){ }^{13} \mathrm{C}$ NMR (125 MHz, $\mathrm{CDCl}_{3}$ ) $\delta$ ppm 159.4 (C1), 137.7 (C4), 133.0 (C6), 131.7 (C7), 129.7 (C8a), 128.1 (C4a), 126.8 (C8), 126.0 (C5), 51.4 (C1'), 31.9 (C6'), 29.1 (C3'), 28.7 (C2'), 26.8 (C5'), 22.7 (C4'), 14.2 (C7'); HRMS-ESI ( $/$ / s) calcd. for $\left[\mathrm{C}_{15} \mathrm{H}_{20} \mathrm{~N}_{2} \mathrm{O}+\mathrm{H}\right]^{+}:$245.1648, obsd.: 245.1646.<smiles>C#C[C]n1ncc2ccccc2c1=O</smiles>

225

2-propargylphthalazin-1(2H)-one (225). Phthalazinone $31(5.1 \mathrm{mg}$, $0.04 \mathrm{mmol})$ was dissolved in DMF $(0.18 \mathrm{~mL})$ before a solution of $\mathrm{NaOEt}(21 \%$ in $\mathrm{EtOH}, 0.05 \mathrm{~mL})$ and propargyl bromide $(80 \%$ in toluene, $0.02 \mathrm{~mL}$ ) were added. The reaction was stirred at room temperature for $2 \mathrm{~d}$, after which time TLC analysis showed complete disappearance of the starting material. Purification by silica gel flash column chromatography (petroleum ether/EtOAc, $1 / 0 \rightarrow 4 / 1, \mathrm{v} / \mathrm{v}$ ) gave the title compound as colourless crystals (4.3 $\mathrm{mg}, 58 \%$ ); $\mathrm{R}_{f}=0.24$ (petroleum/EtOAc, 4/1, v/v); m.p.: $104.8^{\circ} \mathrm{C}$; IR ( $\left.\mathrm{U}_{\max }\right): 2925,1639,1590,1338$, 1102, 905, 767, 683, $583 \mathrm{~cm}^{-1}{ }^{1} \mathrm{H}$ NMR $\left(500 \mathrm{MHz}, \mathrm{CDCl}_{3}\right) \delta \mathrm{ppm} 8.45\left(\mathrm{~d}, J_{6,8}=7.5 \mathrm{~Hz}, 1 \mathrm{H}\right.$, H8), 8.21 (s, 1H, H4), 7.87 - 7.76 (m, 2H, H6, H7), 7.71 (d, J5,6 $=7.4$ Hz, 1H, H5), 5.03 (d, J', $J^{\prime}$ $\left.=2.5 \mathrm{~Hz}, 2 \mathrm{H}, \mathrm{H} 1^{\prime}\right), 2.34\left(\mathrm{t}, J_{1^{\prime}, 3^{\prime}}=2.5 \mathrm{~Hz}, 1 \mathrm{H}, \mathrm{H} 3^{\prime}\right) ;{ }^{13} \mathrm{C} \mathrm{NMR}\left(125 \mathrm{MHz}, \mathrm{CDCl}_{3}\right) \delta \mathrm{ppm} 138.7$ (C4), 133.6 (C6), 132.1 (C7), 129.9 (C8a), 128.0 (C4a), 127.0 (C8), 126.4 (C5), 78.2 (C2'), 72.6 (C3'), 40.7 (C1'); HRMS-ESI (m/ z) calcd. for $\left[\mathrm{C}_{11} \mathrm{H}_{8} \mathrm{~N}_{2} \mathrm{O}+\mathrm{H}\right]^{+}: 185.0709$, obsd.: 185.0707 .

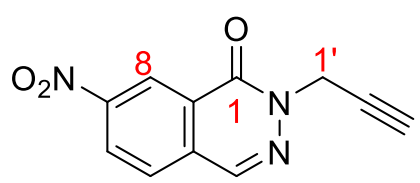

231

\section{7-Nitro-2-propargylphthalazin-1(2H)-one}

(231).

Phthalazinone 31 (72.5 $\mathrm{mg}, 0.38 \mathrm{mmol})$ was dissolved in DMF $(1.89 \mathrm{~mL})$ before a solution of $\mathrm{NaOEt}(21 \%$ in $\mathrm{EtOH}, 0.5 \mathrm{~mL})$ and propargyl bromide $(80 \%$ in toluene, $0.15 \mathrm{~mL})$ were added. The

reaction was stirred at room temperature for $2 \mathrm{~d}$, after which time TLC analysis showed complete disappearance of the starting material. Purification by silica gel flash column chromatography (petroleum ether/EtOAc, $1 / 0 \rightarrow 4 / 1, \mathrm{v} / \mathrm{v}$ ) gave the title compound as yellow crystals (80.1 mg, 92\%); $\mathrm{R}_{f}=0.37$ (EtOAc/petroleum ether, 1/1, v/v); m.p.: $151.4{ }^{\circ} \mathrm{C}$; IR $\left(v_{\max }\right): 3295,1654,1593,1565,1518,1351,1128,920,841,786,772,745,685,642,622,593$, $540 \mathrm{~cm}^{-1} ;{ }^{1} \mathrm{H}$ NMR $\left(500 \mathrm{MHz}, \mathrm{CDCl}_{3}\right) \delta \mathrm{ppm} 9.27\left(\mathrm{~d}, J_{6,8}=2.2 \mathrm{~Hz}, 1 \mathrm{H}, \mathrm{H} 8\right), 8.62\left(\mathrm{dd}, J_{5,6}=\right.$ $\left.8.6 \mathrm{~Hz}, J_{6,8}=2.3 \mathrm{~Hz}, 1 \mathrm{H}, \mathrm{H} 6\right), 8.31$ (s, 1H, H4), 7.91 (d, $\left.J_{5,6}=8.6 \mathrm{~Hz}, 1 \mathrm{H}, \mathrm{H} 5\right), 5.05$ (d, $J_{1^{\prime}, 3^{\prime}}=$ $\left.2.5 \mathrm{~Hz}, 2 \mathrm{H}, \mathrm{H} 1^{\prime}\right), 2.38\left(\mathrm{t}, J_{1^{\prime}, 3^{\prime}}=2.5 \mathrm{~Hz}, 1 \mathrm{H}, \mathrm{H} 3^{\prime}\right) ;{ }^{13} \mathrm{C} \mathrm{NMR}\left(125 \mathrm{MHz}, \mathrm{CDCl}_{3}\right) \delta \mathrm{ppm} 157.6$ 
(C1), 149.4 (C7), 136.8 (C4), 133.2 (C4a), 128.6 (C8a), 128.1 (C5), 127.6 (C6), 123.1 (C8), 77.3 (C2'), 73.1 (C3'), $41.02\left(\mathrm{C}^{\prime}\right)$; HRMS-ESI $(\mathrm{m} / \mathrm{z})$ calcd. for $\left[\mathrm{C}_{11} \mathrm{H}_{7} \mathrm{~N}_{3} \mathrm{O}_{3}+\mathrm{H}\right]^{+}: 230.0560$, obsd.: 230.0558 .

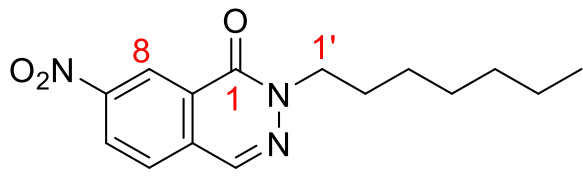

233

\section{2-Heptyl-7-nitro-phthalazin-1(2H)-one}

(233).

Phthalazinone 230 (95.1 mg, $0.49 \mathrm{mmol}$ ) was dissolved in DMF $(2.5 \mathrm{~mL})$ before potassium carbonate $(270.9$ $\mathrm{mg}, 1.96 \mathrm{mmol})$ and bromoheptane $(0.23 \mathrm{~mL}, 1.47$

mmol) were added. The reaction was warmed to $50{ }^{\circ} \mathrm{C}$ and stirred for $12 \mathrm{~h}$, after which time TLC analysis showed complete disappearance of the starting material. Purification by silica gel flash column chromatography (petroleum ether/EtOAc, $1 / 0 \rightarrow 4 / 1, \mathrm{v} / \mathrm{v}$ ) gave the title compound as yellow crystals $(83.6 \mathrm{mg}, 59 \%) ; \mathrm{R}_{f}=0.56($ EtOAc/petroleum ether, $1 / 1, \mathrm{v} / \mathrm{v})$;

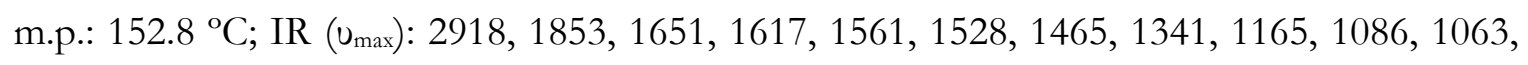
911, 541, 769, 758, 725, 684, 620, 595, $492 \mathrm{~cm}^{-1} ;{ }^{1} \mathrm{H}$ NMR $\left(500 \mathrm{MHz}, \mathrm{CDCl}_{3}\right) \delta$ ppm 9.29 (d, $\left.J_{4,6}=2.3 \mathrm{~Hz}, 1 \mathrm{H}, \mathrm{H} 8\right), 8.60\left(\mathrm{dd}, J_{5,6}=8.6 \mathrm{~Hz}, J_{6,8}=2.3 \mathrm{~Hz}, 1 \mathrm{H}, \mathrm{H} 6\right), 8.28$ (s, 1H, H4), 7.90 (d, $\left.J_{5,6}=8.6 \mathrm{~Hz}, 1 \mathrm{H}, \mathrm{H} 5\right), 4.28$ (t, $\left.J_{1^{\prime}, 2^{\prime}}=7.5 \mathrm{~Hz}, 2 \mathrm{H}, \mathrm{H} 1^{\prime}\right), 1.87$ (pent., $J_{1^{\prime}, 2^{\prime}}=J_{2^{\prime}, 3^{\prime}}=7.4 \mathrm{~Hz}, 2 \mathrm{H}$, H2'), 1.43 - 1.25 (m, 8H, H3', H4', H5', H6'), 0.89 (t, $\left.J_{6^{\prime}, 7^{\prime}}=6.9 \mathrm{~Hz}, 3 \mathrm{H}, \mathrm{H} 7^{\prime}\right) ;{ }^{13} \mathrm{C}$ NMR (125 $\mathrm{MHz}, \mathrm{CDCl}_{3}$ ) $\delta$ ppm 158.2 (C1), 149.3 (C7), 136.0 (C4), 133.2 (C4a), 128.7 (C8a), 127.8 (C5), 127.2 (C6), 123.2 (C8), 51.8 (C1'), 31.8 (C4'), 29.1 (C5'), 28.6 (C2'), 26.8 (C3'), 22.7 (C6'), 14.21 (C7'); HRMS-ESI (m/z) calcd. for $\left[\mathrm{C}_{15} \mathrm{H}_{19} \mathrm{~N}_{3} \mathrm{O}_{3}+\mathrm{H}\right]^{+}: 290.1499$, obsd.: 290.1498.

General procedure for the reduction of nitroaryl groups. Nitro-chromone or nitrophenyl chromone (1 equiv.) was dissolved in $\mathrm{EtOH} / \mathrm{H}_{2} \mathrm{O}(2 / 1, \mathrm{v} / \mathrm{v}, 5 \mathrm{~mL} / \mathrm{mmol})$ and stirred under argon. Fe (10 equiv.) and ammonium formate (8 equiv.) were then added into the reaction flask, and the reaction heated to reflux for $12 \mathrm{~h}$, after which time TLC analysis showed the disappearance of starting materials. The reaction was filtered and rinsed with hot $\mathrm{EtOH}$, and the filtrate was concentrated in vacuo to give a brown solid, which was purified using silica gel column chromatography (petroleum ether/EtOAc, $9 / 1 \rightarrow 1 / 1, \mathrm{v} / \mathrm{v}$ ) to yield the desired aminochromones. 
<smiles>Nc1ccc2cnn(-c3ccccc3)c(=O)c2c1</smiles>

229

7-Nitro-2-phenylphthalazin-1(2H)-one (229). By subjecting nitro-phthalazinone 228 (100 mg, $0.37 \mathrm{mmol}$ ), iron (208.9 mg, 3.74 $\mathrm{mmol})$, and ammonium formate $(356.5 \mathrm{mg}, 2.99 \mathrm{mmol})$ in $\mathrm{EtOH} / \mathrm{H}_{2} \mathrm{O}(2 / 1, \mathrm{v} / \mathrm{v}, 2.1 \mathrm{~mL})$ to the general procedure for the reduction of nitroaryl groups, the title compound was isolated as a white amorphous solid (86.9 mg, 99\%); $\mathrm{R}_{f}=0.38$ (EtOAc/petroleum ether, 2/1, v/v); IR (U $\left.\max \right): 2977,2797,1648$, 1577, 1570, 1510, 1491, 1452, 1425, 1371, 1342, 1293, 1129, 1051, 1027, 773, $758 \mathrm{~cm}^{-1} ;{ }^{1} \mathrm{H}$ NMR (500 MHz, pyridine-d $\left.\mathrm{d}_{5}\right) \delta$ ppm 8.25 (s, 1H, H4), 8.12 (d, J6,8 $=2.4 \mathrm{~Hz}, 1 \mathrm{H}, \mathrm{H} 8$ ), 7.99 (d, $\left.J_{2^{\prime}, 3^{\prime}}=7.5 \mathrm{~Hz}, 2 \mathrm{H}, \mathrm{H} 2^{\prime}\right), 7.61$ (d, $\left.J_{5,6}=8.6 \mathrm{~Hz}, 1 \mathrm{H}, \mathrm{H} 5\right), 7.47$ (t, $J_{2^{\prime}, 3^{\prime}}=J_{3^{\prime}, 4^{\prime}}=7.9 \mathrm{~Hz}, 2 \mathrm{H}, \mathrm{H} 3^{\prime}$ ), $7.38\left(\mathrm{dd}, J_{5,6}=8.5, J_{6,8}=2.4 \mathrm{~Hz}, 1 \mathrm{H}, \mathrm{H} 6\right), 7.32\left(\mathrm{t}, J_{3^{\prime}, 4^{\prime}}=7.5 \mathrm{~Hz}, 1 \mathrm{H}, \mathrm{H} 4^{\prime}\right), 7.00$ (br. s, $2 \mathrm{H}, \mathrm{NH}_{2}$ ); ${ }^{13} \mathrm{C}$ NMR (125 MHz, pyridine-d 5 ) $\delta$ ppm 160.0 (C1), 154.0 (C7), 144.0 (C1'), 139.4 (C4), 131.6 (C8a), 129.2 (C5), 129.2 (C3'), 127.8 (C4'), 127.0 (C2'), 121.6 (C6), 120.8 (C4a), 108.8 (C8); HRMS-ESI $(m / z)$ calcd. for $\left[\mathrm{C}_{14} \mathrm{H}_{11} \mathrm{~N}_{3} \mathrm{O}+\mathrm{H}\right]^{+}: 238.0975$, obsd.: 238.0973. The data obtained for this compound were consistent with those reported in literature. ${ }^{345}$<smiles>C#C[C]n1ncc2ccc(N)cc2c1=O</smiles>

232

7-Amino-2-propargyl-phthalazin-1(2H)-one (232). Вy subjecting nitro-phthalazinone $233(76.8 \mathrm{mg}, 0.34 \mathrm{mmol})$, iron (187.1 mg, $3.35 \mathrm{mmol}$ ), and ammonium formate (319.4 mg, 2.68 $\mathrm{mmol})$ in $\mathrm{EtOH} / \mathrm{H}_{2} \mathrm{O}(2 / 1, \mathrm{v} / \mathrm{v}, 1.68 \mathrm{~mL})$ to the general procedure for the reduction of nitroaryl groups, the title compound was isolated as colourless crystals $(59.6 \mathrm{mg}, 88 \%) ; \mathrm{R}_{f}=0.17$ (petroleum ether/EtOAc, 1/1, v/v); m.p.: $216{ }^{\circ} \mathrm{C}$; IR ( $\left.\mathrm{u}_{\max }\right)$ : 3206, 1636, 1592, 1500, 1450, 1363, 1317, 1250, 1138, 1097, 8728, 706, 604, 587, 511, $461 \mathrm{~cm}^{-}$ ${ }^{1} ;{ }^{1} \mathrm{H}$ NMR (500 MHz, pyridine- $\left.\mathrm{d}_{5}\right) \delta \mathrm{ppm} 8.14$ (s, 1H, H4), 8.03 (d, J6,8 $=2.3 \mathrm{~Hz}, 1 \mathrm{H}, \mathrm{H} 8$ ), $7.52\left(\mathrm{~d}, J_{5,6}=8.5 \mathrm{~Hz}, 1 \mathrm{H}, \mathrm{H} 5\right), 7.32$ (dd, $\left.J_{5,6}=8.5, J_{6,8}=2.3 \mathrm{~Hz}, 1 \mathrm{H}, \mathrm{H} 6\right), 6.97$ (br. s, $1 \mathrm{H}, \mathrm{NH}_{2}$ ), $5.23\left(\mathrm{~d}, J_{1^{\prime}, 3^{\prime}}=2.6 \mathrm{~Hz}, 1 \mathrm{H}, \mathrm{H} 1^{\prime}\right), 3.28\left(\mathrm{~m}, 1 \mathrm{H}, \mathrm{H} 3^{\prime}\right) ;{ }^{13} \mathrm{C}$ NMR $\left(125 \mathrm{MHz}\right.$, pyridine- $\left.\mathrm{d}_{5}\right) \delta \mathrm{ppm}$ 160.5 (C1), 154.8 (C7), 140.0 (C4), 131.8 (C8a), 130.1 (C5), 122.4 (C6), 122.0 (C4a), 109.1 (C8), 81.5 (C2'), 74.8 (C3'), $42.0\left(\mathrm{C}^{\prime}\right)$; HRMS-ESI ( $/$ / z) calcd. for $\left[\mathrm{C}_{11} \mathrm{H}_{9} \mathrm{~N}_{3} \mathrm{O}+\mathrm{H}\right]^{+}: 200.0818$, obsd.: 200.0816.

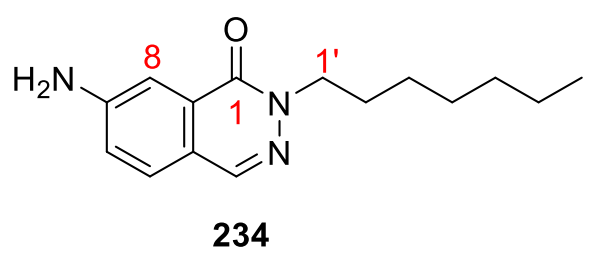

7-Amino-2-heptylphthalazin-1(2H)-one (234). Вy subjecting nitro-phthalazinone 233 (84 mg, $0.29 \mathrm{mmol})$, iron $(52 \mathrm{mg}, 2.9 \mathrm{mmol})$, and ammonium formate $(276.5$ $\mathrm{mg}, 2.32 \mathrm{mmol})$ in $\mathrm{EtOH} / \mathrm{H}_{2} \mathrm{O}(2 / 1, \mathrm{v} / \mathrm{v}, 2.1 \mathrm{~mL})$ to the general procedure for the reduction of nitroaryl groups, the title compound was isolated 
as colourless crystals (73.7 mg, 98\%); $\mathrm{R}_{f}=0.3$ (EtOAc/petroleum ether, 1/1, v/v); m.p.: 116.8 ${ }^{\circ} \mathrm{C}$; IR (U $\left.u_{\max }\right): 2967,2792,2710,1557,1454,1420,1342,1107,1096,773 \mathrm{~cm}^{-1} ;{ }^{1} \mathrm{H}$ NMR $(500$ MHz, pyridine- $\mathrm{d}_{5} / \mathrm{CD}_{3} \mathrm{OD}$ ) $\delta \mathrm{ppm} 8.19$ (s, 1H, H4), 8.12 (d, $\left.J_{6,8}=2.4 \mathrm{~Hz}, 1 \mathrm{H}, \mathrm{H} 8\right), 7.57$ (m, 1H, H5), 7.37 (dd, $\left.J_{5,6}=8.5 \mathrm{~Hz}, J_{6,8}=2.4 \mathrm{~Hz}, 1 \mathrm{H}, \mathrm{H} 6\right), 6.93$ (br. s, $2 \mathrm{H}, \mathrm{NH}_{2}$ ), 4.38 (t, $J_{1^{\prime}, 2^{\prime}}=7.3$ $\left.\mathrm{Hz}, 2 \mathrm{H}, \mathrm{H} 1^{\prime}\right), 1.93$ (pent., $\left.J_{1^{\prime}, 2^{\prime}}=J_{2^{\prime}, 3^{\prime}}=7.4 \mathrm{~Hz}, 2 \mathrm{H}, \mathrm{H} 2^{\prime}\right), 1.37-1.29$ (m, 2H, H3'), $1.28-1.20$ (m, 2H, H4'), 1.20 - 1.10 (m, 4H, H5', H6'), 0.79 (t, $\left.J_{6^{\prime}, 7^{\prime}}=7.0 \mathrm{~Hz}, 3 \mathrm{H}, \mathrm{H} 7^{\prime}\right) ;{ }^{13} \mathrm{C}$ NMR (150 $\mathrm{MHz}$, pyridine- $\mathrm{d}_{5} / \mathrm{CD}_{3} \mathrm{OD}$ ) $\delta$ ppm 160.0 (C1), 153.7 (C7), 138.4 (C4), 131.2 (C8a), 129.0 (C5), 121.4 (C6), 108.3 (C8), 51.5 (C1'), 32.4 (C6'), 29.7 (C4'), 29.5 (C2'), 27.5 (C3'), 23.3 (C5'), 14.7 $\left(\mathrm{C} 7{ }^{\prime}\right)$; HRMS-ESI $(m / z)$ calcd. for $\left[\mathrm{C}_{15} \mathrm{H}_{21} \mathrm{~N}_{3} \mathrm{O}+\mathrm{H}\right]^{+}:$260.1757, obsd.: 260.1761 .

General procedure for the synthesis of pyrimidinyl-phthalazinone. Chloropyrimidine (1.5 equiv.) and aminophthalazinone (1 equiv.) were co-evaporated three times in dry toluene to remove traces of water before being dissolved in 2-ethoxyethanol $(5 \mathrm{~mL} / \mathrm{mmol})$. Concentrated $\mathrm{HCl}$ (0.5 equiv.) was then added, and the reaction was stirred under reflux for $22 \mathrm{~h}$. The precipitate was filtered and washed with $\mathrm{Et}_{2} \mathrm{O}$ to give the title compound as yellow solids.

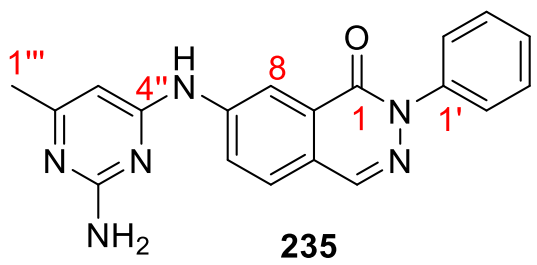

7-((2-Amino-6-methylpyrimidin-4-yl)amino)-2phenylphthalazin-1(2H)-one (235). By subjecting methyl-chloropyrimidine 150 (18.1 $\mathrm{mg}, 0.13 \mathrm{mmol})$ and amino-phthalazinone 229 (20 $\mathrm{mg}, 0.08 \mathrm{mmol}$ ) in conc. $\mathrm{HCl}(0.04 \mathrm{~mL})$ and 2-ethoxyethanol $(0.425 \mathrm{~mL})$ to the general procedure for the synthesis of pyrimidinyl-phthalazinones, the title compound was isolated as a white amorphous solid (17.4 mg, 60\%); $\mathrm{R}_{f}=0.19$ (EtOAc/MeOH, 4/1, v/v); Fluorescence (MeOH): Ex 380 nm, Em 480 nm; IR (Umax): 1640, 1582, 1518, 1496, 1456, 1356, 1341, 1151, 1061, $973 \mathrm{~cm}^{-1}$; ${ }^{1} \mathrm{H}$ NMR (500 MHz, pyridine-d d $\delta$ ppm 10.85 (br. s, $1 \mathrm{H}, \mathrm{NH}$ ), 9.05 (d, J6,8 $=2.2 \mathrm{~Hz}, 1 \mathrm{H}, \mathrm{H} 8), 8.73-8.71$ (m, 1H, H6), 8.35 (s, 1H, H4), 7.98 (d, $J_{2^{\prime}, 3^{\prime}}=7.8$ $\left.\mathrm{Hz}, 2 \mathrm{H}, \mathrm{H} 2^{\prime}\right), 7.67$ (d, $\left.J_{5,6}=8.6 \mathrm{~Hz}, 1 \mathrm{H}, \mathrm{H} 5\right), 7.50$ (t, $\left.J_{2^{\prime}, 3^{\prime}}=J_{3^{\prime}, 4^{\prime}}=7.8 \mathrm{~Hz}, 2 \mathrm{H}, \mathrm{H} 3^{\prime}\right), 7.34$ (t, $J_{3^{\prime}, 4^{\prime}}$ $=7.4 \mathrm{~Hz}, 1 \mathrm{H}, \mathrm{H} 4$ ), 6.29 (s, 1H, H5"), 2.27 (s, 3H, CH $)$; ${ }^{13} \mathrm{C}$ NMR (125 MHz, pyridine- $\left.\mathrm{d}_{5}\right) \delta$ ppm 168.1 (C2"), 164.0 (C6"), 162.9 (C4"), 160.3 (C1), 144.5 (C7), 144.3 (C1'), 139.7 (C4), 131.0 (C8a), 130.1 (C4'), 129.03 (C5), 128.97 (C3'), 128.5 (C6), 127.7 (C2'), 126.9 (C4a), 119.2 (C8), 98.9 (C5"), $23.3\left(\mathrm{CH}_{3}\right)$; HRMS-ESI $(\mathrm{m} / \mathrm{z})$ calcd. for $\left[\mathrm{C}_{19} \mathrm{H}_{16} \mathrm{~N}_{6} \mathrm{O}_{1}+\mathrm{H}\right]^{+}$: 345.1458, obsd.: 345.1456. 


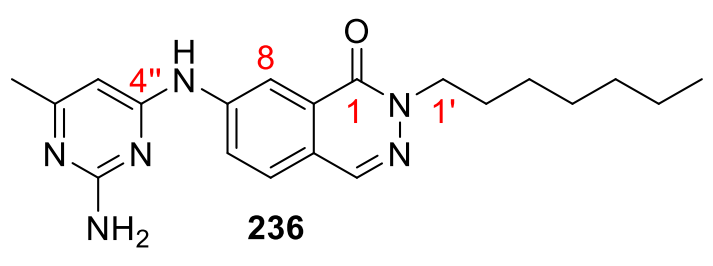

7-((2-Amino-6-methylpyrimidin-4yl)amino)-2-heptylphthalazin-1(2H)-one (236). By subjecting methyl-chloropyrimidine 150 (16.6 mg, $0.12 \mathrm{mmol}$ ) and amino-

phthalazinone $232(20 \mathrm{mg}, 0.08 \mathrm{mmol})$ in conc. $\mathrm{HCl}(0.04 \mathrm{~mL})$ and 2-ethoxyethanol $(0.425$ $\mathrm{mL}$ ) to the general procedure for the synthesis of pyrimidinyl-phthalazinones, the title compound was isolated as a white amorphous solid (23.2 mg, 79\%); $\mathrm{R}_{f}=0.47(\mathrm{EtOAc} / \mathrm{MeOH}$, 4/1, v/v); Fluorescence: Ex 387 nm, 481nm; IR (U $\max ): 2954,2841,1654,1636,1588,1522$, 1518, 1452, 1420, 1104, 599, 578, 541, 516, 472, 462, $455 \mathrm{~cm}^{-1} ;{ }^{1} \mathrm{H}$ NMR (500 MHz, pyridined5) $\delta$ ppm 11.91 (br. s, $1 \mathrm{H}, \mathrm{NH}$ ), 9.05 (d, $J_{6,8}=2.0 \mathrm{~Hz}, 1 \mathrm{H}, \mathrm{H} 8$ ), 8.55 (dd, J5,6 $=8.6, J_{6,8}=2.1$ Hz, 1H, H6), 8.31 (s, 1H, H4), 7.66 (d, J5,6 = 8.6 Hz, 1H, H5), 6.42 (s, 1H, H5"), 4.35 (t, $J_{1^{\prime}, 2^{\prime}}=$ $\left.7.3 \mathrm{~Hz}, 2 \mathrm{H}, \mathrm{H} 1^{\prime}\right), 2.26$ (s, 3H, CH $\left.\mathrm{CH}_{3}\right), 1.91$ (q, $\left.J_{1^{\prime}, 2^{\prime}}=7.4 \mathrm{~Hz}, J_{2^{\prime}, 3^{\prime}}=2 \mathrm{H}, \mathrm{H} 2^{\prime}\right), 1.40-1.09$ (m, 8H, H3', H4', H5', H6'), 0.80 (t, J J $\left.J^{\prime}, 8^{\prime}=7.0 \mathrm{~Hz}, 3 \mathrm{H}, \mathrm{H} 7^{\prime}\right) ;{ }^{13} \mathrm{C}$ NMR (125 MHz, pyridine-d $\left.\mathrm{d}_{5}\right) \delta \mathrm{ppm}$ 163.2 (C6"), 159.8 (C2'), 159.5 (C1), 157.8 (C4"), 143.5 (C7), 137.8 (C4), 129.7 (8a), 128.0 (C5), 127.3 (C6), 126.4 (C4a), 117.7 (C8), 97.9 (C5"), 51.6 (C1'), 32.3 (C2'), 29.7 (C3'), 29.4 (C4'), 27.4 (C5'), $23.3\left(\mathrm{CH}_{3}\right), 20.3\left(\mathrm{C}^{\prime}\right), 14.7\left(\mathrm{C}^{\prime}\right)$; HRMS-ESI $(\mathrm{m} / \mathrm{z})$ calcd. for $\left[\mathrm{C}_{20} \mathrm{H}_{26} \mathrm{~N}_{3} \mathrm{O}+\mathrm{H}\right]^{+}$: 367.2241, obsd.: 367.2239.

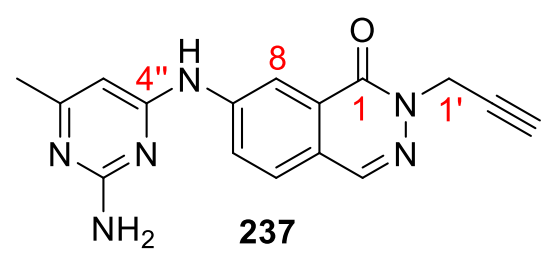

7-(2-Amino-6-methylpyrimidin-4-yl)amino)-2propargylphthalazin-1(2H)-one (237). By subjecting methyl-chloropyrimidine 150 (22.2 $\mathrm{mg}, 0.15 \mathrm{mmol})$ and amino-phthalazinone $232(20.6 \mathrm{mg}, 0.10 \mathrm{mmol})$ in conc.

$\mathrm{HCl}(0.05 \mathrm{~mL})$ and 2-ethoxyethanol $(0.52 \mathrm{~mL})$ to the general procedure for the synthesis of pyrimidinyl-phthalazinones, the title compound was isolated as a white amorphous solid (9.2 $\mathrm{mg}, 29 \%) ; \mathrm{R}_{f}=0.31(\mathrm{EtOAc} / \mathrm{MeOH}, 4 / 1, \mathrm{v} / \mathrm{v}) ;$ Fluorescence $(\mathrm{MeOH}): \mathrm{Ex} 387 \mathrm{~nm}, \mathrm{Em} 484$ $\mathrm{nm}$; IR (U $\left.\mathrm{U}_{\max }\right): 2179,2061,1983,1969,1578,1558,1543,1534,1523,1509,1435,1165,1153$, 1076, 894, 877, 650, 637, 451, 426, $417 \mathrm{~cm}^{-1} ;{ }^{1} \mathrm{H}$ NMR (500 MHz, pyridine-d $\left.\mathrm{d}_{5}\right) \delta \mathrm{ppm} 10.70$ (br. s, 1H, NH), 9.06 (d, J6,8 $=2.2 \mathrm{~Hz}, 1 \mathrm{H}, \mathrm{H} 8$ ), 8.62 (dd, $J_{5,6}=8.7, J_{6,8}=2.3 \mathrm{~Hz}, 1 \mathrm{H}, \mathrm{H} 6$ ), 8.23

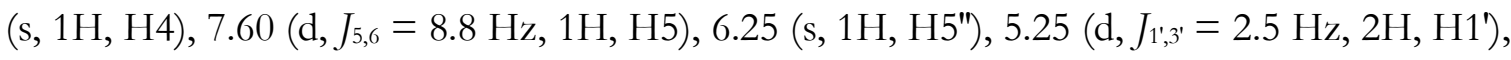
$3.35\left(\mathrm{~d}, J_{1^{\prime}, 3^{\prime}}=2.4 \mathrm{~Hz}, 1 \mathrm{H}, \mathrm{H} 3^{\prime}\right), 2.25\left(\mathrm{~s}, 3 \mathrm{H}, \mathrm{CH}_{3}\right) ;{ }^{13} \mathrm{C}$ NMR $\left(150 \mathrm{MHz}\right.$, pyridine- $\left.\mathrm{d}_{5}\right) \delta \mathrm{ppm}$ 168.4 (C4"), 165.5 (C2"), 163.5 (C6"), 160.2 (C1), 146.8 (C7), 139.6 (C4), 130.6 (C8a), 129.1 
(C5), 126.8 (C6), 125.5 (C4a), 116.0 (C8), 97.8 (C5"), 75.0 (C3'), 42.1 (C1'), 81.2 (C2'), 31.77, $25.2\left(\mathrm{CH}_{3}\right)$; HRMS-ESI calcd. for $\left[\mathrm{C}_{16} \mathrm{H}_{14} \mathrm{~N}_{6} \mathrm{O}+\mathrm{H}\right]^{+}$: 307.1302, obsd.: 307.1304 .

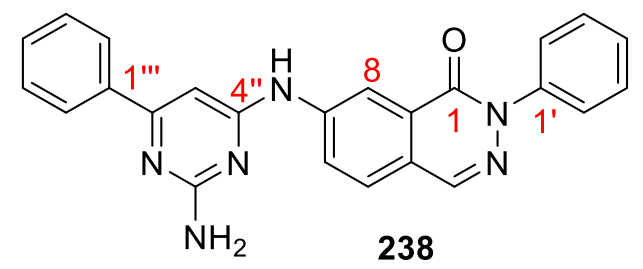

\section{7-((2-Amino-6-phenylpyrimidin-4-yl)amino)-} 2-phenylphthalazin-1(2H)-one (238). By subjecting chloropyrimidine 187 (43.2 $\mathrm{mg}, 0.21$ mmol) and amino-phthalazinone $229(33.5 \mathrm{mg}$, $0.14 \mathrm{mmol})$ in conc. $\mathrm{HCl}(0.07 \mathrm{~mL})$ and 2-ethoxyethanol $(0.7 \mathrm{~mL})$ to the general procedure for the synthesis of pyrimidinyl-phthalazinones, the title compound was isolated as a white amorphous solid (13.1 mg, 23\%); $\mathrm{R}_{f}=0.57$ (EtOAc); Fluorescence (MeOH): Ex $384 \mathrm{~nm}$, Em 432 nm; IR (Umax): 2961, 2851, 1637, 1570, 1518, 1494, 1453, 1260, 1095, 1024, 798, 692, 625, 599, 591, 581, 569, 549, 538, 486, 472, 458, 438, $431 \mathrm{~cm}^{-1} ;{ }^{1} \mathrm{H}$ NMR $(600 \mathrm{MHz}$, pyridine-d $\left.\mathrm{d}_{5}\right) \delta$ ppm 10.82 (br. s, $\left.1 \mathrm{H}, \mathrm{NH}\right), 9.13$ (d, $\left.J_{6,8}=2.0 \mathrm{~Hz}, 1 \mathrm{H}, \mathrm{H} 8\right), 8.76$ (dd, $J_{5,6}=$ 8.6, $\left.J_{6,8}=2.1 \mathrm{~Hz}, 1 \mathrm{H}, \mathrm{H} 6\right), 8.35$ (s, 1H, H4), 8.27 (d, $\left.J_{2^{\prime \prime \prime}, 3^{\prime \prime \prime}}=7.5 \mathrm{~Hz}, 2 \mathrm{H}, \mathrm{H} 2^{\prime \prime \prime}\right), 7.99$ (d, $J_{2^{\prime}, 3^{\prime}}$ $\left.=7.5 \mathrm{~Hz}, 2 \mathrm{H}, \mathrm{H} 2^{\prime}\right), 7.69$ (d, $\left.J_{5,6}=8.7 \mathrm{~Hz}, 1 \mathrm{H}, \mathrm{H} 5\right), 7.51$ (t, $\left.J_{2^{\prime}, 3^{\prime}}=J_{3^{\prime}, 4^{\prime}}=7.8 \mathrm{~Hz}, 2 \mathrm{H}, \mathrm{H} 3^{\prime}\right)$,

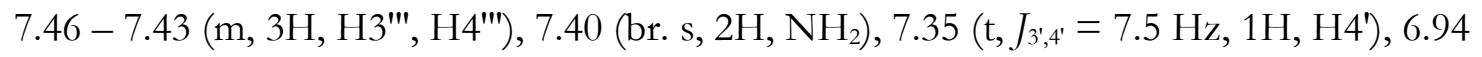
(s, 1H, H5"); ${ }^{13} \mathrm{C}$ NMR (150 MHz, pyridine-d 5 ) $\delta$ ppm 166.1 (C6"), 164.2 (C4"), 160.6 (C1), 146.9 (C7), 144.6 (C1'), 140.2 (C1"'), 139.9 (C3), 131.5 (C4"'), 131.4 (C8a), 130.2 (C3"'), 130.1 (C3'), 129.1 (C5), 128.8 (C4'), 128.5 (C2'"'), 127.7 (C2'), 126.9 (C6), 125.3 (C4a), 116.6 (C8), 95.1 (C5"); HRMS-ESI ( $m / 2)$ calcd. for $\left[\mathrm{C}_{24} \mathrm{H}_{18} \mathrm{~N}_{6} \mathrm{O}+\mathrm{H}\right]^{+}: 407.1615$, obsd.: 407.1613.

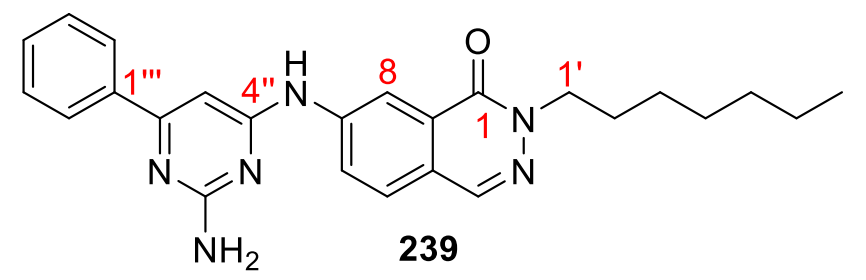

7-((2-Amino-6-phenylpyrimidin-4yl)amino)-2-heptylphthalazin1(2H)-one (239). By subjecting chloropyrimidine $187(56.0 \mathrm{mg}, 0.27$ mmol) and amino-phthalazinone $234(47.0 \mathrm{mg}, 0.18 \mathrm{mmol})$ in conc. $\mathrm{HCl}(0.09 \mathrm{~mL})$ and 2 ethoxyethanol $(0.91 \mathrm{~mL})$ to the general procedure for the synthesis of pyrimidinylphthalazinones, the title compound was isolated as a white amorphous solid (34.9 mg, 45\%);

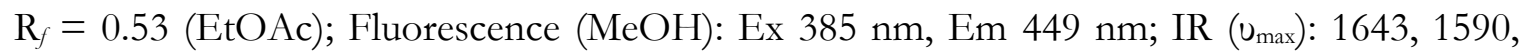
1520, 1497, 1456, 1429, 1357, 1247, 1077, $971 \mathrm{~cm}^{-1} ;{ }^{1} \mathrm{H}$ NMR (600 MHz, pyridine- $\left.\mathrm{d}_{5}\right): \delta \mathrm{ppm}$ 11.07 (s, 1H, NH), 9.19 (d, $J_{6,8}=2.2 \mathrm{~Hz}, 1 \mathrm{H}, \mathrm{H} 8$ ), 8.69 (dd, $J_{5,6}=9.0 \mathrm{~Hz}, J_{6,8}=1.7 \mathrm{~Hz}, 1 \mathrm{H}$, H6), 8.31 - 8.19 (m, 3H, H2"', H8), 7.67 (d, J5,6 = 8.6 Hz, 1H, H5), 7.47 - 7.38 (m, 3H, H3"', H4"'), 6.98 (s, 1H, H5"), 4.38 (t, $J_{1^{\prime}, 2^{\prime}}=7.3 \mathrm{~Hz}, 2 \mathrm{H}, \mathrm{H} 1$ '), 1.94 (pent., $J_{1^{\prime}, 2^{\prime}}=J_{2^{\prime}, 3^{\prime}}=7.5 \mathrm{~Hz}, 2 \mathrm{H}$, 
H2'), 1.34 - 1.18 (m, 8H, H3', H4', H5', H6'), 0.81 (t, $\left.J_{6^{\prime}, 7^{\prime}}=6.9 \mathrm{~Hz}, 3 \mathrm{H}, \mathrm{H} 7^{\prime}\right) ;{ }^{13} \mathrm{C}$ NMR (150 MHz, pyridine-d $\mathrm{d}_{5}$ ): $\delta$ ppm 163.6 (C4"), 162.7 (C6"), 159.0 (C1), 144.6 (C7), 137.4 (C1"'), 137.2 (C4), 130.3 (C4"'), 129.3 (C8a) 128.8 (C3"'), 127.3 (C5), 127.0 (C2'"), 125.4 (C6), 124.4 (C4a), 115.0 (C8), 93.9 (C5"), 50.9 (C1'), 31.7 (C6'), 30.3 (C2'), 29.0 (C4'), 28.8 (C3'), 26.7 (C6'), 14.04 (C7'); HRMS-ESI $(m / 2)$ calcd. for $\left[\mathrm{C}_{25} \mathrm{H}_{28} \mathrm{~N}_{6} \mathrm{O}+\mathrm{H}\right]^{+}: 429.2397$, obsd.: 429.2398 .<smiles>C#CC=[N+]1N=Cc2ccc(Nc3cc(-c4ccccc4)nc(N)n3)cc2C1=O</smiles>

\section{7-((2-Amino-phenylpyrimidin-4-yl)amino)-2-} propargylphthalazin-1(2H)-one (240). By subjecting chloropyrimidine $187(7.7 \mathrm{mg}, 0.04 \mathrm{mmol})$ and amino-phthalazinone $232(12 \mathrm{mg}, 0.06 \mathrm{mmol})$ in

conc. $\mathrm{HCl}(0.02 \mathrm{~mL})$ and 2-ethoxyethanol $(0.2 \mathrm{~mL})$ to the general procedure for the synthesis of pyrimidinyl-phthalazinones, the title compound was isolated as a white amorphous solid (11.8 mg, 83\%); $\mathrm{R}_{f}=0.63$ (EtOAc); Fluorescence (MeOH): Ex 385 nm, Em 436 nm; IR (Umax): 2921, 1645, 1596, 1523, 1453, 1428, 1365, 1180, 1133, 911, 832, 799, 758, $719 \mathrm{~cm}^{-1} ;{ }^{1} \mathrm{H}$ NMR (500 MHz, pyridine-d $\mathrm{d}_{5}$ ) $\delta$ ppm 10.81 (s, 1H, NH), 9.07 (d, J6,8 $\left.=2.4 \mathrm{~Hz}, 1 \mathrm{H}, \mathrm{H} 8\right), 8.67$ (dd, J5,6 $\left.=8.7 \mathrm{~Hz}, J_{6,8}=2.4 \mathrm{~Hz}, 1 \mathrm{H}, \mathrm{H} 6\right), 8.26-8.24$ (m, 3H, H4, H2"'), 7.62 (d, J5,6 = 8.8 Hz, 1H, H5),

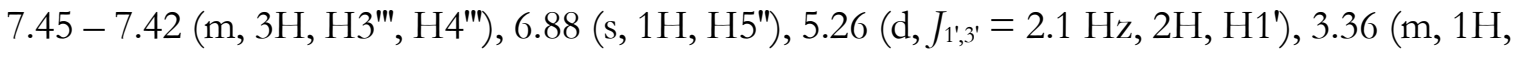
H3'); ${ }^{13} \mathrm{C}$ NMR (150 MHz, pyridine-d 5 ) $\delta$ ppm 165.3 (C4"), 163.3 (C6"), 159.4 (C1), 145.9 (C7), 139.4 (C1"'), 138.8 (C4), 130.7 (C4"'), 129.9 (C8a), 129.4 (C3"'), 128.6 (C5), 127.7 (C2"'), 126.0 (C6), 124.8 (C4a), 115.2 (C8), 94.2 (C5"), 80.4 (C2'), 74.2 (C3'), 41.3 (C1'); HRMS-ESI (m/z) calcd. for $\left[\mathrm{C}_{21} \mathrm{H}_{16} \mathrm{~N}_{6} \mathrm{O}+\mathrm{H}\right]^{+}:$369.1458, obsd.: 369.1453 .

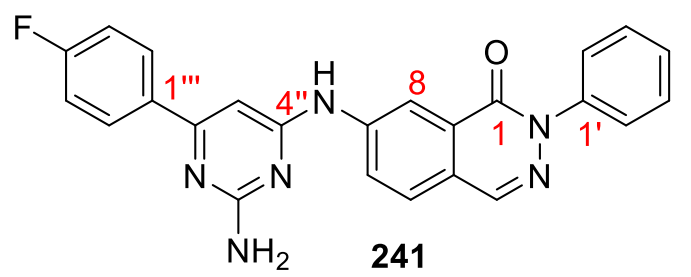

\section{7-((2-Amino-6-(4-fluorophenyl)pyrimidin- 4-yl)amino)-2-phenylphthalazin-1(2H)-} one (241). By subjecting chloropyrimidine 188 (33.5 $\mathrm{mg}, \quad 0.15 \mathrm{mmol})$ and aminophthalazinone 229 (24 mg, $0.1 \mathrm{mmol})$ in conc. $\mathrm{HCl}(0.05 \mathrm{~mL})$ and 2-ethoxyethanol $(0.5 \mathrm{~mL})$ to the general procedure for the synthesis of pyrimidinyl-phthalazinones, the title compound was isolated as a white amorphous solid (16.1 $\mathrm{mg}, 38 \%) ; \mathrm{R}_{f}=0.42$ (EtOAc/petroleum ether, 2/1, v/v); Fluorescence (MeOH): Ex 385 nm, Em 436 nm; IR $\left(u_{\max }\right): 3071,1647,1598,1493,1440,1240,1158,1129,1059,902,813,759,736,703,684$, 567, 582, $507 \mathrm{~cm}^{-1} ;{ }^{1} \mathrm{H}$ NMR (500 MHz, pyridine-d ${ }_{5}$ ) $\delta$ ppm 10.88 (br. s, 1H, NH), 9.14 (s,

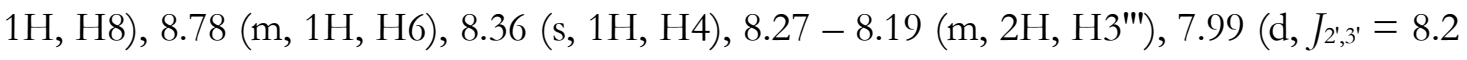


$\left.\mathrm{Hz}, 2 \mathrm{H}, \mathrm{H} 2^{\prime}\right), 7.70$ (d, $\left.J_{5,6}=8.7 \mathrm{~Hz}, 1 \mathrm{H}, \mathrm{H} 5\right), 7.51$ (t, $\left.J_{2^{\prime}, 3^{\prime}}=J_{3^{\prime}, 4^{\prime}}=7.8 \mathrm{~Hz}, 2 \mathrm{H}, \mathrm{H} 3^{\prime}\right), 7.36$ (t, $\left.J_{3^{\prime}, 4^{\prime}}=7.2 \mathrm{~Hz}, 1 \mathrm{H}, \mathrm{H} 4^{\prime}\right), 7.19-7.17$ (m, 2H, H2"), 6.90 (s, 1H, H5"), ${ }^{13} \mathrm{C}$ NMR (125 MHz, pyridine-d $\left.\mathrm{d}_{5}\right) \delta$ ppm 164.06 (d, J4",F $\left.=247.8 \mathrm{~Hz}, \mathrm{C} 4{ }^{\prime \prime \prime}\right), 164.02$ (C6"), 162.7 (C4"'), 162.3 (C4"), 159.1 (C1), 145.2 (C7), 143.0 (C1'), 138.4 (C4), 134.3 (C1"'), 129.8 (C8a), 129.2 (d, $J_{2 " ', F}=8.3$ Hz, C2'"), 128.6 (C3'), 127.6 (C5), 127.4 (C4'), 126.2 (C2'), 125.6 (C6), 123.9 (C4a), 115.6 (d, $\left.J_{3^{\prime \prime \prime}, \mathrm{F}}=21.6 \mathrm{~Hz}, \mathrm{C} 3^{\prime \prime \prime}\right), 115.4$ (C8), 93.6 (C5"); ${ }^{19} \mathrm{~F}$ NMR (282 MHz, pyridine) $\delta \mathrm{ppm}-110.14$; HRMS-ESI $(\mathrm{m} / \mathrm{z})$ calcd. for $\left[\mathrm{C}_{24} \mathrm{H}_{17} \mathrm{FN}_{6} \mathrm{O}+\mathrm{H}\right]^{+}:$425.1521, obsd.: 425.1519.

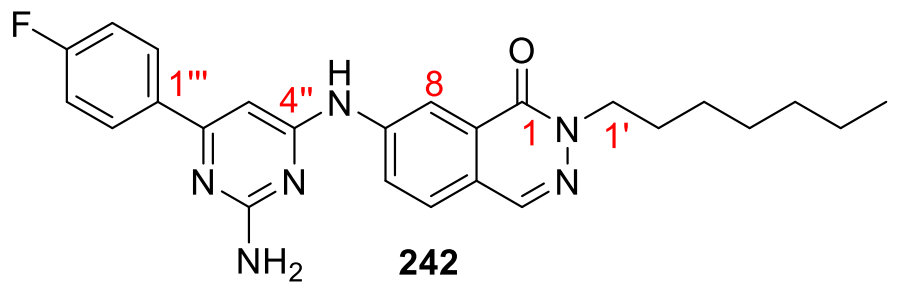

7-((2-Amino-6-(4fluorophenyl)pyrimidin-4yl)amino)-2-heptylphthalazin1(2H)-one (242). By subjecting chloropyrimidine 188 (29.0 mg, 0.13

mmol) and amino-phthalazinone $234(22.4 \mathrm{mg}, 0.09 \mathrm{mmol})$ in conc. $\mathrm{HCl}(0.04 \mathrm{~mL})$ and 2ethoxyethanol $(0.43 \mathrm{~mL})$ to the general procedure for the synthesis of pyrimidinylphthalazinones, the title compound was isolated as a white amorphous solid (10.4 mg, 27\%); $\mathrm{R}_{f}=0.36($ EtOAc/petroleum ether, 2/1, v/v); Fluorescence $(\mathrm{MeOH}): \mathrm{Ex} 283 \mathrm{~nm}, \mathrm{Em} 438$ nm; IR (U $\left.U_{\max }\right): 1638,1599,1571,1510,1499,1440,1378,1356,1229,1204,1156,1075,882$, 839, $601 \mathrm{~cm}^{-1}$; ${ }^{1} \mathrm{H}$ NMR (500 MHz, pyridine-d $)$ ): $\delta$ ppm 10.83 (br. s, $\left.1 \mathrm{H}, \mathrm{NH}\right), 9.16$ (d, J6,8 = $2.3 \mathrm{~Hz}, 1 \mathrm{H}, \mathrm{H} 8$ ), 8.71 (dd, $J_{5,6}=8.7 \mathrm{~Hz}, J_{6,8}=2.3 \mathrm{~Hz}, 1 \mathrm{H}, \mathrm{H} 6$ ), 8.29 (s, 1H, H4), $8.23-8.15$ (m, 2H, H2"'), 7.68 (d, J5,6 = 8.6 Hz, 1H, H5), 7.43 (s, 2H, NH $), 7.20$ - 7.12 (m, 2H, H3"'), 6.86 (s, 1H, H5"), 4.38 (t, $\left.J_{1^{\prime}, 2^{\prime}}=7.3 \mathrm{~Hz}, 2 \mathrm{H}, \mathrm{H} 1^{\prime}\right), 1.94$ (pent., $\left.J_{1^{\prime}, 2^{\prime}}=J_{2^{\prime}, 3^{\prime}}=7.4 \mathrm{~Hz}, 2 \mathrm{H}, \mathrm{H} 2^{\prime}\right), 1.37$ 1.12 (m, 8H, H3', H4', H5', H6'), 0.80 (t, J6, $\left.\sigma^{\prime} 7^{\prime}=6.9 \mathrm{~Hz}, 3 \mathrm{H}, \mathrm{H} 7^{\prime}\right) ;{ }^{13} \mathrm{C}$ NMR (150 MHz, pyridine-

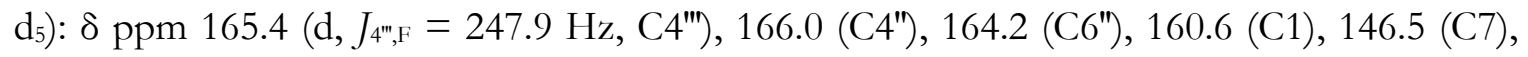
138.8 (C4), 136.4 (C1"'), 130.9 (C8a), 130.5 (d, J2",F$=8.4$ Hz, C2"'), 128.2 (C4a), 125.05 (C5), 126.0 (C6), 116.9 (d, J3",F= 22.1 Hz, C3"'), 116.1 (C8), 94.7 (C5"), 52.4 (C1'), 33.2 (C6'), 30.5 (C2'), 30.3 (C4'), 28.3 (C3'), 24.1 (C5'), 15.5 (C7'); ${ }^{19} \mathrm{~F}$ NMR (282 MHz, pyridine-d $\left.\mathrm{d}_{5}\right) \delta \mathrm{ppm}$ 111.71; HRMS-ESI $(m / z)$ calcd. for $\left[\mathrm{C}_{25} \mathrm{H}_{24} \mathrm{FN}_{6} \mathrm{O}+\mathrm{H}\right]^{+}$: 447.2303, obsd.: 447.2305.

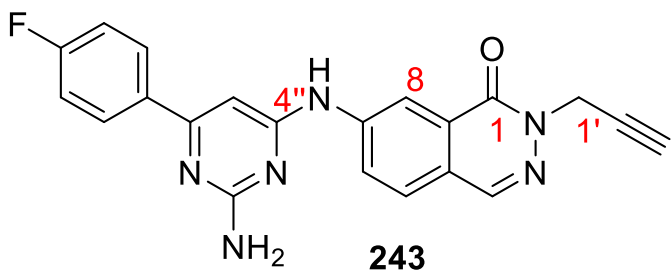

2-ethoxyethanol $(0.2 \mathrm{~mL})$ to the general procedure for the synthesis of pyrimidinyl-

\section{7-((2-Amino-(4-fluorophenyl)pyrimidin-4-} yl)amino-2-propargylphthalazin-1(2H)-one (243). By subjecting chloropyrimidine 188 (6.4 $\mathrm{mg}, 0.03 \mathrm{mmol}$ ) and amino-phthalazinone 232 $(10.7 \mathrm{mg}, 0.05 \mathrm{mmol})$ in conc. $\mathrm{HCl}(0.02 \mathrm{~mL})$ and 
phthalazinones, the title compound was isolated as a white amorphous solid (6.1 mg, 53\%); $\mathrm{R}_{f}$ $=0.44\left(\right.$ EtOAc); IR (U (max $\left._{\text {max }}\right): 2925,1648,1595,1501,1360,1108,1034,928,847,798,754,719$ $\mathrm{cm}^{-1} ;{ }^{1} \mathrm{H}$ NMR (600 MHz, pyridine-d $\left.\mathrm{d}_{5}\right) \delta$ ppm 10.77 (br. s, $\left.1 \mathrm{H}, \mathrm{NH}\right), 9.08$ (d, J6,8 $=2.2 \mathrm{~Hz}, 1 \mathrm{H}$, H8), 8.62 (dd, $J_{5,6}=8.5 \mathrm{~Hz}, J_{6,8}=2.2 \mathrm{~Hz}, 1 \mathrm{H}, \mathrm{H} 6$ ), 8.18 - 8.13 (m, 3H, H4, H2"'), 7.60 (d, J5,6

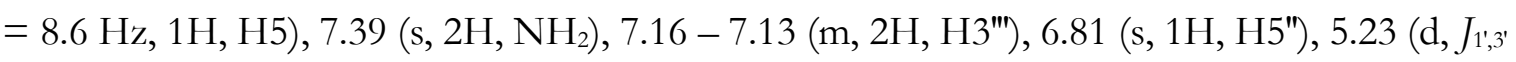
$=2.5 \mathrm{~Hz}, 2 \mathrm{H}), 3.31\left(\mathrm{~m}, 1 \mathrm{H}, \mathrm{H} 3^{\prime}\right) ;{ }^{13} \mathrm{C}$ NMR $\left(150 \mathrm{MHz}\right.$, pyridine- $\left.\mathrm{d}_{5}\right) \delta \mathrm{ppm} 164.7\left(\mathrm{~d}, J_{4^{\mathrm{m}}, \mathrm{F}}=\right.$ 247.9 Hz, C4"'), 165.2 (C4"), 164.0 (C6"), 163.3 (C2"), 159.4 (C1), 145.9 (C7), 138.8 (C4), 135.6 (C1"'), 129.8 (C8a), 129.7 (d, J2",F = 8.4 Hz, C2"'), 128.3 (C5), 126.0 (C6), 124.9 (C4a), 116.2 (C8), 116.2 (d, $\left.J_{3^{\prime \prime \prime}, \mathrm{F}}=21.5 \mathrm{~Hz}, \mathrm{C} 3^{\prime \prime}\right), 94.0$ (C5"), 80.4 (C2'), $74.2\left(\mathrm{C}^{\prime}\right), 41.3$ (C1'); ${ }^{19} \mathrm{~F}$ NMR (282 $\mathrm{MHz}$, pyridine-d $\left.\mathrm{d}_{5}\right) \delta \mathrm{ppm}-111.64$; HRMS-ESI $(\mathrm{m} / \mathrm{z})$ calcd. for $\left[\mathrm{C}_{21} \mathrm{H}_{15} \mathrm{FN}_{6} \mathrm{O}+\mathrm{H}\right]^{+}: 387.1364$, obsd.: 387.1365.

\subsubsection{Biology}

Samples. Each compound was dissolved in sterile DMSO to make $10 \mathrm{mM}$ stock solutions.

Obtaining MIC values. In clear-bottomed 96-well plates, two-fold serial dilutions of each compound were added to volumes of $100 \mu \mathrm{L}$ of HEPES-NaOH buffer ( $\mathrm{pH}$ 7.5). The buffer consisted of $10 \mathrm{mM}$ HEPES-NaOH, $5 \mathrm{mM} \mathrm{MgCl}_{2}$, and $100 \mathrm{mM} \mathrm{KCl}$. The last column of each plate did not contain any compound and served as a negative control. Previously prepared $M t b$ $\mathrm{mc}^{2} 6230$ inocula were diluted to $5 \%$ with HEPES-NaOH buffer to achieve $\mathrm{OD}_{600}$. The inocula were then added to each well and the plates incubated at $37^{\circ} \mathrm{C}$ for four days. After incubation, $20 \mu \mathrm{L}$ of resazurin was added to the wells, and the plates incubated at $37^{\circ} \mathrm{C}$ for 24 hours. The wells that remained blue were deemed to contain non-replicating bacteria, while those that underwent a colour change to purple or pink were deemed to contain replicating bacteria. MIC values were ascertained by visual judgment of colour.

Measuring rates of NADH oxidation. To make a stock solution of $M$. smegmatis IMVs, $1 \mu \mathrm{L}$ of IMV solution (20 mg/mL protein) was added to $1 \mathrm{~mL}$ of HEPES-NaOH buffer (pH 7.5). The HEPES-NaOH buffer is composed of $10 \mathrm{mM}$ HEPES-NaOH, $5 \mathrm{mM} \mathrm{MgCl}, 100 \mathrm{mM}$ $\mathrm{KCl}$. In clear-bottomed 96-well plates, $20 \mu \mathrm{L}$ of the IMV/buffer solution was added to each well, followed by $2 \mu \mathrm{L}$ of $10 \mathrm{mM}$ (in DMSO) solutions of each compound. NADH (10 $\mu \mathrm{L}$, $400 \mu \mathrm{M}$ ) was then added to each well. The solution in the well was mixed with a pipette and put into the UV spectrometer. Each plate had two wells without any drug, to serve as negative controls. The vehicle control contained IMV/buffer solution $(20 \mu \mathrm{L})$ and DMSO $(2 \mu \mathrm{L})$ while the second negative control consisted of IMV/buffer solution $(20 \mu \mathrm{L})$ and NADH $(10 \mu \mathrm{L})$ to 
obtain the basal rate of NADH oxidation. Absorbance values were recorded every minute for twenty minutes at $340 \mathrm{~nm}$ to allow for calculations of the NADH consumption rate. The experiments were undertaken three times, and the average rate of reaction was taken for each compound. 


\section{CHAPTER SIX}

\section{Conclusion and future prospects}

The overall objective of the research in this thesis was to develop new classes of heterocyclic compounds as anti-tuberculosis agents using NDH-II as the drug target. NDH-II is an attractive therapeutic target against $M t b$ as it is essential for the viability of $M t b$, yet it is absent in mammalian mitochondria. To date, the crystal structures of NDH-II in several organisms have been solved, providing insight into the binding sites of known substrates NADH, FAD and menaquinone, as highlighted in Chapter 1. Accordingly, considerations of potential binding interactions with NDH-II were taken during the exploration of the four classes of heterocyclic compounds discussed in this dissertation 
Ascidiathiazone A and ascidiathiazone B are tricyclic natural products that have been shown to possess potent anti-inflammatory activity. ${ }^{104}$ It has been proposed that ascidiathiazones A and B could also exhibit anti-tubercular activity as they contain the QQ scaffold which, as previously described, is a promising backbone used in TB drugs. Here, QQs are thought to exert their biological properties through the activation of NDH-II and consequent overproduction of ROS in $M t b .{ }^{64,65}$ Accordingly, Chapter 2 described a divergent synthetic strategy to access ascidiathiazone A and ascidiathiazone B. Here, the first total synthesis of ascidiathiazone $\mathrm{B}$ was achieved in 2.5\% yield over six steps from the same starting reagent used for the synthesis of ascidiathiazone A ( $6 \%$ over 5 steps). In addition, the crystal structure of ascidiathiazone A was obtained and solved, while further work is required to obtain the crystal structure of ascidiathiazone B. The two synthetic procedures presented also allowed for the preparation of a library of related analogues of the natural products.

The biological screening of ascidiathiazones $\mathrm{A}$ and $\mathrm{B}$, and derivatives thereof, led to the identification of ascidiathiazone $\mathrm{A}$ as a promising anti-tuberculosis agent with an MIC of 1.6 $\mu \mathrm{M}$, as well as previously noted low cytotoxicity in mammalian cells. ${ }^{105}$ Insight into the mode of action of the ascidiathiazone analogues demonstrated the ability of 7-chloro-6chloroethylamino-2-methyl-QQ to increase $\mathrm{NADH}$ oxidation rates in mycobacteria, while ascidiathiazone A did not affect NADH oxidation. This finding indicated that ascidiathiazone A exhibited a respiration-independent mode of inhibition. With this in mind, investigations to determine the mechanism(s) by which ascidiathiazone A inhibits mycobacterial growth are required. Here, genetic target-identification methods could be applied, whereby the DNA of ascidiathiazone A-resistant $M t b$ mutants could be sequenced and analysed to identify the protein affected by the drug. ${ }^{346}$

Given the anti-tubercular activity of QQs ${ }^{64,65}$ Chapter 3 discussed the preparation of a library of amine-substituted QQs, whereby the synthesised compounds were screened for their ability to inhibit Mtb growth. Along with the identification of a new lead compound, 7-chloro-6propargylamino-QQ $(\mathrm{MIC}=8 \mu \mathrm{M})$, SAR data of QQ library was extrapolated. Here, it was observed that a short C3 alkyl chain on the QQ scaffold was insufficient to effectively inhibit $M t b$ growth, though installing a tetradecylamine on the QQ also led to a loss of inhibitory activity. Notwithstanding, QQs bearing moderate chain lengths, such as octylamino-QQs and heptylanilino-QQs exhibited high anti-tubercular activity. Moreover, the redox activity of QQs were measured and while no correlation was found between tuberculostatic activity and redox potentials in CVs conducted in acetonitrile, a near linear relationship was observed for those 
QQs that were able to be measured in a microemulsion system. Here, QQs with redox potentials between -0.5 and $-0.6 \mathrm{~V}$ typically exhibited high anti-tubercular activity.

In addition to the redox activity of QQs, it was proposed that electrophilic substituents on the QQ scaffold, i.e. acetylene and chloroethylamine, could enhance the anti-tubercular activity of QQs by participating in covalent inhibition with nucleophilic amino acids within NDH-II. To explore this hypothesis, other electrophiles such as $\alpha, \beta$-unsaturated ketone and thiocyanide could be incorporated into the QQ scaffold at the 6-position (i.e. QQ 244 and QQ 245, Figure 6.1) and the effect of these drugs on the anti-tubercular activity explored.<smiles>C=CC(=O)C1=C(Cl)C(=O)c2ncccc2C1=O</smiles>

244<smiles>O=C1C(Cl)=C(N=C=S)C(=O)c2ncccc21</smiles>

245

Figure 6.1: Proposed QQs bearing electrophilic substituents to be synthesised.

In Chapter 4, the anti-tubercular activity of two libraries of chromonyl-pyrimidines, which have a structural resemblance to $M t b$ NDH-II inhibitors quinolinyl pyrimidines, was investigated. The synthesis of chromonyl-pyrimidines involved a convergent synthesis, whereby functionalised pyrimidines and chromones were condensed to give di-substituted chromonyl-pyrimidines in good (20\%-87\%) yields. Docking studies demonstrated that chromonyl-pyrimidines could occupy the quinone binding tunnel surrounded by the hydrophobic residues, Q317 and I379. Moreover, a hydrogen bond between the primary amine protons of the pyrimidine scaffold with a glutamic acid side chain in the NDH-II Q-site was observed. Accordingly, an eleven-member set of chromonyl-pyrimidines was synthesised and screened against $M t b$. SAR data for this library showed that the instalment of aromatic substituents led to enhanced inhibitory activity. Subsequently, a second generation library of chromonyl-pyrimidines bearing aromatic substituents was prepared and found to exhibit improved anti-tubercular activity.

When compared to quinolinyl pyrimidines, chromonyl-pyrimidines exhibited poor $M t b$ growth inhibition, despite both showing similar binding modes within the NDH-II Q-site, as was observed via docking studies. The marked difference in anti-tubercular activity between the 
two scaffolds suggests that they may target different proteins. Notwithstanding, future work in this area should focus on the quinolinyl pyrimidine scaffold due to its high efficacy against $M t b$. SAR analysis of aryl-substituted quinolinyl pyrimidines found that when different phenyl substituents were installed on the quinolinyl pyrimidine scaffold, i.e. fluorophenyl and methoxyphenyl, the same degree of growth inhibition was achieved. While altering the electronic properties of the aromatic substituents on the quinolinyl pyrimidine scaffold does not affect the compounds' anti-tubercular activity, it is postulated that increasing the $\pi$ conjugation of the substituents may improve the ability of the quinolinyl pyrimidines to bind. To explore this hypothesis, a second generation library of quinolinyl pyrimidines bearing functional groups with various degrees of conjugation such as naphthalene, heptatriene, biphenyl, or styrylbenzene, at the 6'- and 2-positions (i.e. quinolinyl pyrimidines 246, Figure 6.2) could be synthesised. In addition, analysis of how lead quinolinyl pyrimidines affect NADH oxidation in IMVs that overexpress NDH-II could further support the speculation that NDH-II is the target protein of quinolinyl pyrimidines.<smiles>[R]c1cc(Nc2ccc3nc([R])cc(N)c3c2)nc(N)n1</smiles>

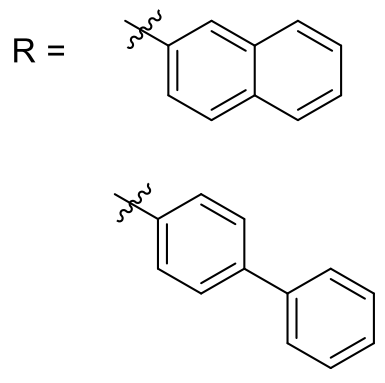<smiles>C=C/C=C\C=C/C(C)(C)C</smiles><smiles>Cc1ccc(/C=C/c2ccccc2)cc1</smiles>

Figure 6.2: Proposed structures of quinolinyl pyrimidines bearing highly aromatic substituents to be prepared.

Finally, Chapter $\mathbf{5}$ concerned the synthesis and evaluation of phthalazinones and pyrimidinylphthalazinones as anti-tubercular agents. Functionalised phthalazinones were readily synthesised in 3-4 steps in good overall (7\%-38\%) yields from phthalic anhydride, while pyrimidinyl-phthalazinones were prepared via the condensation of functionalised phthalazinones and pyrimidines (24\%-83\% yield). Here, the biological screening of 
phthalazinones identified $N$-tert-butyl- and nitro-phthalazinones as lead compounds (MIC $=3$ $\mu \mathrm{M})$, while 4-fluorophenyl-pyrimidinyl- $N$-heptylphthalazinone displayed the highest potency of the library against $M t b(\mathrm{MIC}=1.6 \mu \mathrm{M})$. Investigations into the effect of phthalazinones on $\mathrm{NADH}$ oxidation rates showed that several phthalazinones decreased the rate of NADH oxidation in IMVs, which could be due to redox cycling or uncoupling activity, ${ }^{37}$ while a number of pyrimidinyl-phthalazinones increased the oxidation rate, which could result in the generation of lethal levels of ROS. ${ }^{64}$ These findings suggest that the two classes of compounds have different modes of inhibition, though the mechanism of action of the lead inhibitors appeared to be independent of mycobacterial respiration.

To investigate the respiration-independent inhibition mechanism of the lead tuberculostatic phthalazinone, phthalazinone-resistant populations of $M t b$ could be generated and their genetic material examined to determine the mycobacterial proteins affected. ${ }^{346,347}$ Moreover, a second generation library of functionalised phthalazinones could be prepared. From the SAR data of the phthalazinone library, it was noted that the instalment of hydrocarbons on the hydrazone nitrogen, as well as a nitro-group on the 7-position generally increased the compounds' ability to inhibit Mtb growth. To this end, investigations into how the instalment of different hydrocarbons, i.e. allyl, cyclopropyl, dodecyl, on the hydrazone nitrogen (i.e. phthalazinones 247, 248, 249, Figure 6.3) and modifying the position of the electronwithdrawing nitro substituent affect the anti-tubercular activity of phthalazinones are required.

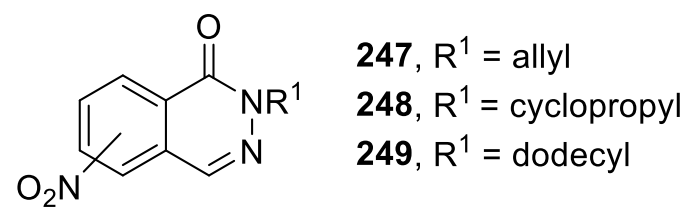

Figure 6.3: Proposed phthalazinones to synthesise

Overall, the ascidiathiazone analogues and phthalazinones showed promise as future antituberculosis agents, with lead compounds ascidiathiazone $\mathrm{A}$ and 7-((2-amino-6-(4fluorophenyl)pyrimidin-4-yl)amino)2-heptylphthalazin-1(2H)-one, both exhibiting an MIC of 1.6 $\mu \mathrm{M}$. Here, tuberculostatic drugs have a place in the ongoing search for new TB drugs as they can be included in combination therapies with other more cytotoxic drugs. This drug synergism has been demonstrated with the bacteriostatic agent linezolid (250, Figure 6.4). ${ }^{348-350}$ While linezolid (250) has low MIC values against $M t b(\leq 0.125 \mathrm{mg} / \mathrm{L}),{ }^{351}$ it has limited cidal 
activity during the exponential growth phase of the bacteria. ${ }^{352}$ Notwithstanding, the WHO has listed linezolid (250) as a second-line TB drug ${ }^{350}$ as linezolid (250) has been reported to enhance the sterilizing ability of bedaquiline and pretomanid in a three-drug regimen. ${ }^{353}$ Accordingly, effort should be focused on the mode of inhibition exerted by lead compounds ascidiathiazone $\mathrm{A}$ and 7-((2-amino-6-(4-fluorophenyl)pyrimidin-4-yl)amino)2heptylphthalazin-1(2H)-one as their potent inhibitory activity could lead to the discovery of new $M t b$ drug targets and new TB drug regimens.

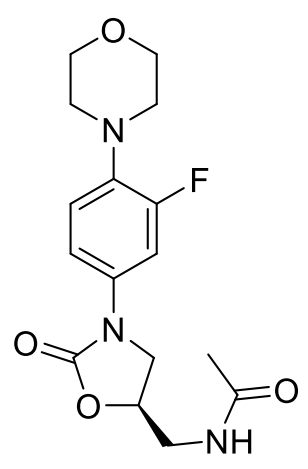

250

Figure 6.4: Linezolid (250), a bacteriostatic TB drug. 


\section{References}

1. I. G. Sia and M. L. Wieland, Mayo Clin. Proc., 2011, 86, 348-361.

2. A. Zumla, P. Nahid and S. T. Cole, Nat. Rev. Drug Discov., 2013, 12, 388-404.

3. WHO, Global tuberculosis report 2018, World Health Organisation, 2018.

4. S. E. Weis, P. C. Slocum, F. X. Blais, B. King, M. Nunn, G. B. Matney, E. Gomez and B. H. Foresman, New Engl. J. Med., 1994, 330, 1179-1184.

5. Y. L. Janin, Bioorg. Med. Chem., 2007, 15, 2479-2513.

6. $\quad$ S. Ahmad, Clin. Dev. Immunol., 2011, DOI: 10.1155/2011/814943, 17.

7. E. M. Dangerfield, A. L. Win-Mason, M. S. M. Timmer and B. L. Stocker, Chemistry in Australia, 2010, May, 17-21.

8. $\quad$ S. Schwander and K. Dheda, Am. J. Respir. Cell Mol. Biol., 2011, 183, 696-707.

9. M. J. Marakalala, L. M. Graham and G. D. Brown, Clin. Dev. Immunol., 2010, DOI: $10.1155 / 2010 / 567571$

10. P. Constantoulakis, E. Filiou, N. Rovina, G. Chras, A. Hamhougia, S. Karabela, A. Sotiriou, C. Roussos and N. Poulakis, BMC Infect. Dis., 2010, 10, 243.

11. J. Zuñiga, D. Torres-García, T. Santos-Mendoza, T. S. Rodriguez-Reyna, J. Granados and E. J. Yunis, Clin. Dev. Immunol., 2012, 18.

12. D. G. Russell, Nat. Rev. Mol. Cell Biol., 2001, 2, 569-586.

13. B. M. Saunders and W. J. Britton, Immunol. Cell Biol., 2007, 85, 103-111.

14. D. G. Russell, P.-J. Cardona, M.-J. Kim, S. Allain and F. Altare, Nat. Immunol., 2009, 10, 943948.

15. M. I. Sadek, E. Sada, Z. Toossi, S. K. Schwander and E. A. Rich, Am. J. Respir. Cell Mol. Biol., 1998, 19, 513-521.

16. P. Peyron, J. Vaubourgeix, Y. Poquet, F. Levillain, C. Botanch, F. Bardou, M. Daffé, J.-F. Emile, B. Marchou, P.-J. Cardona, C. de Chastellier and F. Altare, PLoS Path., 2008, 4, e1000204.

17. J. E. Gomez and J. D. McKinney, Tuberculosis, 2004, 84, 29-44.

18. A. Koch, V. Mizrahi and D. F. Warner, Emerg. Microbes Infect., 2014, 3, e17.

19. S. H. Gillespie, Antimicrob. Agents Chemother., 2002, 46, 267-274.

20. T. Smith, K. A. Wolff and L. Nguyen, Curr. Top. Microbiol. Immunol., 2013, 374, 53-80.

21. L. Schrager, O. Oleson, J. Vekemans, D. Lewisnsohn, J. Shea, W. Hanekom and N. Drager, Global report on tuberculosis vaccines, 2018.

22. A. Schatz and S. A. Waksman, Exp. Biol. Med., 1944, 57, 244-248.

23. J. Lehmann, The Lancet, 1946, 247, 15-16.

24. S. Füresz and M. T. Timbal, Chemotherapia, 1963, 7, 200-208.

25. P. Klee, Br. Med. J., 1952, 2, 764-765.

26. K. D. Mc, L. Malone and et al., J. Lab. Clin. Med., 1948, 33, 1249-1253.

27. S. H. Ferebee, B. E. Doster and F. J. Murray, Ann. N. Y. Acad. Sci., 1966, 135, 910-920.

28. G. L. Hobby and T. F. Lenert, Am. Rev. Tuberc., 1952, 65, 771-774.

29. S.-J. Cheng, L. Thibert, T. Sanchez, L. Heifets and Y. Zhang, Antimicrob. Agents Chemother., 2000, 44, 528-532.

30. R. Johnson, E. M. Streicher, G. E. Louw, R. M. Warren, P. D. van Helden and T. C. Victor, Curr. Issues Mol. Biol., 2006, 8, 97-111.

31. A. Scorpio and Y. Zhang, Nat. Med., 1996, 2, 662-667.

32. K. Hirano, M. Takahashi, Y. Kazumi, Y. Fukasawa and C. Abe, Tuberc. Lung Dis., 1997, 78, 117-122.

33. K. Hards, J. R. Robson, M. Berney, L. Shaw, D. Bald, A. Koul, K. Andries and G. M. Cook, J. Antimicrob. Chemother., 2015, DOI: 10.1093/jac/dkv054.

34. J. Debnath, S. Siricilla, B. Wan, D. C. Crick, A. J. Lenaerts, S. G. Franzblau and M. Kurosu, J. Med. Chem., 2012, 55, 3739-3755.

35. E. A. Weinstein, T. Yano, L.-S. Li, D. Avarbock, A. Avarbock, D. Helm, A. A. McColm, K. Duncan, J. T. Lonsdale and H. Rubin, Proc. Natl. Acad. Sci. U.S.A., 2005, 102, 4548-4553. 
36. B. Lechartier and S. T. Cole, Antimicrob. Agents Chemother., 2015, 59, 4457-4463.

37. T. Yano, S. Kassovska-Bratinova, J. S. Teh, J. Winkler, K. Sullivan, A. Isaacs, N. M. Schechter and H. Rubin, J. Biol. Chem., 2011, 286, 10276-10287.

38. T. Yano, L.-S. Li, E. Weinstein, J.-S. Teh and H. Rubin, J. Biol. Chem., 2006, 281, 11456-11463.

39. R. H. Garrett and C. M. Grisham, Biochemistry, Mary Finch, Boston, USA, 2010.

40. B. A. Haddock and C. W. Jones, Bacteriol. Rev., 1977, Mar., 47-99.

41. G. M. Cook, C. Greening, K. Hards and M. Berney, in Adv. Microb. Physiol., ed. K. P. Robert, Academic Press, 2014, vol. Volume 65, pp. 1-62.

42. D. L. Piddington, A. Kashkouli and N. A. Buchmeier, Infect. Immun., 2000, 68, 4518-4522.

43. P. R. Jensen and O. Michelsen, J. Bacteriol., 1992, 174, 7635-7641.

44. M. Santana, M. S. Ionescu, A. Vertes, R. Longin, F. Kunst, A. Danchin and P. Glaser, J. Bacteriol., 1994, 176, 6802-6811.

45. S. L. Tran, M. Rao, C. Simmers, S. Gebhard, K. Olsson and G. M. Cook, Microbiology, 2005, 151, 665-672.

46. M. Rao, T. L. Streur, F. E. Aldwell and G. M. Cook, Microbiology, 2001, 147, 1017-1024.

47. S. L. Tran and G. M. Cook, J. Bacteriol., 2005, 187, 5023-5028.

48. D. Jones, Nat. Rev. Drug Discov., 2013, 12, 175-176.

49. K. Andries, P. Verhasselt, J. Guillemont, H. W. H. Göhlmann, J.-M. Neefs, H. Winkler, J. Van Gestel, P. Timmerman, M. Zhu, E. Lee, P. Williams, D. d. Chaffoy, E. Huitric, S. Hoffner, E. Cambau, C. Truffot-Pernot, N. Lounis and V. Jarlier, Science, 2005, 307, 223-227.

50. A. Koul, N. Dendouga, K. Vergauwen, B. Molenberghs, L. Vranckx, R. Willebrords, Z. Ristic, H. Lill, I. Dorange, J. Guillemont, D. Bald and K. Andries, Nat. Chem. Biol., 2007, 3, 323-324.

51. L. Formentini, M. Sánchez-Aragó, L. Sánchez-Cenizo and José M. Cuezva, Mol. Cell, 2012, 45, 731-742.

52. S. P. S. Rao, S. Alonso, L. Rand, T. Dick and K. Pethe, Proc. Natl. Acad. Sci. U.S.A., 2008, 105, 11945-11950.

53. A. Heikal, Y. Nakatani, E. Dunn, M. R. Weimar, C. L. Day, E. N. Baker, J. S. Lott, L. A. Sazanov and G. M. Cook, Mol. Microbiol., 2014, 91, 950-964.

54. A. M. P. Melo, T. M. Bandeiras and M. Teixeira, Microbiol. Mol. Biol. Rev., 2004, 68, 603-616.

55. D. Awasthy, A. Ambady, A. Narayana, S. Morayya and U. Sharma, Gene, 2014, 550, 110-116.

56. A. J. Warman, T. S. Rito, N. E. Fisher, D. M. Moss, N. G. Berry, P. M. O'Neill, S. A. Ward and G. A. Biagini, J. Antimicrob. Chemother., 2013, 68, 869-880.

57. J. Knuuti, G. Belevich, V. Sharma, D. A. Bloch and M. Verkhovskaya, Mol. Microbiol., 2013, 90, 1190-1200.

58. Y. Nakatani, W. Jiao, D. Aragao, Y. Shimaki, J. Petri, E. J. Parker and G. M. Cook, Acta Crystallogr. Sect. F, 2017, 73, 541-549.

59. M. Iwata, Y. Lee, T. Yamashita, T. Yagi, S. Iwata, A. D. Cameron and M. J. Maher, Proc. Natl. Acad. Sci. U.S.A., 2012, 109, 15247-15252.

60. F. V. Sena, A. P. Batista, T. Catarino, J. A. Brito, M. Archer, M. Viertler, T. Madl, E. J. Cabrita and M. M. Pereira, Mol. Microbiol., 2015, 98, 272-288.

61. Y. Yang, Y. Yu, X. Li, J. Li, Y. Wu, J. Yu, J. Ge, Z. Huang, L. Jiang, Y. Rao and M. Yang, J. Med. Chem., 2017, 60, 1994-2005.

62. J. Petri, Y. Shimaki, W. Jiao, H. R. Bridges, E. R. Russell, E. J. Parker, D. Aragão, G. M. Cook and Y. Nakatani, Biochim. Biophys. Acta, 2018, 1859, 482-490.

63. Y. Feng, W. Li, J. Li, J. Wang, J. Ge, D. Xu, Y. Liu, K. Wu, Q. Zeng, J. W. Wu, C. Tian, B. Zhou and M. Yang, Nature, 2012, 491, 478-482.

64. A. Heikal, K. Hards, C.-Y. Cheung, A. Menorca, M. S. M. Timmer, B. L. Stocker and G. M. Cook, J. Antimicrob. Chemother., 2016, 71, 2840-2847.

65. B. J. Mulchin, C. G. Newton, J. W. Baty, C. H. Grasso, W. J. Martin, M. C. Walton, E. M. Dangerfield, C. H. Plunkett, M. V. Berridge, J. L. Harper, M. S. M. Timmer and B. L. Stocker, Bioorg. Med. Chem., 2010, 18, 3238-3251.

66. C.-X. He, H. Meng, X. Zhang, H.-Q. Cui and D.-L. Yin, Chin. Chem. Lett., 2015, 26, 951-954.

67. L. Amaral, A. Martins, J. Molnar, J. E. Kristiansen, M. Martins, M. Viveiros, L. Rodrigues, G. Spengler, I. Couto, J. Ramos, S. Dastidar, S. Fanning, M. McCusker and J.-M. Pages, In Vivo, 2010, 24, 409-424. 
68. D. V. Gadre, V. Talwar, H. C. Gupta and R. Murthy, Int. Clin. Pyschopharmacol., 1998, 13, 129132.

69. L. Amaral, J. E. Kristiansen, M. Viveiros and J. Atouguia, J. Antimicrob. Chemother., 2001, 47, 505-511.

70. S. Sellamuthu, M. Singh, A. Kumar and S. K. Singh, Expert Opin. Ther. Targets, 2017, 21, 559570 .

71. L. Amaral and M. Viveiros, Int. J. Antimicrob. Agents, 2012, 39, 376-380.

72. L. Amaral and M. Viveiros, Antibiotics, 2017, 6, 3.

73. D. van Soolingen, R. Hernandez-Pando, H. Orozco, D. Aguilar, C. Magis-Escurra, L. Amaral, J. van Ingen and M. J. Boeree, PLoS ONE, 2010, 5.

74. L. Amaral, J. E. Kristiansen, L. S. Abebe and W. Millett, J. Antimicrob. Chemother., 1996, 38, 1049-1053.

75. D. Ordway, M. Viveiros, C. Leandro, R. Bettencourt, J. Almeida, M. Martins, J. E. Kristiansen, J. Molnar and L. Amaral, Antimicrob. Agents Chemother., 2003, 47, 917-922.

76. L. Amaral and J. Molnar, In Vivo, 2014, 28, 267-271.

77. L. Amaral, M. Martins and M. Viveiros, J. Antimicrob. Chemother., 2007, 59, 1237-1246.

78. A. J. Crowle, G. S. Douvas and M. H. May, Chemother., 1992, 38, 410-419.

79. P. S. Shirude, B. Paul, N. Roy Choudhury, C. Kedari, B. Bandodkar and B. G. Ugarkar, ACS Med. Chem. Lett., 2012, 3, 736-740.

80. T. R. Ioerger, T. O’Malley, R. Liao, K. M. Guinn, M. J. Hickey, N. Mohaideen, K. C. Murphy, H. I. M. Boshoff, V. Mizrahi, E. J. Rubin, C. M. Sassetti, C. E. Barry, III, D. R. Sherman, T. Parish and J. C. Sacchettini, PLoS ONE, 2013, 8, e75245.

81. M. B. Harbut, B. Yang, R. Liu, T. Yano, C. Vilchèze, B. Cheng, J. Lockner, H. Guo, C. Yu, S. G. Franzblau, H. M. Petrassi, W. R. Jacobs, H. Rubin, A. K. Chatterjee and F. Wang, Angew. Chem. Int. Ed., 2018, 57, 3478-3482.

82. D. Murugesan, P. C. Ray, T. Bayliss, G. A. Prosser, J. R. Harrison, K. Green, C. Soares de Melo, T.-S. Feng, L. J. Street, K. Chibale, D. F. Warner, V. Mizrahi, O. Epemolu, P. Scullion, L. Ellis, J. Riley, Y. Shishikura, L. Ferguson, M. Osuna-Cabello, K. D. Read, S. R. Green, D. A. Lamprecht, P. M. Finin, A. J. C. Steyn, T. R. Ioerger, J. Sacchettini, K. Y. Rhee, K. Arora, C. E. Barry, P. G. Wyatt and H. I. M. Boshoff, ACS Infect. Dis., 2018, 4, 954-969.

83. A. S. Ginsburg, J. H. Grosset and W. R. Bishai, The Lancet Infect. Dis., 2003, 3, 432-442.

84. K. J. Aldred, R. J. Kerns and N. Osheroff, Biochem., 2014, 53, 1565-1574.

85. S. C. Leung, P. Gibbons, R. Amewu, G. L. Nixon, C. Pidathala, W. D. Hong, B. Pacorel, N. G. Berry, R. Sharma, P. A. Stocks, A. Srivastava, A. E. Shone, S. Charoensutthivarakul, L. Taylor, O. Berger, A. Mbekeani, A. Hill, N. E. Fisher, A. J. Warman, G. A. Biagini, S. A. Ward and P. M. O’Neill, J. Med. Chem., 2012, 55, 1844-1857.

86. C. Pidathala, R. Amewu, B. Pacorel, G. L. Nixon, P. Gibbons, W. D. Hong, S. C. Leung, N. G. Berry, R. Sharma, P. A. Stocks, A. Srivastava, A. E. Shone, S. Charoensutthivarakul, L. Taylor, O. Berger, A. Mbekeani, A. Hill, N. E. Fisher, A. J. Warman, G. A. Biagini, S. A. Ward and P. M. O'Neill, J. Med. Chem., 2012, 55, 1831-1843.

87. W. D. Hong, P. D. Gibbons, S. C. Leung, R. Amewu, P. A. Stocks, A. Stachulski, P. Horta, M. L. S. Cristiano, A. E. Shone, D. Moss, A. Ardrey, R. Sharma, A. J. Warman, P. T. P. Bedingfield, N. E. Fisher, G. Aljayyoussi, S. Mead, M. Caws, N. G. Berry, S. A. Ward, G. A. Biagini, P. M. O’Neill and G. L. Nixon, J. Med. Chem., 2017, 60, 3703-3726.

88. L. Z. Holland, Curr. Biol., 2016, 26, R146-R152.

89. C. Bertanha, A. Januário, T. Alvarenga, L. Pimenta, M. Silva, W. Cunha and P. Pauletti, Mar. Drugs, 2014, 12, 3608.

90. A. Aiello, E. Fattorusso, P. Luciano, A. Macho, M. Menna and E. Muñoz, J. Med. Chem., 2005, 48, 3410-3416.

91. A. Aiello, E. Fattorusso, P. Luciano, A. Mangoni and M. Menna, Eur. J. Org. Chem., 2005, 2005, 5024-5030.

92. M. Broggini, S. V. Marchini, E. Galliera, P. Borsotti, G. Taraboletti, E. Erba, M. Sironi, J. Jimeno, G. T. Faircloth, R. Giavazzi and M. d'Incalci, Leukemia, 2003, 17, 52.

93. D. J. Newman and G. M. Cragg, J. Nat. Prod., 2004, 67, 1216-1238.

94. M. Gompel, M. Leost, E. B. De Kier Joffe, L. Puricelli, L. H. Franco, J. Palermo and L. Meijer, Bioorg. Med. Chem. Lett., 2004, 14, 1703-1707. 
95. L. Núñez-Pons, R. M. Nieto, C. Avila, C. Jiménez and J. Rodríguez, J. Mass Spectrom., 2015, 50, 103-111.

96. S. Yin, G. M. Boyle, A. R. Carroll, M. Kotiw, J. Dearnaley, R. J. Quinn and R. A. Davis, J. Nat. Prod., 2010, 73, 1586-1589.

97. D. R. Appleton, C. S. Chuen, M. V. Berridge, V. L. Webb and B. R. Copp, J. Org. Chem., 2009, 74, 9195-9198.

98. M. Menna, C. Imperatore, F. Aniello and A. Aiello, Mar. Drugs, 2013, 11, 1602.

99. B. S. Davidson, Chem. Rev., 1993, 93, 1771-1791.

100. C. S. Bertanha, A. H. Januário, T. A. Alvarenga, L. P. Pimenta, M. L. A. E. Silva, W. R. Cunha and P. M. Pauletti, Mar. Drugs, 2014, 12, 3608-3633.

101. A. Aiello, E. Fattorusso, P. Luciano, M. Menna, M. A. Calzado, E. Muñoz, F. Bonadies, M. Guiso, M. F. Sanasi, G. Cocco and R. Nicoletti, Bioorg. Med. Chem., 2010, 18, 719-727.

102. A. Aiello, E. Fattorusso, P. Luciano, M. Menna, G. Esposito, T. Iuvone and D. Pala, Eur. J. Org. Chem., 2003, 2003, 898-900.

103. M. Menna, A. Aiello, F. D'Aniello, C. Imperatore, P. Luciano, R. Vitalone, C. Irace and R. Santamaria, Eur. J. Org. Chem., 2013, 2013, 3241-3246.

104. A. N. Pearce, E. W. Chia, M. V. Berridge, G. R. Clark, J. L. Harper, L. Larsen, E. W. Maas, M. J. Page, N. B. Perry, V. L. Webb and B. R. Copp, J. Nat. Prod., 2007, 70, 936-940.

105. E. W. Chia, A. N. Pearce, M. V. Berridge, L. Larsen, N. B. Perry, C. E. Sansom, C. A. Godfrey, L. R. Hanton, G.-L. Lu, M. Walton, W. A. Denny, V. L. Webb, B. R. Copp and J. L. Harper, Bioorg. Med. Chem., 2008, 16, 9432-9442.

106. J. E. Nycz and G. J. Malecki, J. Mol. Struct., 2013, 1032, 159-168.

107. W.-H. Sun, P. Hao, S. Zhang, Q. Shi, W. Zuo, X. Tang and X. Lu, Organometallics, 2007, 26, 2720-2734.

108. V. Chandrasekhar, S. Hossain, S. Das, S. Biswas and J.-P. Sutter, Inorg. Chem., 2013, 52, 63466353.

109. G. Black, E. Depp and B. B. Corson, J. Org. Chem., 1949, 14, 14-21.

110. T. M. Ali Shaikh, L. Emmanuvel and A. Sudalai, J. Org. Chem., 2006, 71, 5043-5046.

111. F. Z. Basha, S. Hibino, D. Kim, W. E. Pye, T.-T. Wu and S. M. Weinreb, J. Am. Chem. Soc., 1980, 102, 3962-3964.

112. S. M. Weinreb, F. Z. Basha, S. Hibino, N. A. Khatri, D. Kim, W. E. Pye and T. T. Wu, J. Am. Chem. Soc., 1982, 104, 536-544.

113. A. S. Kende, D. P. Lorah and R. J. Boatman, J. Am. Chem. Soc., 1981, 103, 1271-1273.

114. S. Mukhopadhyay and S. B. Chandalia, Org. Process Res. Dev., 1999, 3, 455-459.

115. V. Verones, N. Flouquet, M. Lecoeur, A. Lemoine, A. Farce, B. Baldeyrou, C. Mahieu, N. Wattez, A. Lansiaux, J.-F. Goossens, P. Berthelot and N. Lebegue, Eur. J. Med. Chem., 2013, 59, 39-47.

116. N. Sharma, R. Gupta, M. Kumar and R. R. Gupta, J. Fluorine Chem., 1999, 98, 153-157.

117. D. V. Kalinin, S. Wagner, B. Riemann, S. Hermann, F. Schmidt, C. Becker-Pauly, S. RoseJohn, M. Schäfers and R. Holl, J. Med. Chem., 2016, 59, 9541-9559.

118. K. S. Battula, S. Narsimha, R. K. Thatipamula, Y. N. Reddy and V. R. Nagavelli, ChemistrySelect, 2017, 2, 4001-4005.

119. X. Huang, D. Chen, N. Wu, A. Zhang, Z. Jia and X. Li, Bioorg. Med. Chem. Lett., 2009, 19, 41304133.

120. E. Badetti, A. Bonetto, F. Romano, L. Marchiò, C. Zonta and G. Licini, Catal. Lett., 2017, 147, 2313-2318.

121. M. T. Gallego, E. Brunet, J. L. Garcia Ruano and E. L. Eliel, J. Org. Chem., 1993, 58, 39053911.

122. R. A. Henry and W. M. Dehn, J. Am. Chem. Soc., 1950, 72, 2280-2281.

123. S. Dhanju and D. Crich, Org. Lett., 2016, 18, 1820-1823.

124. J. B. Lambert and R. G. Keske, J. Org. Chem., 1966, 31, 3429-3431.

125. H. P. Yennawar, Z. Yang and L. J. Silverberg, Acta Crystallogr. Sect. E, 2016, 72, 1541-1543.

126. E. Hough, E. Wold and S. G. Dahl, Acta Crystallogr. Sect. C, 1985, 41, 386-389.

127. Q. Wang, Z. Xu and Y. Sun, Acta Crystallogr. Sect. E, 2010, 66, o1422.

128. M. R. Johnson, M. J. Fazio, D. L. Ward and L. R. Sousa, J. Org. Chem., 1983, 48, 494-499. 
129. H. P. Yennawar, J. Tierney, P. D. Hullihen and L. J. Silverberg, Acta Crystallogr. Sect. E, 2015, 71, 264-267.

130. R. M. Silverstein, F. X. Webster and D. J. Kiemle, Spectrometric identification of organic compounds, John Wiley \& Sons, Inc., New Jersey, USA, 2005.

131. R. H. Pouwer, S. M. Deydier, P. V. Le, B. D. Schwartz, N. C. Franken, R. A. Davis, M. J. Coster, S. A. Charman, M. D. Edstein, T. S. Skinner-Adams, K. T. Andrews, I. D. Jenkins and R. J. Quinn, ACS Med. Chem. Lett., 2014, 5, 178-182.

132. C. Vilchèze, B. Weinrick, L. W. Leung and W. R. Jacobs, Proc. Natl. Acad. Sci. U.S.A., 2018, 115, 1599-1604.

133. R. O. Akinola, G. K. Mazandu and N. J. Mulder, J. Bacteriol. Parasitol., 2013, 4, 173.

134. Z. He and J. De Buck, BMC Microbiol., 2010, 10, 121.

135. T. Lu and K. Drlica, J. Antimicrob. Chemother., 2003, 52, 1025-1028.

136. V. Chaturvedi, N. Dwivedi, R. P. Tripathi and S. Sinha, The Journal of General and Applied Microbiology, 2007, 53, 333-337.

137. K. Andries, P. Verhasselt, J. Guillemont, H. W. H. Göhlmann, J.-M. Neefs, H. Winkler, J. Van Gestel, P. Timmerman, M. Zhu, E. Lee, P. Williams, D. de Chaffoy, E. Huitric, S. Hoffner, E. Cambau, C. Truffot-Pernot, N. Lounis and V. Jarlier, Science, 2005, 307, 223-227.

138. C. Lam, A. Pearce, S. Tan, M. Kaiser and B. Copp, Mar. Drugs, 2013, 11, 3472.

139. T. Fryatt, H. I. Pettersson, W. T. Gardipee, K. C. Bray, S. J. Green, A. M. Z. Slawin, H. D. Beall and C. J. Moody, Bioorg. Med. Chem., 2004, 12, 1667-1687.

140. Y. Wang, D. Oxer and S. Hekimi, Nat. Comm., 2015, 6, 6393.

141. B. Nowicka and J. Kruk, Biochim. Biophys. Acta, 2010, 1797, 1587-1605.

142. T. Hasegawa, A. Bando, K. Tsuchiya, S. Abe, M. Okamoto, K. Kirima, S. Ueno, M. Yoshizumi, H. Houchi and T. Tamaki, Biocbim. Biophys. Acta, 2004, 1670, 19-27.

143. Y. Zu, R. J. Shannon and J. Hirst, J. Am. Chem. Soc., 2003, 125, 6020-6021.

144. J. W. Lown and S.-K. Sim, Can. J. Chem., 1976, 54, 2563-2572.

145. L. Kussmaul and J. Hirst, Proc. Natl. Acad. Sci. U.S.A., 2006, 103, 7607-7612.

146. M. P. Murphy, Biochem. J., 2009, 417, 1-13.

147. J. Imlay and S. Linn, Science, 1988, 240, 1302-1309.

148. A. Alfadhli, A. Mack, L. Harper, S. Berk, C. Ritchie and E. Barklis, Bioorg. Med. Chem., 2016, 24, 5618-5625.

149. A. Chakraborty, P. R. Gangadharam, P. Damle, P. Pratt, P. Wright and P. T. Davidson, Tubercle, 1981, 62, 37-41.

150. S. A. Egu, A. Ibezim, E. A. Onoabedje and U. C. Okoro, Chemistry Select, 2017, 2, 9222-9226.

151. R. Cone, S. K. Hasan, J. W. Lown and A. R. Morgan, Can. J. Biochem., 1976, 54, 219-223.

152. K. V. Rao, K. Biemann and R. B. Woodward, J. Am. Chem. Soc., 1963, 85, 2532-2533.

153. D. L. Boger, M. Yasuda, L. A. Mitscher, S. D. Drake, P. A. Kitos and S. C. Thompson, J. Med. Chem., 1987, 30, 1918-1928.

154. H. D. Beall, Y. Liu, D. Siegel, E. M. Bolton, N. W. Gibson and D. Ross, Biochem. Pharmacol., 1996, 51, 645-652.

155. C.-K. Ryu, H.-J. Jeong, S. K. Lee, H.-J. You, K. U. Choi, J.-Y. Shim, Y. H. Heo and C.-O. Lee, Arch. Pharmacal Res., 2001, 24, 390.

156. H. A. Kontos and E. P. Wei, Stroke, 1993, 24, 427-434.

157. A. Mülsch, R. Busse, S. Liebau and U. Förstermann, J. Pharmacol. Exp. Ther., 1988, 247, $283-$ 288.

158. P. R. Gangadharam, P. F. Pratt, P. B. Damle, P. T. Davidson, T. H. Porter and K. Folkers, Am. Rev. Res. Dis., 1978, 118, 467-473.

159. P. Claes, D. Cappoen, C. Uythethofken, J. Jacobs, B. Mertens, V. Mathys, L. Verschaeve, K. Huygen and N. De Kimpe, Eur. J. Med. Chem., 2014, 77, 409-421.

160. B. R. Copp, H. C. Christiansen, B. S. Lindsay and S. G. Franzblau, Bioorg. Med. Chem. Lett., 2005, 15, 4097-4099.

161. X. Wei, W. Xu, J. Huang, L. Zhang, E. Walter, C. Lawrence, M. Vijayakumar, W. A. Henderson, T. Liu, L. Cosimbescu, B. Li, V. Sprenkle and W. Wang, Angew. Chem. Int. Ed., 2015, 54, 8684-8687. 
162. J. Huang, B. Pan, W. Duan, X. Wei, R. S. Assary, L. Su, F. R. Brushett, L. Cheng, C. Liao, M. S. Ferrandon, W. Wang, Z. Zhang, A. K. Burrell, L. A. Curtiss, I. A. Shkrob, J. S. Moore and L. Zhang, Sci. Rep., 2016, 6, 32102.

163. Y. Song and G. R. Buettner, Free Radical Biol. Med., 2010, 49, 919-962.

164. F. C. de Abreu, P. A. de L. Ferraz and M. O. F. Goulart, J. Braz. Chem. Soc., 2002, 13, 19-35.

165. S. Er, C. Suh, M. P. Marshak and A. Aspuru-Guzik, Chem. Sci., 2015, 6, 885-893.

166. S. Ergun, C. F. Elliott, A. P. Kaur, S. R. Parkin and S. A. Odom, Chem. Comm., 2014, 50, 53395341.

167. M.-H. Livertoux, P. Lagrange and A. Minn, Brain Res., 1996, 725, 207-216.

168. P. S. Guin, S. Das and P. C. Mandal, Int. J. Electrochem., 2011, DOI: 10.4061/2011/816202, 22.

169. M. G. Evans and J. De Heer, Q. Rev. Chem. Soc., 1950, 4, 94-114.

170. C. J. O'Connor, D. J. McLennan, B. M. Sutton, W. A. Denny and W. R. Wilson, J. Chem. Soc., 1991, DOI: 10.1039/P29910000951, 951-954.

171. P. Gopinath, A. Mahammed, S. Ohayon, Z. Gross and A. Brik, Chem. Sci., 2016, 7, 7079-7086.

172. S. Ohayon, M. Refua, A. Hendler, A. Aharoni and A. Brik, Angew. Chem. Int. Ed., 2015, 54, 599-603.

173. T. Yano, M. Rahimian, K. K. Aneja, N. M. Schechter, H. Rubin and C. P. Scott, Biochem., 2014, 53, 1179-1190.

174. H. Zimmer, D. C. Lankin and S. W. Horgan, Chem. Rev., 1971, 71, 229-246.

175. C. M. Keyari, A. K. Kearns, N. S. Duncan, E. A. Eickholt, G. Abbott, H. D. Beall and P. Diaz, J. Med. Chem., 2013, 56, 3806-3819.

176. K. Nepali, S. Kumar, H.-L. Huang, F.-C. Kuo, C.-H. Lee, C.-C. Kuo, T.-K. Yeh, Y.-H. Li, J.Y. Chang, J.-P. Liou and H.-Y. Lee, Org. Biomol. Chem., 2016, 14, 716-723.

177. R. H. J. Hargreaves, C. L. David, L. J. Whitesell, D. V. LaBarbera, A. Jamil, J. C. Chapuis and E. B. Skibo, J. Med. Chem., 2008, 51, 2492-2501.

178. Y. Wada, Y. Harayama, D. Kamimura, M. Yoshida, T. Shibata, K. Fujiwara, K. Morimoto, H. Fujioka and Y. Kita, Org. Biomol. Chem., 2011, 9, 4959-4976.

179. K. Majerz-Maniecka, R. Musiol, A. Skórska-Stania, D. Tabak, P. Mazur, B. J. Oleksyn and J. Polanski, Bioorg. Med. Chem., 2011, 19, 1606-1612.

180. K. Yoshida, M. Yamamoto and M. Ishiguro, Chem. Lett., 1986, 1059-1062.

181. A. Orzeł, R. Podsiadły, K. Podemska, R. Strzelczyk, J. Kolińska and J. Sokołowska, Color. Technol., 2014, 130, 185-190.

182. E. Y. Yoon, H. Y. Choi, K. J. Shin, K. H. Yoo, D. Y. Chi* and D. J. Kim, Tetrahedron Lett., 2000, 41, 7475-7480.

183. X. Xiao and B. Chen, Environ. Sci. Technol., 2017, 51, 5473-5482.

184. F. H. Allen, O. Kennard, D. G. Watson, L. Brammer, A. G. Orpen and R. Taylor, J. Chem. Soc,, 1987, DOI: 10.1039/P298700000S1, S1-S19.

185. M. Fatollahpour and H. Tahermansouri, C. R. Chim., 2017, 20, 942-951.

186. M. Kadela-Tomanek, M. Jastrzębska, E. Bębenek, E. Chrobak, M. T. Latocha, J. Kusz and D. Tarnawska, Crystals, 2017.

187. M. Jastrzebska, S. Boryczka, M. Kadela, R. Wrzalik, J. Kusz and M. Nowak, J. Mol. Struct., 2014, 1067, 160-168.

188. S. S. Jhamb, A. Goyal and P. P. Singh, Bra\%. J. Infect. Dis., 2014, 18, 336-340.

189. S. S. Lin, S. Kerscher, A. Saleh, U. Brandt, U. Groß and W. Bohne, Biochim. Biophys. Acta, 2008, 1777, 1455-1462.

190. L. Jeftić and G. Manning, J. Electroanal. Chem. Interfacial Electrochem., 1970, 26, 195-200.

191. M. W. Lehmann and D. H. Evans, J. Electroanal. Chem., 2001, 500, 12-20.

192. S. G. Bratsch, J. Chem. Educ., 1985, 62, 101.

193. J. Mullay, J. Am. Chem. Soc., 1985, 107, 7271-7275.

194. D. W. Smith, J. Chem. Soc., Faraday Trans., 1998, 94, 201-205.

195. 2018.

196. S. Kishi, K. Saito, Y. Kato and H. Ishikita, Photosynthesis Res., 2017, 134, 193-200.

197. E. Bębenek, E. Chrobak, J. Wietrzyk, M. Kadela, A. Chrobak, J. Kusz, M. Ksiazżek, M. Jastrzębska and S. Boryczka, J. Mol. Struct., 2016, 1106, 210-219.

198. W. Mól, M. Matyja, B. Filip, J. Wietrzyk and S. Boryczka, Bioorg. Med. Chem., 2008, 16, 81368141. 
199. S. J. Senior, P. A. Illarionov, S. S. Gurcha, I. B. Campbell, M. L. Schaeffer, D. E. Minnikin and G. S. Besra, Bioorg. Med. Chem. Lett., 2004, 14, 373-376.

200. M. Kögler, B. Vanderhoydonck, S. De Jonghe, J. Rozenski, K. Van Belle, J. Herman, T. Louat, A. Parchina, C. Sibley, E. Lescrinier and P. Herdewijn, J. Med. Chem., 2011, 54, 4847-4862.

201. R. Ekkebus, S. I. van Kasteren, Y. Kulathu, A. Scholten, I. Berlin, P. P. Geurink, A. de Jong, S. Goerdayal, J. Neefjes, A. J. R. Heck, D. Komander and H. Ovaa, J. Am. Chem. Soc., 2013, 135, 2867-2870.

202. F. Brotzel and H. Mayr, Org. Biomol. Chem., 2007, 5, 3814-3820.

203. D. Tull, S. G. Withers, N. R. Gilkes, D. G. Kilburn, R. A. Warren and R. Aebersold, J. Biol. Chem., 1991, 266, 15621-15625.

204. S. Miao, L. Ziser, R. Aebersold and S. G. Withers, Biochem., 1994, 33, 7027-7032.

205. A. Heikal, Y. Nakatani, W. Jiao, C. Wilson, D. Rennison, M. R. Weimar, E. J. Parker, M. A. Brimble and G. M. Cook, Bioorg. Med. Chem. Lett., 2018, 28, 2239-2243.

206. I. Smith, Clin. Microbiol. Rev., 2003, 16, 463-496.

207. A. Gaspar, M. J. Matos, J. Garrido, E. Uriarte and F. Borges, Chem. Rev., 2014, 114, 4960-4992.

208. J. R. Mays, S. A. Hill, J. T. Moyers and B. S. J. Blagg, Bioorg. Med. Chem., 2010, 18, 249-266.

209. W. Huang, M.-Z. Liu, Y. Li, Y. Tan and G.-F. Yang, Bioorg. Med. Chem., 2007, 15, 5191-5197.

210. M. Isaka, M. Sappan, P. Auncharoen and P. Srikitikulchai, Phytochem. Lett., 2010, 3, 152-155.

211. D. H. Nam, K. Y. Lee, C. S. Moon and Y. S. Lee, Eur. J. Med. Chem., 2010, 45, 4288-4292.

212. P. Singh, M. Kaur and W. Holzer, Eur. J. Med. Chem., 2010, 45, 4968-4982.

213. S. Zhang, J. Ma, Y. Bao, P. Yang, L. Zou, K. Li and X. Sun, Bioorg. Med. Chem., 2008, 16, $7127-$ 7132.

214. A. Gaspar, J. Reis, A. Fonseca, N. Milhazes, D. Viña, E. Uriarte and F. Borges, Bioorg. Med. Chem. Lett., 2011, 21, 707-709.

215. C. A. V., G. D. S., S. S. Bhagat and G. C. H., J. Heterocyclic Chem., 2013, 50, 149-154.

216. P. Cano, A. Islas-Jácome, Á. Rangel-Serrano, F. Anaya-Velázquez, F. Padilla-Vaca, E. TrujilloEsquivel, P. Ponce-Noyola, A. Martínez-Richa and R. Gámez-Montaño, Molecules, 2015, 20, 12436.

217. L. Feng, M. M. Maddox, M. Z. Alam, L. S. Tsutsumi, G. Narula, D. F. Bruhn, X. Wu, S. Sandhaus, R. B. Lee, C. J. Simmons, Y.-C. Tse-Dinh, J. G. Hurdle, R. E. Lee and D. Sun, J. Med. Chem., 2014, 57, 8398-8420.

218. D. R. Ferry, A. Smith, J. Malkhandi, D. W. Fyfe, P. G. deTakats, D. Anderson, J. Baker and D. J. Kerr, Clin. Cancer. Res., 1996, 2, 659-668.

219. R. B. Weiss, R. F. Greene, R. D. Knight, J. M. Collins, J. J. Pelosi, A. Sulkes and G. A. Curt, Cancer Res., 1988, 48, 5878-5882.

220. A. M. Senderowicz, Inves. New Drugs, 1999, 17, 313-320.

221. S. Dianqing, H. J. G., L. Robin, L. Richard, C. Mark and P. J. M., ChemMedChem, 2012, 7, 15411545.

222. D. D. Haveliwala, N. R. Kamdar, P. T. Mistry and S. K. Patel, J. Sulfur Chem., 2011, 32, 451462.

223. D. Sriram, P. Yogeeswari, M. Dinakaran, D. Banerjee, P. Bhat and S. Gadhwal, Eur. J. Med. Chem., 2010, 45, 120-123.

224. D. E. Stevenson and R. D. Hurst, Cell. Mol. Life Sci., 2007, 64, 2900-2916.

225. C. Proença, H. M. T. Albuquerque, D. Ribeiro, M. Freitas, C. M. M. Santos, A. M. S. Silva and E. Fernandes, Eur. J. Med. Chem., 2016, 115, 381-392.

226. S. de Vries and L. A. Grivell, Eur. J. Biochem., 1988, 176, 377-384.

227. A. M. O. Amoussa, M. Bourjot, L. Lagnika, C. Vonthron-Sénécheau and A. Sanni, BMC Complement. Altern. Med., 2016, 16, 506.

228. K. M. Hatzade, V. S. Taile, P. K. Gaidhane, V. D. Umare, A. G. M. Haldar and V. N. Ingle, Indian J. Chem., 2009, 48B, 1548-1557.

229. H. Liu, C. Jiang, C. Xiong and J. Ruan, Toxicol. In Vitro, 2012, 26, 16-23.

230. Y. M. Ahn, L. Vogeti, C.-J. Liu, H. K. R. Santhapuram, J. M. White, V. Vasandani, L. A. Mitscher, G. H. Lushington, P. R. Hanson, D. R. Powell, R. H. Himes, K. F. Roby, Q. Ye and G. I. Georg, Bioorg. Med. Chem., 2007, 15, 702-713.

231. J. Xi, X. Zhu, Y. Feng, N. Huang, G. Luo, Y. Mao, X. Han, W. Tian, G. Wang, X. Han, R. Luo, Z. Huang and J. An, Mol. Cancer Res., 2013, 11, 856-864. 
232. V. J. Watts, Neuropsychopharmacology, 2017, 43, 215.

233. L. Huang, X. Yuan, D. Yu, K. H. Lee and C. Ho Chen, Virology, 2005, 332, 623-628.

234. C. F. M. Silva, D. C. G. A. Pinto and A. M. S. Silva, ChemMedChem, 2016, 11, 2252-2260.

235. R. S. Keri, S. Budagumpi, R. K. Pai and R. G. Balakrishna, Eur. J. Med. Chem., 2014, 78, 340374.

236. K. Pia, N. Olivia, K. Olga, S. Gregor and W. S. R., Eur. J. Org. Chem., 2010, DOI: doi:10.1002/ejoc.201000957, 6417-6422.

237. W. Baker, J. Chem. Soc., 1933, DOI: 10.1039/JR9330001381, 1381-1389.

238. L. Costantino, G. Rastelli, M. C. Gamberini, J. A. Vinson, P. Bose, A. Iannone, M. Staffieri, L. Antolini, A. Del Corso, U. Mura and A. Albasini, J. Med. Chem., 1999, 42, 1881-1893.

239. A. Gomes, O. Neuwirth, M. Freitas, D. Couto, D. Ribeiro, A. G. P. R. Figueiredo, A. M. S. Silva, R. S. G. R. Seixas, D. C. G. A. Pinto, A. C. Tomé, J. A. S. Cavaleiro, E. Fernandes and J. L. F. C. Lima, Bioorg. Med. Chem., 2009, 17, 7218-7226.

240. A. Y. Shaw, C.-Y. Chang, H.-H. Liau, P.-J. Lu, H.-L. Chen, C.-N. Yang and H.-Y. Li, Eur. J. Med. Chem., 2009, 44, 2552-2562.

241. I. Goodall and A. Robertson, J. Chem. Soc., 1936, 426-428.

242. A. Robertson, Nature, 1931, 128, 908.

243. M. Hadjeri, M. Barbier, X. Ronot, A.-M. Mariotte, A. Boumendjel and J. Boutonnat, J. Med. Chem., 2003, 46, 2125-2131.

244. R. A. Appleton, J. R. Bantick, T. R. Chamberlain, D. N. Hardern, T. B. Lee and A. D. Pratt, J. Med. Chem., 1977, 20, 371-379.

245. P. Kumar and M. S. Bodas, Org. Lett., 2000, 2, 3821-3823.

246. L. Yet, Privileged structures in drug discovery: Medicinal chemistry and synthesis, John Wiley \& Sons, 2018.

247. E. Vitaku, D. T. Smith and J. T. Njardarson, J. Med. Chem., 2014, 57, 10257-10274.

248. P. A. Harris, A. Boloor, M. Cheung, R. Kumar, R. M. Crosby, R. G. Davis-Ward, A. H. Epperly, K. W. Hinkle, R. N. Hunter, J. H. Johnson, V. B. Knick, C. P. Laudeman, D. K. Luttrell, R. A. Mook, R. T. Nolte, S. K. Rudolph, J. R. Szewczyk, A. T. Truesdale, J. M. Veal, L. Wang and J. A. Stafford, J. Med. Chem., 2008, 51, 4632-4640.

249. B. J. Druker, M. Talpaz, D. J. Resta, B. Peng, E. Buchdunger, J. M. Ford, N. B. Lydon, H. Kantarjian, R. Capdeville, S. Ohno-Jones and C. L. Sawyers, New Engl. J. Med., 2001, 344, 1031 1037.

250. G. L. Plosker and D. M. Robinson, Drugs, 2008, 68, 449-459.

251. K. M. Amin, F. M. Awadalla, A. A. M. Eissa, S. M. Abou-Seri and G. S. Hassan, Bioorg. Med. Chem., 2011, 19, 6087-6097.

252. N. A. Powell, J. K. Hoffman, F. L. Ciske, J. T. Kohrt, S. M. Baxi, Y.-W. Peng, M. Zhong, C. Catana, J. Ohren, L. A. Perrin and J. J. Edmunds, Bioorg. Med. Chem. Lett., 2013, 23, 1051-1055.

253. S. Yang, C. Pannecouque, D. Daelemans, X.-D. Ma, Y. Liu, F.-E. Chen and E. De Clercq, Eur. J. Med. Chem., 2013, 65, 134-143.

254. Q. Choo, G. Kuo, A. Weiner, L. Overby, D. Bradley and M. Houghton, Science, 1989, 244, 359362 .

255. A. Arasappan, F. Bennett, V. Girijavallabhan, Y. Huang, R. Huelgas, C. Alvarez, L. Chen, S. Gavalas, S.-H. Kim, A. Kosinski, P. Pinto, R. Rizvi, R. Rossman, B. Shankar, L. Tong, F. Velazquez, S. Venkatraman, V. A. Verma, J. Kozlowski, N.-Y. Shih, J. J. Piwinski, M. MacCoss, C. D. Kwong, J. L. Clark, A. T. Fowler, F. Geng, H. S. Kezar, A. Roychowdhury, R. C. Reynolds, J. A. Maddry, S. Ananthan, J. A. Secrist, C. Li, R. Chase, S. Curry, H.-C. Huang, X. Tong and F. G. Nioroge, Bioorg. Med. Chem. Lett., 2012, 22, 3229-3234.

256. N. Ingarsal, G. Saravanan, P. Amutha and S. Nagarajan, Eur. J. Med. Chem., 2007, 42, 517-520.

257. J. Morgan, R. Haritakul and P. A. Keller, Bioorg. Med. Chem. Lett., 2003, 13, 1755-1757.

258. A. R. Trivedi, D. K. Dodiya, N. R. Ravat and V. H. Shah, Arch. Org. Chem., 2008, 131-141.

259. E. V. Verbitskiy, E. M. Cheprakova, P. A. Slepukhin, M. A. Kravchenko, S. N. Skornyakov, G. L. Rusinov, O. N. Chupakhin and V. N. Charushin, Eur. J. Med. Chem., 2015, 97, 225-234.

$260 . \quad$ H. Sohn, K.-S. Lee, Y.-K. Ko, J.-W. Ryu, J.-C. Woo, D.-W. Koo, S.-J. Shin, S.-J. Ahn, A. R. Shin, C.-H. Song, E.-K. Jo, J.-K. Park and H.-J. Kim, Int. J. Antimicrob. Agents, 2008, 31, $567-$ 571. 
261. M. L. Read, M. Brændvang, P. O. Miranda and L.-L. Gundersen, Bioorg. Med. Chem., 2010, 18, 3885-3897.

262. V. Virsodia, R. R. S. Pissurlenkar, D. Manvar, C. Dholakia, P. Adlakha, A. Shah and E. C. Coutinho, Eur. J. Med. Chem., 2008, 43, 2103-2115.

263. A. A. Siddiqui, R. Rajesh, M. Ul-Islam, V. Alagarsamy and E. de Clercq, Arch. pharm., 2007, 340, 95-102.

264. N. C. Srivastav, D. Rai, C. Tse, B. Agrawal, D. Y. Kunimoto and R. Kumar, J. Med. Chem., 2010, 53, 6180-6187.

265. M. Johar, T. Manning, C. Tse, N. Desroches, B. Agrawal, D. Y. Kunimoto and R. Kumar, J. Med. Chem., 2007, 50, 3696-3705.

266. N. C. Srivastav, T. Manning, D. Y. Kunimoto and R. Kumar, Bioorg. Med. Chem., 2007, 15, 2045-2053.

267. A. L. B. Gerum, J. E. Ulmer, D. P. Jacobus, N. P. Jensen, D. R. Sherman and C. H. Sibley, Antimicrob. Agents Chemother., 2002, 46, 3362-3369.

268. A. M. Dhople, Int. J. Antimicrob. Agents, 2002, 19, 71-74.

269. W. J. Suling and J. A. Maddry, J. Antimicrob. Chemother., 2001, 47, 451-454.

270. A. K. Debnath, J. Med. Chem., 2002, 45, 41-53.

271. A. A. Joshi, S. S. Narkhede and C. L. Viswanathan, Bioorg. Med. Chem. Lett., 2005, 15, 73-76.

272. K. M. Khan, S. Iqbal, M. A. Bashir, N. Ambreen, S. Perveen and W. Voelter, Tetrabedron Lett., 2015, 56, 1179-1182.

273. P. R. Patel, C. Ramalingan and Y.-T. Park, Bioorg. Med. Chem. Lett., 2007, 17, 6610-6614.

274. P. G. Baraldi, A. Bovero, F. Fruttarolo, R. Romagnoli, M. A. Tabrizi, D. Preti, K. Varani, P. A. Borea and A. R. Moorman, Bioorg. Med. Chem., 2003, 11, 4161-4169.

275. R. Goldschmidt, P. M. Arce, O. M. Khdour, V. C. Collin, S. Dey, J. Jaruvangsanti, D. M. Fash and S. M. Hecht, Bioorg. Med. Chem., 2013, 21, 969-978.

276. H. Chen, J. Bai, L. Jiao, Z. Guo, Q. Yin and X. Li, Bioorg. Med. Chem., 2009, 17, 3980-3986.

277. R. K. Rawal, R. Tripathi, S. B. Katti, C. Pannecouque and E. De Clercq, Bioorg. Med. Chem., 2007, 15, 3134-3142.

278. A. S. Komendantova, A. V. Komkov, Y. A. Volkova and I. V. Zavarzin, Eur. J. Org. Chem., 2017, DOI: doi:10.1002/ejoc.201700737, 4260-4264.

279. A. V. Erkin, V. V. Gurzhii and V. I. Krutikov, Russ. J. Gen. Chem., 2015, 85, 79-87.

280. F. Wakita, Y. Ando, K. Ohmori and K. Suzuki, Org. Lett., 2018, 20, 3928-3932.

281. X.-Y. Meng, H.-X. Zhang, M. Mezei and M. Cui, Curr. Comput.-Aided Drug Des., 2011, 7, 146157.

282. E. F. Pettersen, T. D. Goddard, C. C. Huang, G. S. Couch, D. M. Greenblatt, E. C. Meng and T. E. Ferrin, J. Comput. Chem., 2004, 25, 1605-1612.

283. W. J. Allen, T. E. Balius, S. Mukherjee, S. R. Brozell, D. T. Moustakas, P. T. Lang, D. A. Case, I. D. Kuntz and R. C. Rizzo, J. Comput. Chem., 2015, 36, 1132-1156.

284. A. V. Erkin and V. I. Krutikov, Russ. J. Gen. Chem., 2011, 81, 1699.

285. B. M. Savall, S. P. Meduna, J. Venable, J. Wei, R. C. Smith, M. D. Hack, R. L. Thurmond, P. McGovern and J. P. Edwards, Bioorg. Med. Chem. Lett., 2014, 24, 5489-5492.

286. W. A. Velema, M. van der Toorn, W. Szymanski and B. L. Feringa, J. Med. Chem., 2013, 56, 4456-4464.

287. Z. Dongbing, B. Bernhard and G. Frank, Angew. Chem. Int. Ed., 2013, 52, 8454-8458.

288. J. Rocha-Pereira, R. Cunha, D. C. G. A. Pinto, A. M. S. Silva and M. S. J. Nascimento, Bioorg. Med. Chem., 2010, 18, 4195-4201.

289. E. Venkateswararao, M. Manickam, P. Boggu, Y. Kim and S.-H. Jung, Bioorg. Med. Chem., 2015, 23, 2498-2504.

290. P. Pérez, L. R. Domingo, M. Duque-Noreña and E. Chamorro, J. Mol. Struct., 2009, 895, 8691.

291. M. Cárdenas, M. Marder, V. C. Blank and L. P. Roguin, Bioorg. Med. Chem., 2006, 14, 29662971.

292. A. O. Fitton and B. T. Hatton, J. Chem. Soc. C: Org., 1970, DOI: 10.1039/J39700002518, 25182522.

293. A. Patel, W. Lewis, M. S. Searle, M. F. G. Stevens and C. J. Moody, Tetrahedron, 2015, 71, 73397343. 
294. V. Kolman, F. Kalčic, P. Jansa, Z. Zídek and Z. Janeba, Eur. J. Med. Chem., 2018, 156, 295-301.

295. N. Thasana and S. Ruchirawat, Tetrahedron Lett., 2002, 43, 4515-4517.

296. P. Kahnberg, E. Lager, C. Rosenberg, J. Schougaard, L. Camet, O. Sterner, E. Ø. Nielsen, M. Nielsen and T. Liljefors, J. Med. Chem., 2002, 45, 4188-4201.

297. S. S. Roy, P. Chakraborty and S. Bhattacharya, Eur. J. Med. Chem., 2014, 73, 195-209.

298. U. Uciechowska-Kaczmarzyk, I. Chauvot de Beauchene and S. A. Samsonov, J. Mol. Graphics Model., 2019, 90, 42-50.

299. B. Zhu, Z. Feng, Z. Zheng and X. Wang, J. Appl. Polym. Sci., 2012, 123, 1755-1763.

300. K. Radhakrishnan, N. Sharma and L. M. Kundu, RSC Adv., 2014, 4, 15087-15090.

301. P. Ghosh, A. K. Nandi and S. Das, Tetrahedron Lett., 2018, 59, 2025-2029.

302. R. A. Joshi, M. K. Gurjar, N. K. Tripathy and M. S. Chorghade, Org. Process Res. Dev., 2001, 5, 176-178.

303. V. K. Rai, F. Verma, G. P. Sahu, M. Singh and A. Rai, Eur. J. Org. Chem., 2018, DOI: doi:10.1002/ejoc.201701435, 537-544.

304. G. Casano, M. Robin, P. Barbier, V. Peyrot and R. Faure, Magn. Reson. Chem., 2010, 48, 738744.

305. H. Terada, Environ. Health Perspect., 1990, 87, 213-218.

306. V. P. Skulachev, A. A. Sharaf and E. A. Liberman, Nature, 1967, 216, 718.

307. T. Ubbink-Kok, J. A. Anderson and W. N. Konings, Antimicrob. Agents Chemother, 1986, 30, 147-151.

308. L. A. Schurig-Briccio, T. Yano, H. Rubin and R. B. Gennis, Biochim. Biophys. Acta, 2014, 1837, 954-963.

309. P. Skrobecki, A. Chmielińska, P. Bonarek, P. Stepien, A. Wisniewska-Becker, M. DziedzickaWasylewska and A. Polit, ACS Chem. Neurosci., 2017, 8, 1543-1553.

310. U. Domańska, A. Pelczarska and A. Pobudkowska, Int. J. Pharm., 2011, 421, 135-144.

311. J. S. Modica-Napolitano, C. J. Lagace, W. A. Brennan and J. R. Aprille, Arch. Pharmacal Res., 2003, 26, 951-959.

312. X. Feng, W. Zhu, L. A. Schurig-Briccio, S. Lindert, C. Shoen, R. Hitchings, J. Li, Y. Wang, N. Baig, T. Zhou, B. K. Kim, D. C. Crick, M. Cynamon, J. A. McCammon, R. B. Gennis and E. Oldfield, Proc. Natl. Acad. Sci. U.S.A., 2015, 112, E7073-E7082.

313. M. Kurosu, P. Narayanasamy, K. Biswas, R. Dhiman and D. C. Crick, J. Med. Chem., 2007, 50, 3973-3975.

314. K. Li, L. A. Schurig-Briccio, X. Feng, A. Upadhyay, V. Pujari, B. Lechartier, F. L. Fontes, H. Yang, G. Rao, W. Zhu, A. Gulati, J. H. No, G. Cintra, S. Bogue, Y.-L. Liu, K. Molohon, P. Orlean, D. A. Mitchell, L. Freitas-Junior, F. Ren, H. Sun, T. Jiang, Y. Li, R.-T. Guo, S. T. Cole, R. B. Gennis, D. C. Crick and E. Oldfield, J. Med. Chem., 2014, 57, 3126-3139.

315. S. S. Grant, B. B. Kaufmann, N. S. Chand, N. Haseley and D. T. Hung, Proc. Natl. Acad. Sci. U.S.A., 2012, 109, 12147-12152.

316. W. Li, A. Sanchez-Hidalgo, V. Jones, V. C. N. de Moura, E. J. North and M. Jackson, Antimicrob. Agents Chemother., 2017, 61, e02399-02316.

317. T. V. A. Nguyen, R. M. Anthony, A.-L. Bañuls, T. V. A. Nguyen, D. H. Vu and J.-W. C. Alffenaar, Clin. Infect. Dis., 2018, 66, 1625-1630.

318. K. A. Sacksteder, M. Protopopova, C. E. Barry, K. Andries and C. A. Nacy, Future Microbiology, 2012, 7, 823-837.

319. M. I. Marzouk, S. A. Shaker, A. A. Abdel Hafiz and K. Z. El-Baghdady, Biol. Pharm. Bull., 2016, 39, 239-251.

320. L. Yang, W. Wang, Q. Sun, F. Xu, Y. Niu, C. Wang, L. Liang and P. Xu, Bioorg. Med. Chem. Lett., 2016, 26, 2801-2805.

321. A. D. Hameed, S. Ovais, R. Yaseen, P. Rathore, M. Samim, S. Singh, K. Sharma, M. Akhtar and K. Javed, Arch. pharm., 2016, 349, 150-159.

322. P. A. Procopiou, A. J. Ford, P. M. Gore, B. E. Looker, S. T. Hodgson, D. S. Holmes, S. Vile, K. L. Clark, K. A. Saunders, R. J. Slack, J. E. Rowedder and C. J. Watts, ACS Med. Chem. Lett., 2017, 8, 577-581.

323. P. A. Procopiou, C. Browning, P. M. Gore, S. M. Lynn, S. A. Richards, R. J. Slack and S. L. Sollis, Bioorg. Med. Chem., 2012, 20, 6097-6108. 
324. P. A. Procopiou, C. Browning, J. M. Buckley, K. L. Clark, L. Fechner, P. M. Gore, A. P. Hancock, S. T. Hodgson, D. S. Holmes, M. Kranz, B. E. Looker, K. M. L. Morriss, D. L. Parton, L. J. Russell, R. J. Slack, S. L. Sollis, S. Vile and C. J. Watts, J. Med. Chem., 2011, 54, 2183-2195.

325. T. Önkol, D. S. Doğruer, L. Uzun, S. Adak, S. Özkan and M. Fethi Şahin, J. Enžyme Inhib. Med. Chem., 2008, 23, 277-284.

326. N. Vila, P. Besada, D. Viña, M. Sturlese, S. Moro and C. Terán, RSC Adv., 2016, 6, 4617046185.

327. S. K. Gorla, Y. Zhang, M. M. Rabideau, A. Qin, S. Chacko, A. L. House, C. R. Johnson, K. Mandapati, H. M. Bernstein, E. S. McKenney, H. Boshoff, M. Zhang, I. J. Glomski, J. B. Goldberg, G. D. Cuny, B. J. Mann and L. Hedstrom, Antimicrob. Agents Chemother., 2017, 61.

328. A. M. Sridhara, K. R. Venugopala Reddy, J. Keshavayya, P. S. Kumar Goud, B. C. Somashekar, P. Bose, S. K. Peethambar and S. K. Gaddam, Eur. J. Med. Chem., 2010, 45, 4983-4989.

329. C. C. Gunderson and K. N. Moore, Future Oncology, 2015, 11, 747-757.

330. N. Chand and R. D. Sofia, J. Asthma, 1995, 32, 227-234.

331. T. Tomita, I. Yonekura, Y. Shirasaki, E. Hayashi and F. Numano, Jpn. J. Pharmacol., 1980, 30, 65-73.

332. A. Melikian, G. Schlewer, J. P. Chambon and C. G. Wermuth, J. Med. Chem., 1992, 35, 40924097.

333. S. Nath, Biophys. Chem., 2018, 242, 15-21.

334. U. Domańska, A. Pobudkowska and A. Pelczarska, J. Phys. Chem. B, 2011, 115, 2547-2554.

335. ChemAxon, Chemicalize).

336. Tokyo Pat., EP0580074B1, 1995.

337. X. Qiang, Y. Li, X. Yang, L. Luo, R. Xu, Y. Zheng, Z. Cao, Z. Tan and Y. Deng, Bioorg. Med. Chem. Lett., 2017, 27, 718-722.

338. C. Walling, A. L. Rieger and D. D. Tanner, J. Am. Chem. Soc., 1963, 85, 3129-3134.

339. D. Sroczyński and Z. Malinowski, J. Mol. Struct., 2017, 1150, 614-628.

340. H. Gökce and S. Bahçeli, J. Mol. Struct., 2010, 967, 42-46.

341. V. M. Outerbridge, S. M. Landge, H. Tamaki and B. Török, Synthesis, 2009, 2009, 1801-1806.

342. H. Wang, J. Cai, H. Huang and G.-J. Deng, Org. Lett., 2014, 16, 5324-5327.

343. X.-F. Wu, H. Neumann, S. Neumann and M. Beller, Chem. Eur. J., 2012, 18, 8596-8599.

344. D. E. Beck, K. Agama, C. Marchand, A. Chergui, Y. Pommier and M. Cushman, J. Med. Chem., 2014, 57, 1495-1512.

345. A. Sugimoto, K. Sakamoto, Y. Fujino, Y. Takashima and M. Ishikawa, Chem. Pharm. Bull. (Tokyo), 1985, 33, 2809-2820.

346. M. Schenone, V. Dančík, B. K. Wagner and P. A. Clemons, Nat. Chem. Biol., 2013, 9, 232-240.

347. S. A. Wacker, B. R. Houghtaling, O. Elemento and T. M. Kapoor, Nat. Chem. Biol., 2012, 8, 235-237.

348. G. Corti, R. Cinelli and F. Paradisi, Int. J. Antimicrob. Agents, 2000, 16, 527-530.

349. G. E. Zurenko, B. H. Yagi, R. D. Schaadt, J. W. Allison, J. O. Kilburn, S. E. Glickman, D. K. Hutchinson, M. R. Barbachyn and S. J. Brickner, Antimicrob. Agents Chemother., 1996, 40, 839845.

350. S. Wasserman, G. Meintjes and G. Maartens, Expert Rev. Anti-infect. Ther., 2016, 14, 901-915.

351. L. Alcalá, M. J. Ruiz-Serrano, C. Pérez-Fernández Turégano, D. García de Viedma, M. DíazInfantes, M. Marín-Arriaza and E. Bouza, Antimicrob. Agents Chemother., 2003, 47, 416-417.

352. R. Cremades, J. C. Rodriguez, E. Garcia-Pachon, A. Galiana, M. Ruiz-Garcia, P. Lopez and G. Royo, APMIS, 2011, 119, 304-308.

353. X. Zhang, M. E. Falagas, K. Z. Vardakas, R. Wang, R. Qin, J. Wang and Y. Liu, J. Thoracic Dis., 2015, 7, 603-615. 


\section{APPENDIX: CHAPTER TWO}

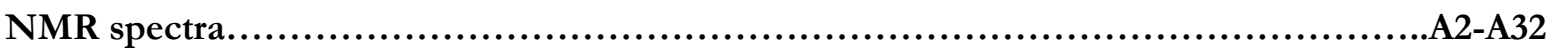




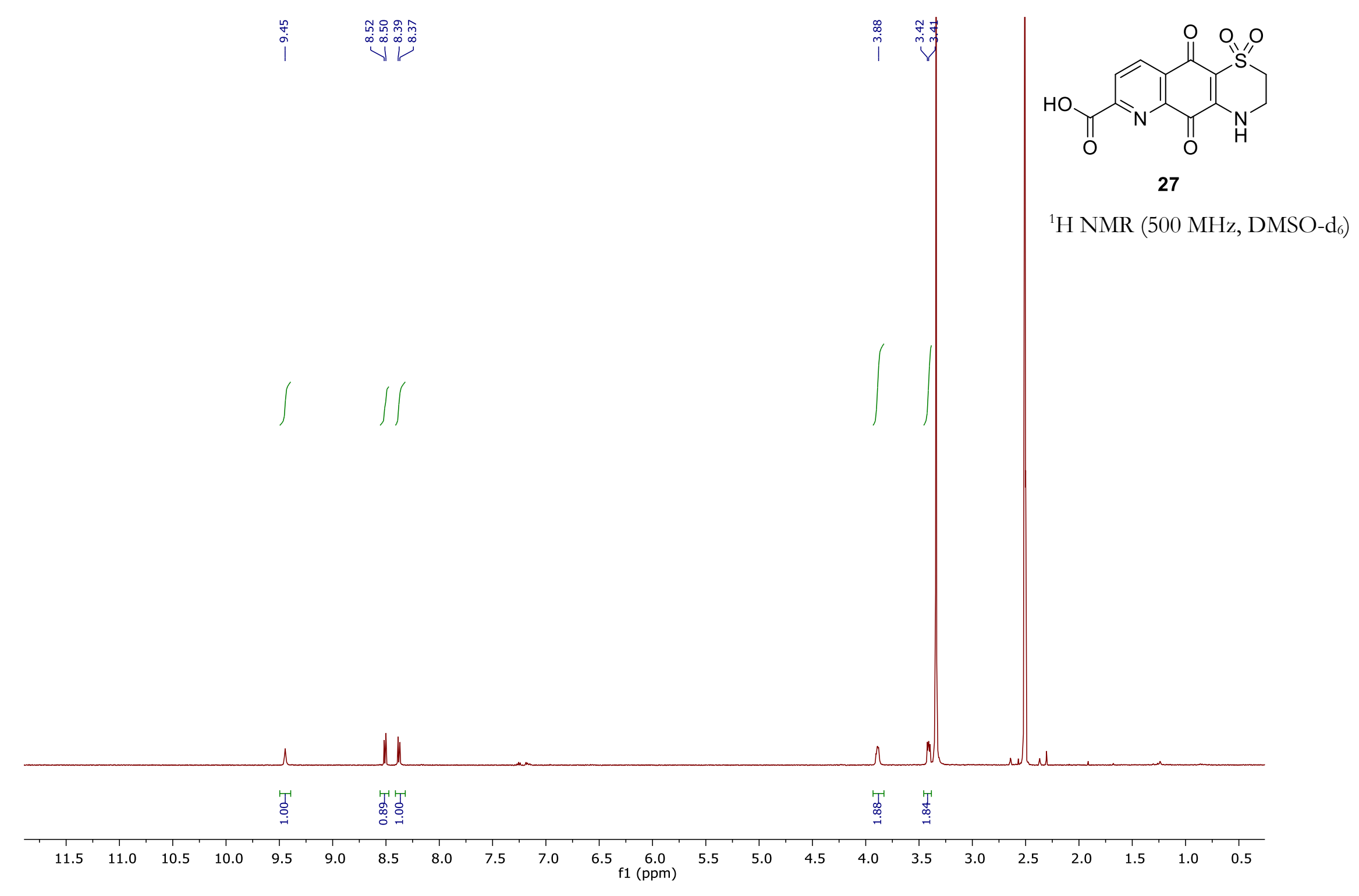




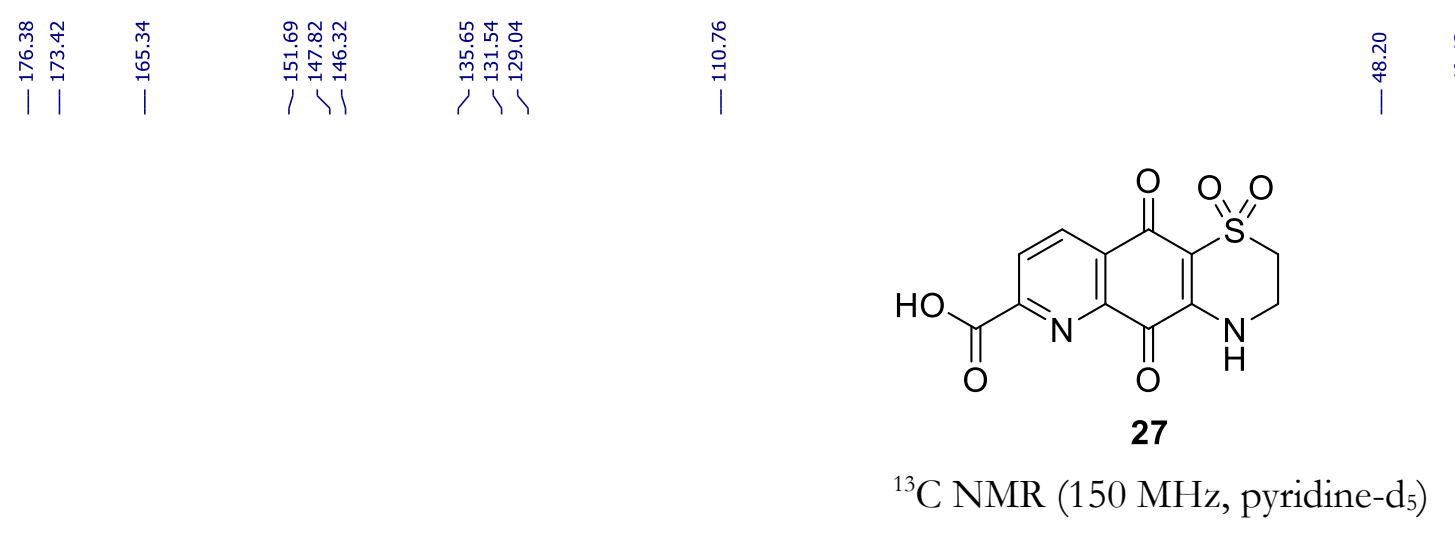

${ }^{13} \mathrm{C}$ NMR (150 MHz, pyridine-d $\left.\mathrm{d}_{5}\right)$
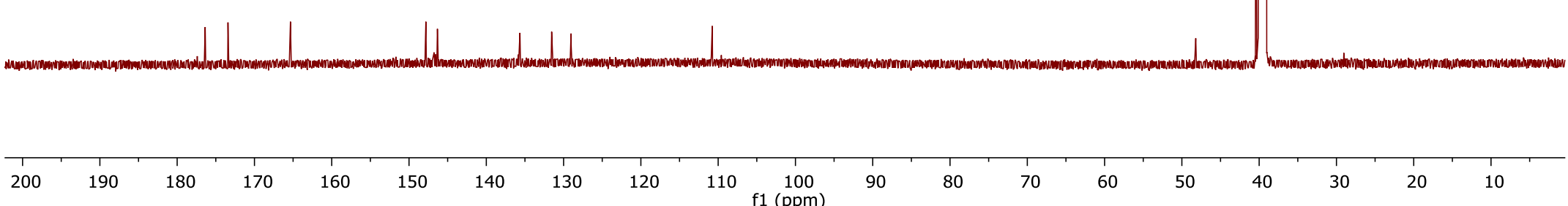


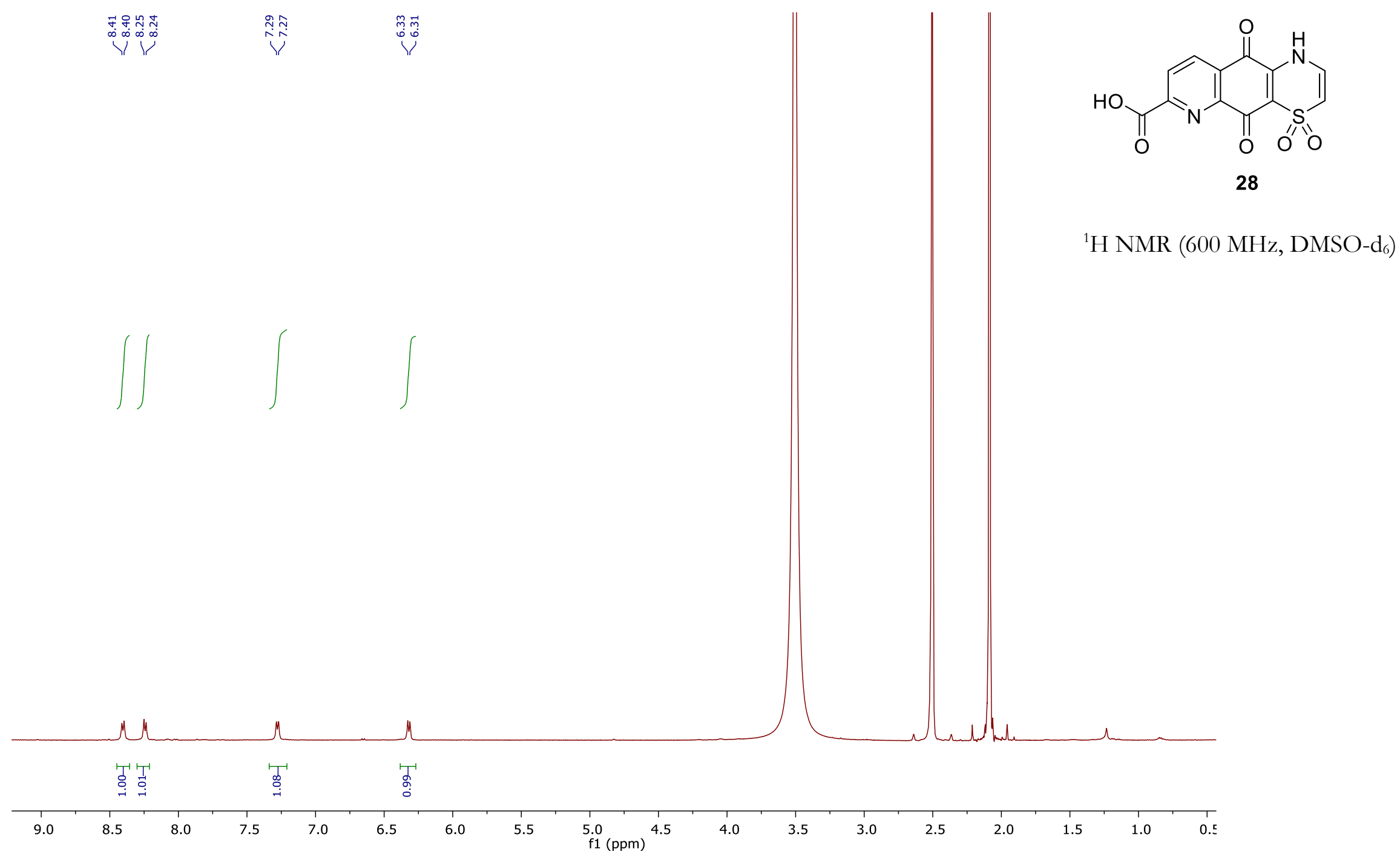




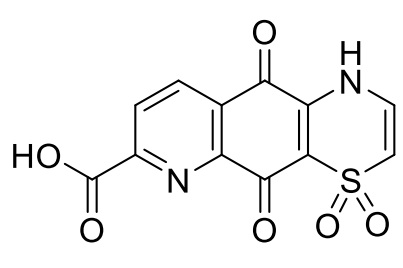

28

${ }^{13} \mathrm{C}$ NMR $\left(150 \mathrm{MHz}, \mathrm{DMSO}-\mathrm{d}_{6}\right)$

andminmmanum

$\begin{array}{lllllllllllllllllllllllll}230 & 220 & 210 & 200 & 190 & 180 & 170 & 160 & 150 & 140 & 130 & 120 & 110 & 100 & 90 & 80 & 70 & 60 & 50 & 40 & 30 & 20 & 10 & 0 & -10\end{array}$




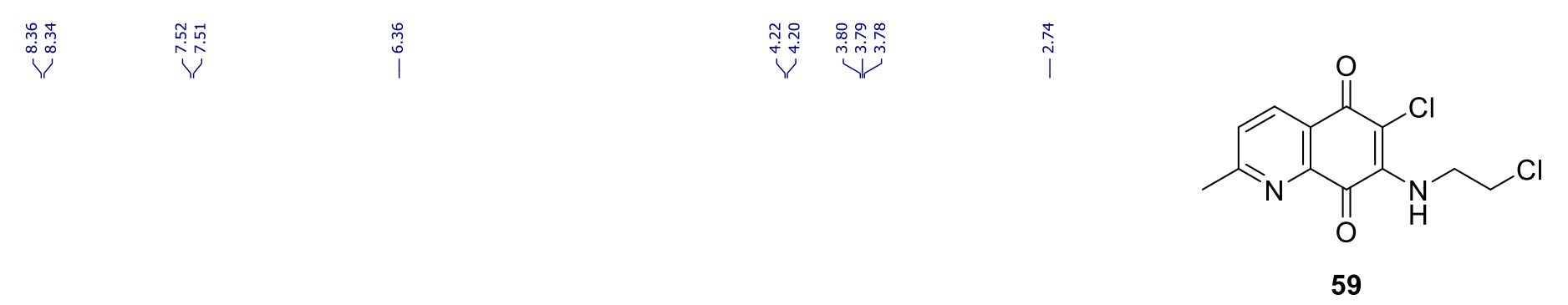

${ }^{1} \mathrm{H}$ NMR (500 MHz, $\mathrm{CDCl}_{3}$ )

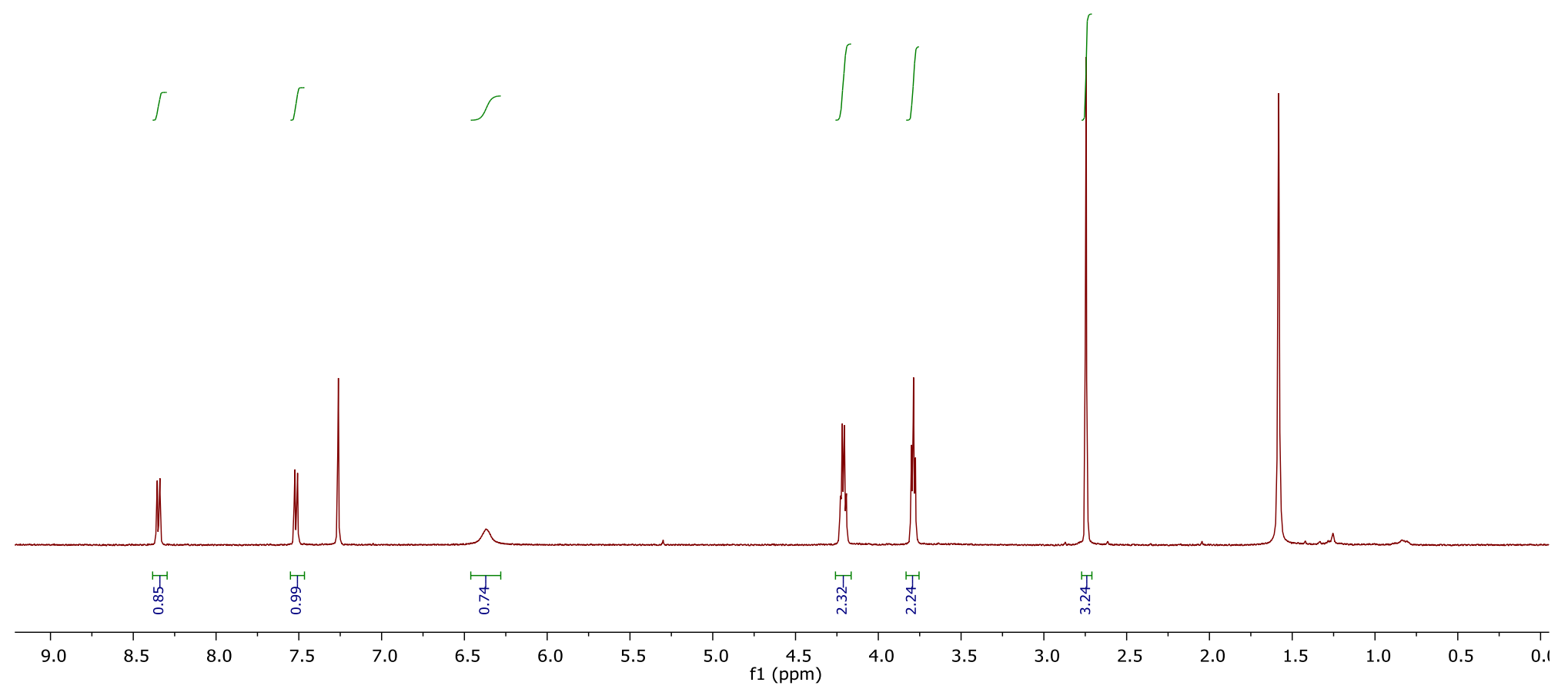




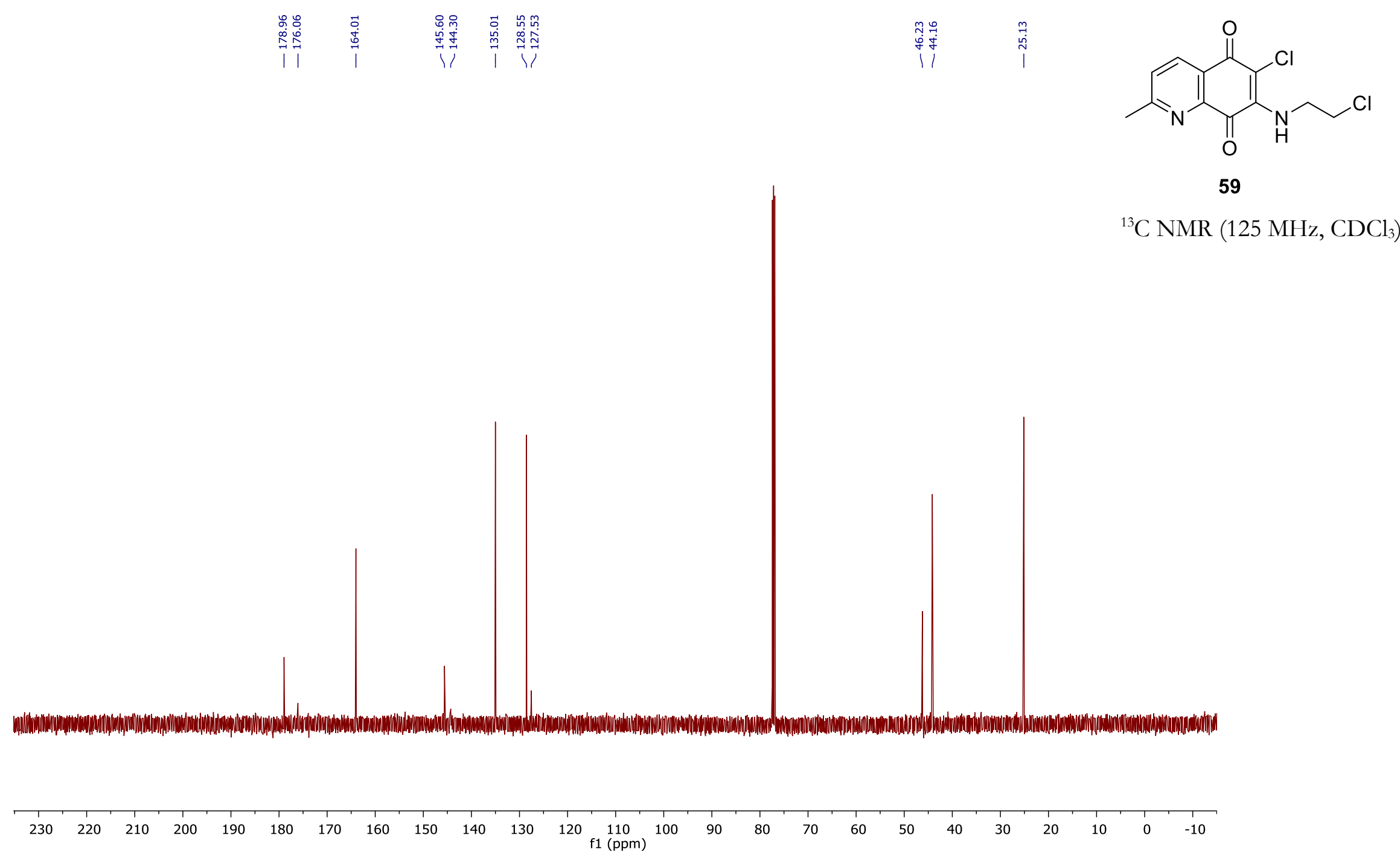




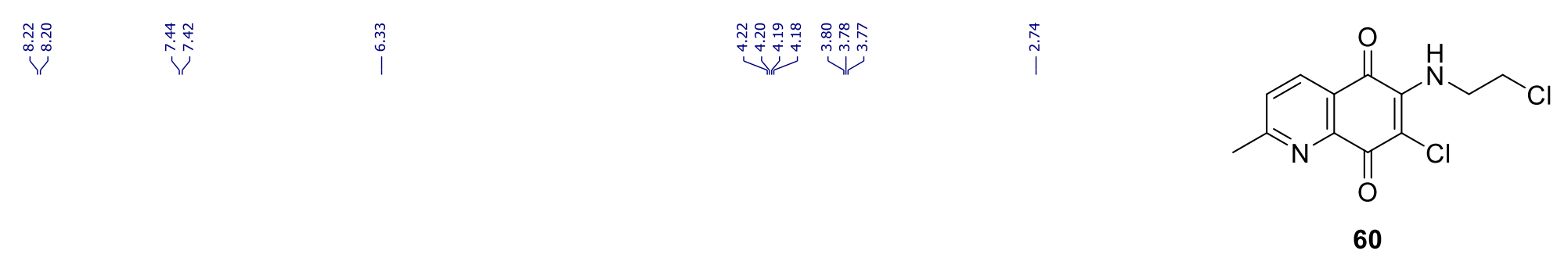

${ }^{1} \mathrm{H}$ NMR $\left(500 \mathrm{MHz}, \mathrm{CDCl}_{3}\right)$

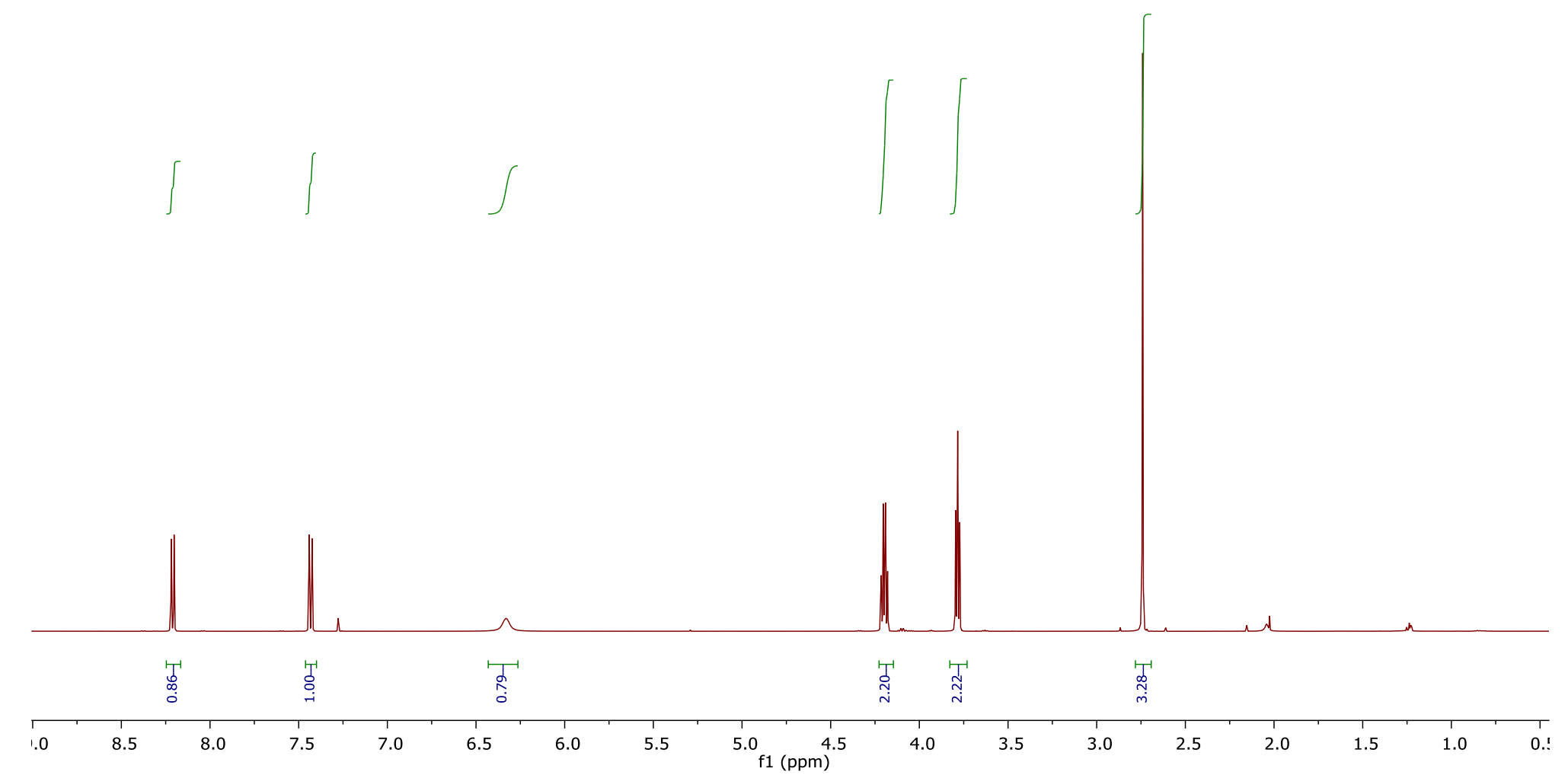




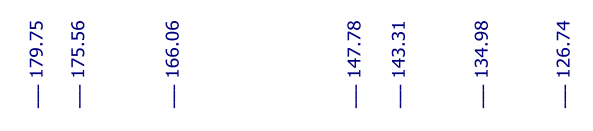

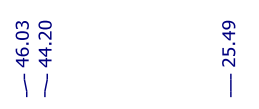

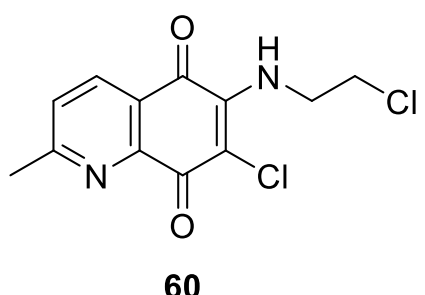

${ }^{13} \mathrm{C} \mathrm{NMR}\left(125 \mathrm{MHz}, \mathrm{CDCl}_{3}\right)$

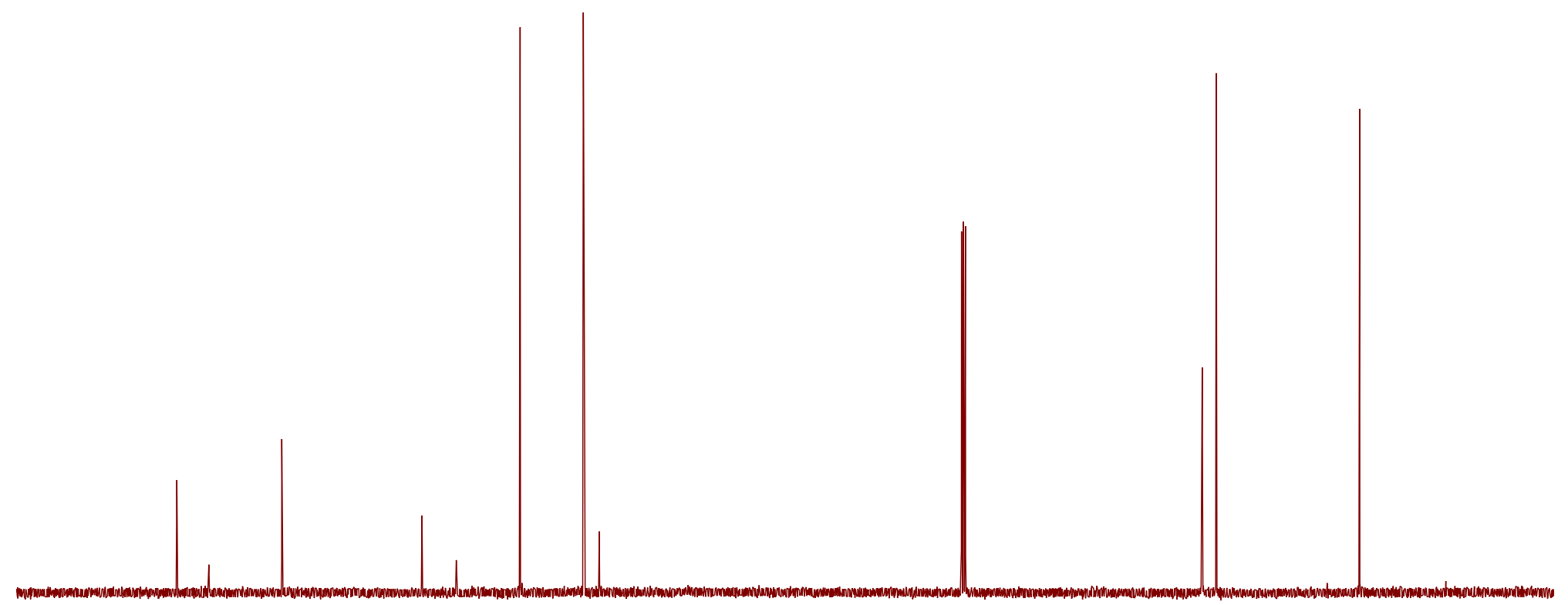

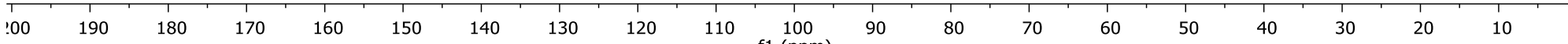




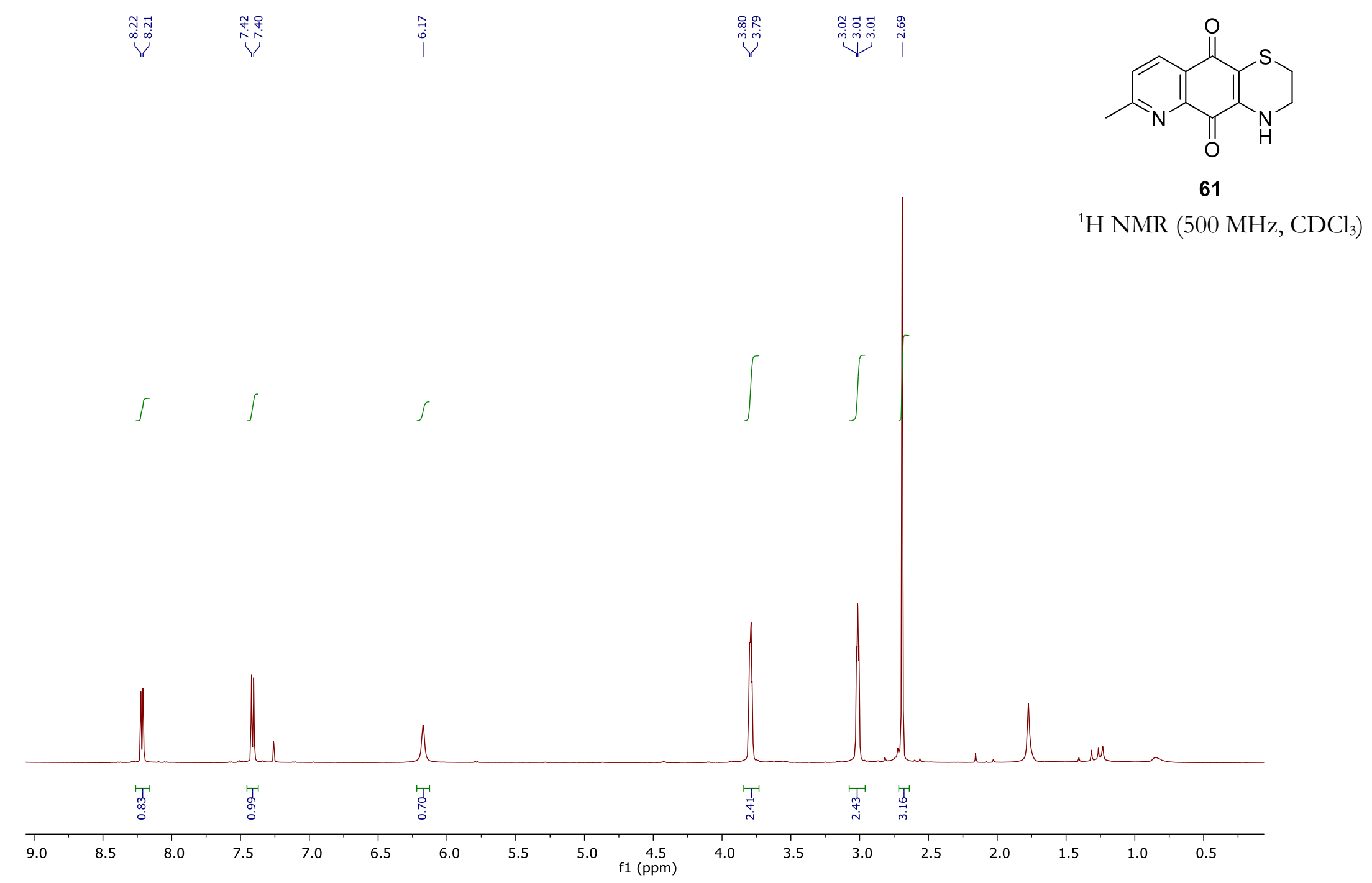




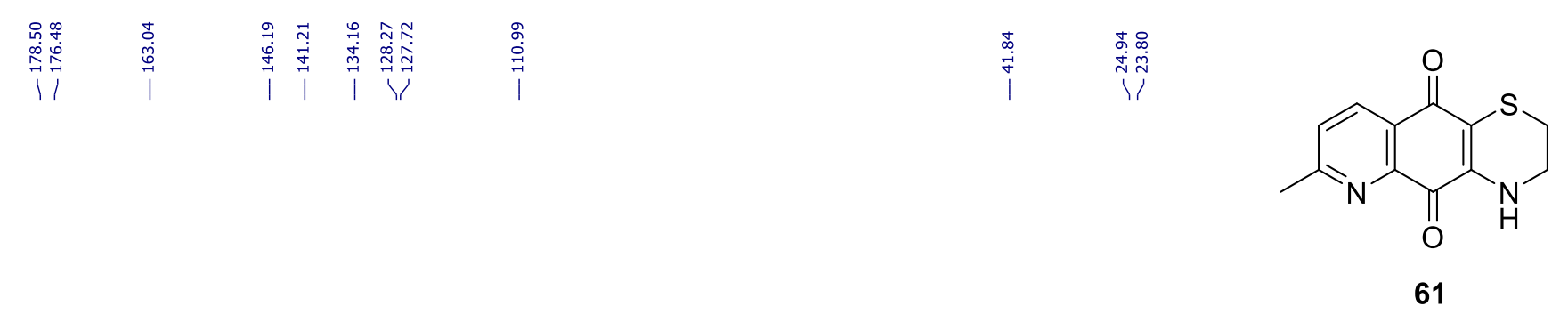

${ }^{13} \mathrm{C}$ NMR (125 MHz, $\left.\mathrm{CDCl}_{3}\right)$

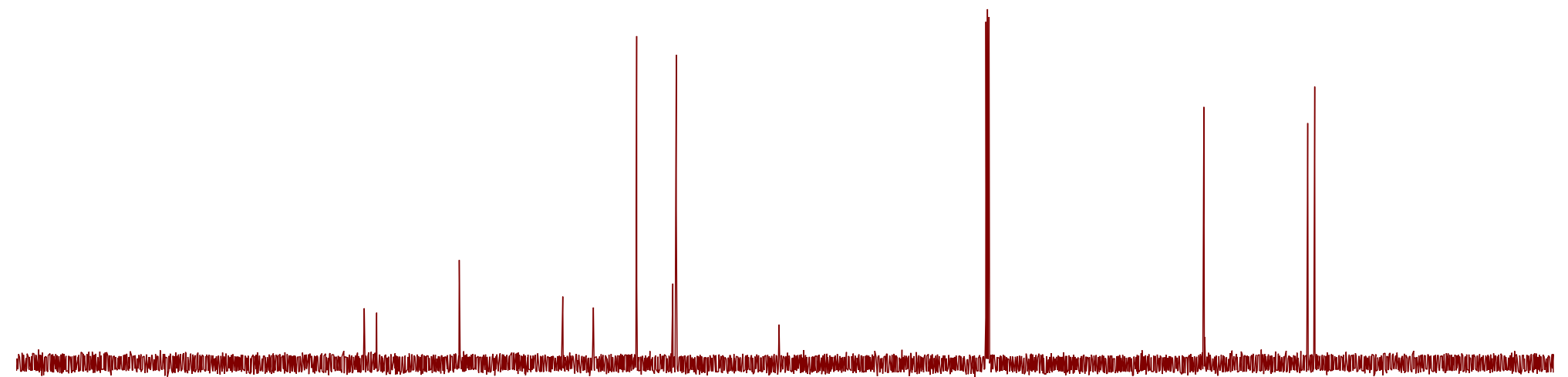




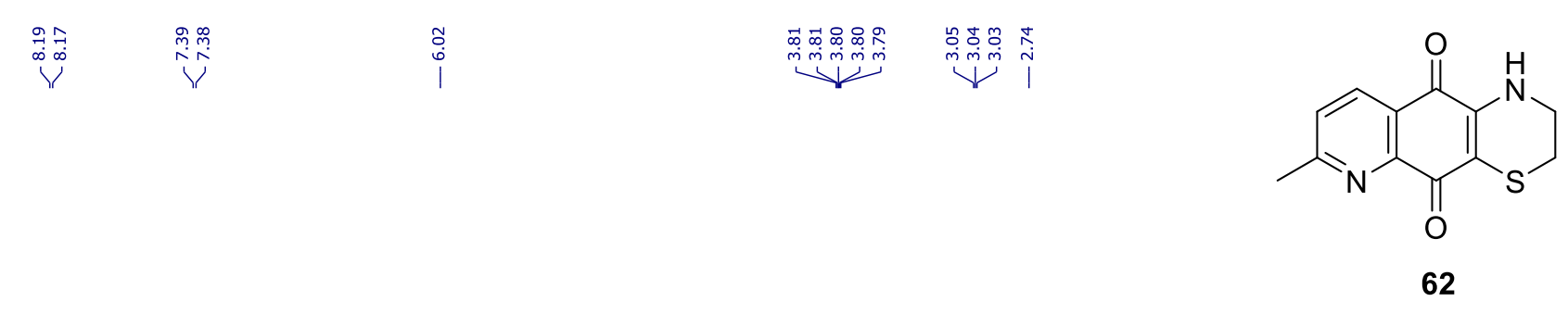

${ }^{1} \mathrm{H}$ NMR $\left(500 \mathrm{MHz}, \mathrm{CDCl}_{3}\right)$

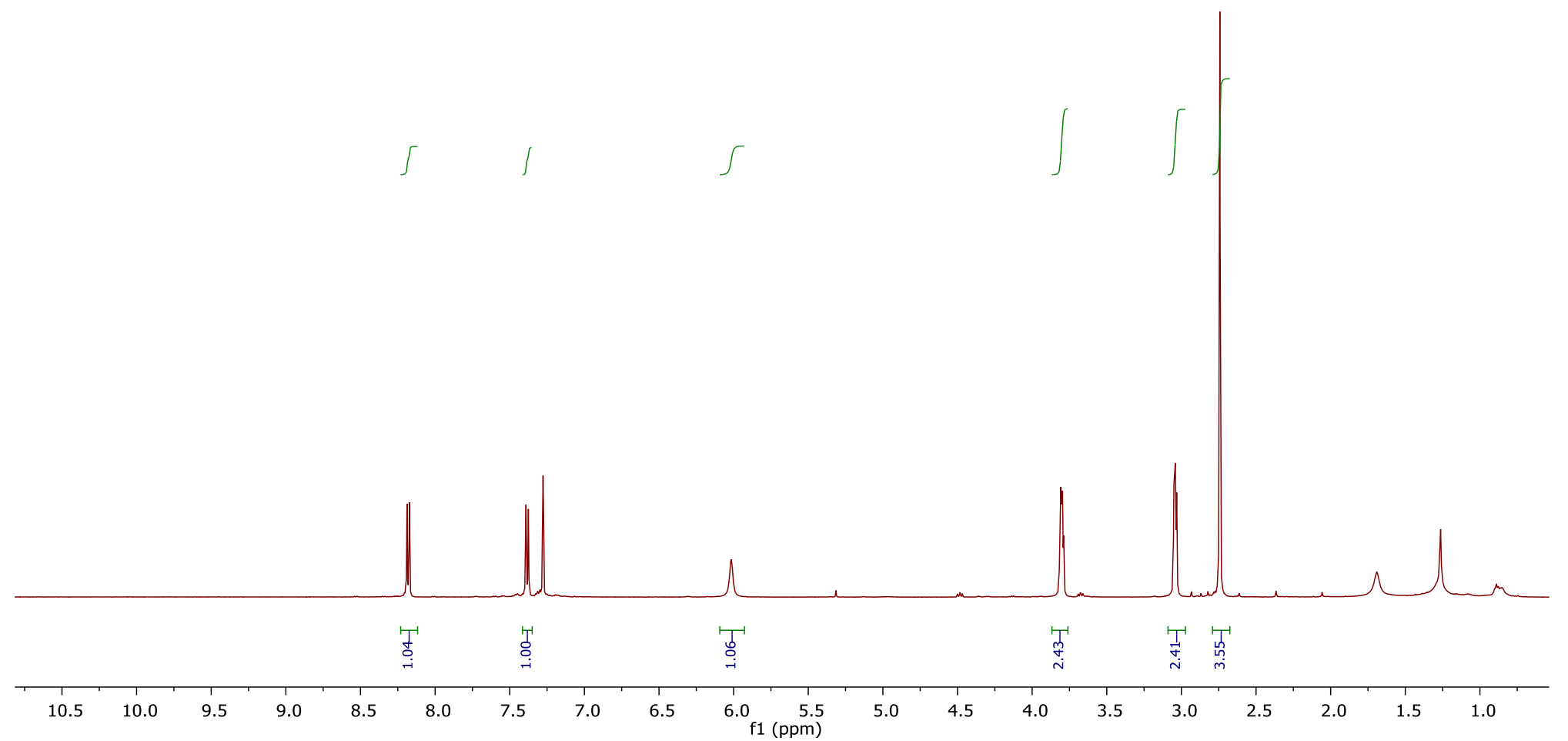




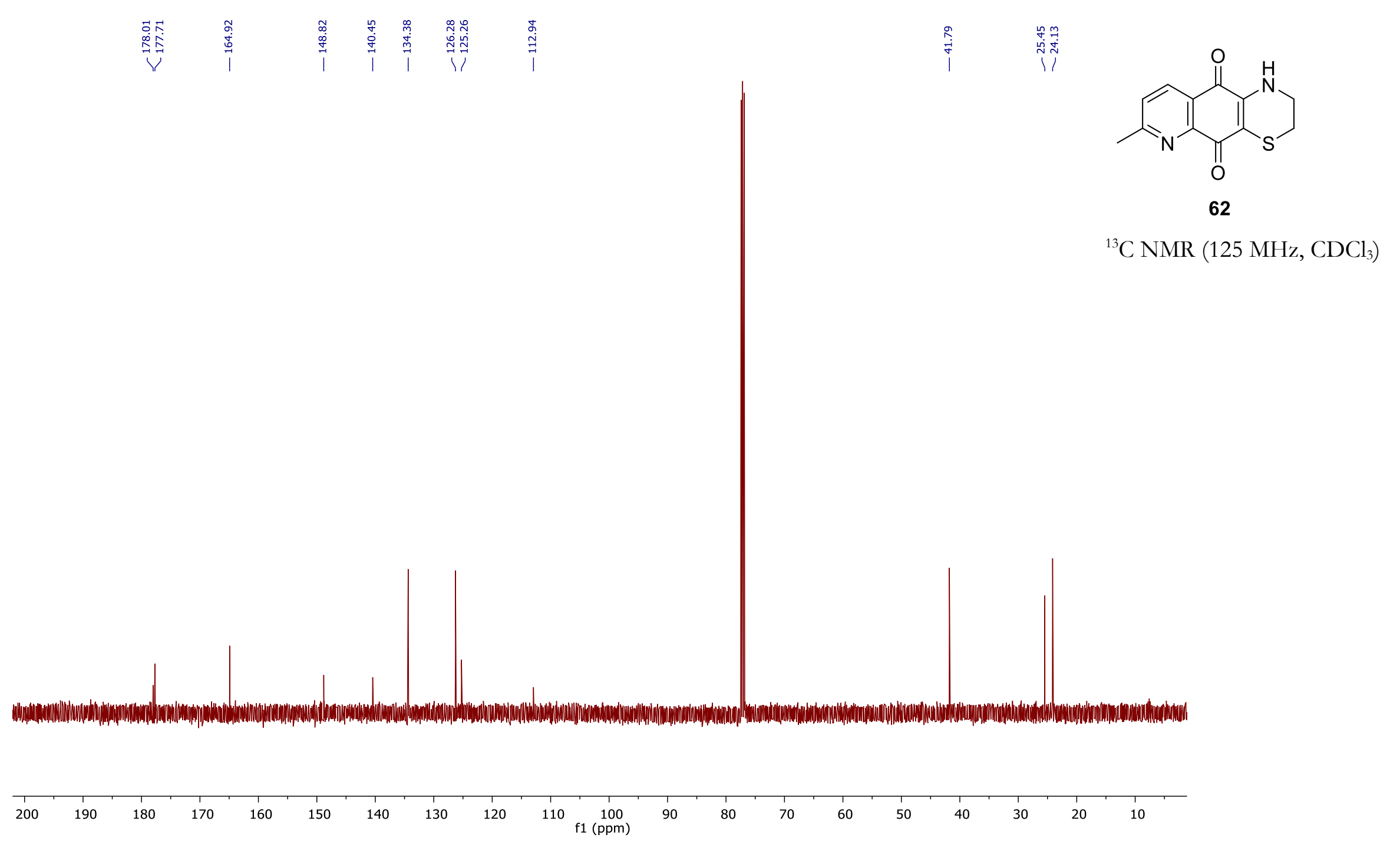




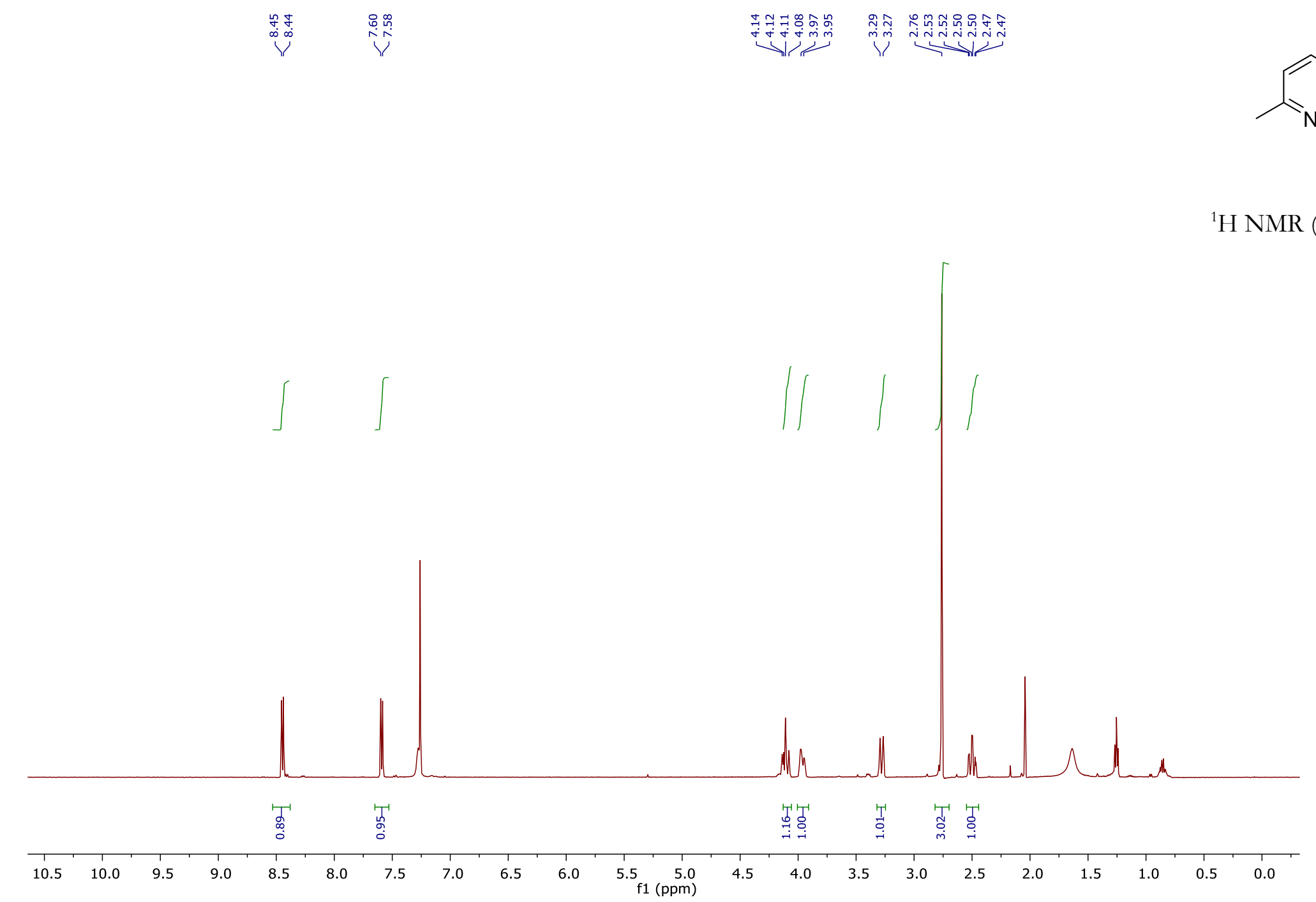




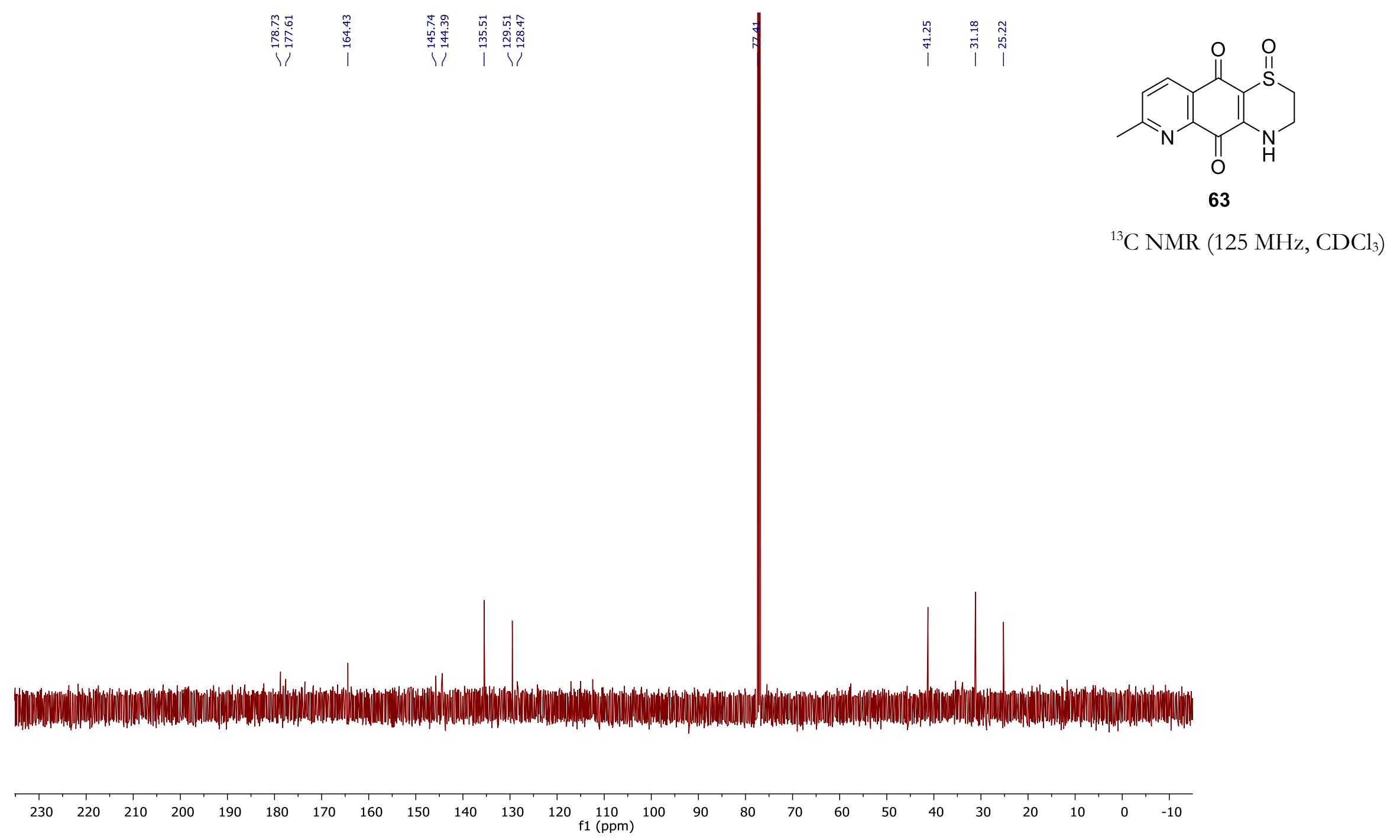




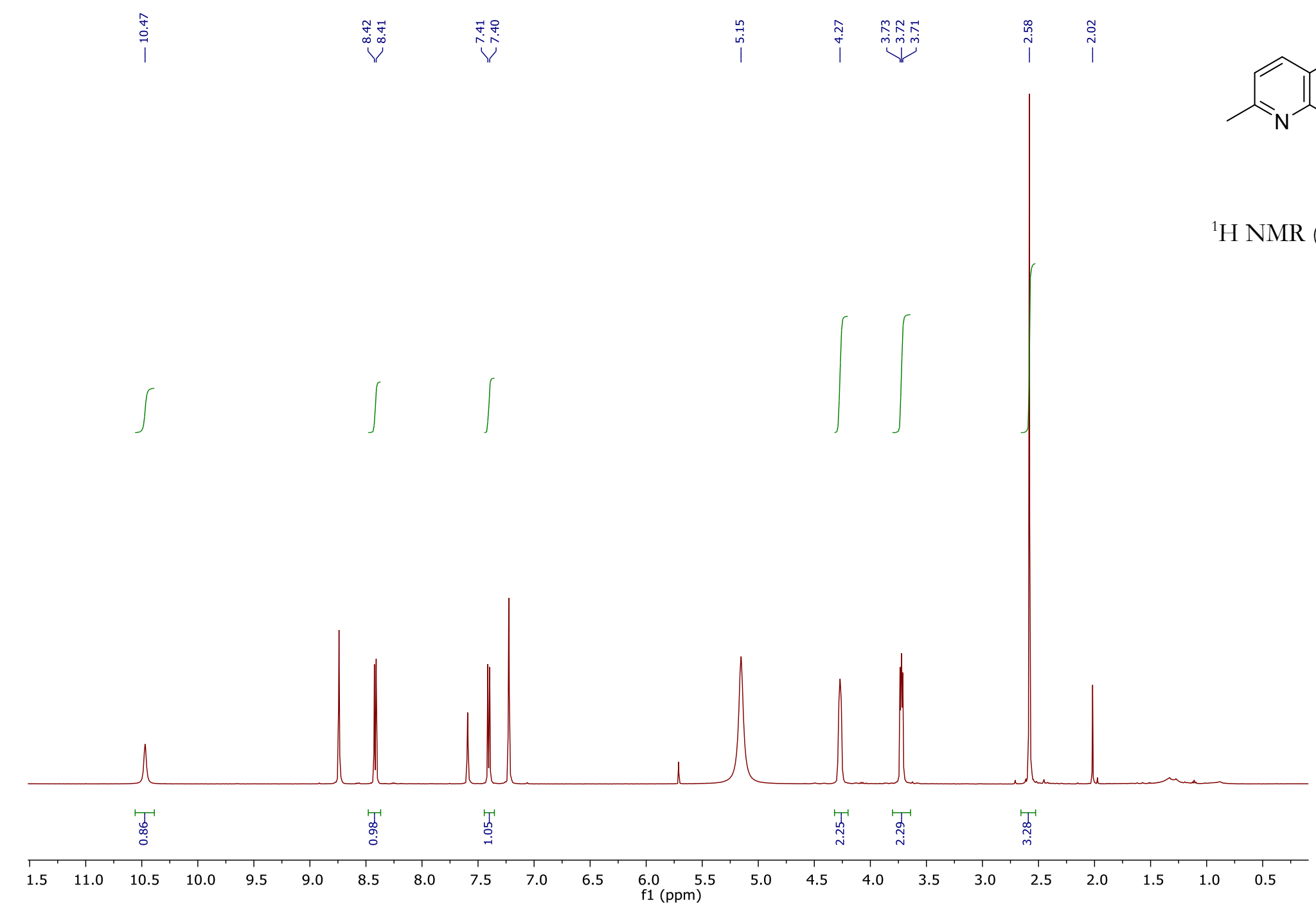




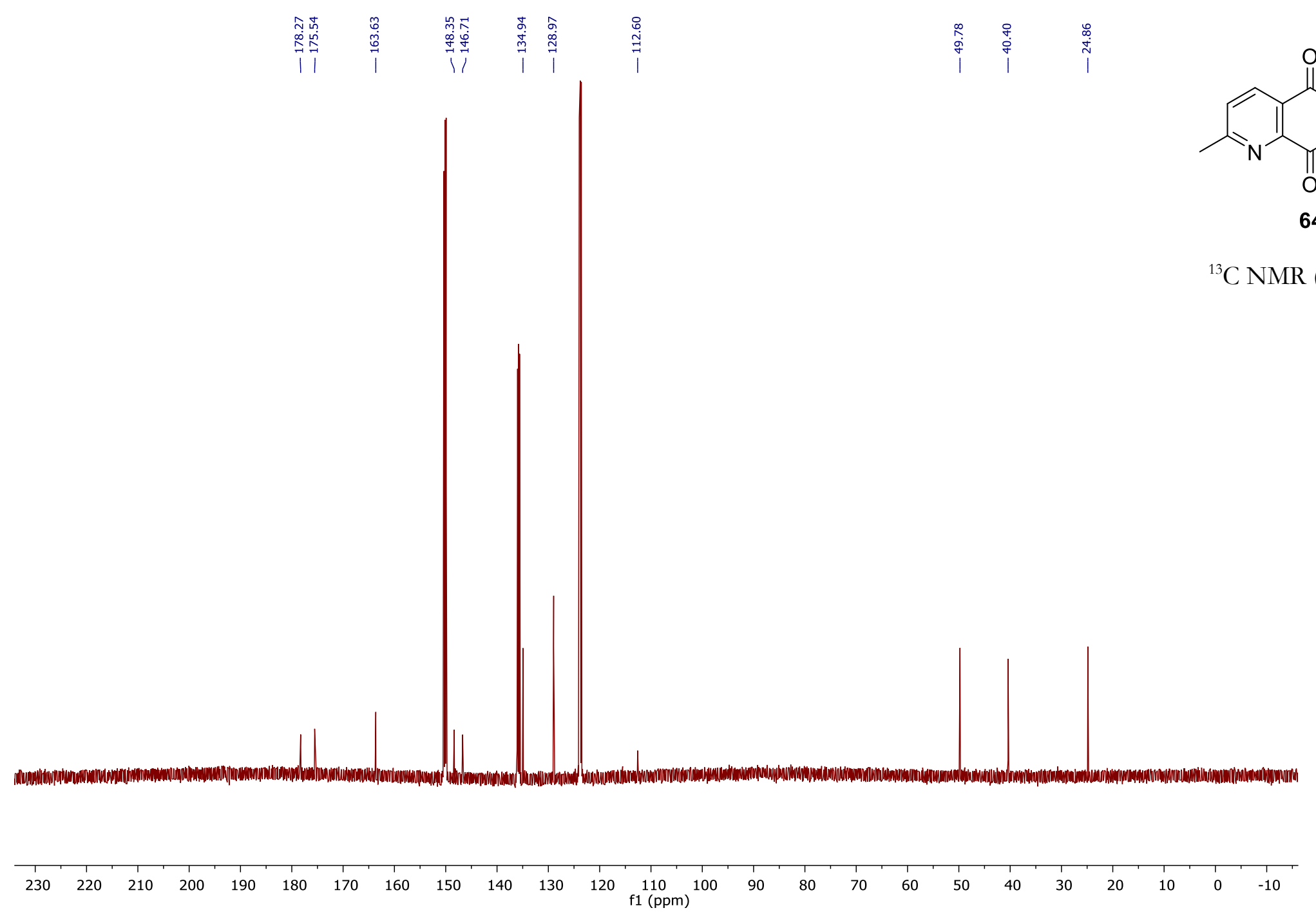




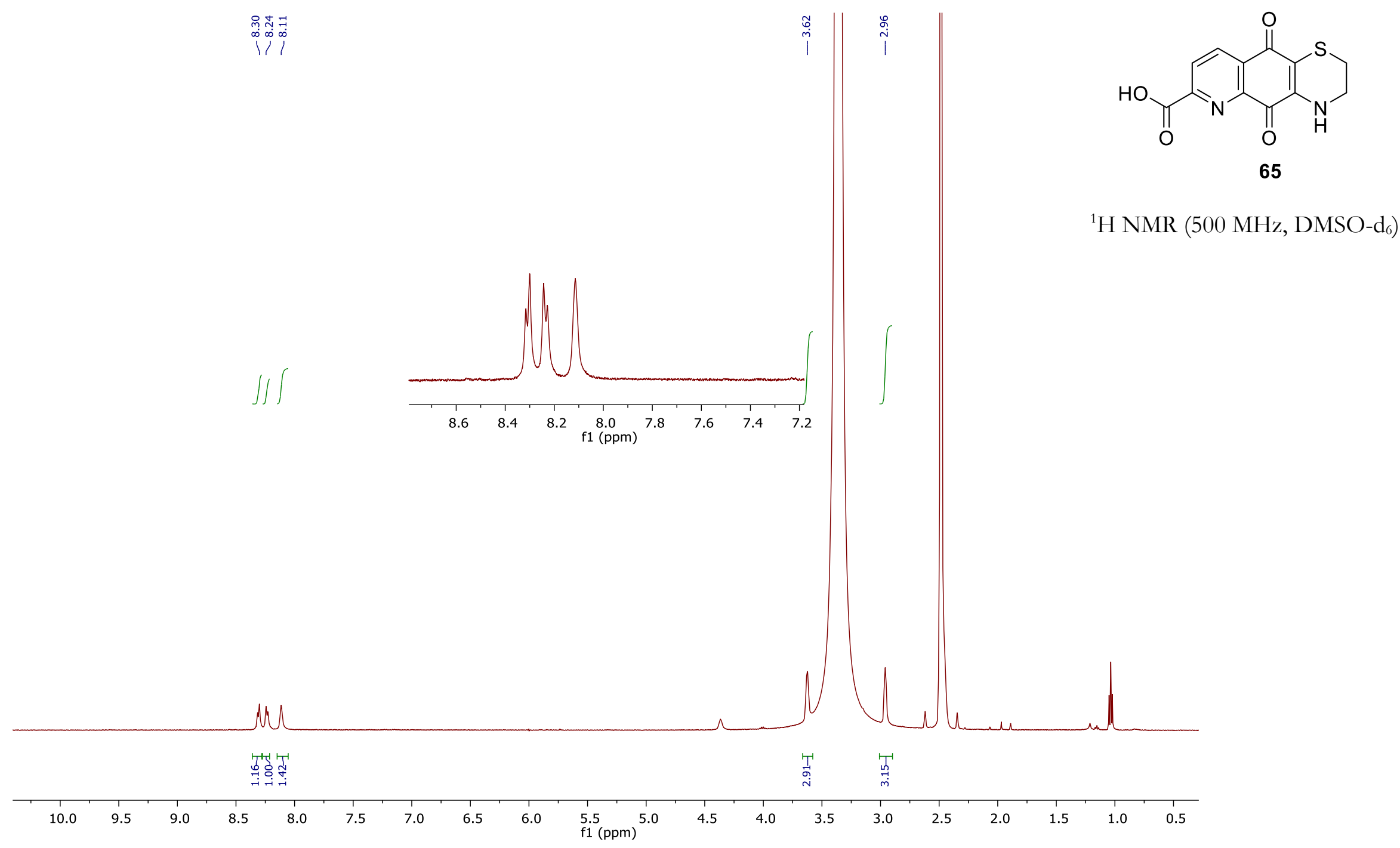




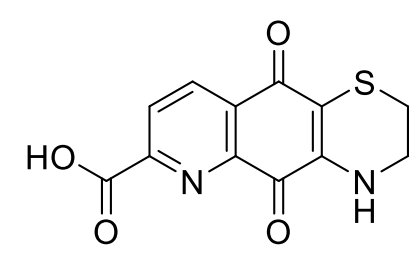

65

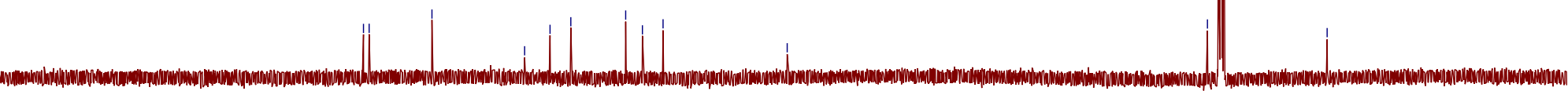
$210 \quad 200$ $\begin{array}{lll}190 & 180 & 170\end{array}$ $\begin{array}{lll}160 & 150 \quad 140\end{array}$ $30 \quad 120 \quad 110 \quad 100$ 


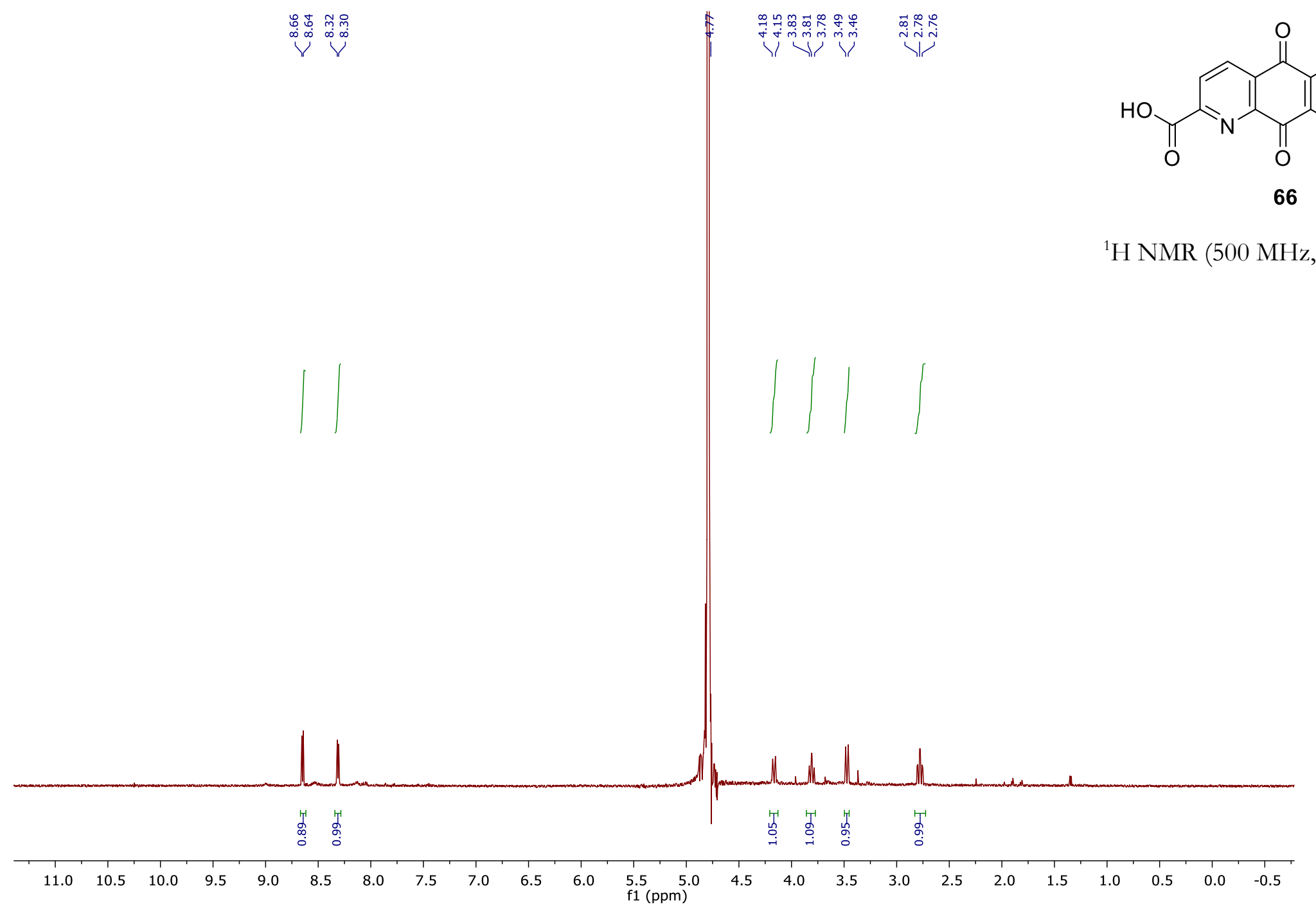



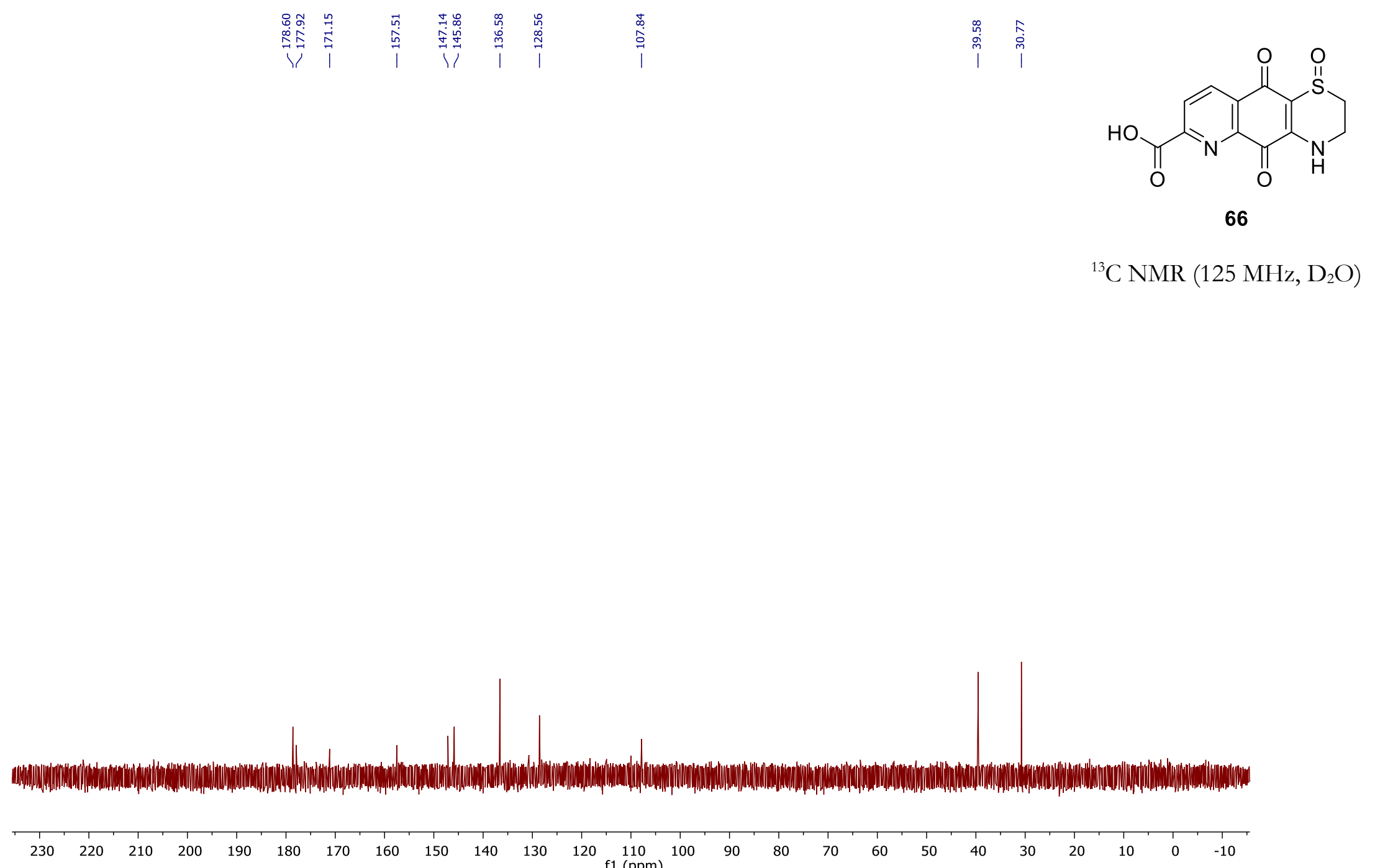


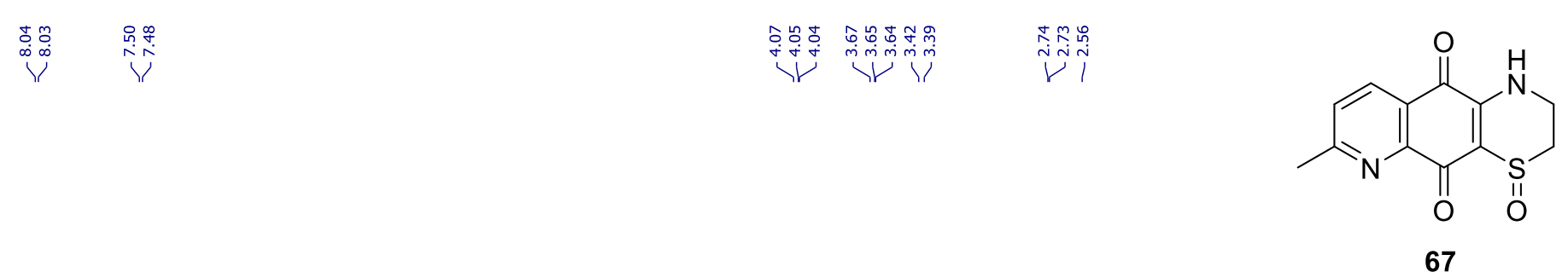

${ }^{1} \mathrm{H}$ NMR $\left(500 \mathrm{MHz}, \mathrm{D}_{2} \mathrm{O}\right)$

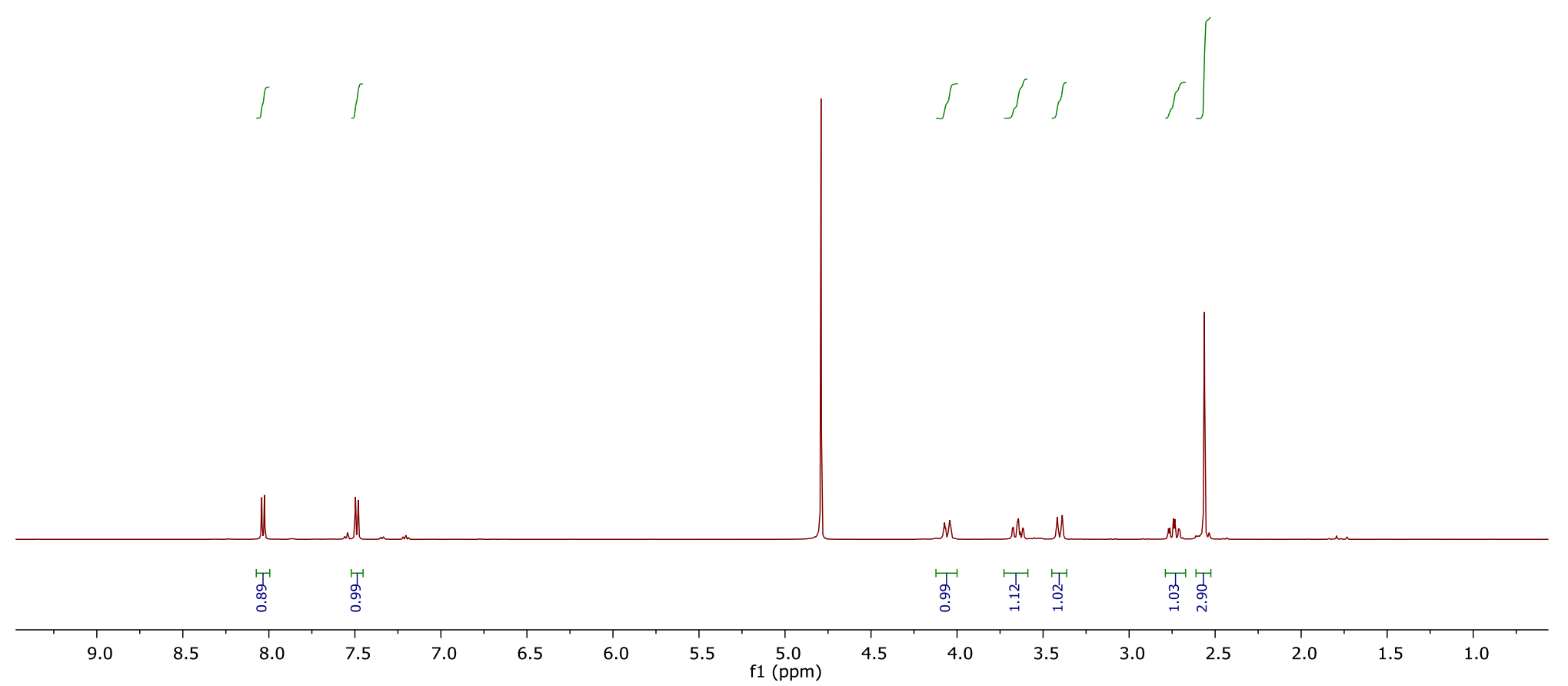




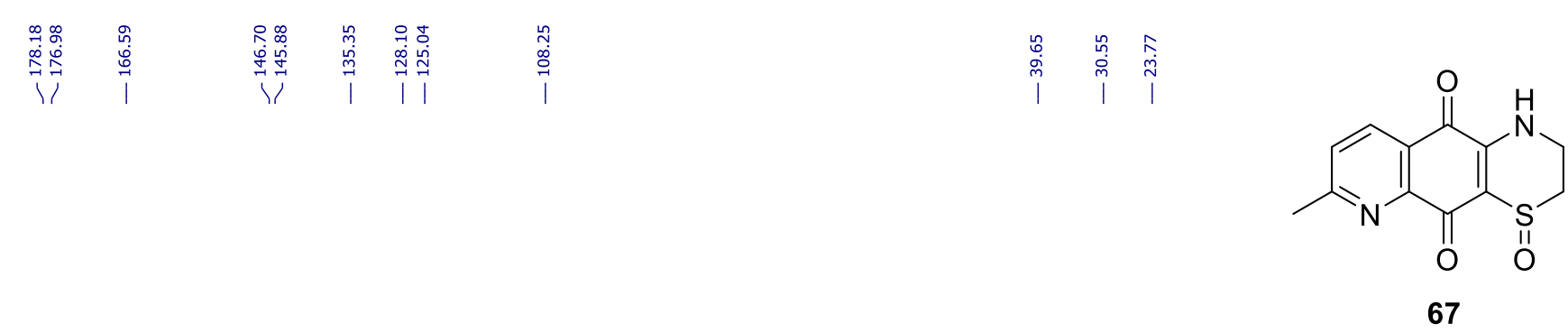

${ }^{13} \mathrm{C}$ NMR $\left(125 \mathrm{MHz}, \mathrm{D}_{2} \mathrm{O}\right)$
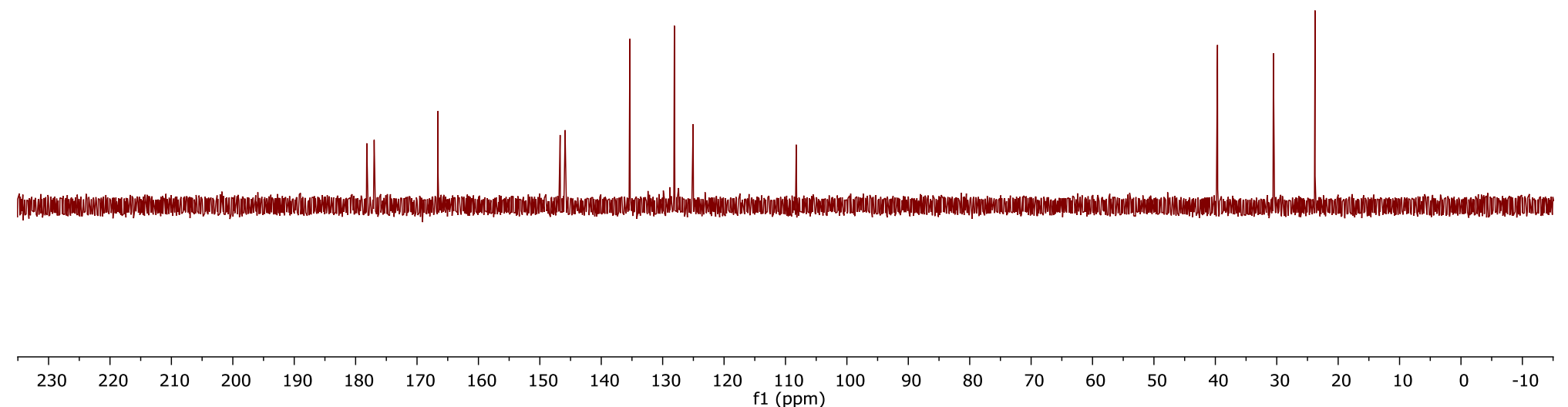


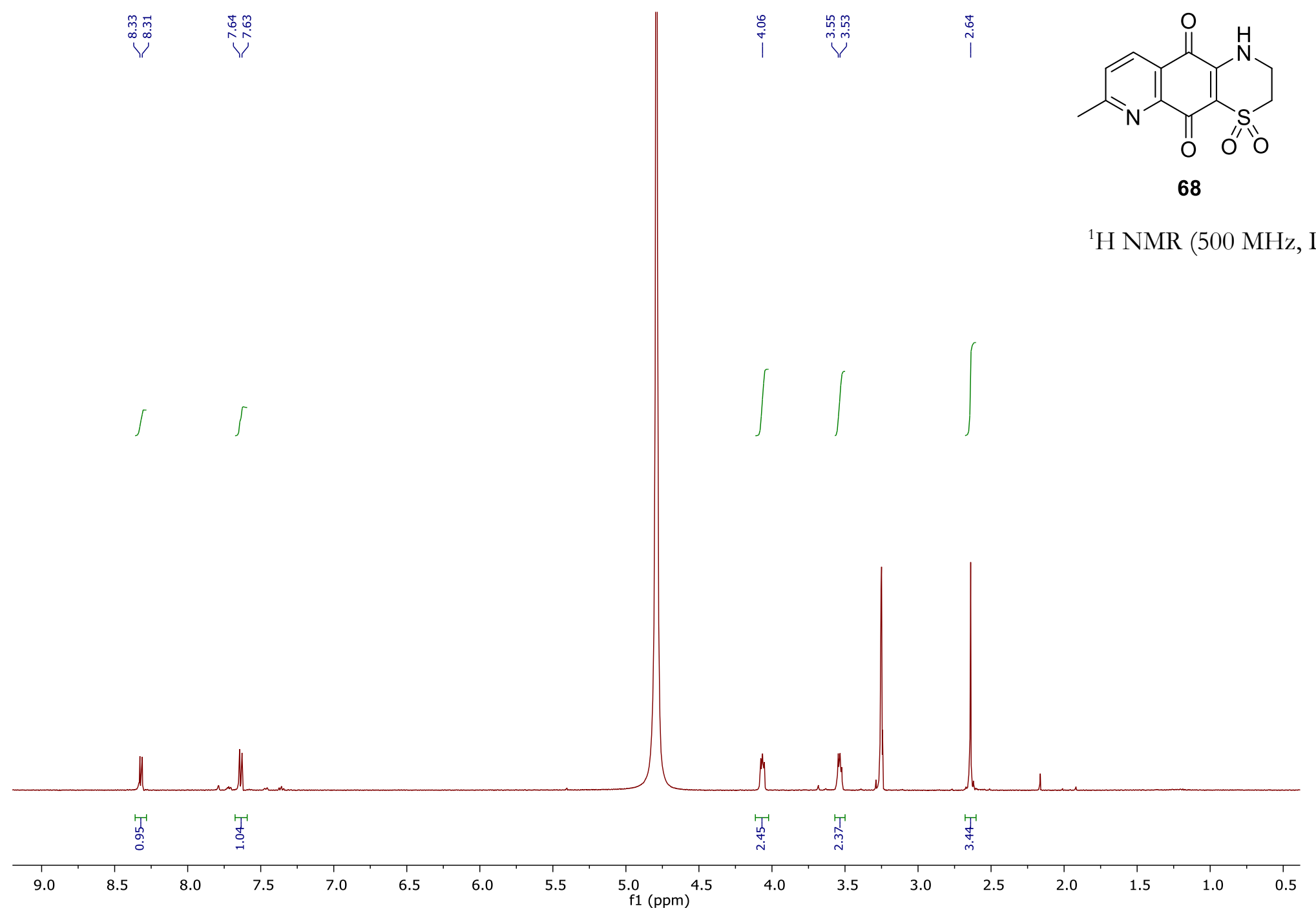




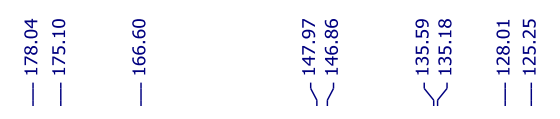

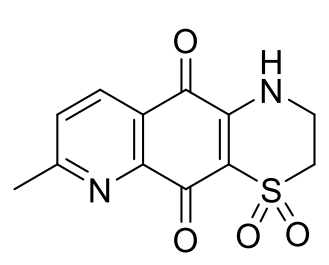

68

${ }^{13} \mathrm{C}$ NMR $\left(125 \mathrm{MHz}, \mathrm{D}_{2} \mathrm{O} / \mathrm{CD}_{3} \mathrm{OD}\right)$

. 


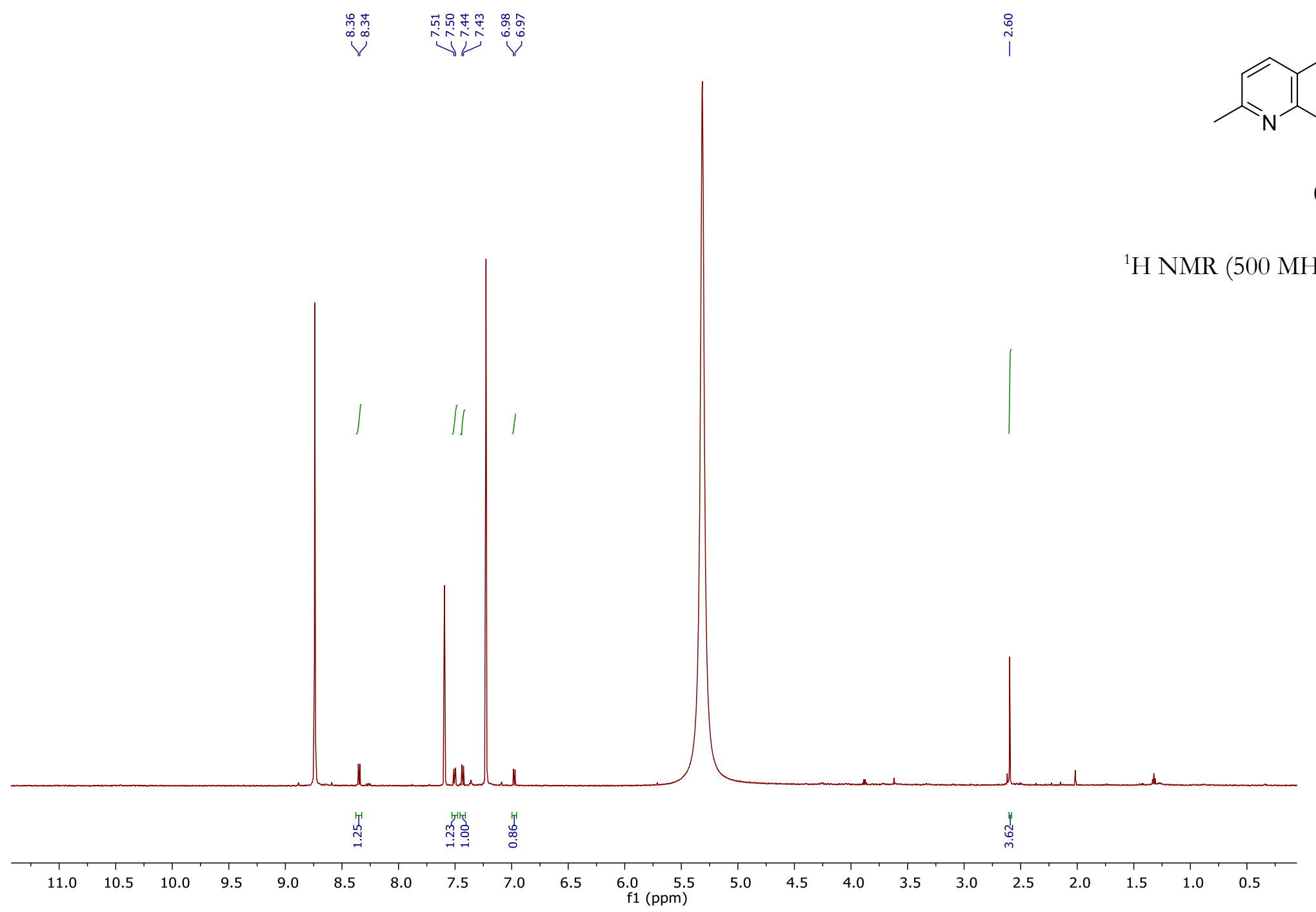




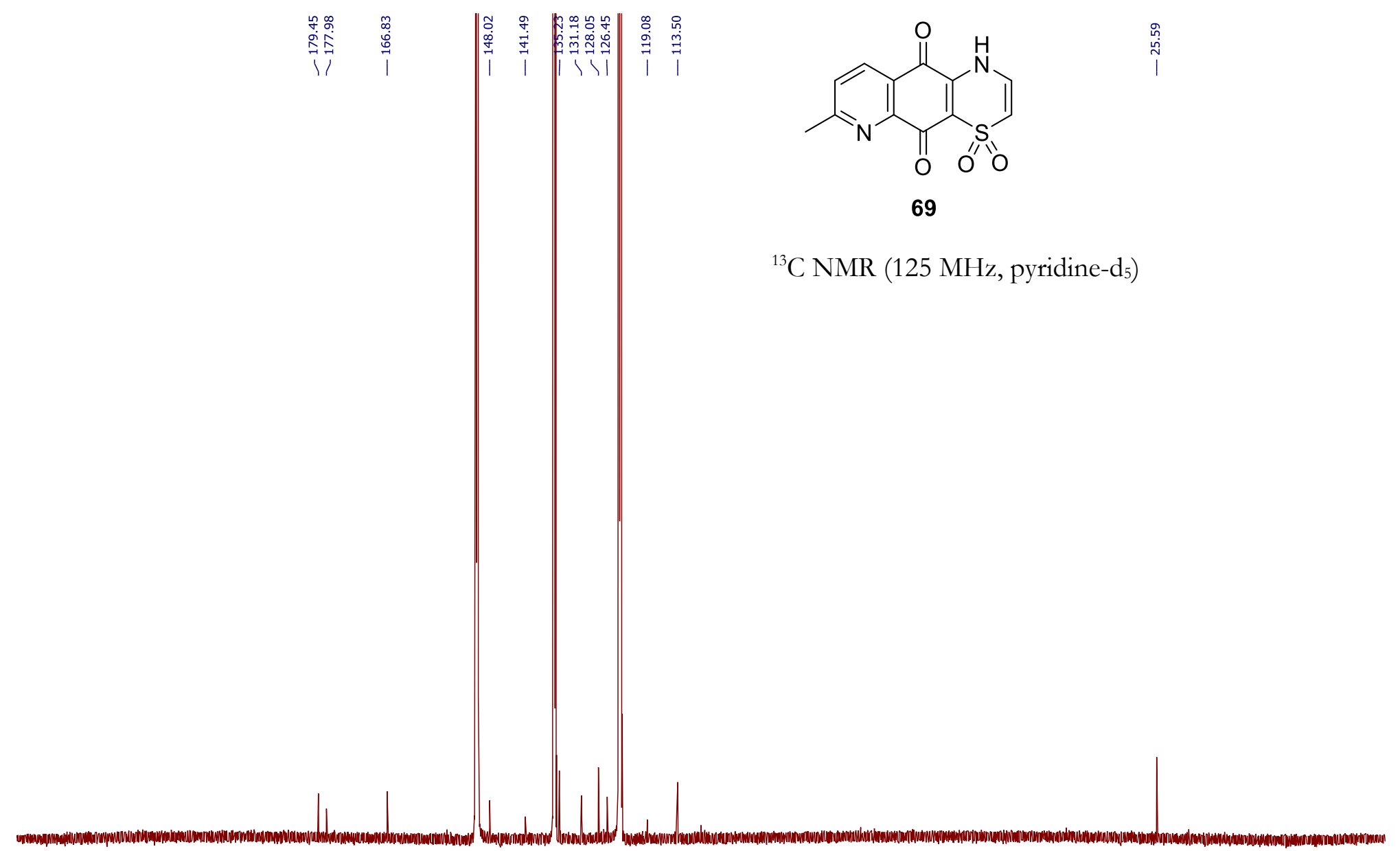

$\begin{array}{llllllllllllllllllllllllll}230 & 220 & 210 & 200 & 190 & 180 & 170 & 160 & 150 & 140 & 130 & 120 & 110 & 100 & 90 & 80 & 70 & 60 & 50 & 40 & 30 & 20 & 10 & 0 & -10\end{array}$ 


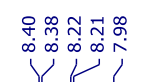

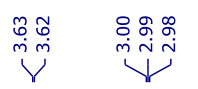

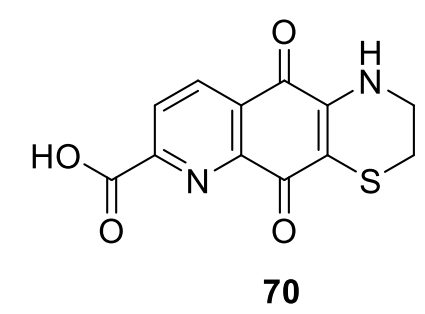

${ }^{1} \mathrm{H}$ NMR (500 MHz, DMSO-d 6 )

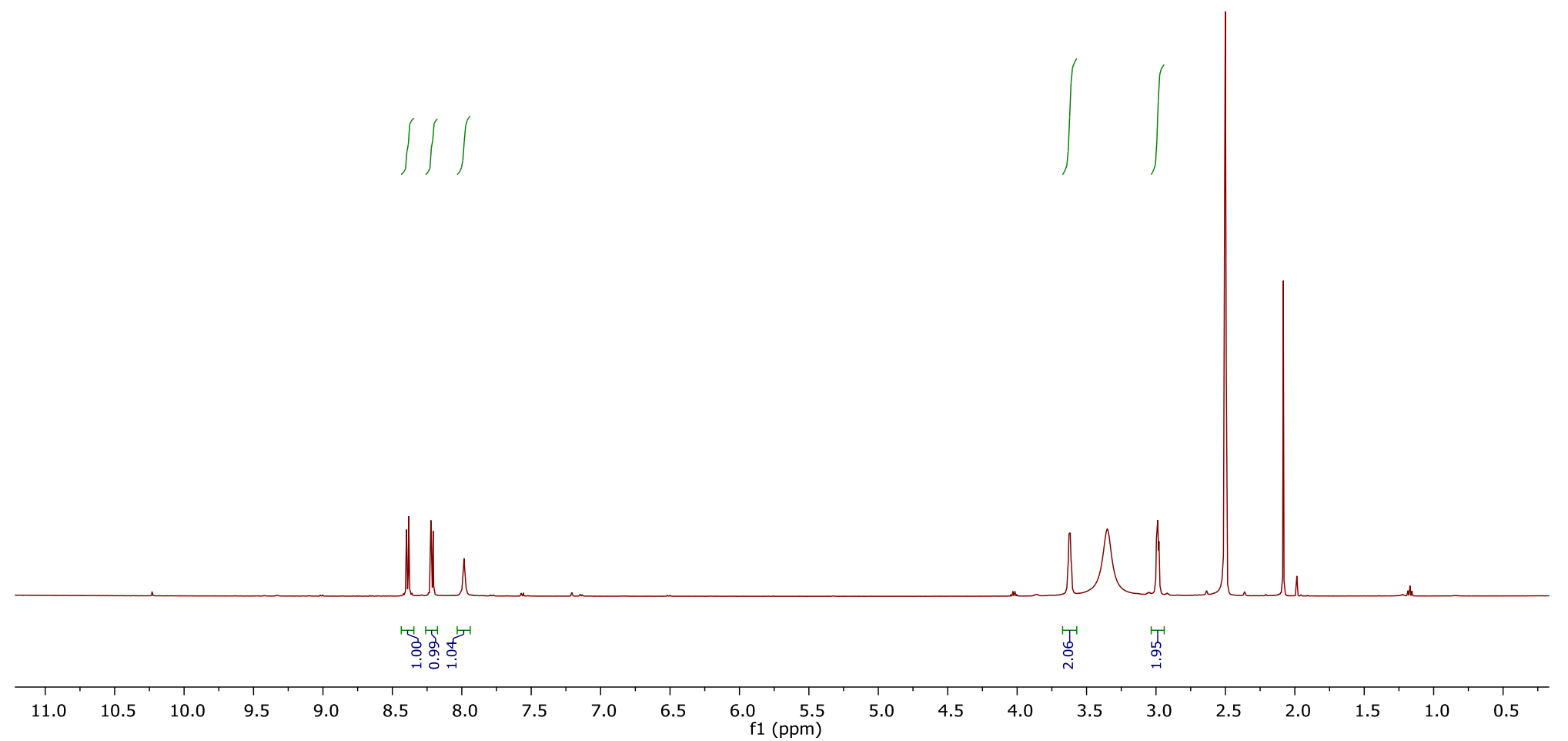




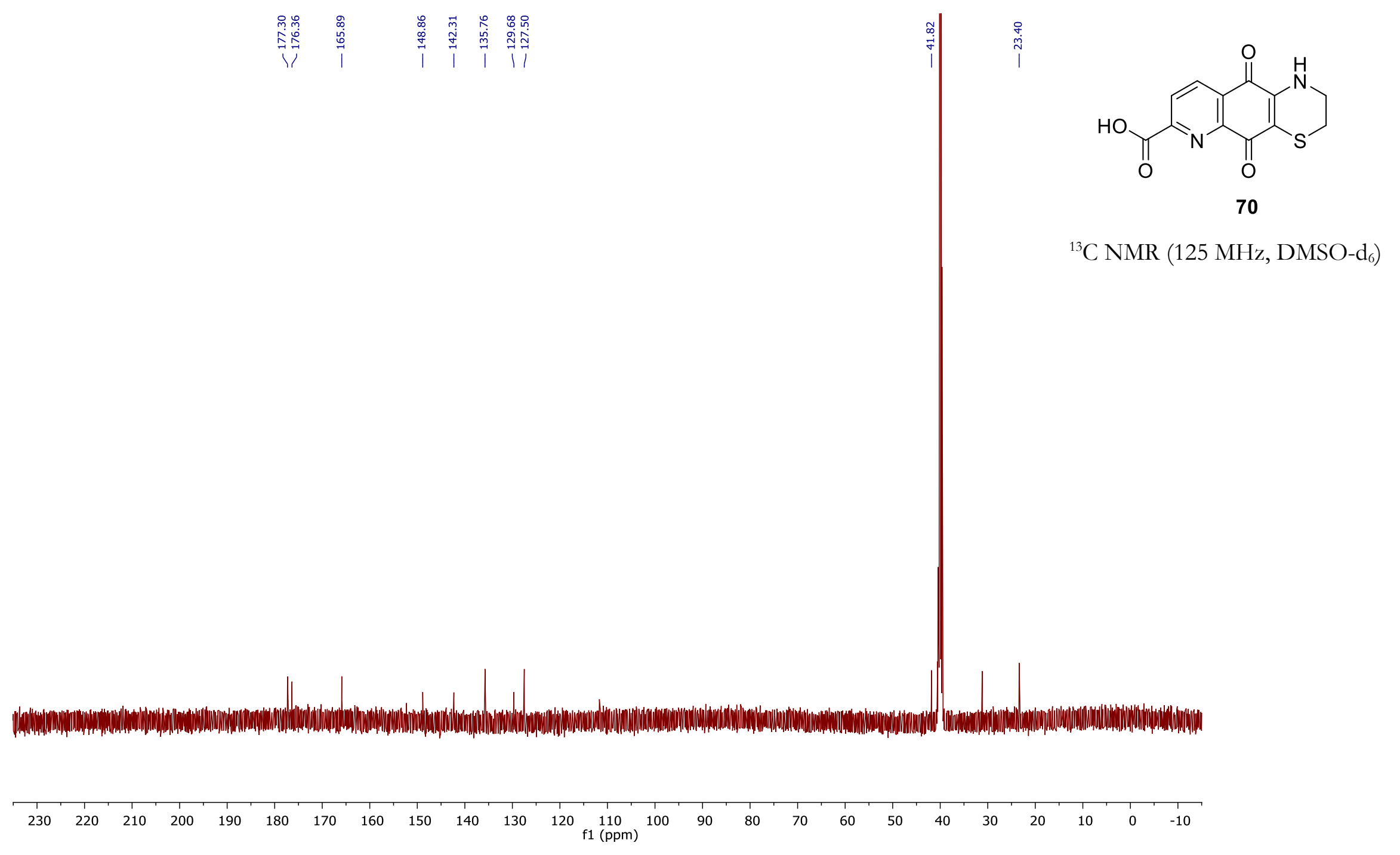




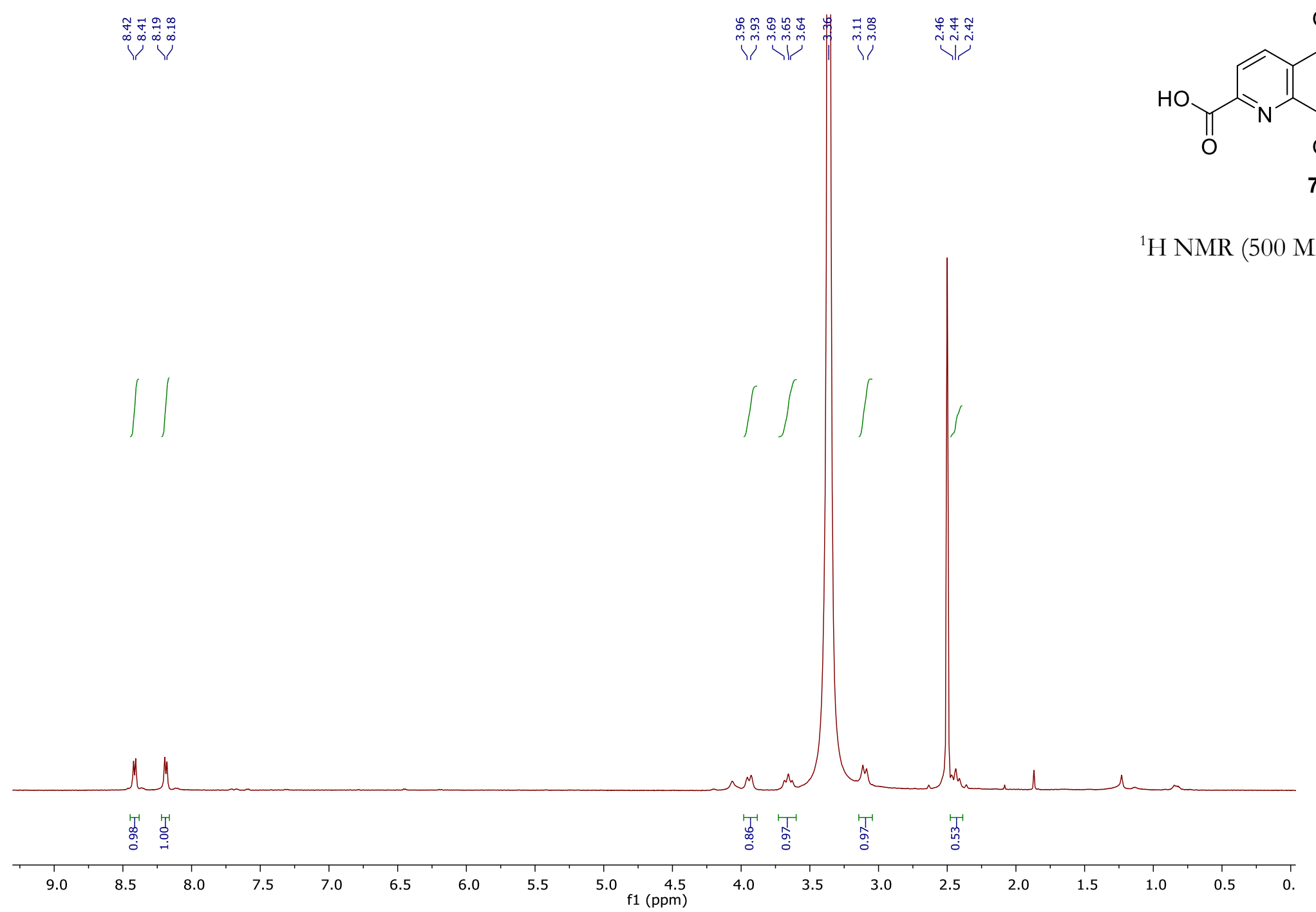




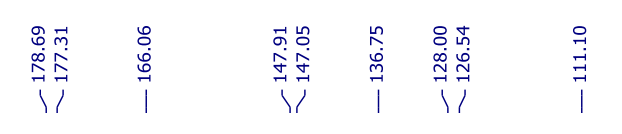

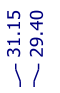

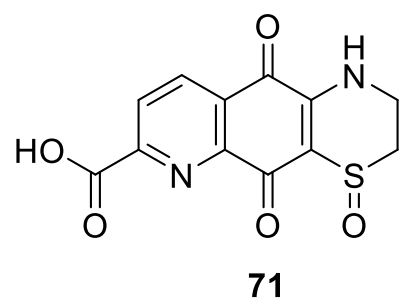

${ }^{13} \mathrm{C}$ NMR $\left(125 \mathrm{MHz}, \mathrm{DMSO}-\mathrm{d}_{6}\right)$

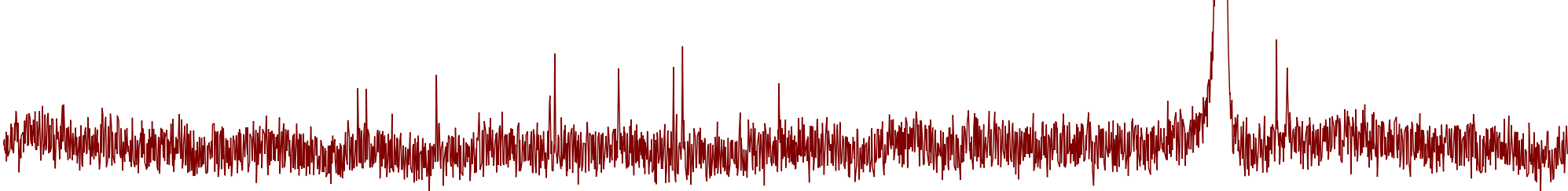

$\begin{array}{lllllllllllll}230 & 220 & 210 & 200 & 190 & 180 & 170 & 160 & 150 & 140 & 130 & 120 & 110 \\ 100\end{array}$ 


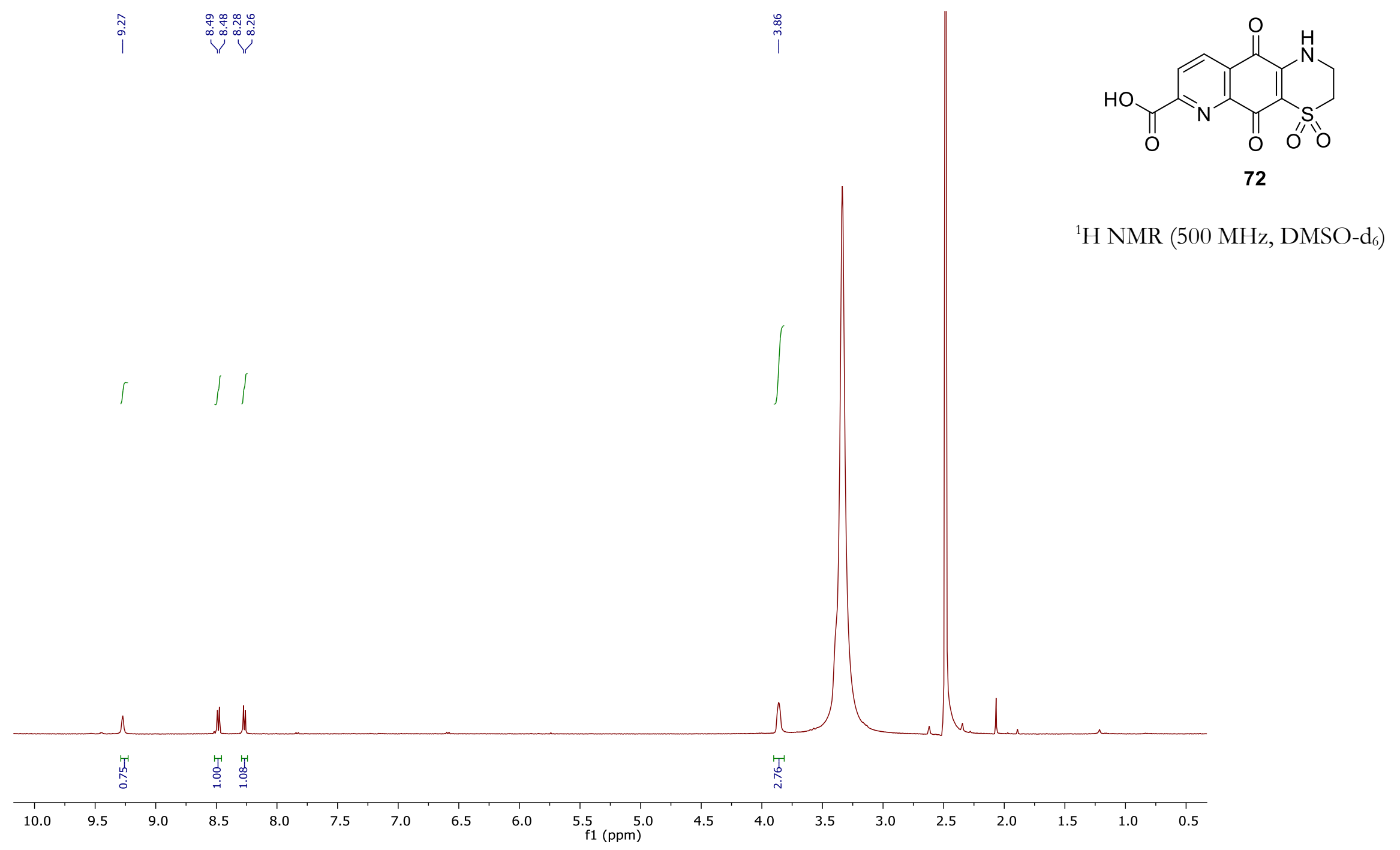




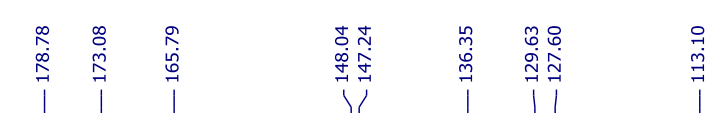

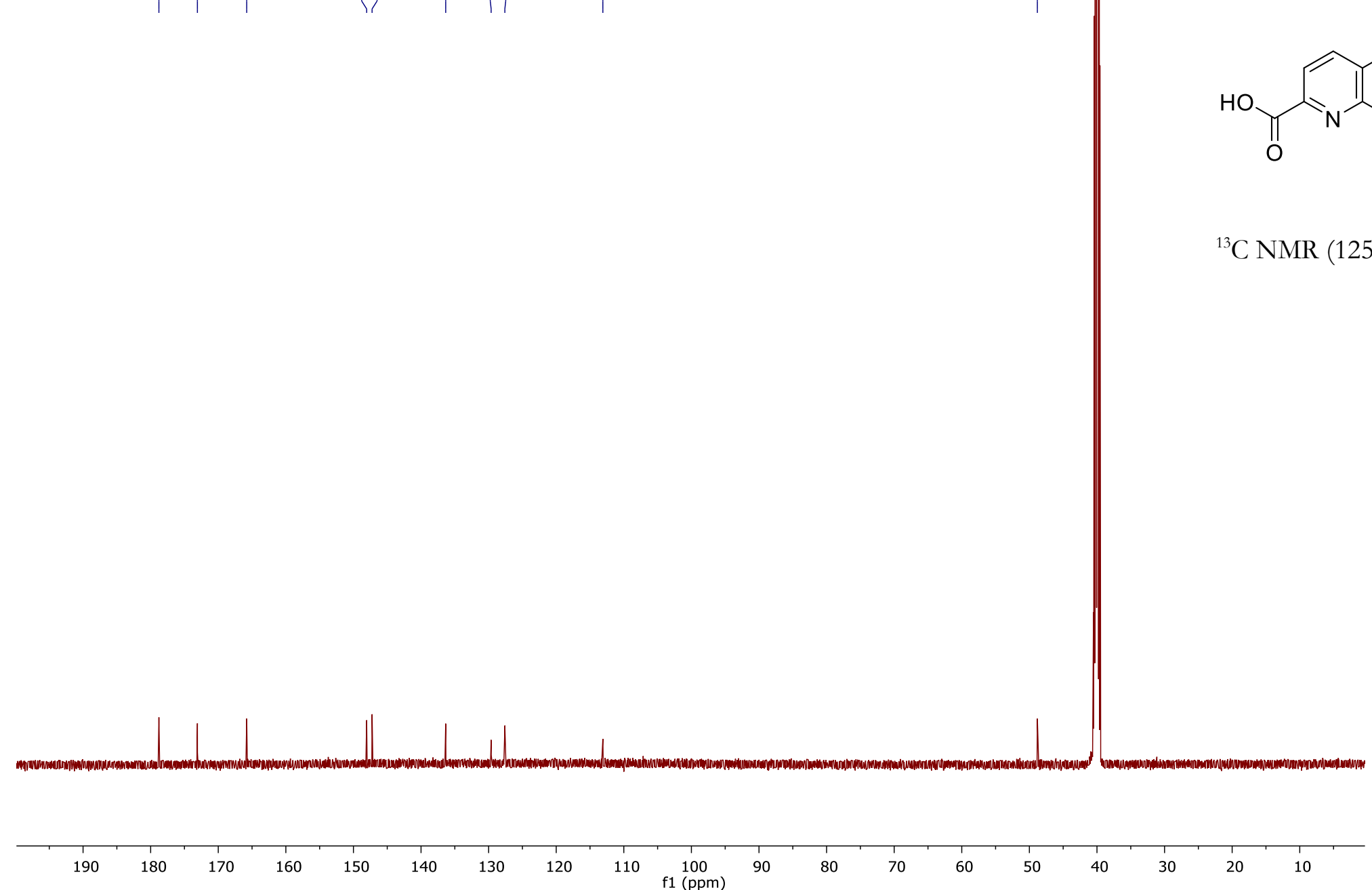




\section{APPENDIX: CHAPTER THREE}

Table A.3.1. Selected bond lenths of QQ 104b..............................................................................A35

Table A.3.2. Intermolecular hydrogen bonds in QQ 104................................ 35

Table A.3.3. Redox potentials of selected QQs in DMF..................................A35-A37

Figure A.3.1. Redox potentials (in DMF) vs. MIC of selected QQs........................A37

NMR spectra:

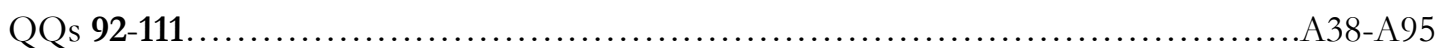


Table A.3.2: Selected bond lengths of 6-anilino-2-methylquinolinequinone (104b).

\begin{tabular}{llll} 
Entry & Atom $\mathbf{1}$ & Atom $\mathbf{2}$ & Bond length (̊̊) \\
\hline 1 & $\mathrm{C} 2$ & $\mathrm{C} 3$ & $1.404(2)$ \\
2 & $\mathrm{C} 3$ & $\mathrm{C} 4$ & $1.373(2)$ \\
3 & $\mathrm{C} 4$ & $\mathrm{C} 4 \mathrm{a}$ & $1.396(2)$ \\
4 & $\mathrm{C} 4 \mathrm{a}$ & $\mathrm{C} 8 \mathrm{a}$ & $1.3908(18)$ \\
5 & $\mathrm{~N} 1$ & $\mathrm{C} 8 \mathrm{a}$ & $1.3387(18)$ \\
6 & $\mathrm{~N} 1$ & $\mathrm{C} 2$ & $1.3423(18)$ \\
7 & $\mathrm{C} 6$ & $\mathrm{C} 7$ & $1.3756(18)$ \\
8 & $\mathrm{C} 4 \mathrm{a}$ & $\mathrm{C} 5$ & $1.4752(19)$ \\
9 & $\mathrm{C} 5$ & $\mathrm{C} 6$ & $1.5085(19)$ \\
10 & $\mathrm{C} 7$ & $\mathrm{C} 8$ & $1.4613(19)$ \\
11 & $\mathrm{C} 8$ & $\mathrm{C} 8 \mathrm{a}$ & $1.5070(19)$
\end{tabular}

Table A.3.3: Intermolecular hydrogen bonds in 6-anilino-2-methylquinolinequinone (104b).

\begin{tabular}{llll} 
Entry & H-bond donor & $\mathbf{d}(\mathbf{H} \cdots \mathbf{A})(\mathbf{\AA})$ & H-bond acceptor \\
\hline 1 & $\mathrm{~N} 2-\mathrm{H} 2 \mathrm{n}$ & 2.539 & $\mathrm{O} 1$ \\
2 & $\mathrm{~N} 2-\mathrm{H} 2 \mathrm{n}$ & 2.223 & $\mathrm{~N} 1$
\end{tabular}

Table A.3.3: Redox potentials of selected QQs in DMF.

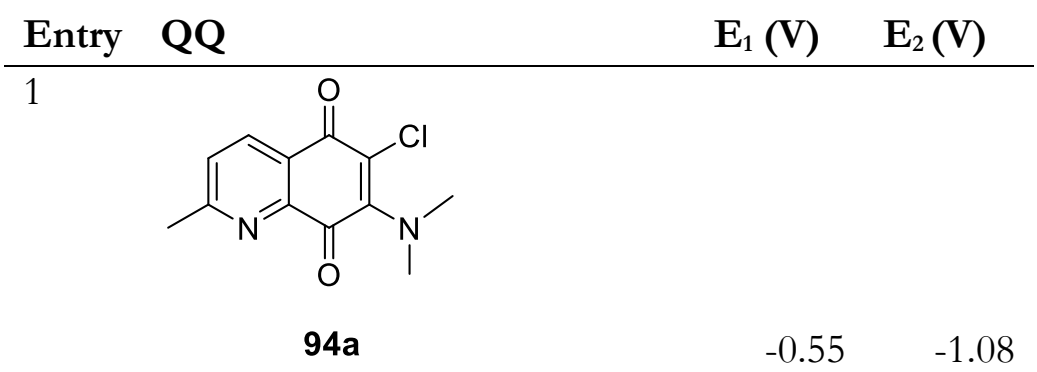

2<smiles>O=C1C(Cl)=C(N2CCCCC2)C(=O)c2ncccc21</smiles>

95a 
3<smiles>CCCNC1=C(Cl)C(=O)c2ncccc2C1=O</smiles>

98b

$-0.57 \quad-1.10$

4<smiles>CCCNC1=C(Cl)C(=O)c2ccc(C)nc2C1=O</smiles>

99a

$\begin{array}{ll}-0.59 & -1.12\end{array}$

5<smiles>[3H]C(C)(C)NC1=C(Cl)C(=O)c2ncccc2C1=O</smiles>

102b

$\begin{array}{ll}-0.56 & -1.11\end{array}$

6<smiles>O=C1C(Cl)=C(Nc2ccccc2)C(=O)c2ncccc21</smiles>

104b

$\begin{array}{ll}-0.45 & -1.10\end{array}$

7<smiles>O=C1C(Cl)=C(NCCc2ccccc2)C(=O)c2ncccc21</smiles>

106a

$\begin{array}{ll}-0.56 & -1.09\end{array}$

8<smiles>O=C1C(Cl)=C(NCCc2ccccc2)C(=O)c2ncccc21</smiles>

106b

$\begin{array}{ll}-0.56 & -1.09\end{array}$

9<smiles>Cc1ccc2c(n1)C(=O)C(NCCc1ccccc1)=C(Cl)C2=O</smiles>

107a

$\begin{array}{ll}-0.56 & -1.10\end{array}$ 
10<smiles>CC(C)(C)c1ccc(NC2=C(Cl)C(=O)c3cccnc3C2=O)cc1</smiles>

$109 a$

$-0.47 \quad-0.99$

11<smiles>COc1ccc(CNC2=C(Cl)C(=O)c3cccnc3C2=O)cc1</smiles>

$110 a$

$-0.56-1.08$

12<smiles>COc1ccc(CNC2=C(Cl)C(=O)c3ccc(C)nc3C2=O)cc1</smiles>

111a

$-0.56-1.12$

Redox potential (determined by CV in DMF) vs. MIC of selected QQs

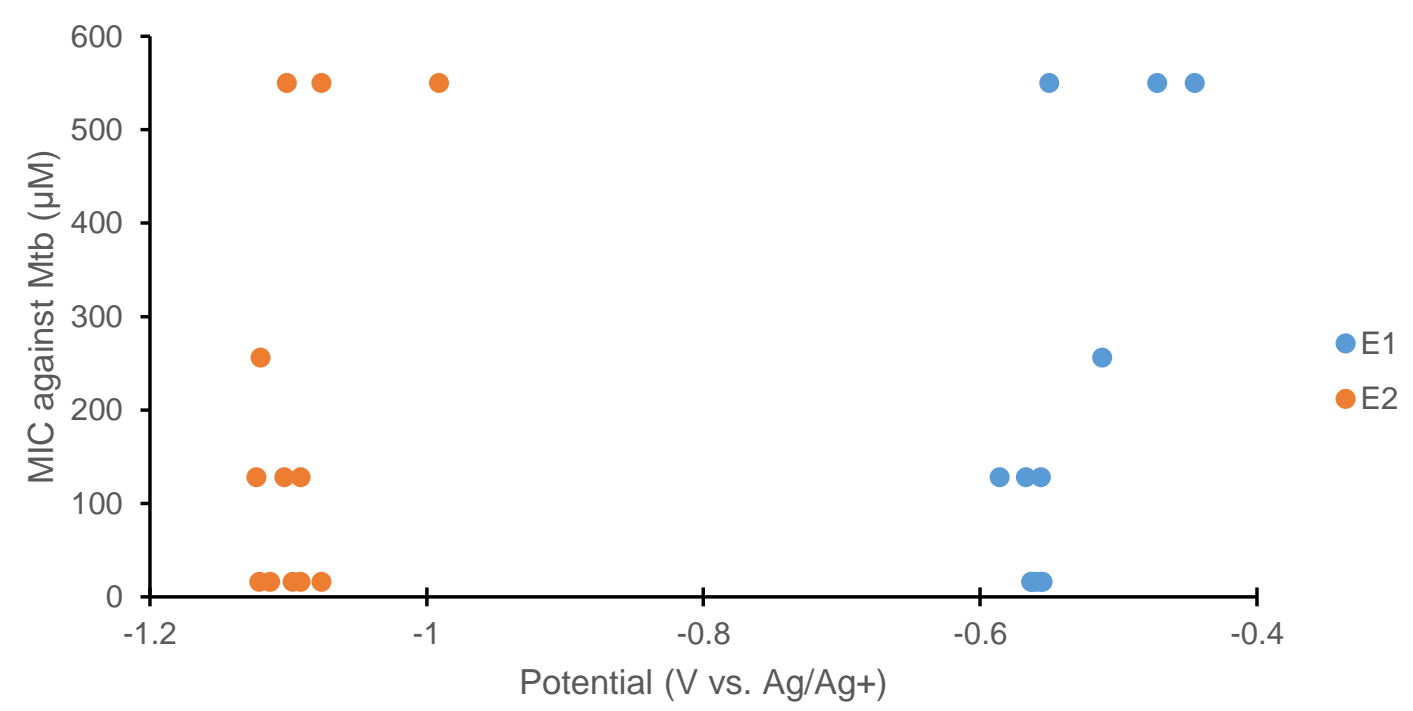

Figure A.3.1.: Lack of correlation between redox potentials obtained for a selection of QQs via CV experiments in DMF and their anti-tubercular activity. 
${ }^{1} \mathrm{H}$ NMR $\left(500 \mathrm{MHz}, \mathrm{CDCl}_{3}\right)$
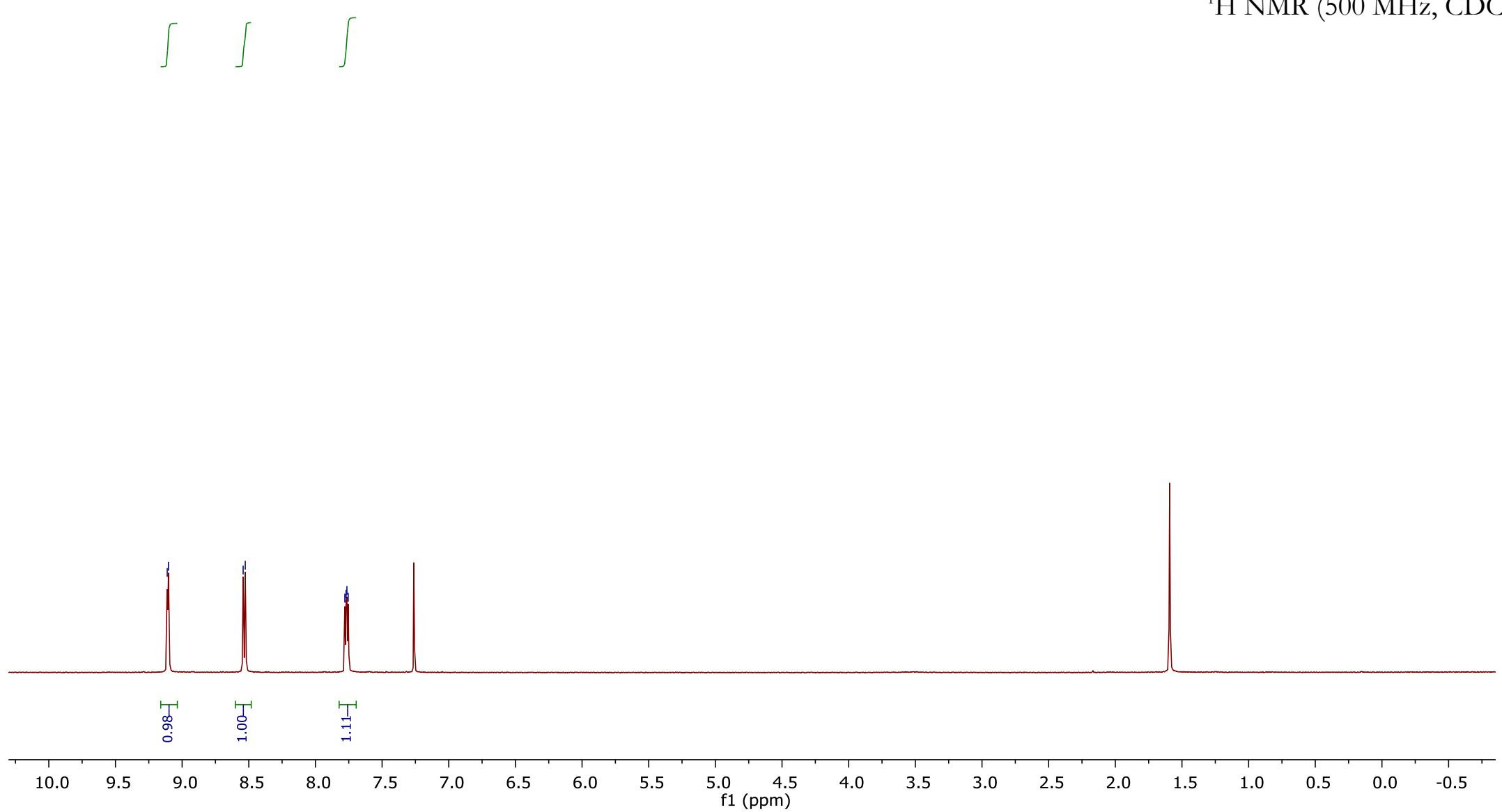


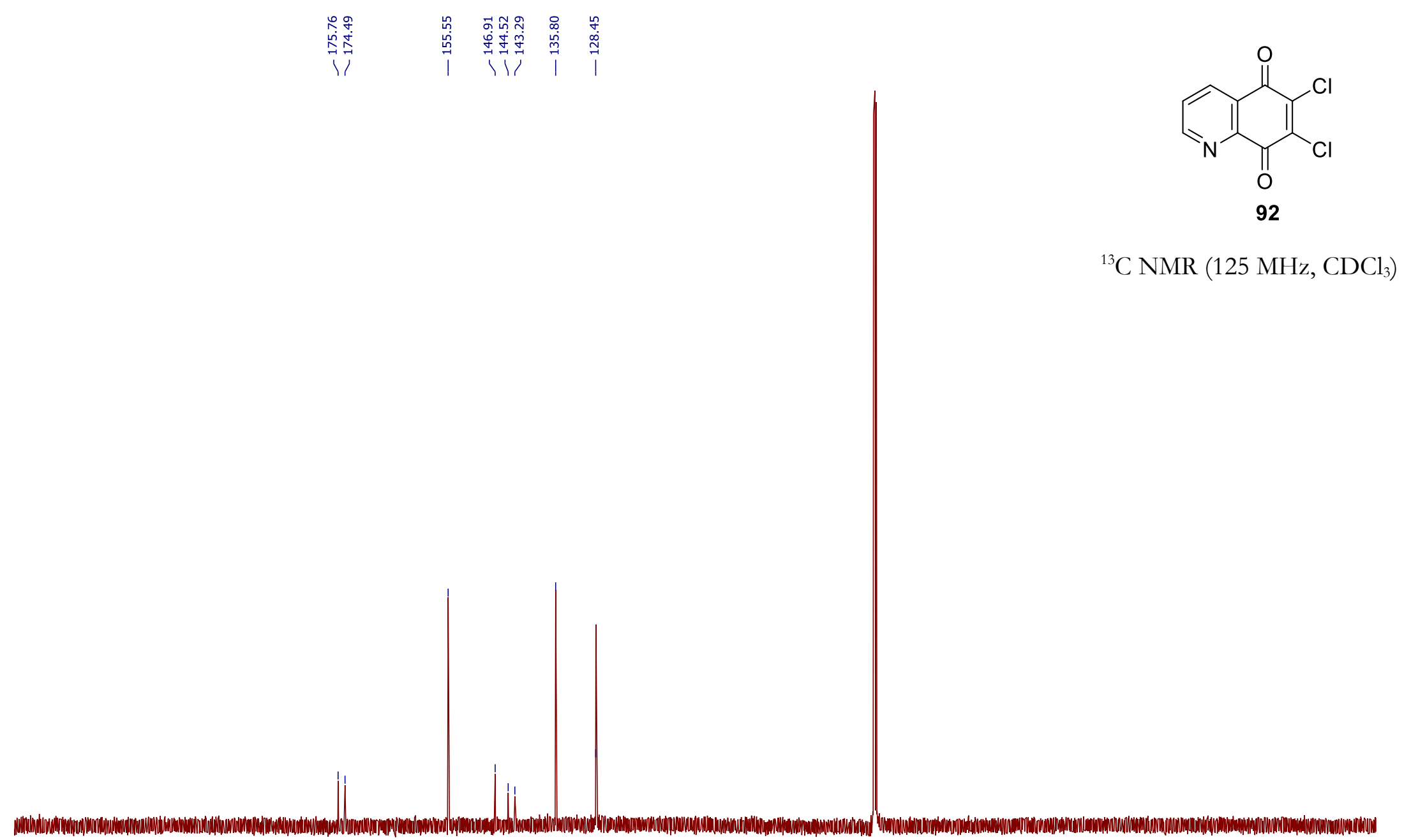

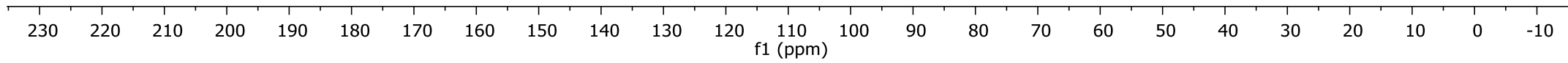




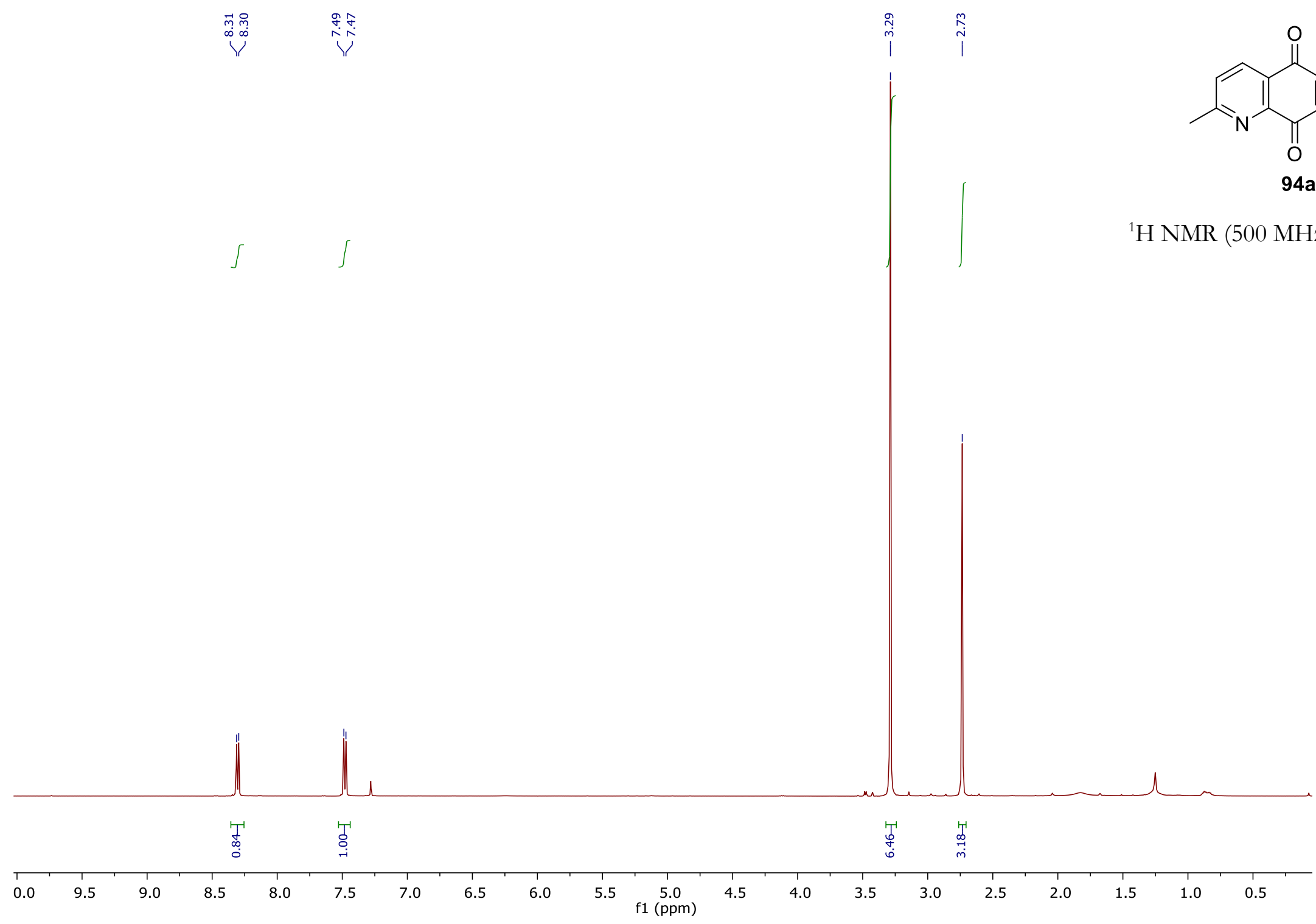




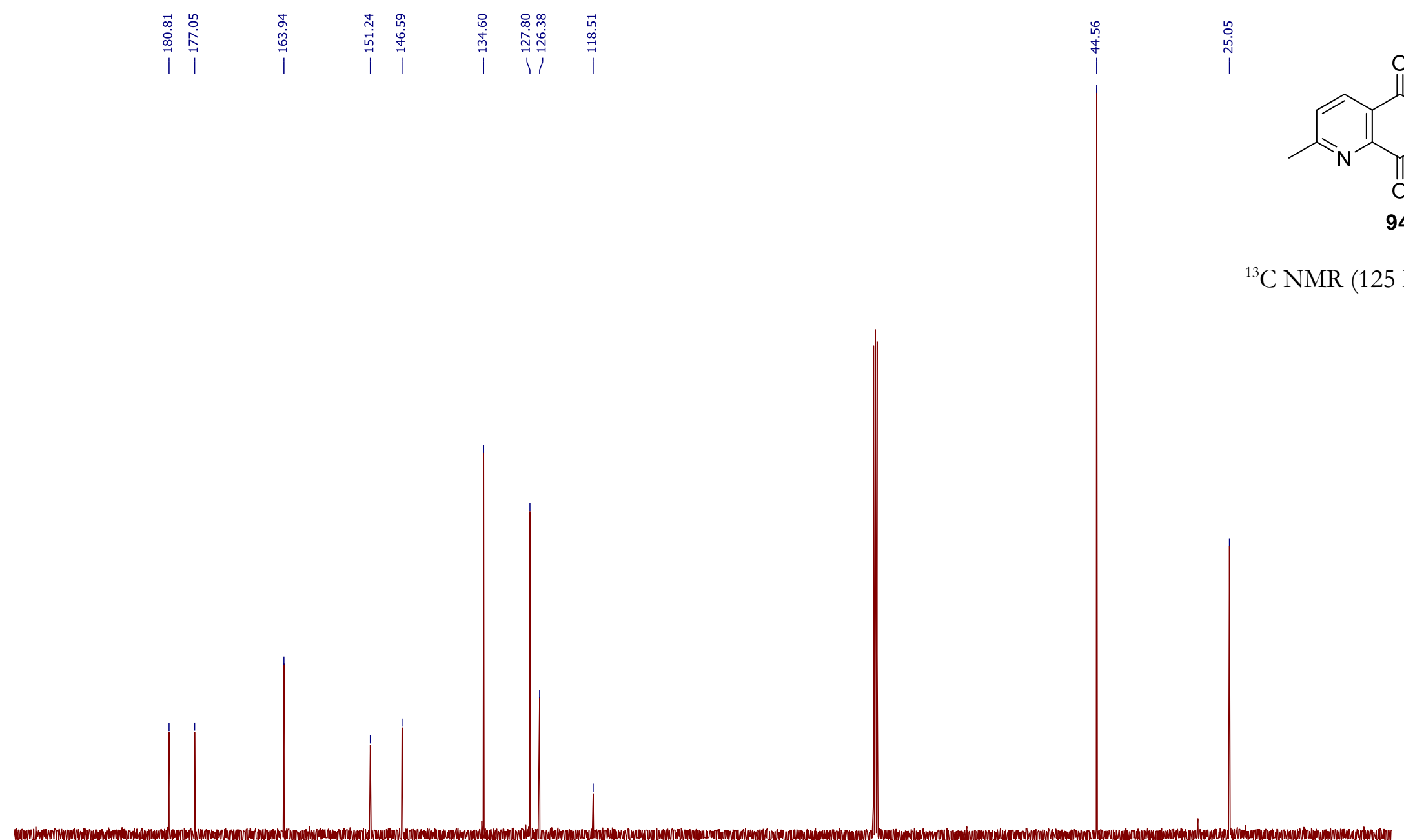

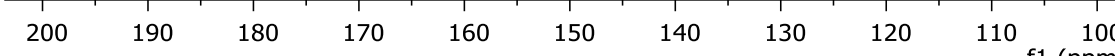
$90 \quad 80$ $70 \quad 60$ $50 \quad 40$

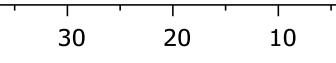




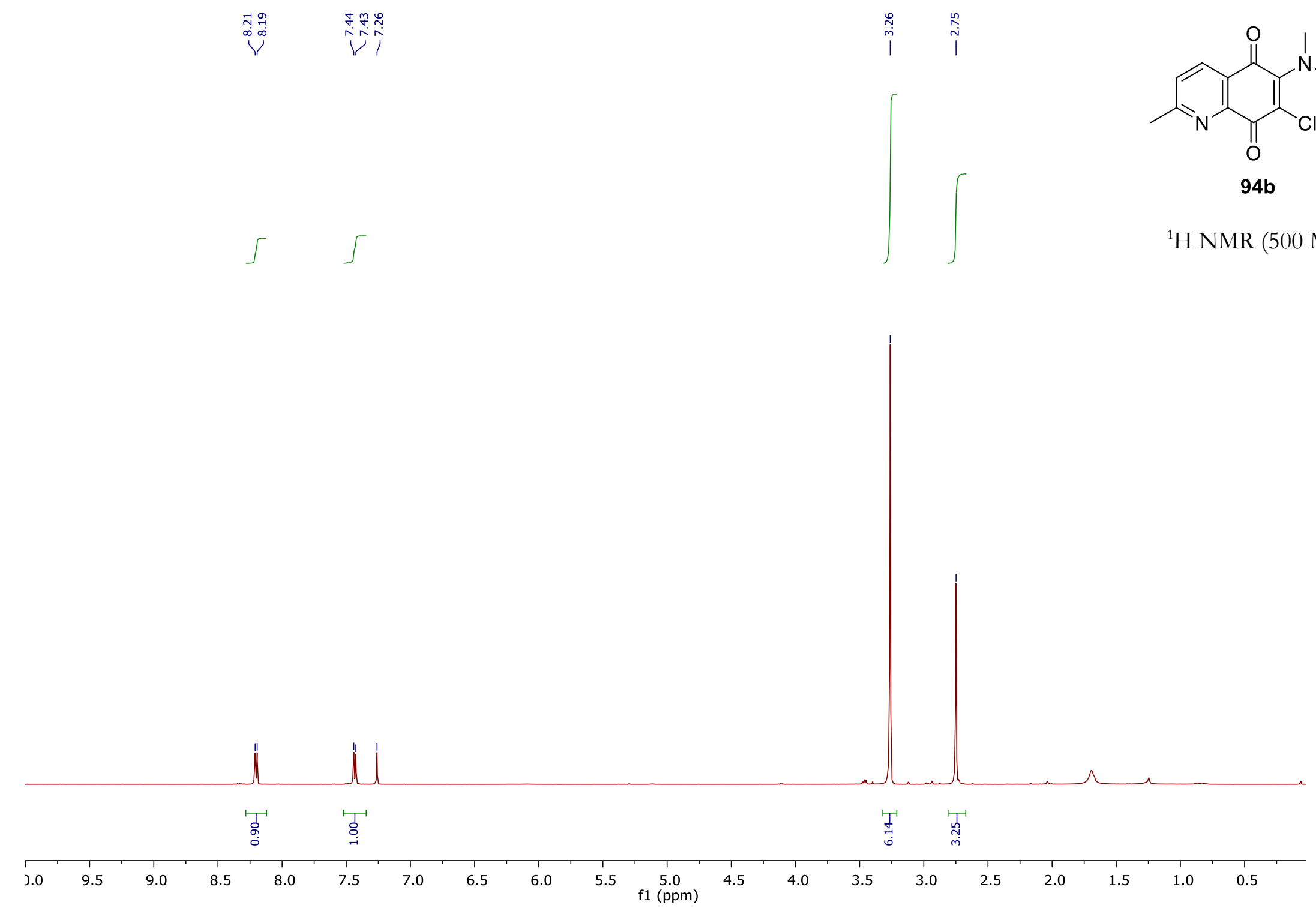




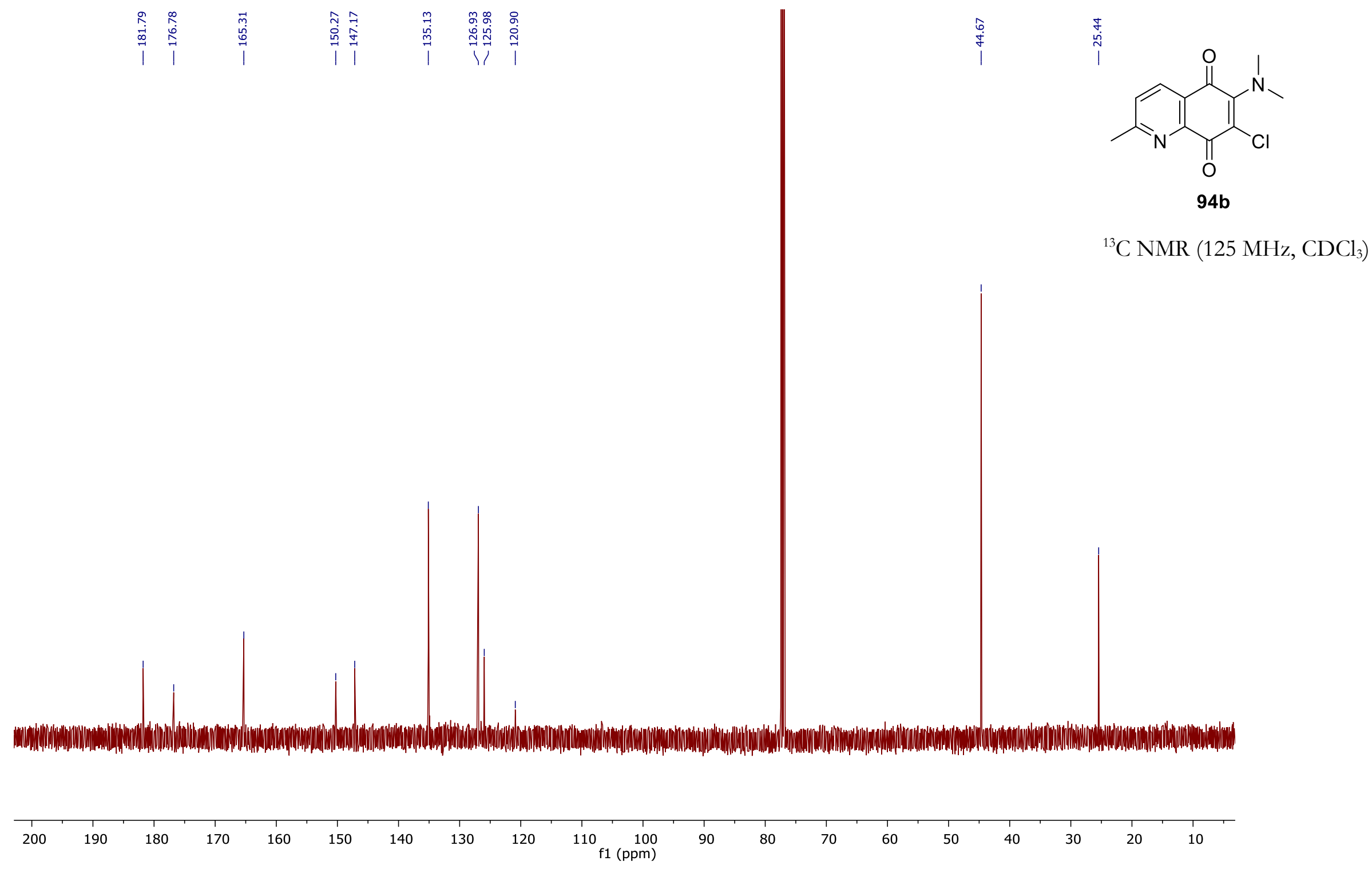




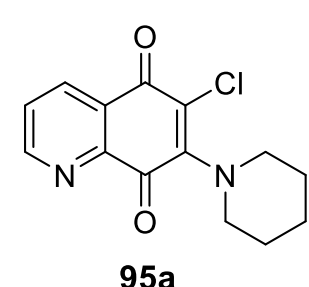

${ }^{1} \mathrm{H}$ NMR $\left(500 \mathrm{MHz}, \mathrm{CDCl}_{3}\right)$

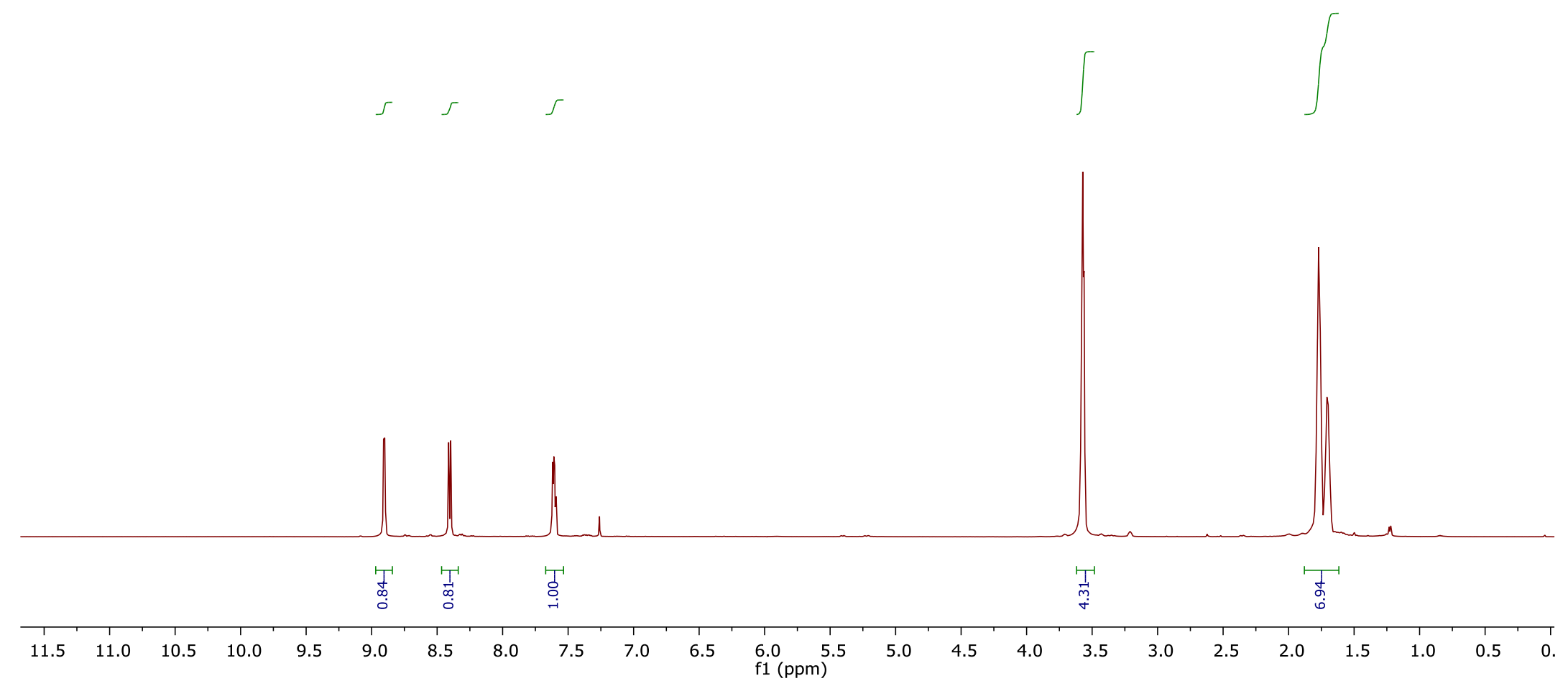



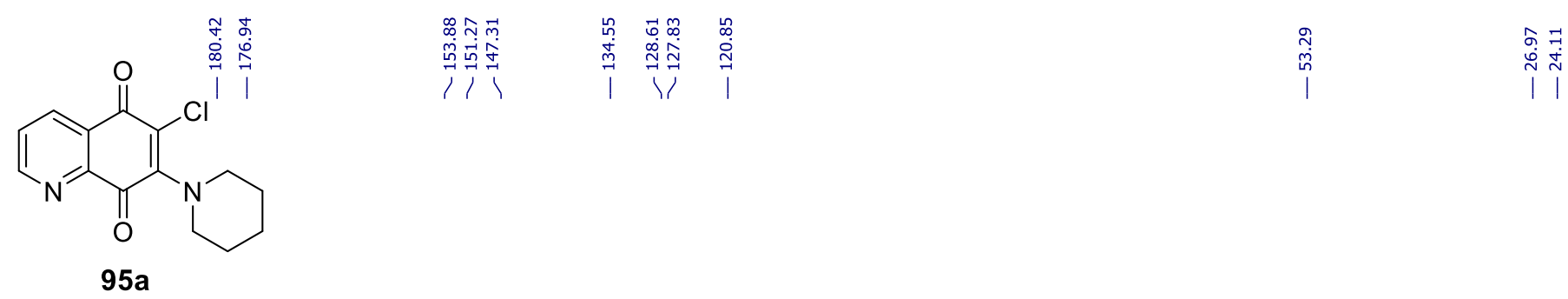

${ }^{13} \mathrm{C}$ NMR $\left(125 \mathrm{MHz}, \mathrm{CDCl}_{3}\right)$

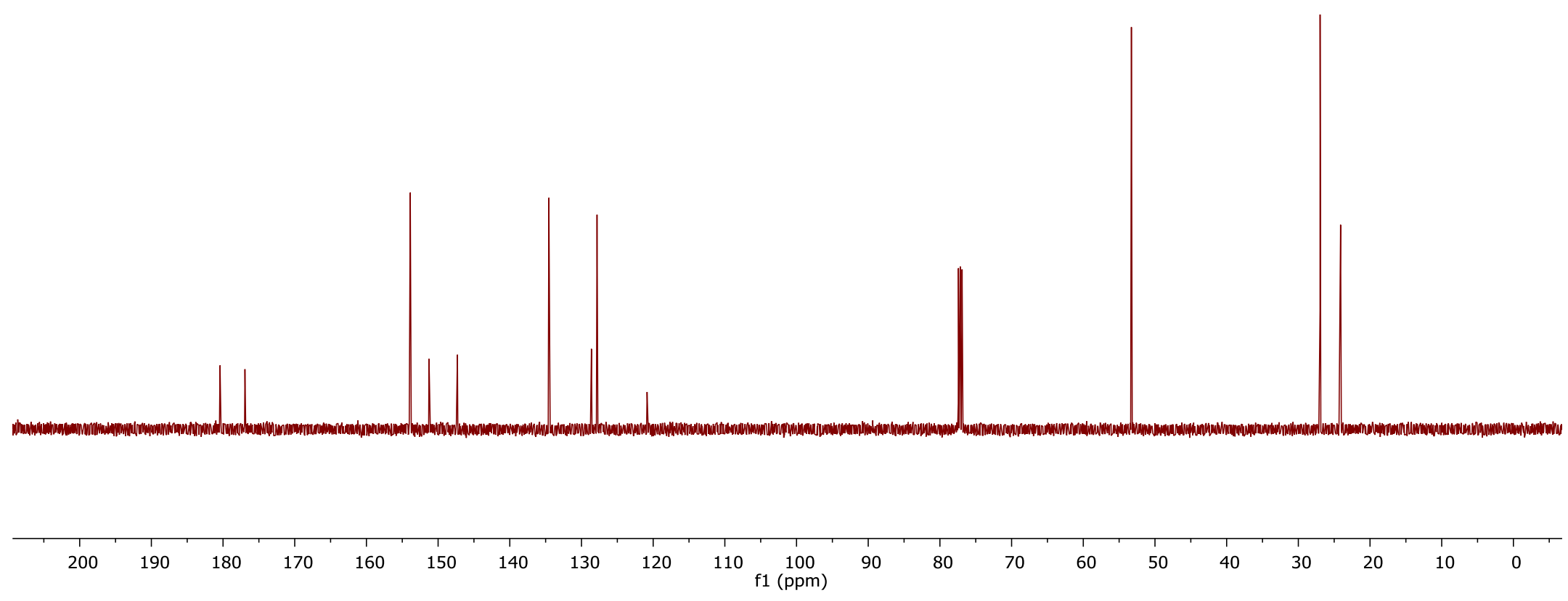




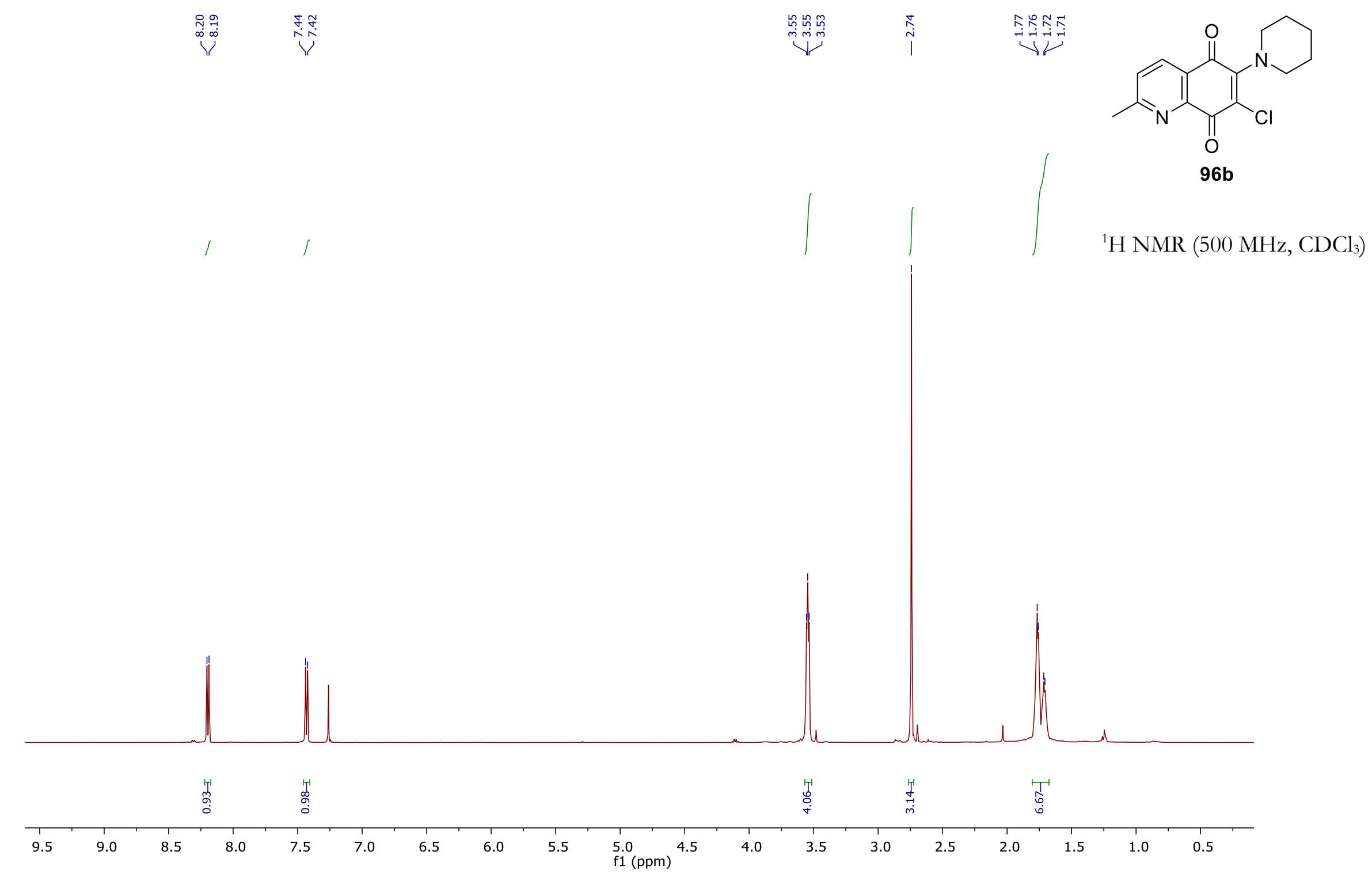




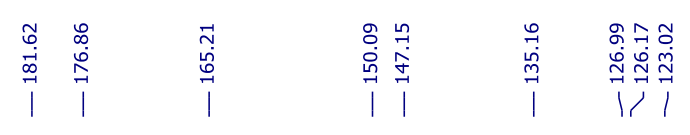

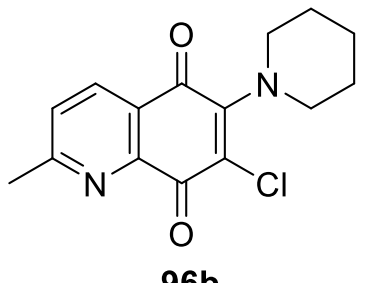

96b

${ }^{13} \mathrm{C}$ NMR $\left(125 \mathrm{MHz}, \mathrm{CDCl}_{3}\right)$

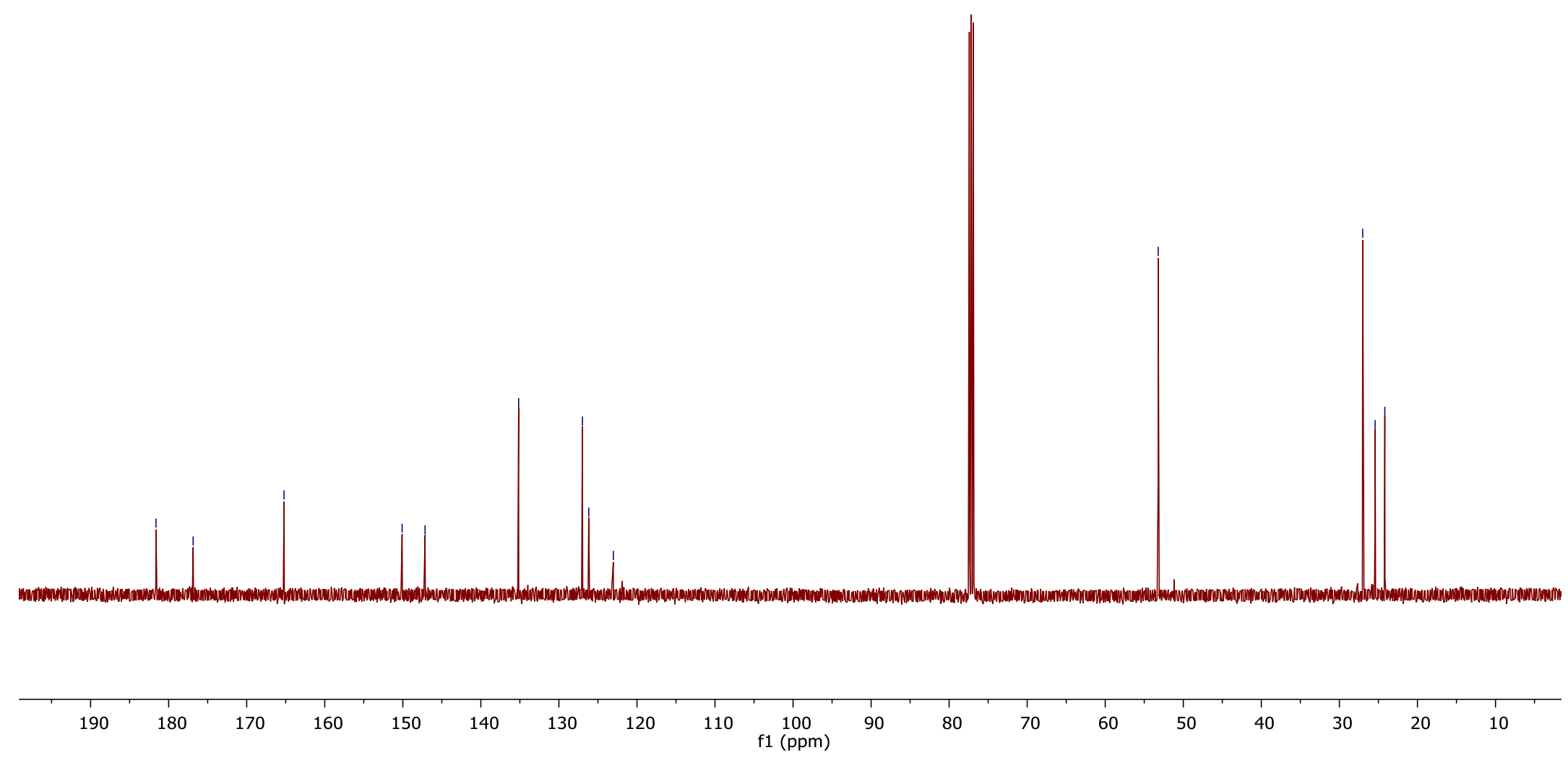




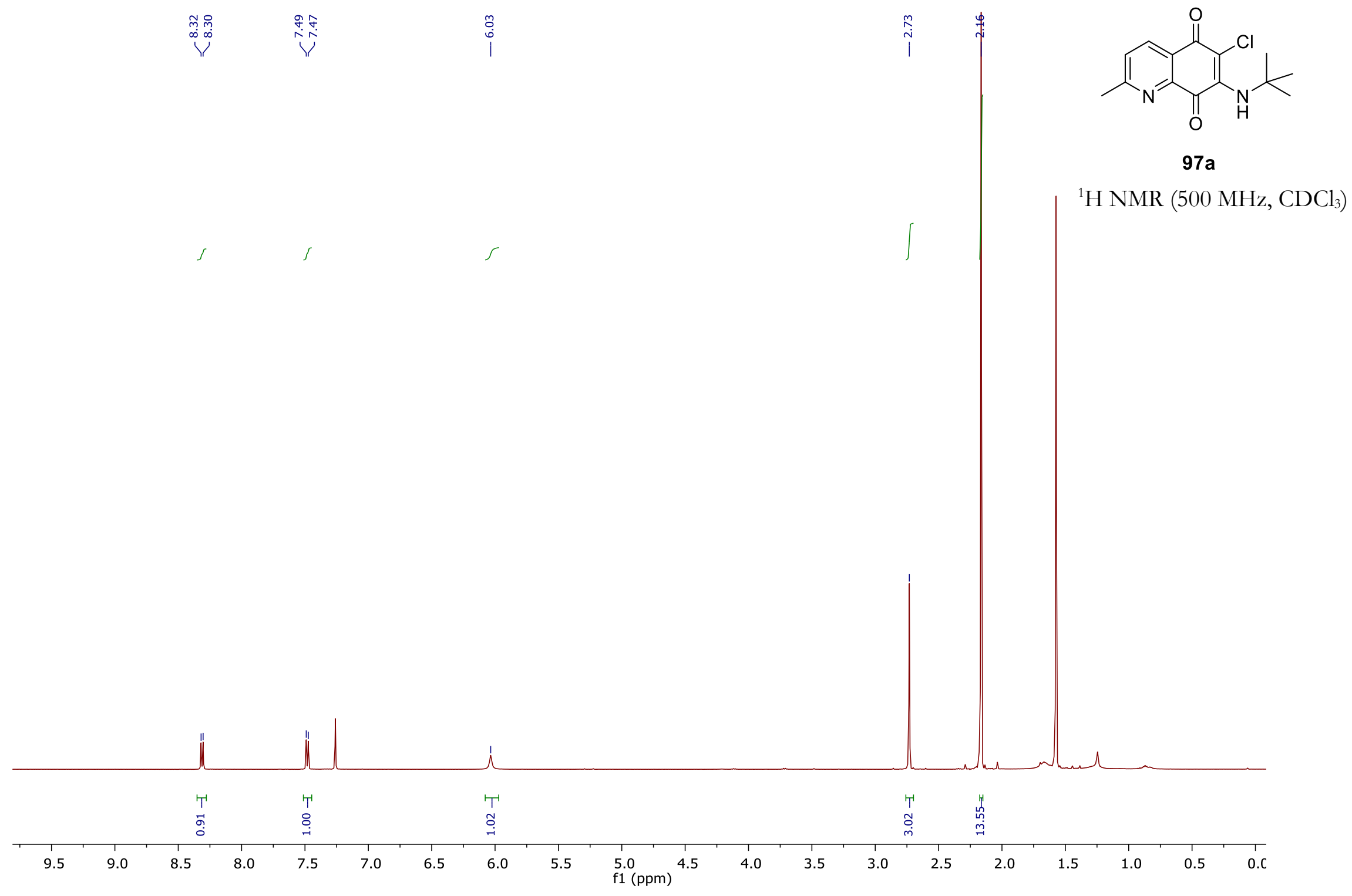



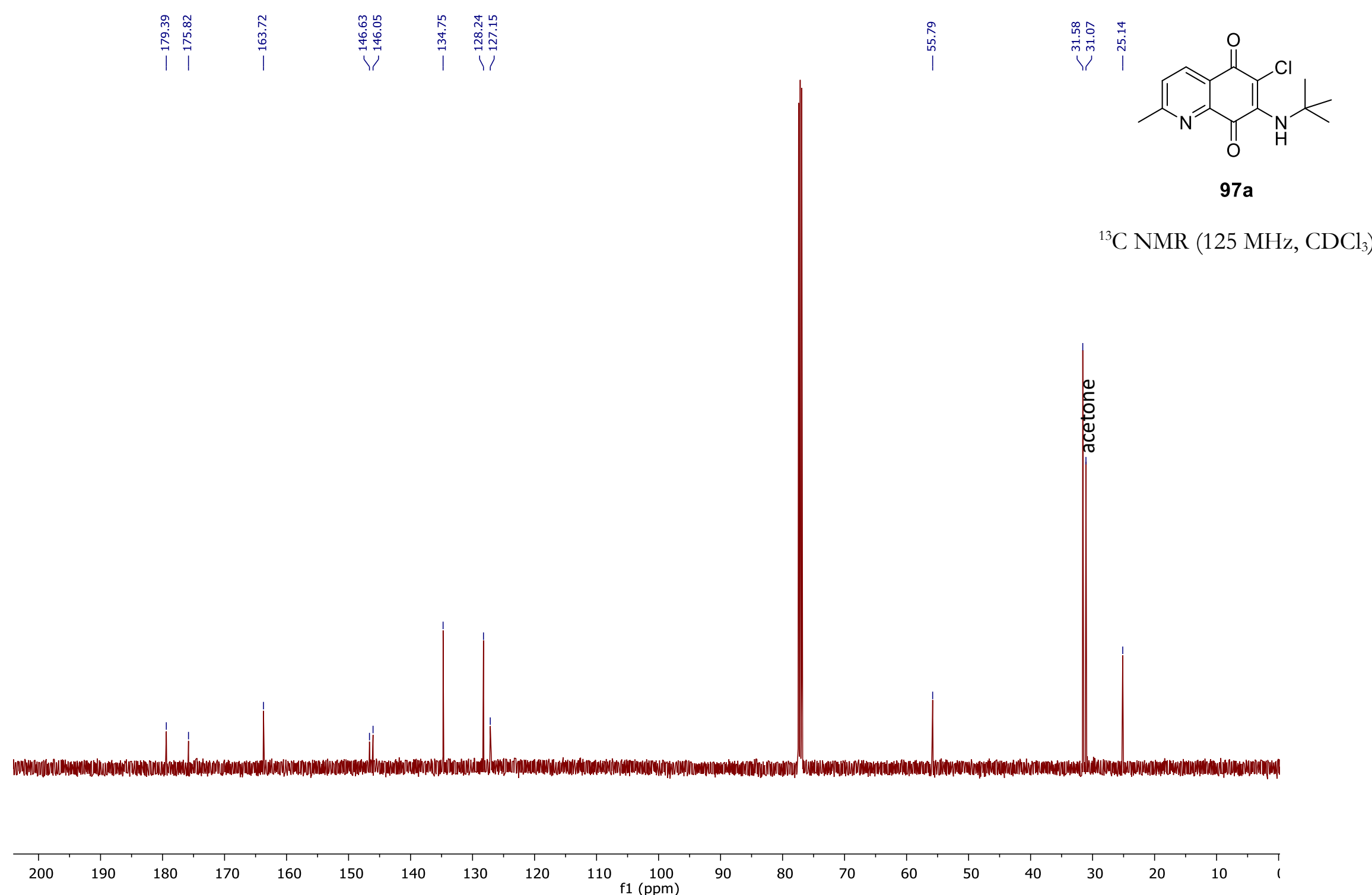


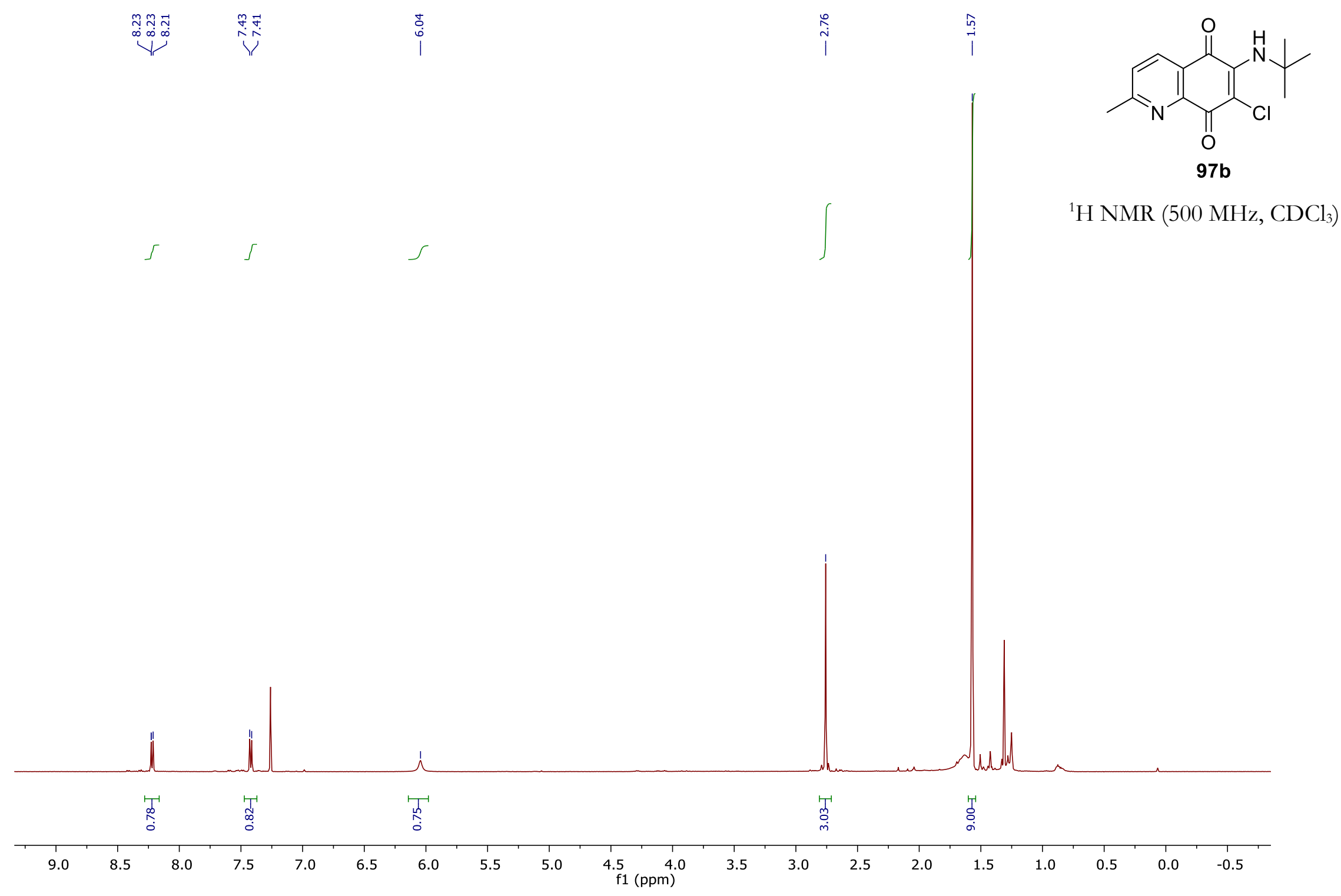




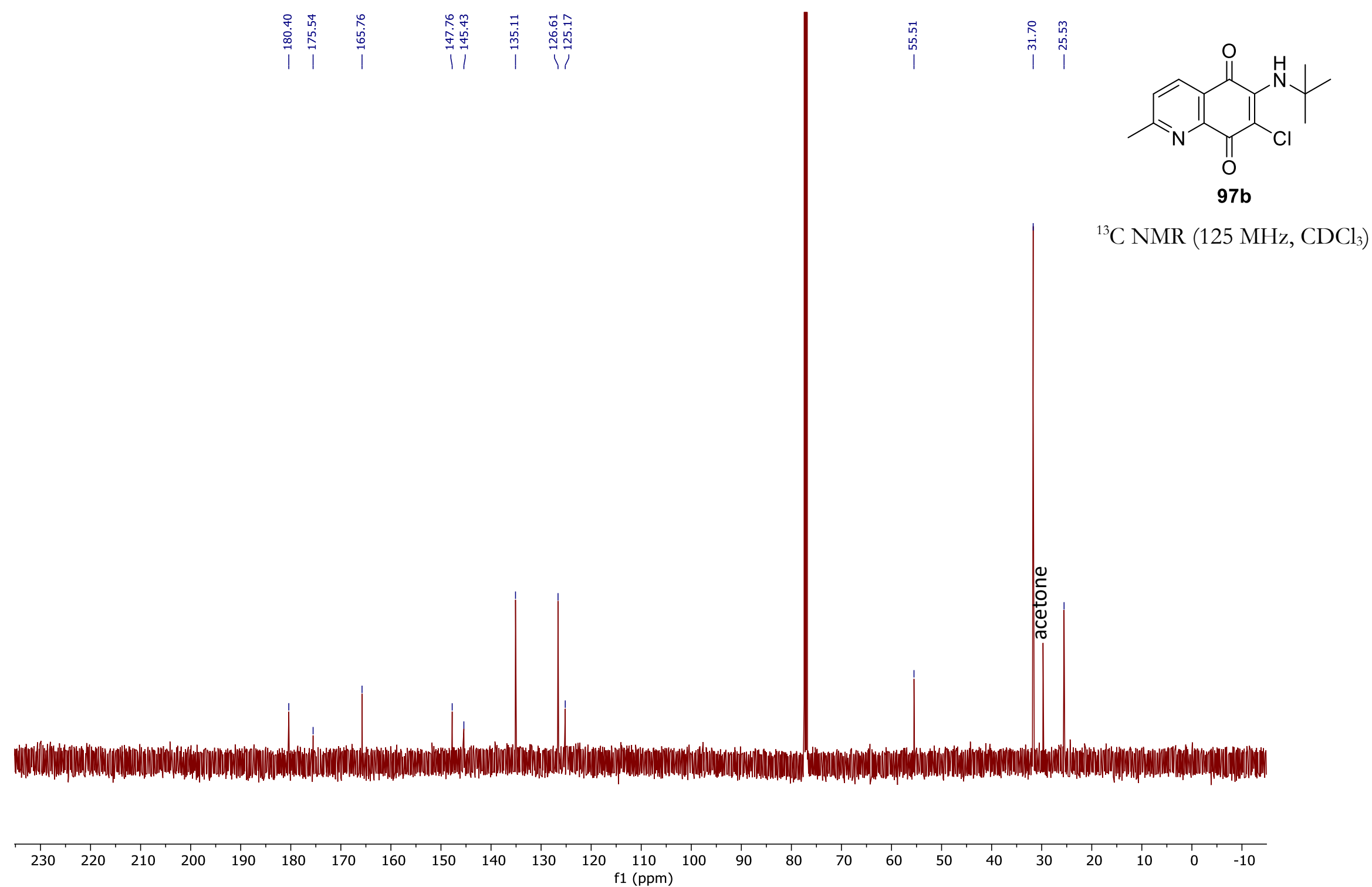




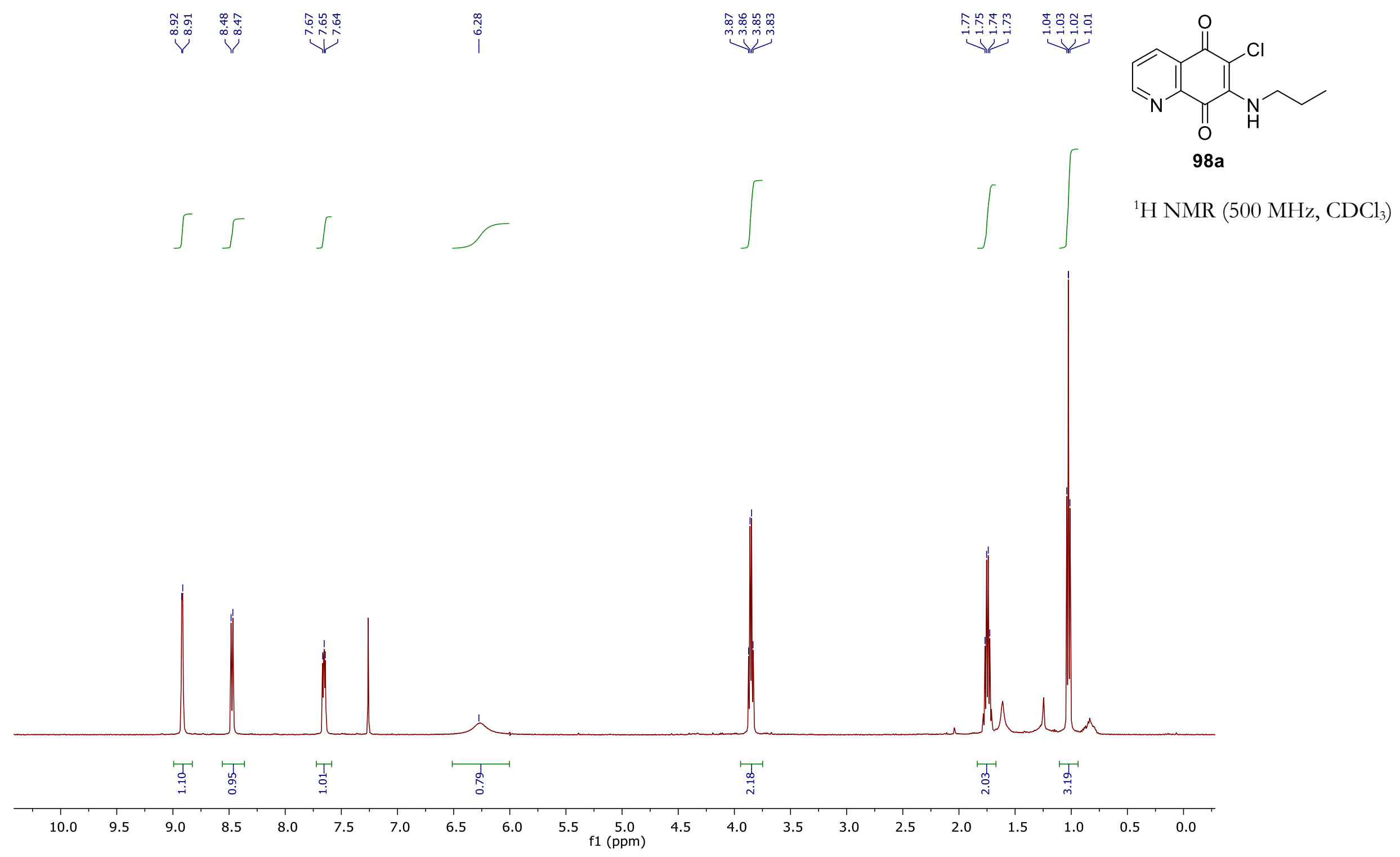



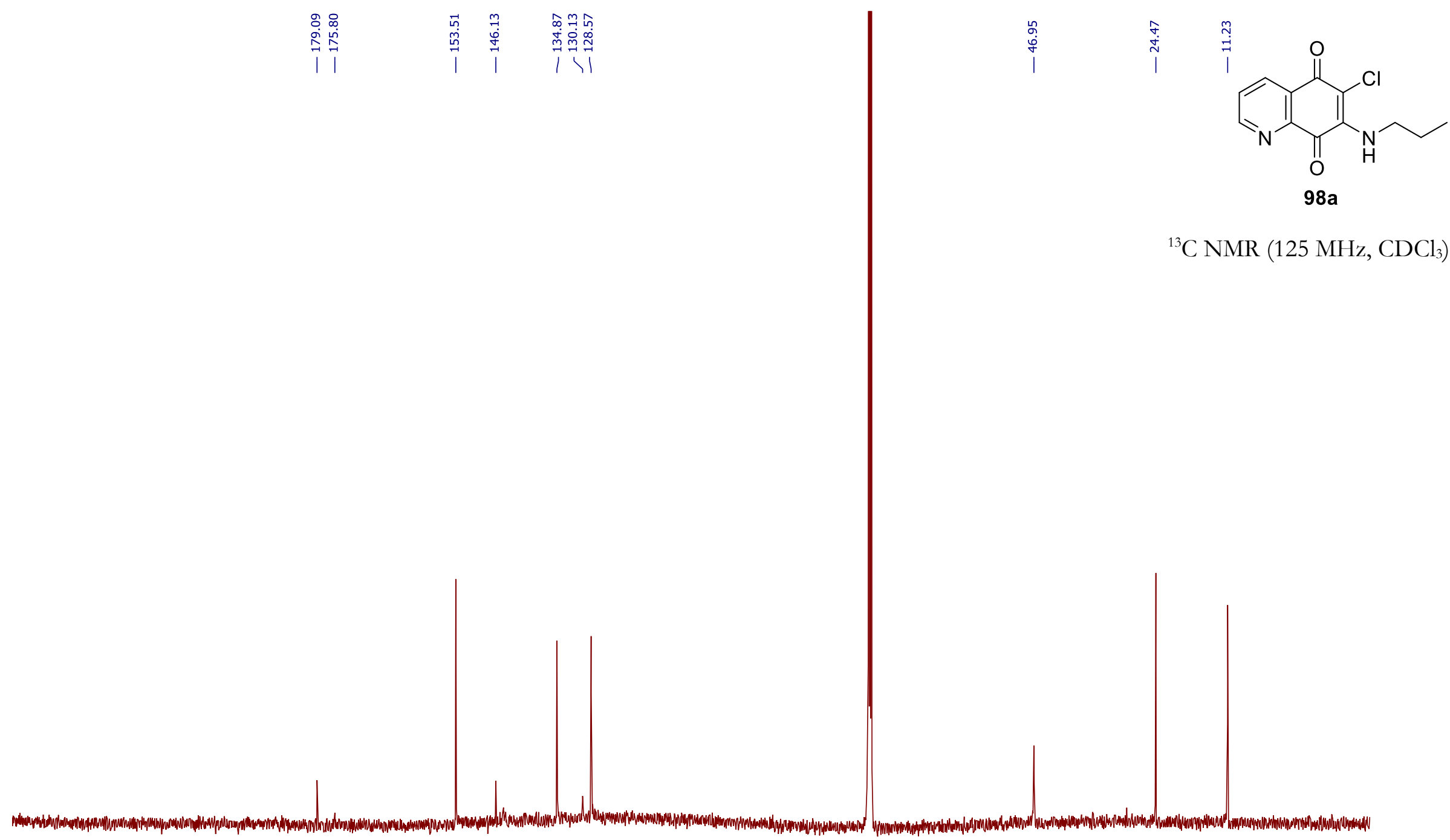

98a

${ }^{13} \mathrm{C}$ NMR (125 MHz, $\left.\mathrm{CDCl}_{3}\right)$

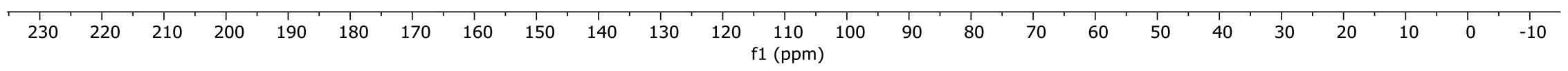

A53 


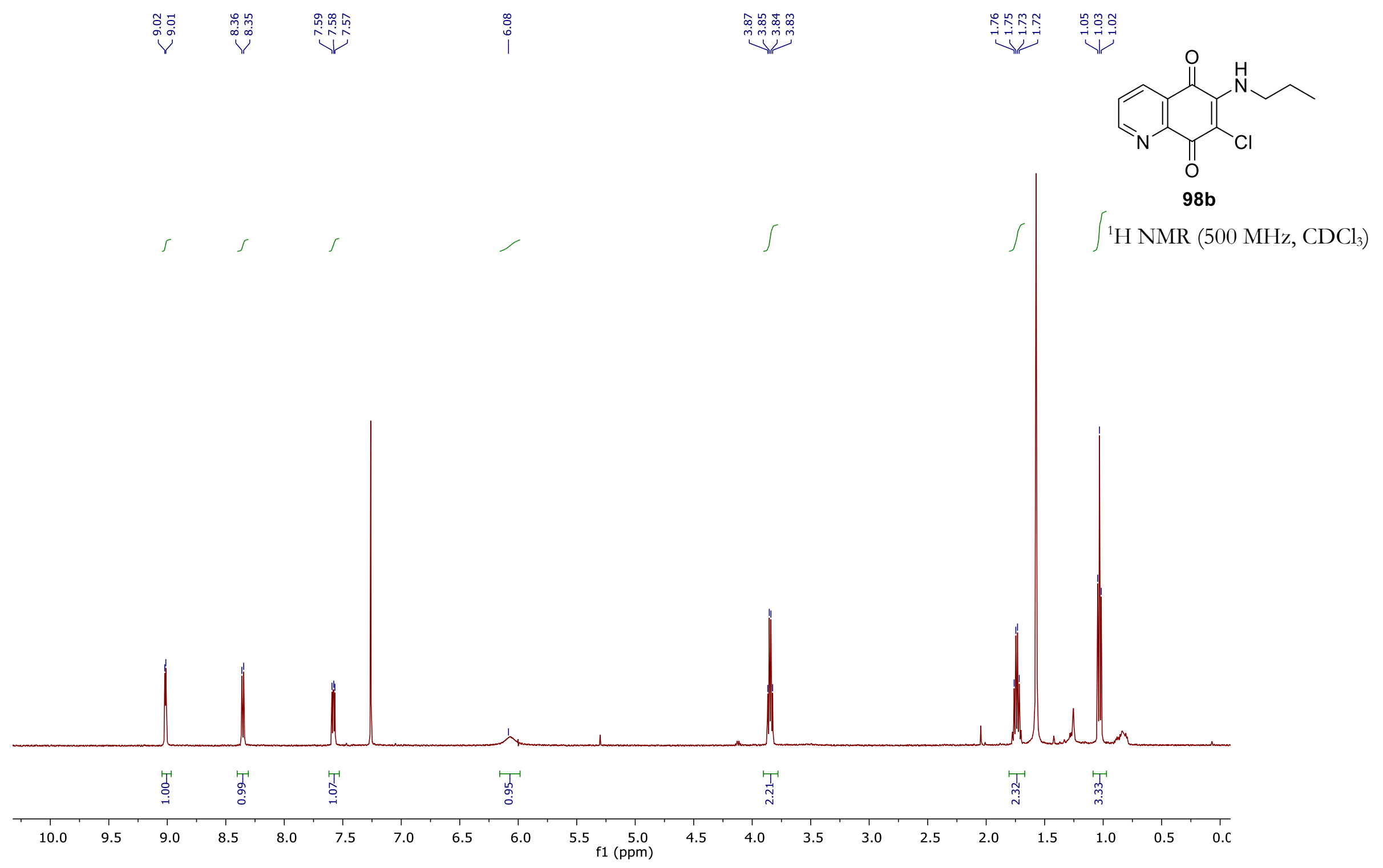




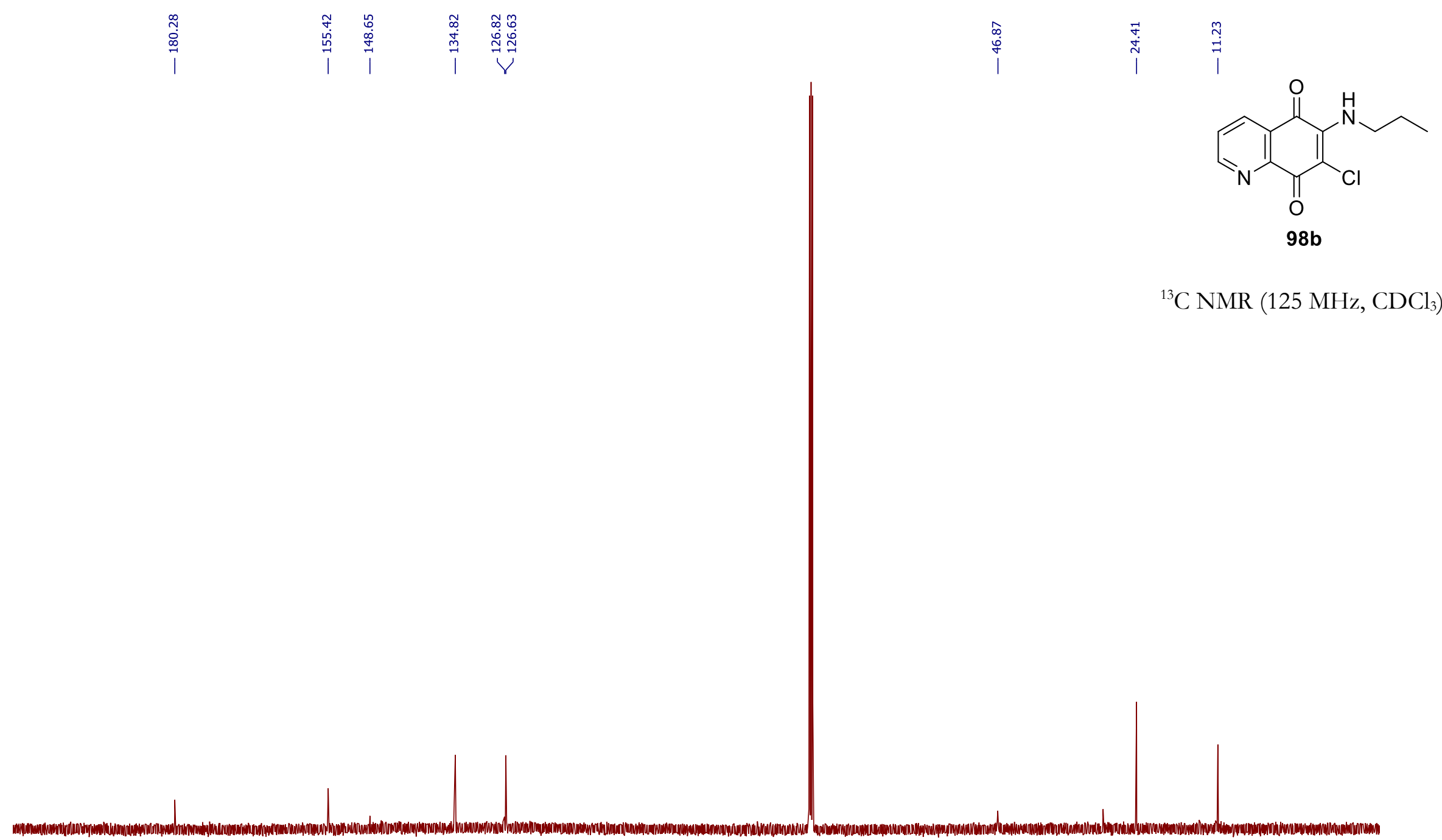

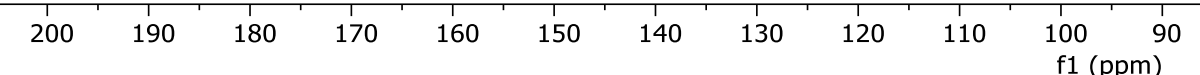




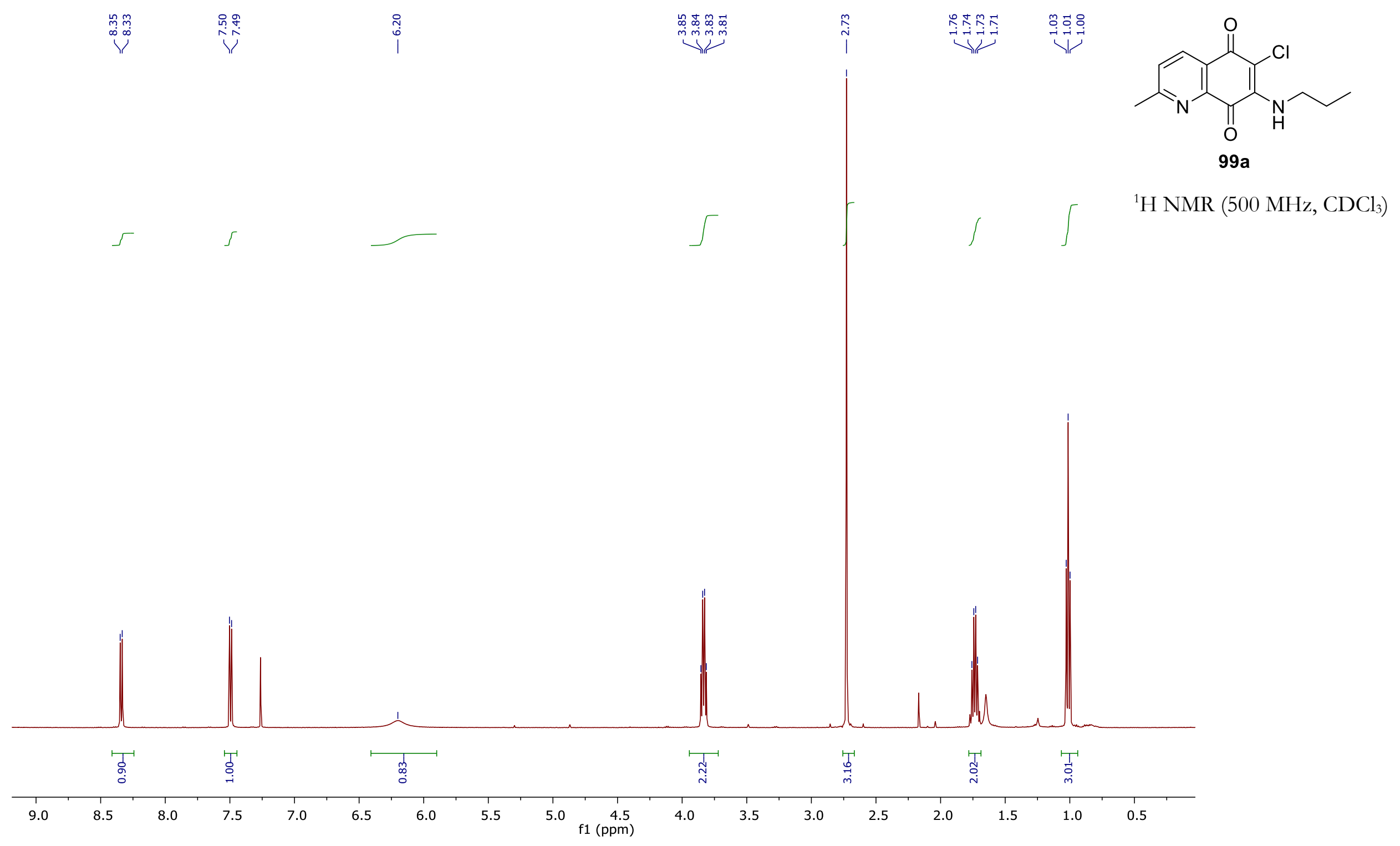



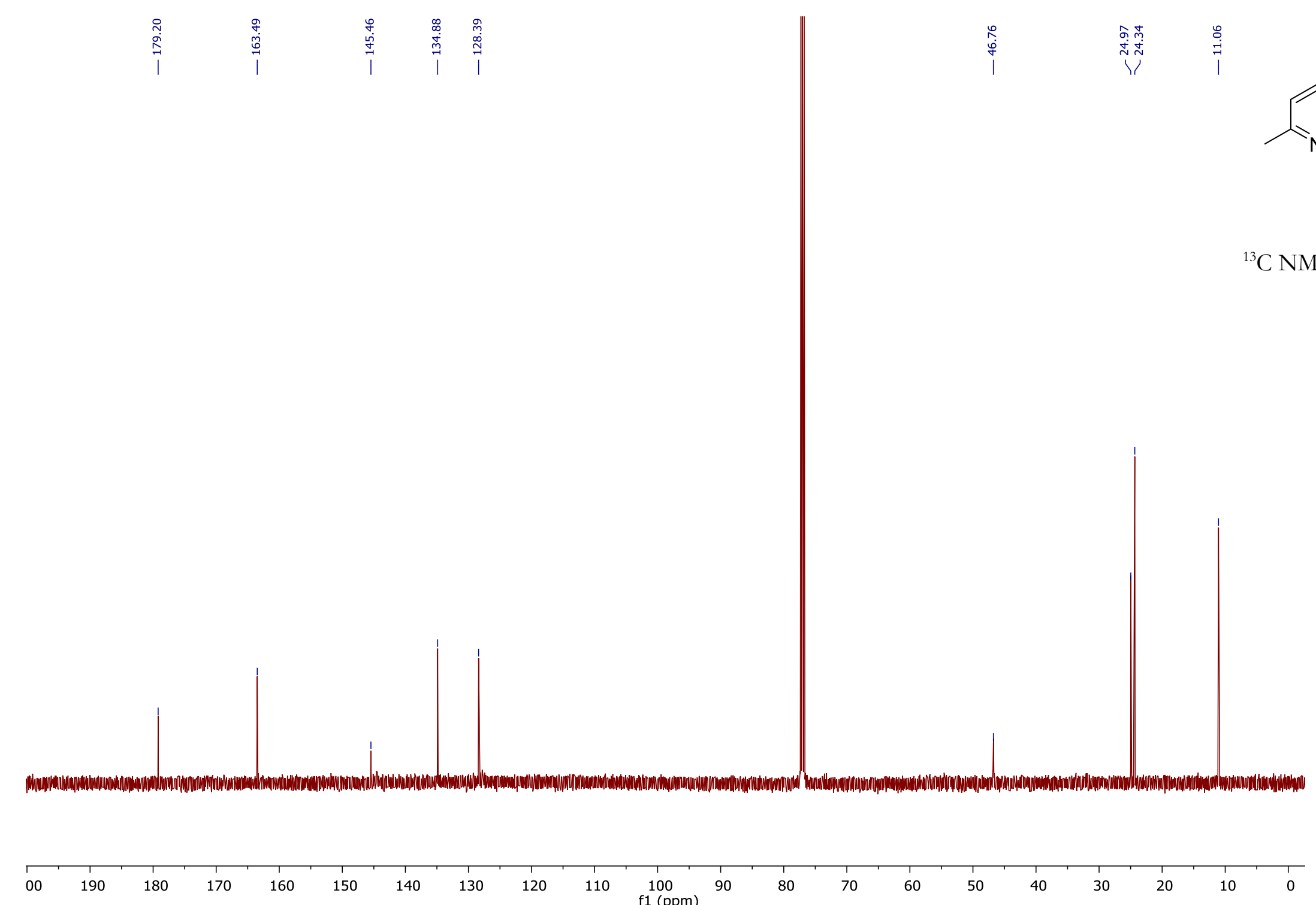


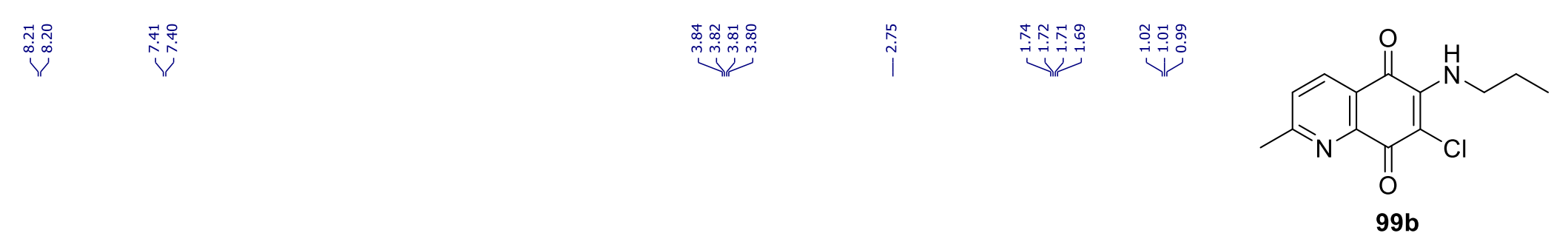

${ }^{1} \mathrm{H}$ NMR $\left(500 \mathrm{MHz}, \mathrm{CDCl}_{3}\right)$

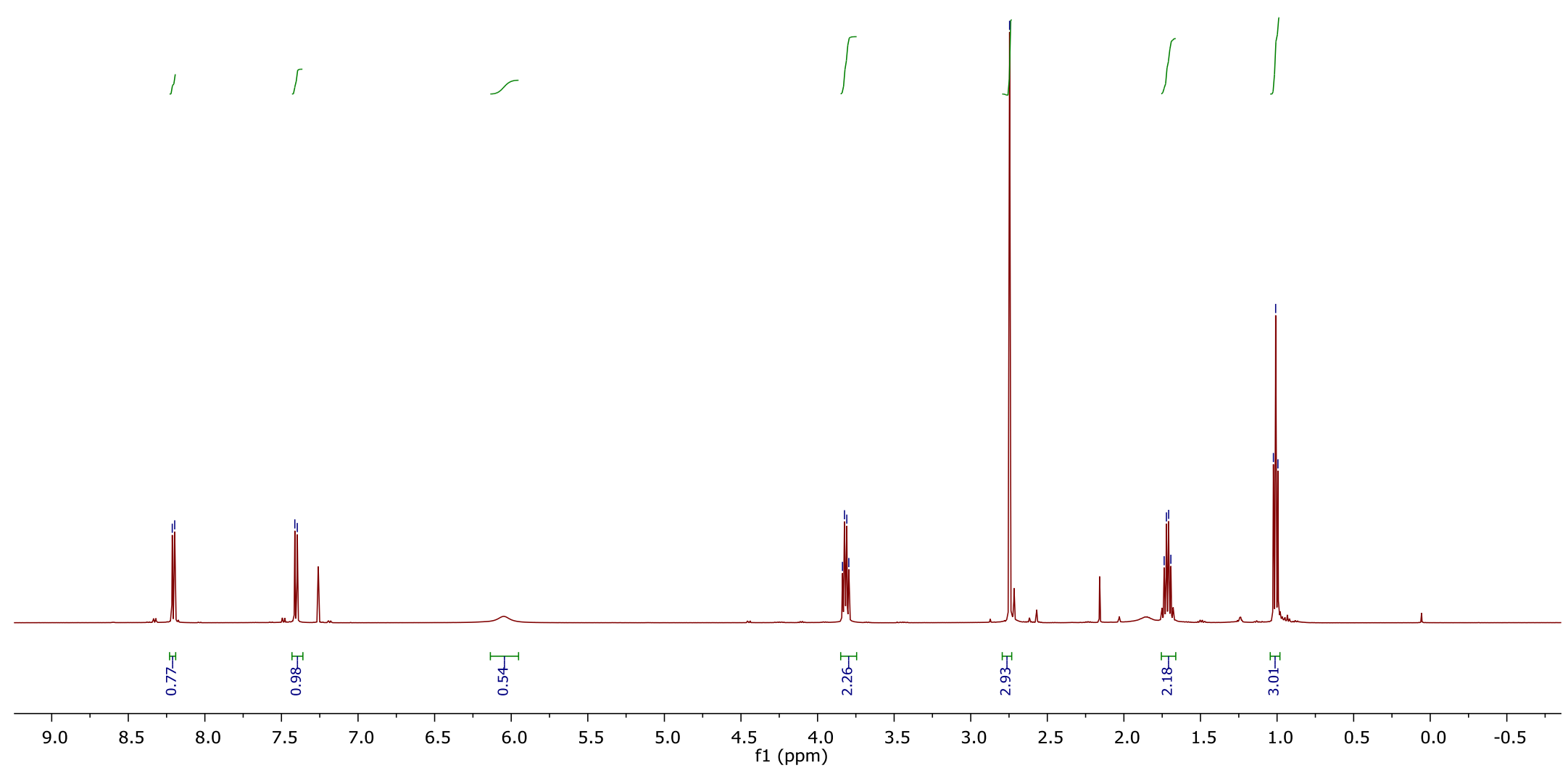




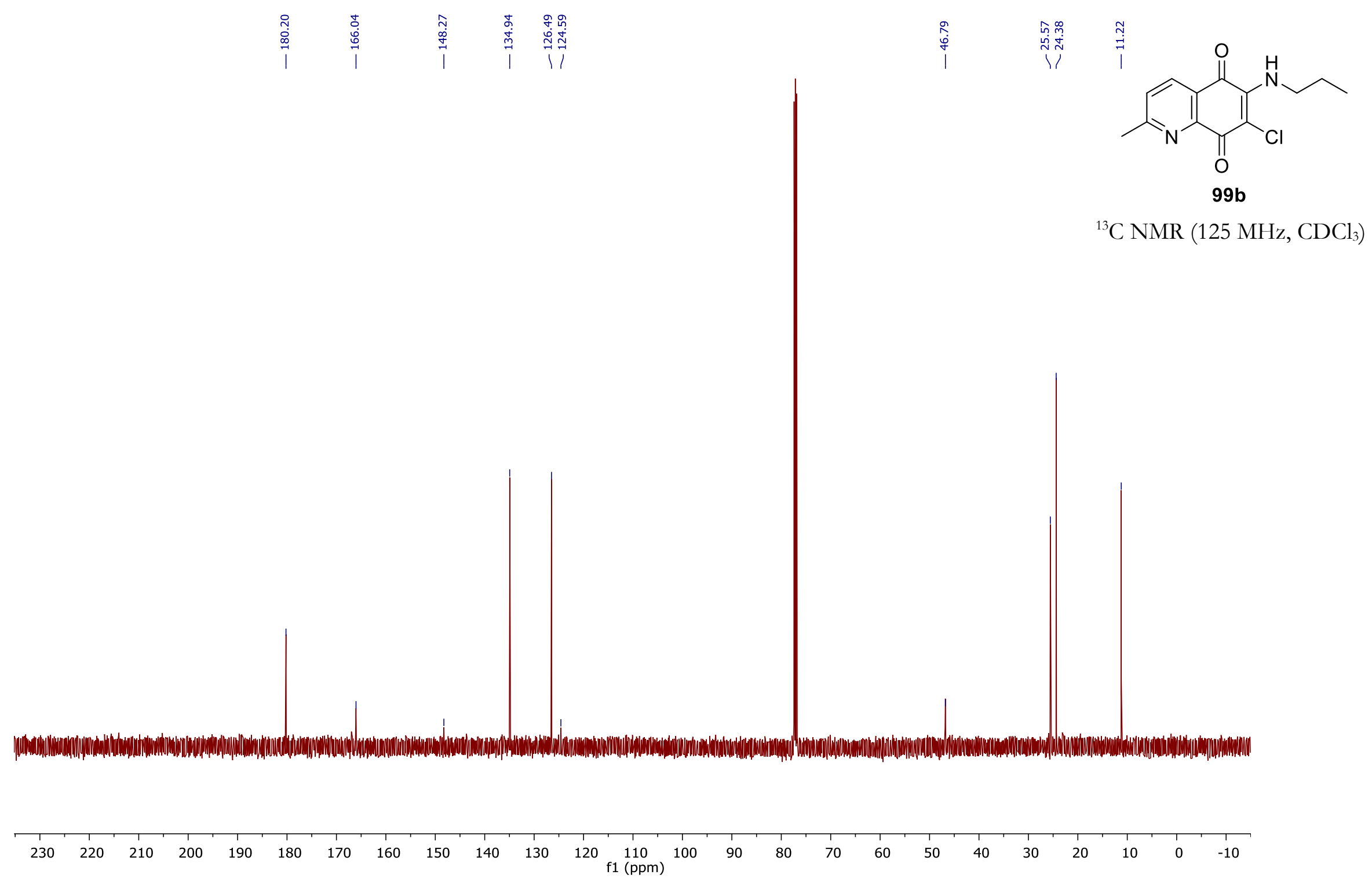




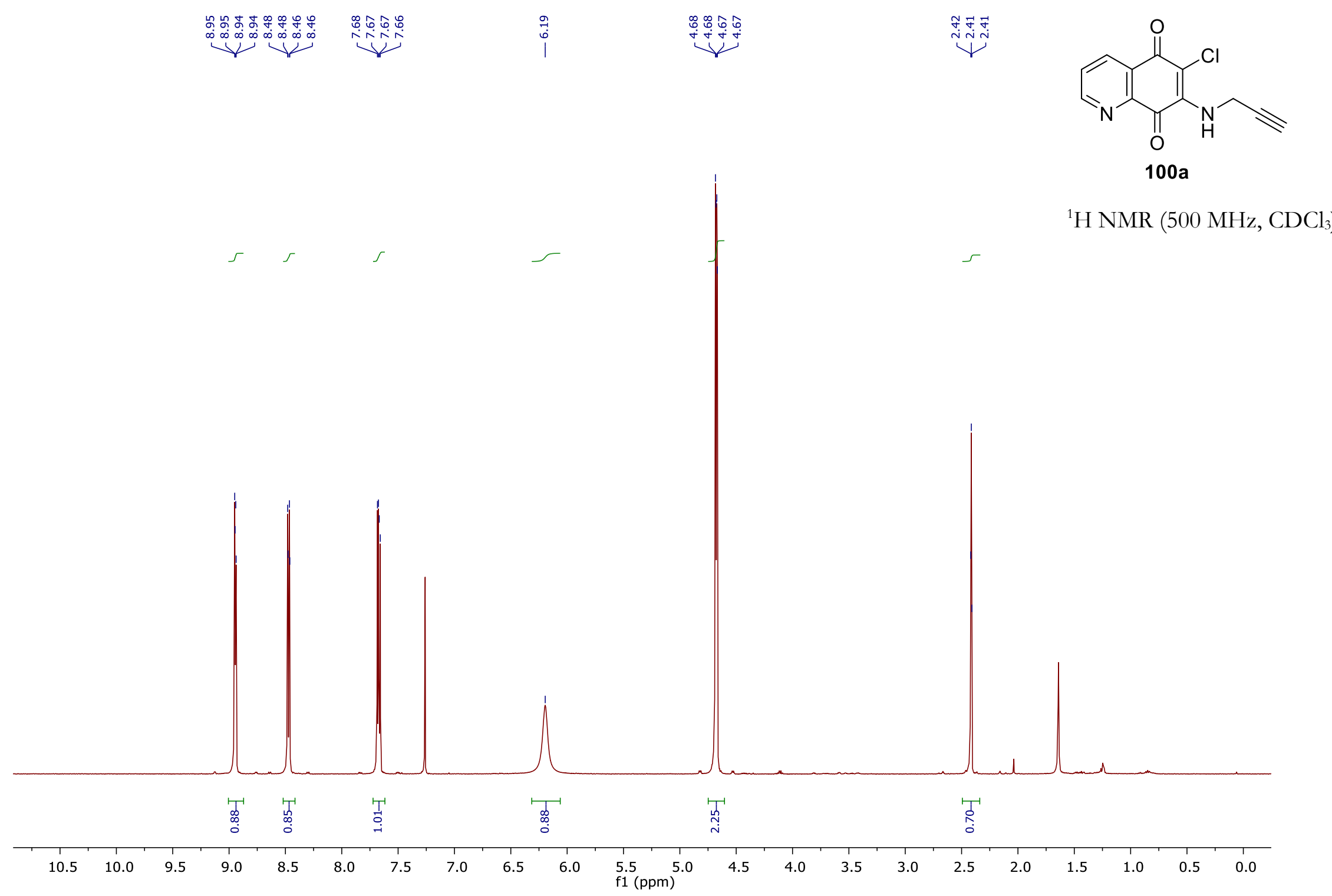




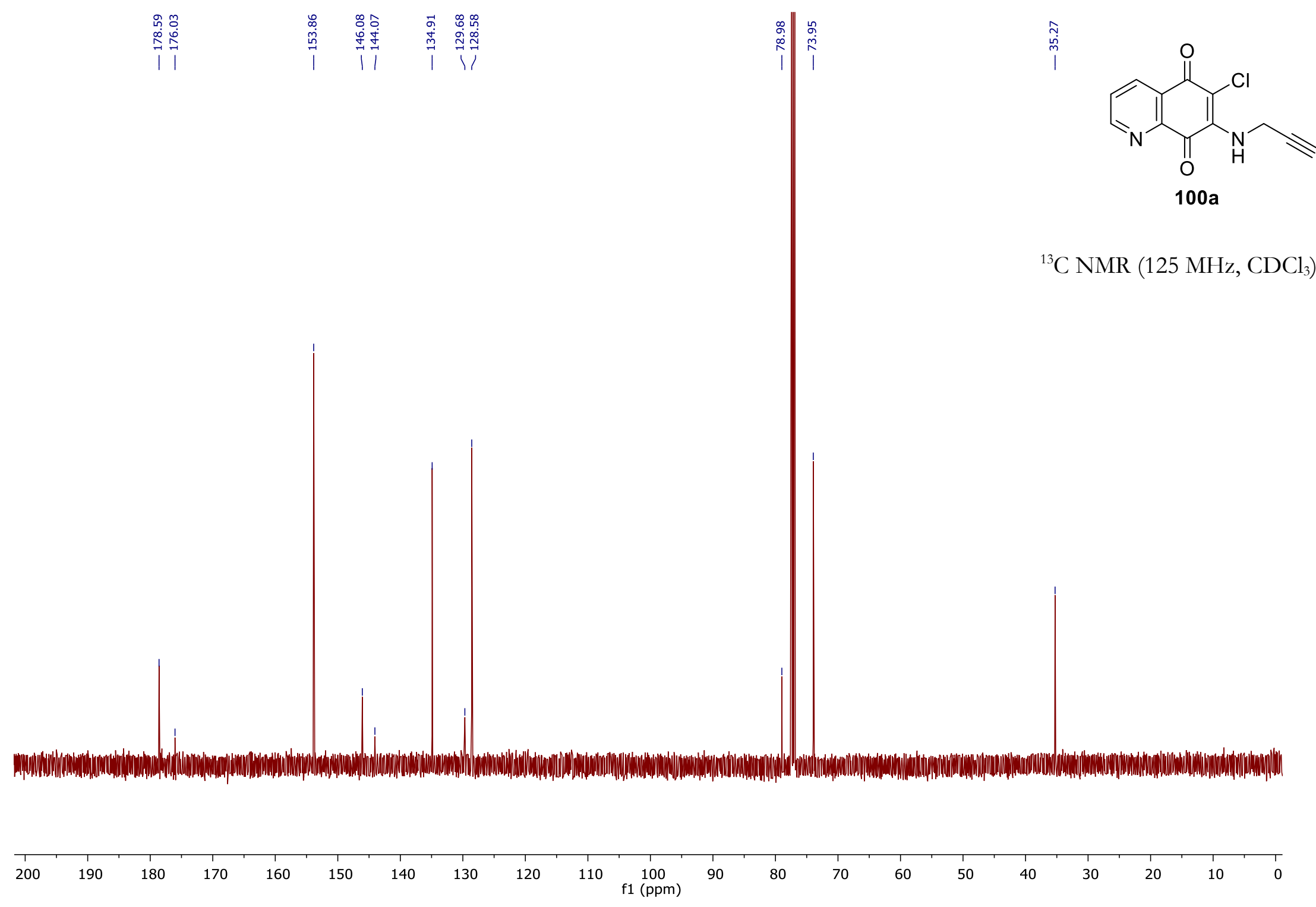




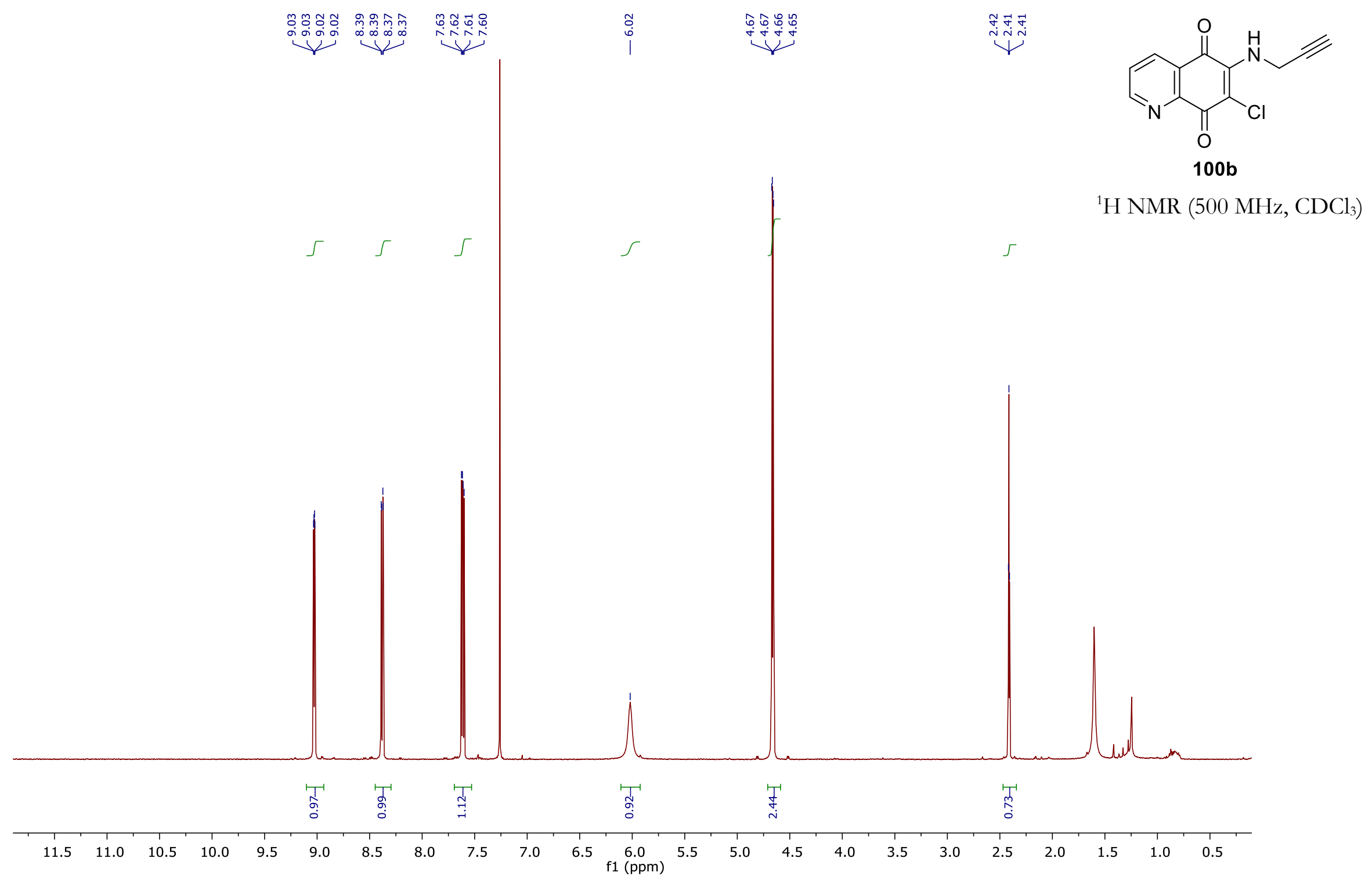




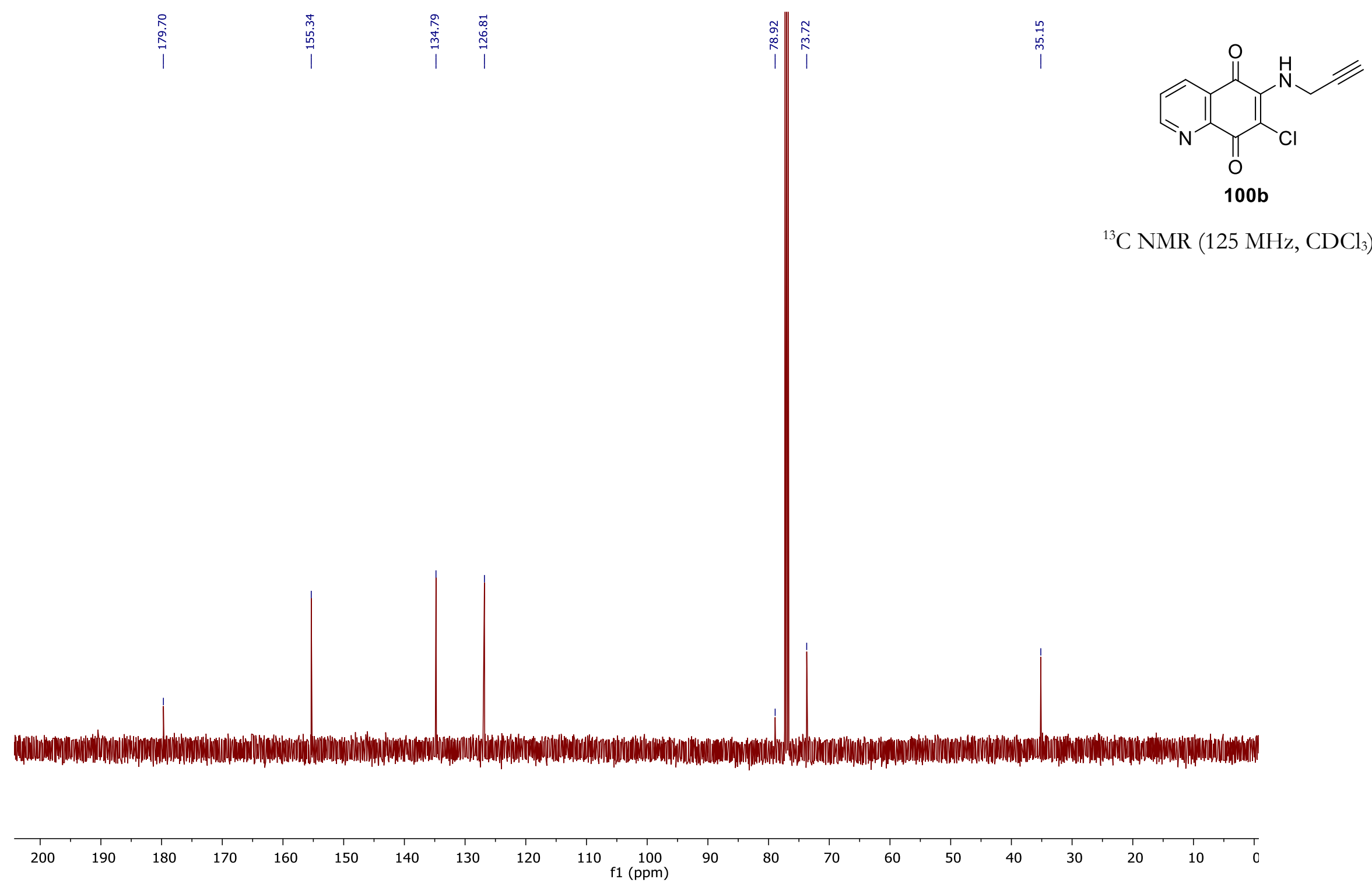




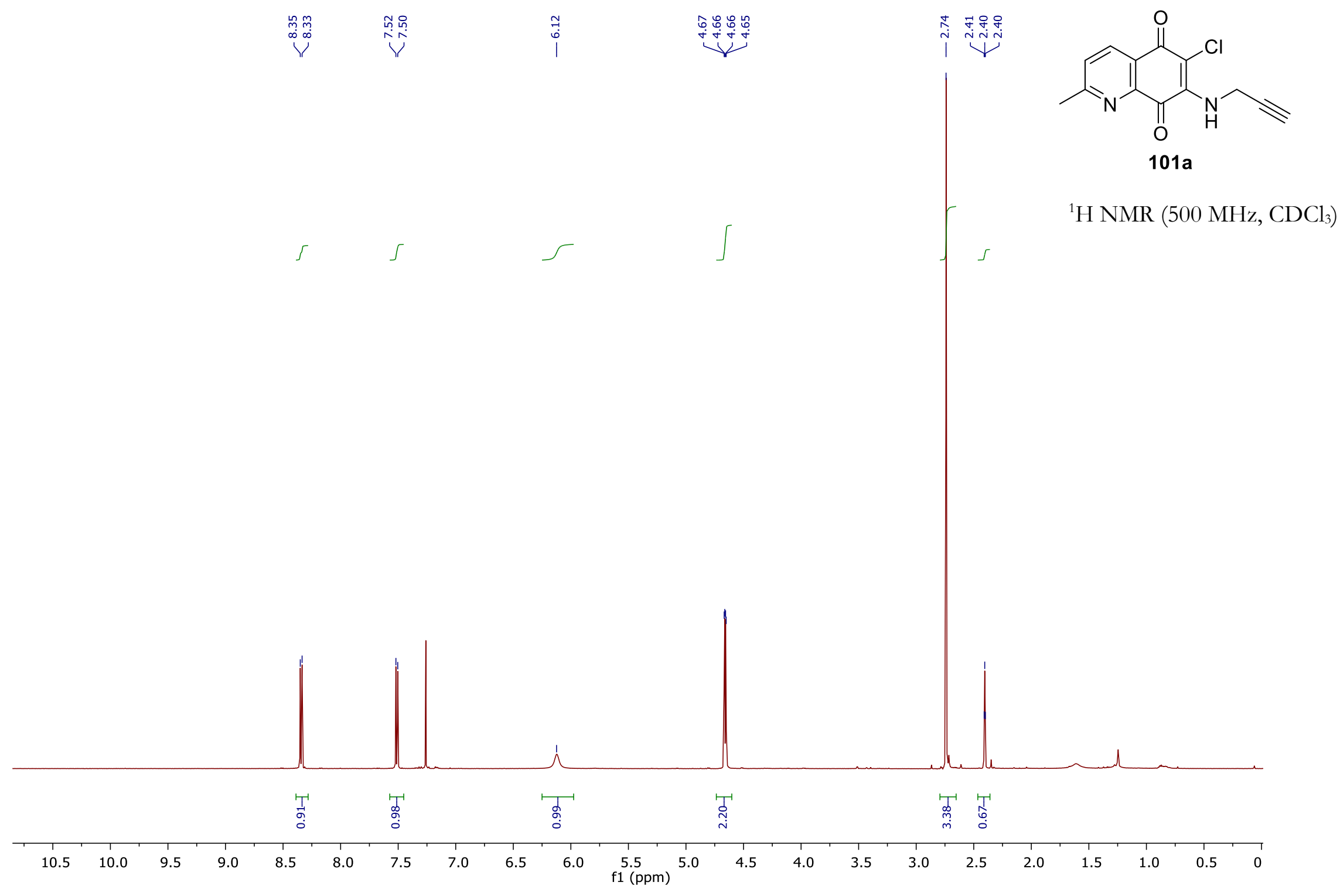




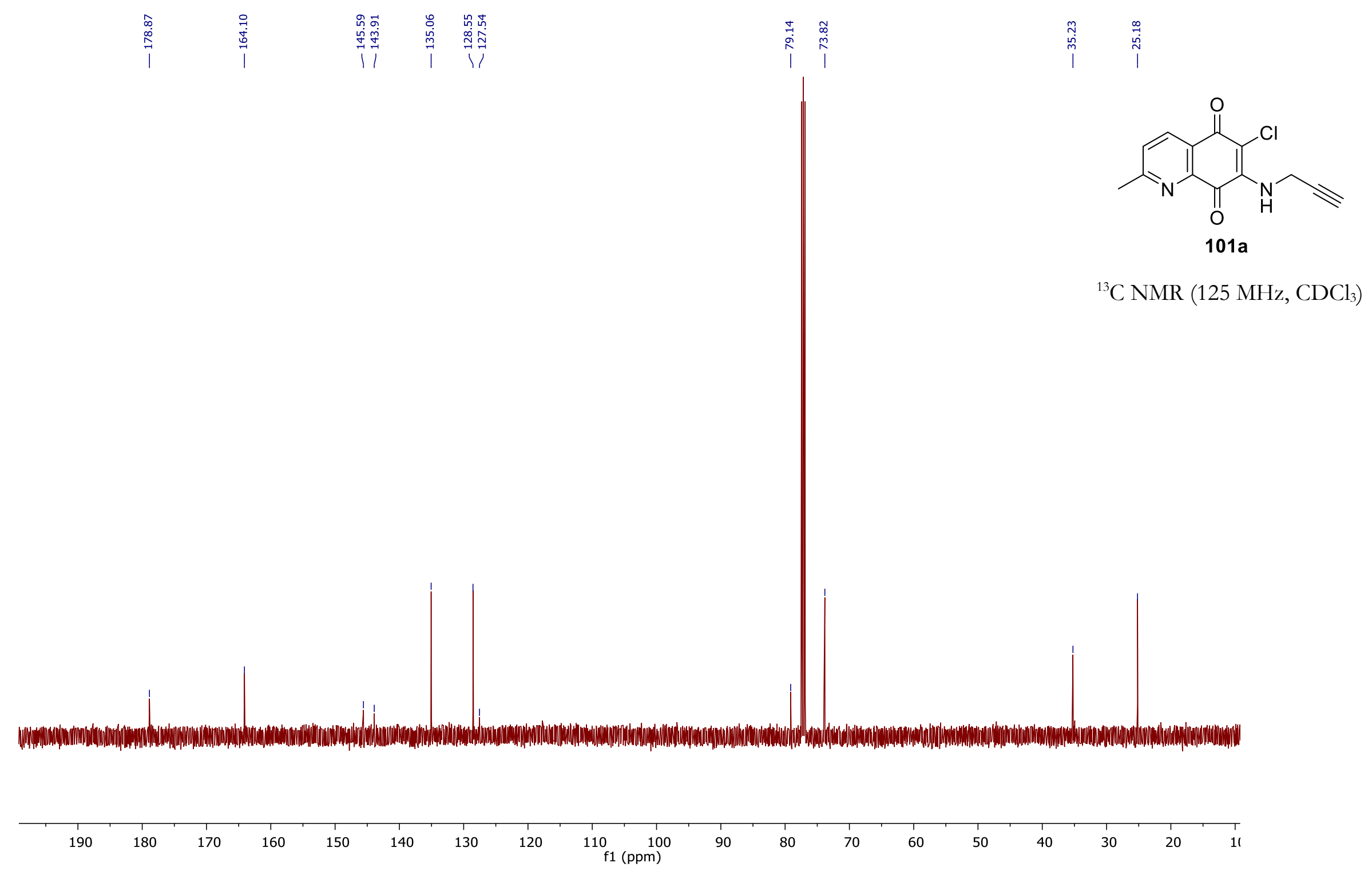




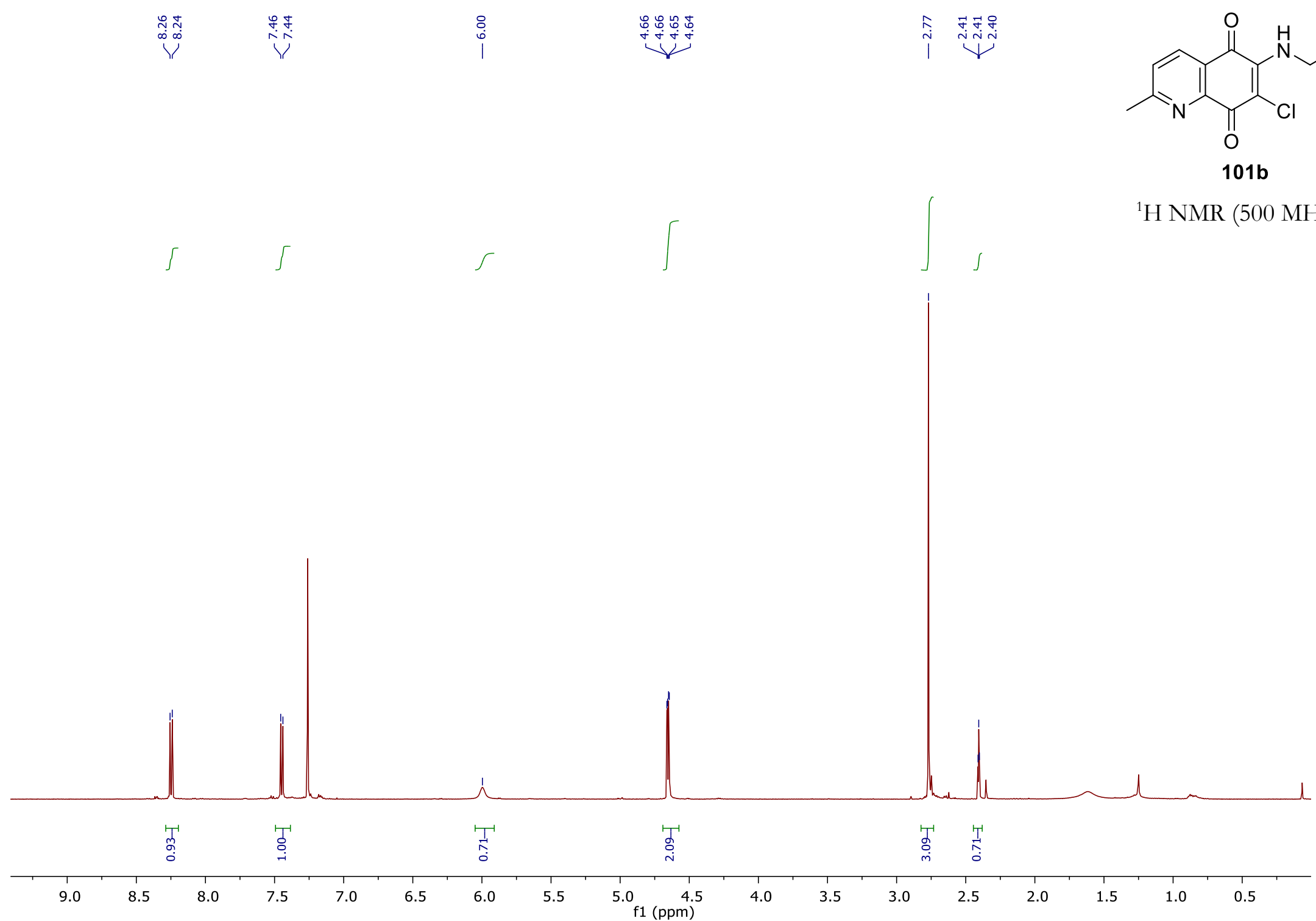




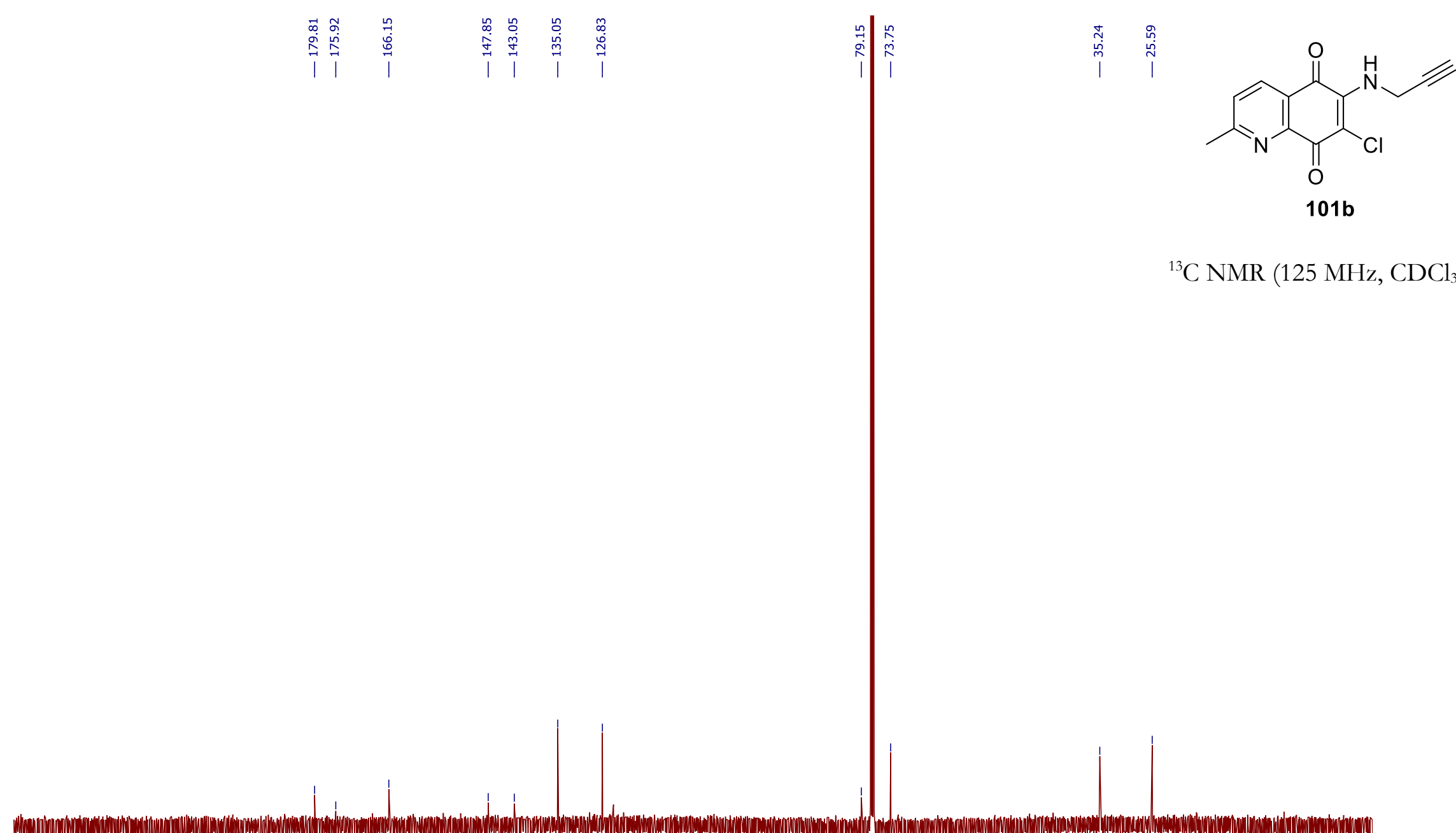

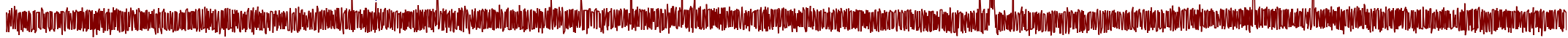

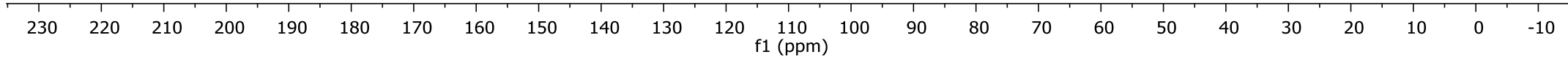



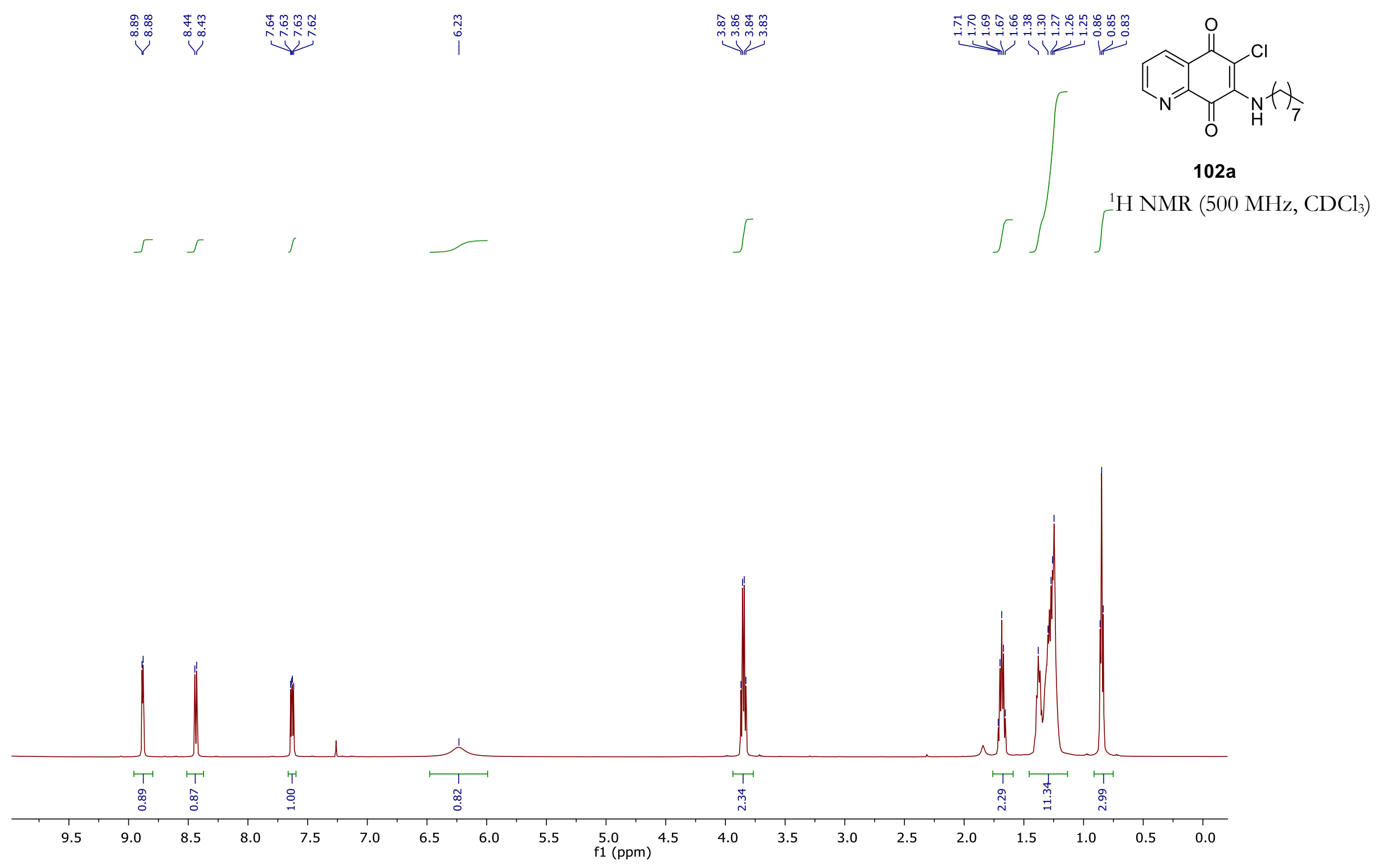


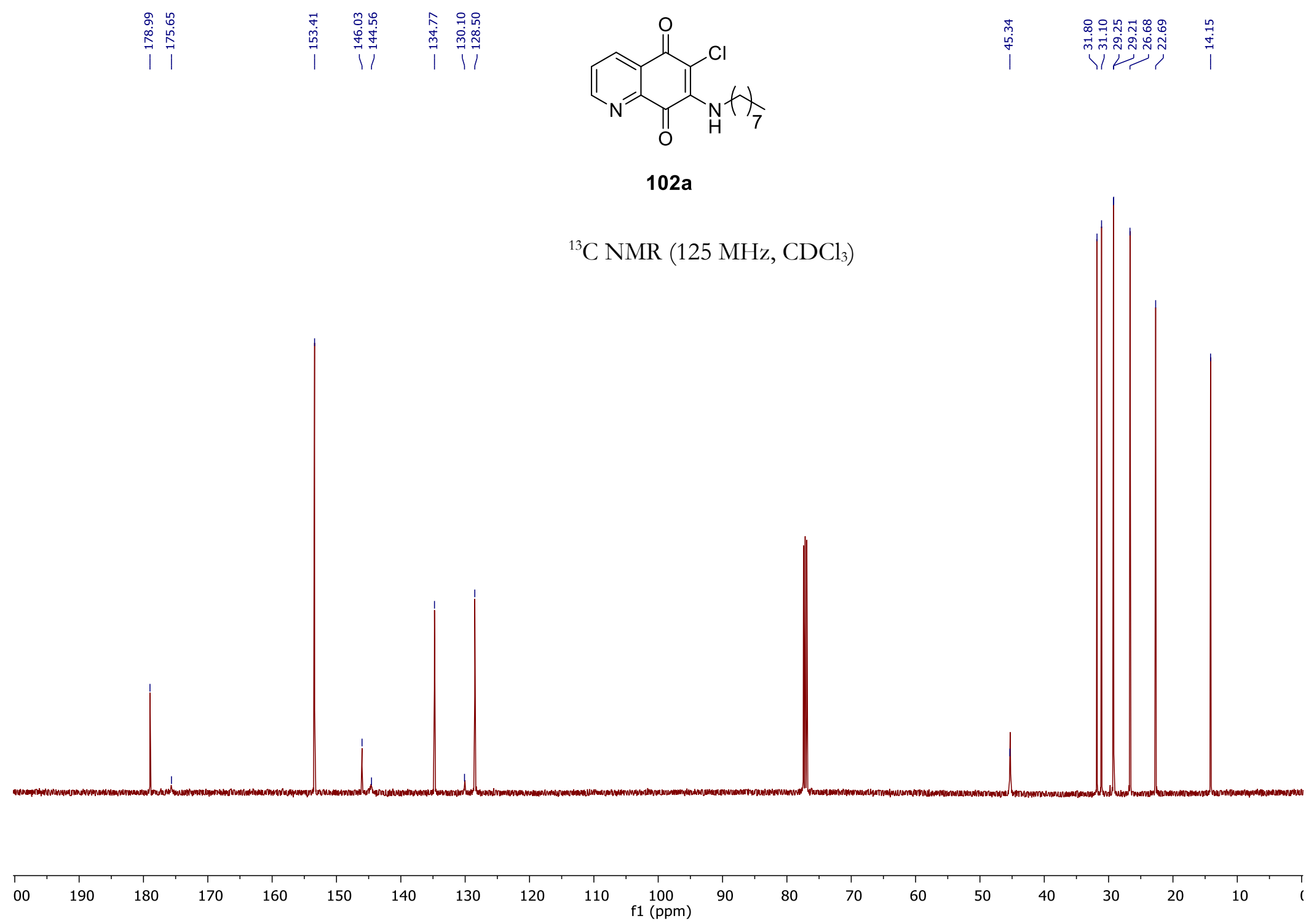




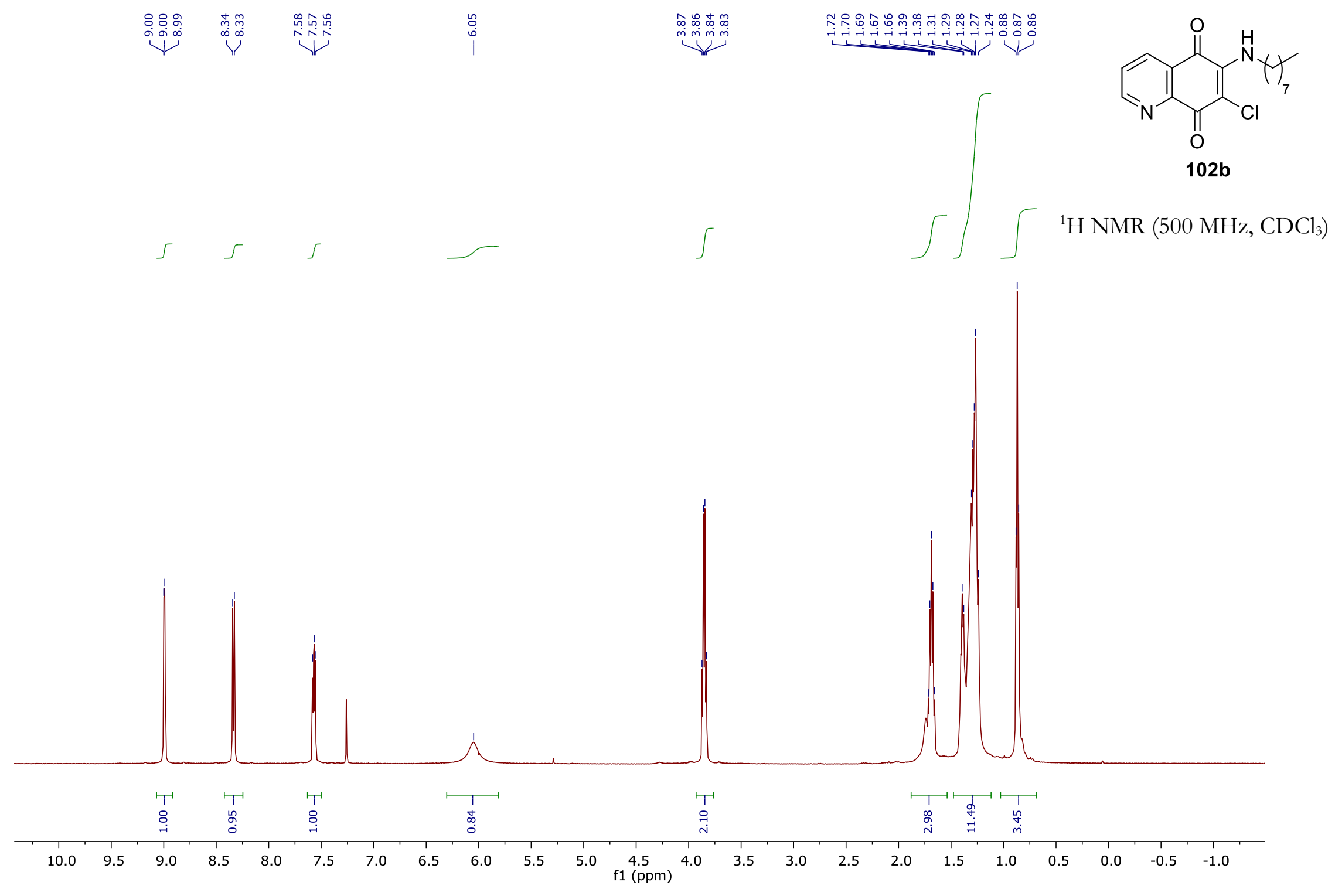




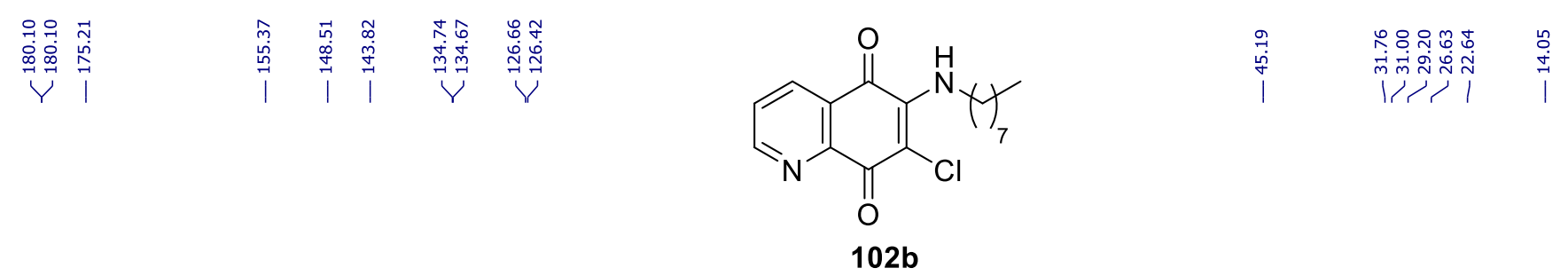

${ }^{13} \mathrm{C}$ NMR $\left(125 \mathrm{MHz}, \mathrm{CDCl}_{3}\right)$
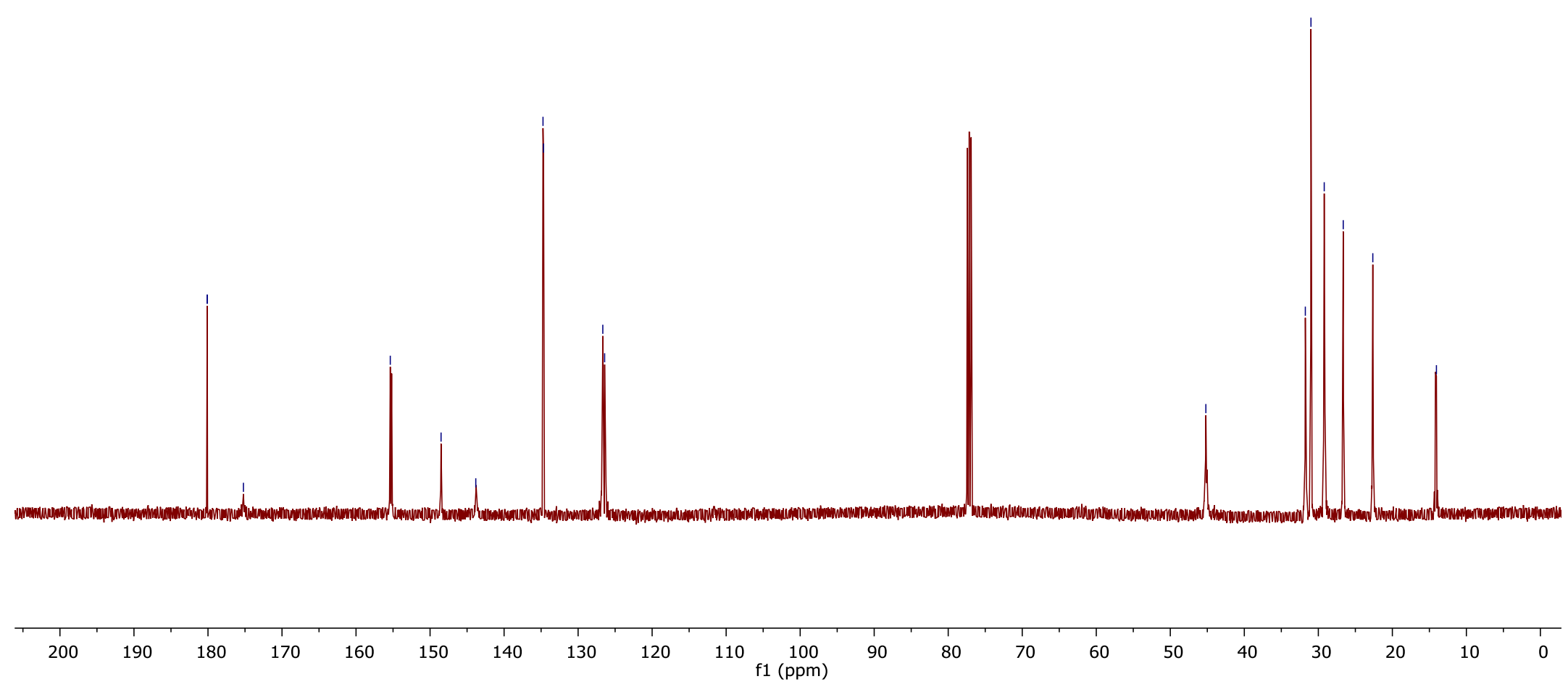


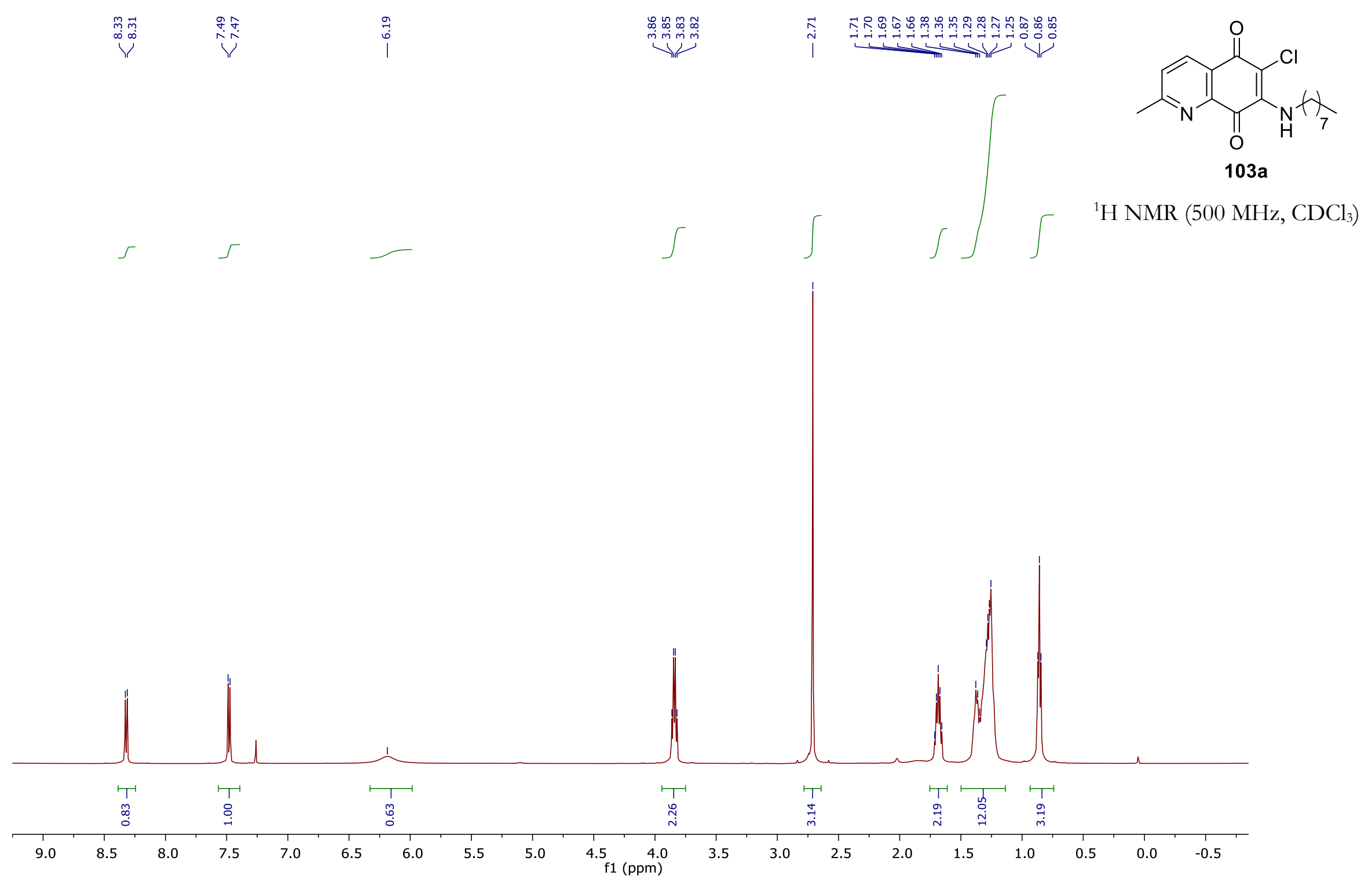




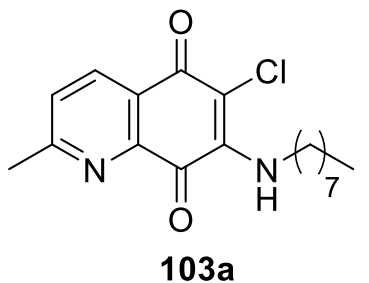

${ }^{13} \mathrm{C}$ NMR $\left(125 \mathrm{MHz}, \mathrm{CDCl}_{3}\right)$

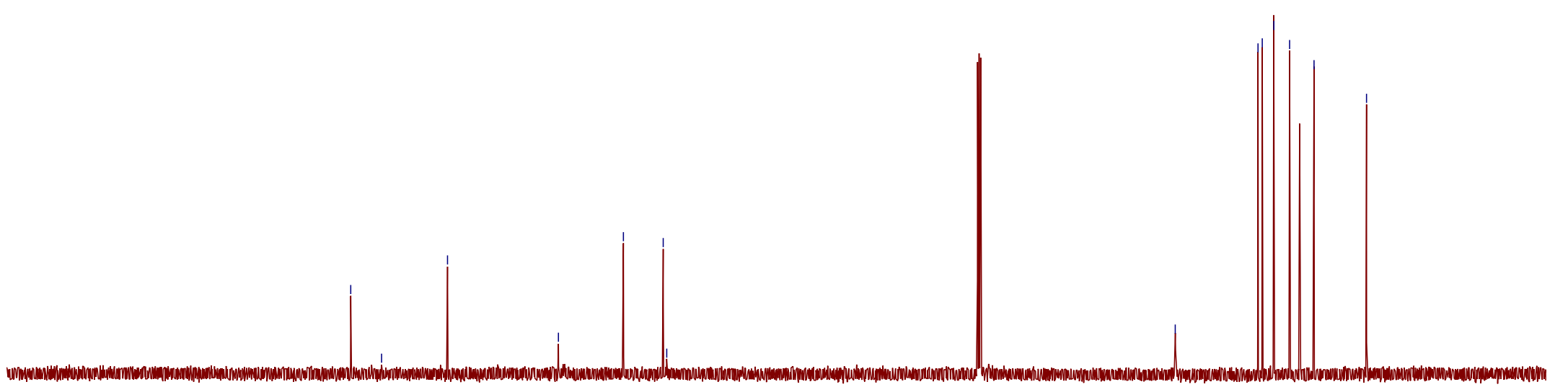

$\begin{array}{llllllllllll}230 & 220 & 210 & 200 & 190 & 180 & 170 & 160 & 150 & 140 & 130 & 120 \\ \mathrm{f} 1(\mathrm{ppm}) & 110 & 100\end{array}$ 


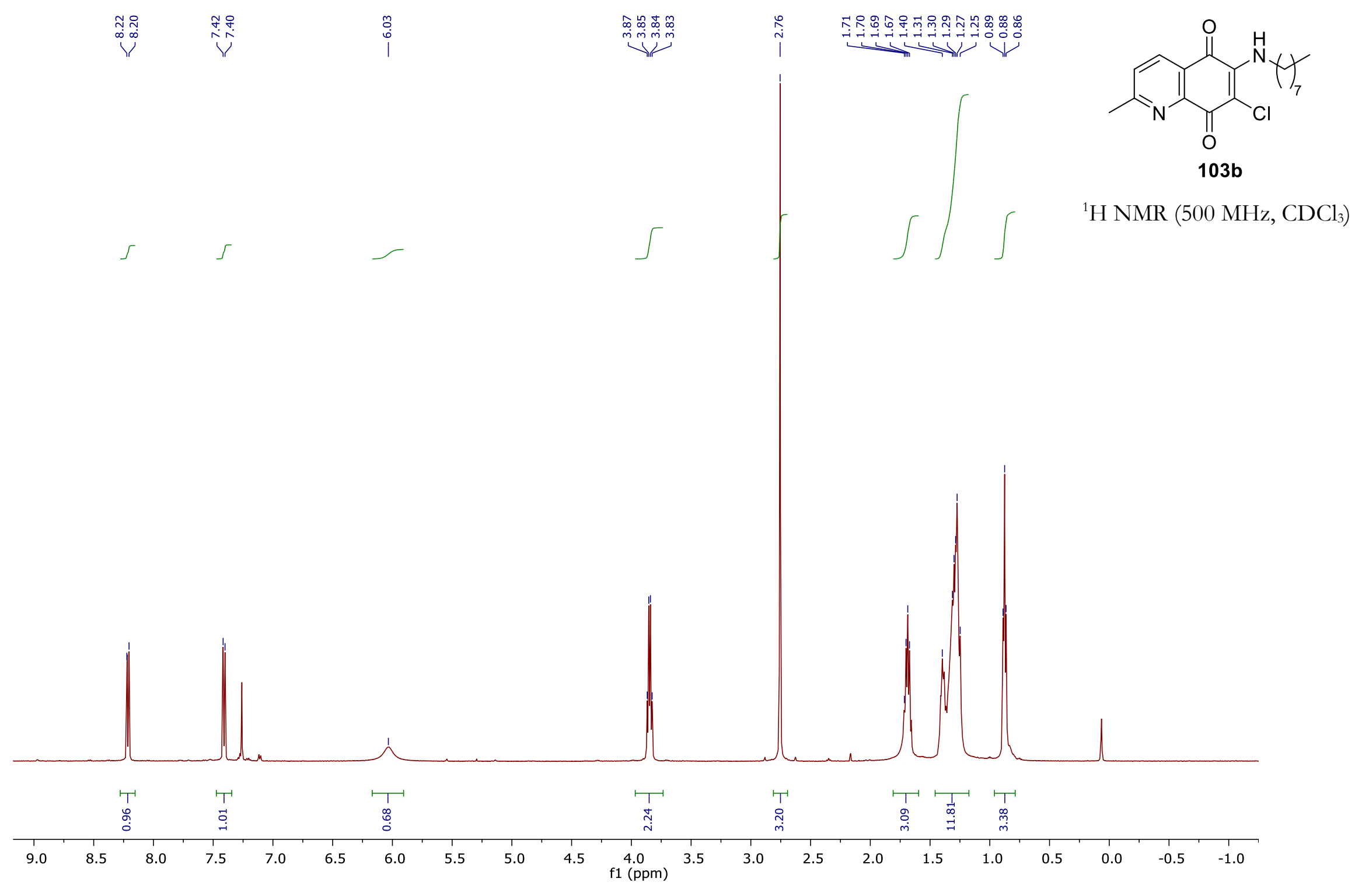


章

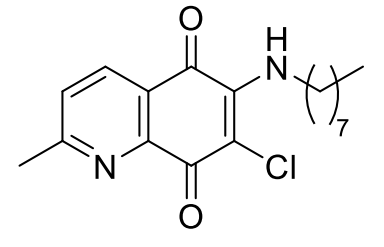

$103 b$

${ }^{13} \mathrm{C} \mathrm{NMR}\left(125 \mathrm{MHz}, \mathrm{CDCl}_{3}\right)$

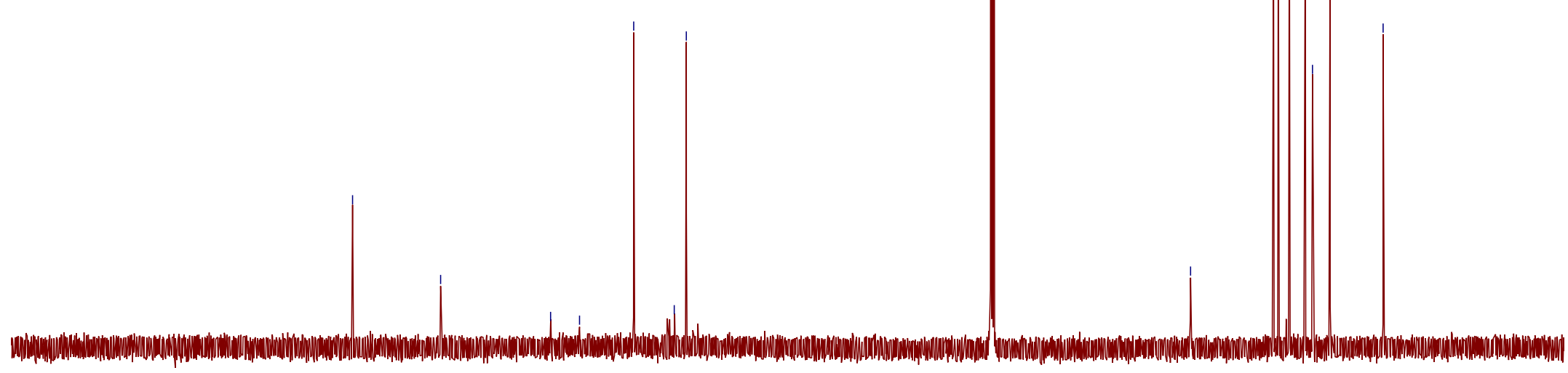

$\begin{array}{lllllllllllll}230 & 220 & 210 & 200 & 190 & 180 & 170 & 160 & 150 & 140 & 130 & 120 & 110 \\ \mathrm{f} 1(\mathrm{ppm}) & 100\end{array}$ 
ע

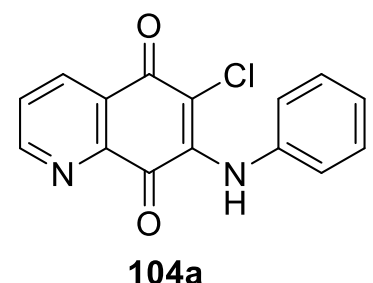

${ }^{1} \mathrm{H}$ NMR $\left(500 \mathrm{MHz}, \mathrm{CDCl}_{3}\right)$
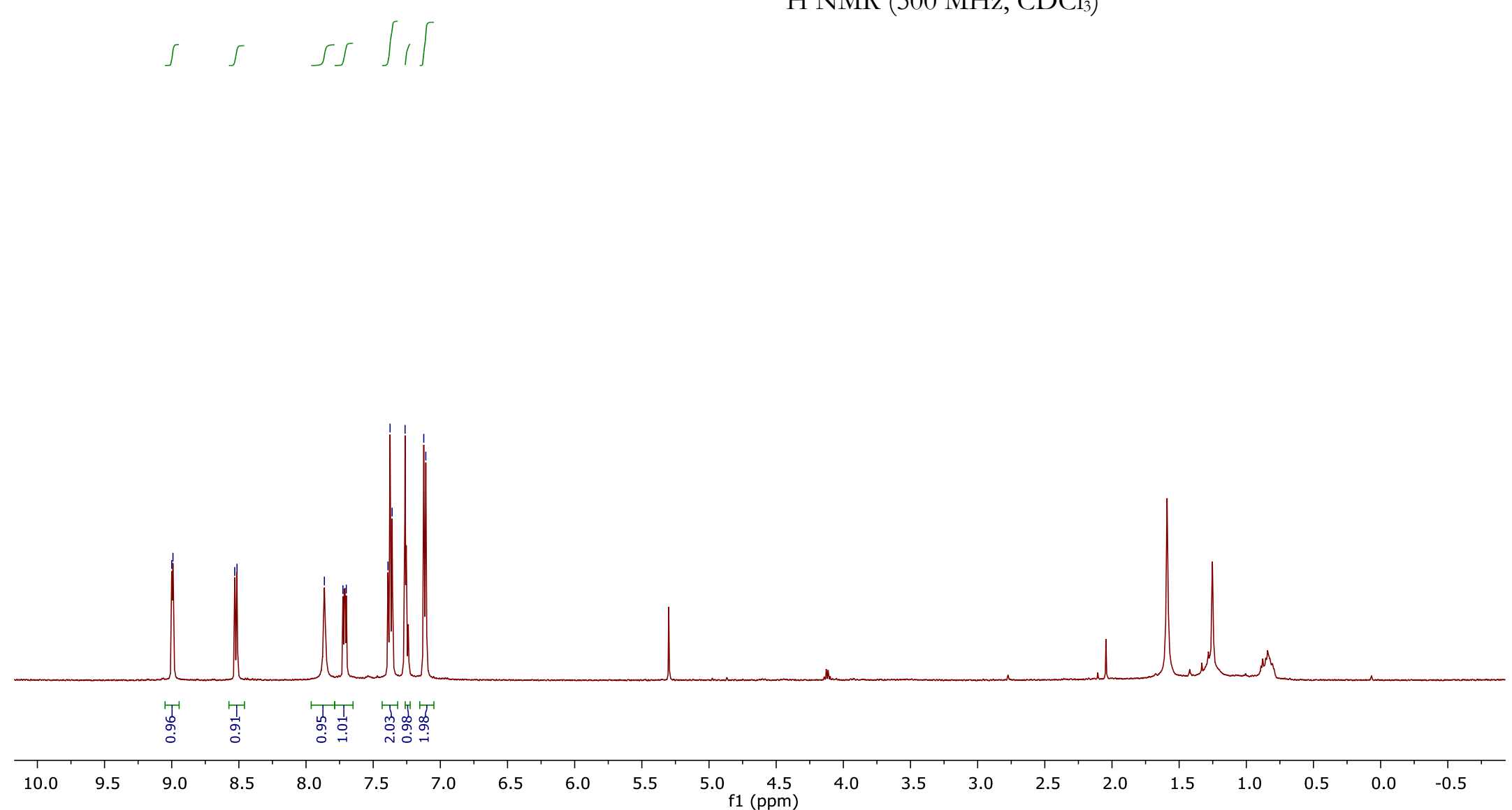

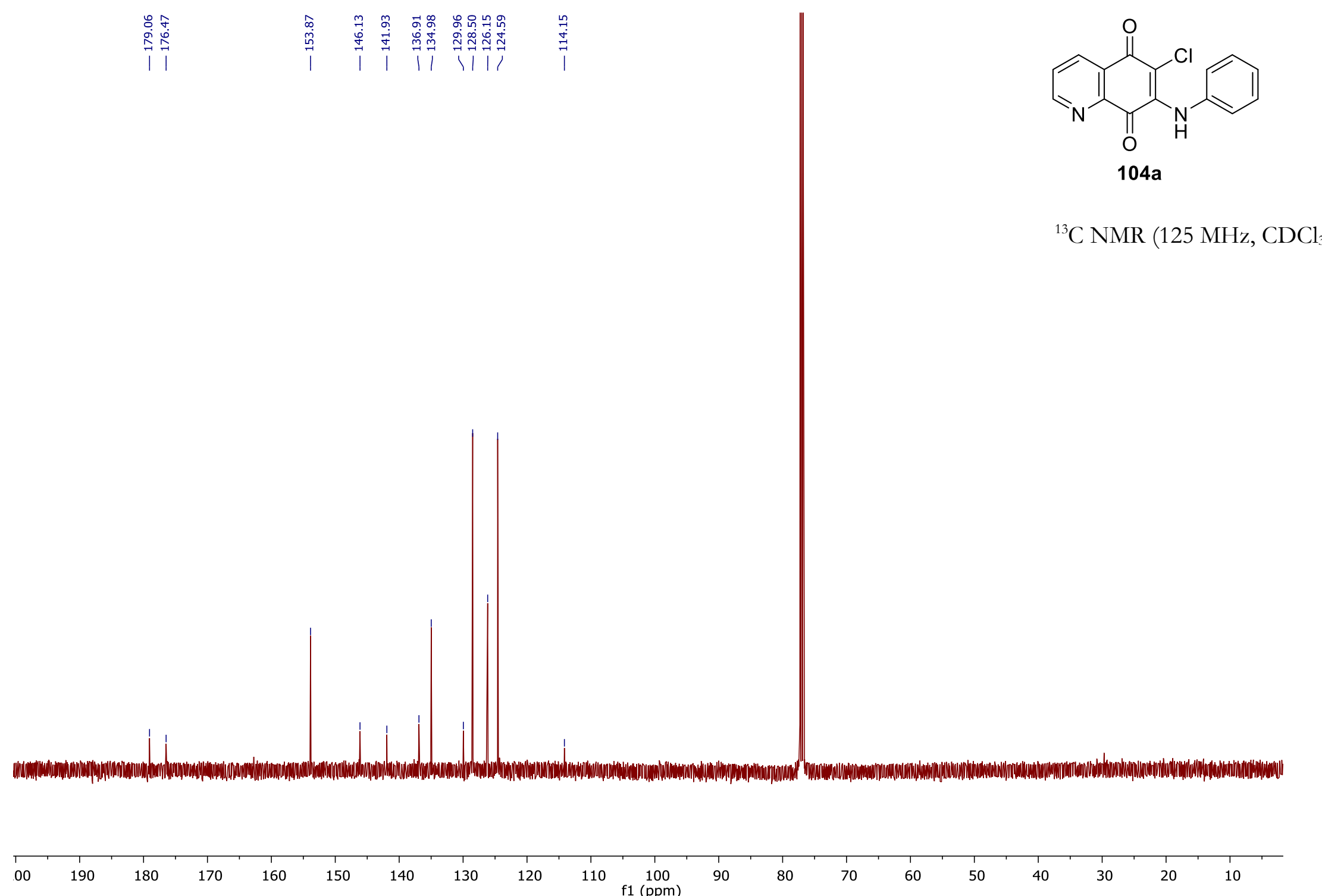


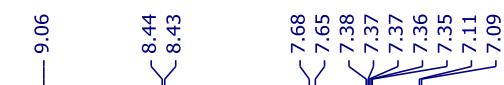

$\iiint \iint$

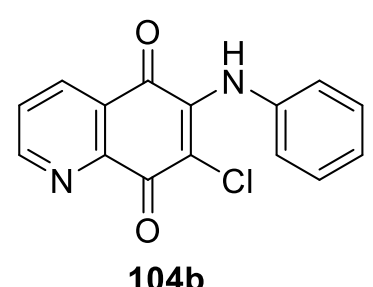

${ }^{1} \mathrm{H}$ NMR $\left(500 \mathrm{MHz}, \mathrm{CDCl}_{3}\right)$

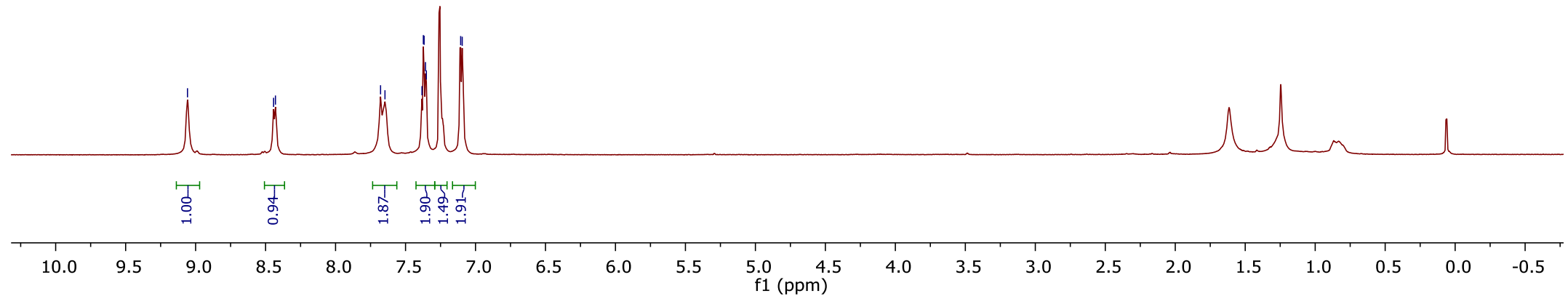




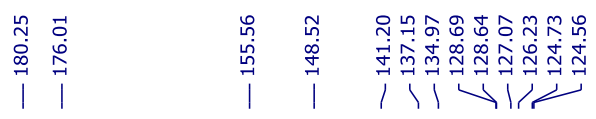

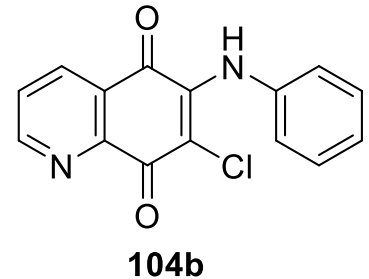

${ }^{13} \mathrm{C}$ NMR $\left(125 \mathrm{MHz}, \mathrm{CDCl}_{3}\right)$

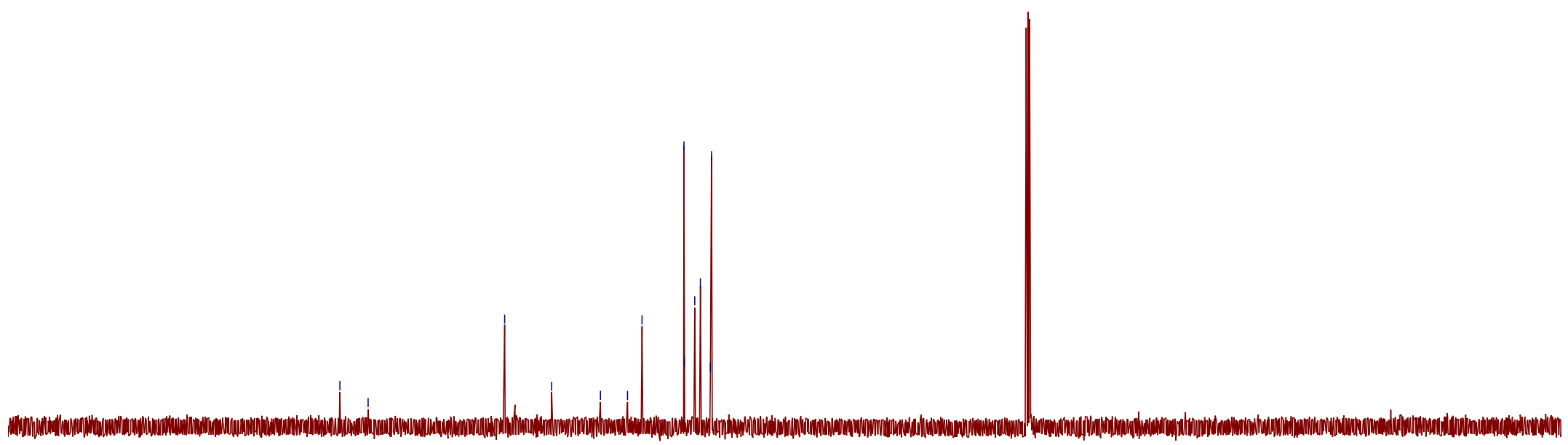




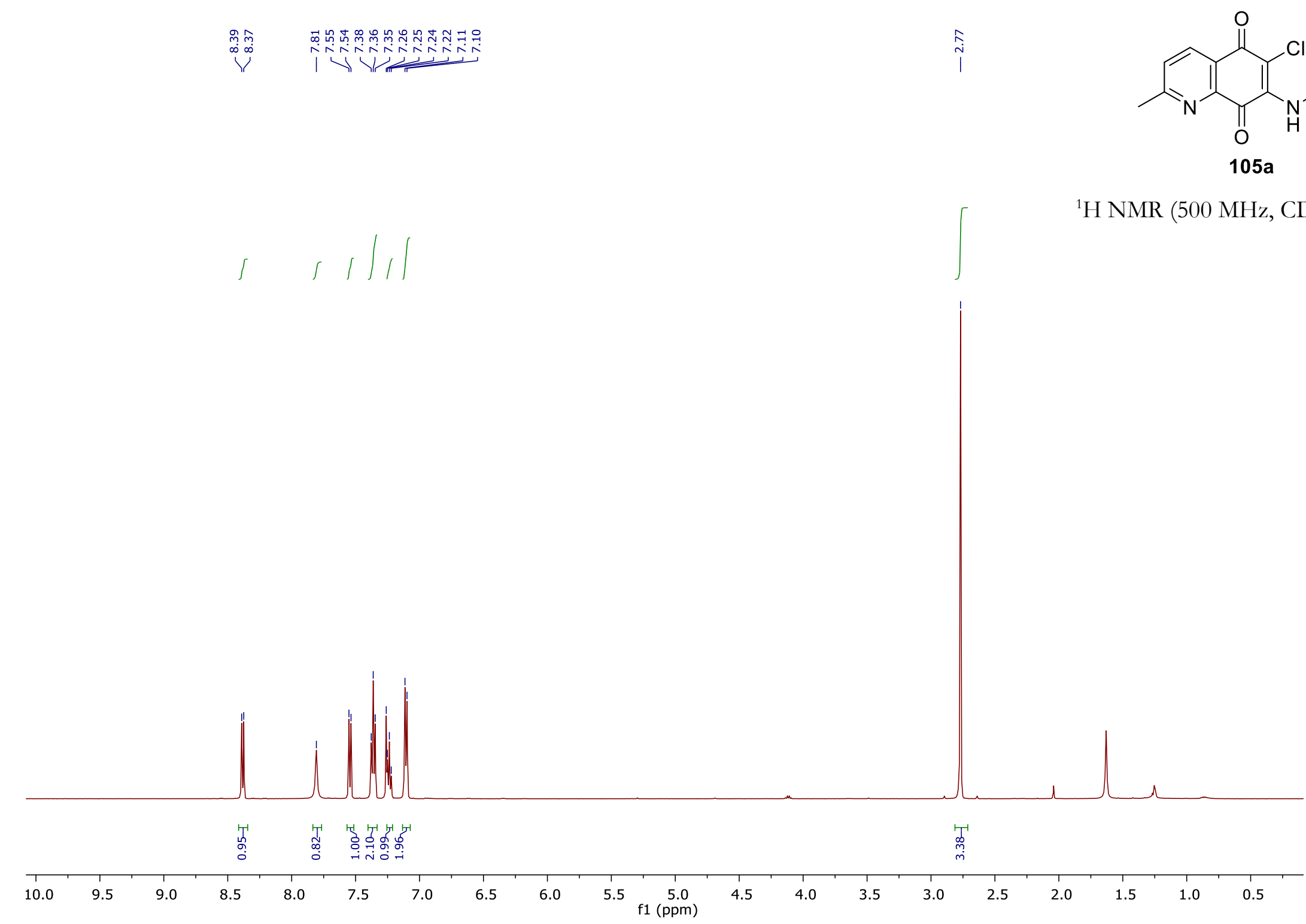




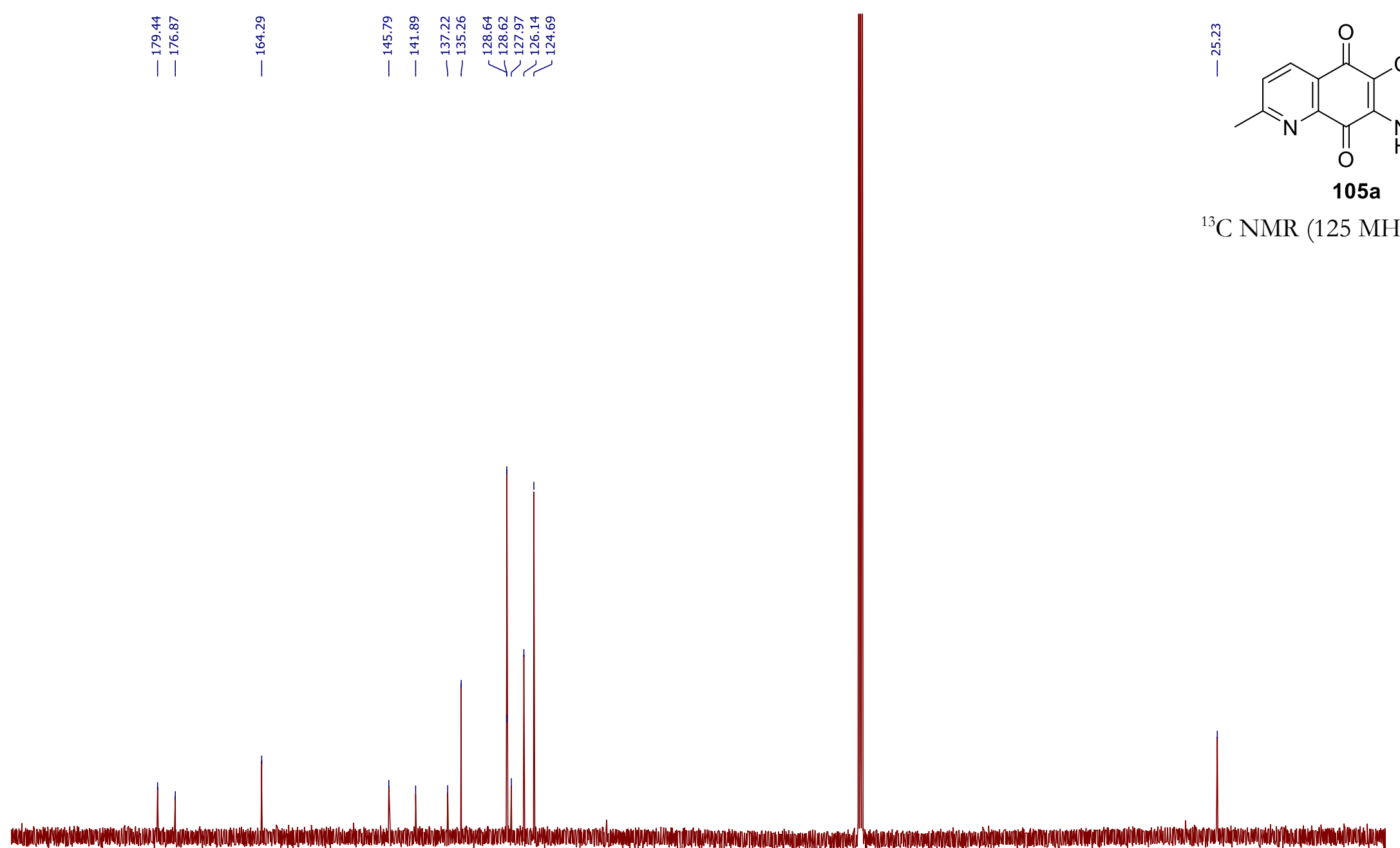

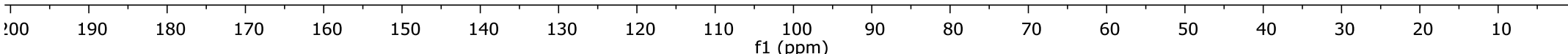


|

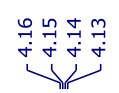

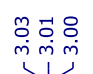

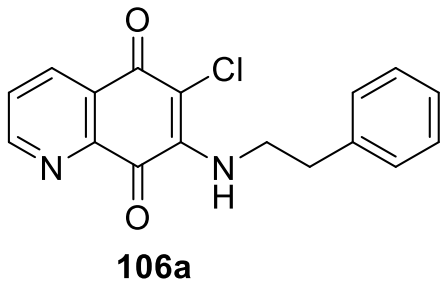

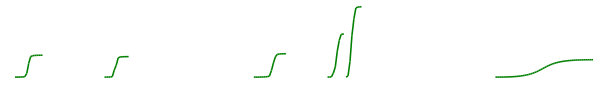

${ }^{1} \mathrm{H} \mathrm{NMR}\left(500 \mathrm{MHz}, \mathrm{CDCl}_{3}\right)$

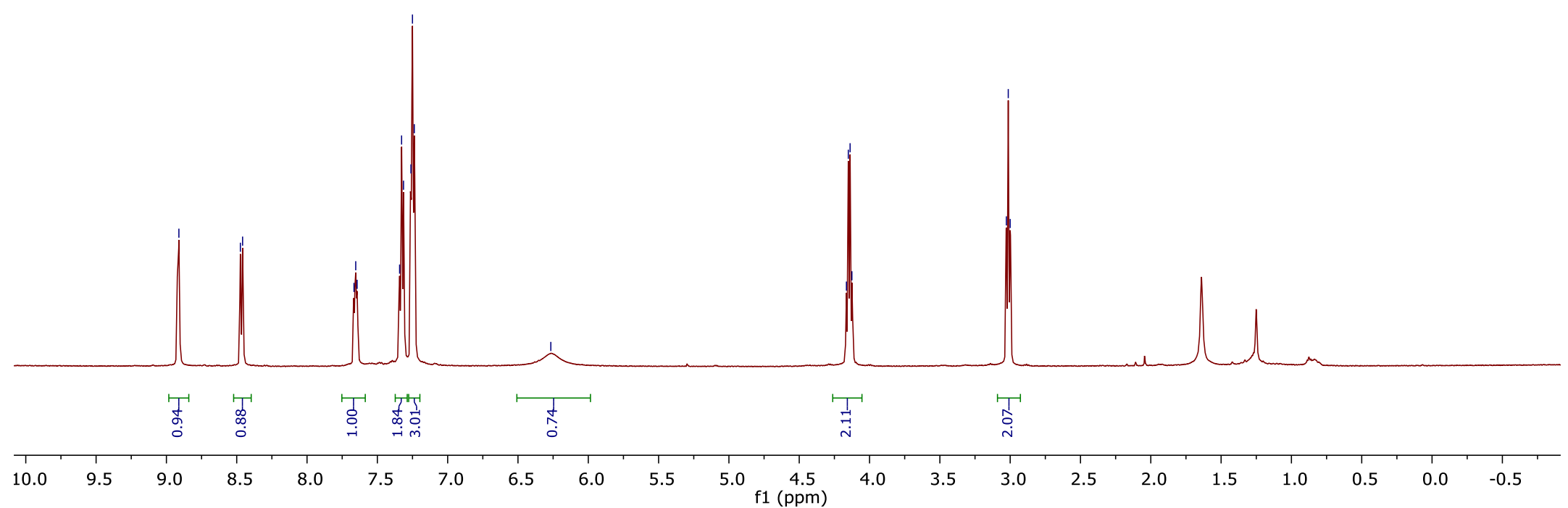




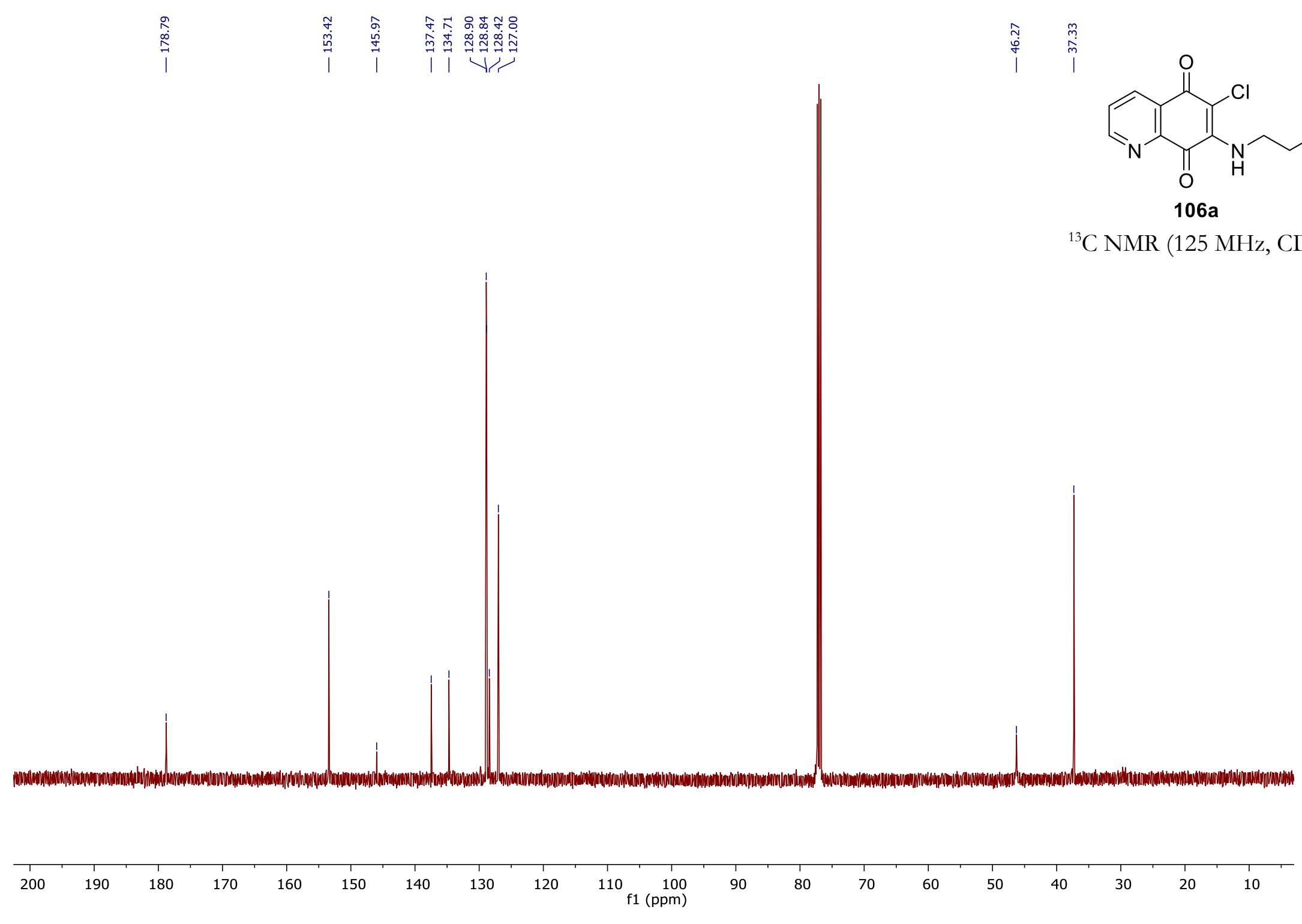




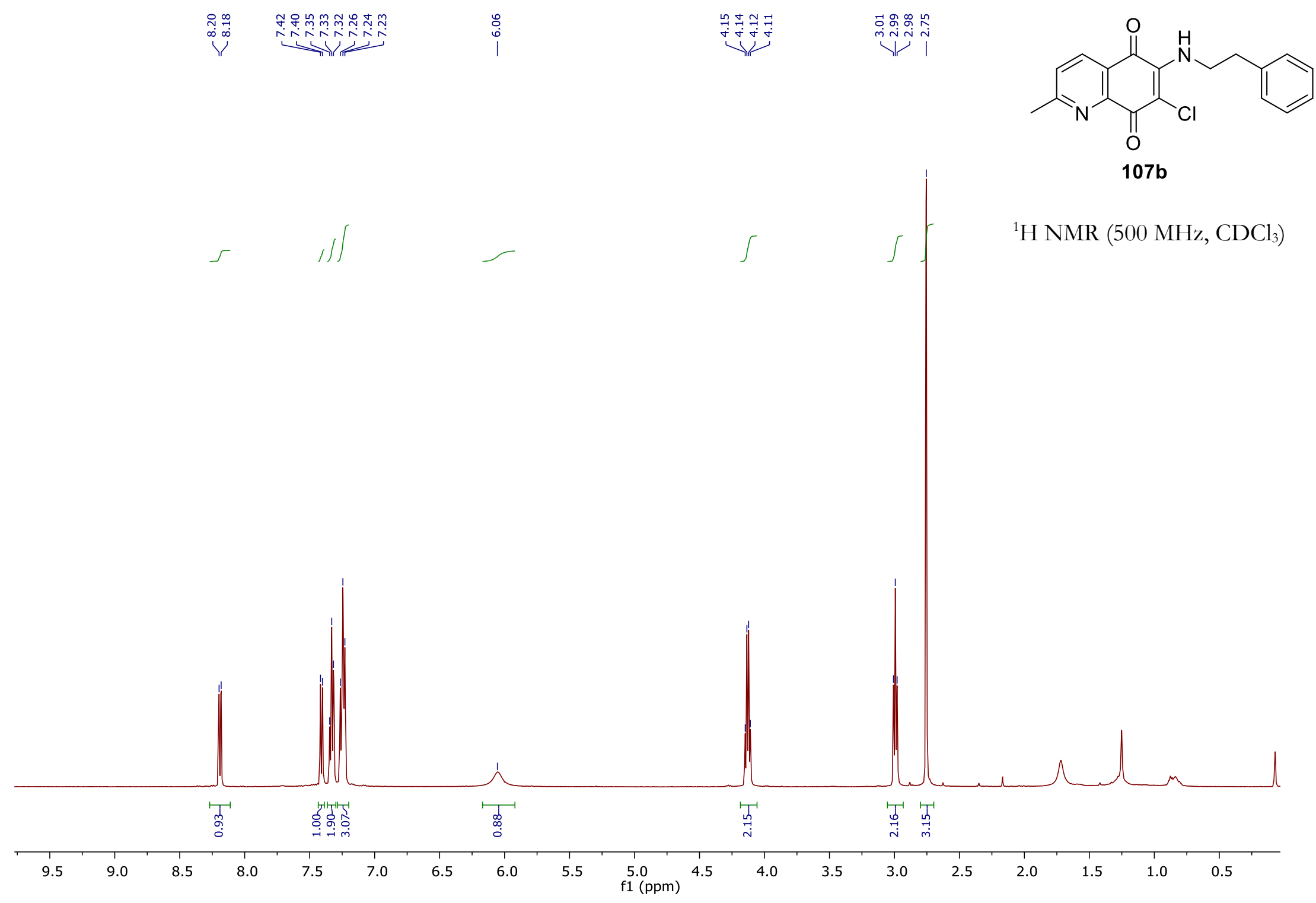



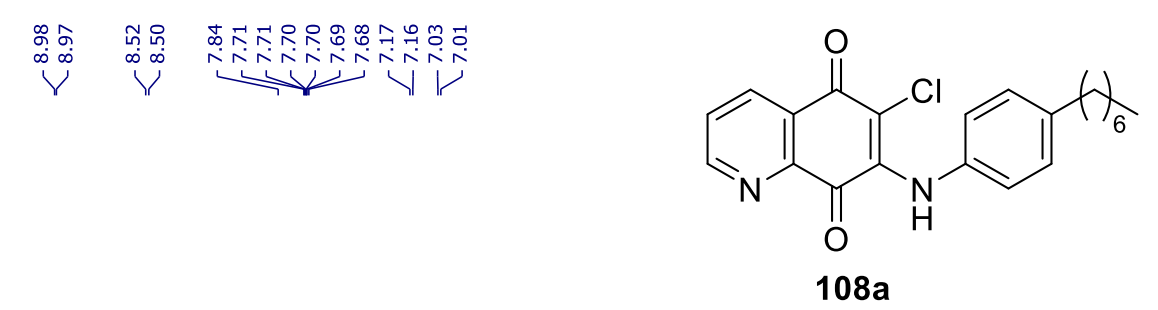

บับ

${ }^{1} \mathrm{H}$ NMR $\left(500 \mathrm{MHz}, \mathrm{CDCl}_{3}\right)$

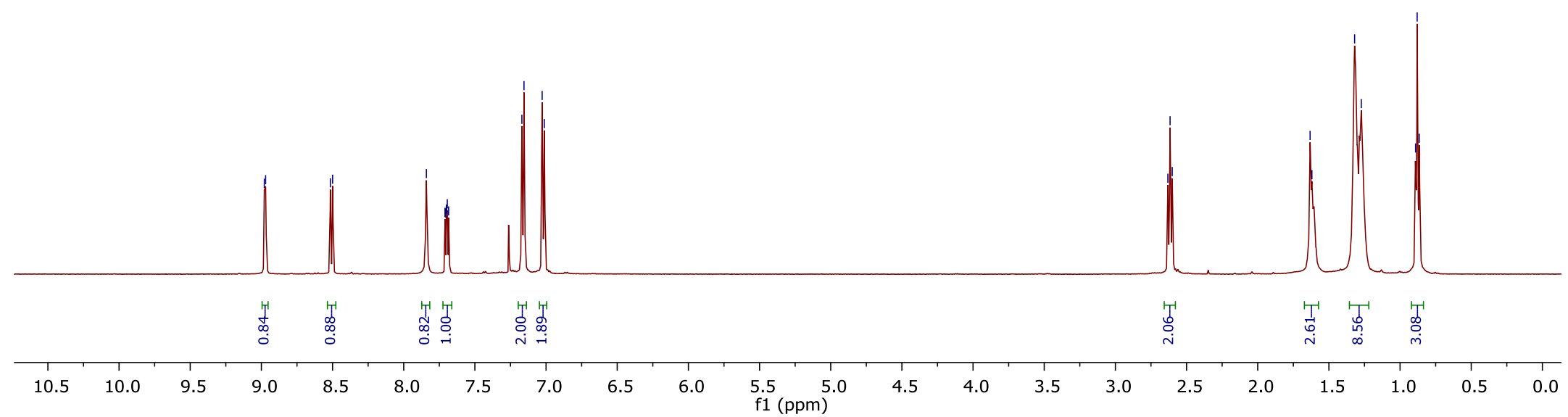




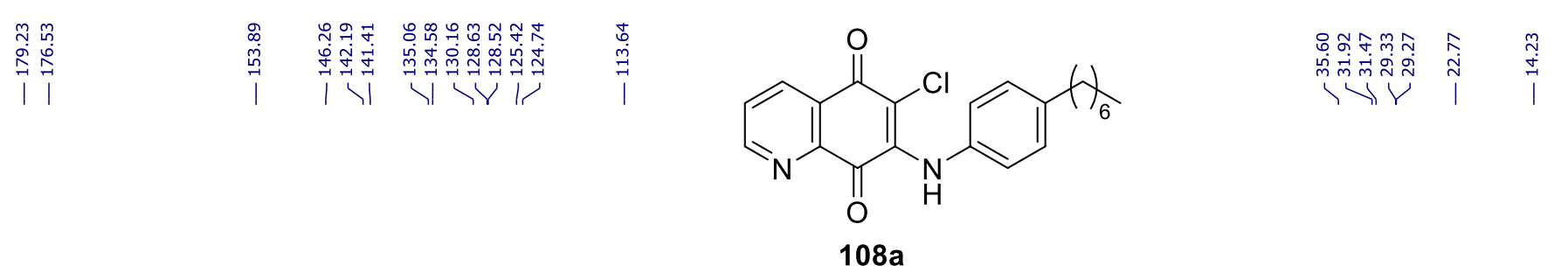

${ }^{13} \mathrm{C}$ NMR (125 MHz, $\mathrm{CDCl}_{3}$ )

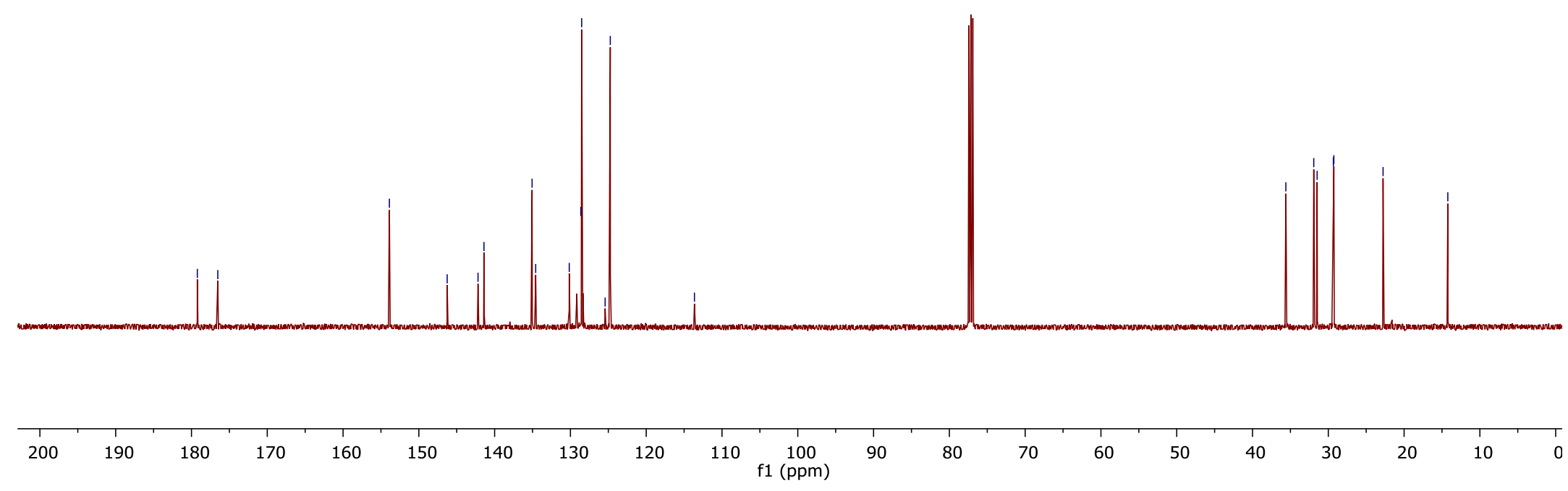




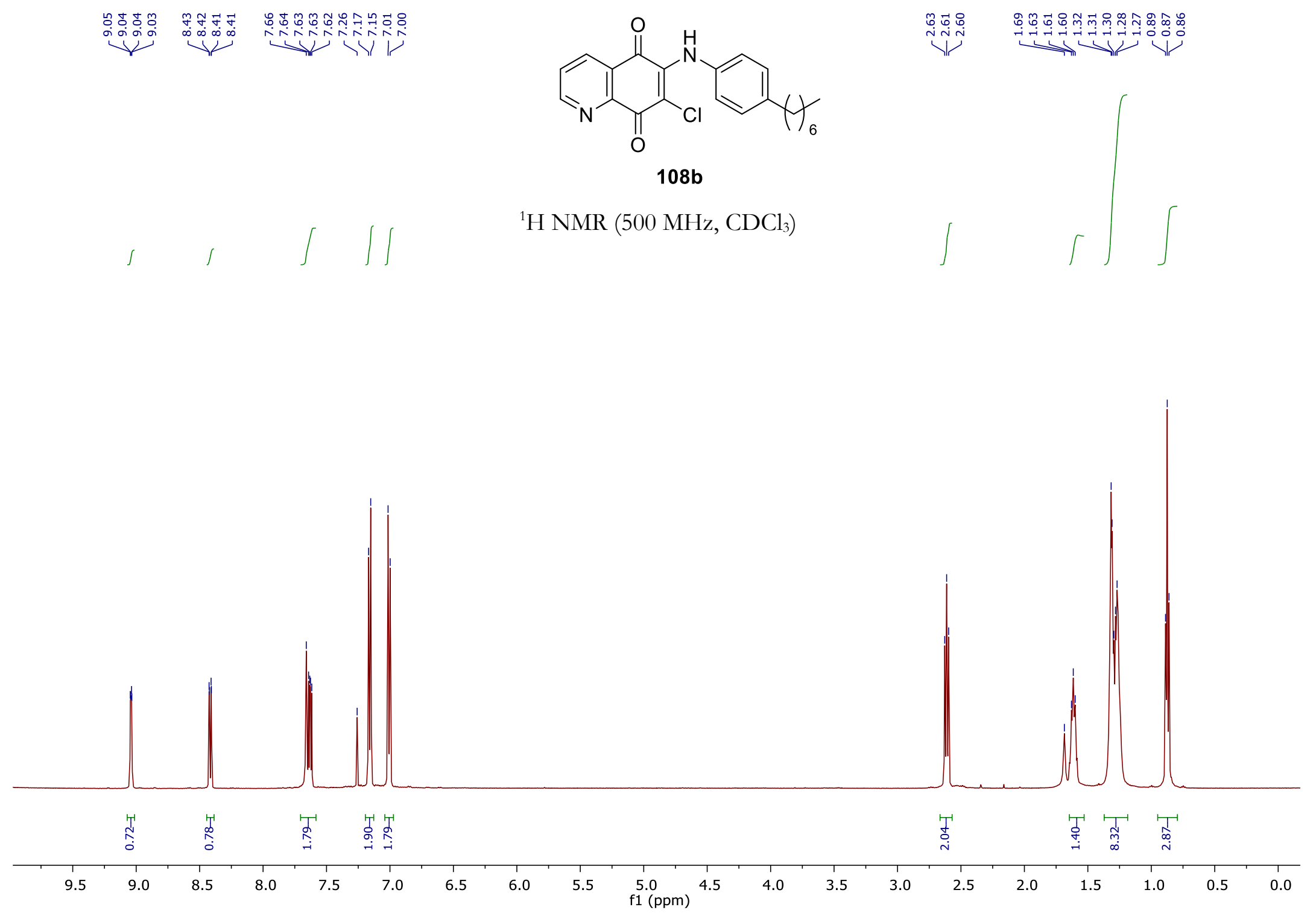




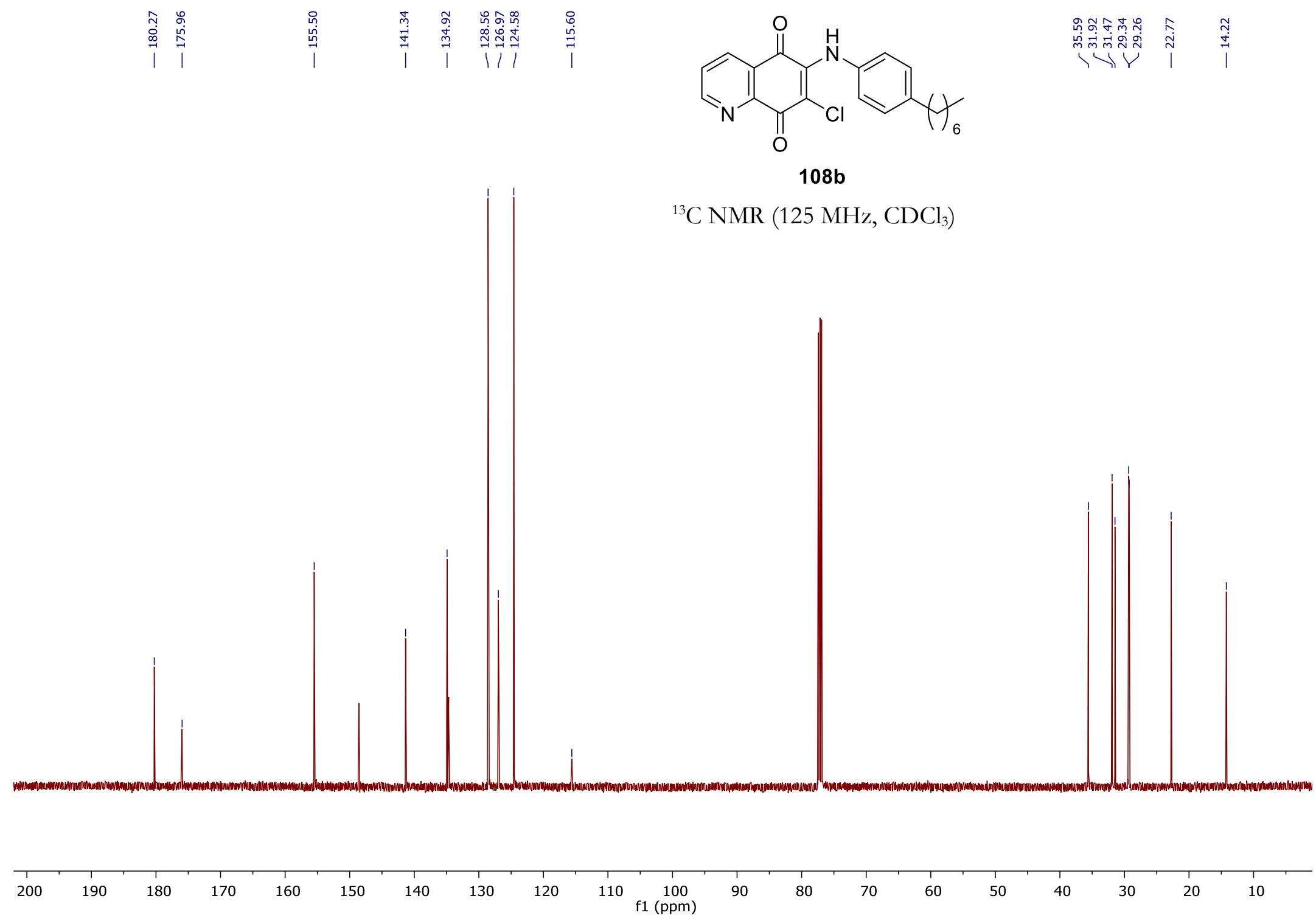




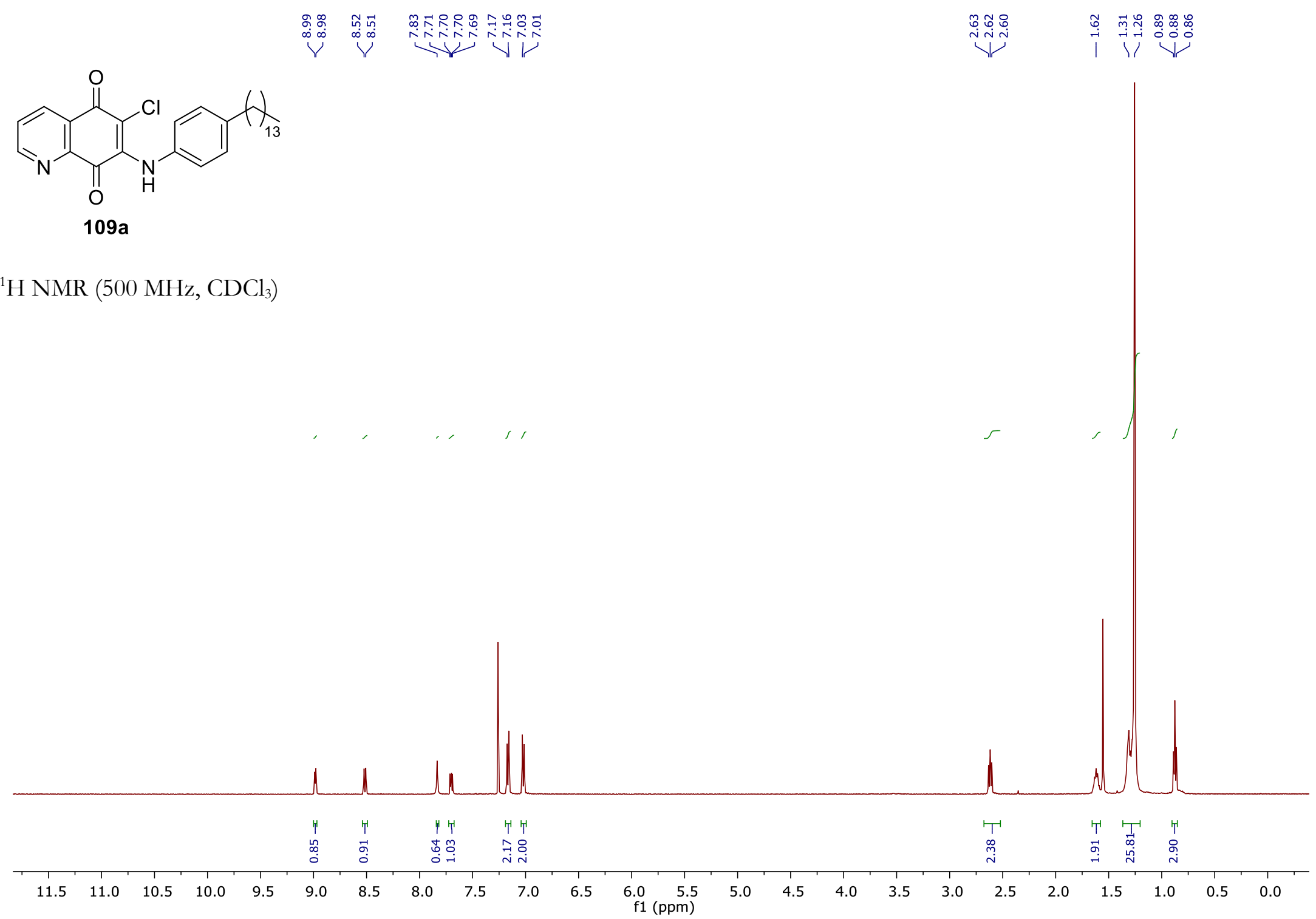




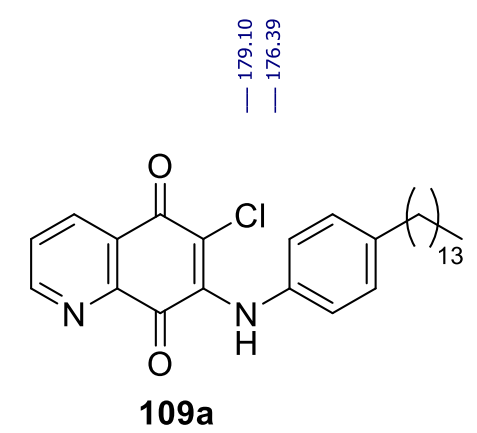

| |

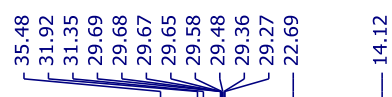

${ }^{13} \mathrm{C}$ NMR $\left(125 \mathrm{MHz}, \mathrm{CDCl}_{3}\right)$

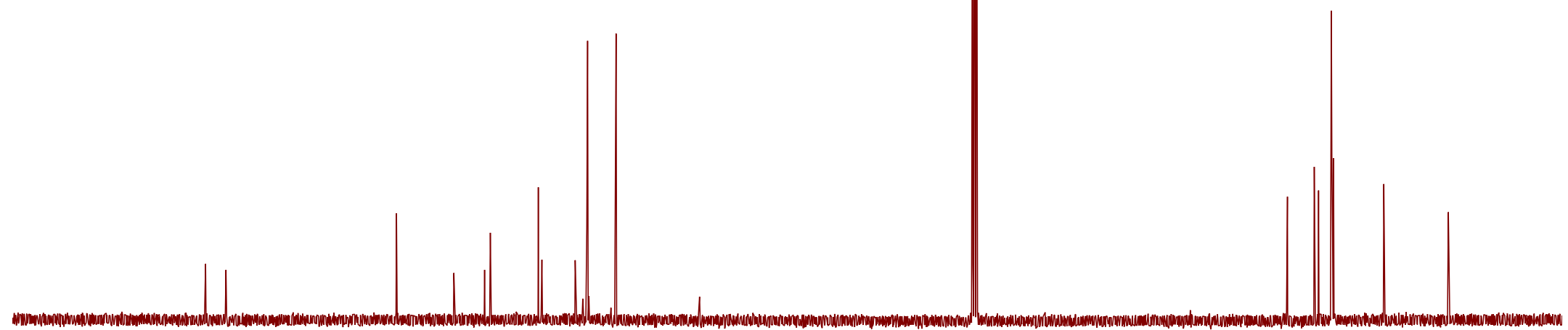

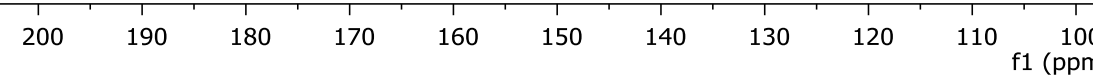




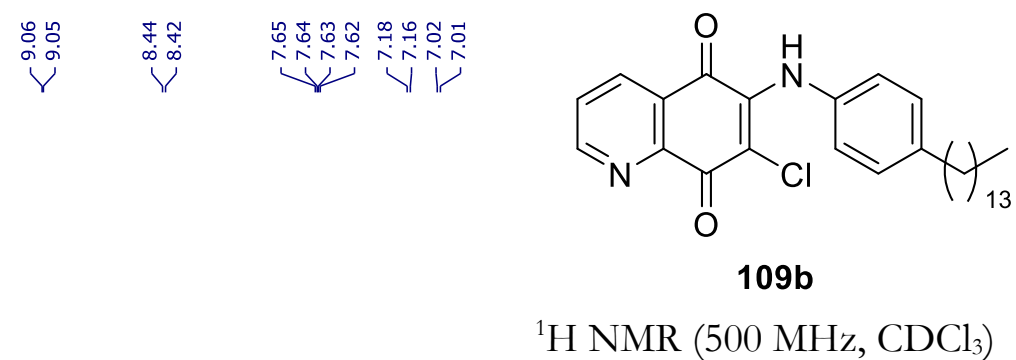

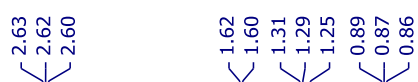

H NMR (500 MHz, $\left.\mathrm{CDCl}_{3}\right)$

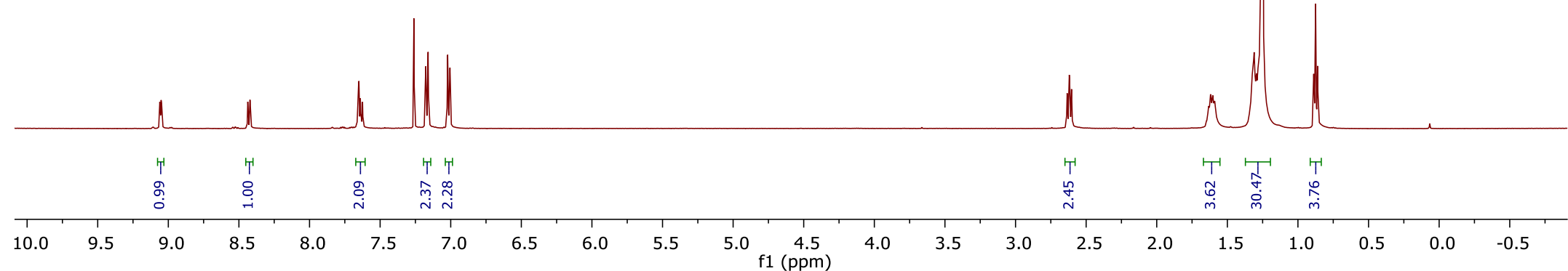




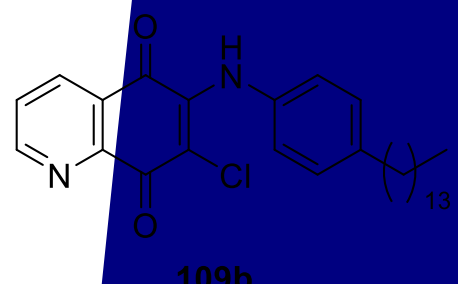

${ }^{13} \mathrm{C}$ NMR $\left(125 \mathrm{MHz}, \mathrm{CDCl}_{3}\right)$
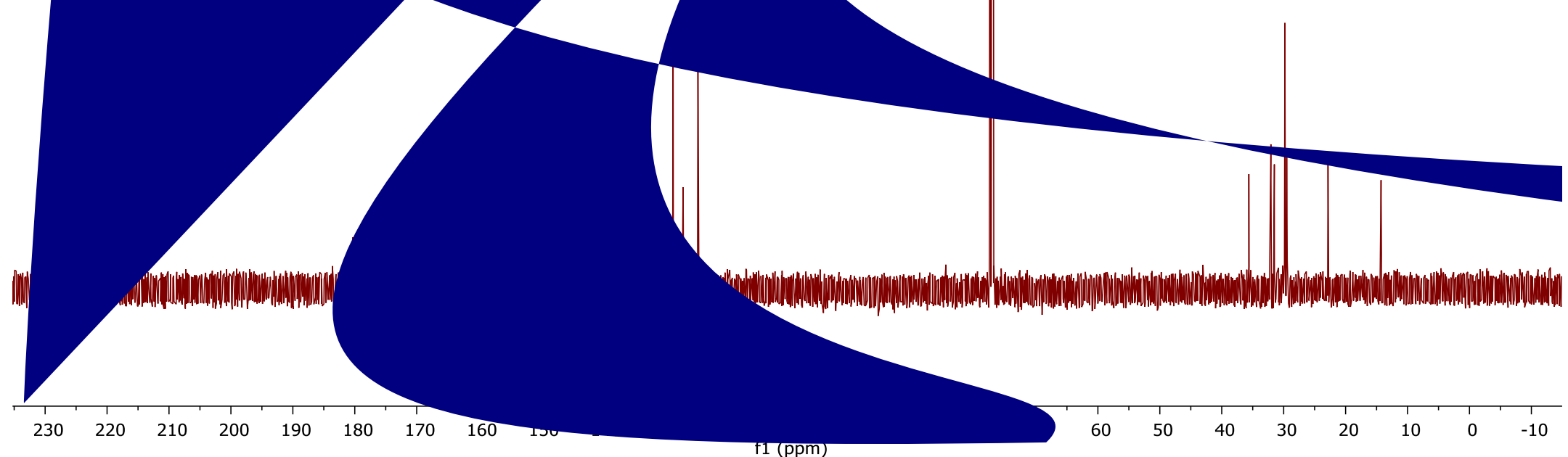


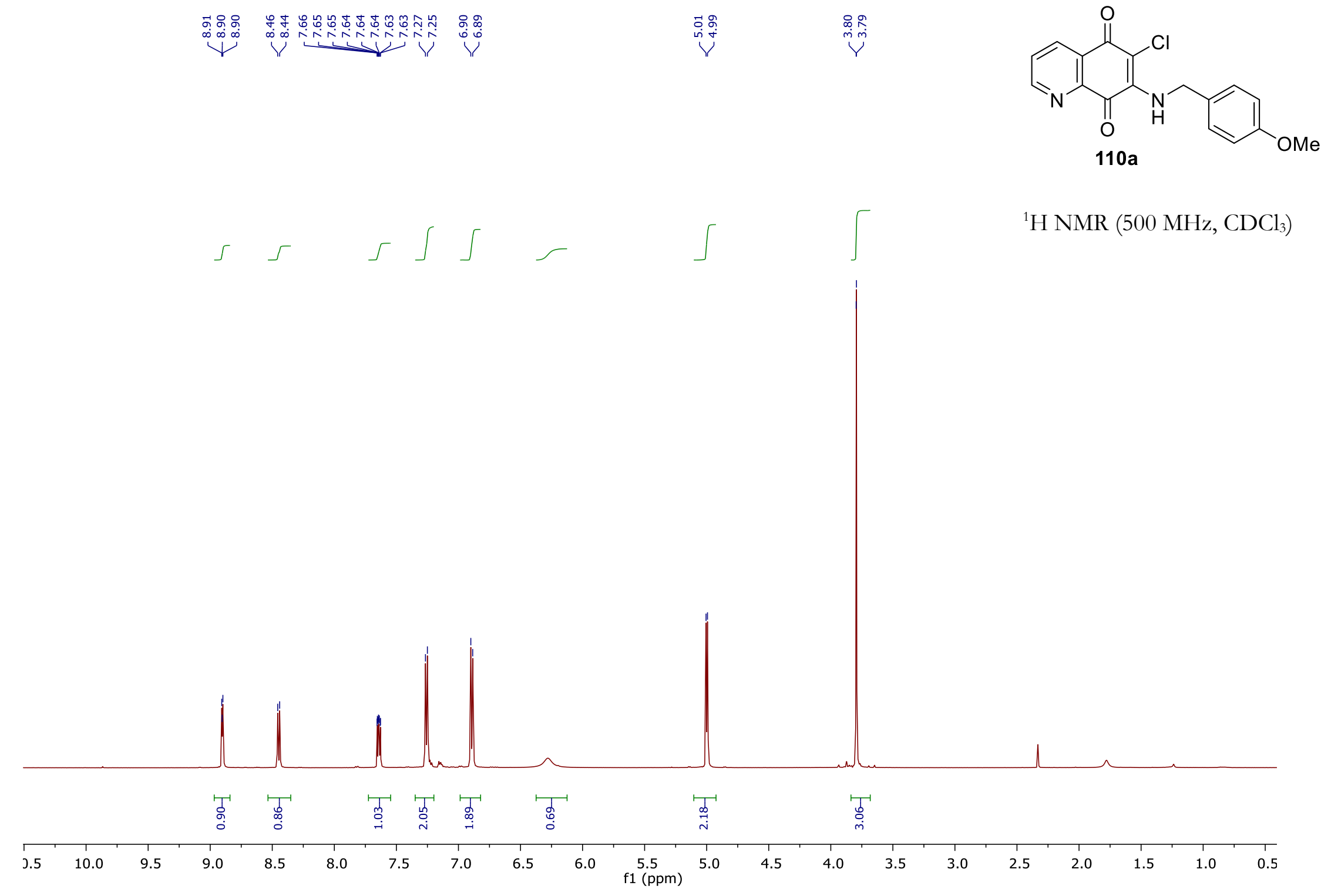




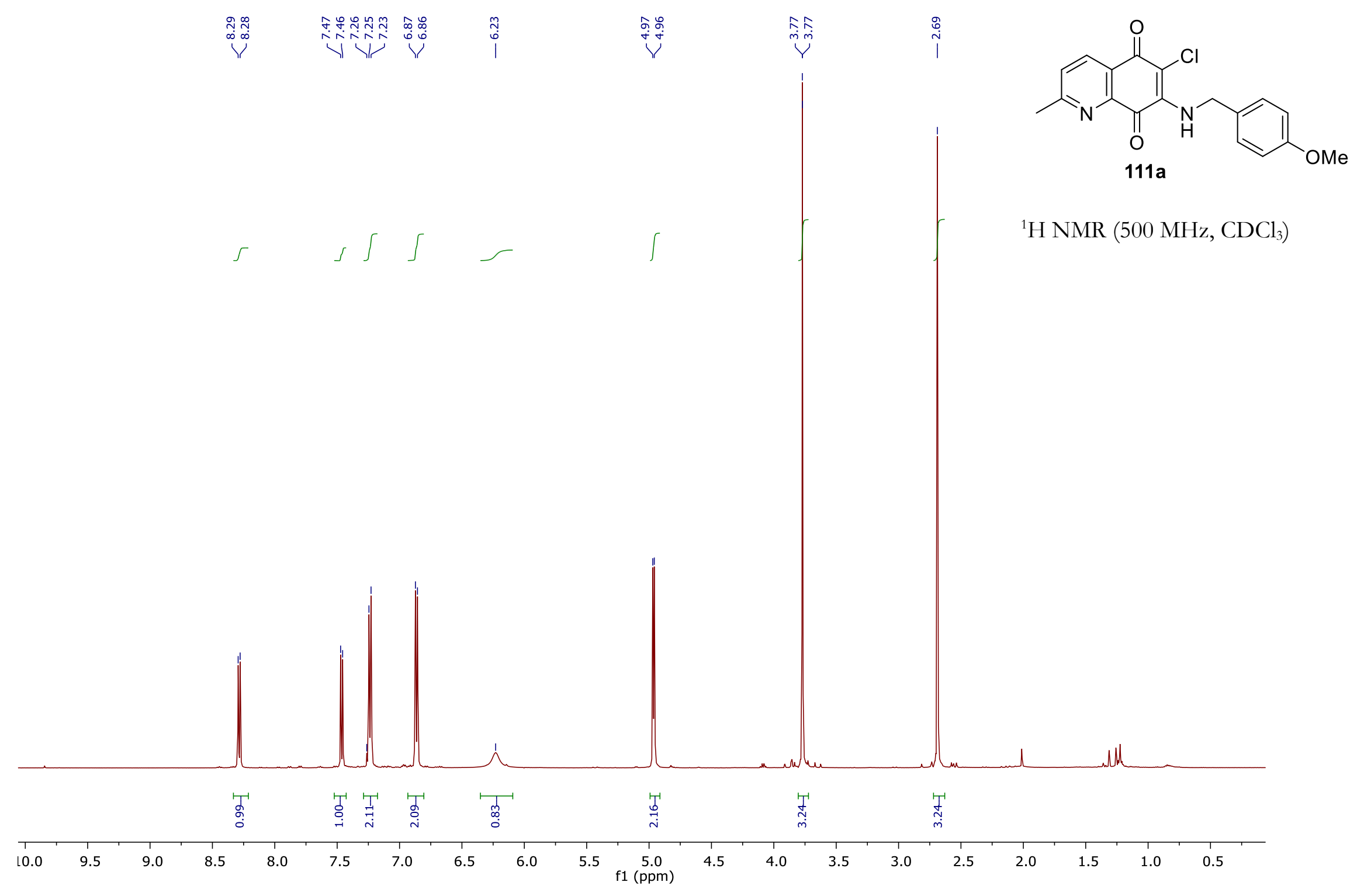




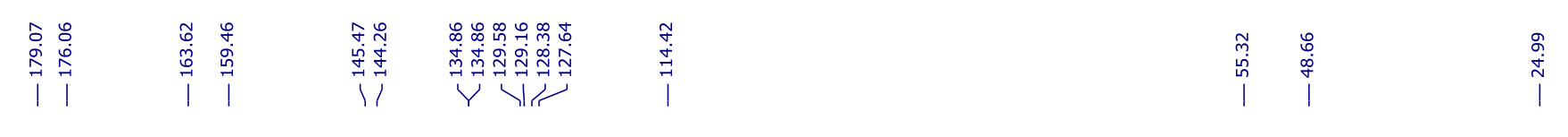

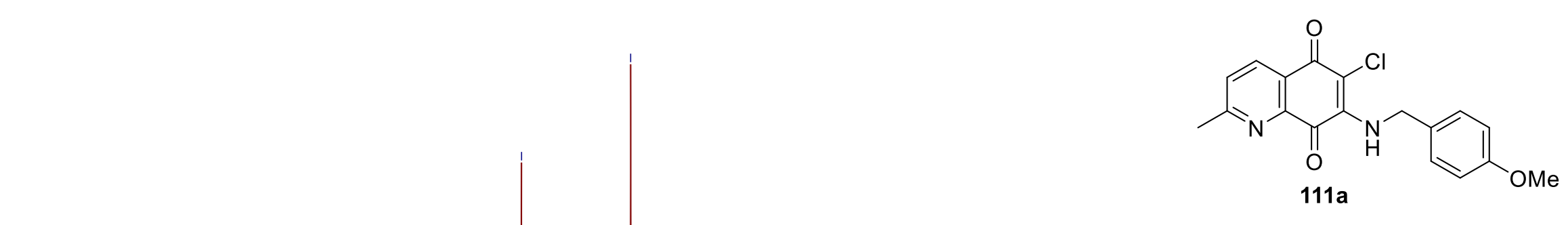

${ }^{13} \mathrm{C}$ NMR $\left(125 \mathrm{MHz}, \mathrm{CDCl}_{3}\right)$

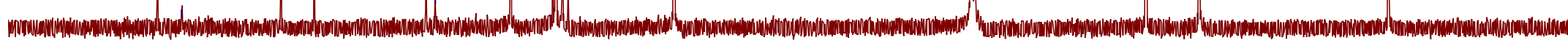

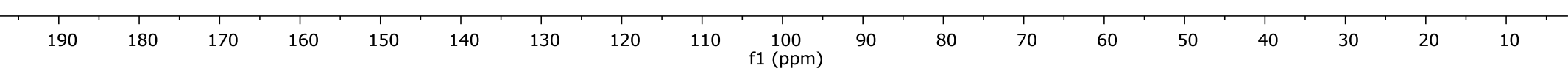




\section{APPENDIX: CHAPTER FOUR}

Figure A.4.1. Additional docking studies ...

A97

NMR spectra:

A98-A153 
A.<smiles>Nc1nc(Nc2ccc3oc(-c4ccccc4)cc(=O)c3c2)cc(-c2ccccc2)n1</smiles>

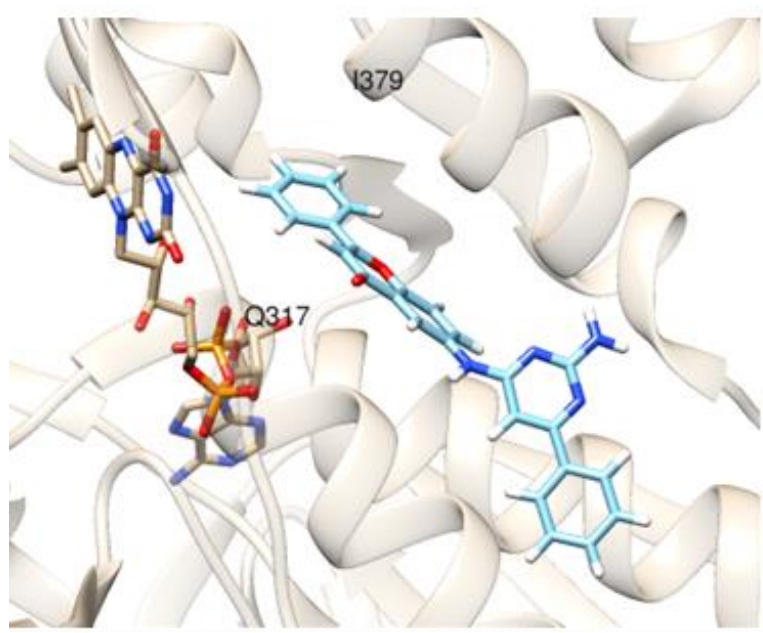

B.<smiles>Nc1nc(Nc2ccc3nc(-c4ccccc4)cc(N)c3c2)cc(-c2ccccc2)n1</smiles>

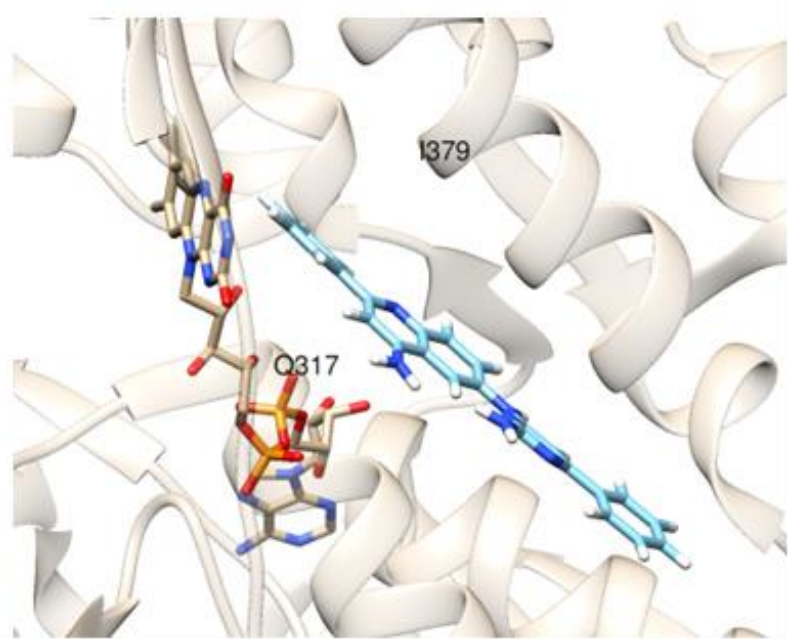

Figure A.4.18: Docking studies of 6-((2-amino-6-phenylpyrimidin-4-yl)amino)-2-phenyl-4Hchromen-4-one (196) (A) and N-(2-amino-6-phenylpyrimidin-4-yl)-2-phenylquinoline-4,6-diamine (A.1.) (B) in C. thermarum NDH-II (PDB: 6BDO), calculated using USCF Chimera/DOCK6. In both cases, chromonyl-pyrimidine 196 and quinolinyl pyrimidine A.1. are well-placed in the quinone binding site between Q317 and I317. Here, distant edge-to-face $\pi-\pi$ interactions are possible between the phenyl substituents on the chromone and quinoline scaffolds in diphenyls 196 and A.1., respectively and the FAD isoalloxazine ring.. 


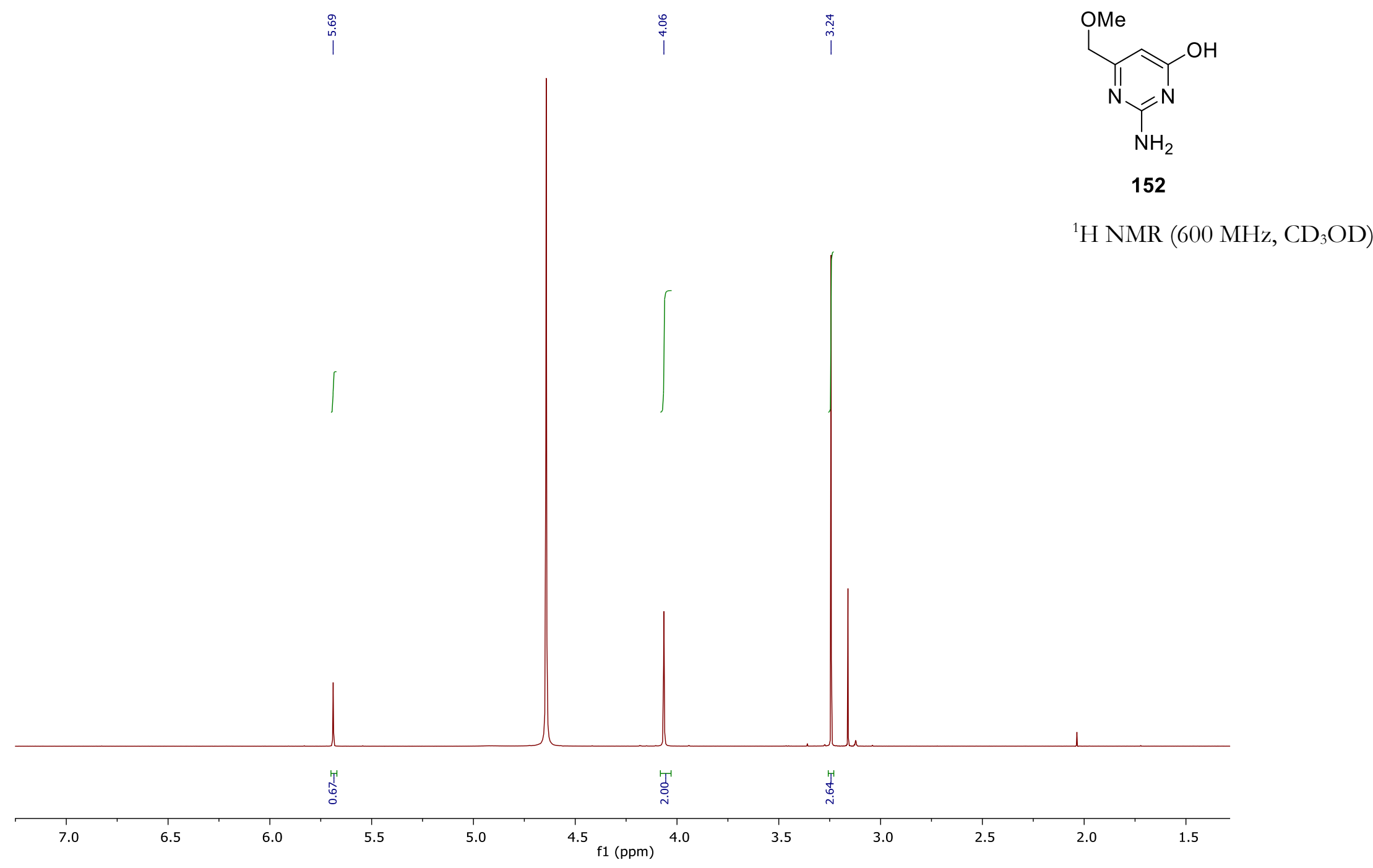




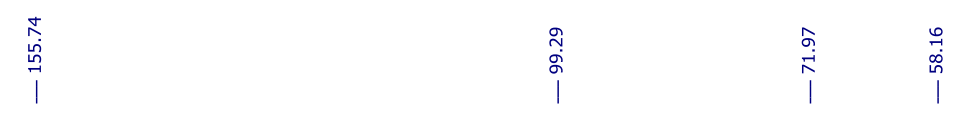

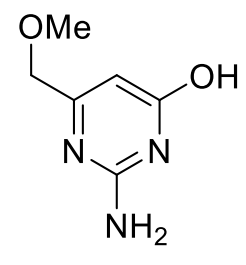

152

${ }^{13} \mathrm{C}$ NMR $\left(150 \mathrm{MHz}, \mathrm{CD}_{3} \mathrm{OD}\right)$
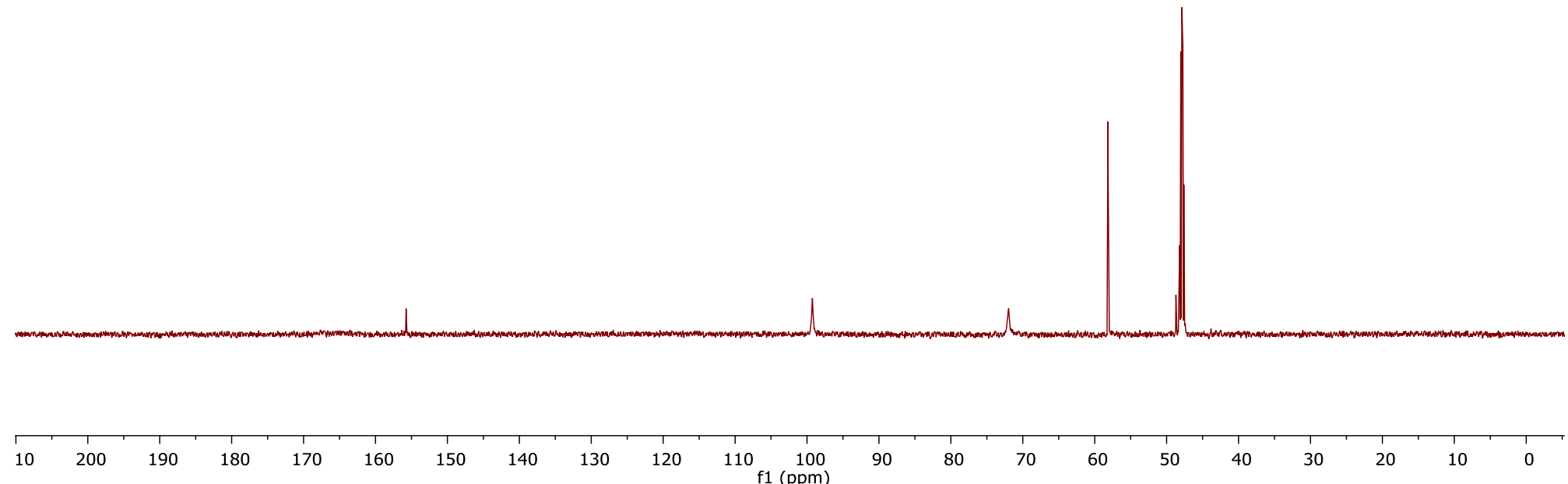


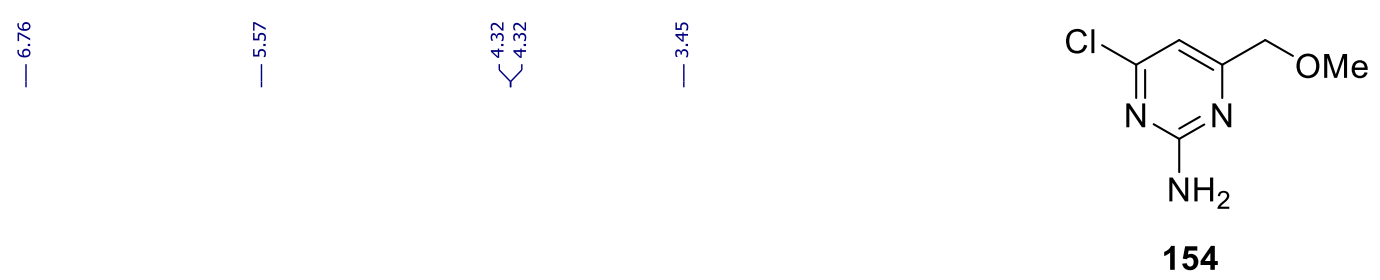

${ }^{1} \mathrm{H}$ NMR $\left(500 \mathrm{MHz}, \mathrm{CDCl}_{3}\right)$

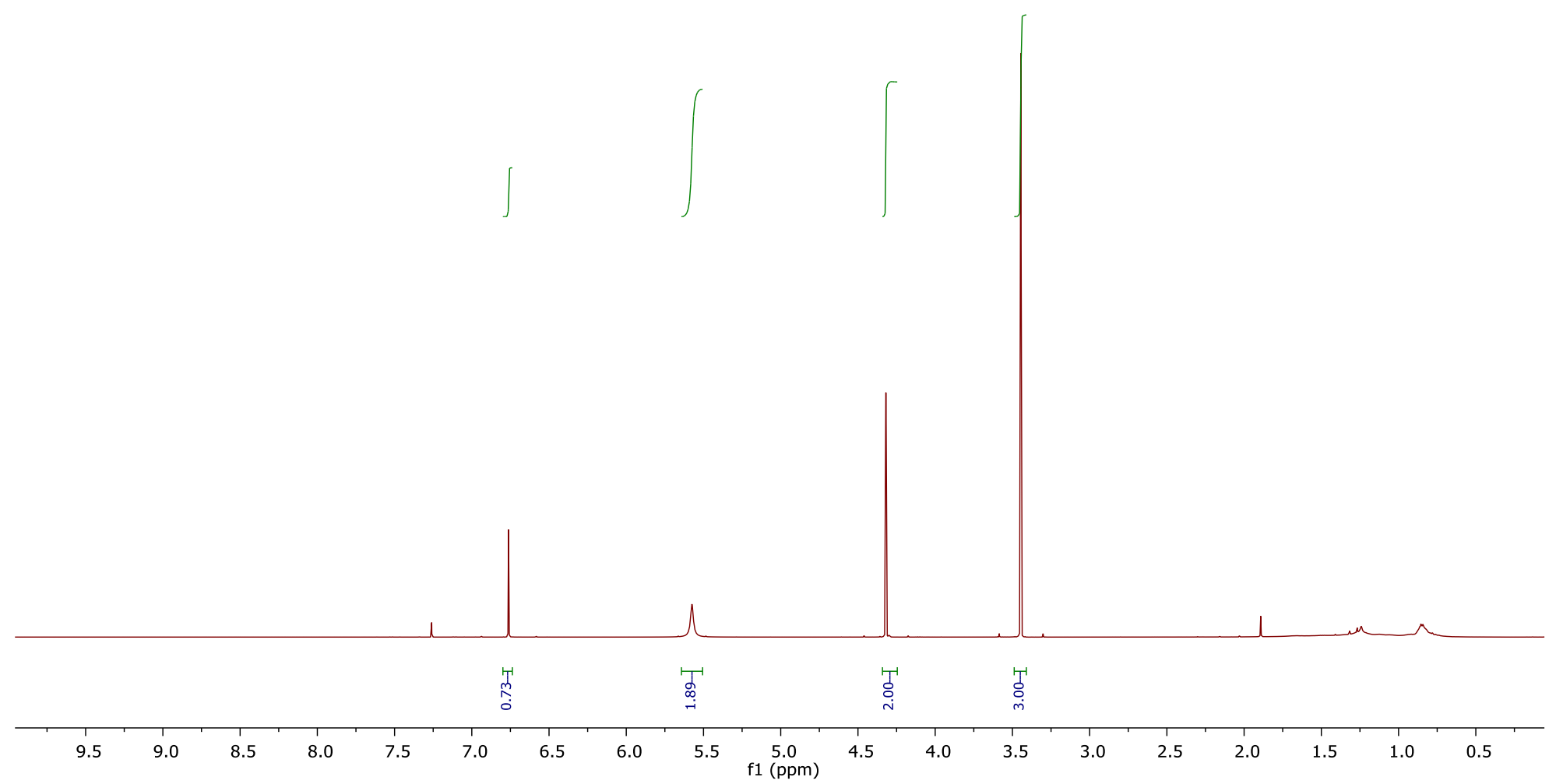




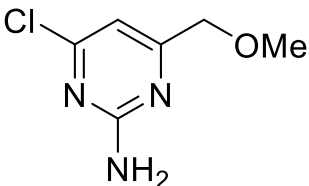

154

${ }^{13} \mathrm{C}$ NMR $\left(125 \mathrm{MHz}, \mathrm{CDCl}_{3}\right)$
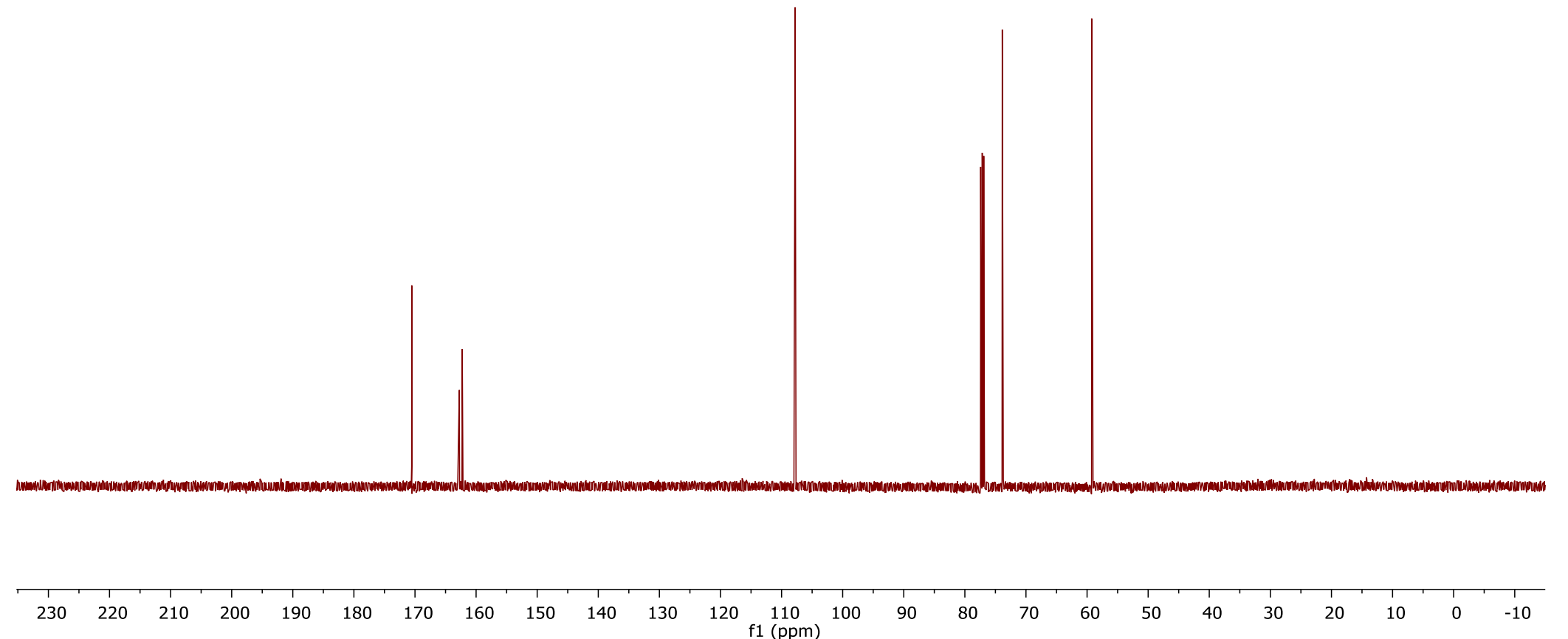


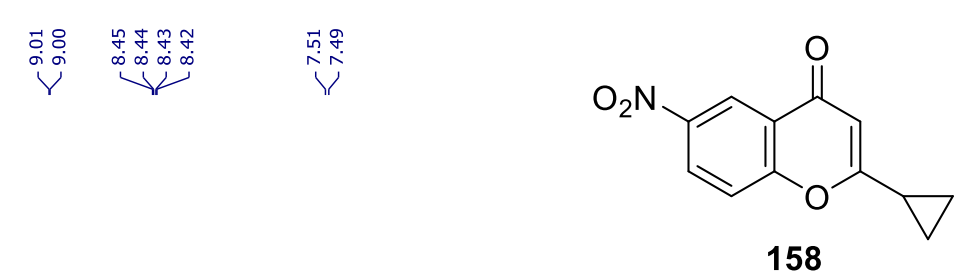

$\underbrace{\underbrace{}_{0}}$

${ }^{1} \mathrm{H}$ NMR (500 MHz, $\left.\mathrm{CDCl}_{3}\right)$

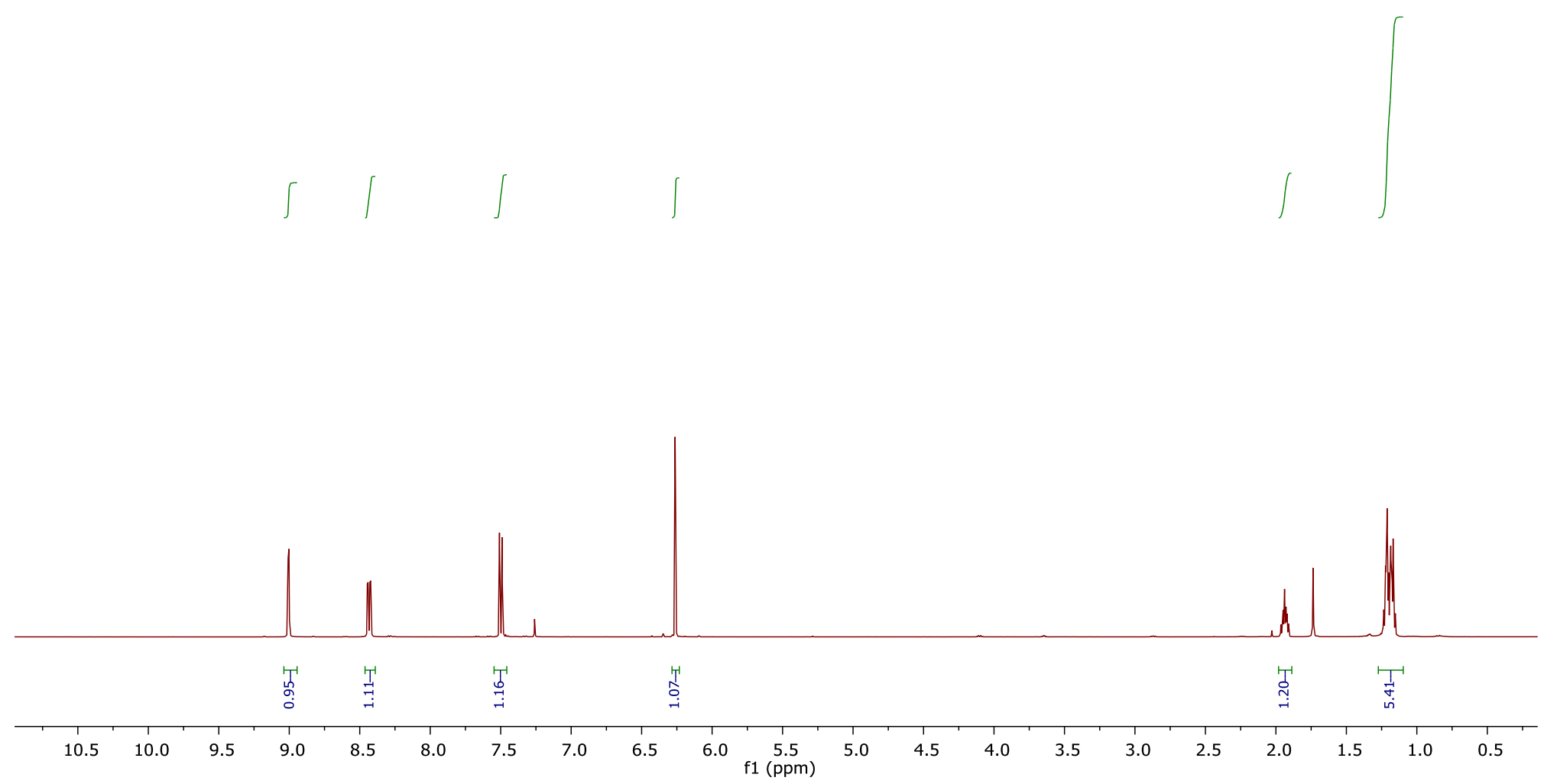




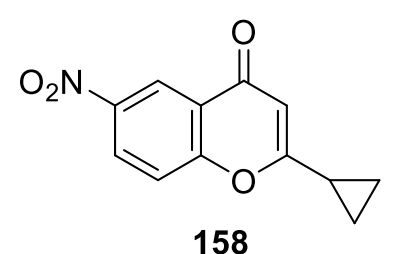

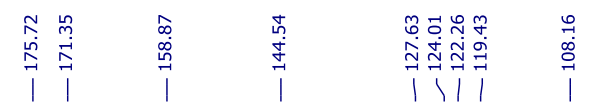

${ }^{13} \mathrm{C}$ NMR $\left(125 \mathrm{MHz}, \mathrm{CDCl}_{3}\right)$

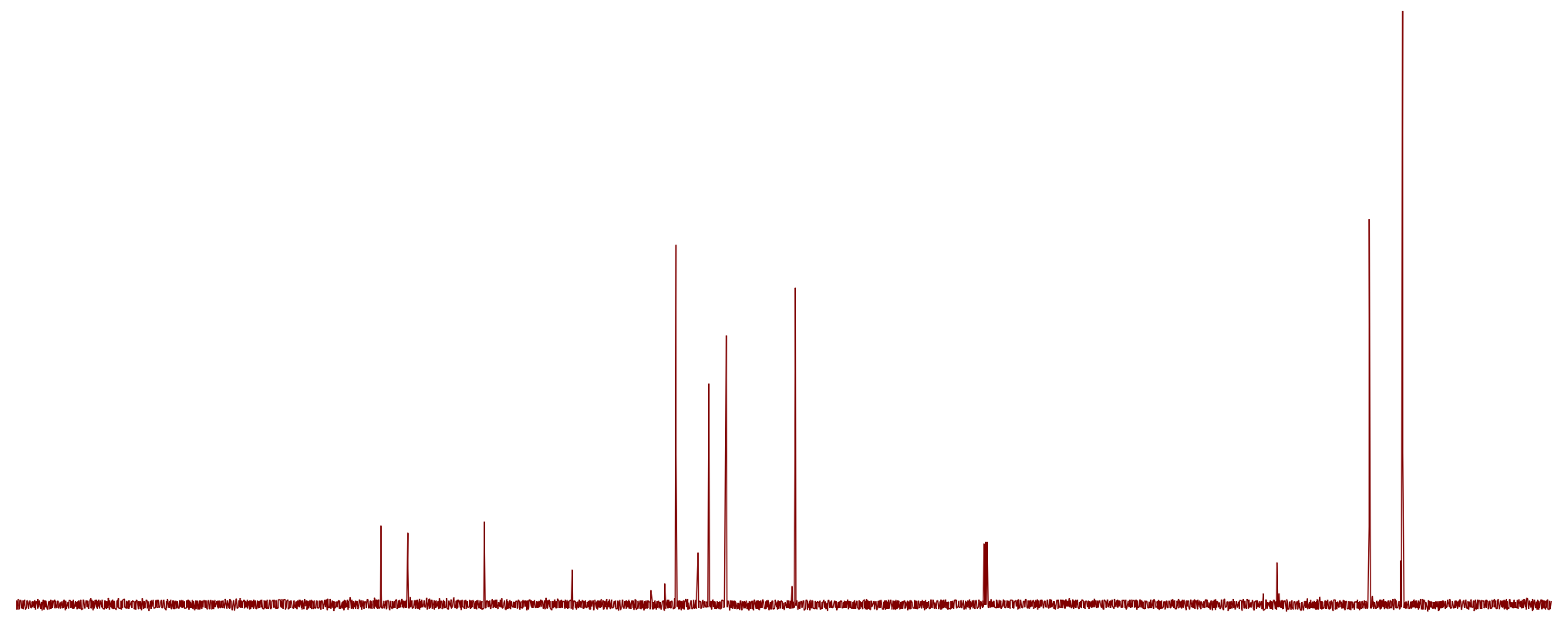

\begin{tabular}{lllllllllllllllllllllllllllllllllll}
\hline 230 & 220 & 210 & 200 & 190 & 180 & 170 & 160 & 150 & 140 & 130 & 120 & 110 & 100 & 90 & 80 & 70 & 60 & 50 & 40 & 30 & 20 & 10 & 0 & -10
\end{tabular} 


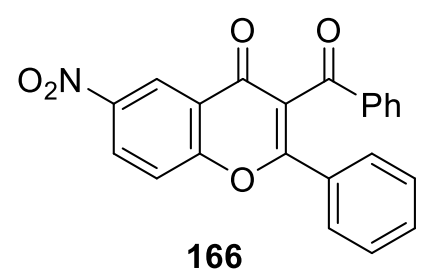

${ }^{1} \mathrm{H}$ NMR (500 MHz, $\mathrm{CDCl}_{3}$ )
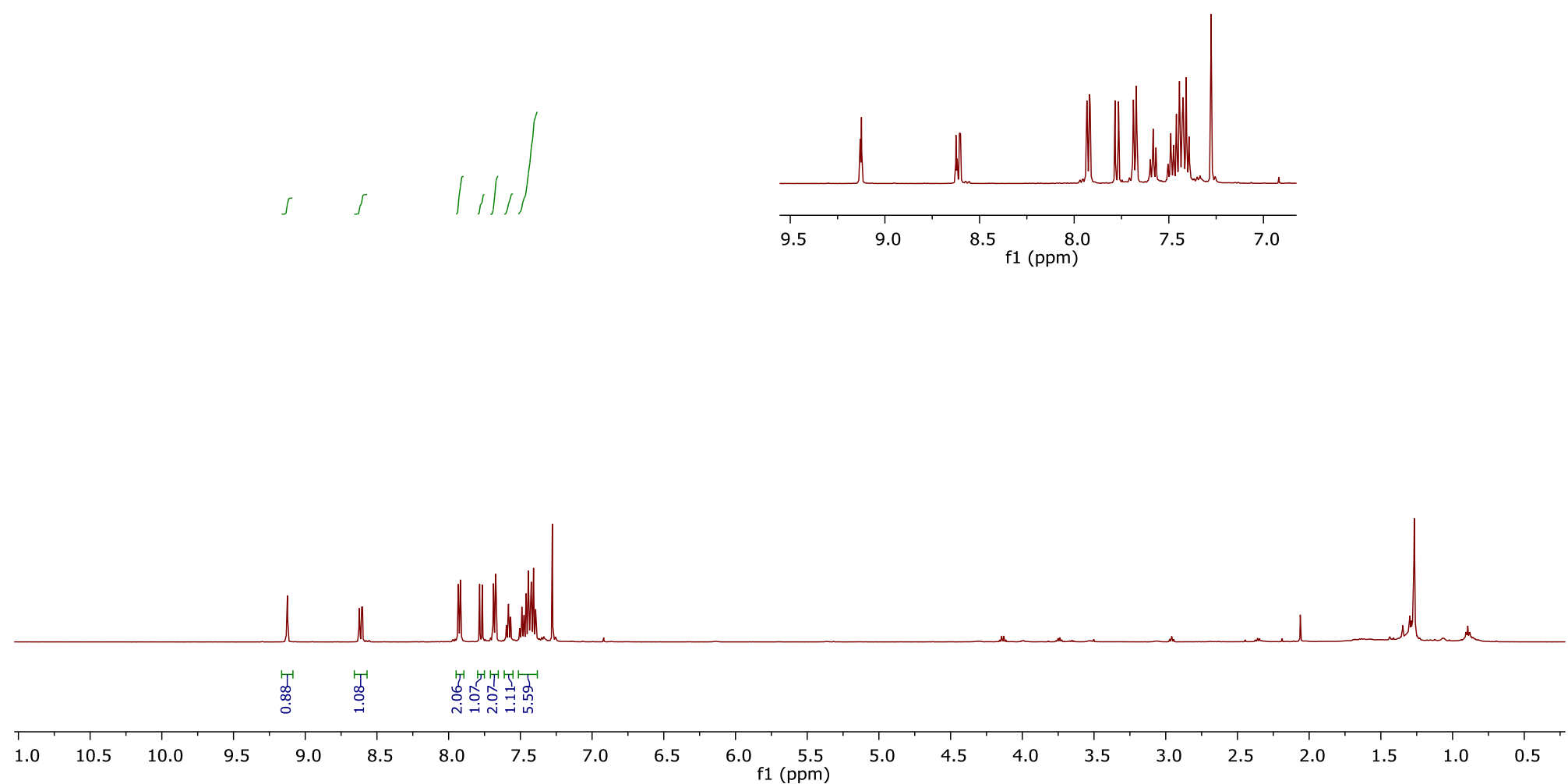


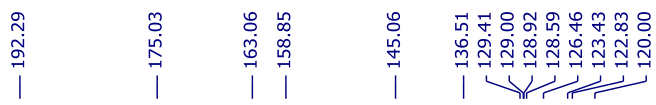

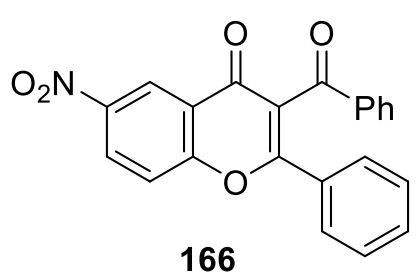

${ }^{13} \mathrm{C}$ NMR $\left(125 \mathrm{MHz}, \mathrm{CDCl}_{3}\right)$

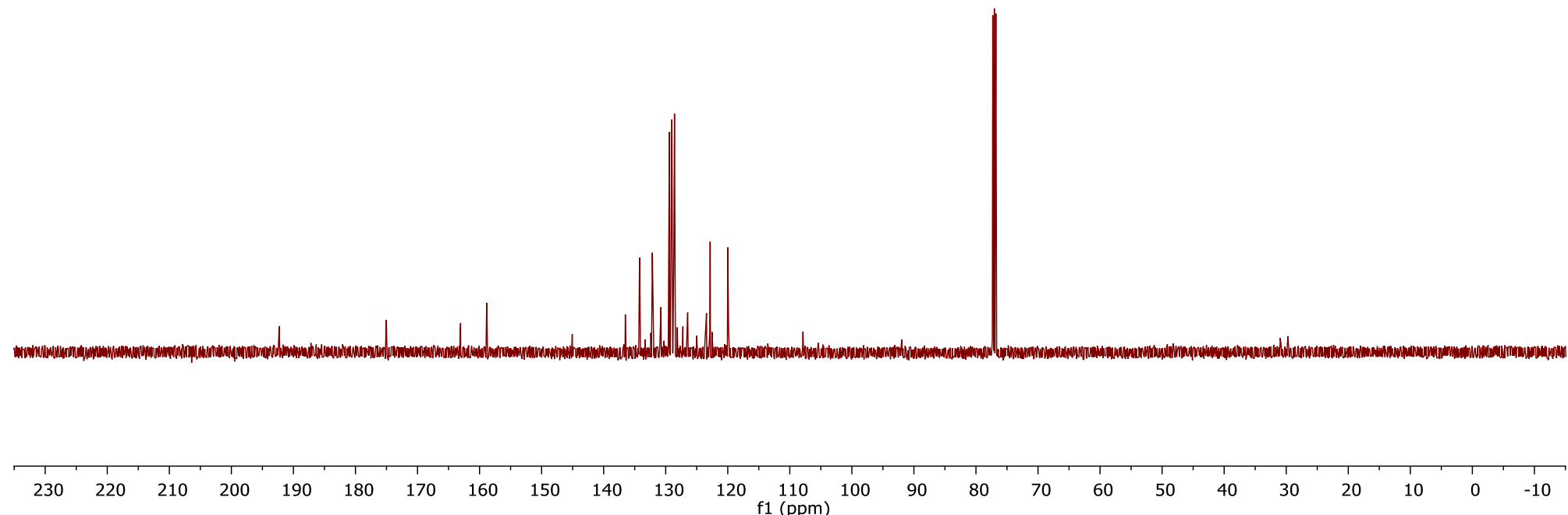




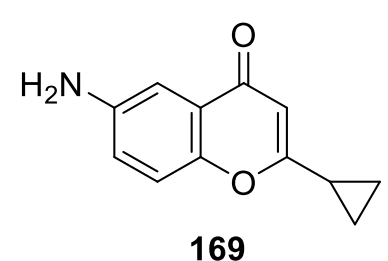

${ }^{1} \mathrm{H}$ NMR $\left(500 \mathrm{MHz}, \mathrm{CDCl}_{3}\right)$

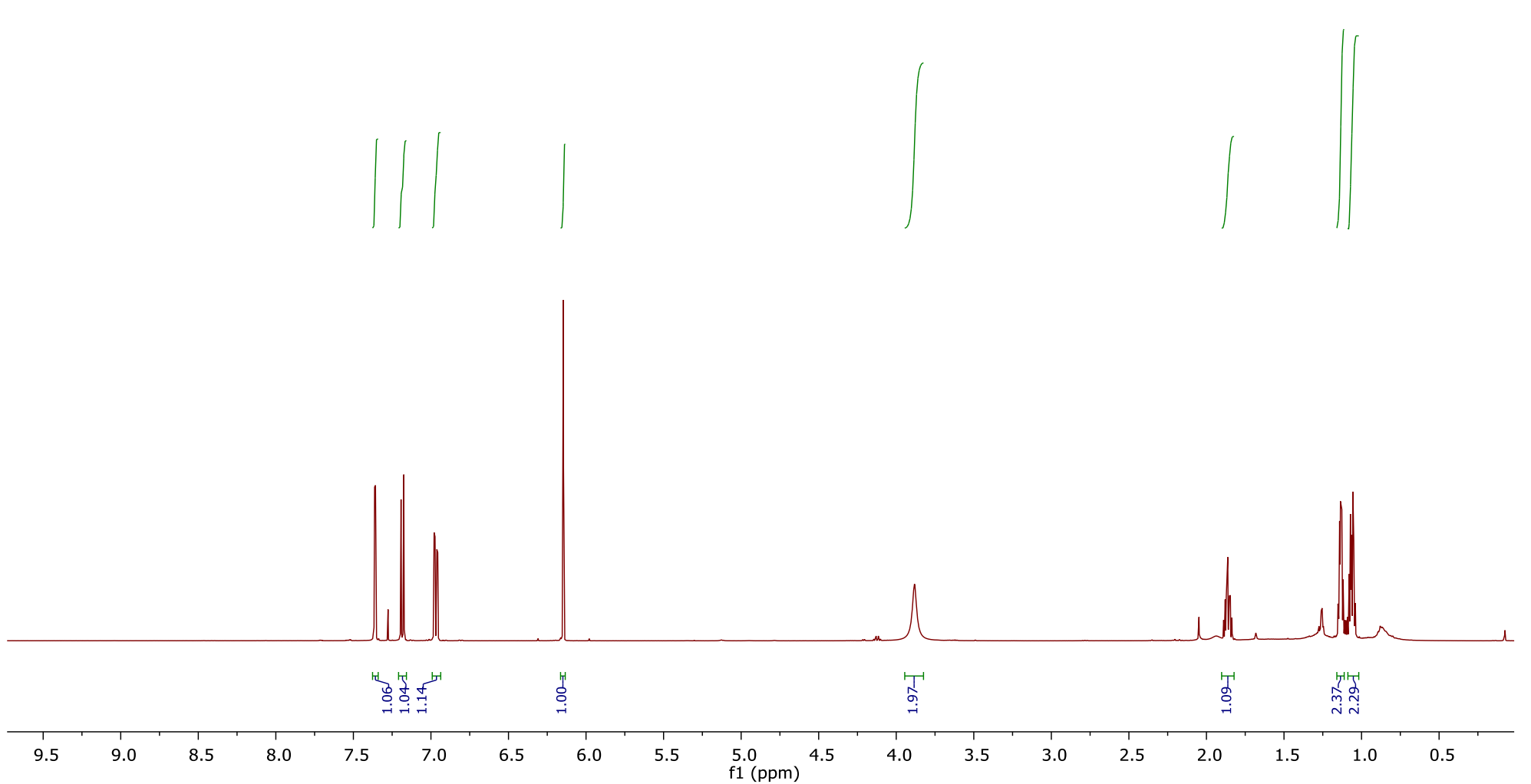



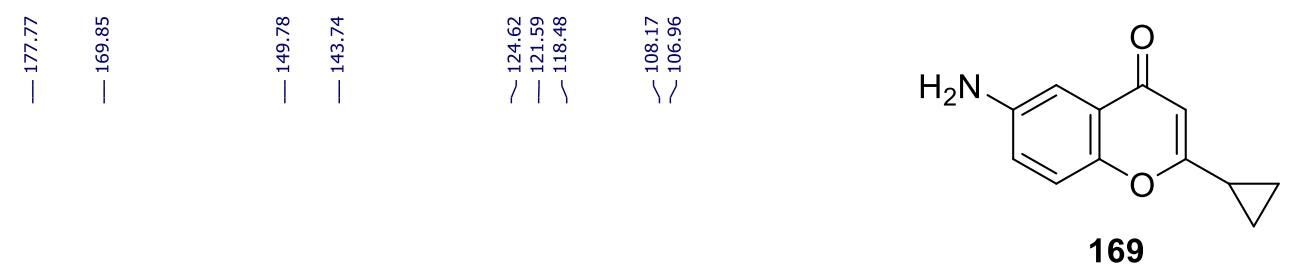

1
1

169

${ }^{13} \mathrm{C}$ NMR $\left(125 \mathrm{MHz}, \mathrm{CDCl}_{3}\right)$

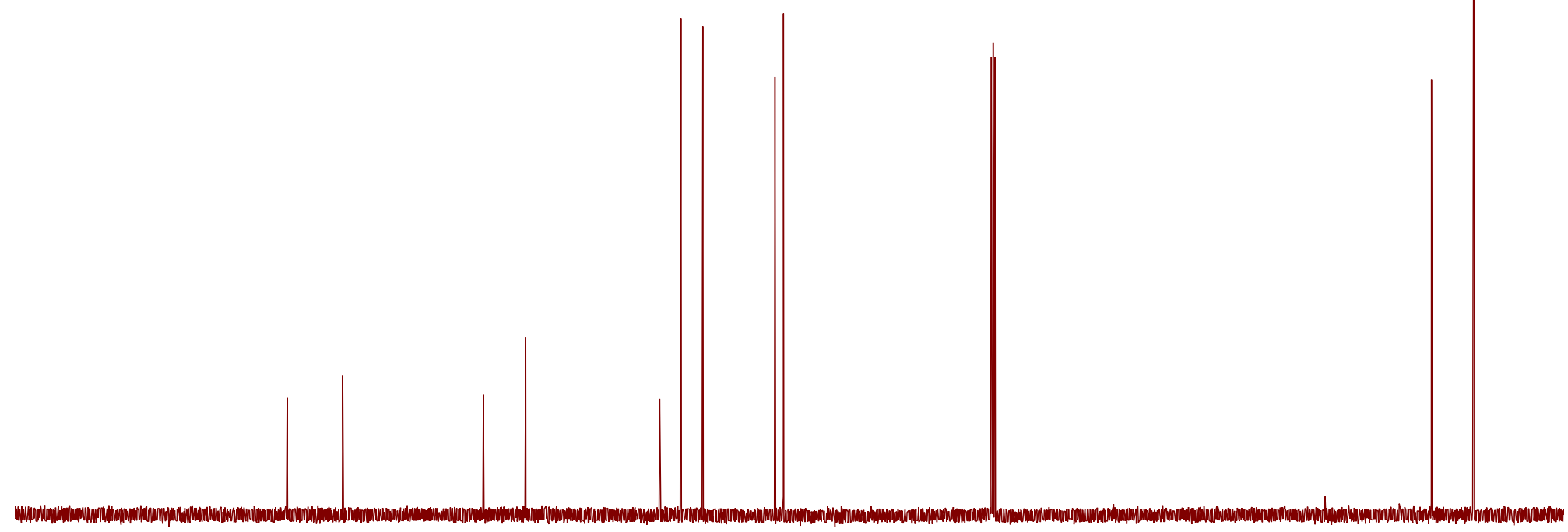




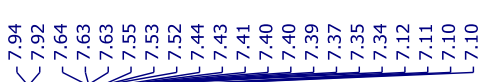

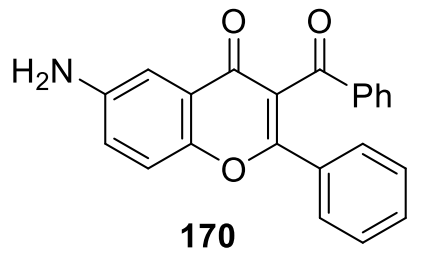

${ }^{1} \mathrm{H}$ NMR $\left(500 \mathrm{MHz}, \mathrm{CDCl}_{3}\right)$
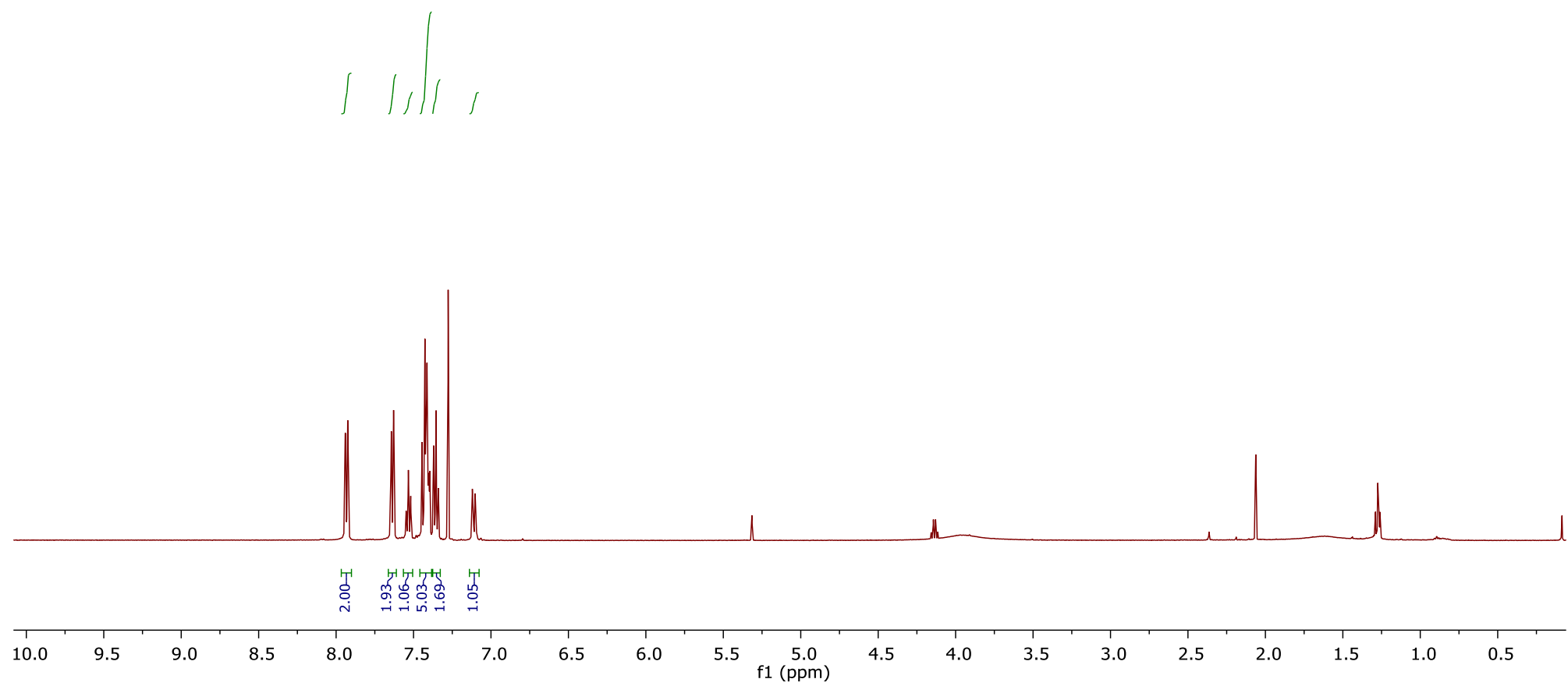


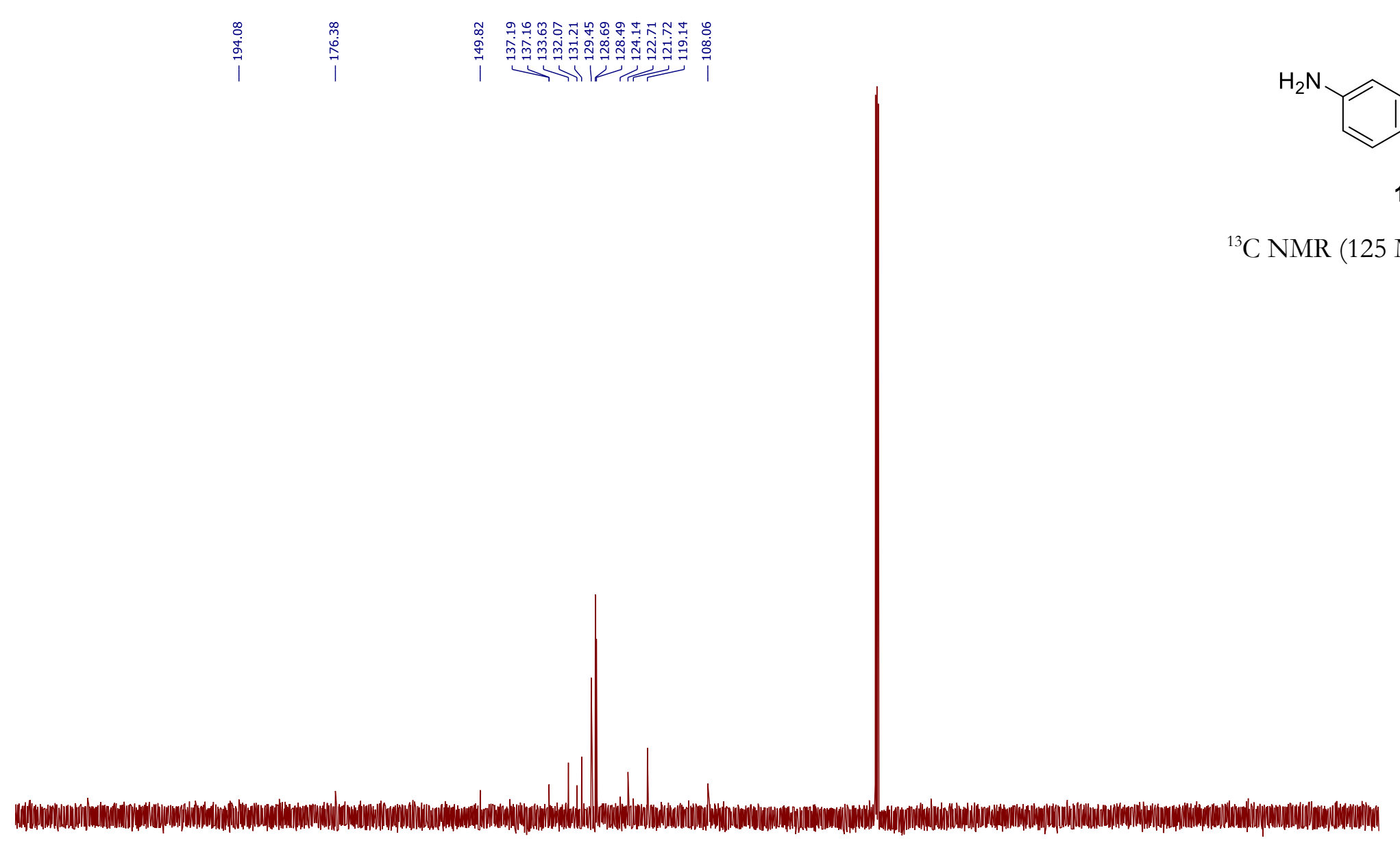




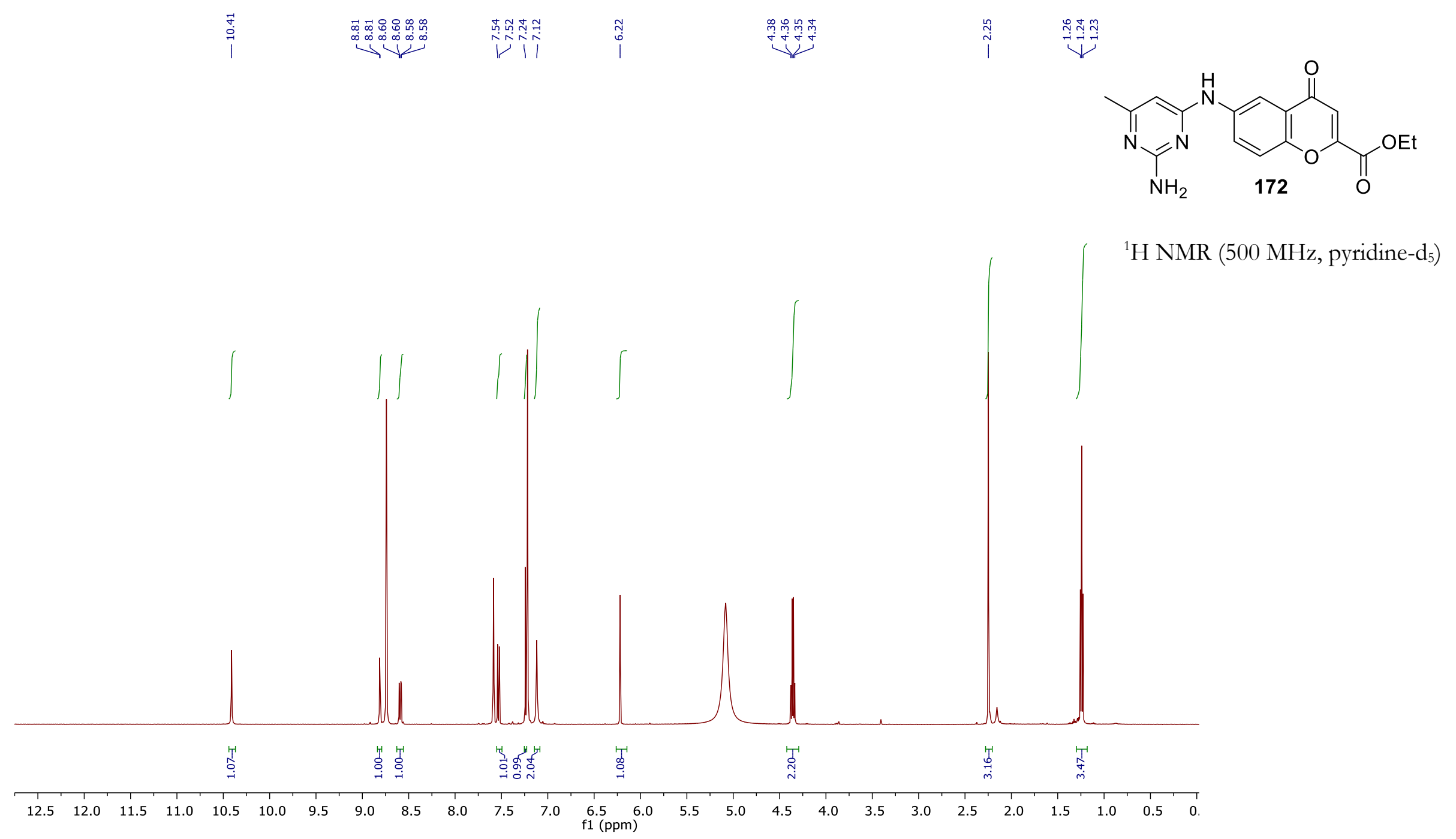



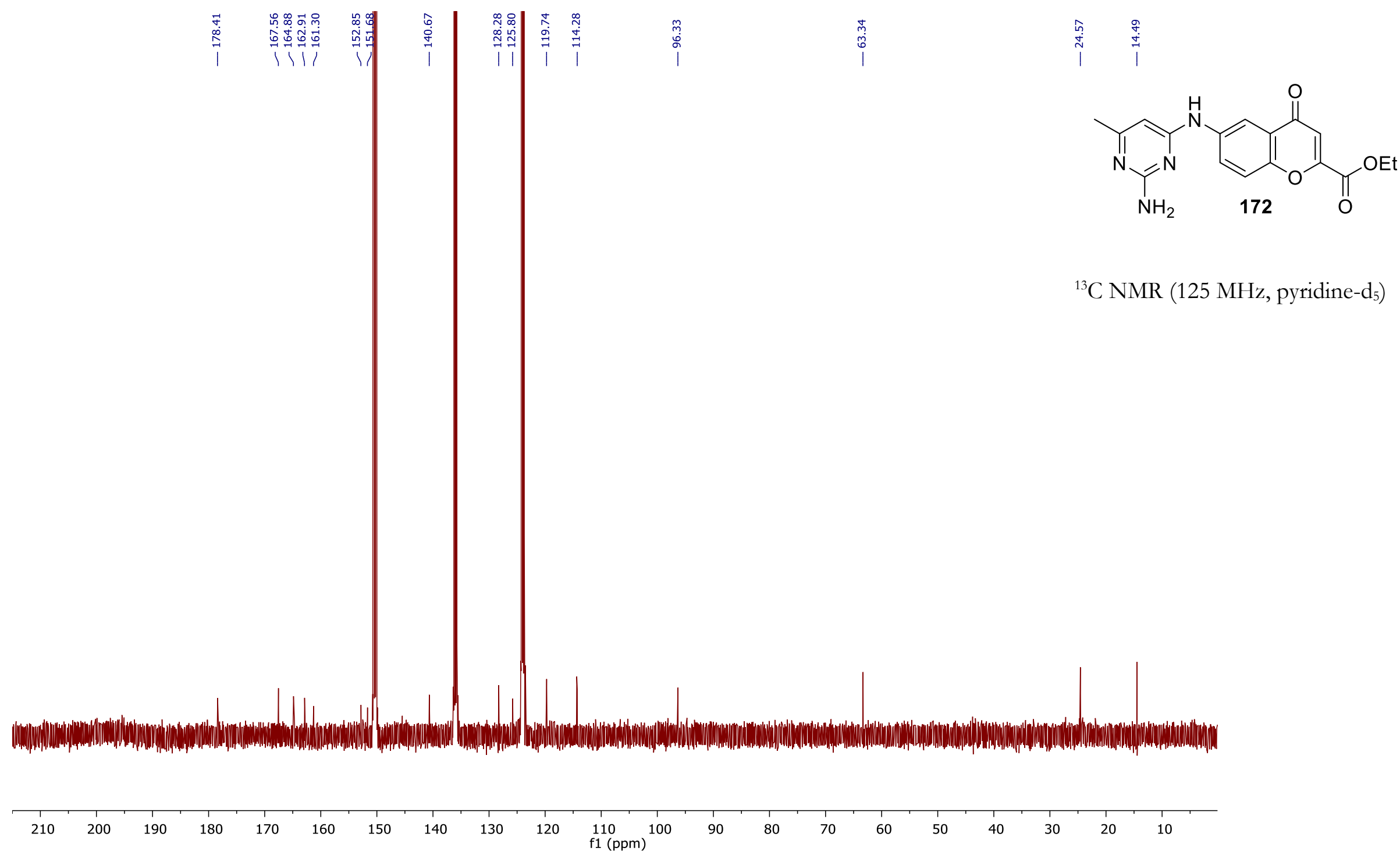


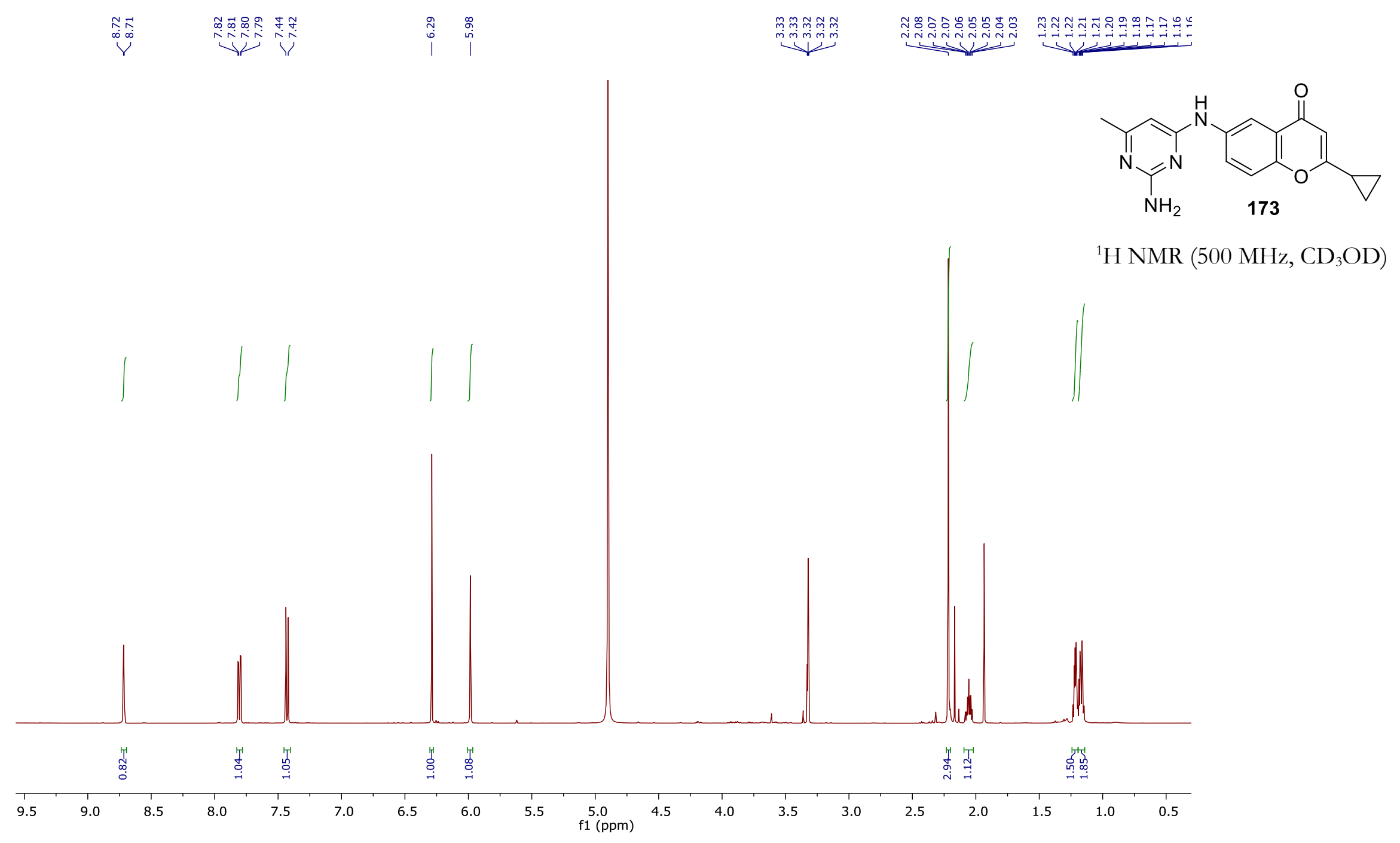




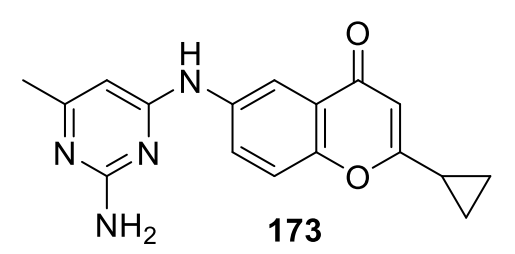

${ }^{13} \mathrm{C}$ NMR $\left(125 \mathrm{MHz}, \mathrm{CD}_{3} \mathrm{OD}\right)$
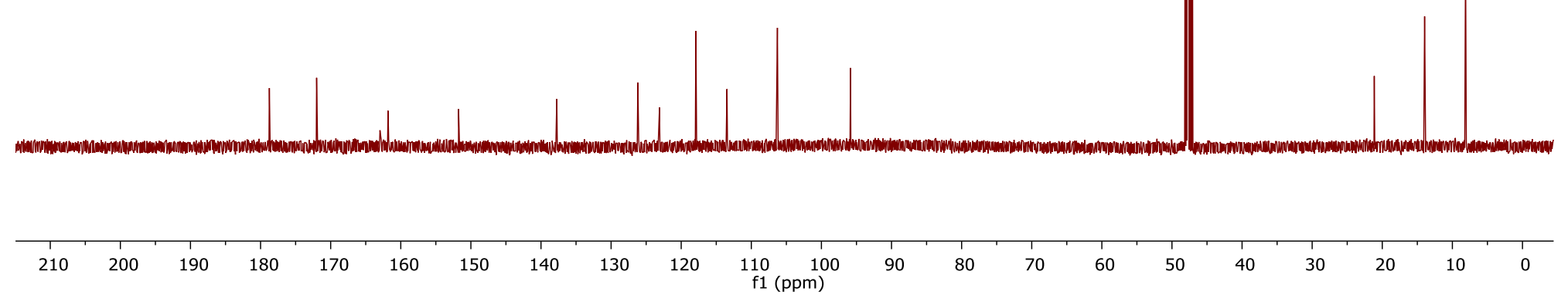


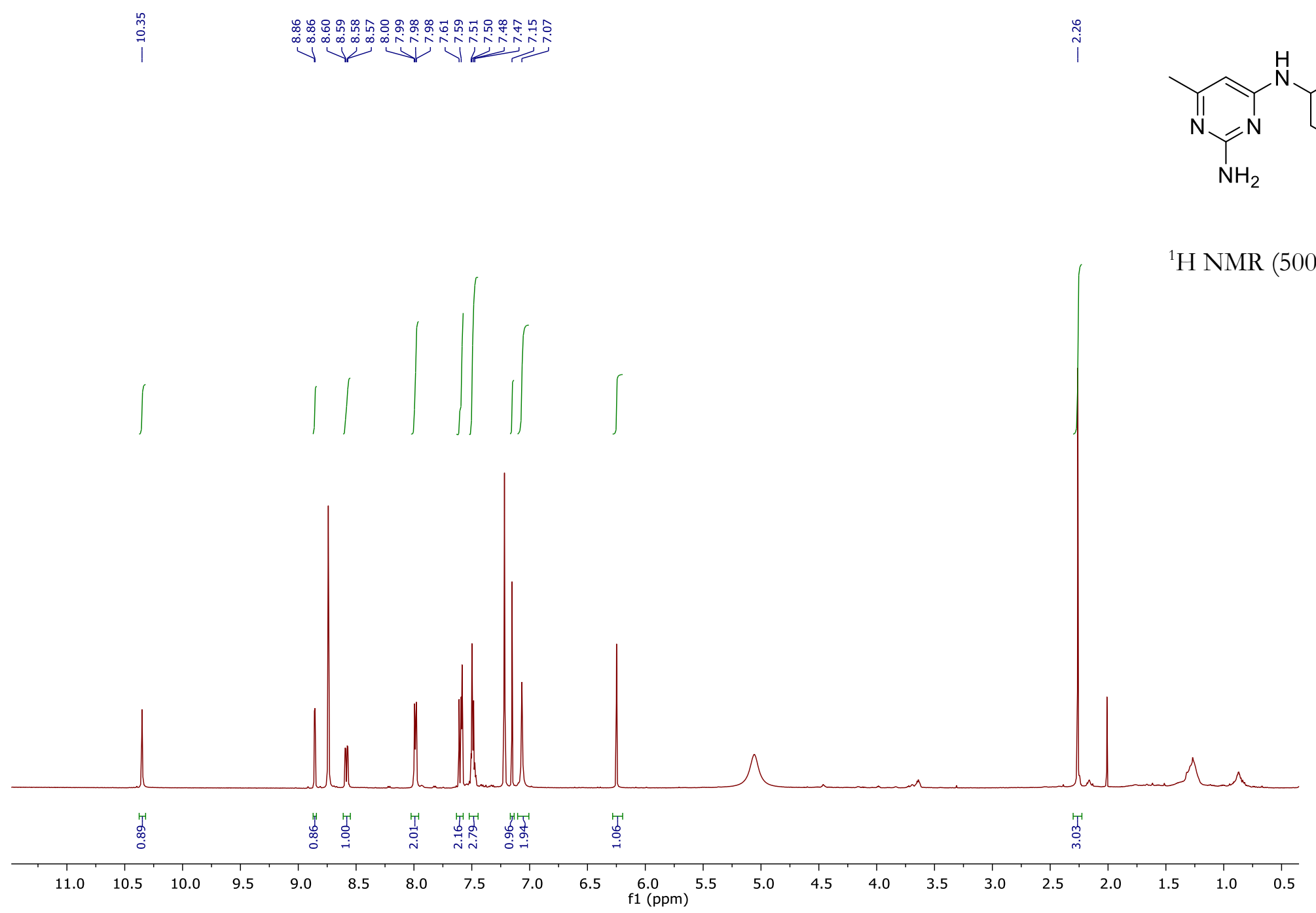




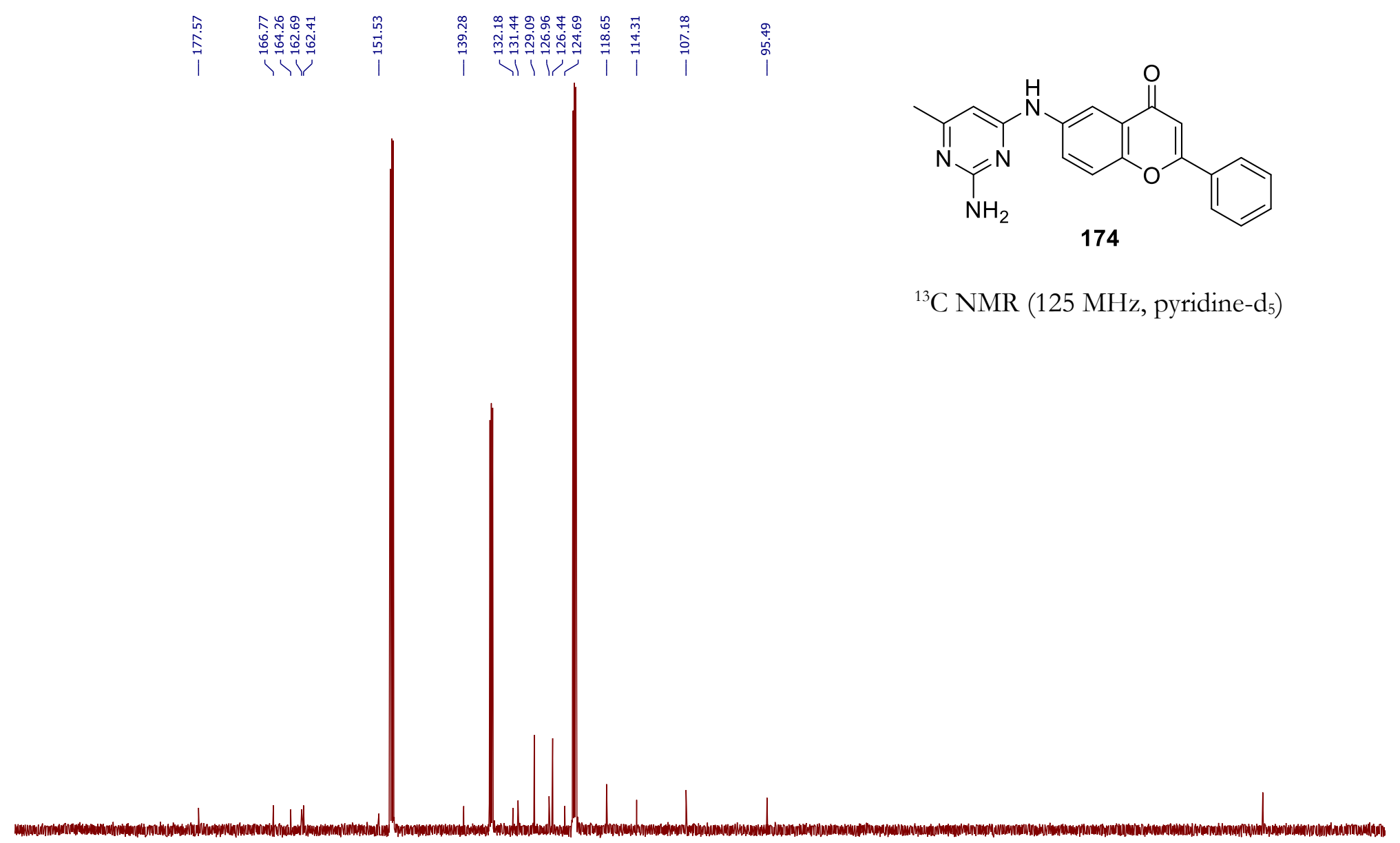




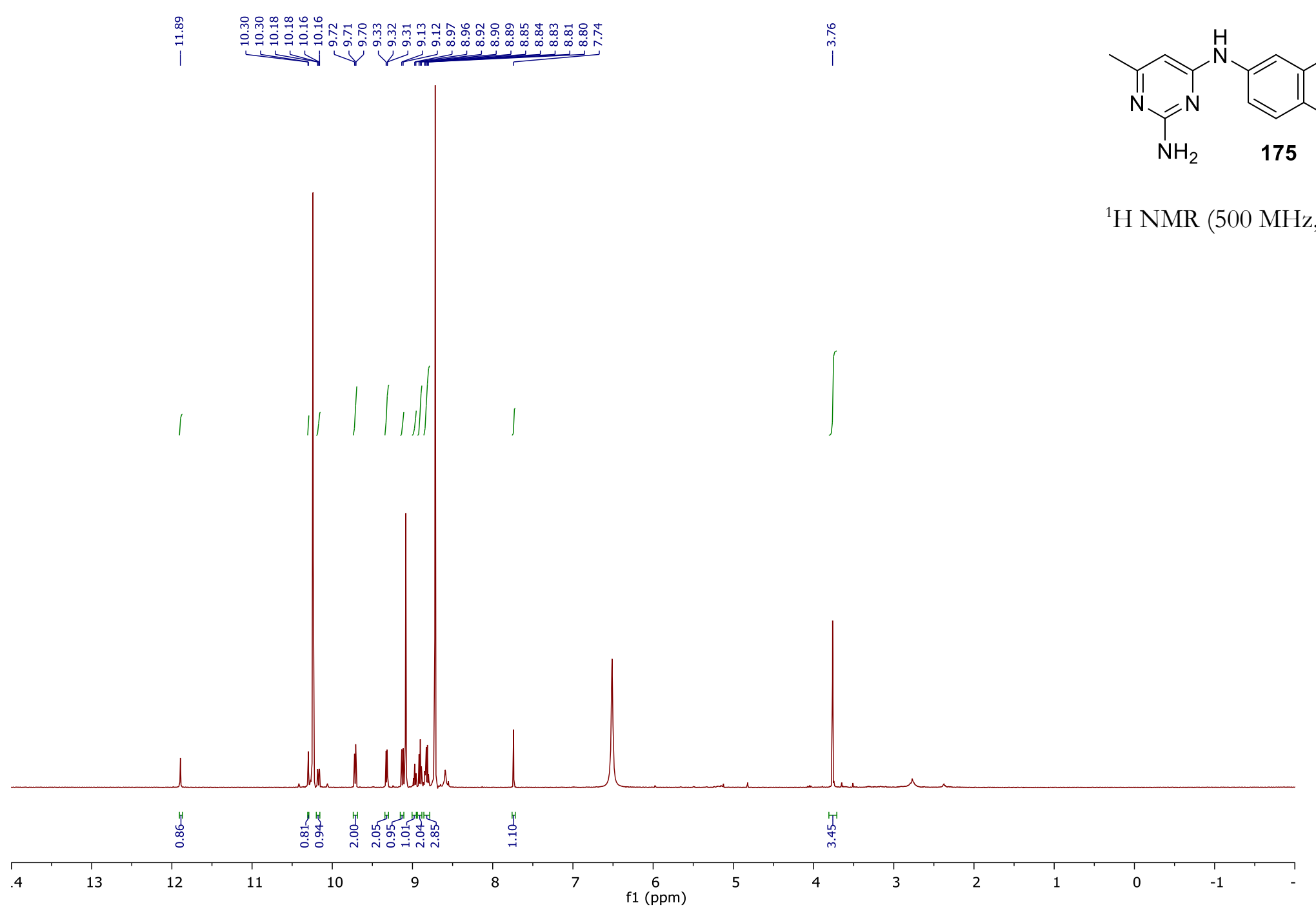




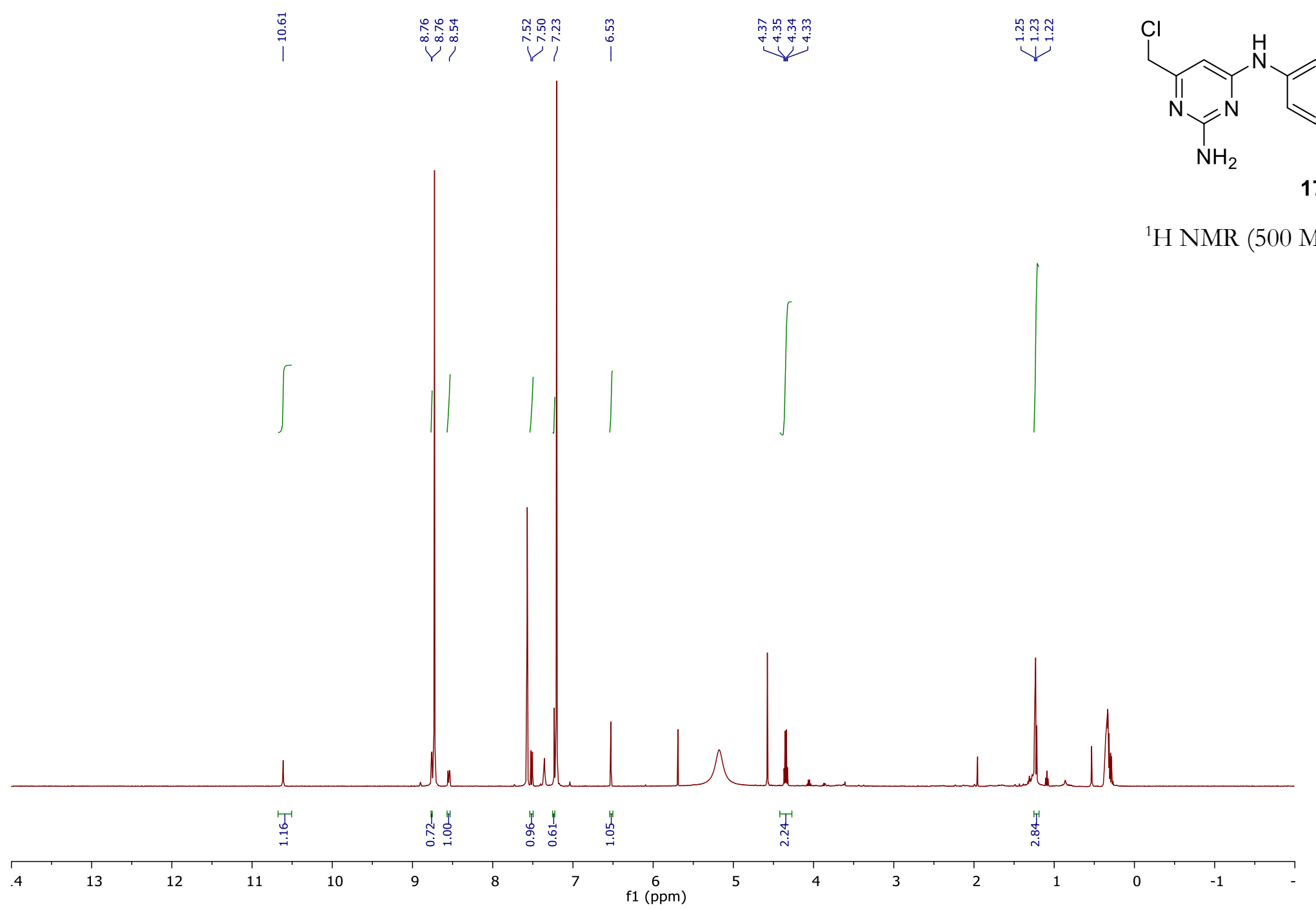



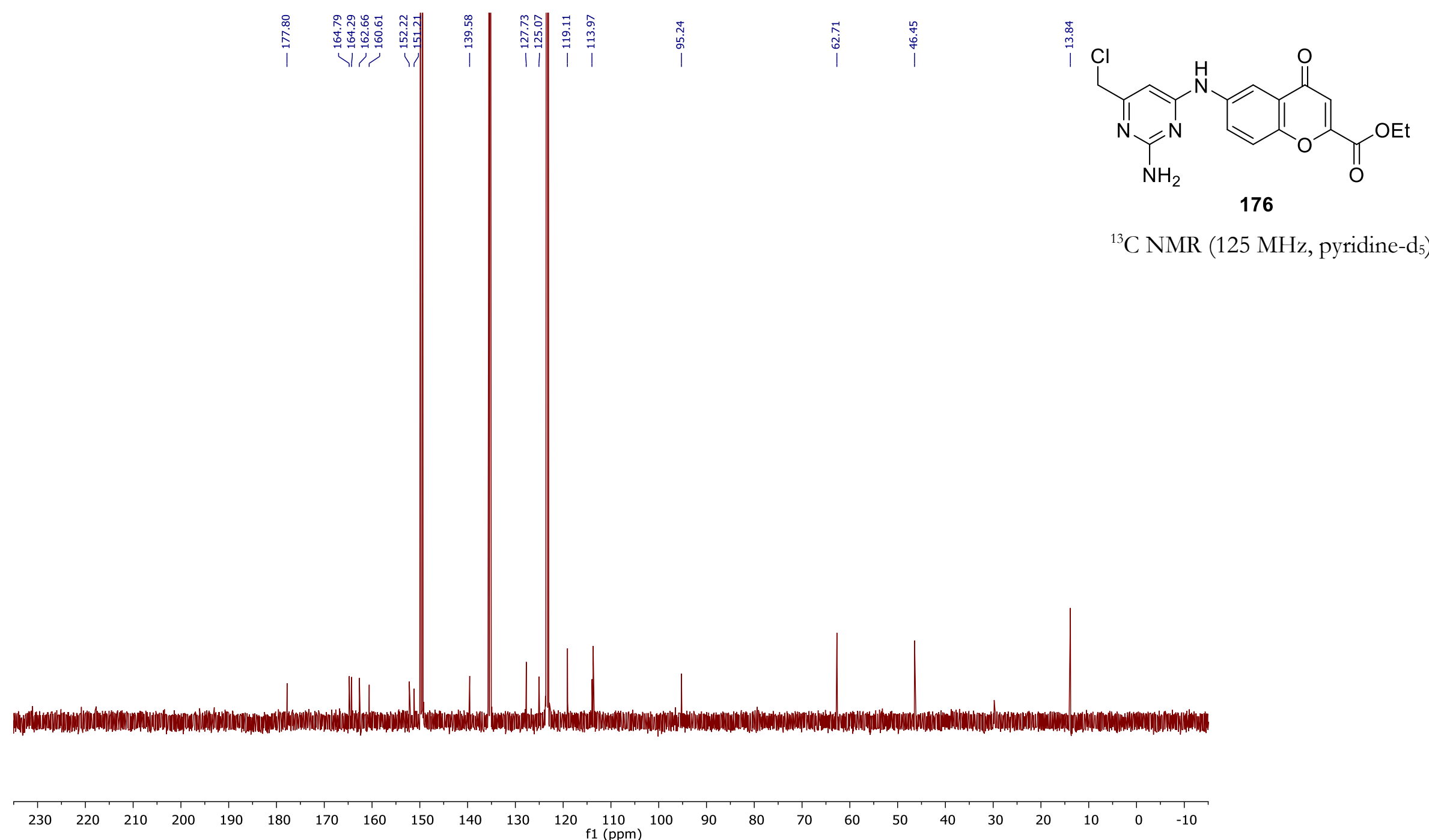


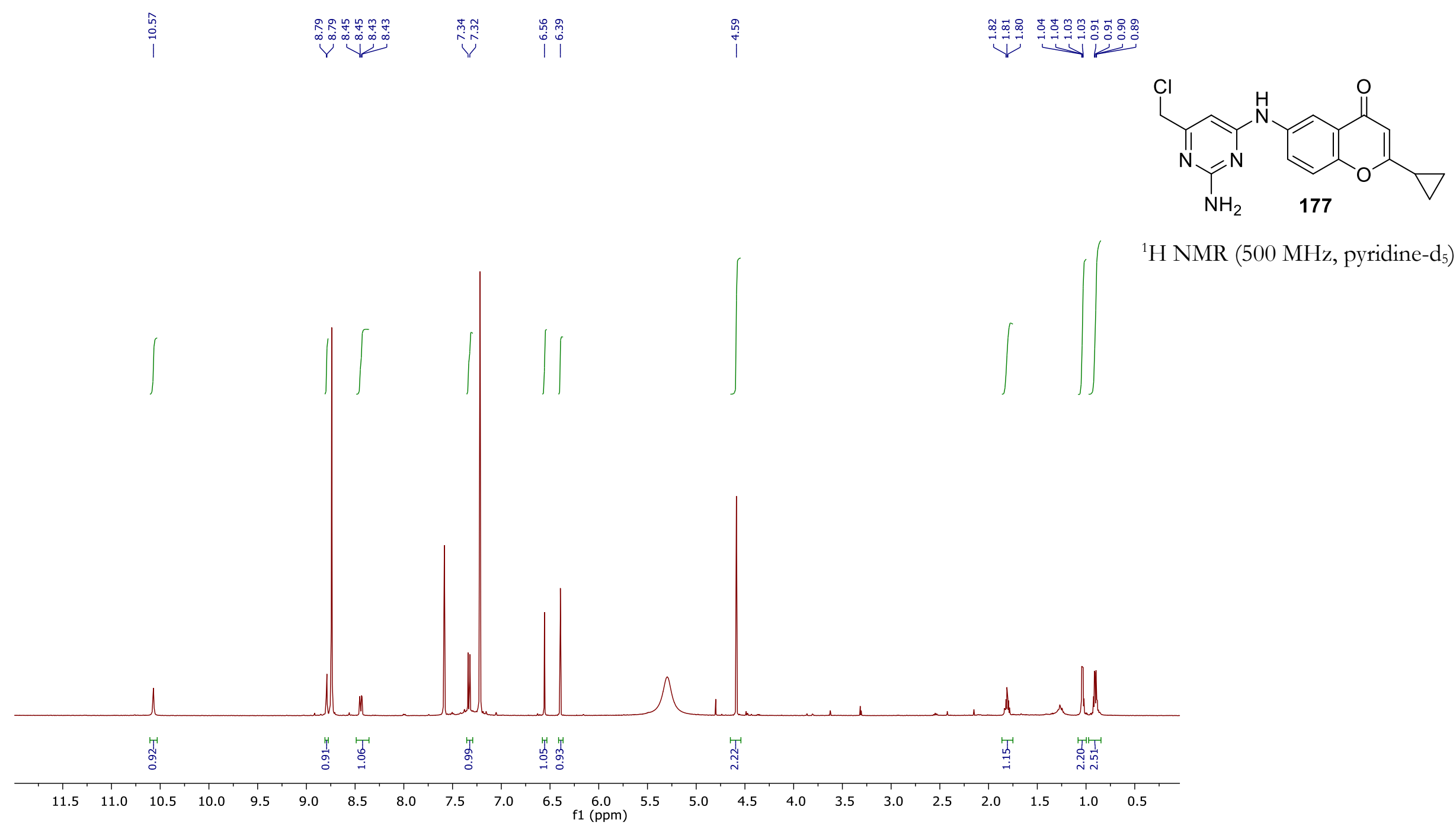




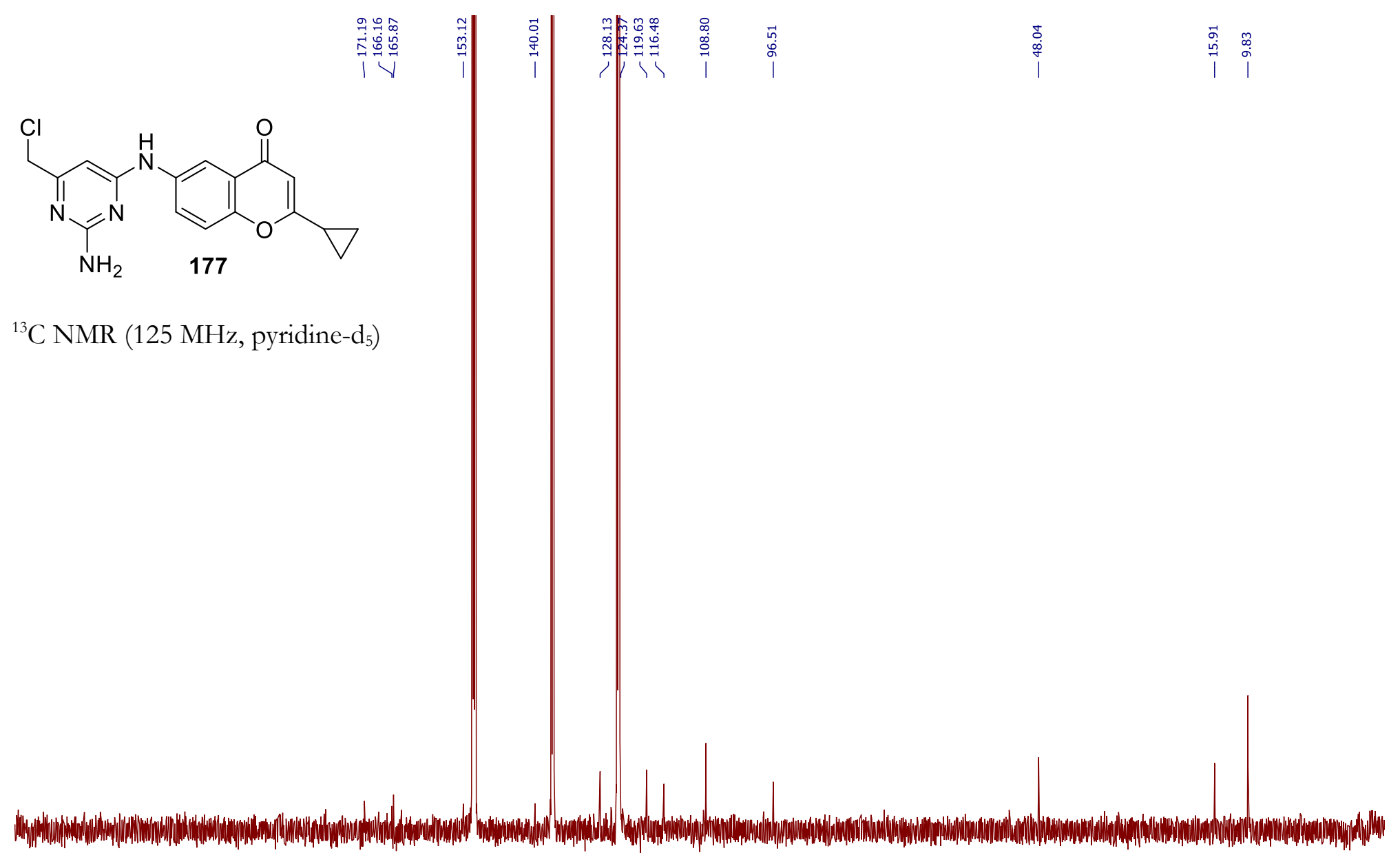

$\begin{array}{lllllllllllllllllllllllllllllllll}230 & 220 & 210 & 200 & 190 & 180 & 170 & 160 & 150 & 140 & 130 & 120 & 110 & 100 & 90 & 80 & 70 & 60 & 50 & 40 & 30 & 20 & 10 & 0 & -10\end{array}$ 


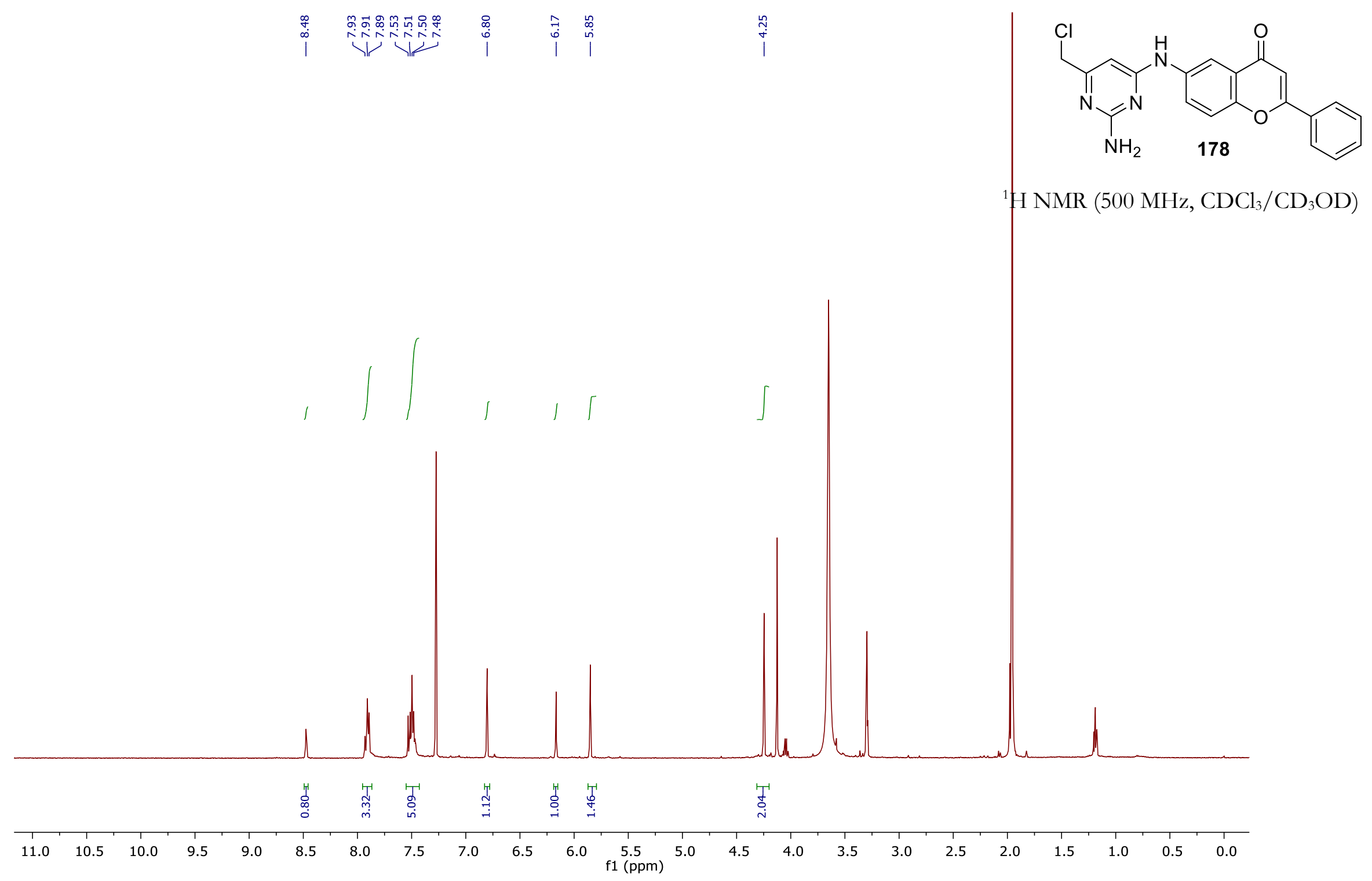




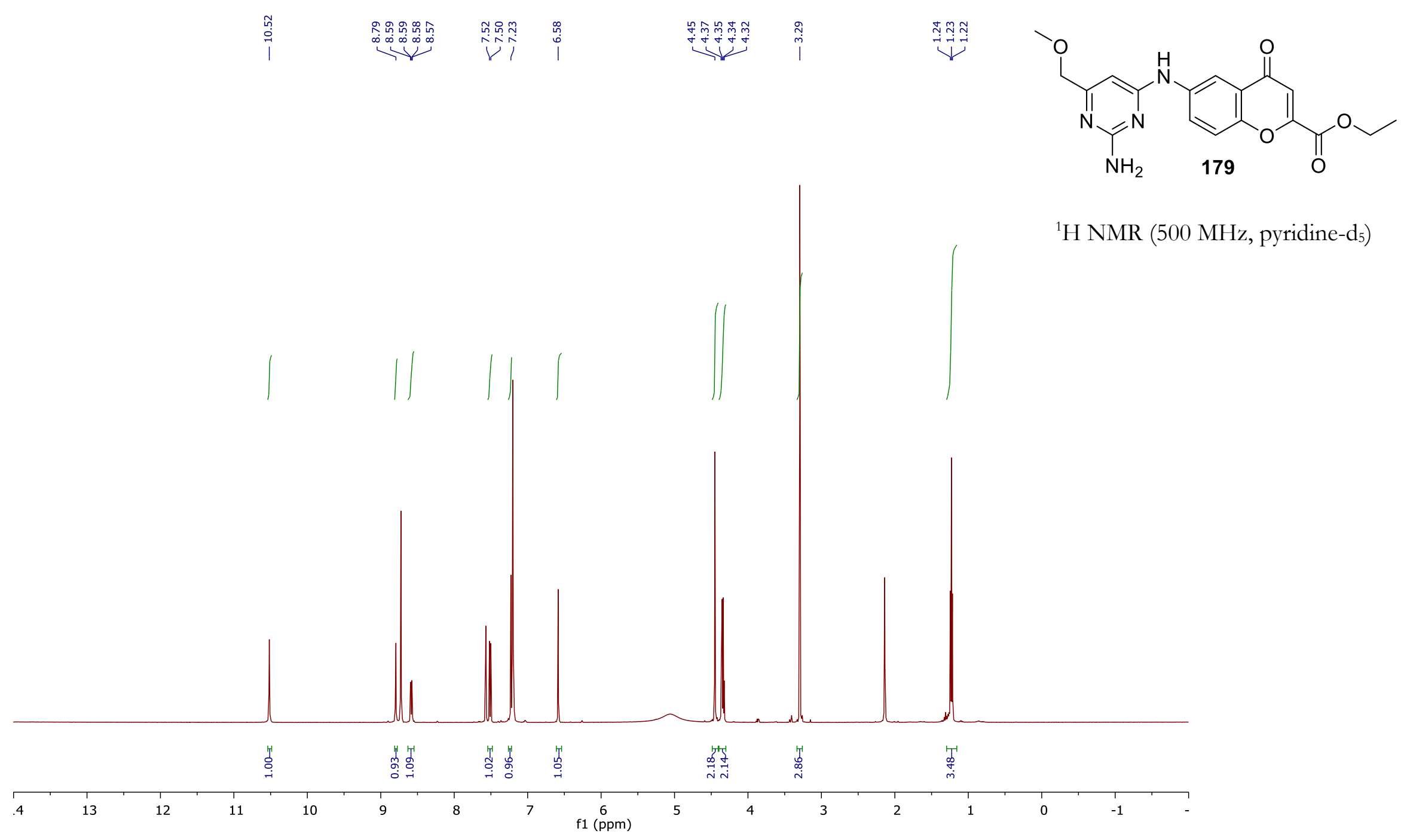




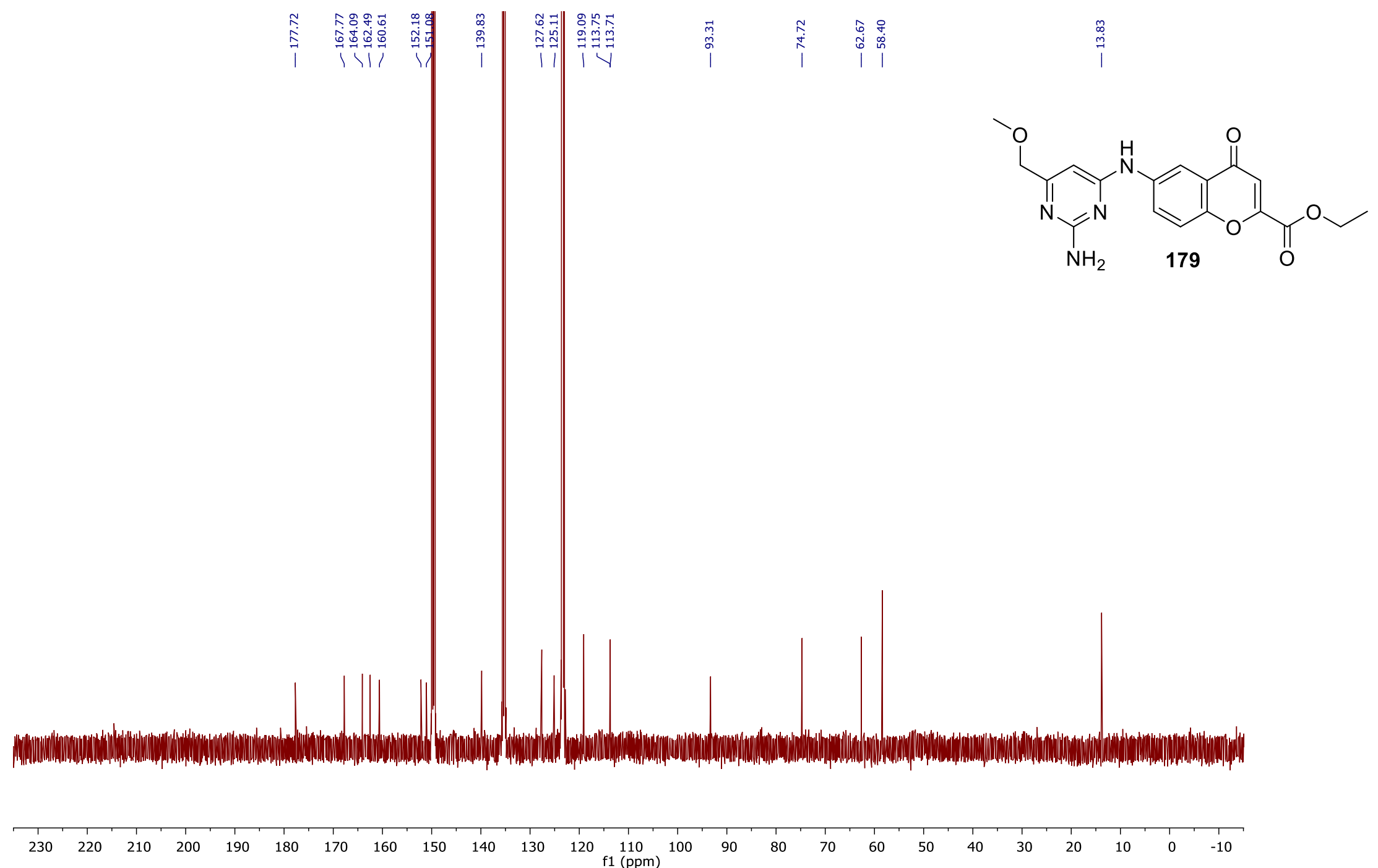




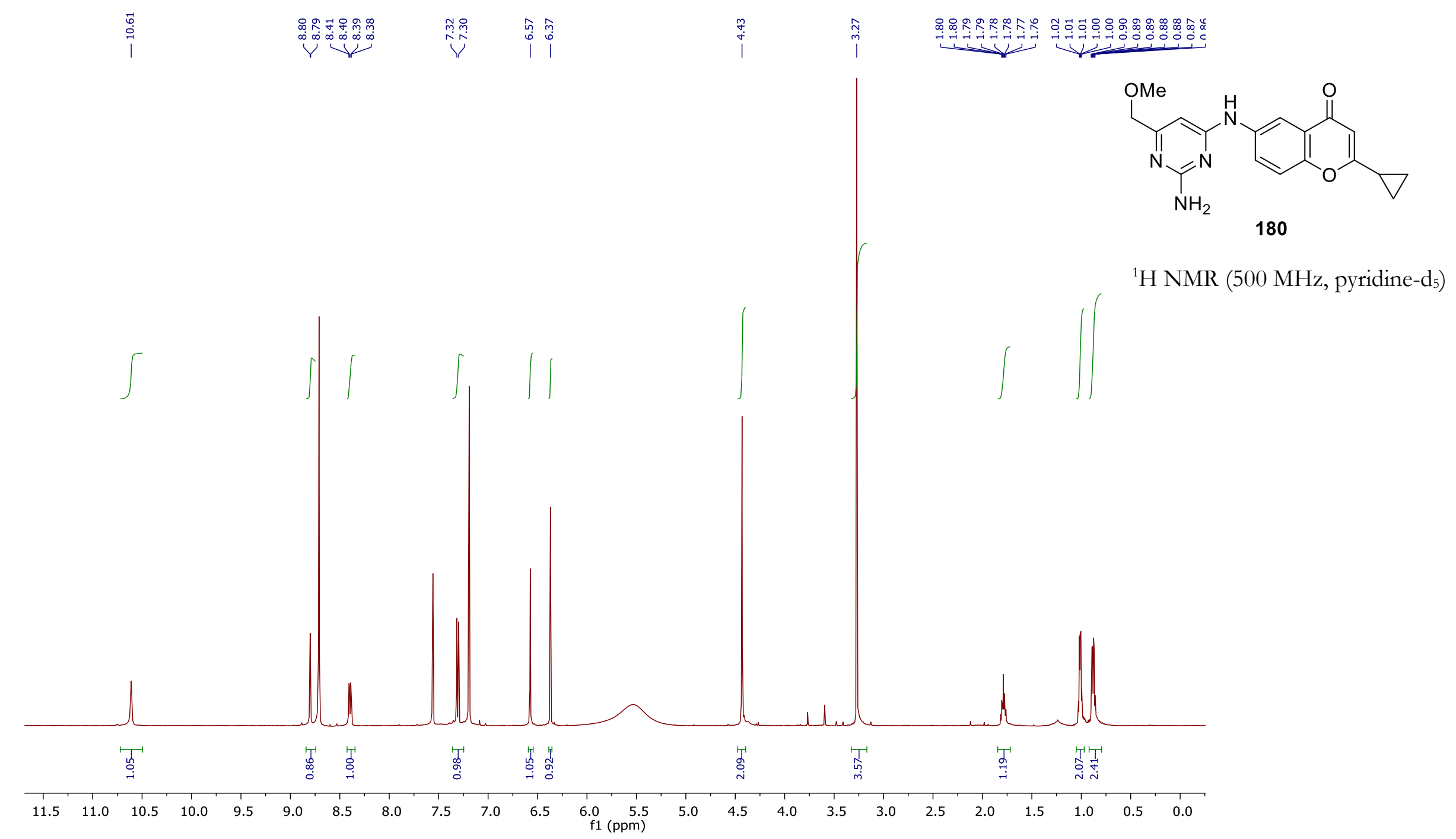




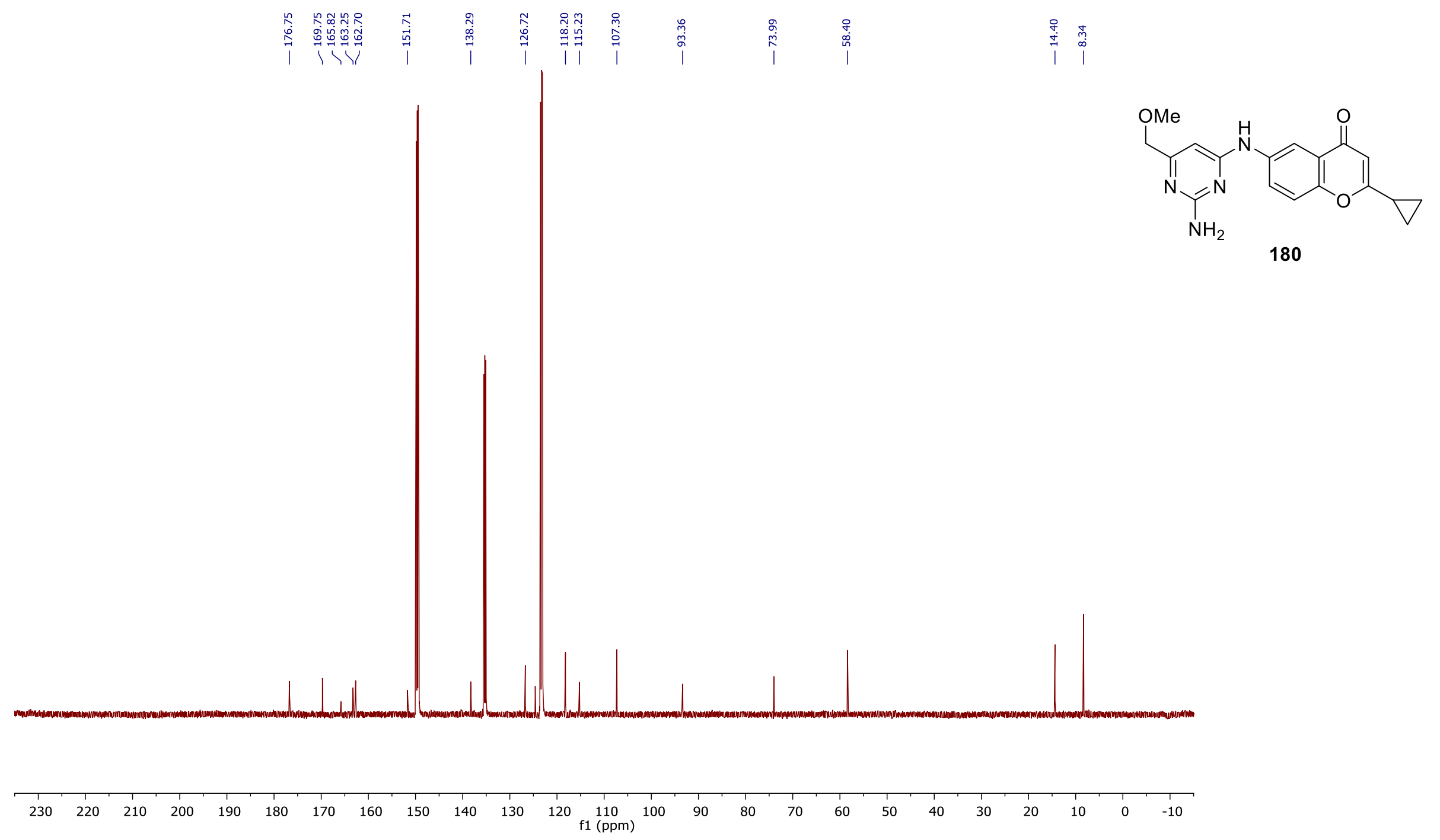




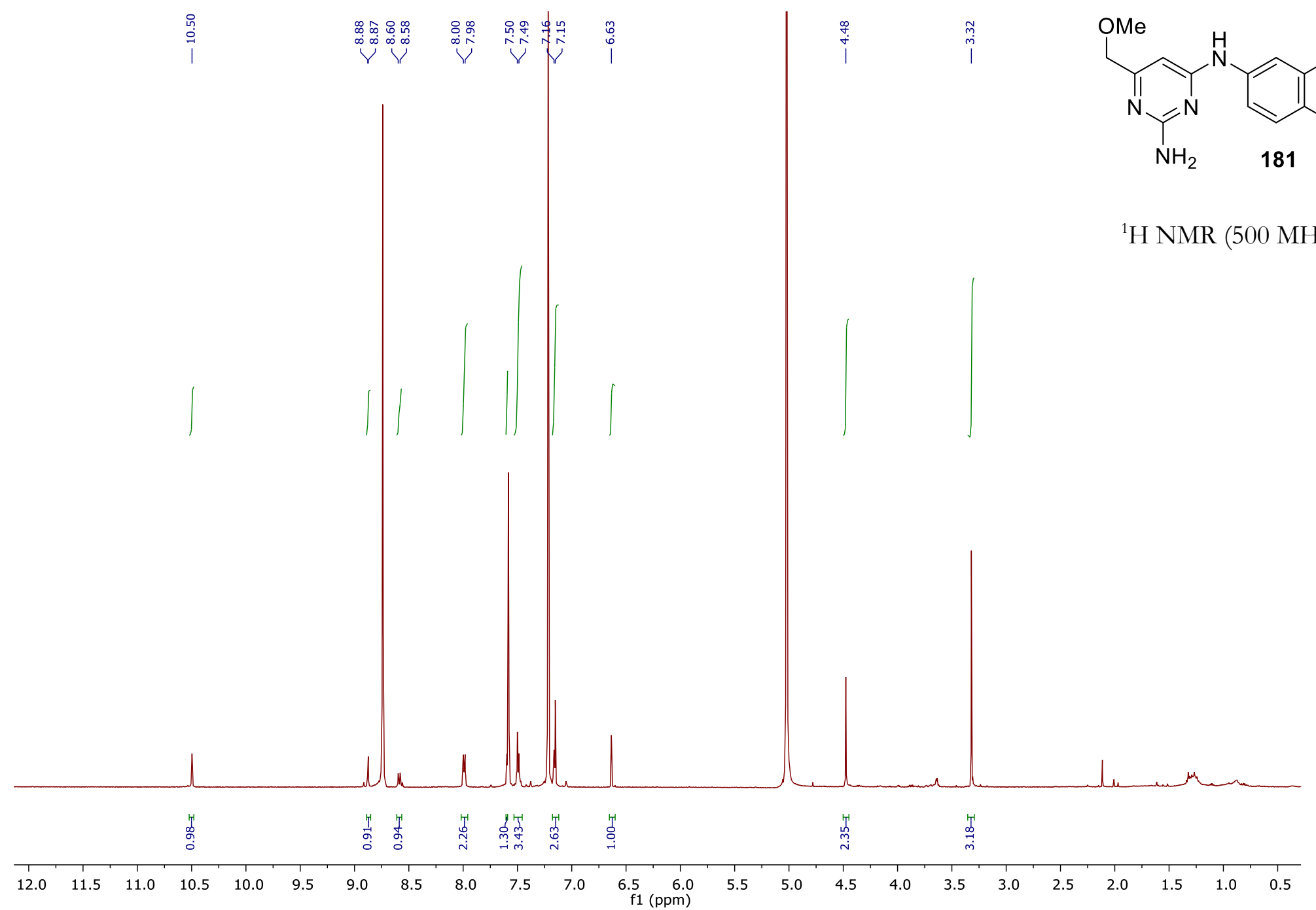




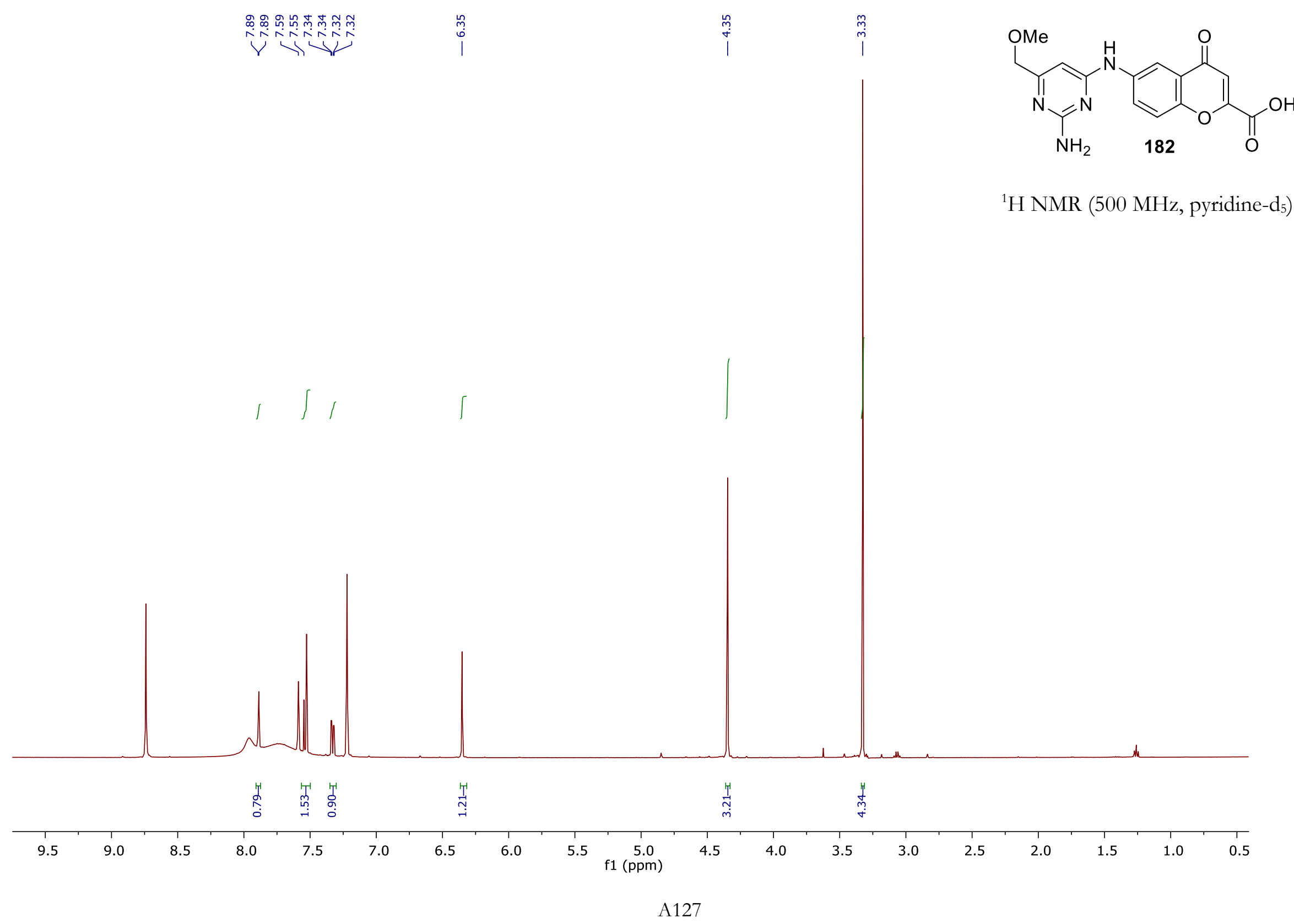




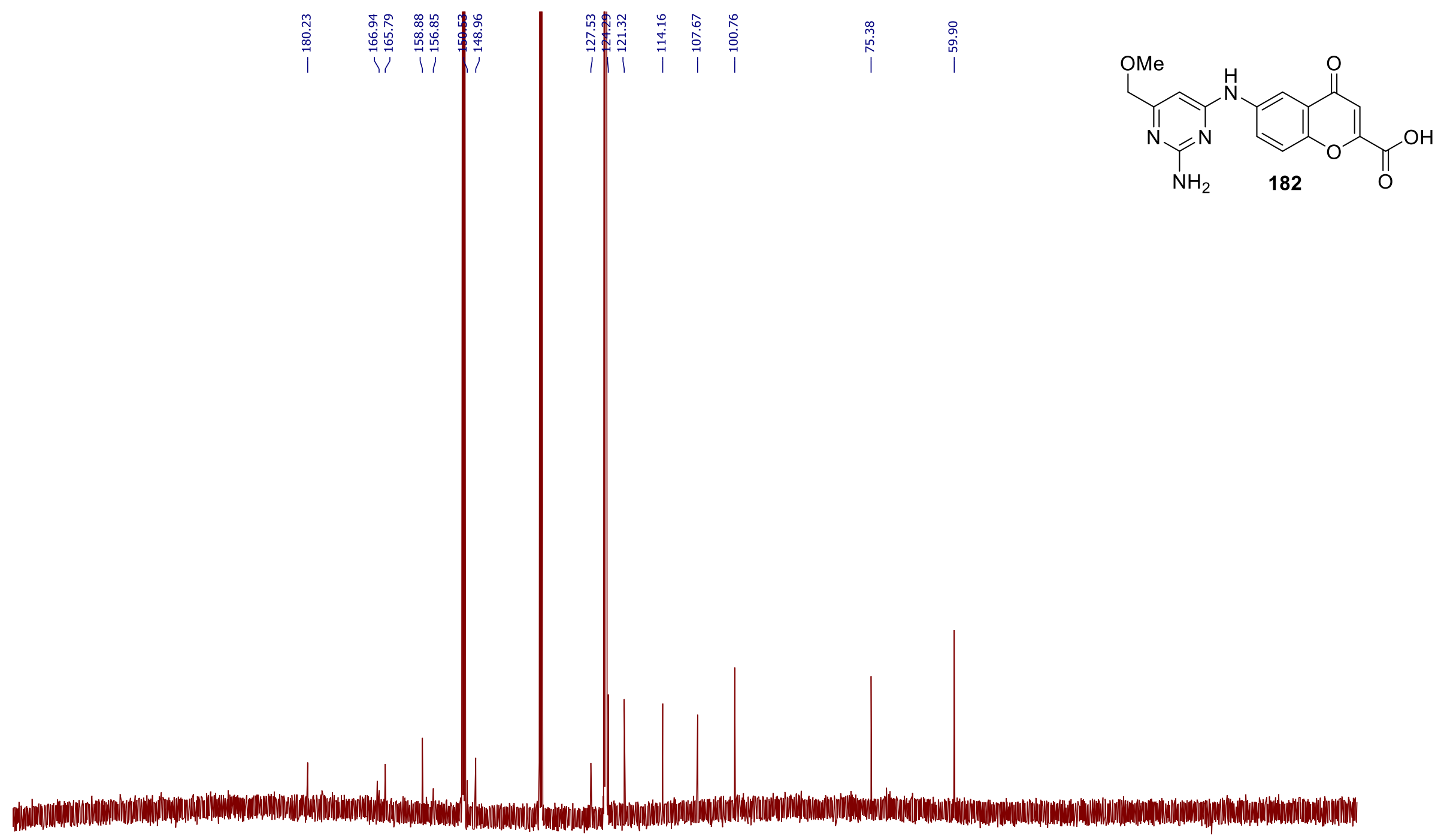

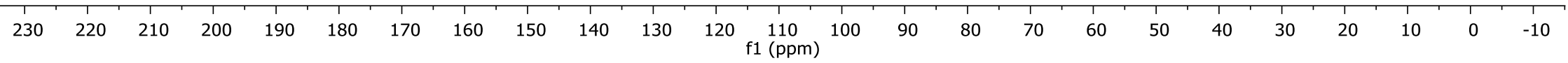



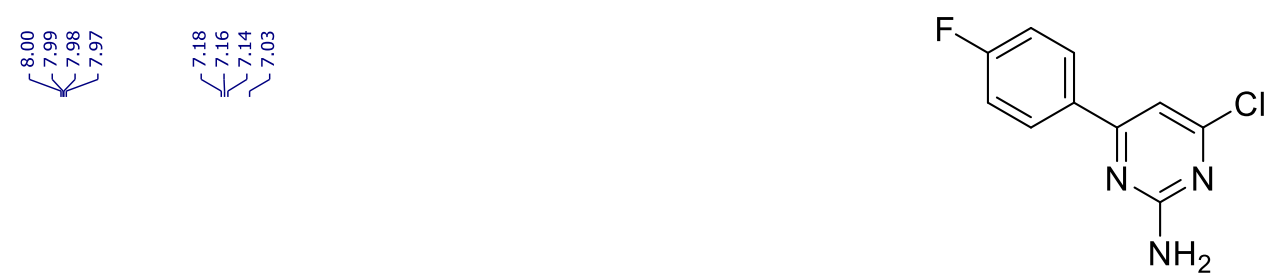

188

${ }^{1} \mathrm{H}$ NMR $\left(500 \mathrm{MHz}, \mathrm{CDCl}_{3}\right)$
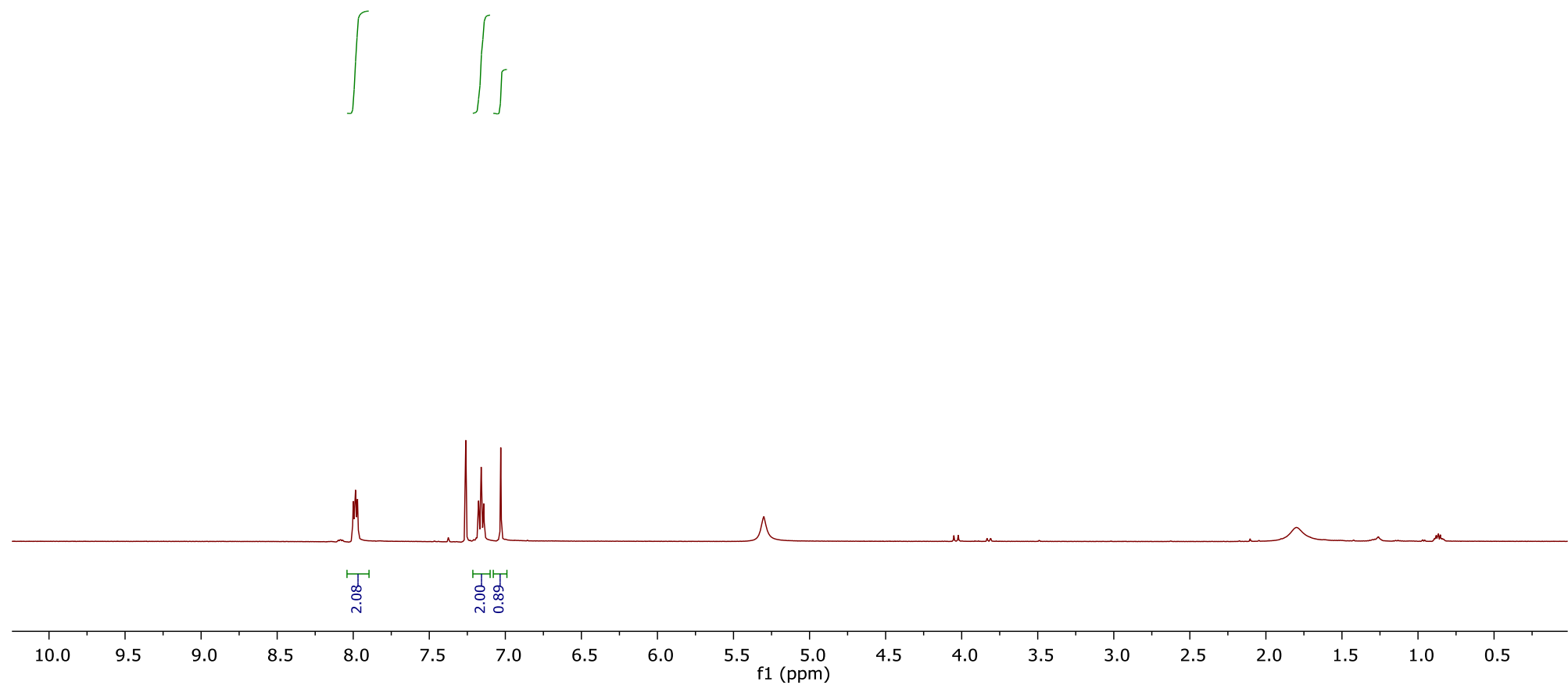


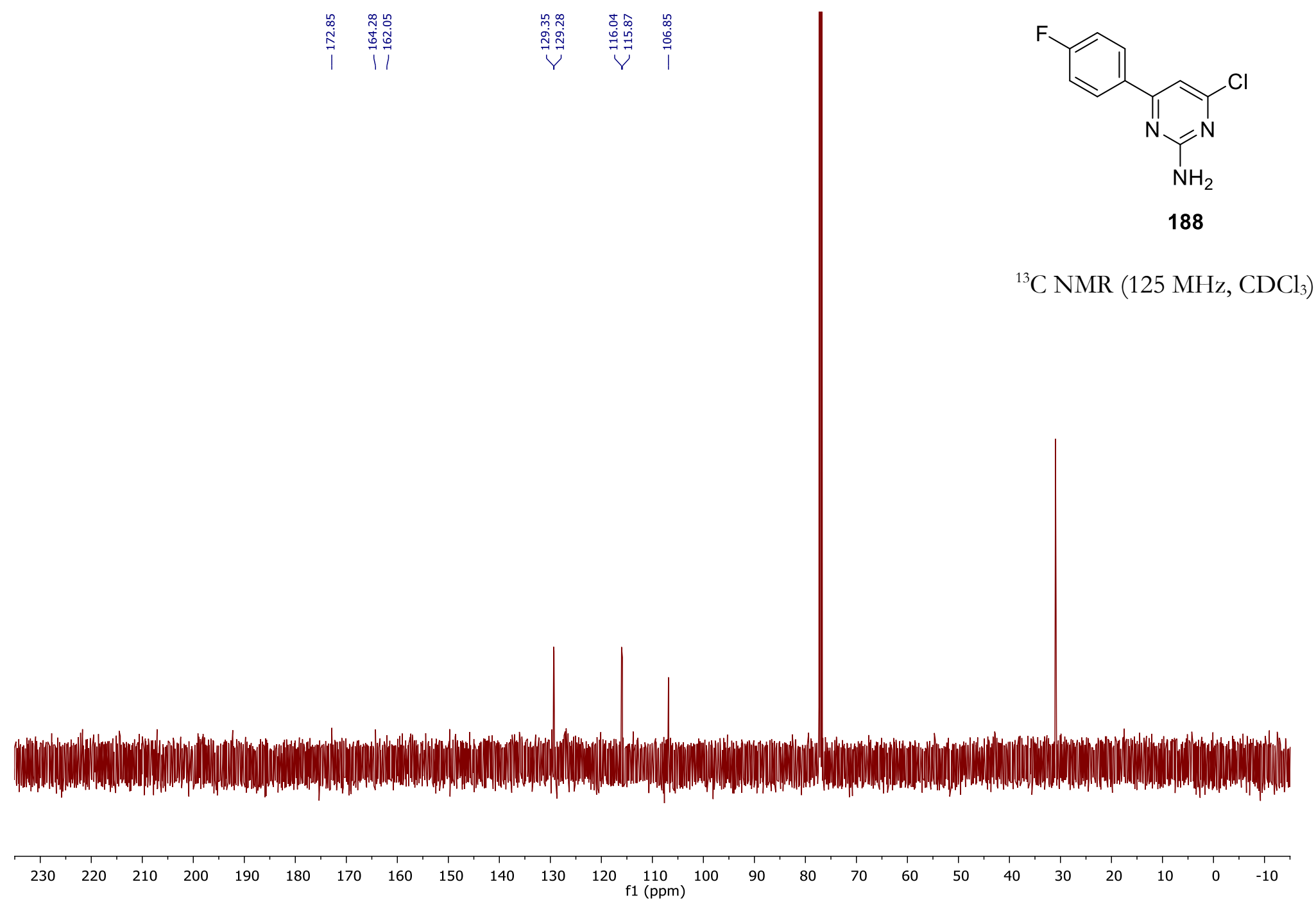




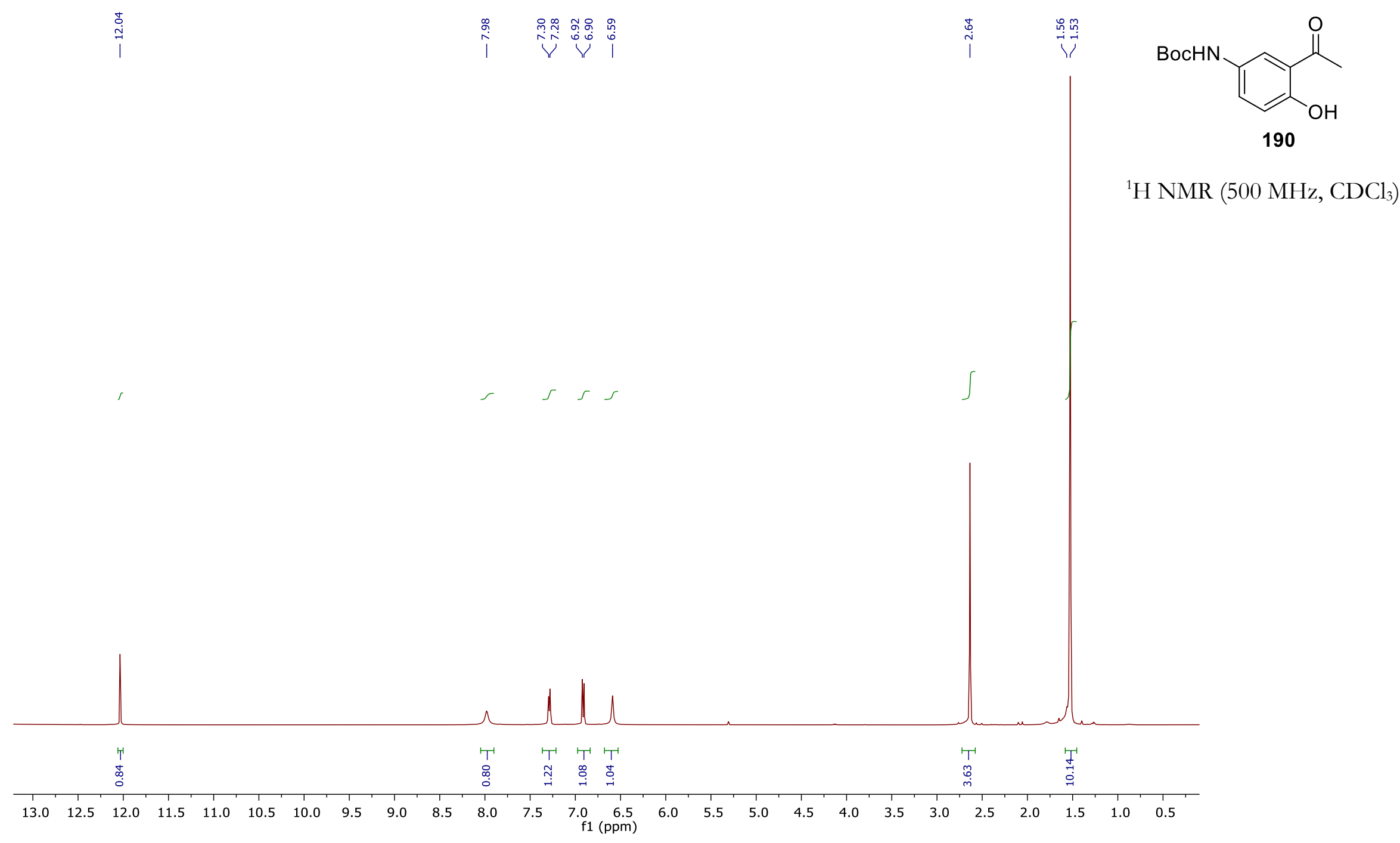



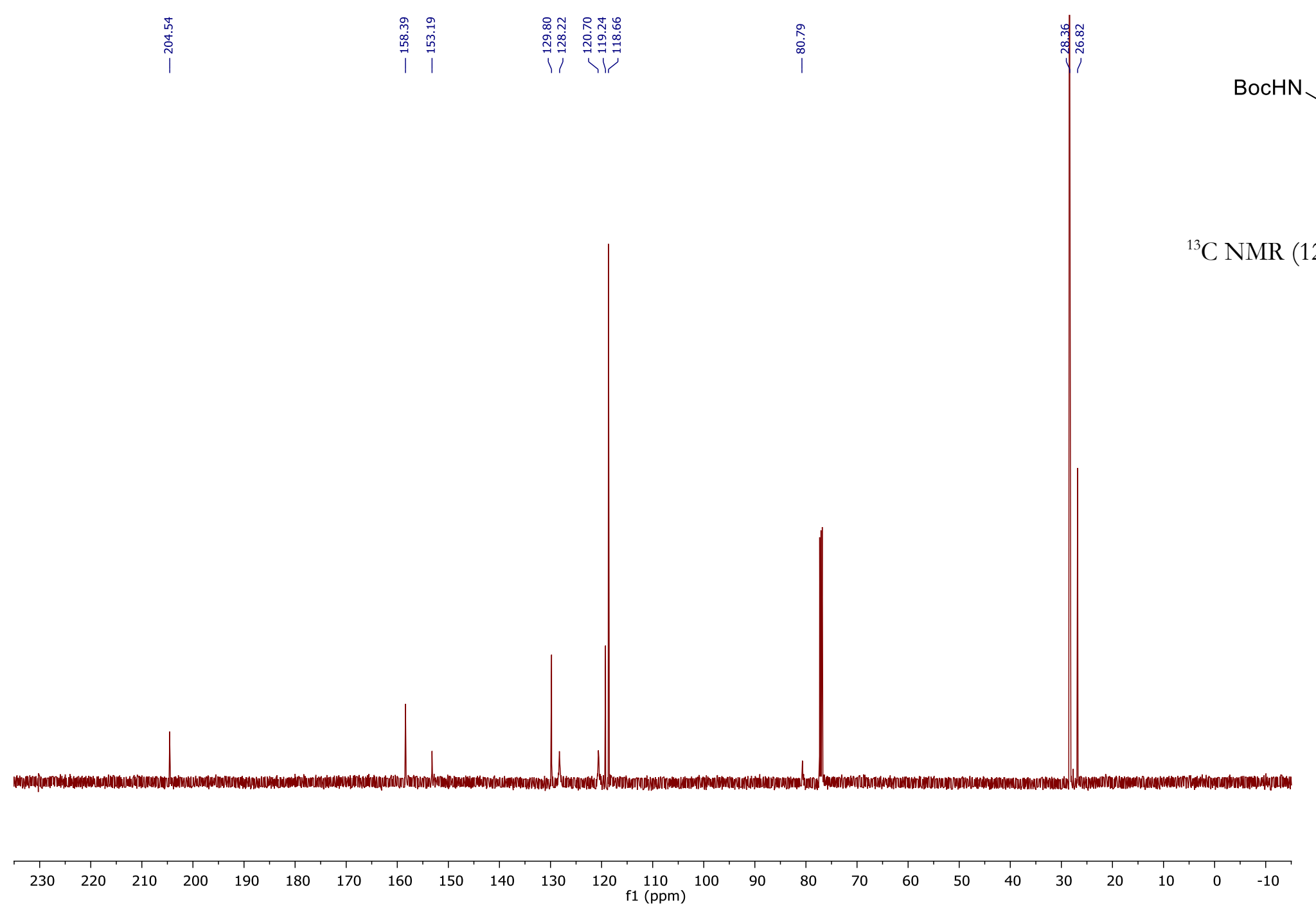


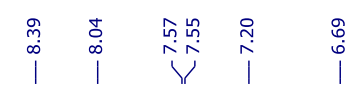

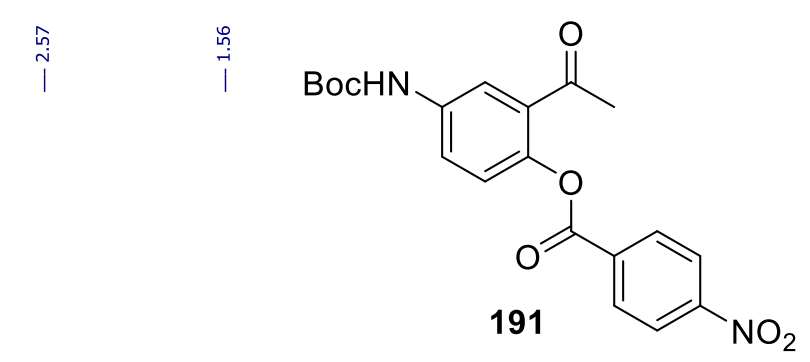

${ }^{1} \mathrm{H}$ NMR $\left(500 \mathrm{MHz}, \mathrm{CDCl}_{3}\right)$

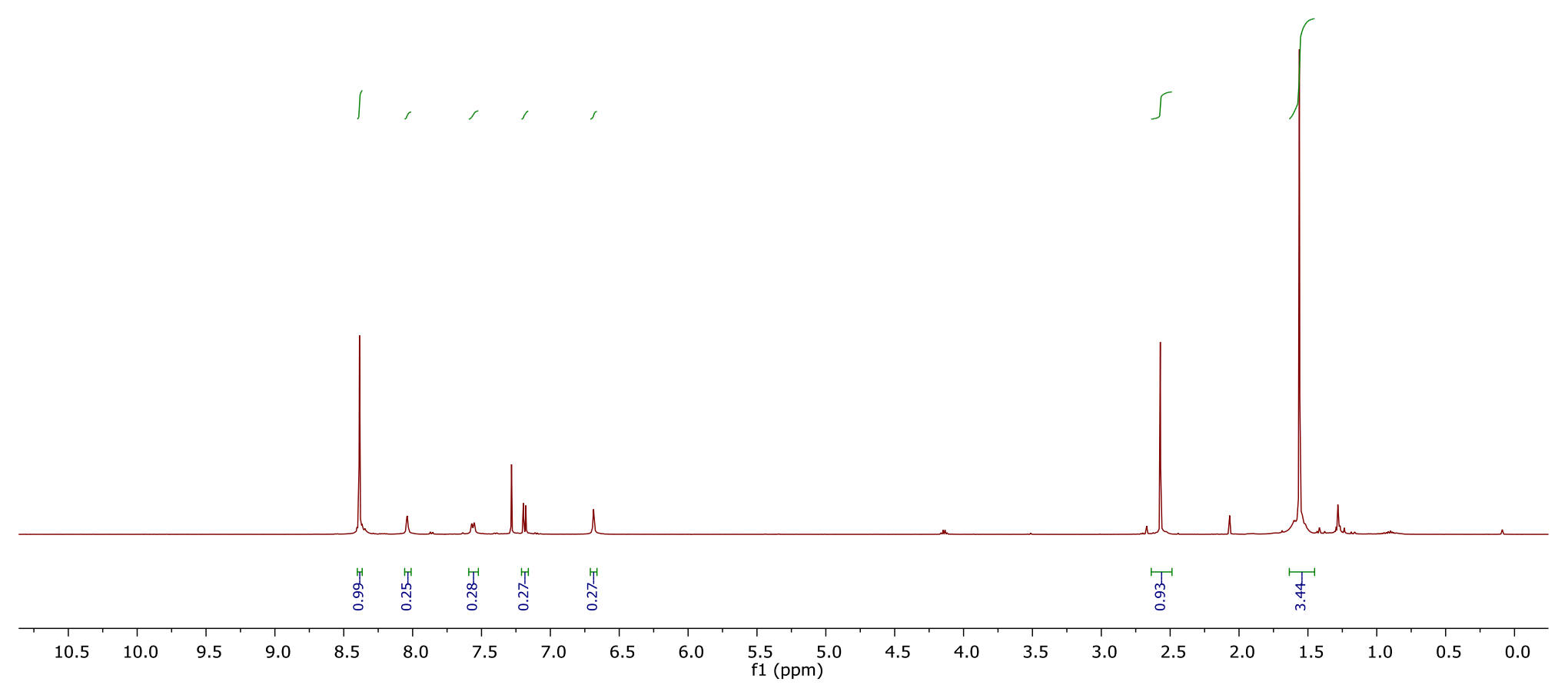




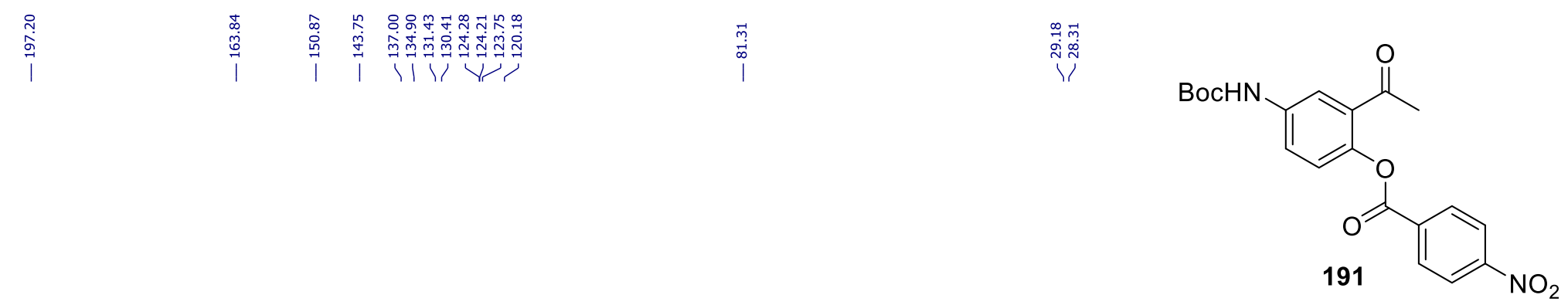

${ }^{13} \mathrm{C}$ NMR $\left(125 \mathrm{MHz}, \mathrm{CDCl}_{3}\right)$

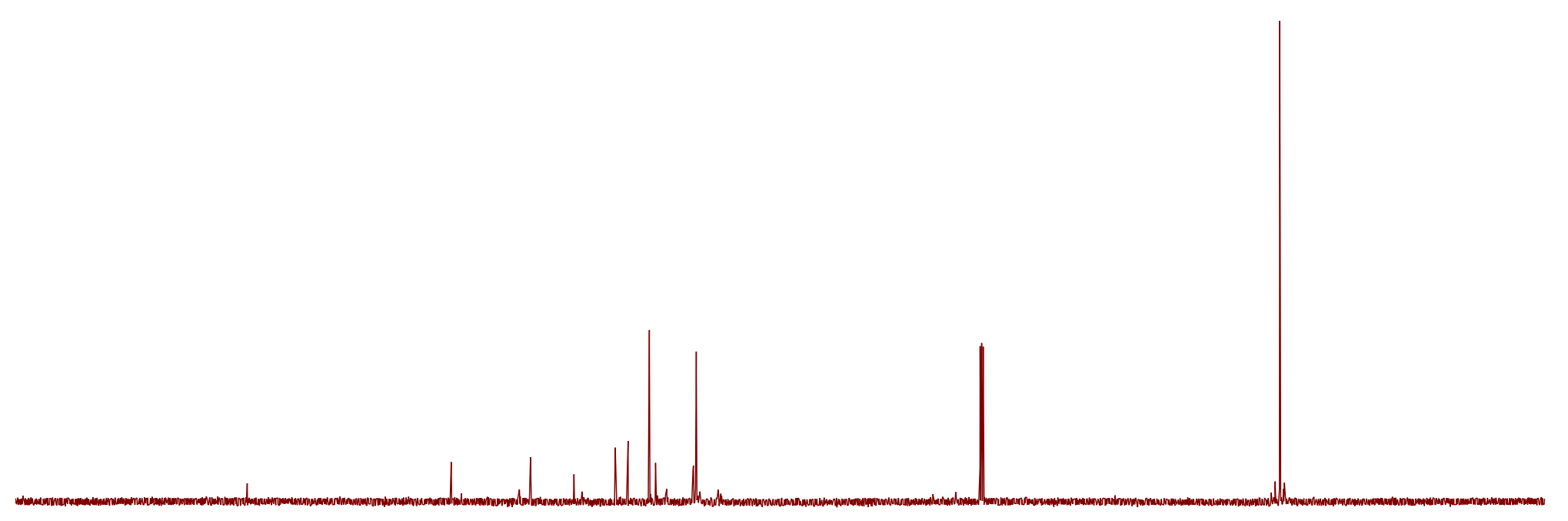

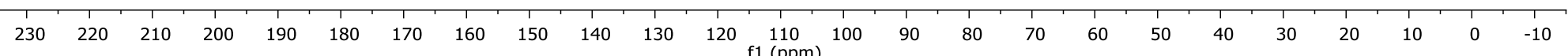



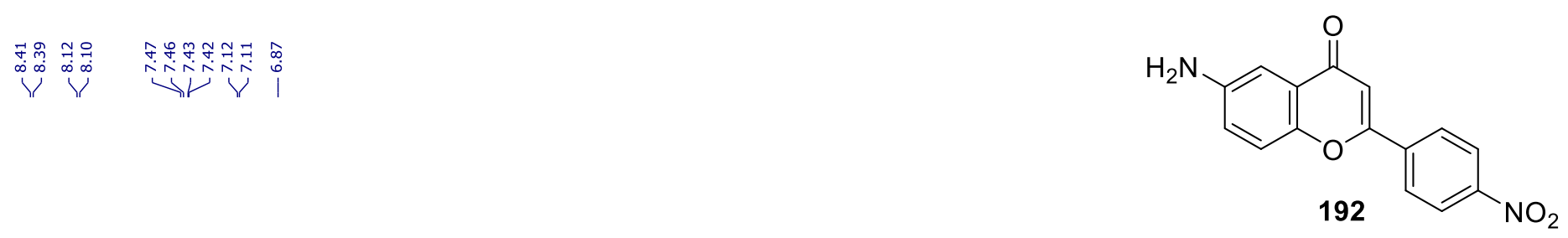

${ }^{1} \mathrm{H}$ NMR $\left(500 \mathrm{MHz}, \mathrm{CDCl}_{3}\right)$

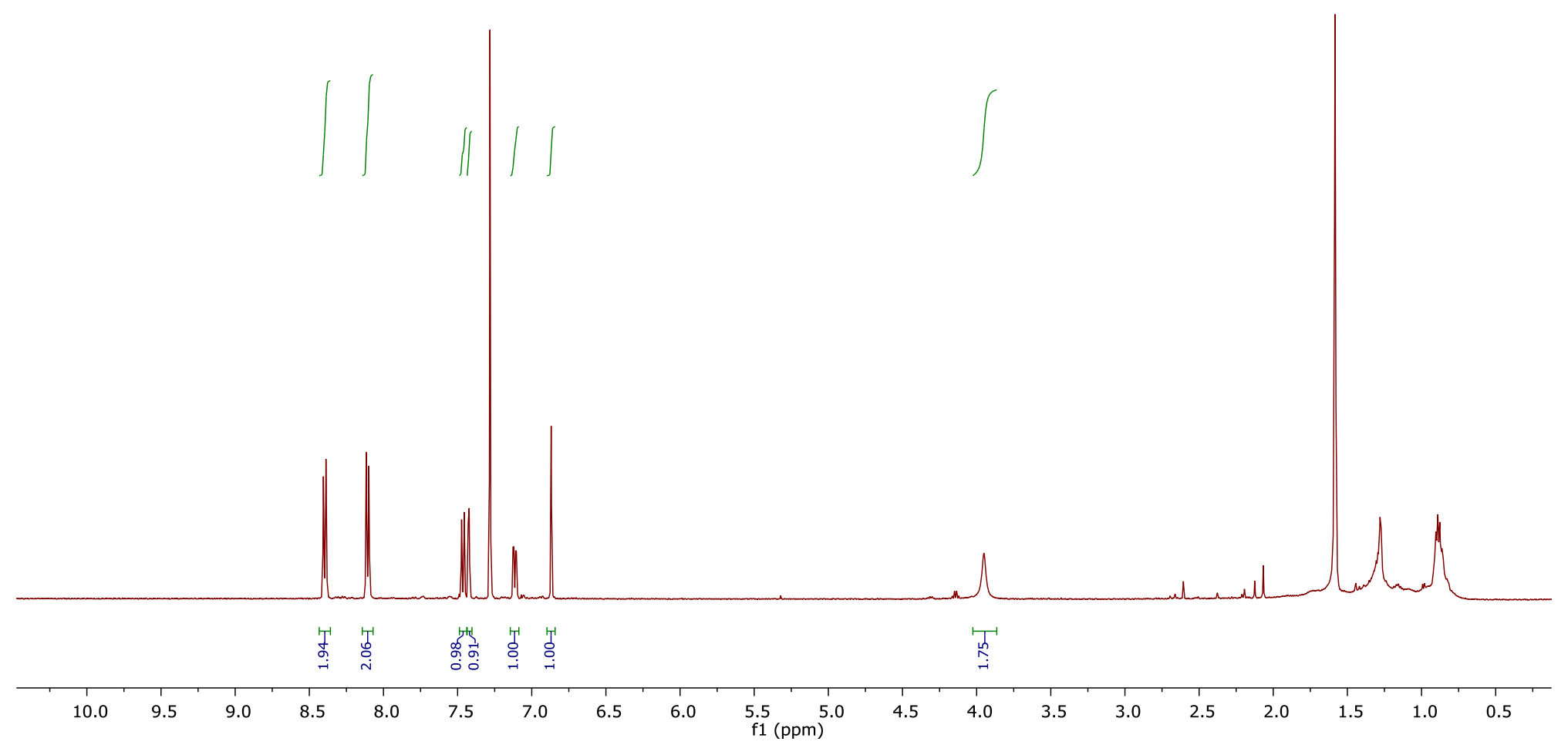




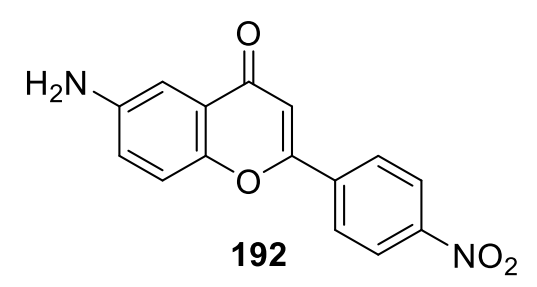

${ }^{13} \mathrm{C}$ NMR $\left(125 \mathrm{MHz}, \mathrm{CDCl}_{3}\right)$

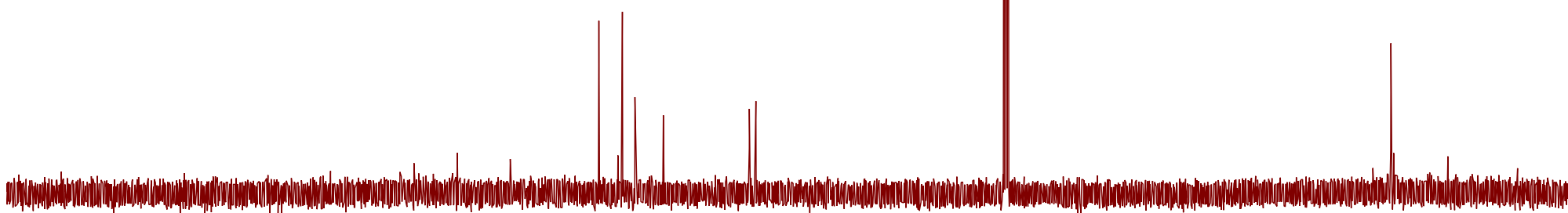




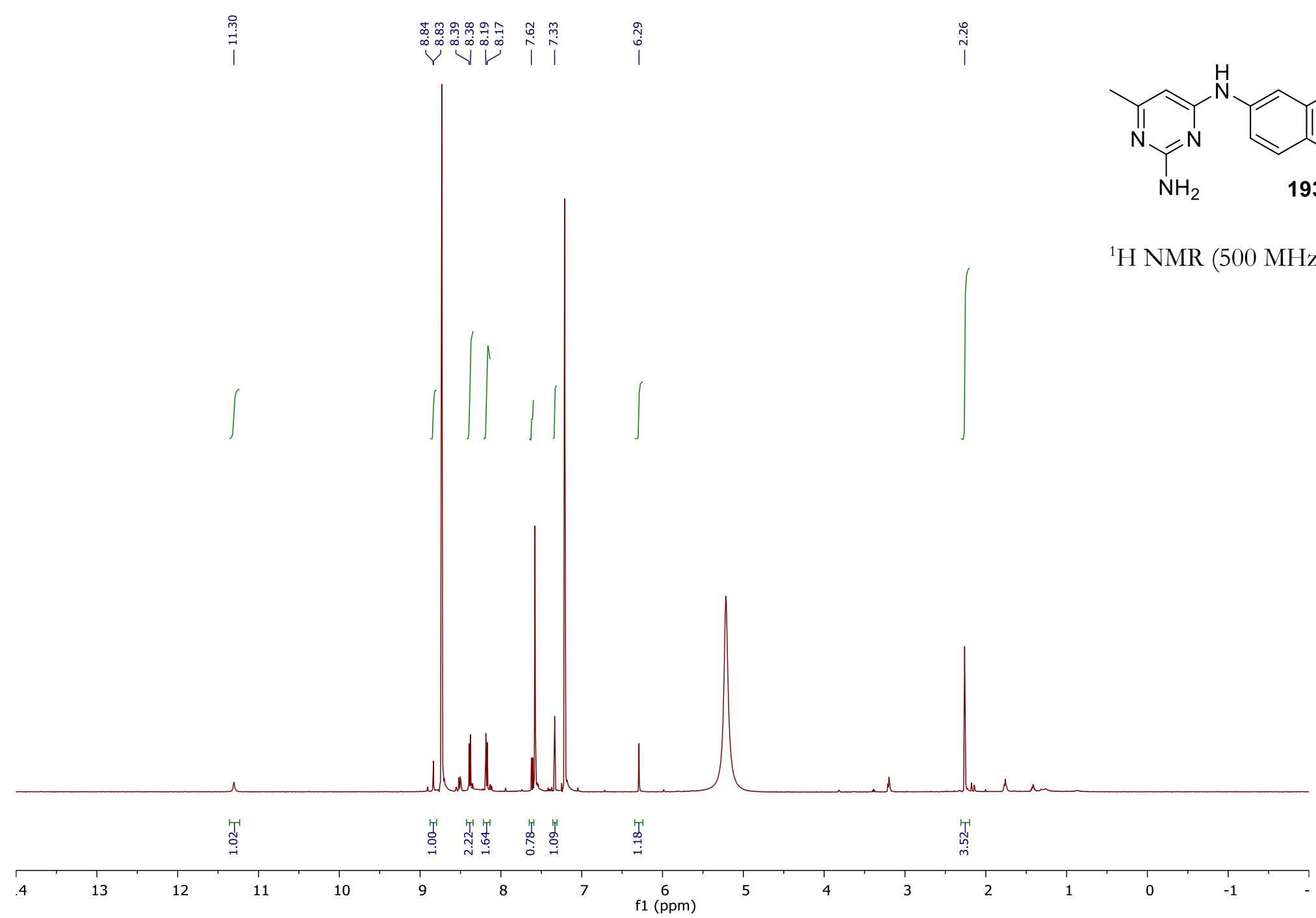




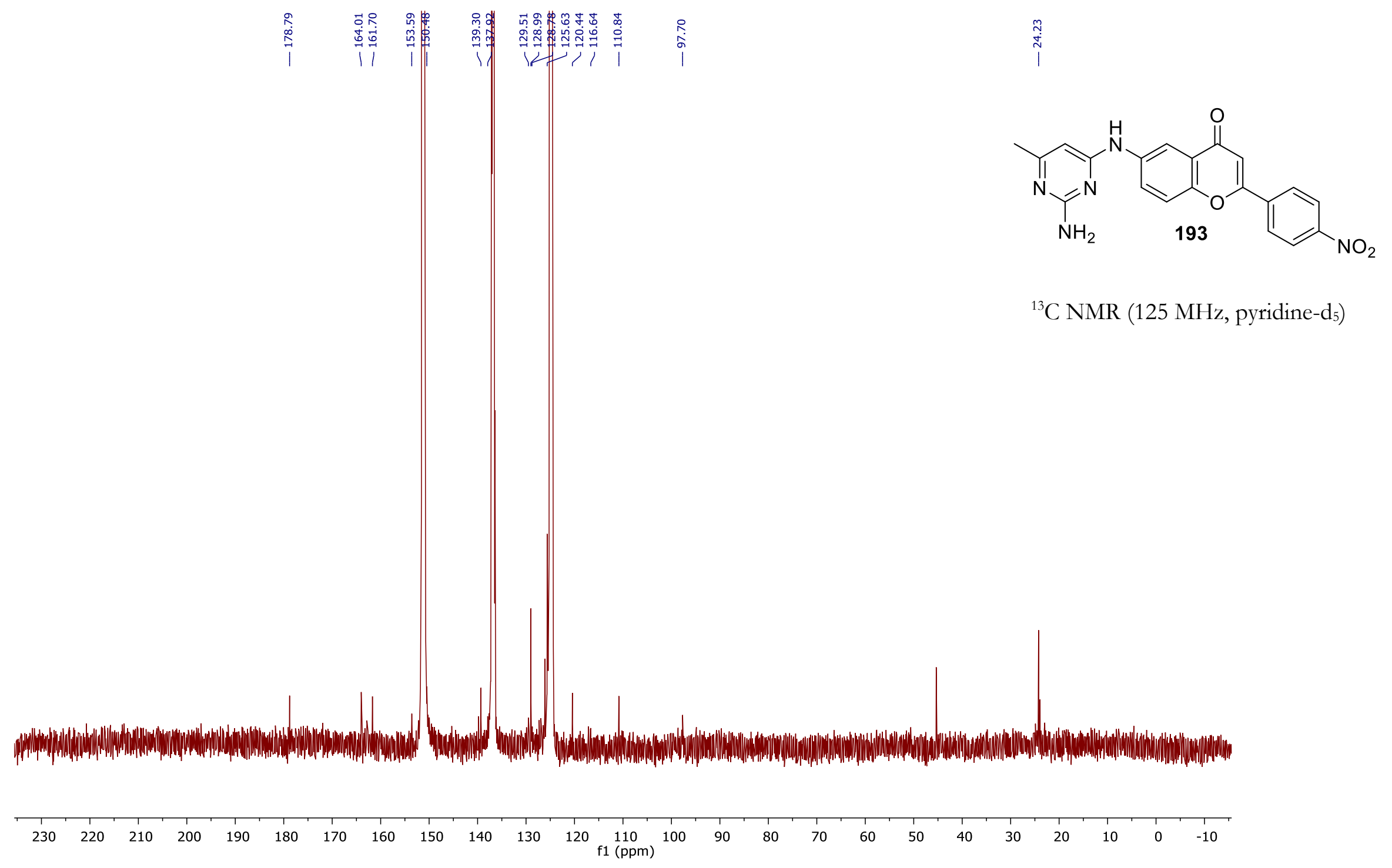



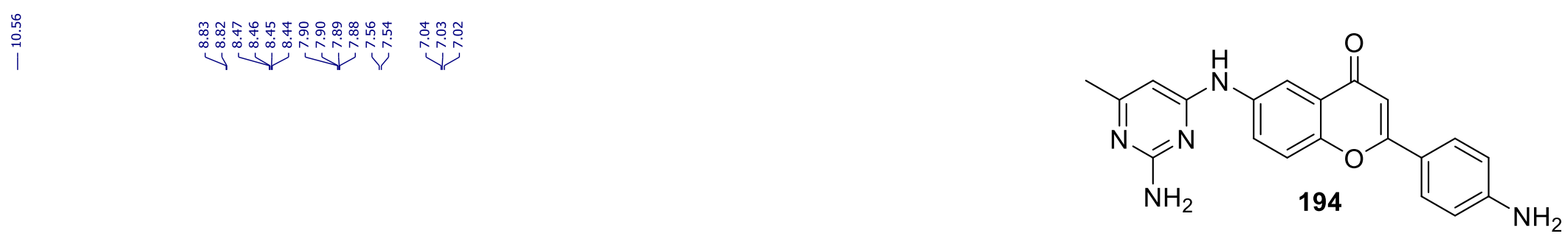

${ }^{1} \mathrm{H}$ NMR $\left(500 \mathrm{MHz}\right.$, pyridine- $\left.\mathrm{d}_{5}\right)$

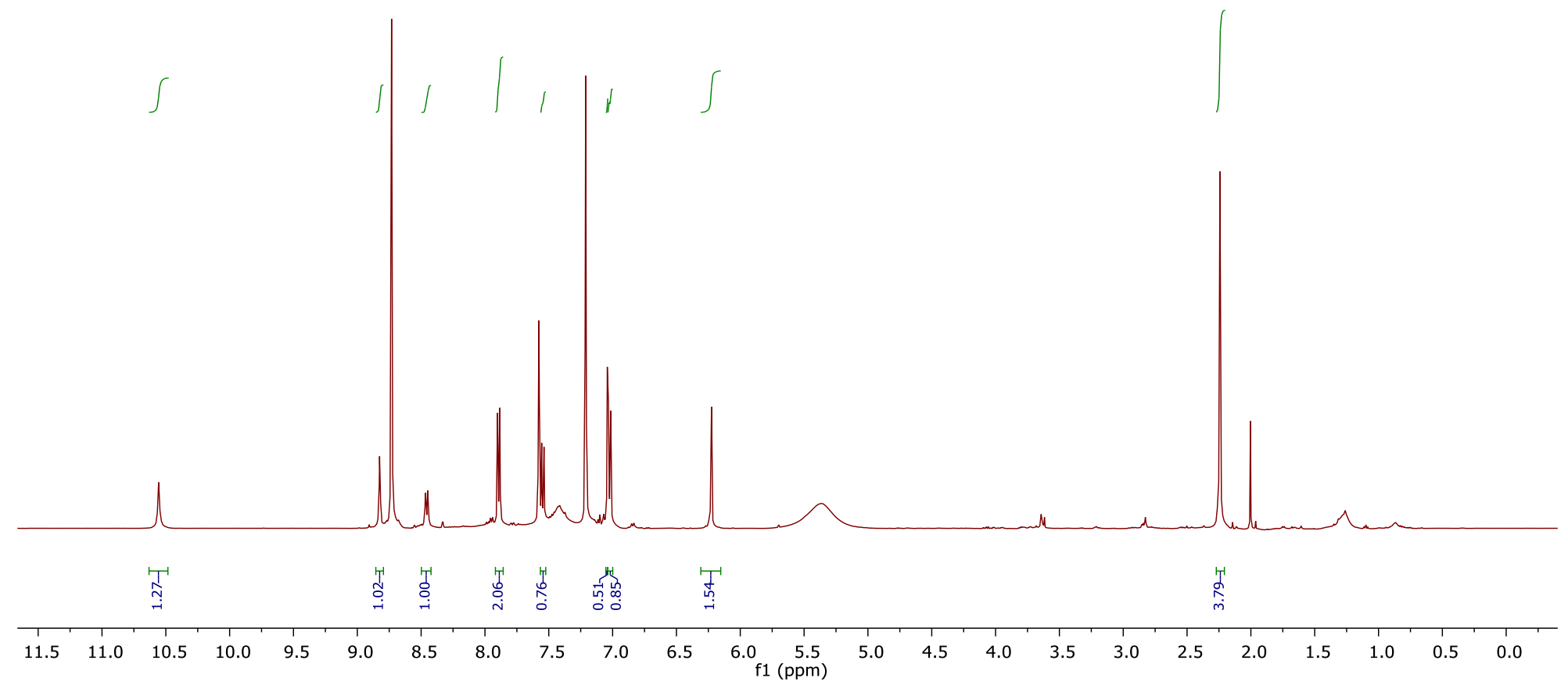




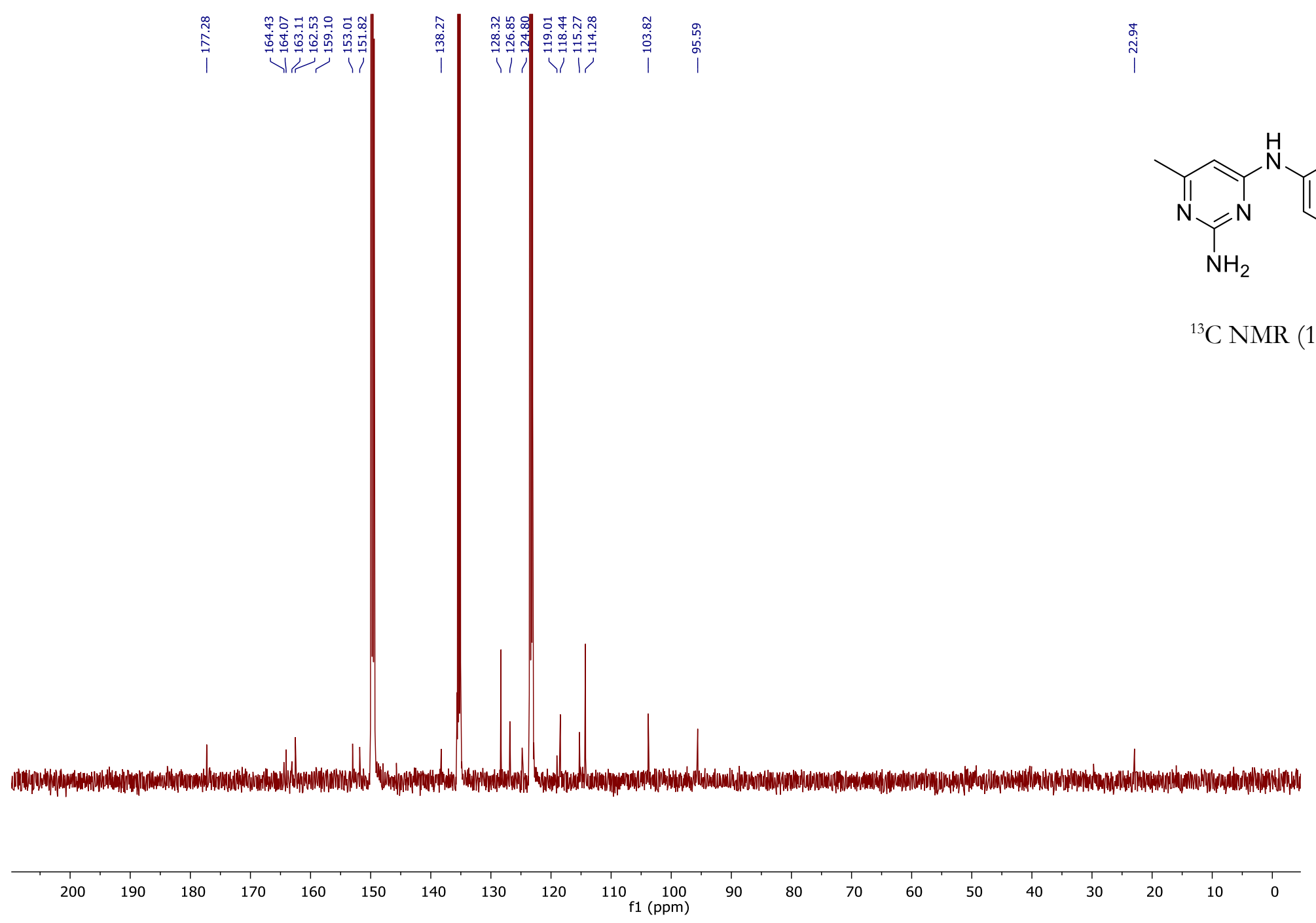




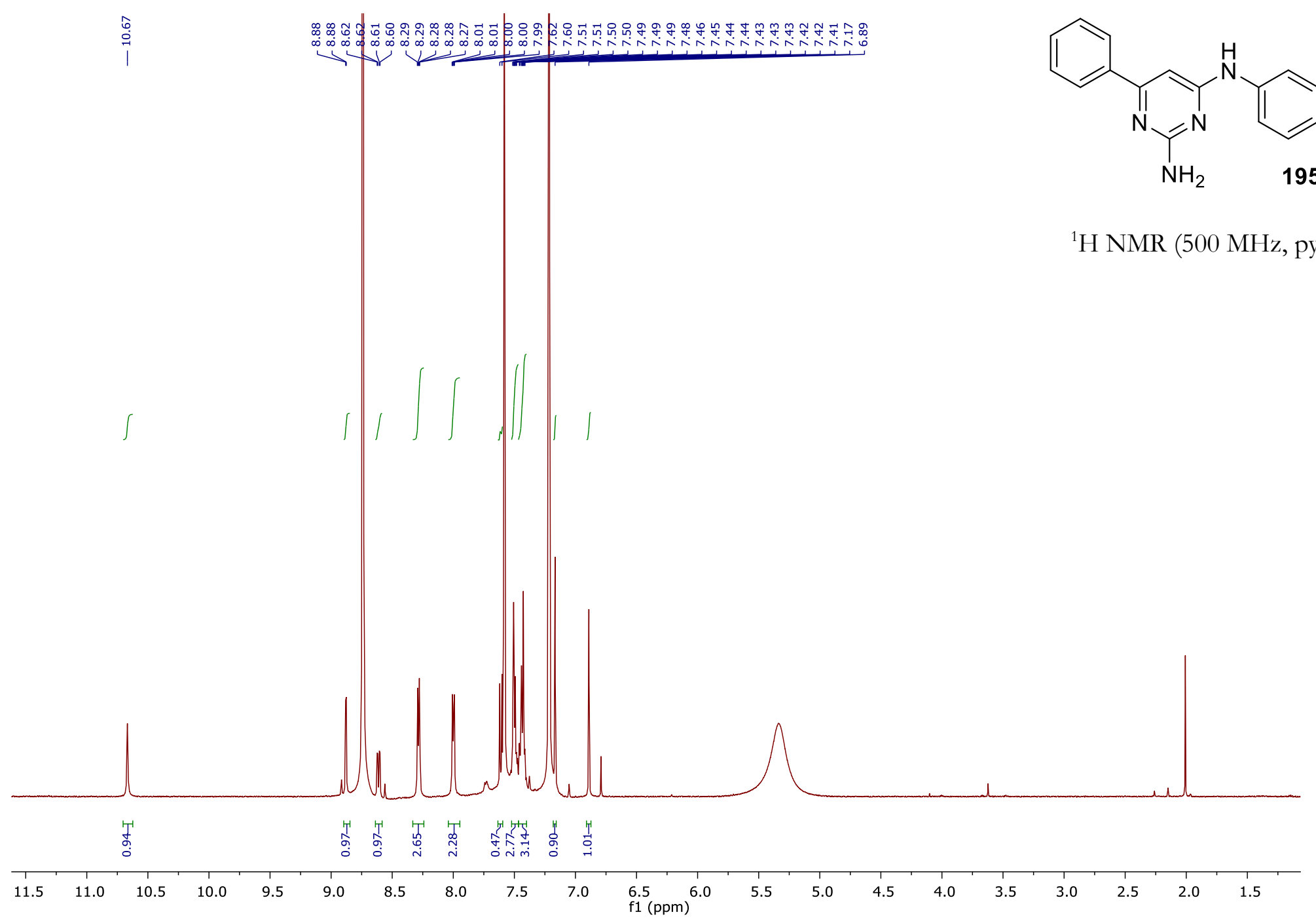



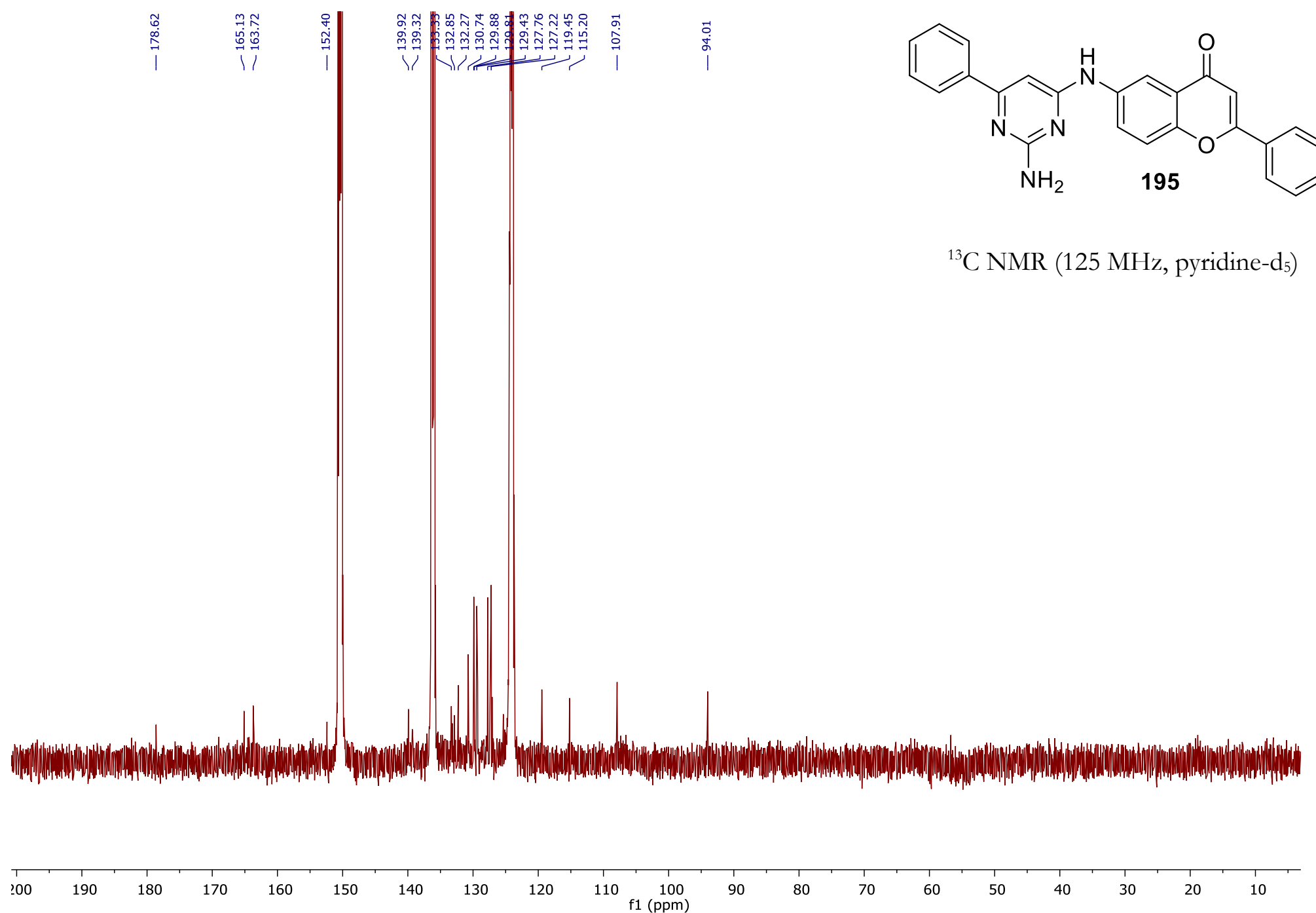


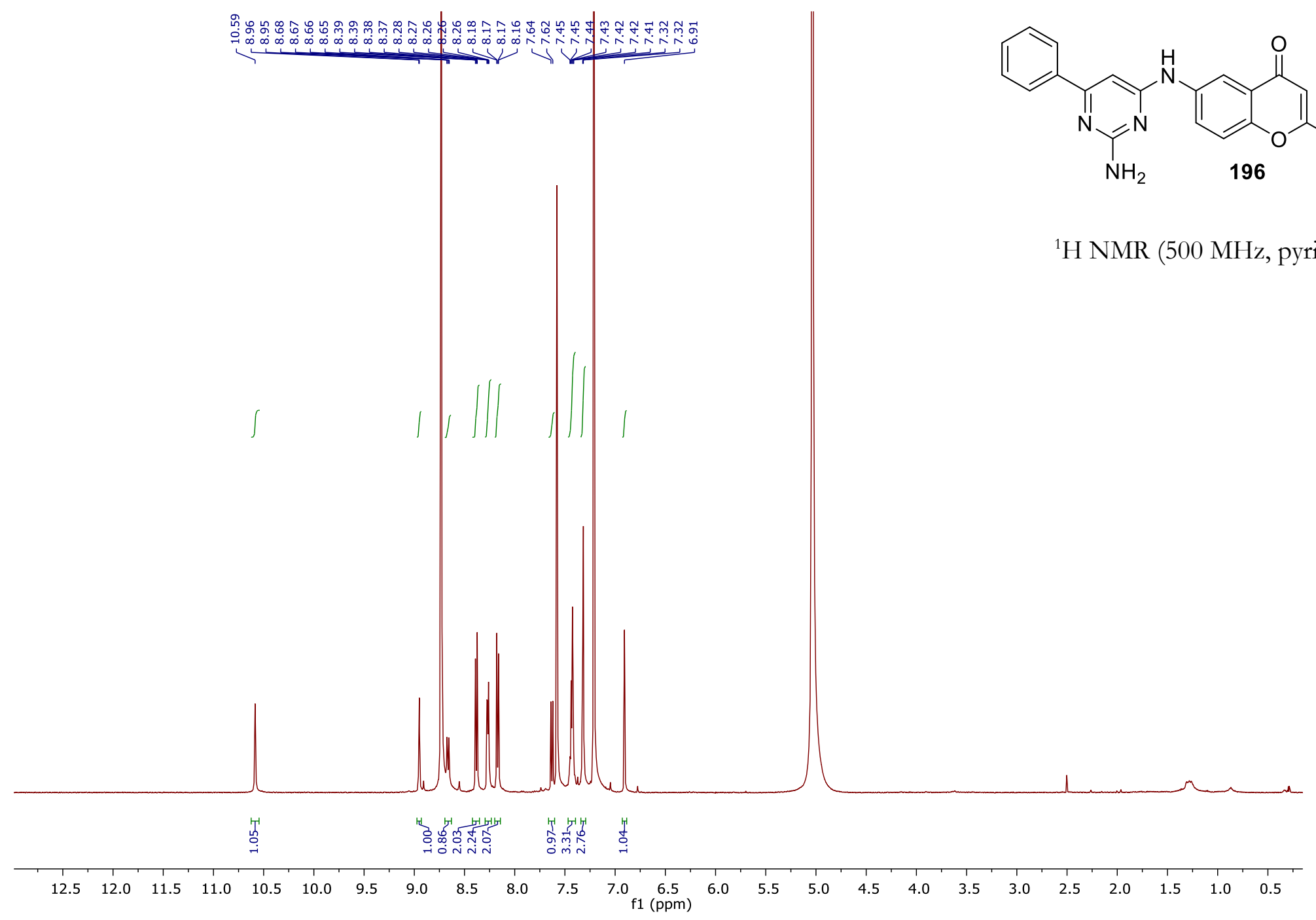




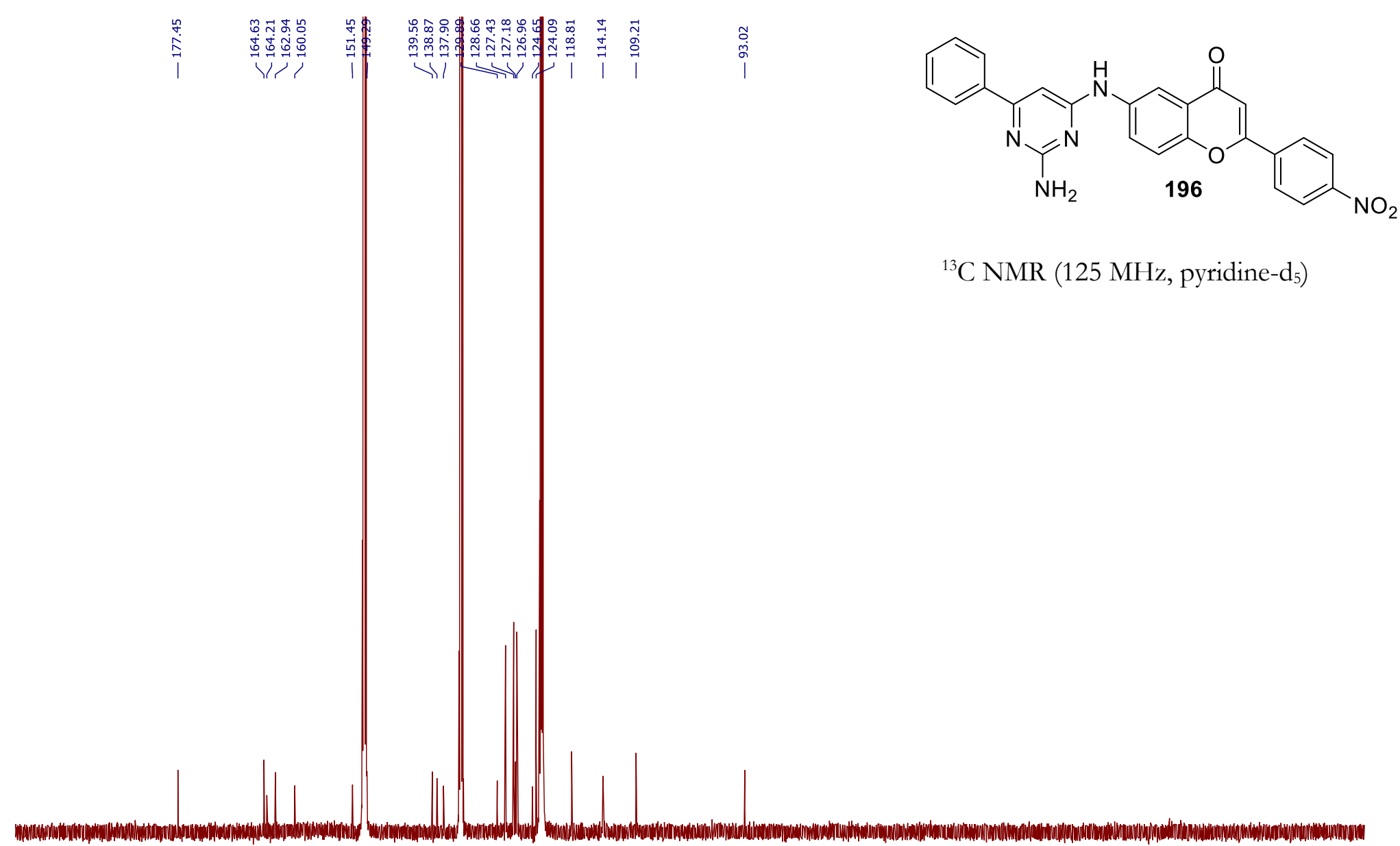




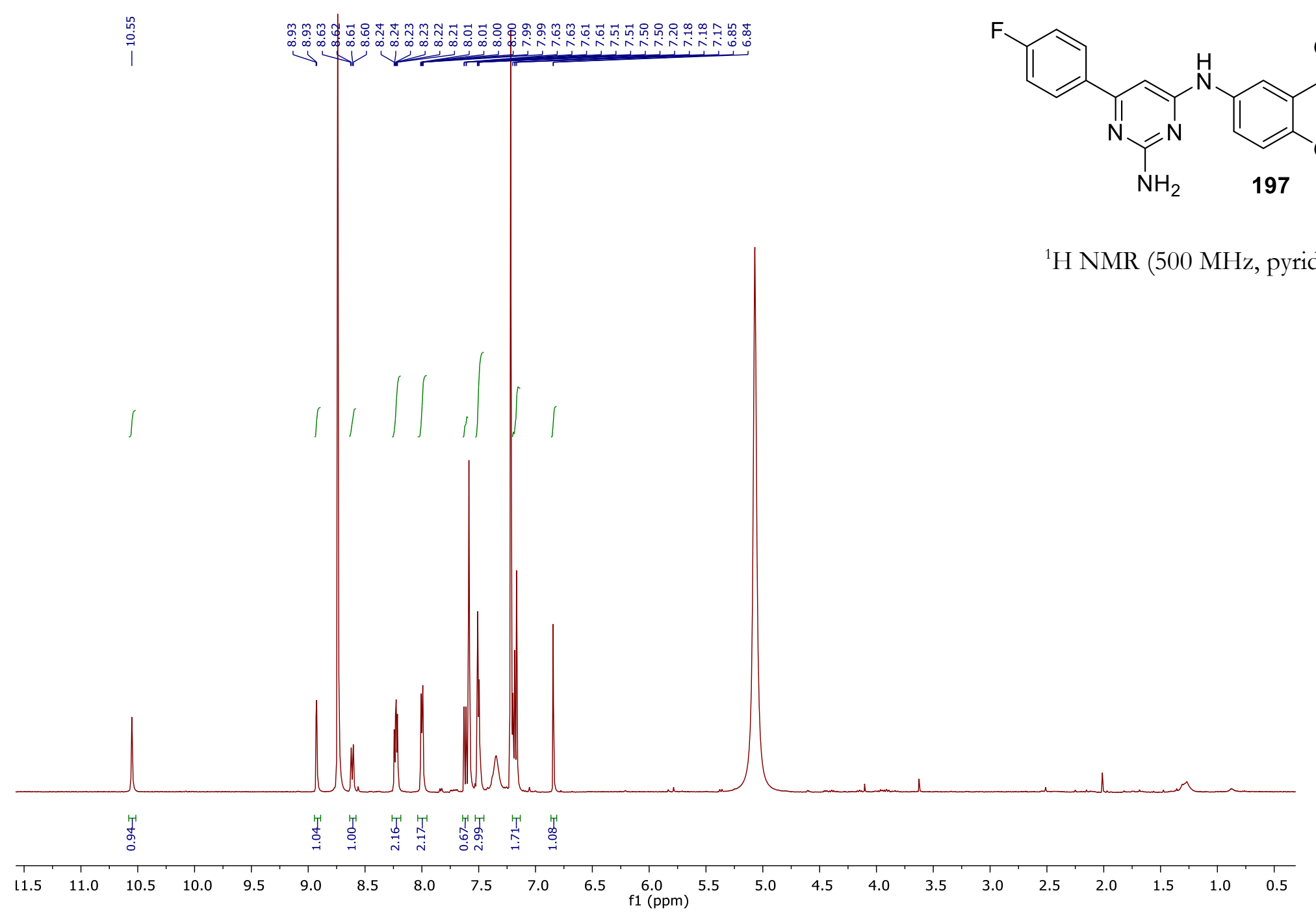




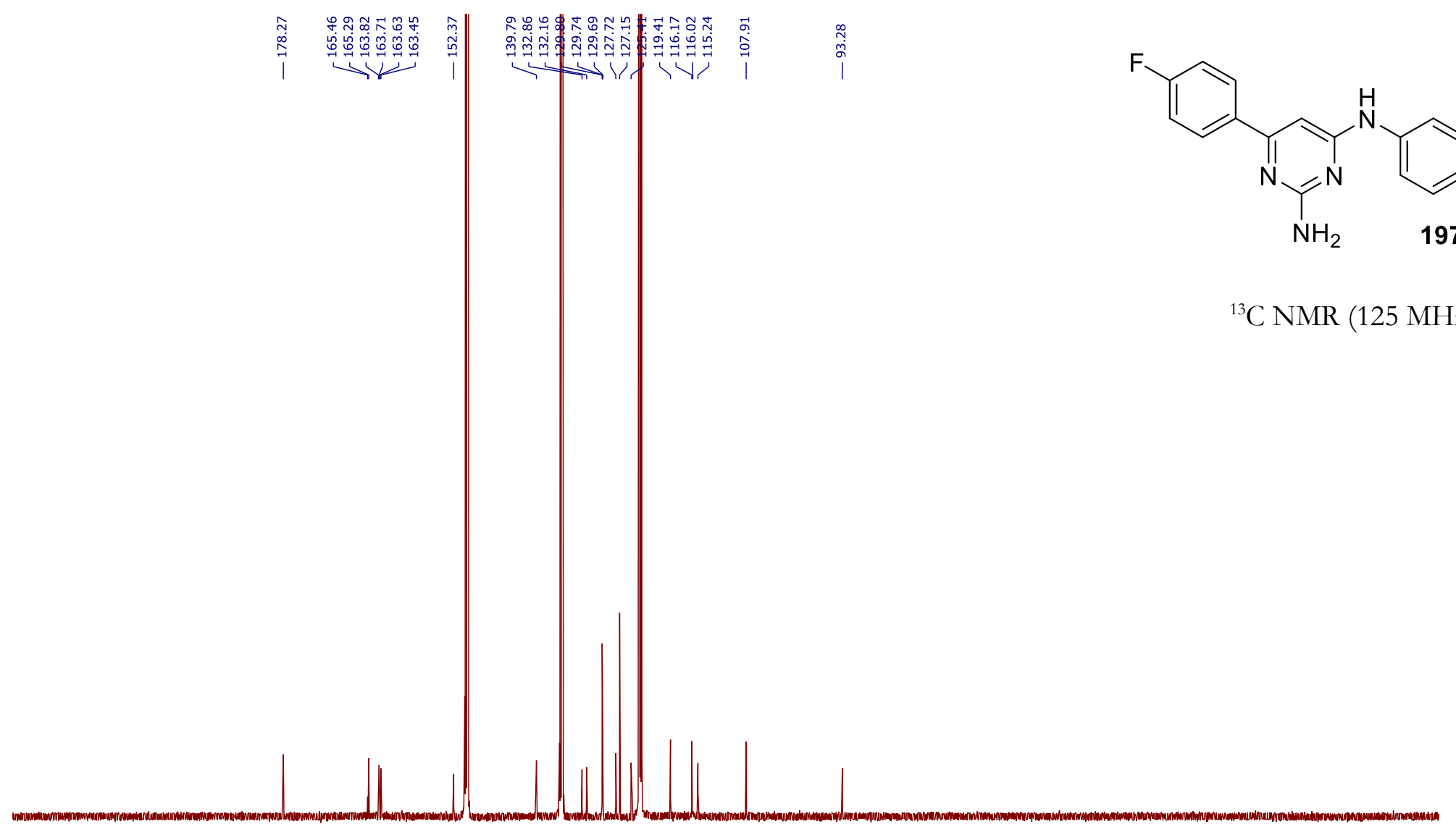




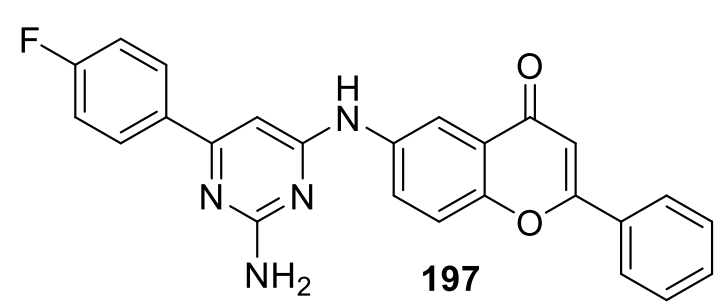

${ }^{19} \mathrm{~F}$ NMR (282 MHz, pyridine- $\mathrm{d}_{5}$ )

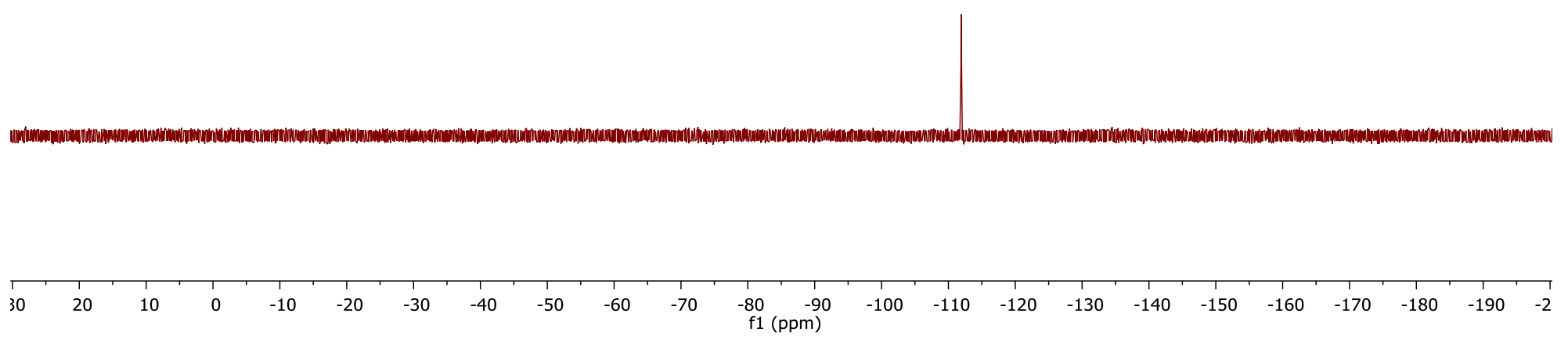




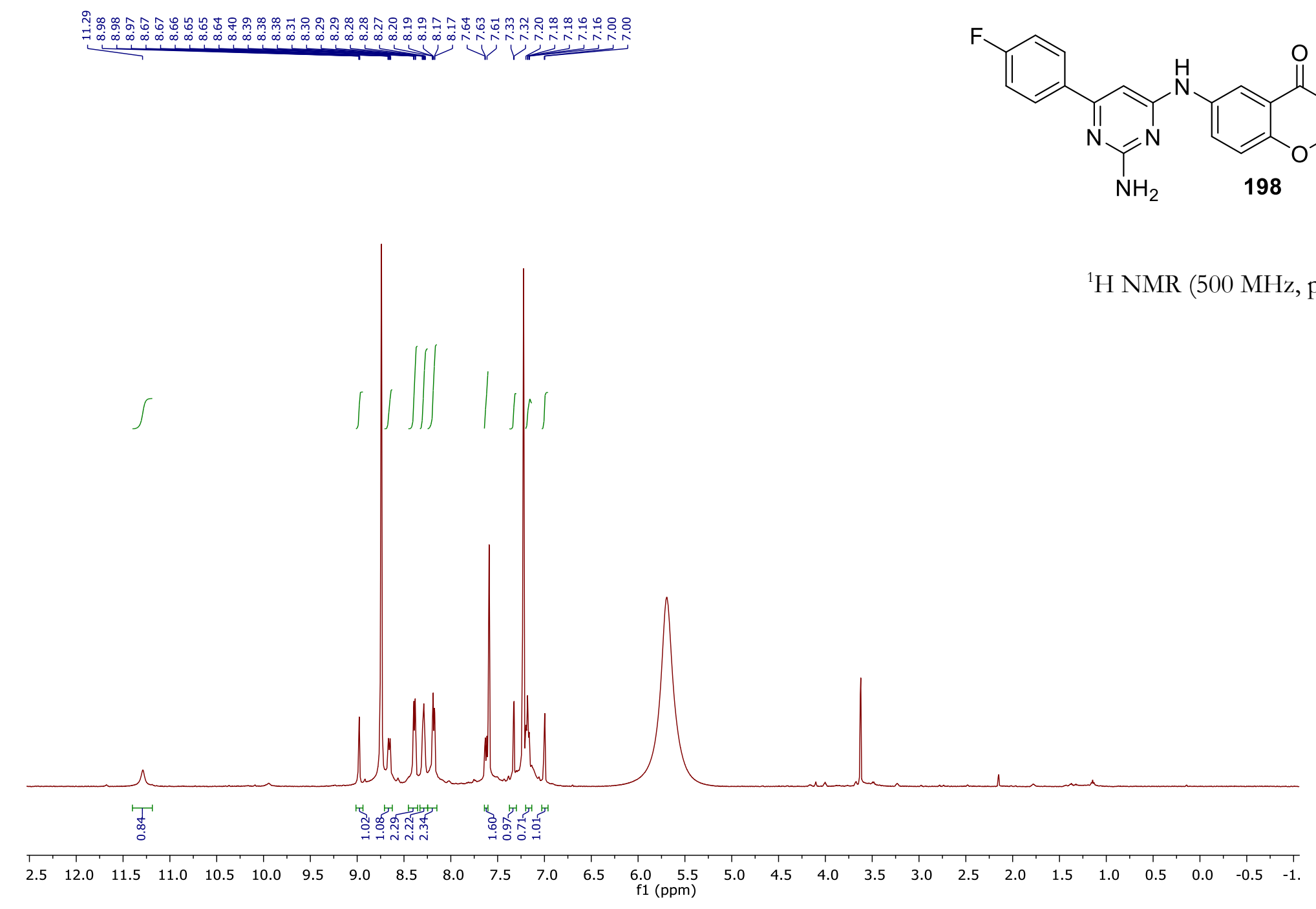




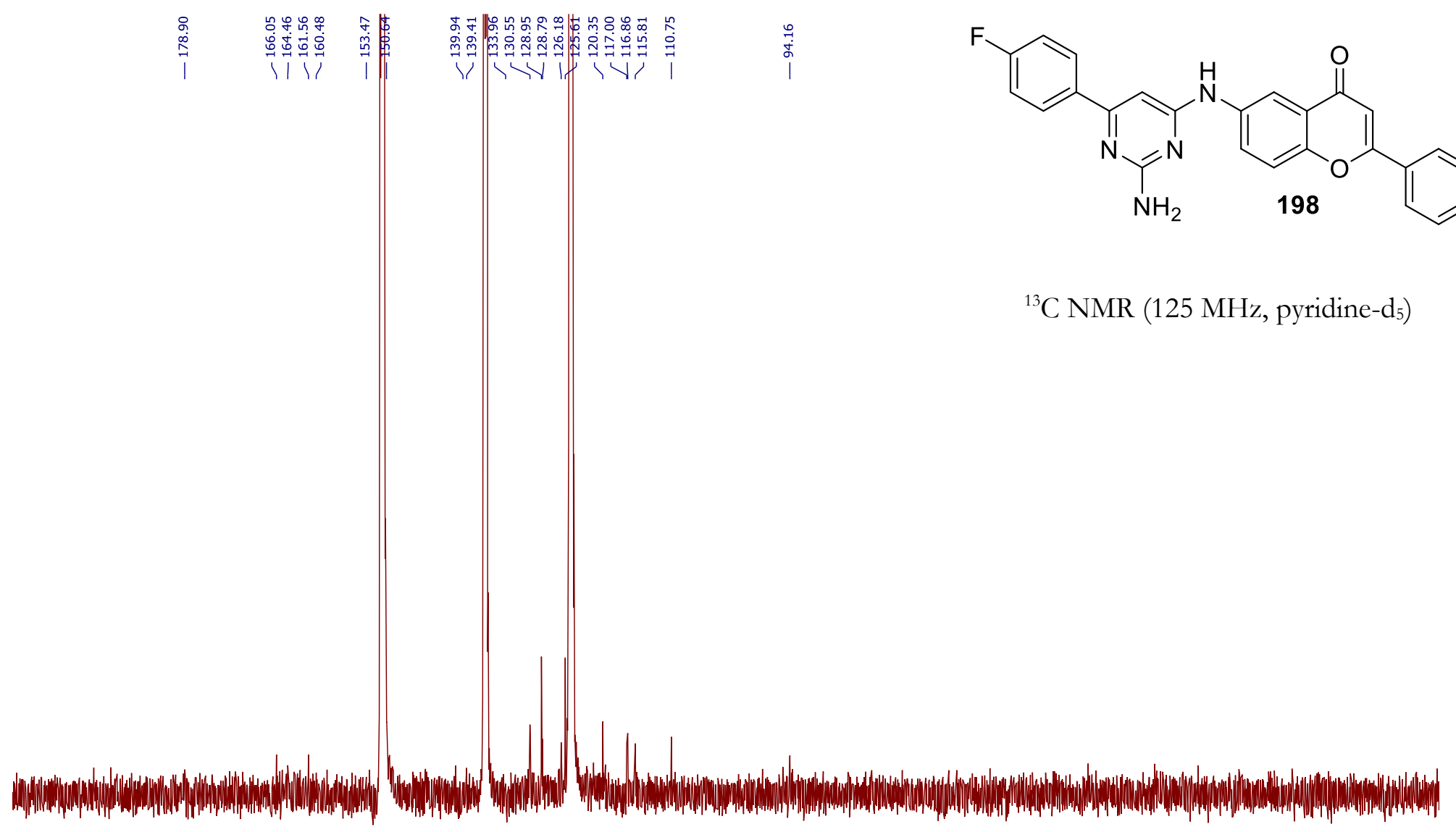

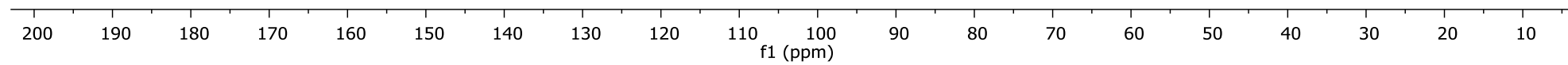




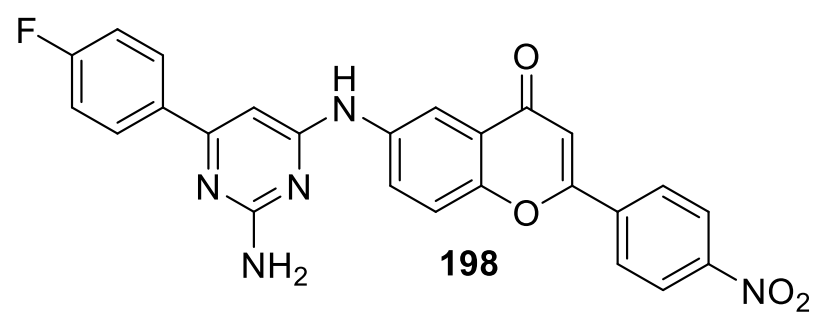

${ }^{19}$ F NMR (282 MHz, pyridine-d $\left.\mathrm{d}_{5}\right)$
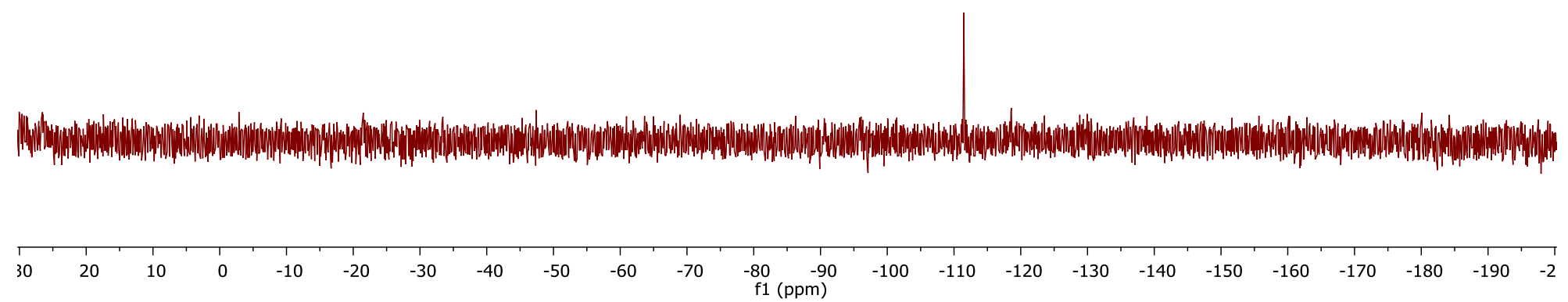


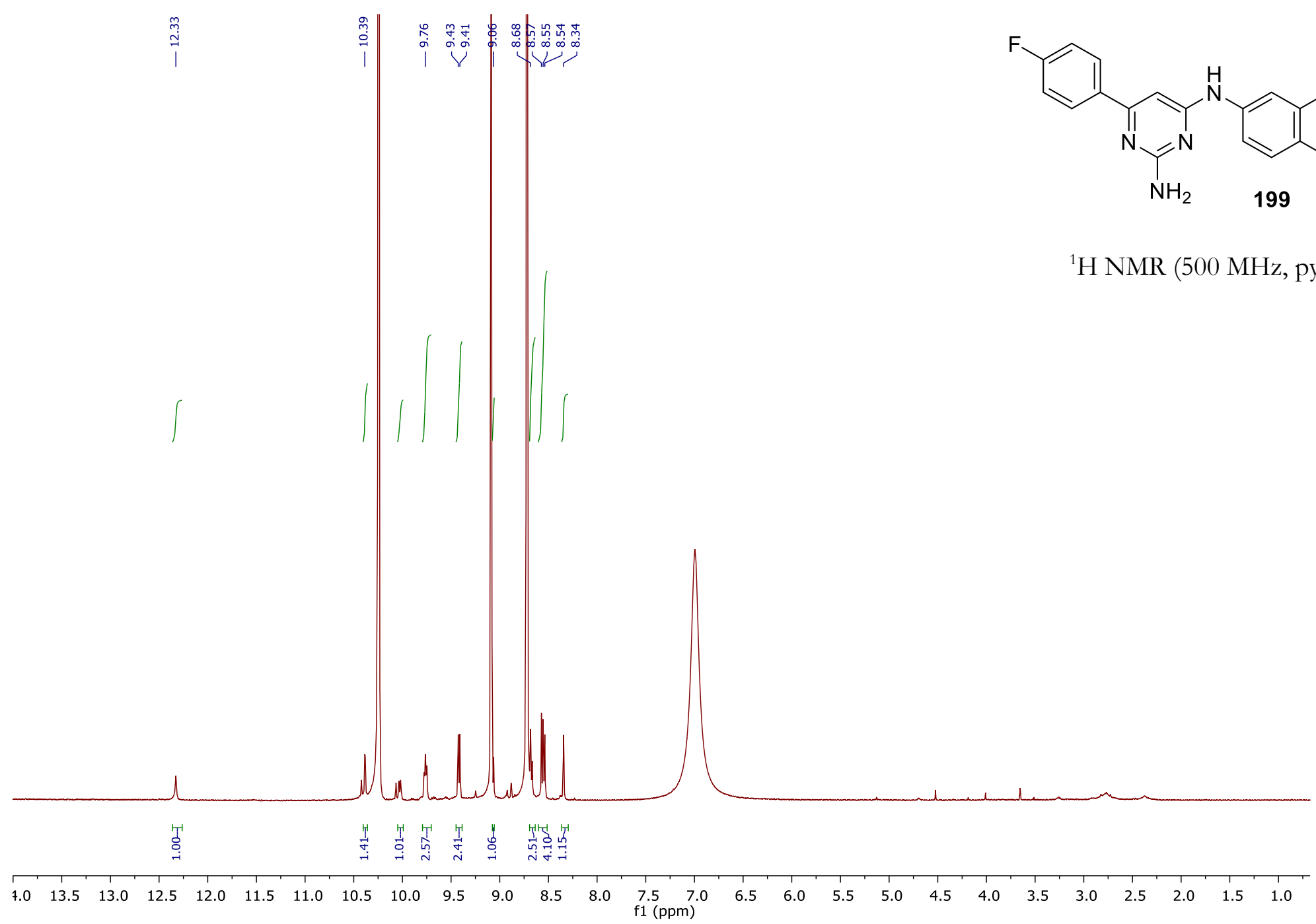




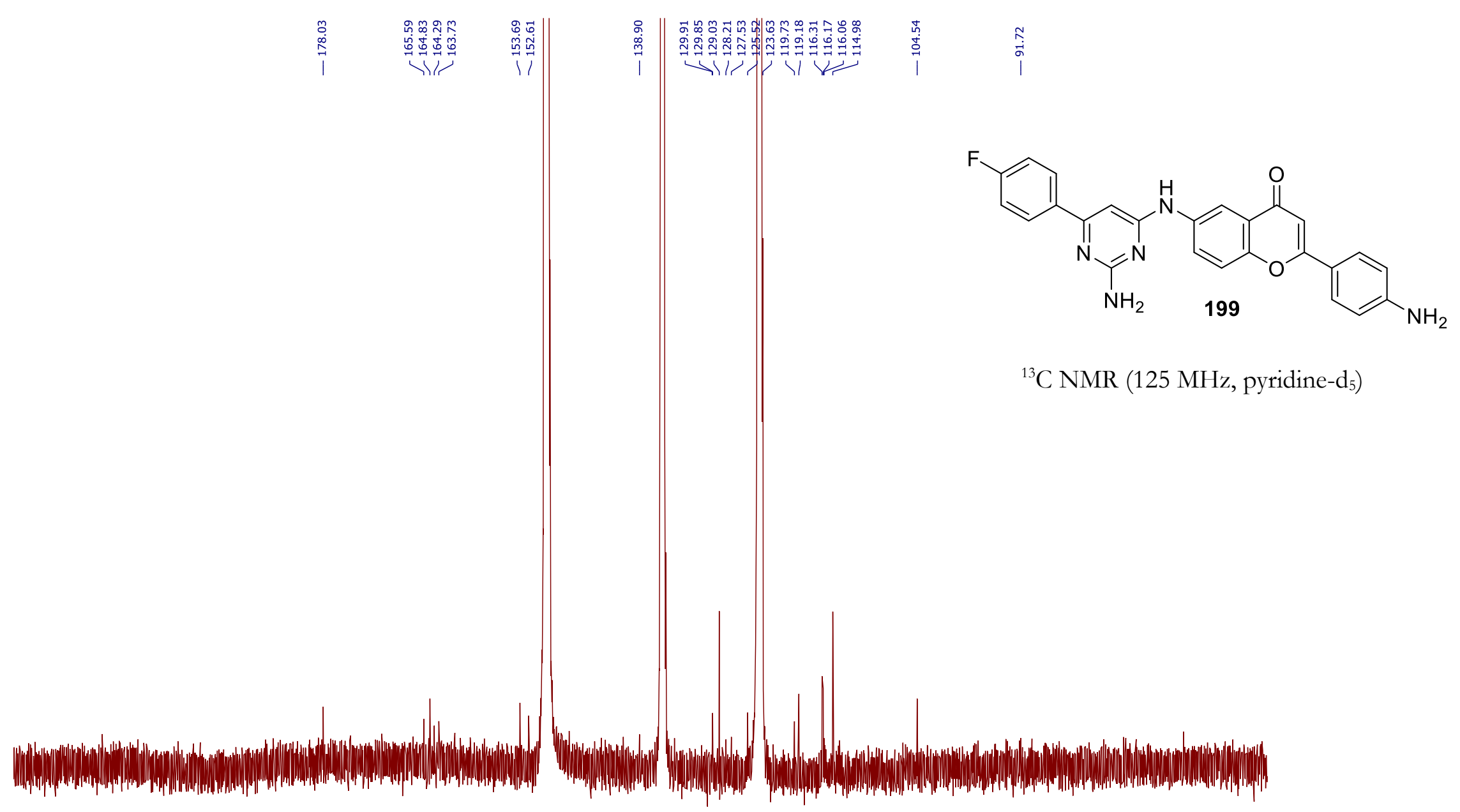

$\begin{array}{llllllllllllllllllllllllllllllllllllllllllllllll} & 215 & 210 & 205 & 200 & 195 & 190 & 185 & 180 & 175 & 170 & 165 & 160 & 155 & 150 & 145 & 140 & 135 & 130 & 125 & 120 & 115 & 110 & 105 & 100 & 95 & 90 & 85 & 80 & 75 & 70 & 65\end{array}$ 


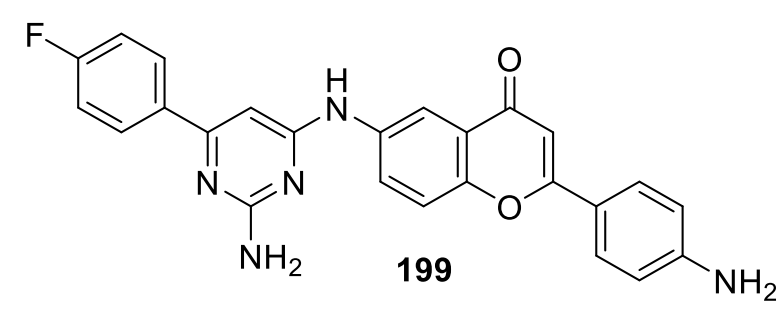

${ }^{19} \mathrm{~F}$ NMR (282 MHz, pyridine- $\left.\mathrm{d}_{5}\right)$

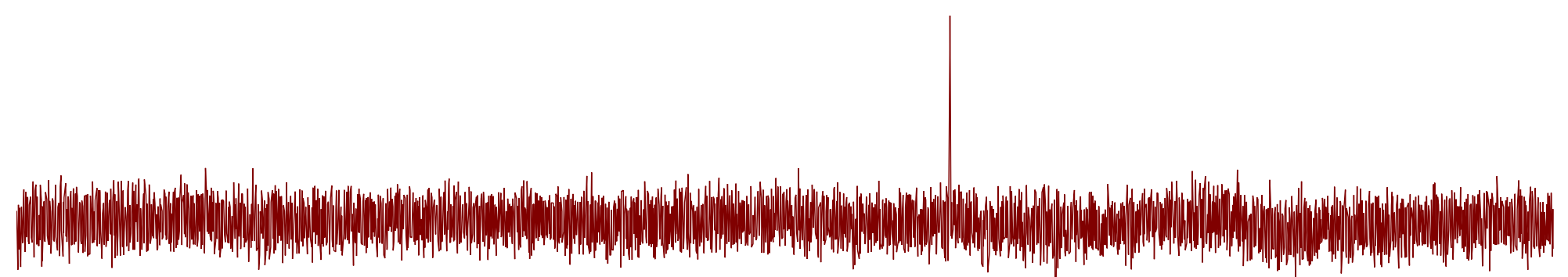

$\begin{array}{llllll}30 & 20 & 10 & 0 & -10 & -20\end{array}$ $-40$ $\begin{array}{cccc}-60 & -70 & -80 & -90 \\ & & \text { f1 } & \left(\mathrm{ppm}^{2}\right)\end{array}$ $\begin{array}{llllllllllll}-100 & -110 & -120 & -130 & -140 & -150 & -160 & -170 & -180 & -190 & -2\end{array}$ 


\section{APPENDIX: CHAPTER FIVE}

NMR spectra:

A155-A213 


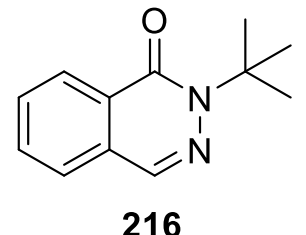

${ }^{1} \mathrm{H}$ NMR $\left(500 \mathrm{MHz}, \mathrm{CDCl}_{3}\right)$

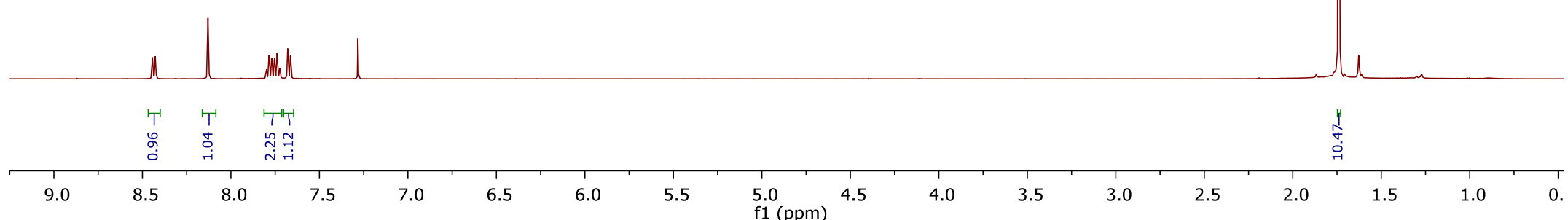




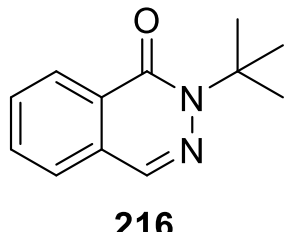

${ }^{13} \mathrm{C}$ NMR $\left(125 \mathrm{MHz}, \mathrm{CDCl}_{3}\right)$

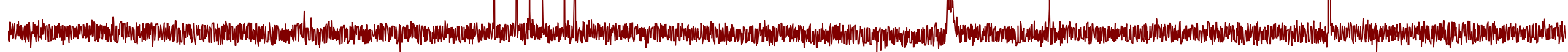
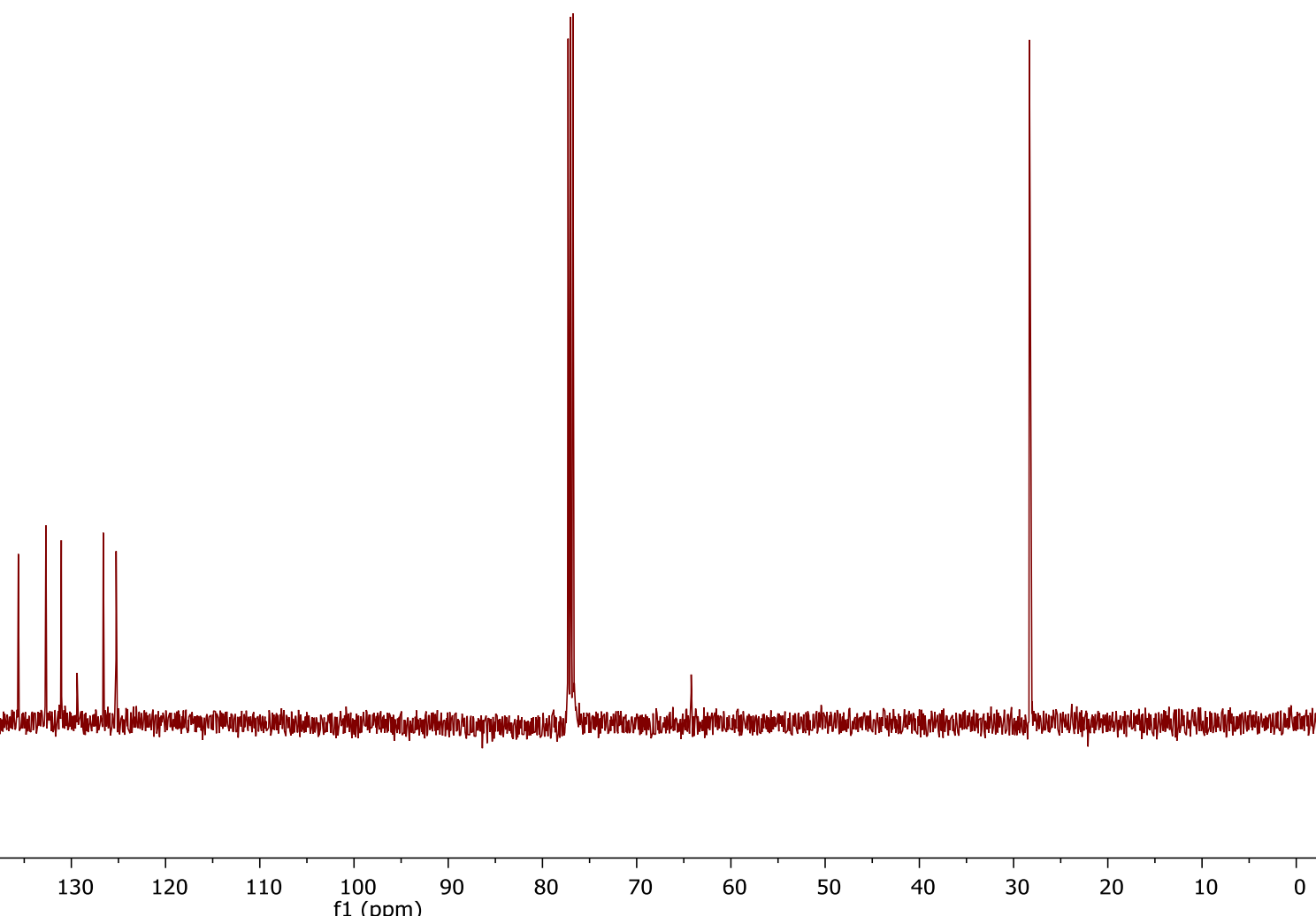


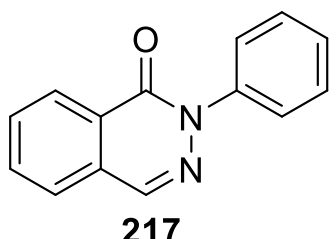

${ }^{1} \mathrm{H}$ NMR $\left(500 \mathrm{MHz}, \mathrm{CDCl}_{3}\right)$
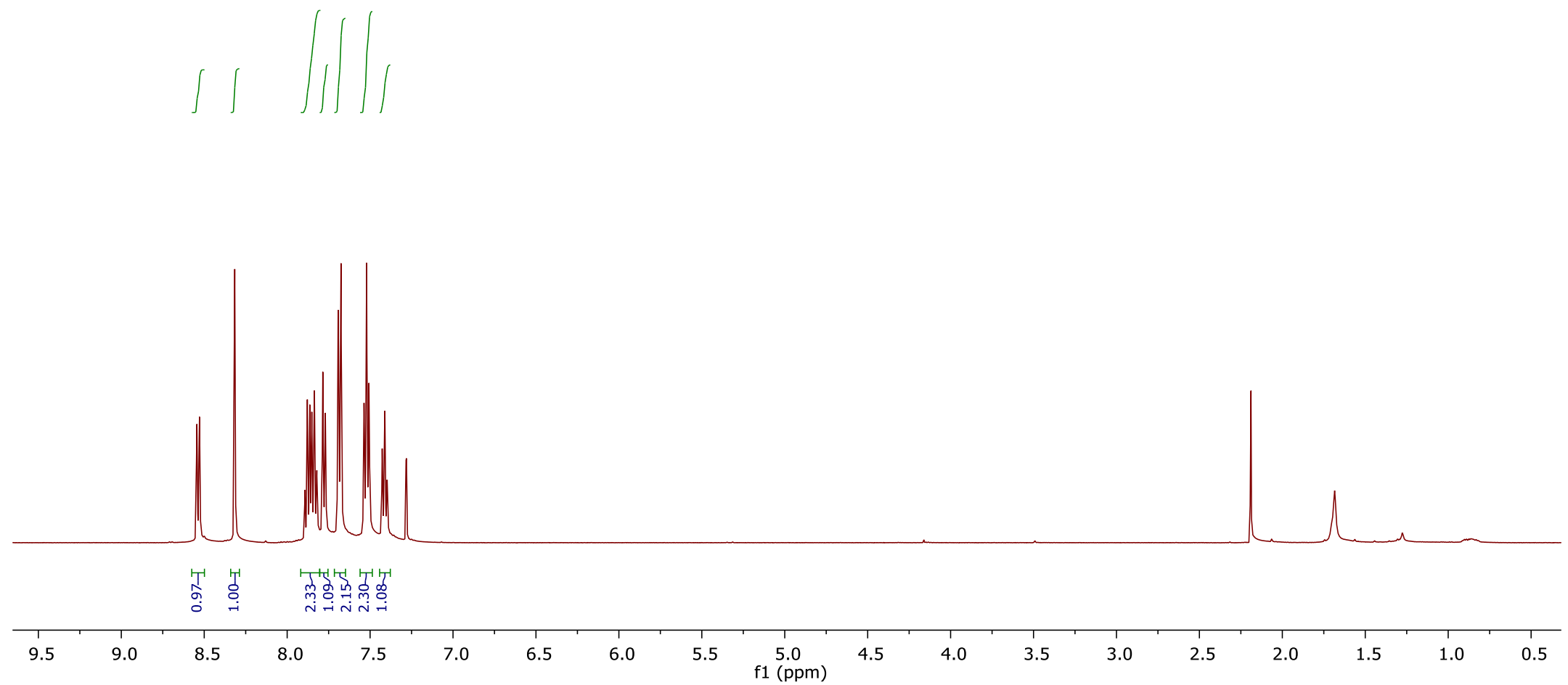

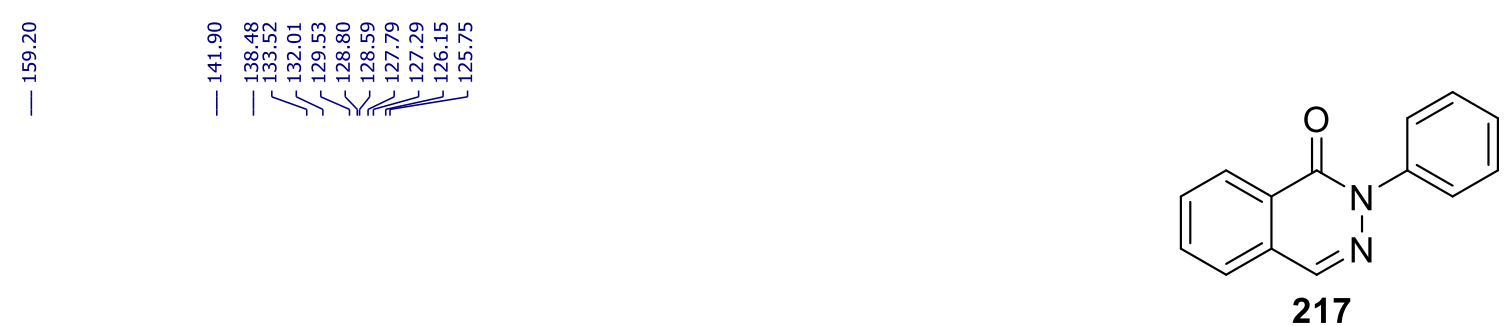

${ }^{13} \mathrm{C}$ NMR $\left(125 \mathrm{MHz}, \mathrm{CDCl}_{3}\right)$

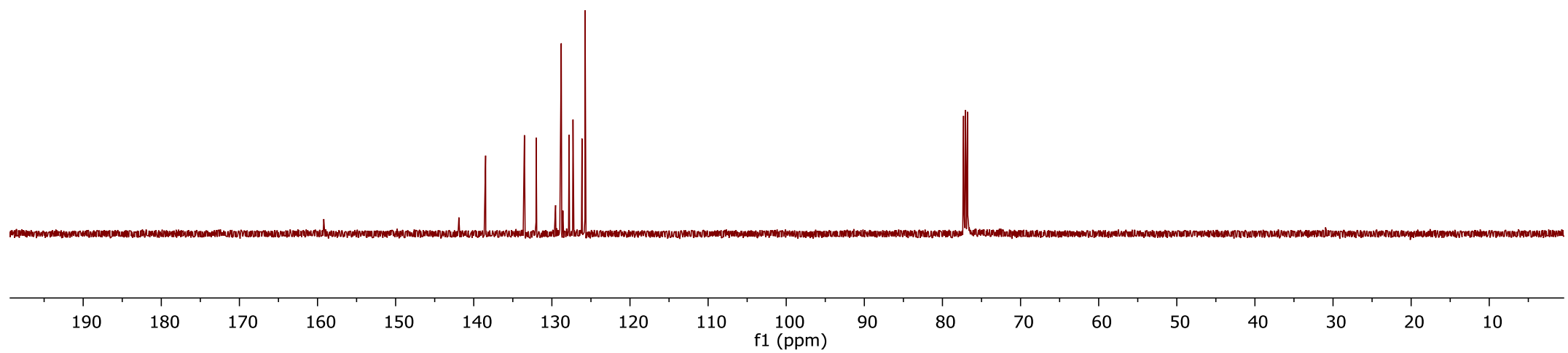




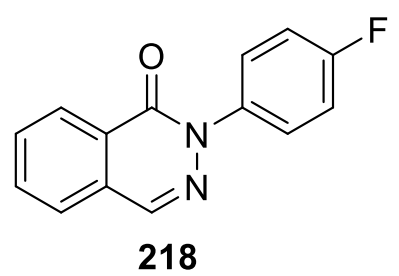

${ }^{1} \mathrm{H} \mathrm{NMR}\left(500 \mathrm{MHz}, \mathrm{CDCl}_{3}\right)$
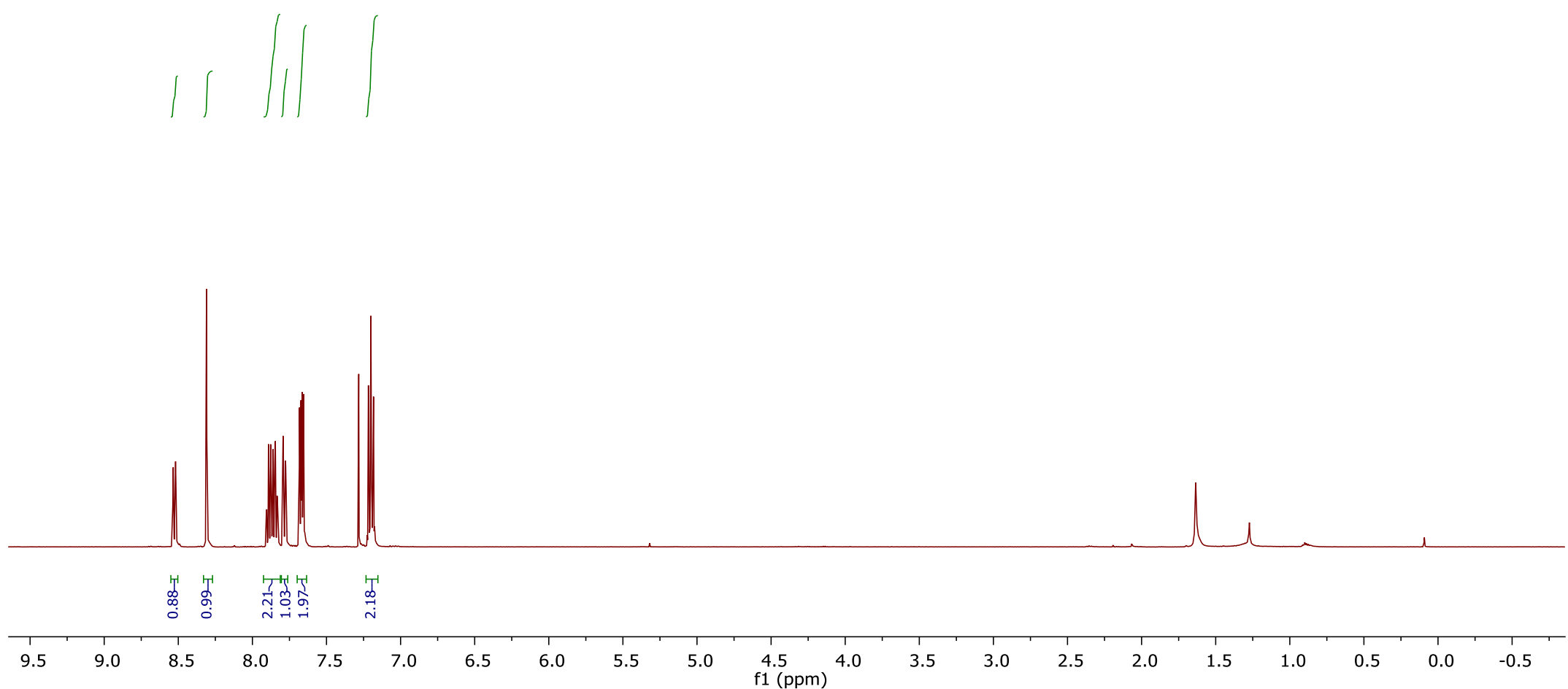


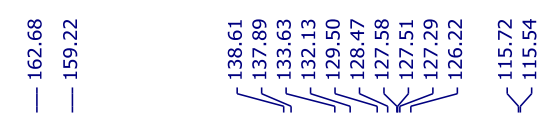

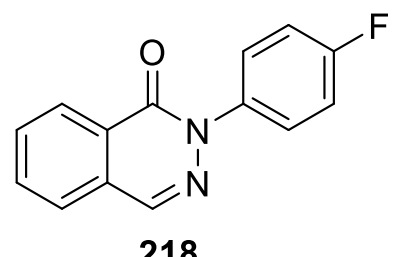

218

${ }^{13} \mathrm{C}$ NMR $\left(125 \mathrm{MHz}, \mathrm{CDCl}_{3}\right)$

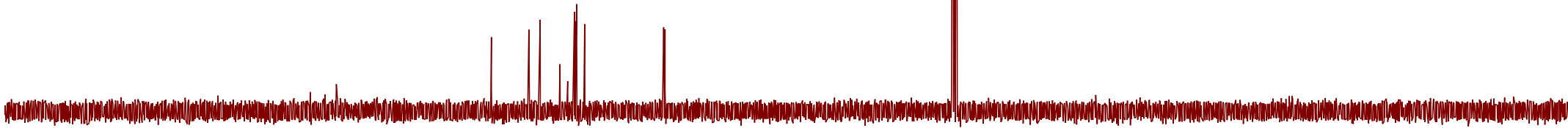

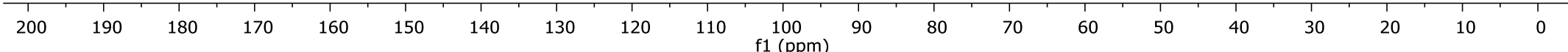




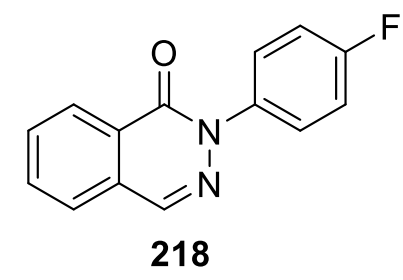

${ }^{19} \mathrm{~F}$ NMR (282 $\left.\mathrm{MHz}, \mathrm{CDCl}_{3}\right)$

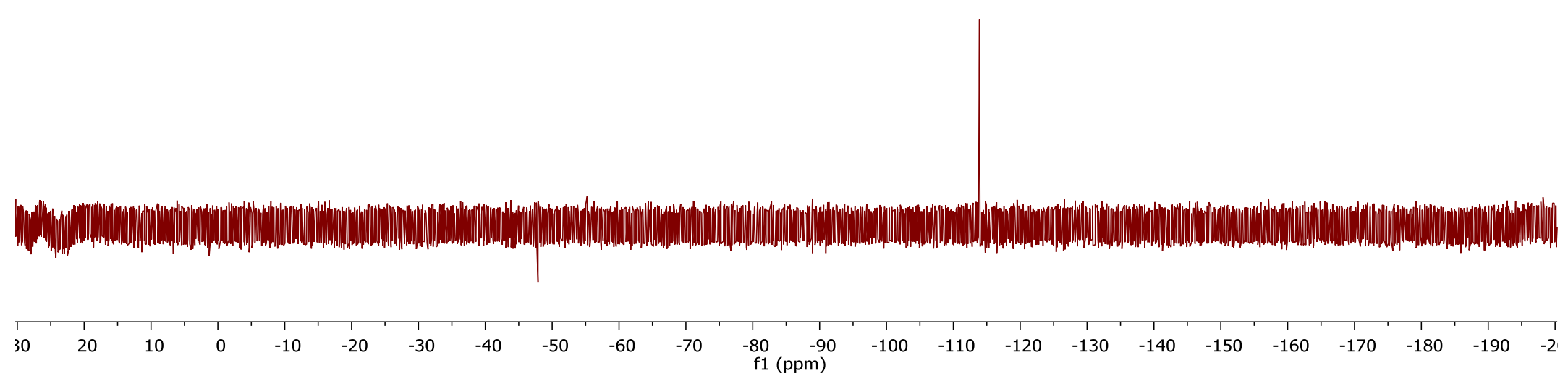




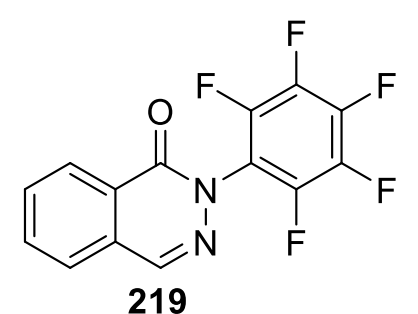

${ }^{1} \mathrm{H}$ NMR $\left(500 \mathrm{MHz}, \mathrm{CDCl}_{3}\right)$
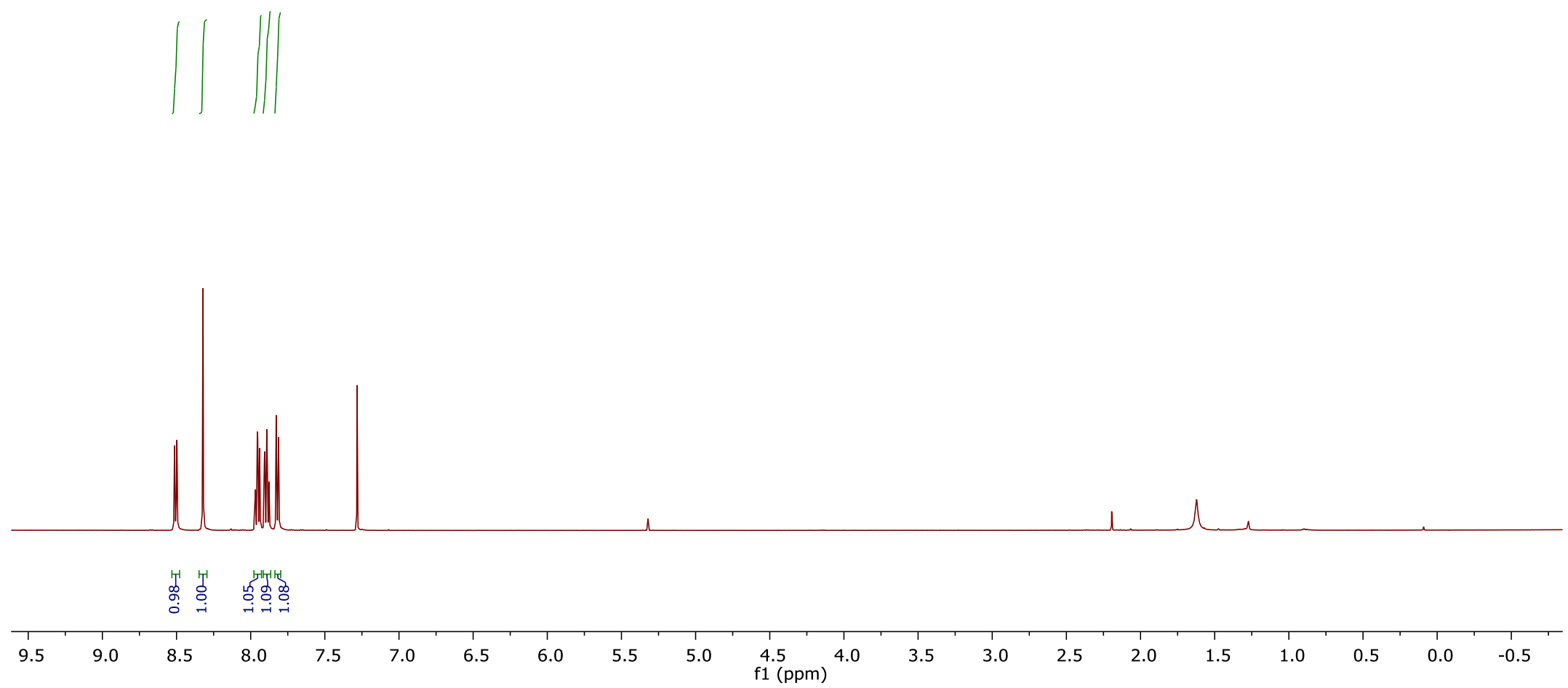


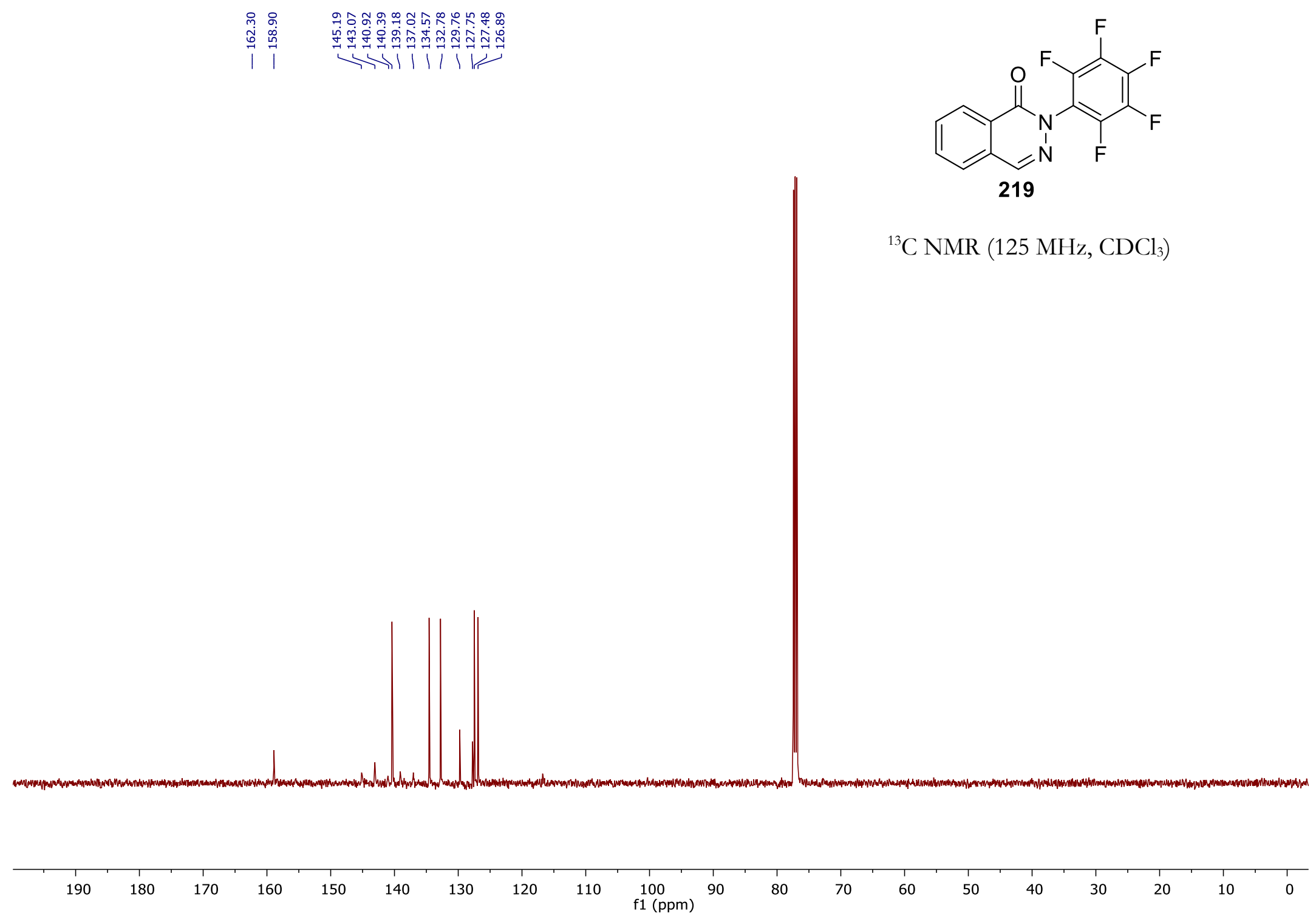




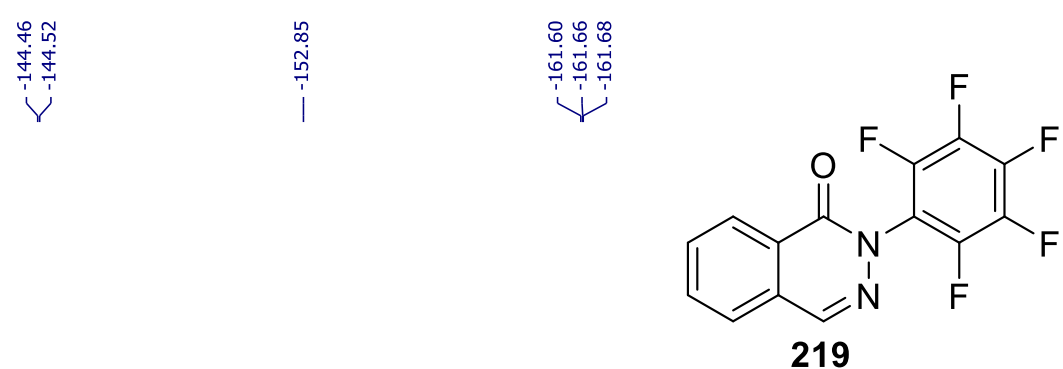

${ }^{19} \mathrm{~F}$ NMR $\left(282 \mathrm{MHz}, \mathrm{CDCl}_{3}\right)$
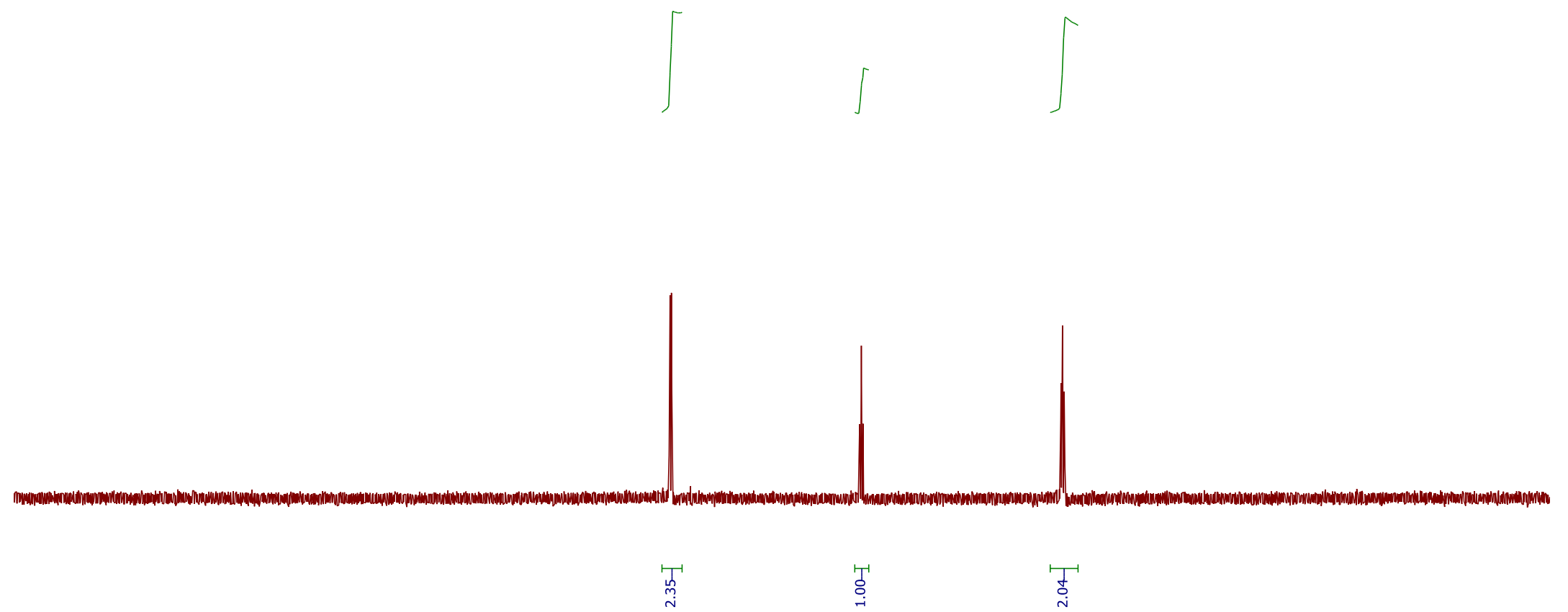

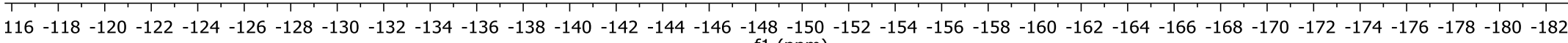
f1 (ppm) 


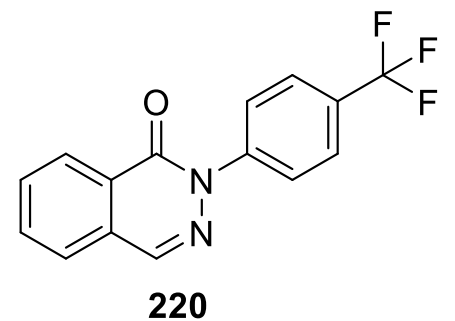

${ }^{1} \mathrm{H}$ NMR $\left(500 \mathrm{MHz}, \mathrm{CDCl}_{3}\right)$

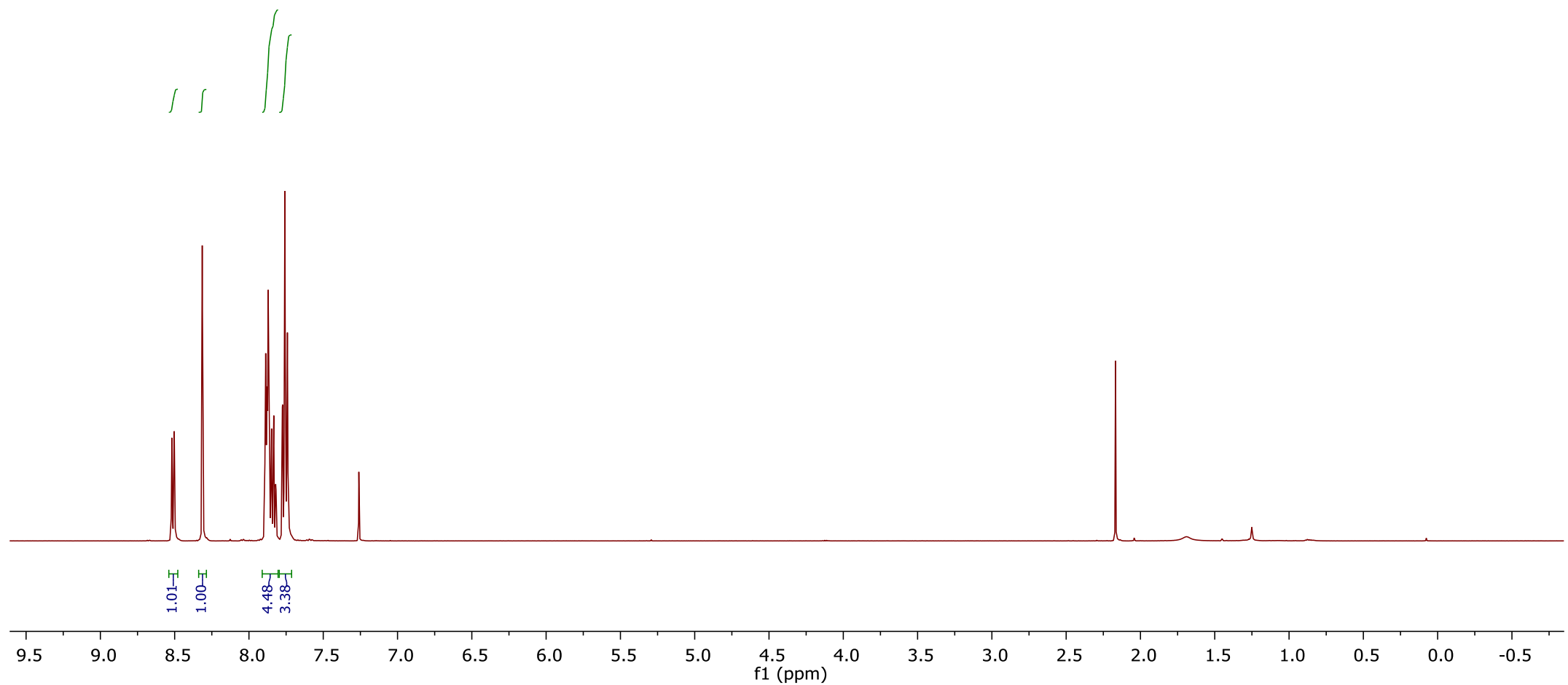



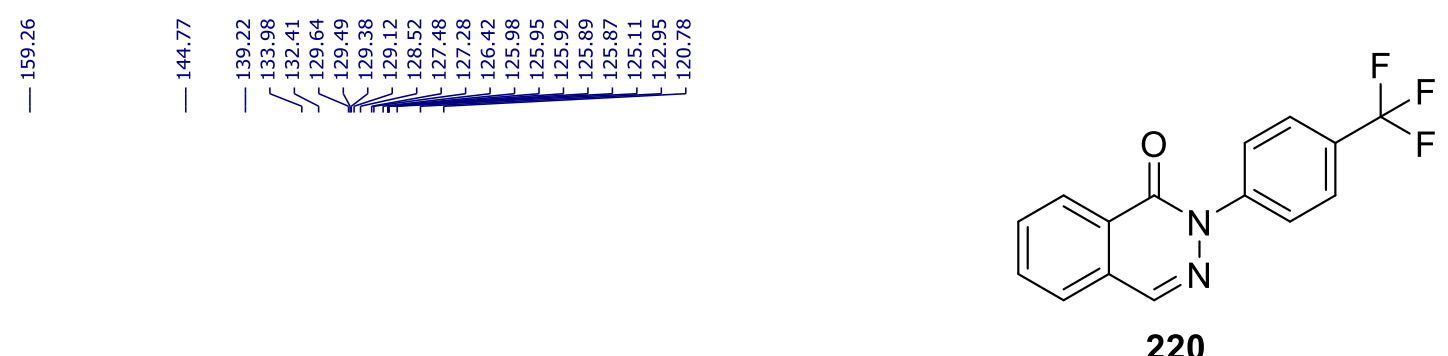

${ }^{13} \mathrm{C}$ NMR $\left(125 \mathrm{MHz}, \mathrm{CDCl}_{3}\right)$

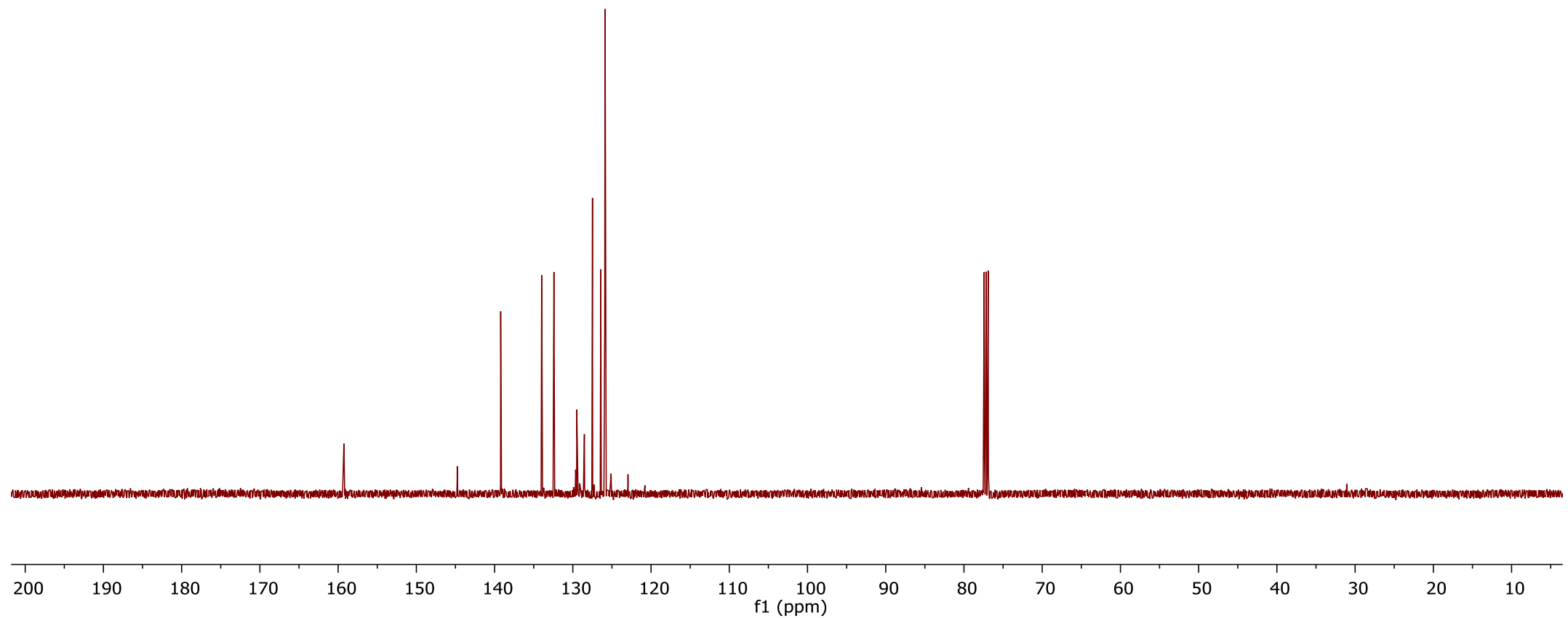




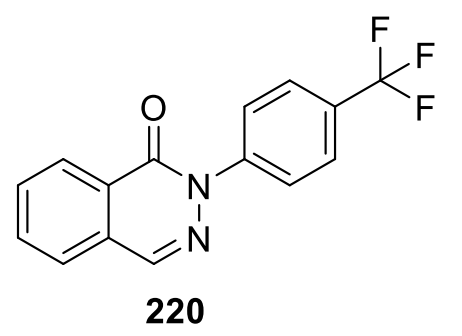

${ }^{19} \mathrm{~F}$ NMR $\left(282 \mathrm{MHz}, \mathrm{CDCl}_{3}\right)$

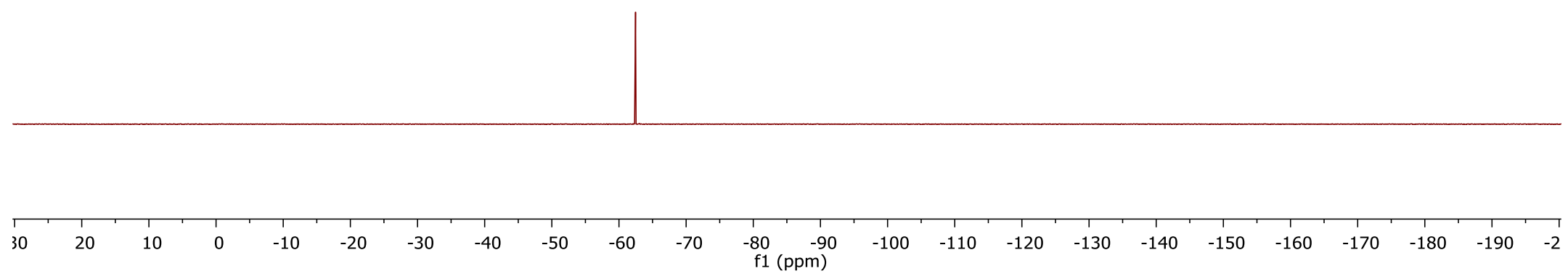




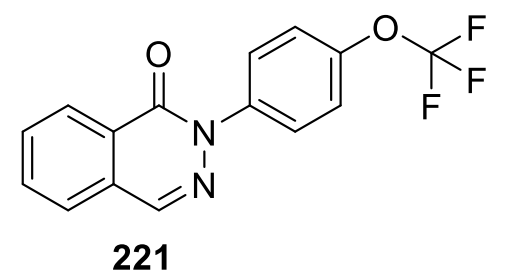

${ }^{1} \mathrm{H}$ NMR $\left(500 \mathrm{MHz}, \mathrm{CDCl}_{3}\right)$

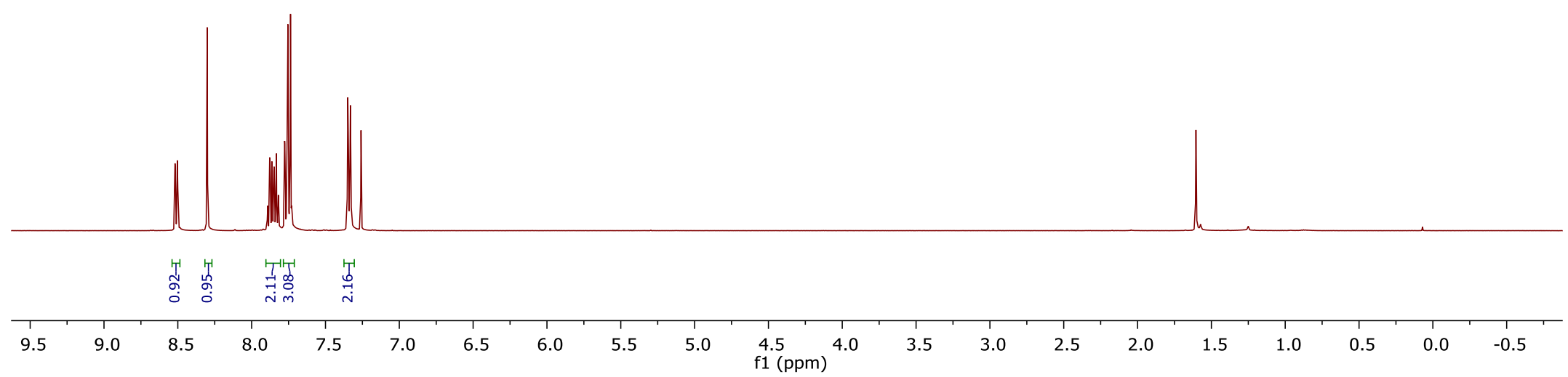




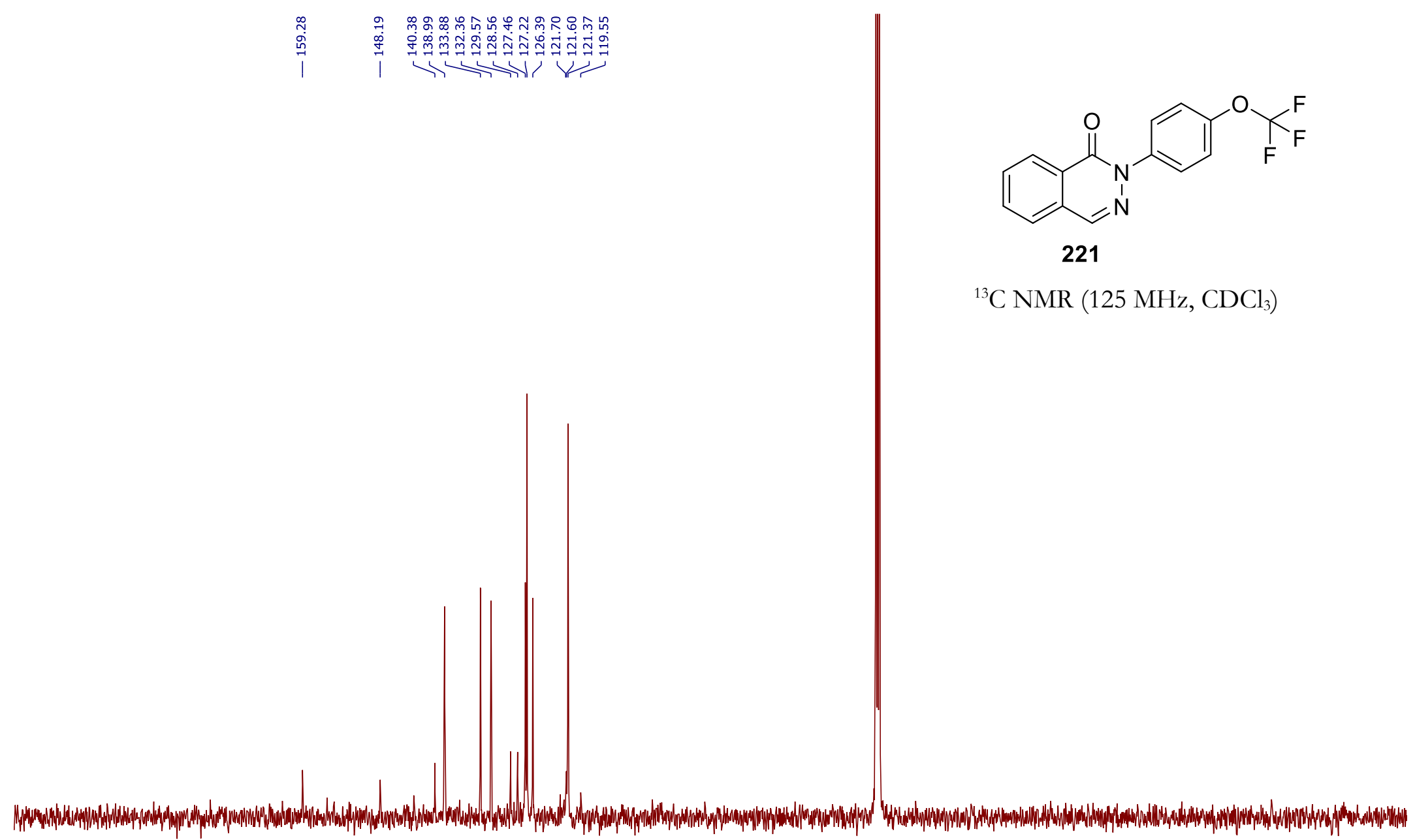




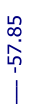

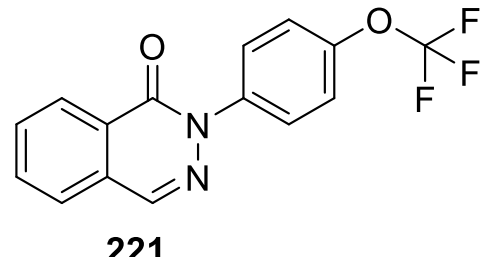

${ }^{19} \mathrm{~F}$ NMR $\left(282 \mathrm{MHz}, \mathrm{CDCl}_{3}\right)$

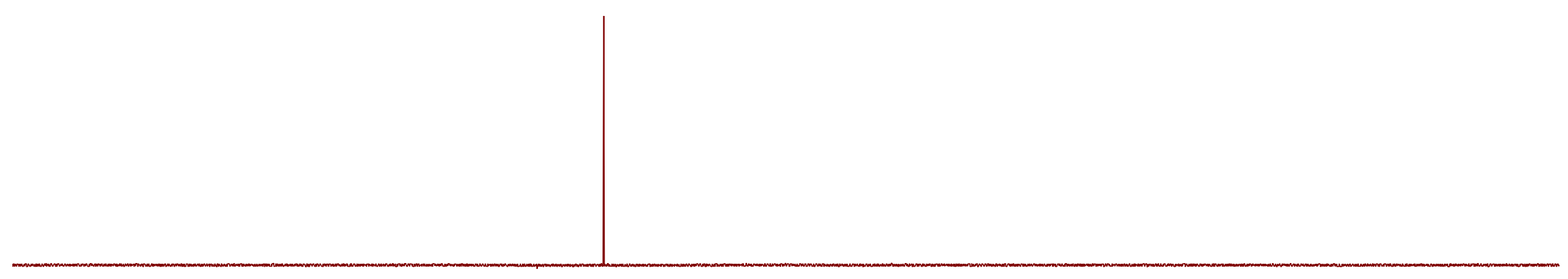

$\begin{array}{lllllllllllllllllllllllllllll} & 20 & 20 & 10 & 0 & -10 & -20 & -30 & -40 & -50 & -60 & -70 & -80 & -90 & -100 & -110 & -120 & -130 & -140 & -150 & -160 & -170 & -180 & -190 & -2\end{array}$ 


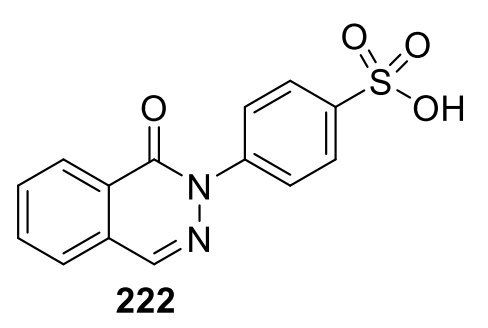

${ }^{1} \mathrm{H}$ NMR (500 MHz, DMSO-d $\left.\mathrm{d}_{6}\right)$

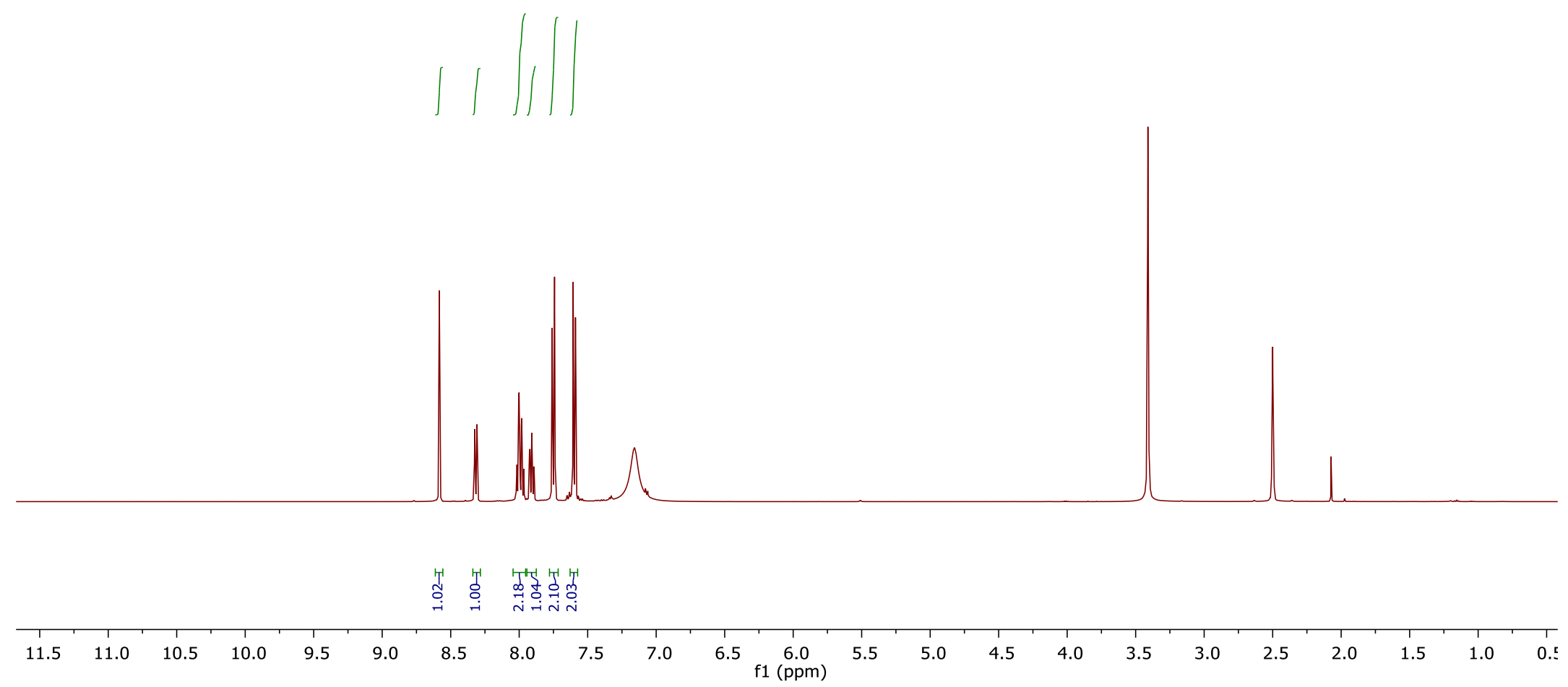




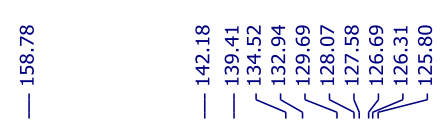

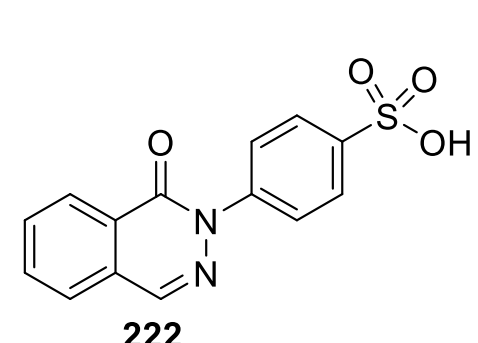

${ }^{13} \mathrm{C}$ NMR (125 MHz, DMSO-d 6 )

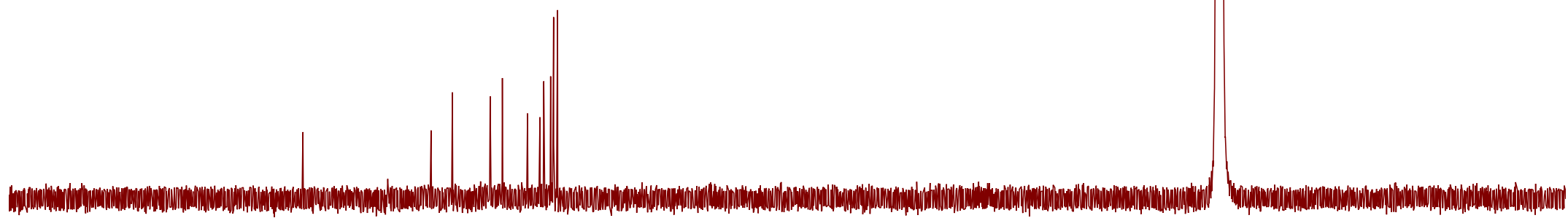




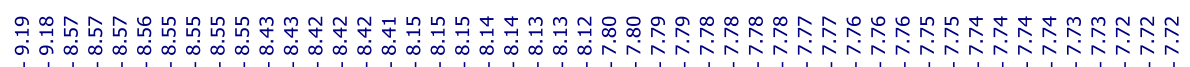

$Y \longrightarrow$

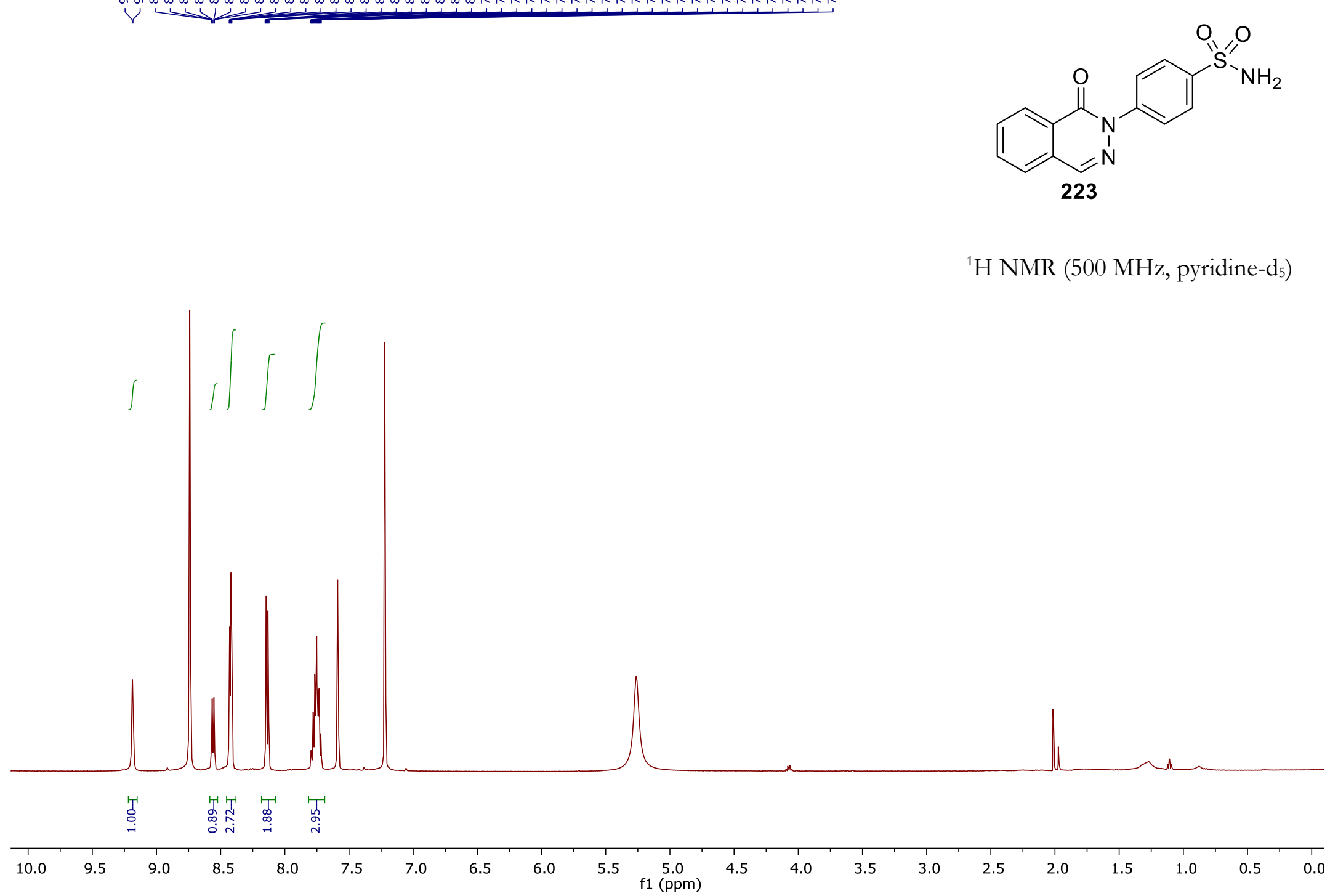




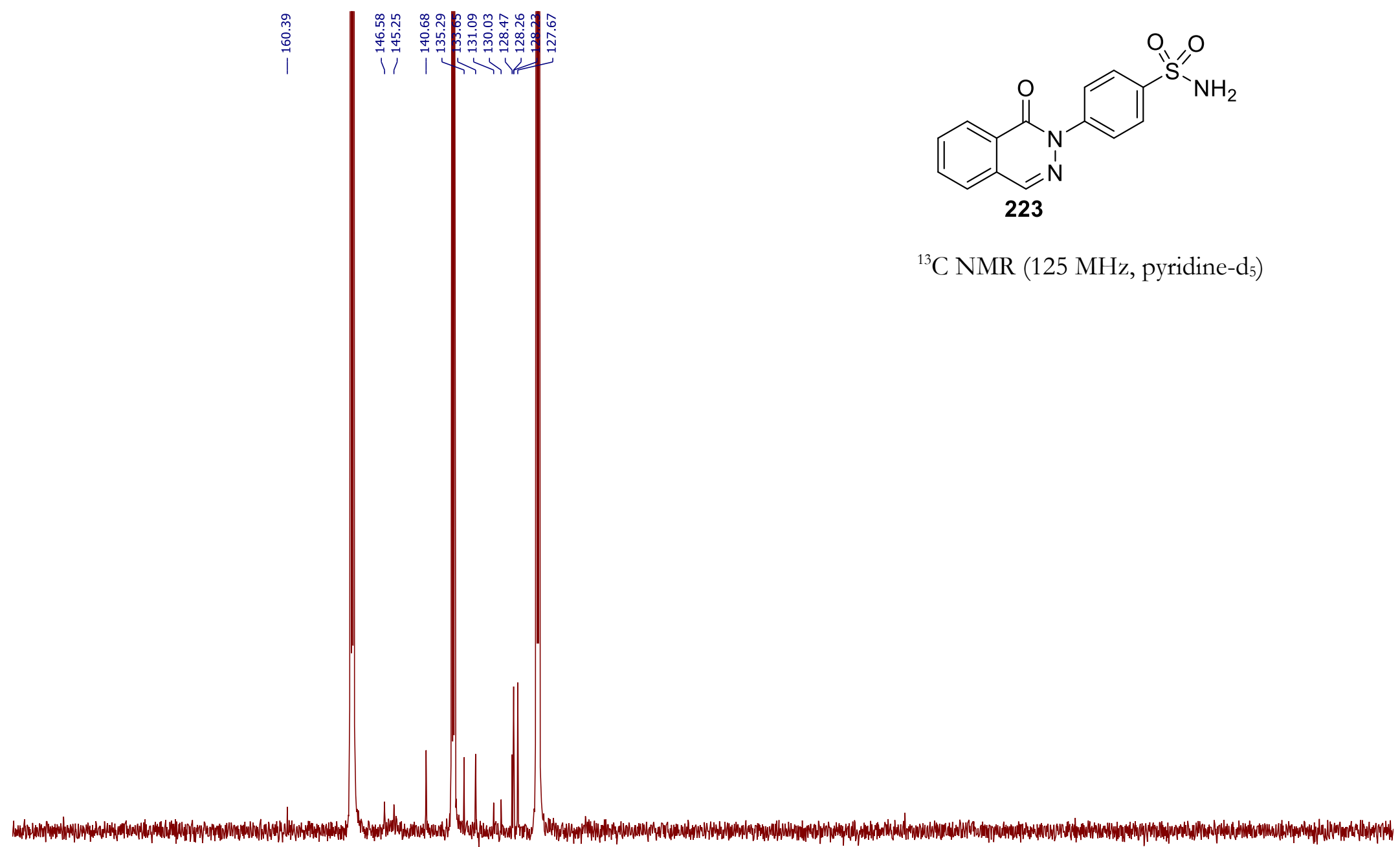

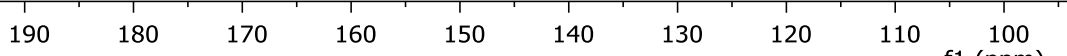

$90 \quad 80$

$70 \quad 60$

50

$40 \quad 30$

20 10 


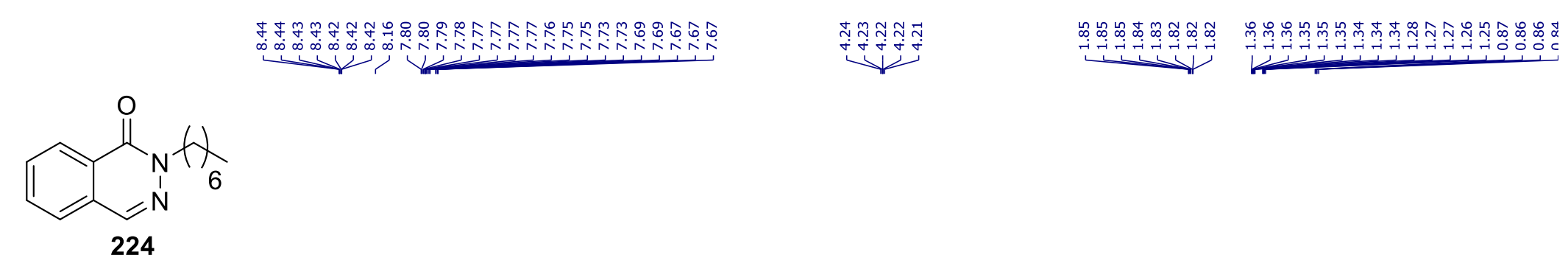

${ }^{1} \mathrm{H}$ NMR $\left(500 \mathrm{MHz}, \mathrm{CDCl}_{3}\right)$

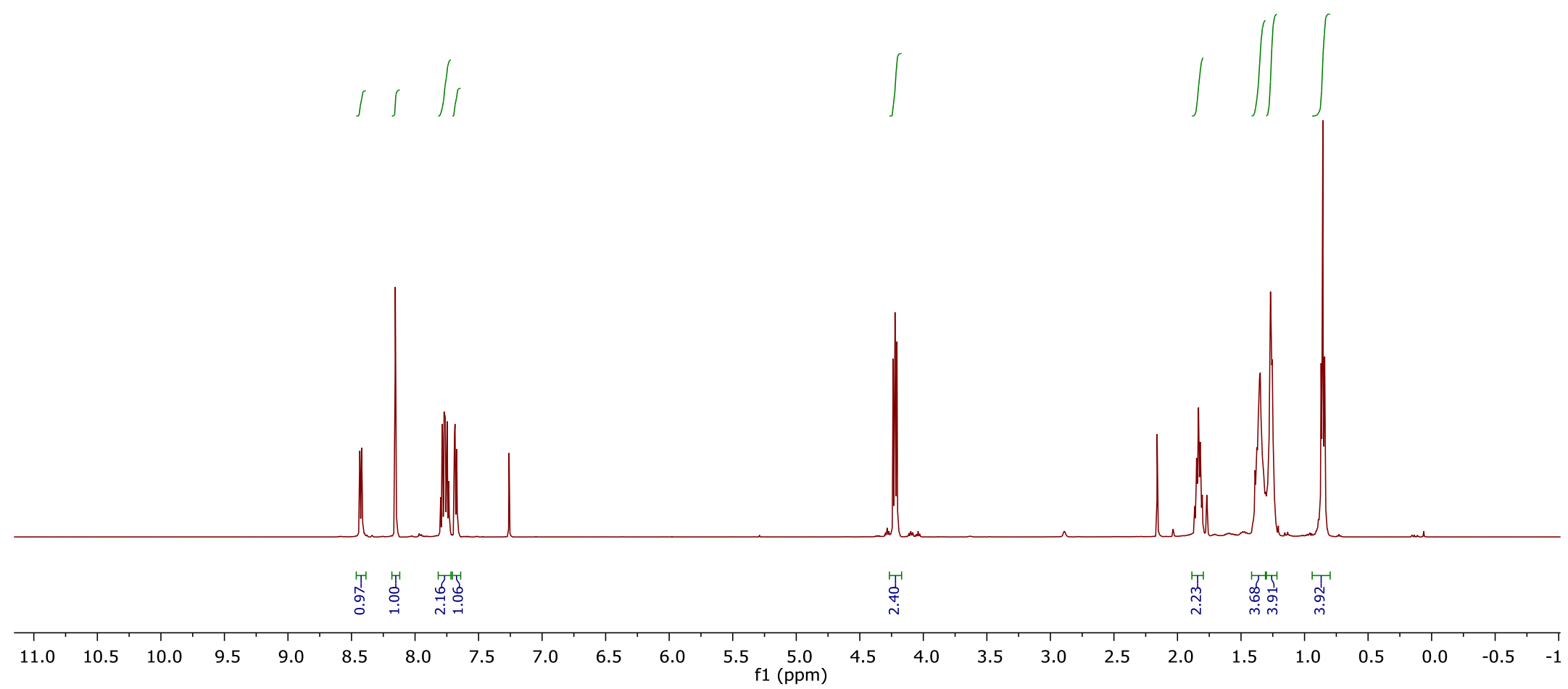




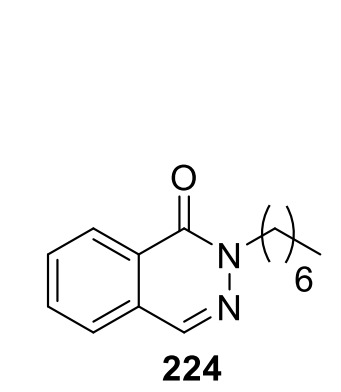

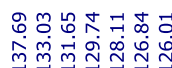

昰牙

至

${ }^{13} \mathrm{C}$ NMR $\left(125 \mathrm{MHz}, \mathrm{CDCl}_{3}\right)$

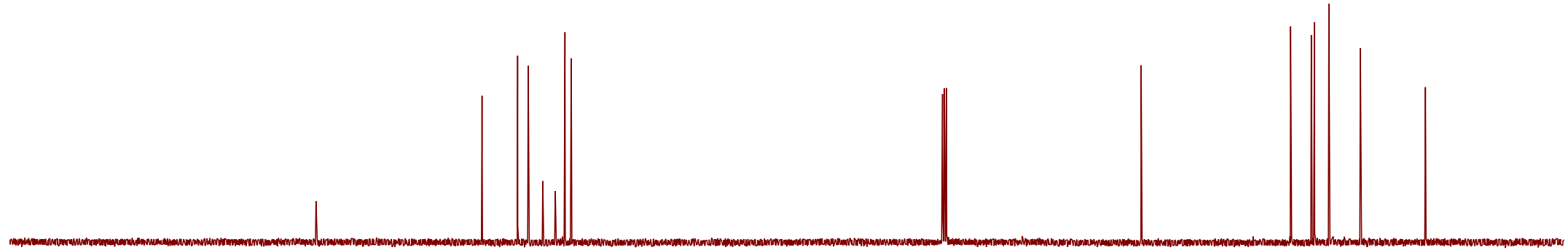

$\begin{array}{llllllllllllllllllllll}190 & 180 & 170 & 160 & 150 & 140 & 130 & 120 & 110 & 100 & 90 & 80 & 70 & 60 & 50 & 40 & 30 & 20 & 10 & 0\end{array}$ 


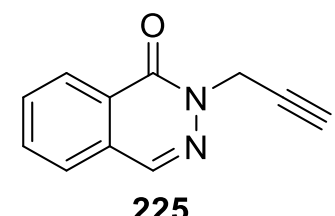

${ }^{1} \mathrm{H} \mathrm{NMR}\left(500 \mathrm{MHz}, \mathrm{CDCl}_{3}\right)$

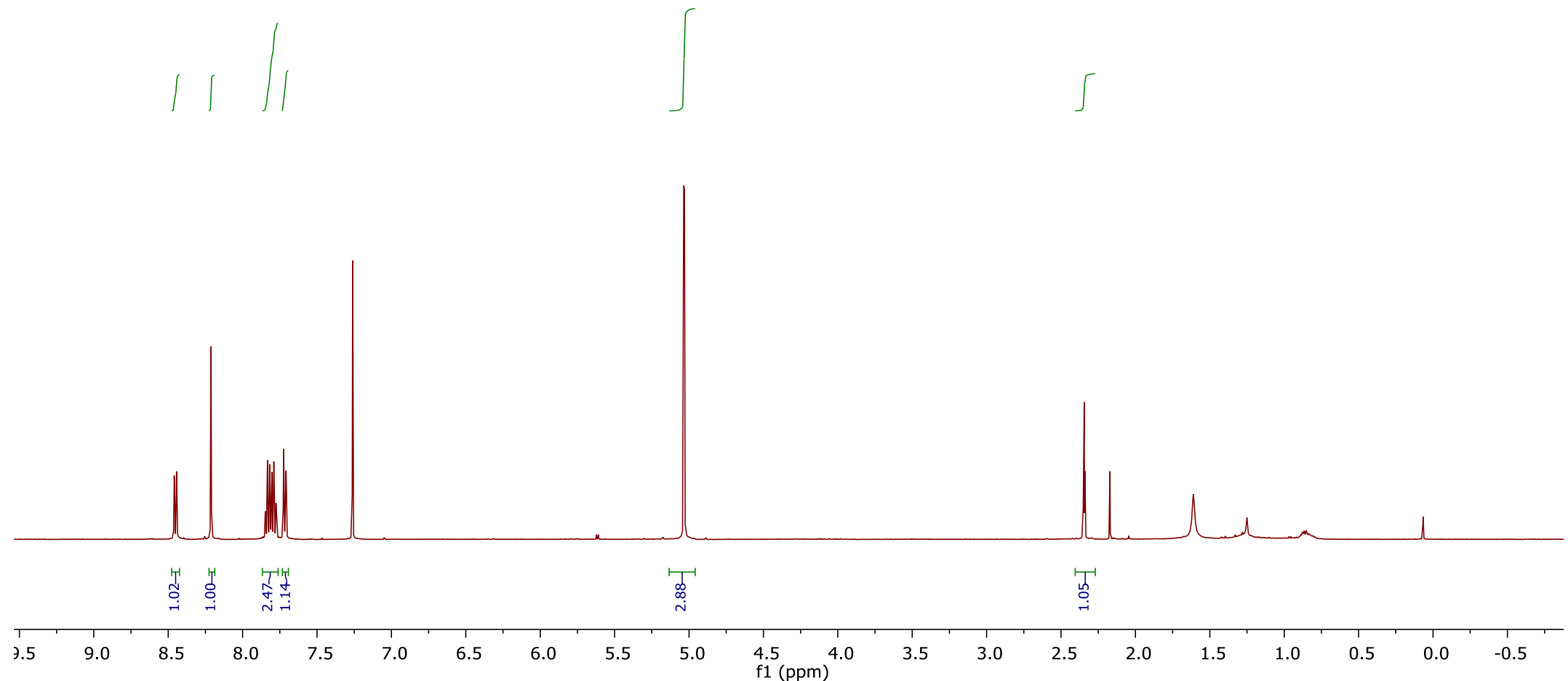




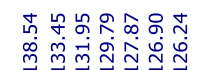

|

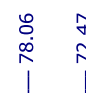

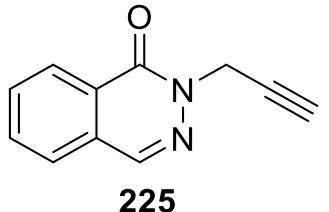

${ }^{13} \mathrm{C}$ NMR $\left(125 \mathrm{MHz}, \mathrm{CDCl}_{3}\right)$

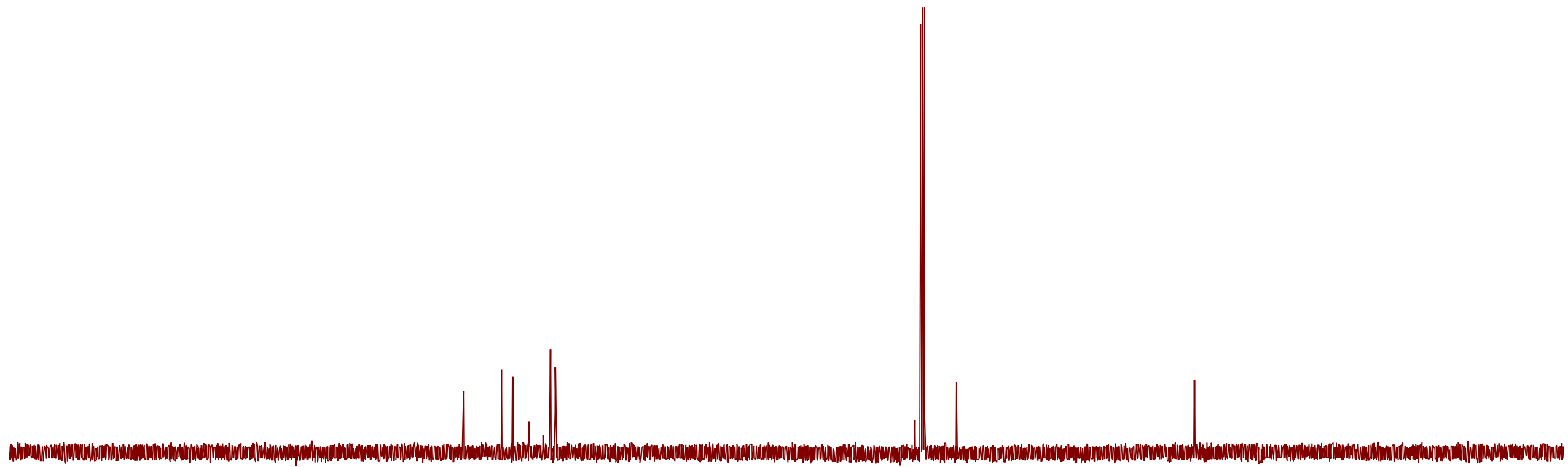



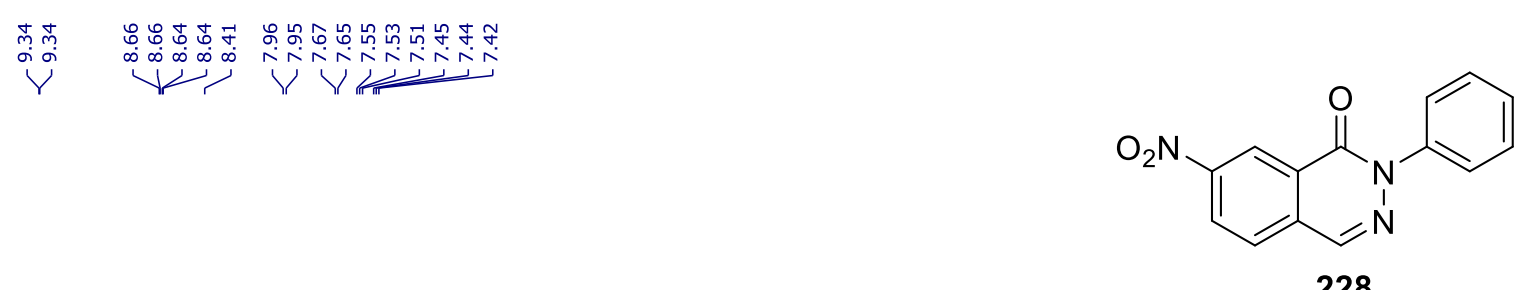

228

${ }^{1} \mathrm{H}$ NMR (500 MHz, $\left.\mathrm{CDCl}_{3}\right)$

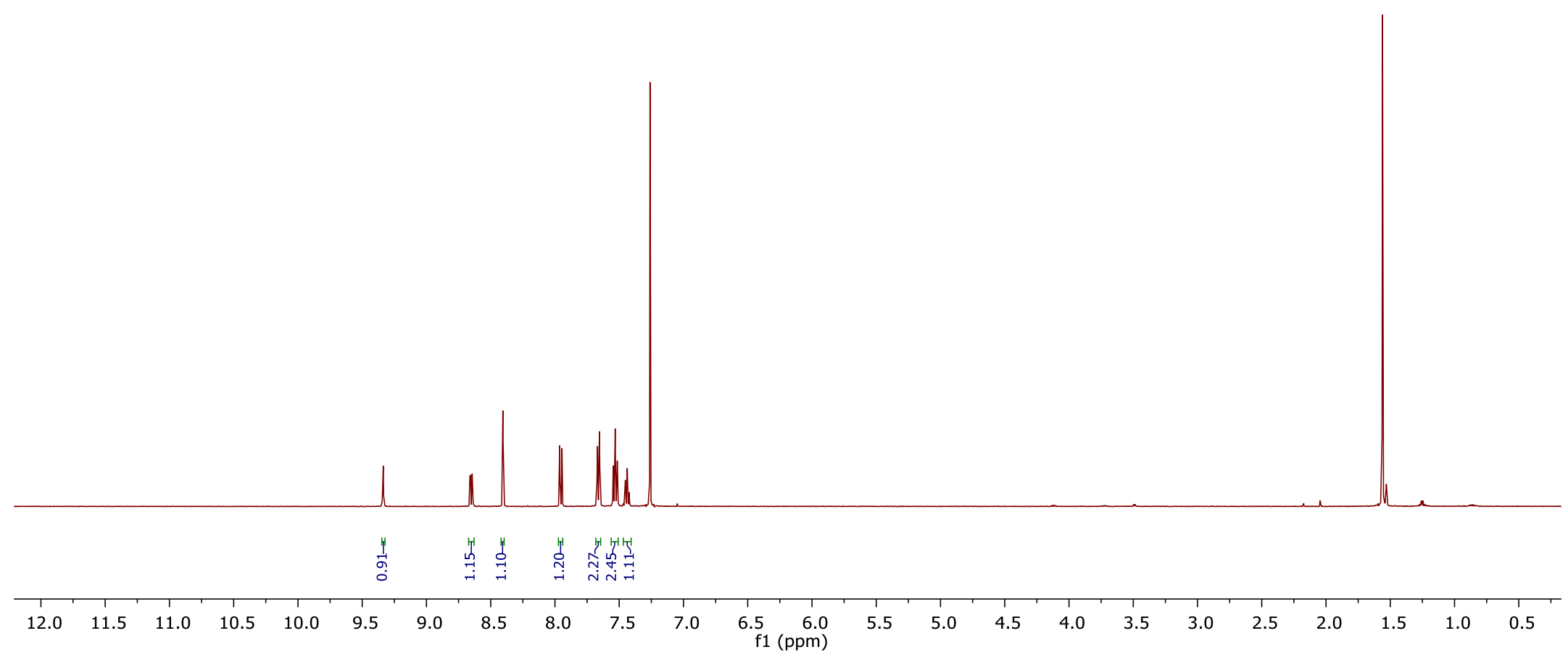




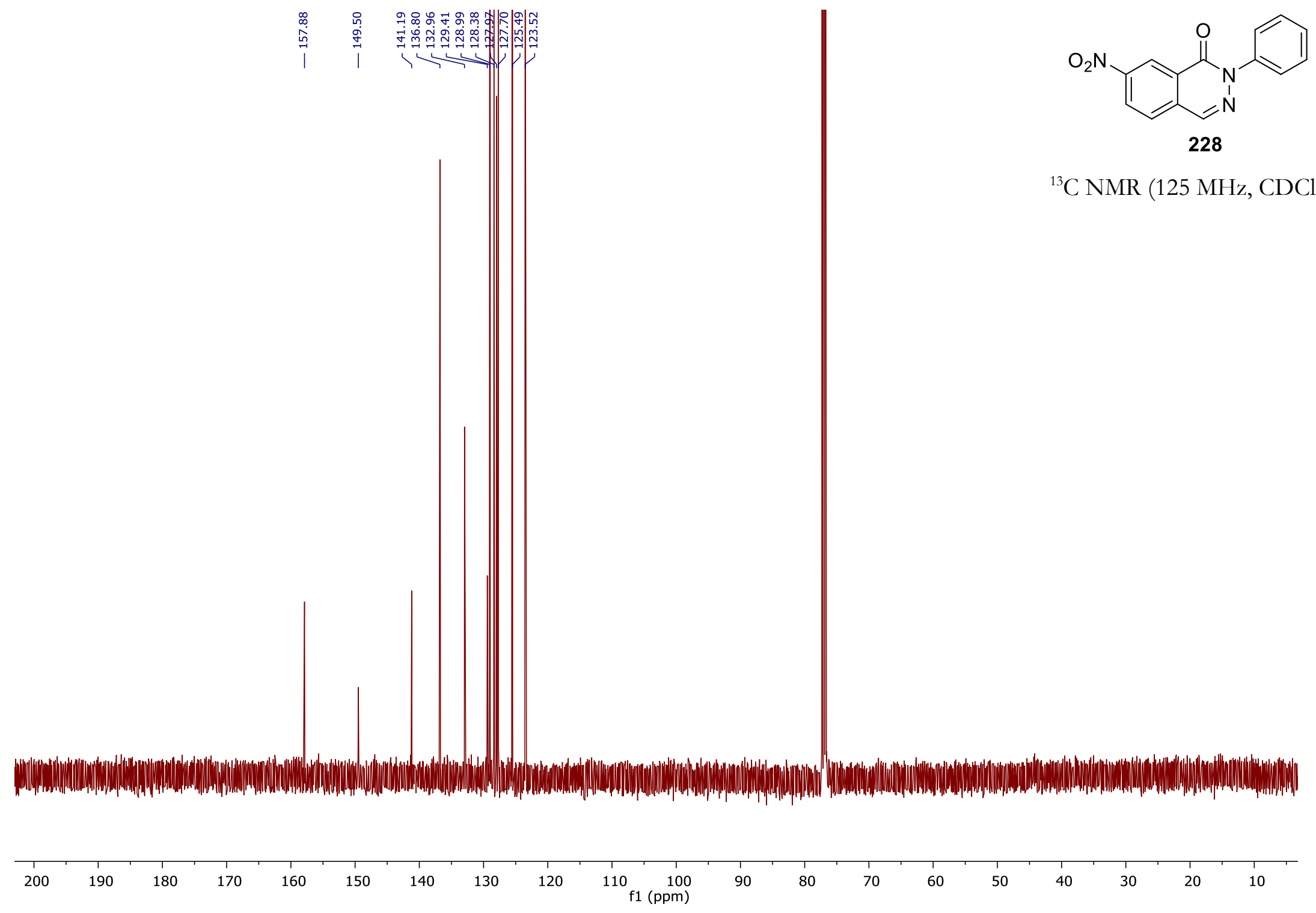




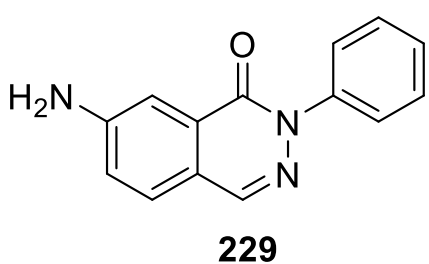

${ }^{1} \mathrm{H}$ NMR (500 MHz, pyridine- $\left.\mathrm{d}_{5}\right)$

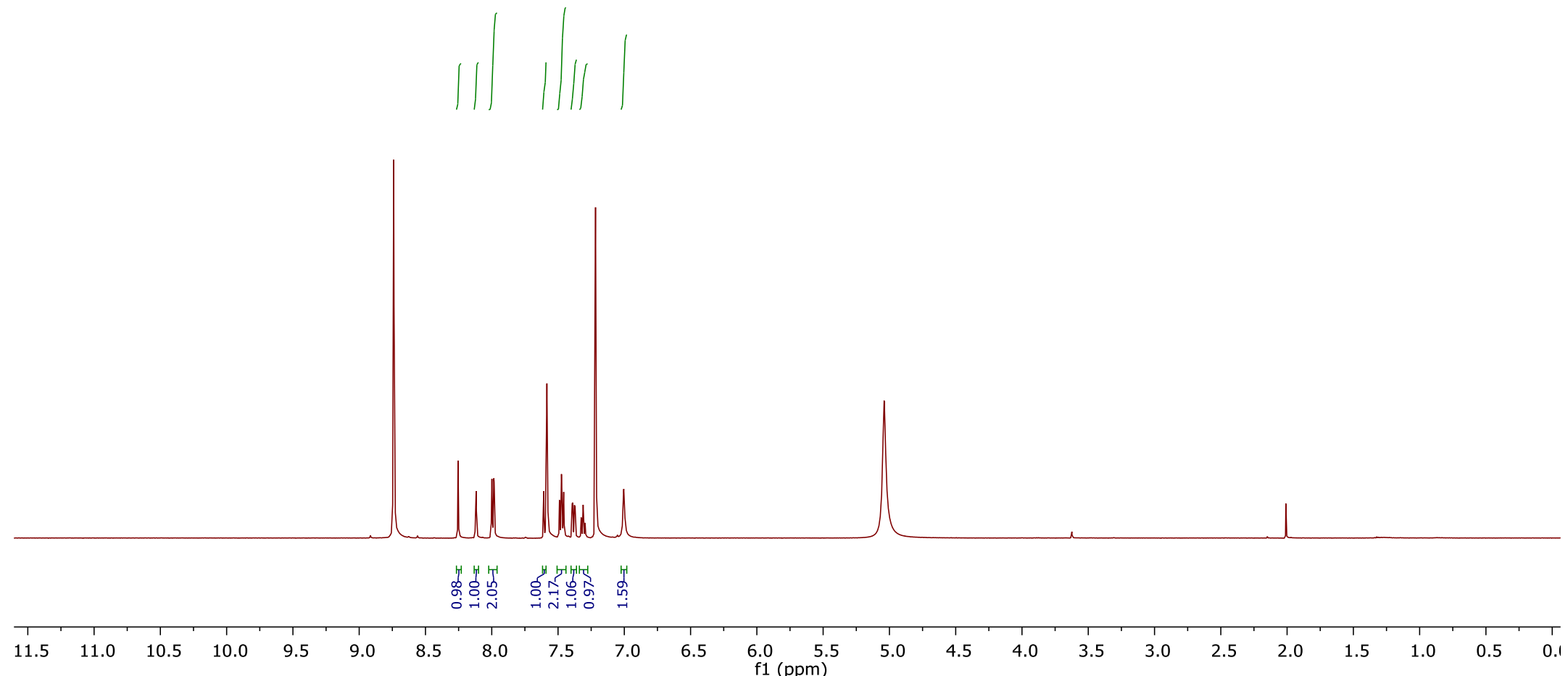



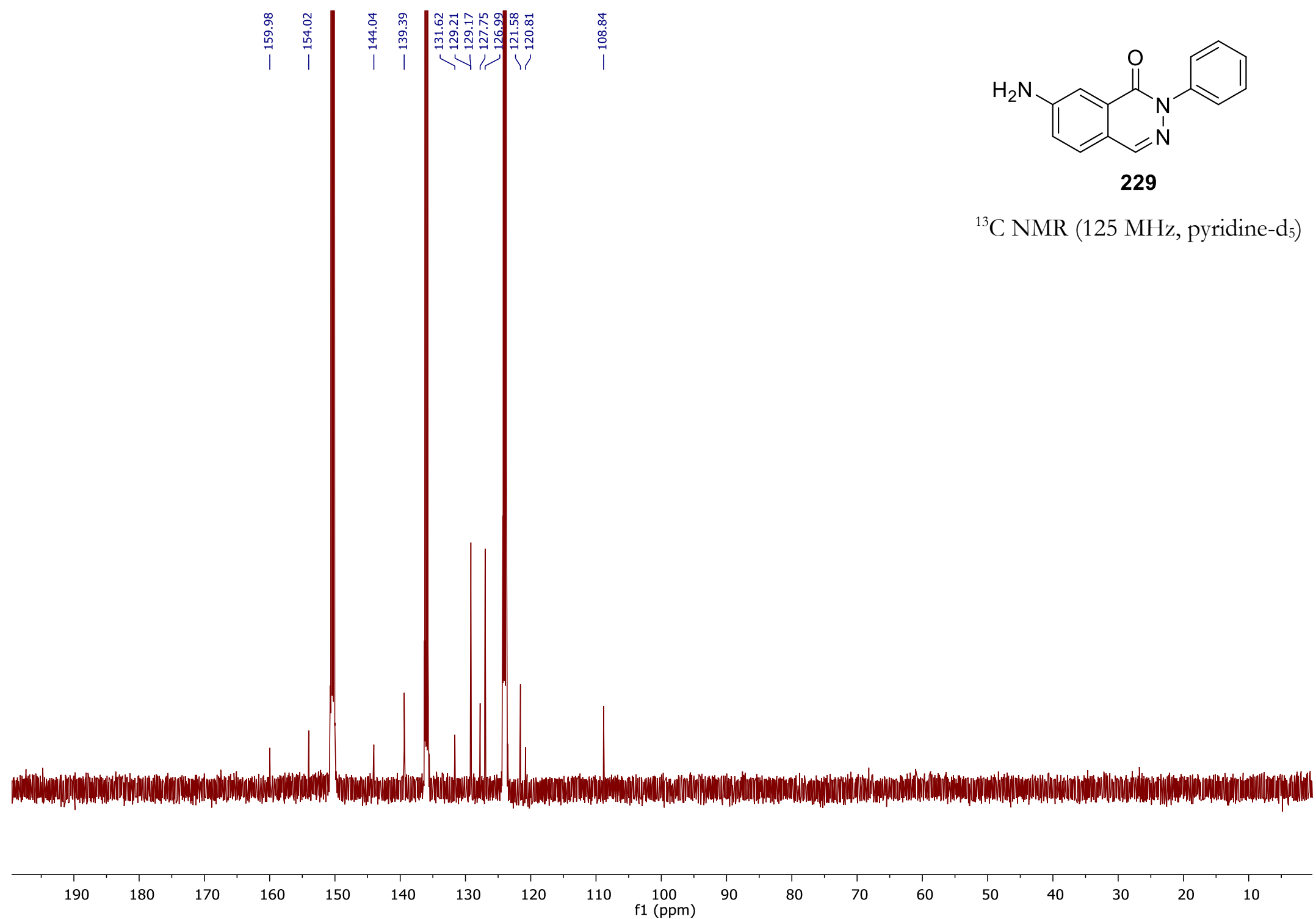


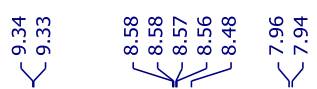

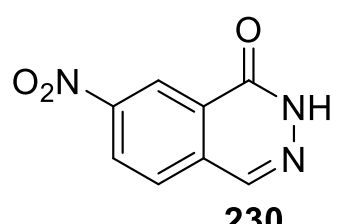

${ }^{1} \mathrm{H}$ NMR (500 MHz, pyridine- $\mathrm{d}_{5}$ )

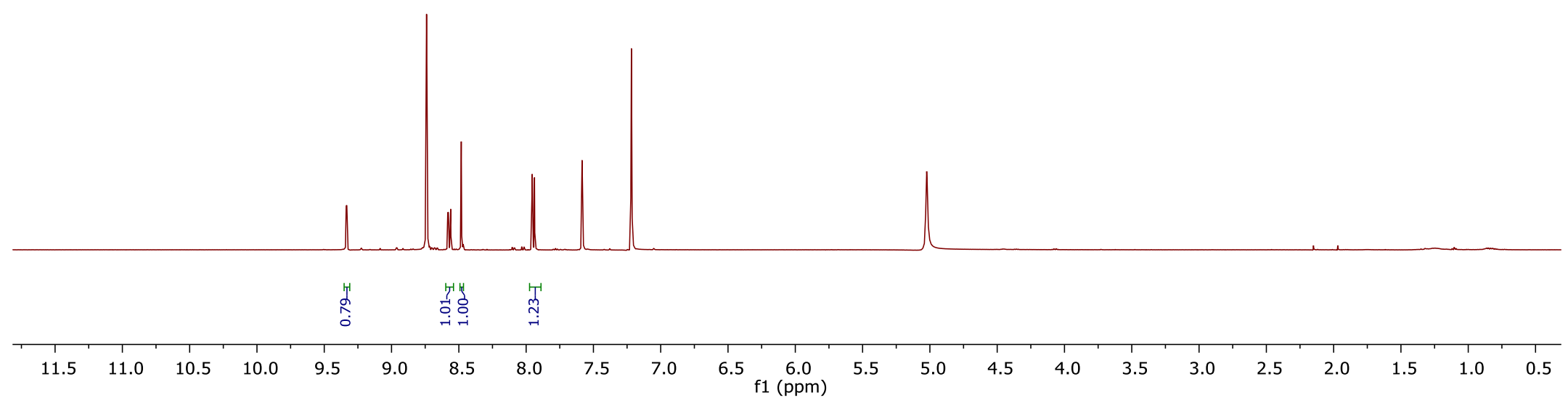



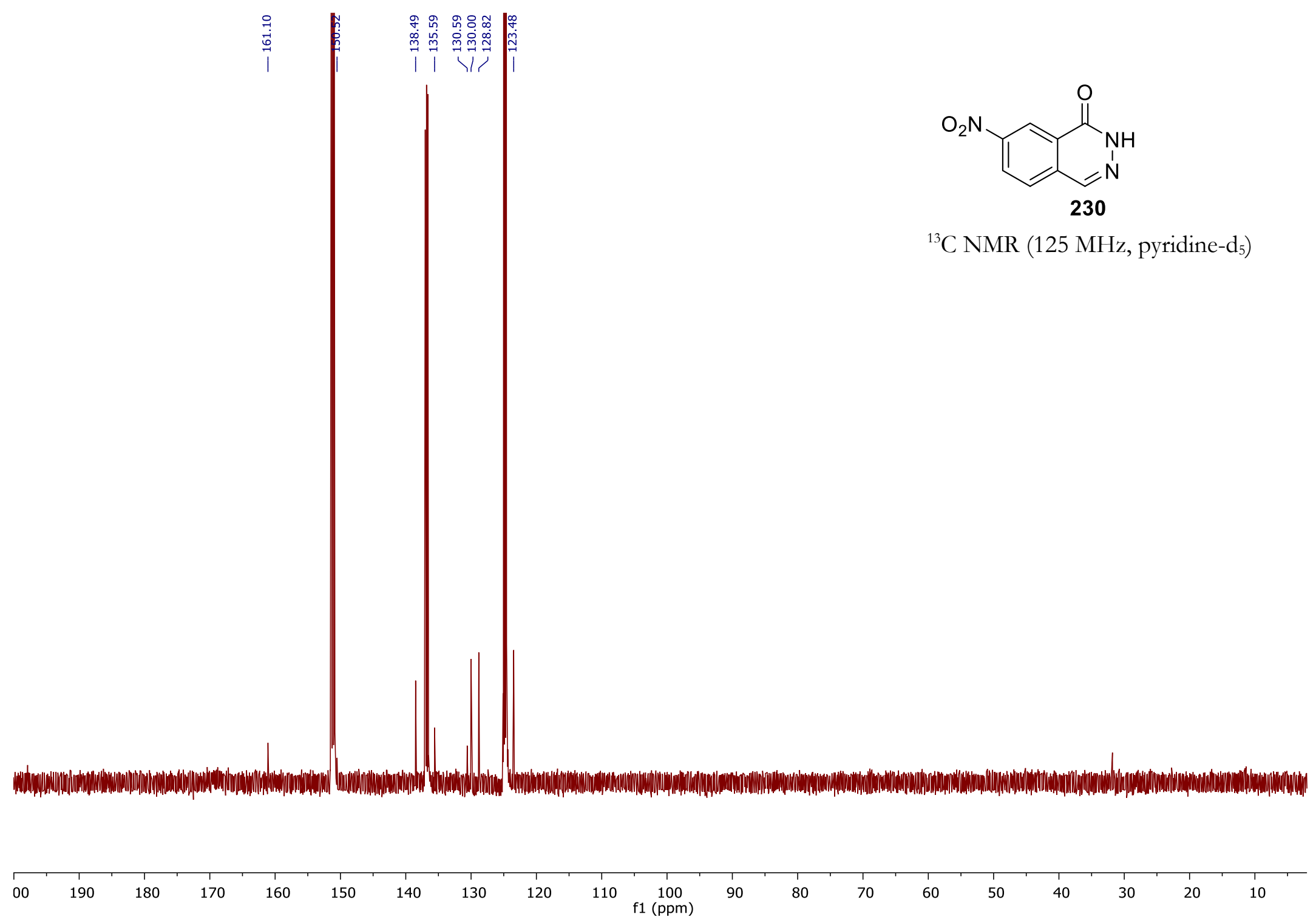


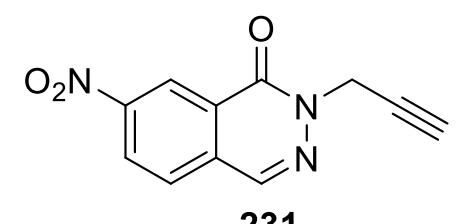

${ }^{1} \mathrm{H}$ NMR $\left(500 \mathrm{MHz}, \mathrm{CDCl}_{3}\right)$

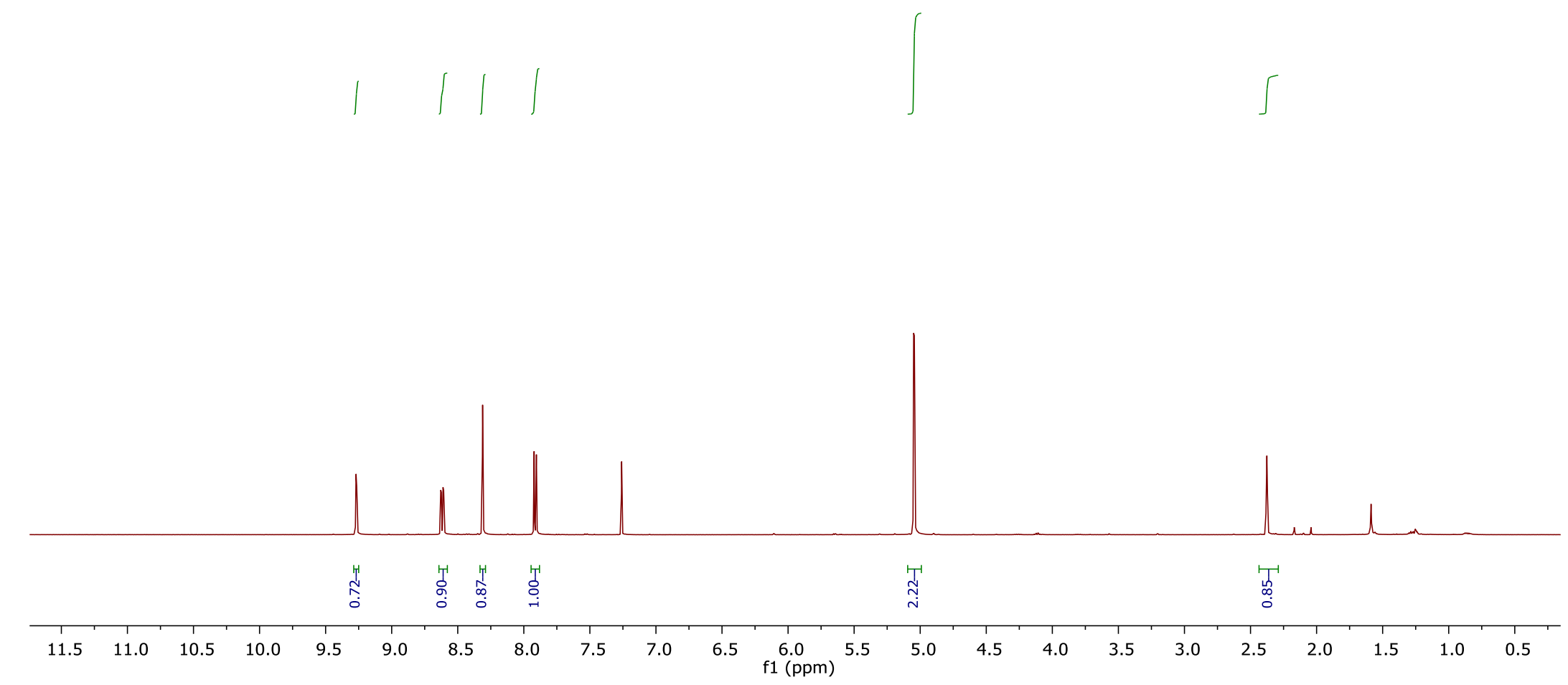




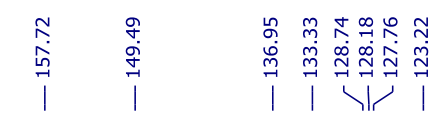

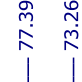

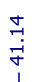

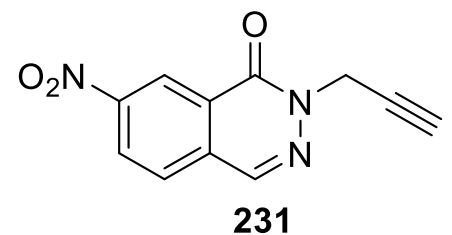

${ }^{13} \mathrm{C}$ NMR $\left(125 \mathrm{MHz}, \mathrm{CDCl}_{3}\right)$

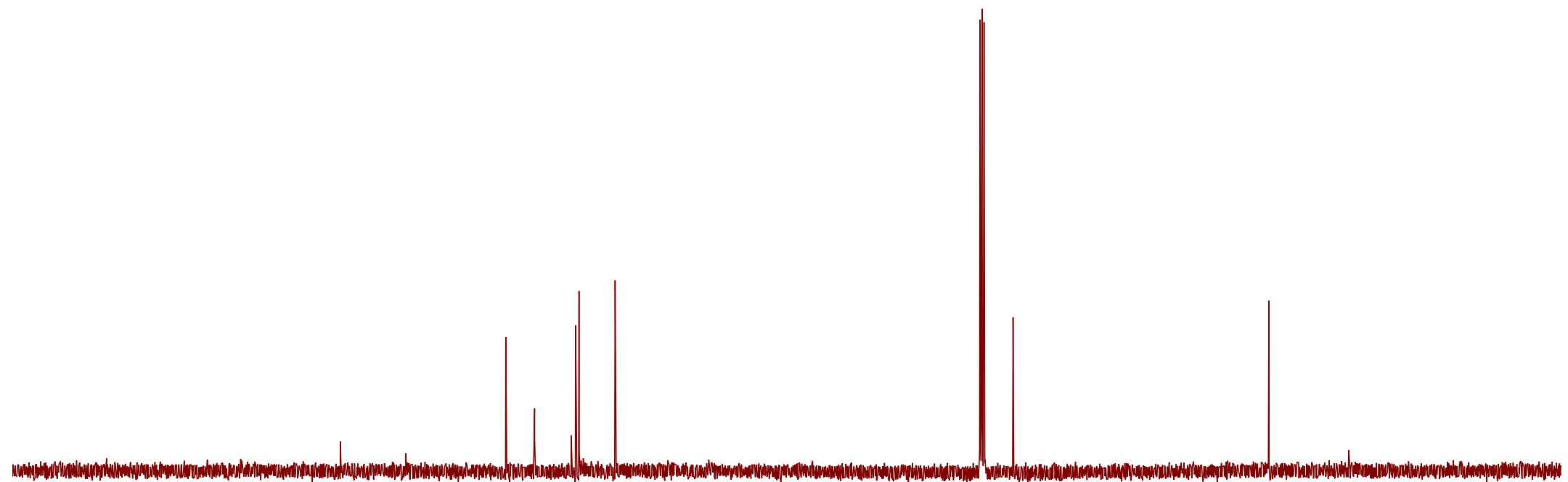




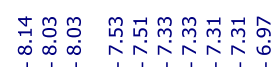

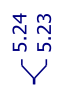

ָֻׁ

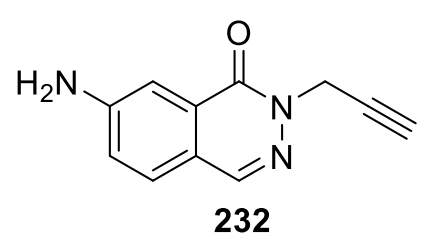

${ }^{1} \mathrm{H}$ NMR (500 MHz, pyridine- $\mathrm{d}_{5}$ )

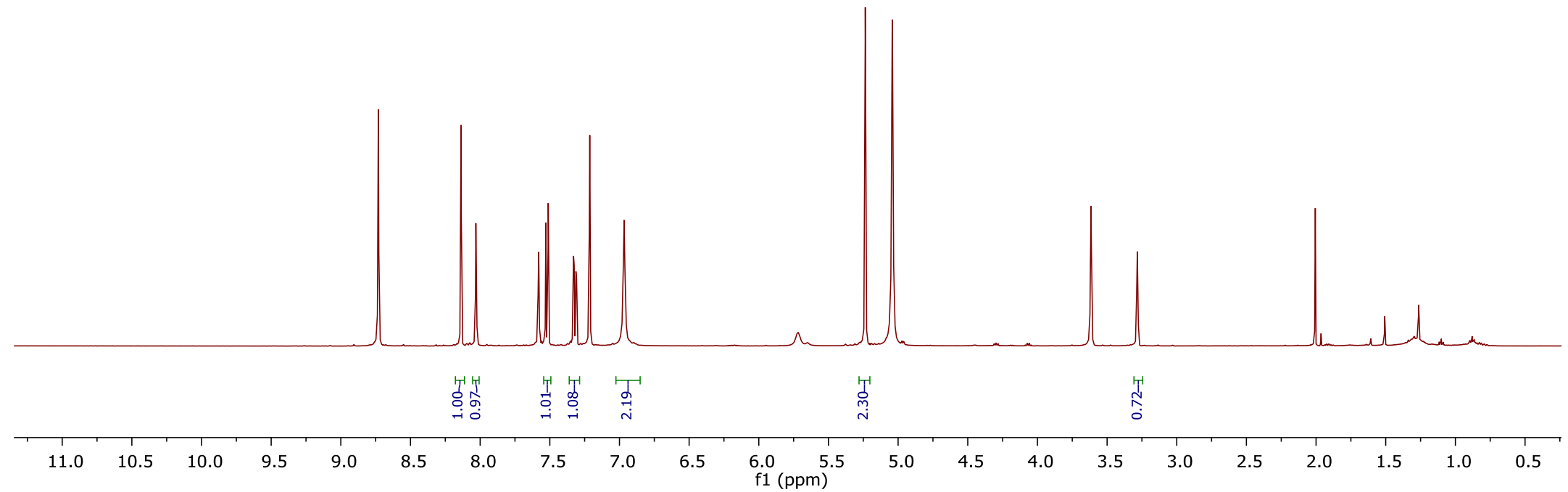




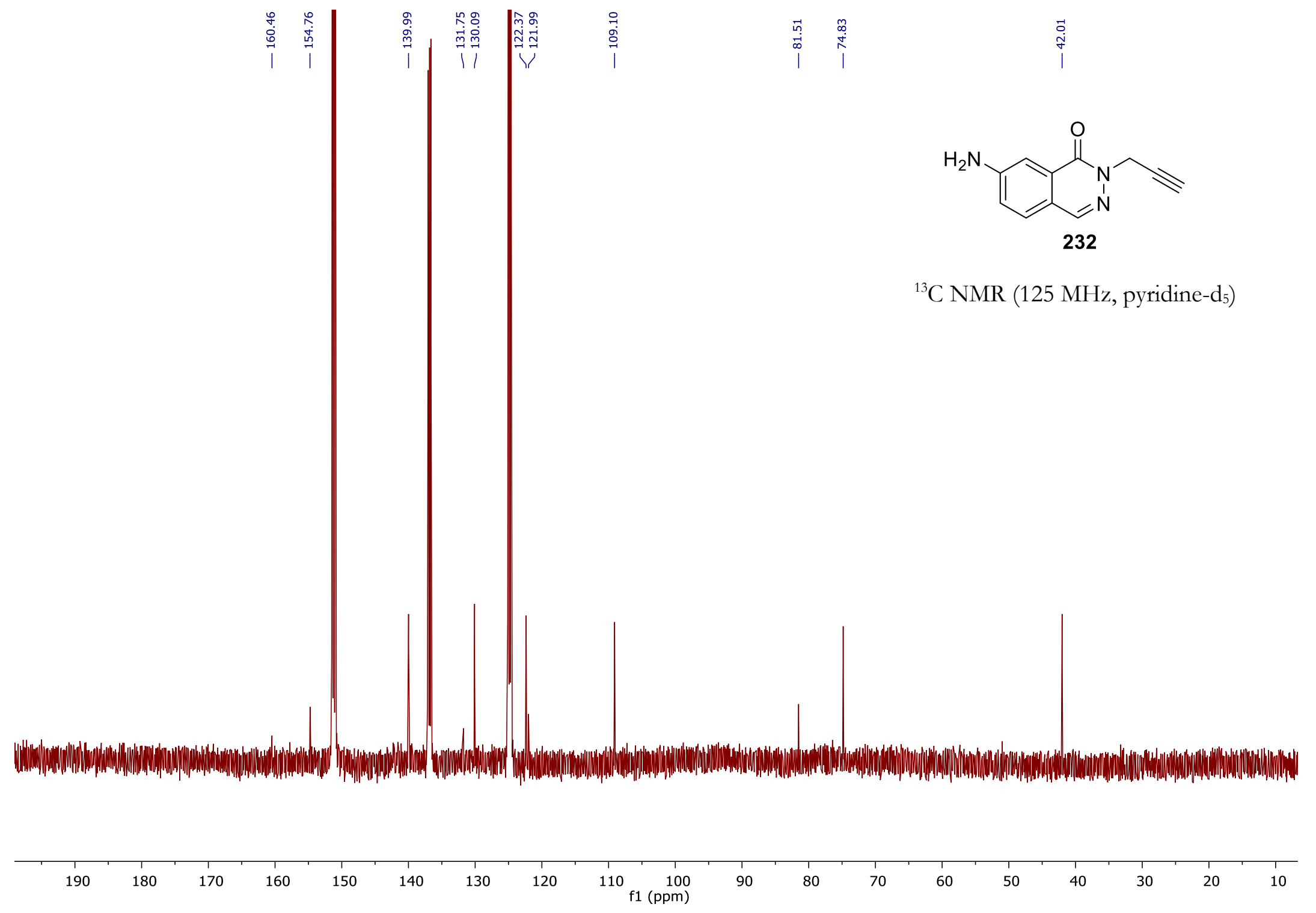




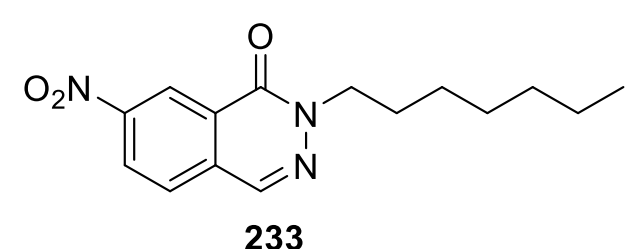

${ }^{1} \mathrm{H}$ NMR $\left(500 \mathrm{MHz}, \mathrm{CDCl}_{3}\right)$

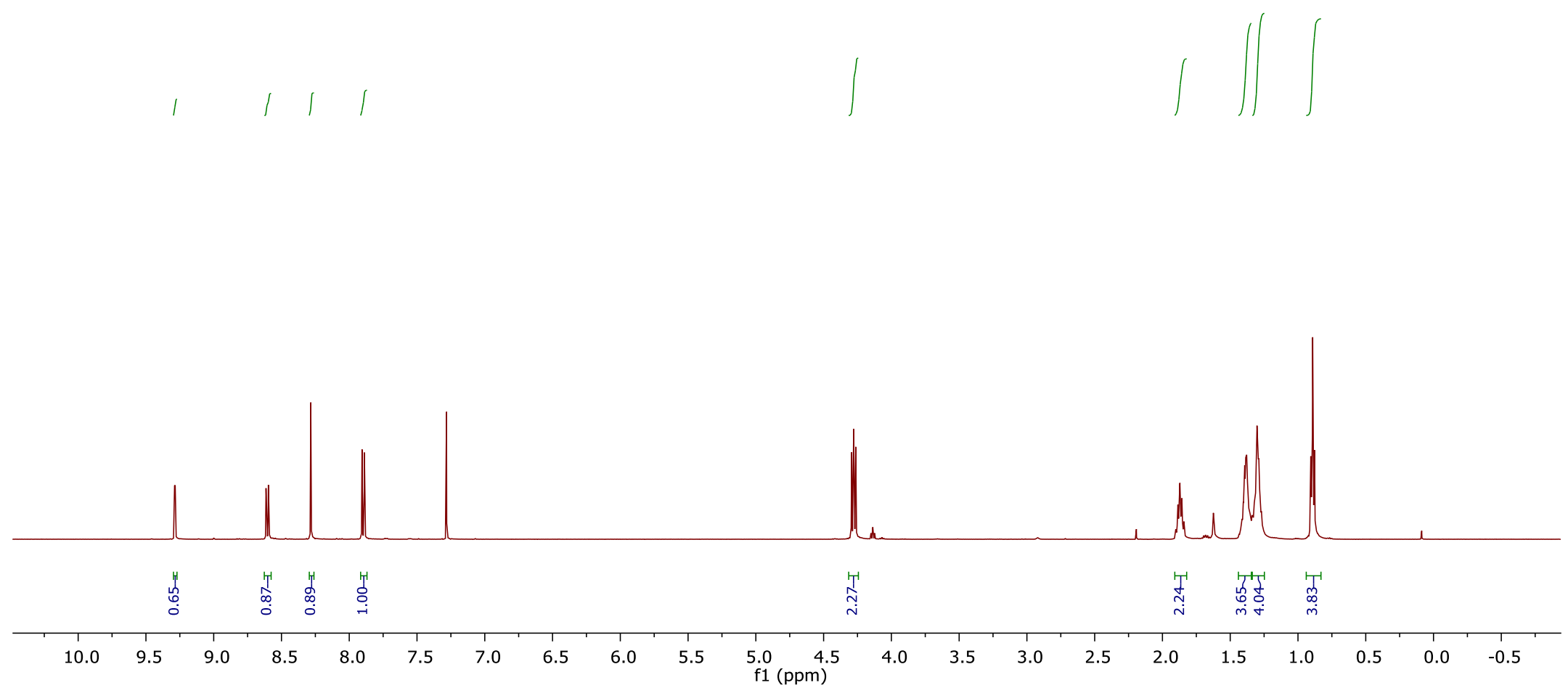




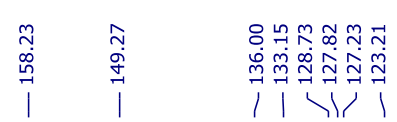

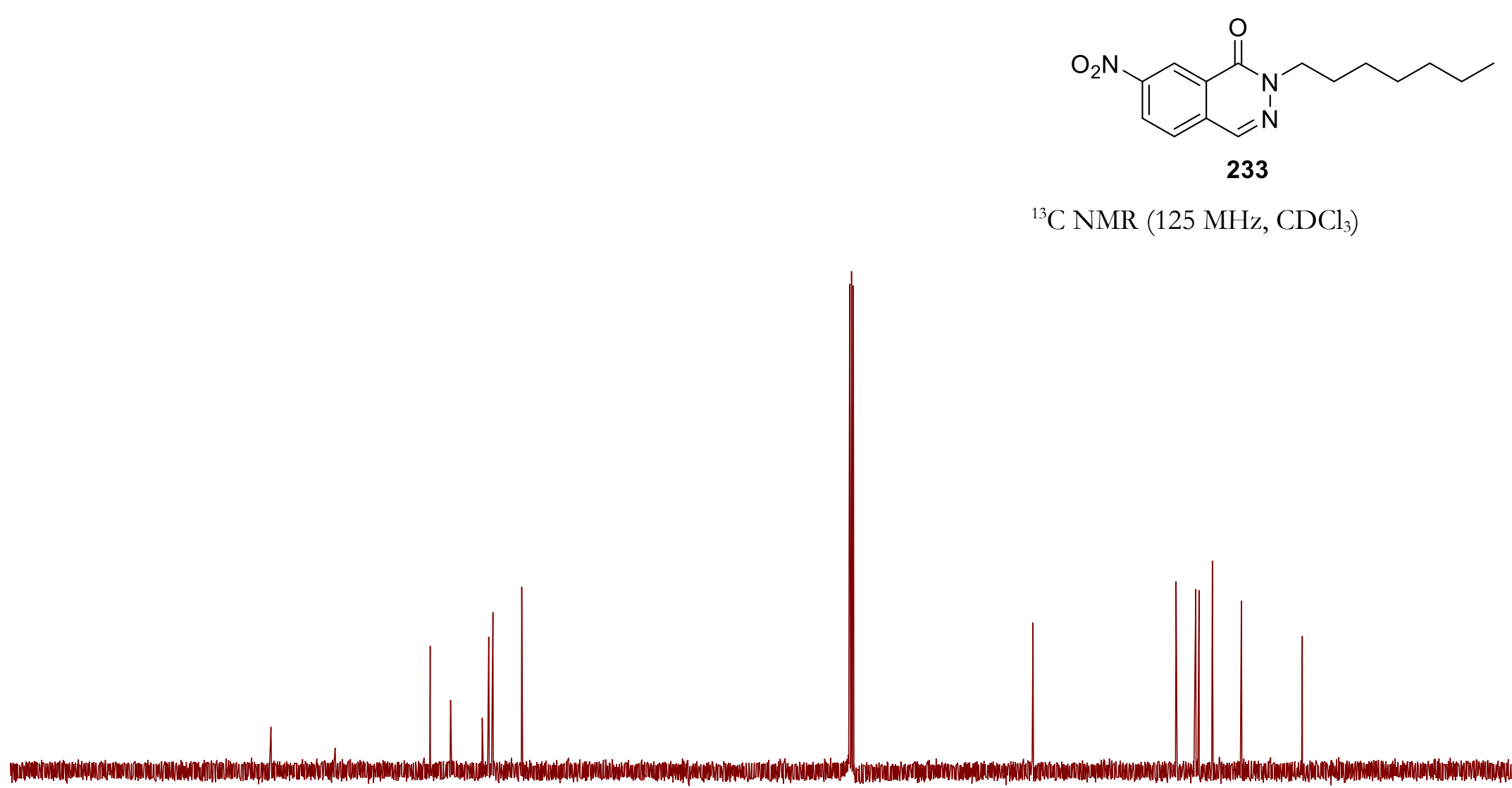

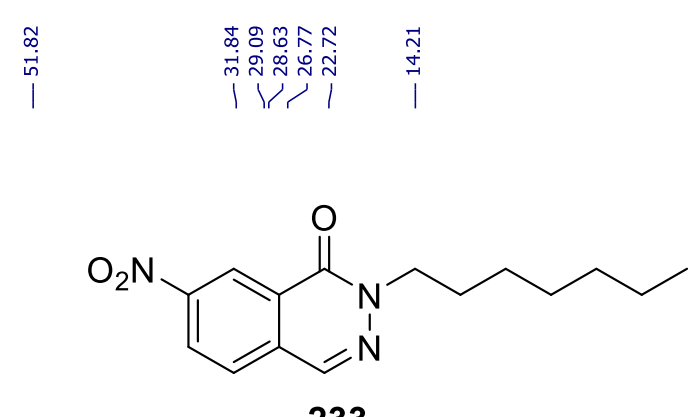

${ }^{13} \mathrm{C}$ NMR $\left(125 \mathrm{MHz}, \mathrm{CDCl}_{3}\right)$ 


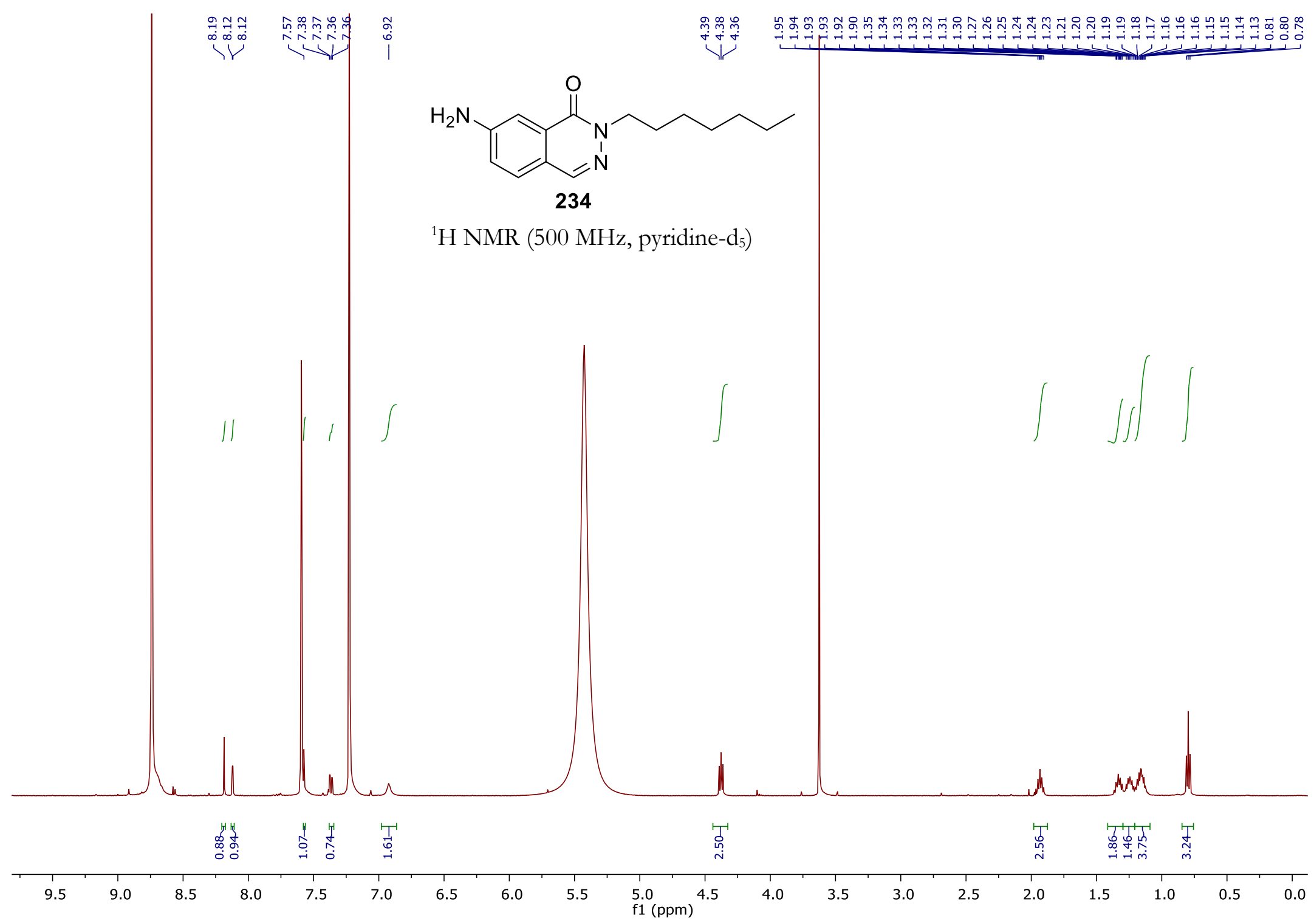




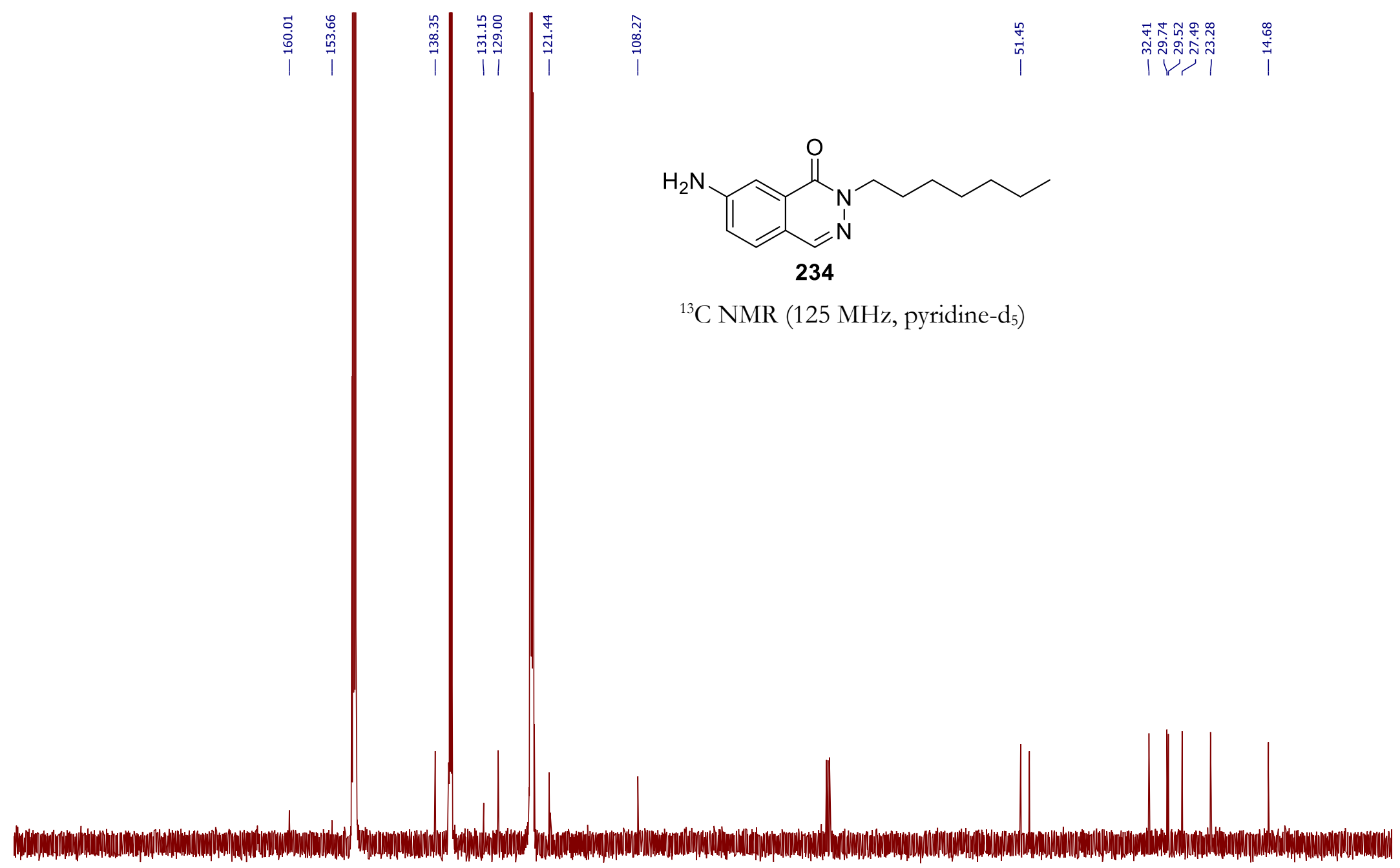

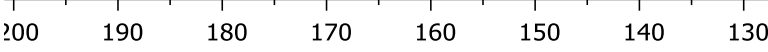

120

10090

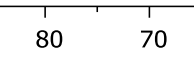

$60 \quad 50$

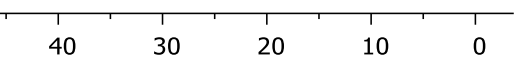




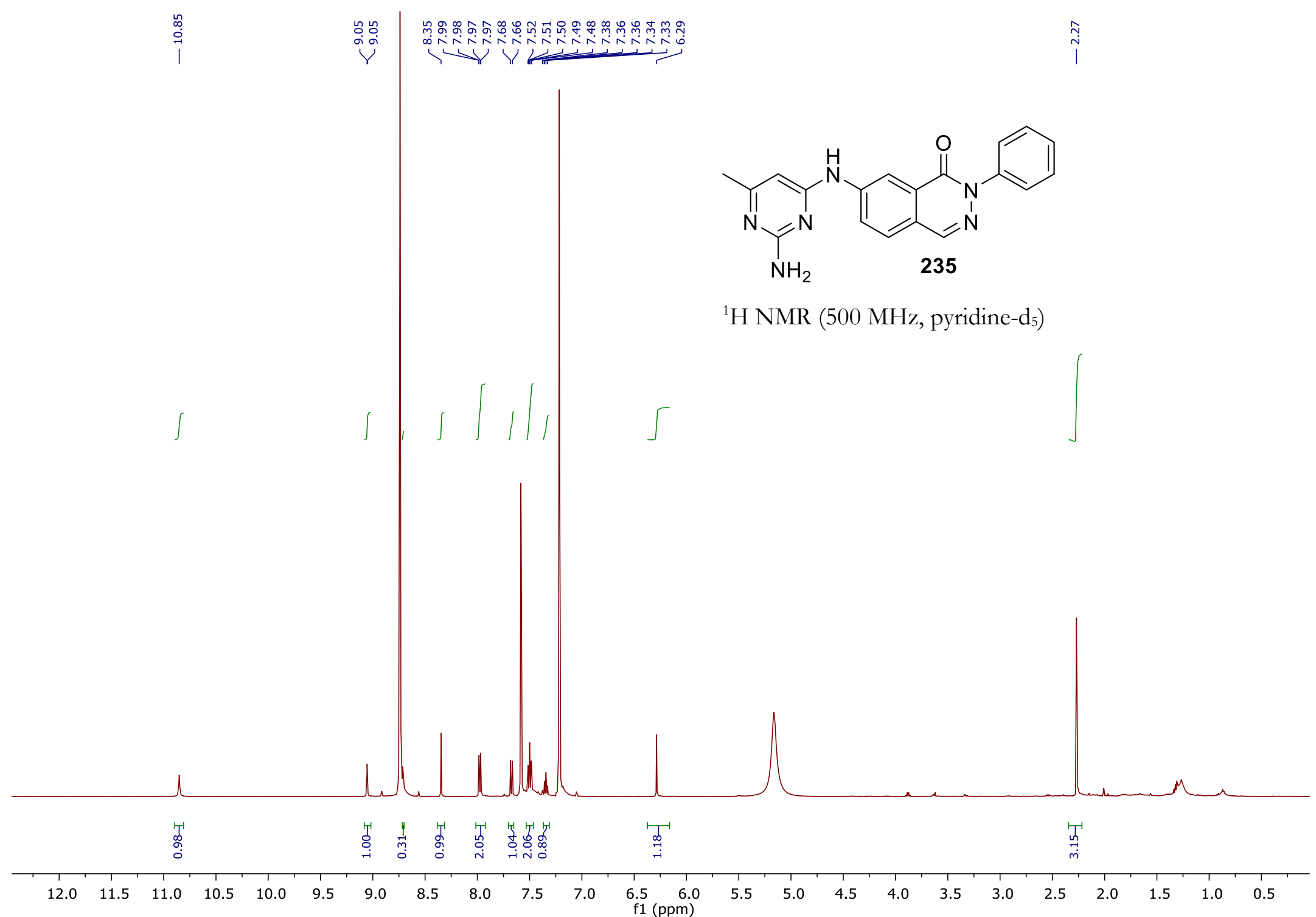




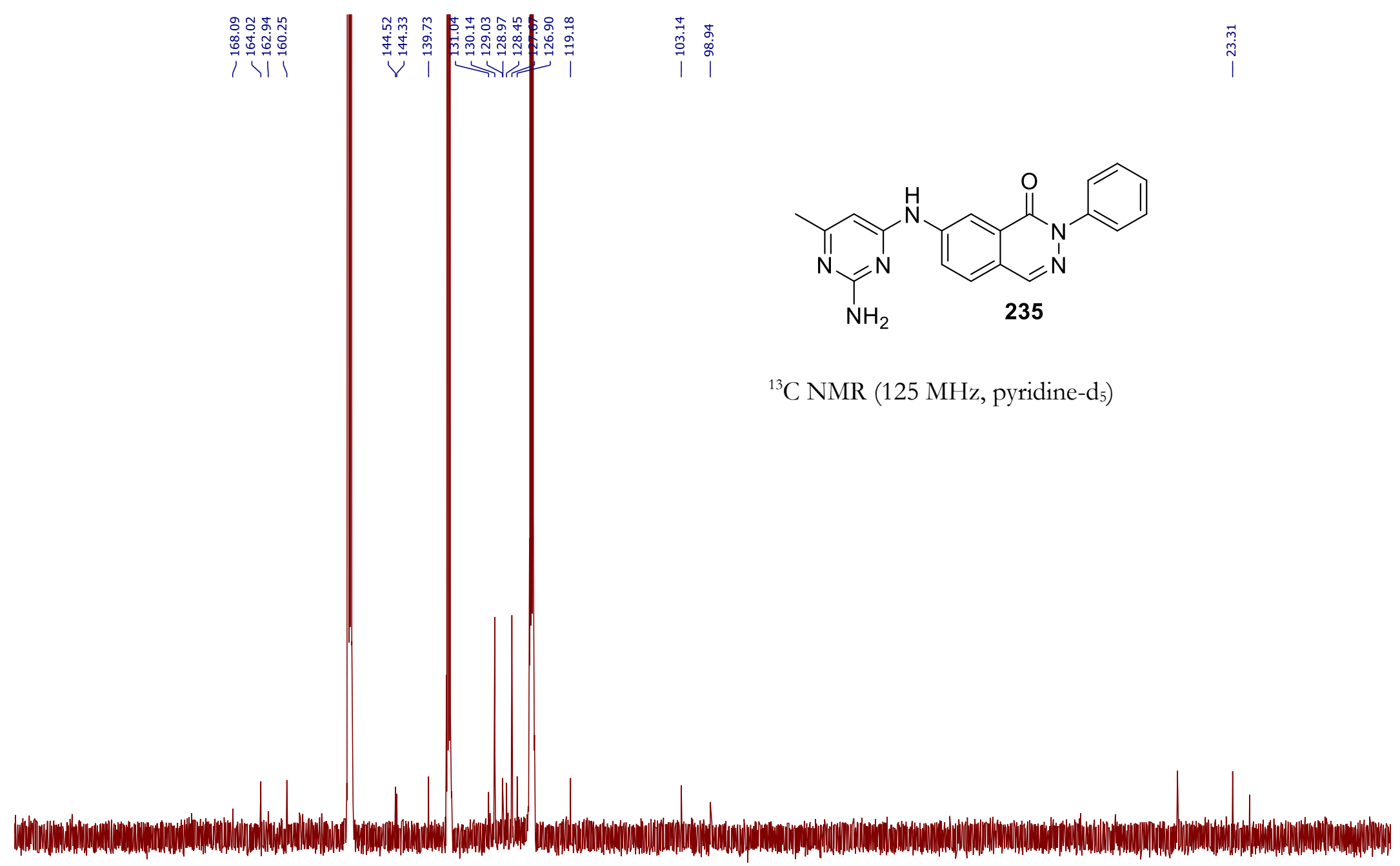

$190 \quad 180$

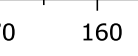

150

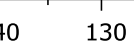

120

$110 \quad 100$

$90 \quad 80$

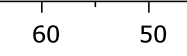

40

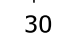

20 


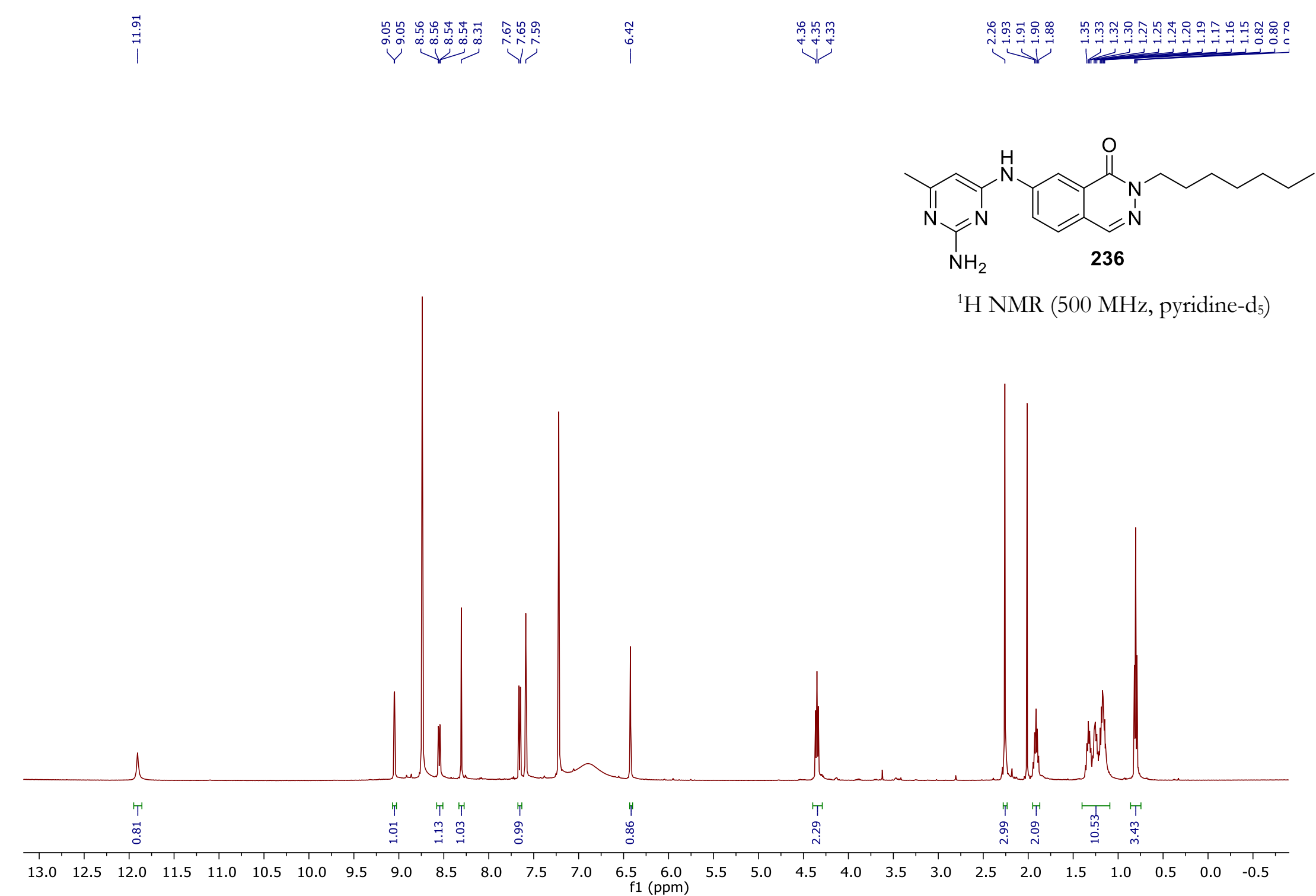




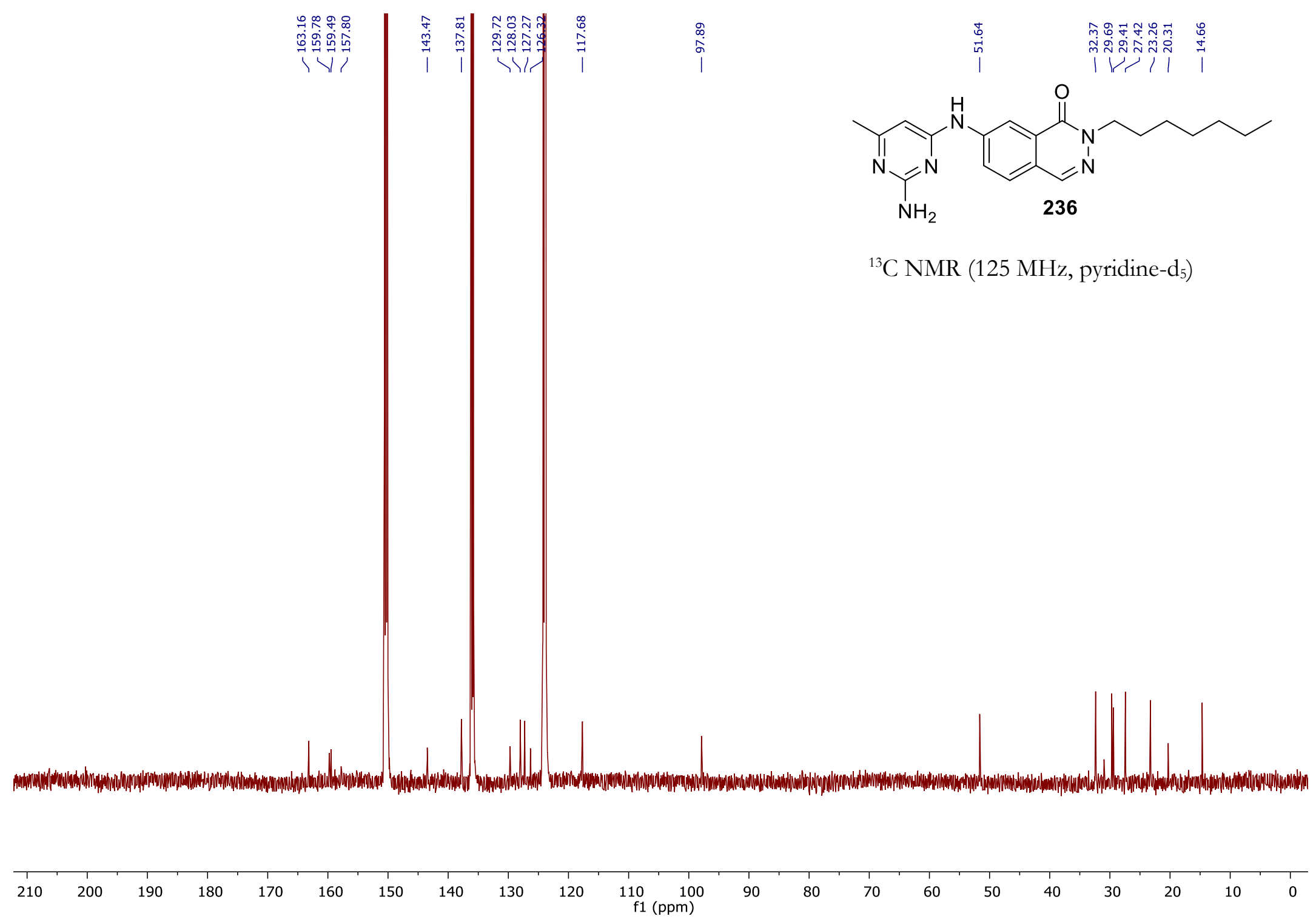




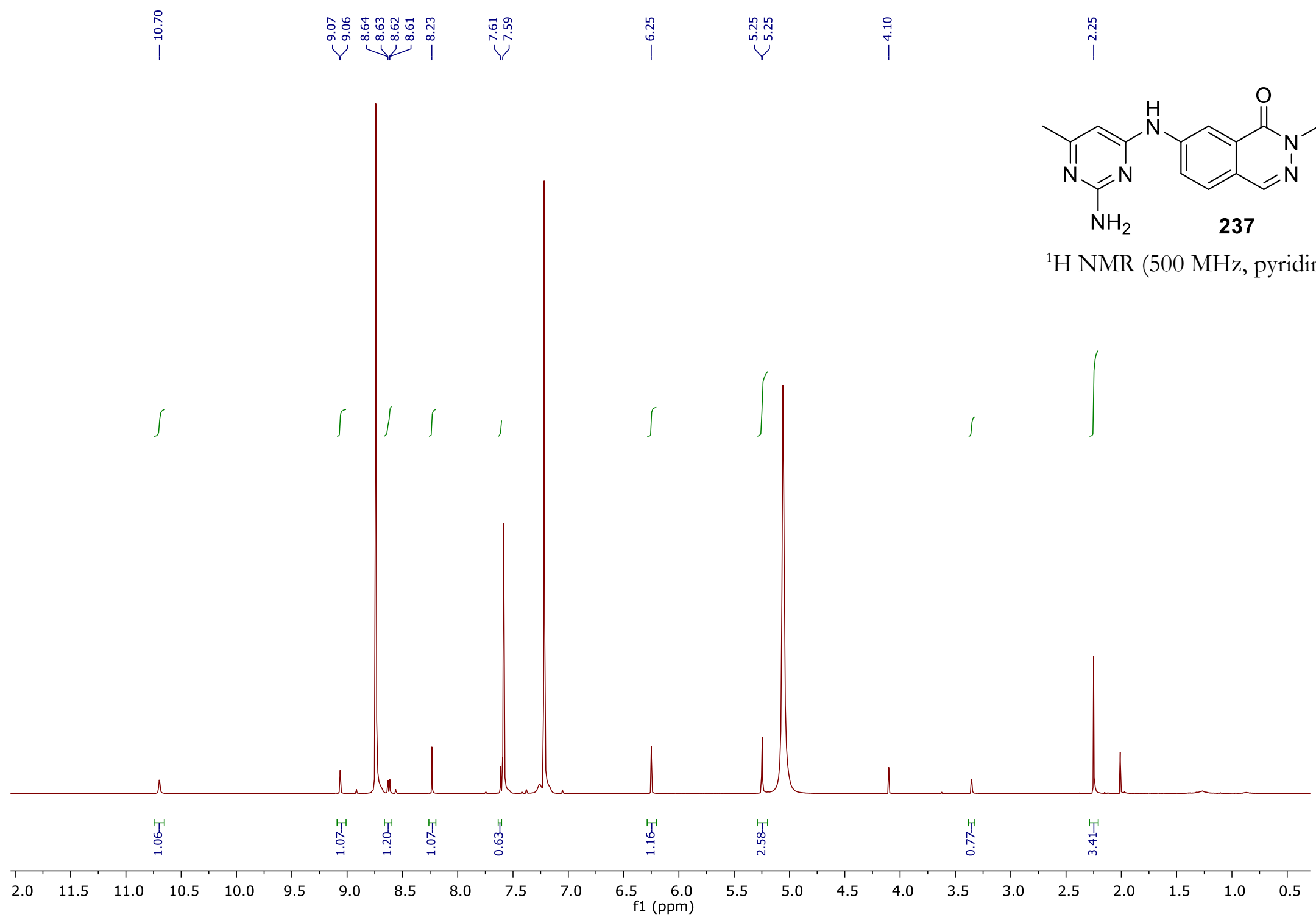




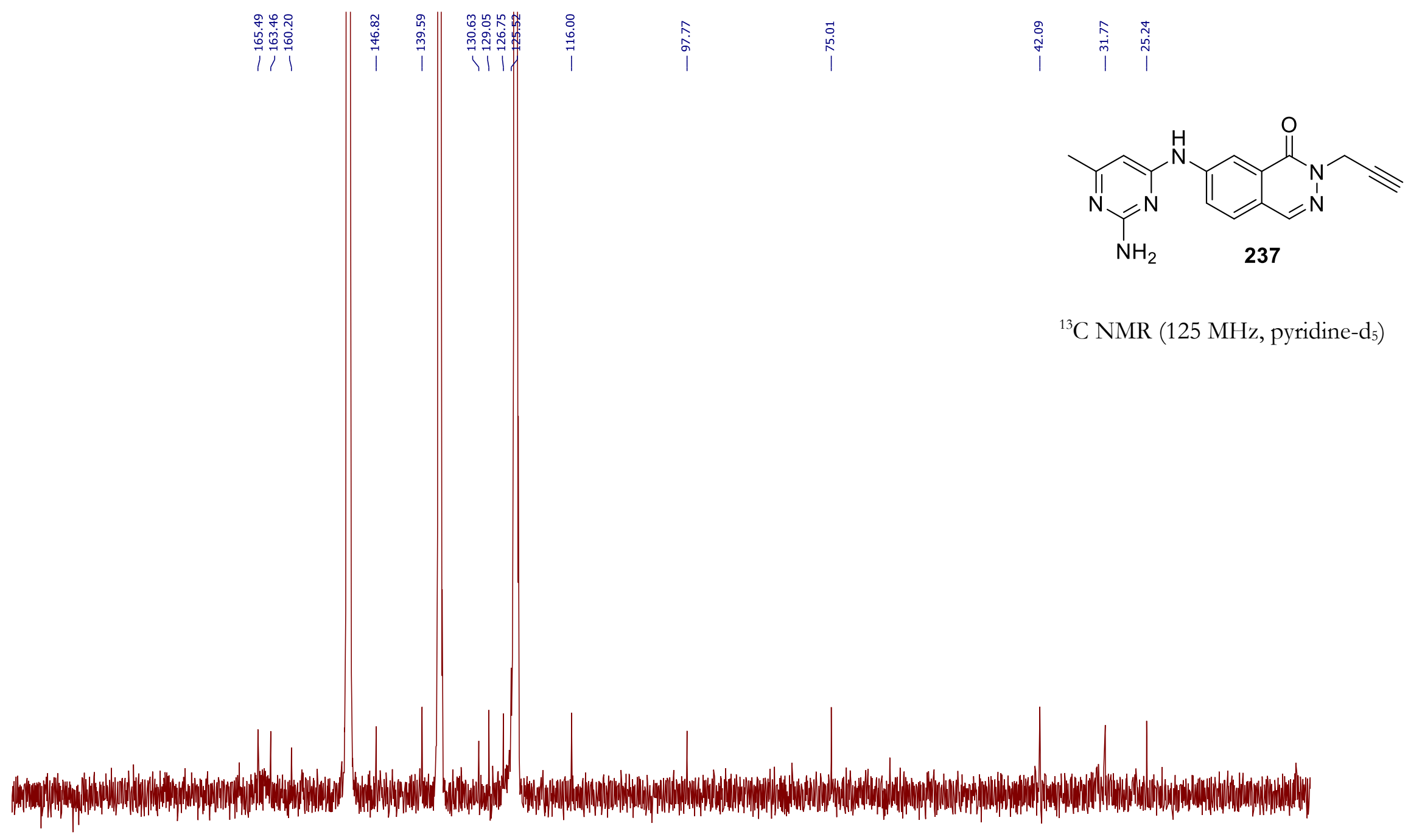

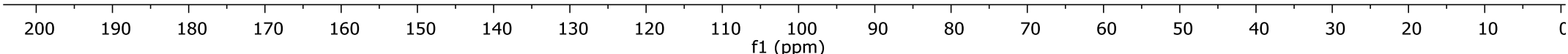



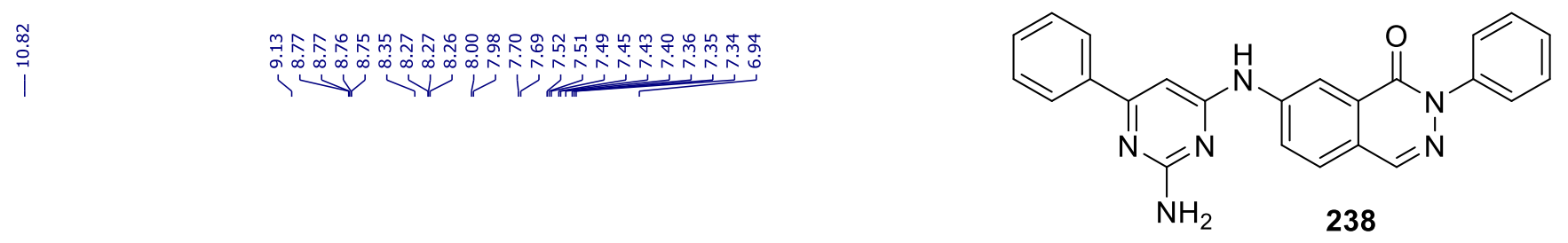

${ }^{1} \mathrm{H}$ NMR (500 MHz, pyridine- $\mathrm{d}_{5}$ )

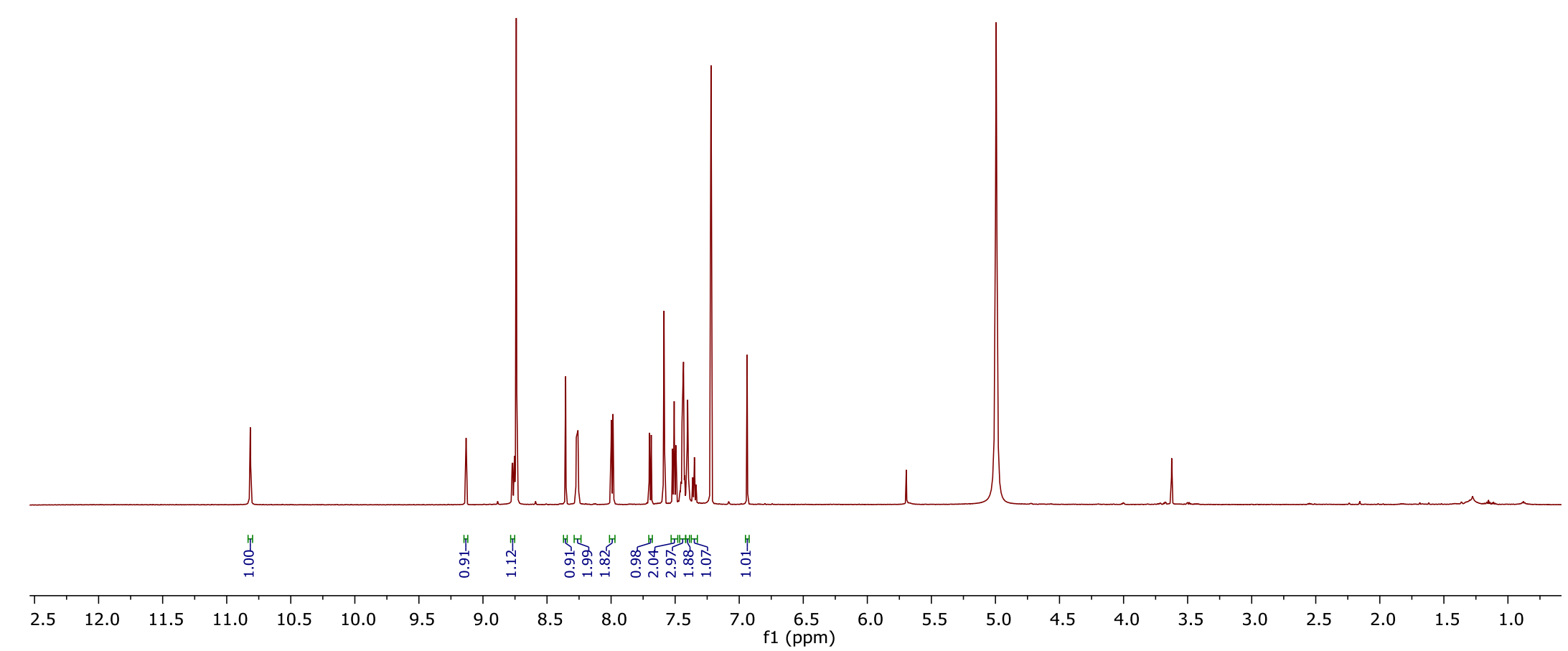




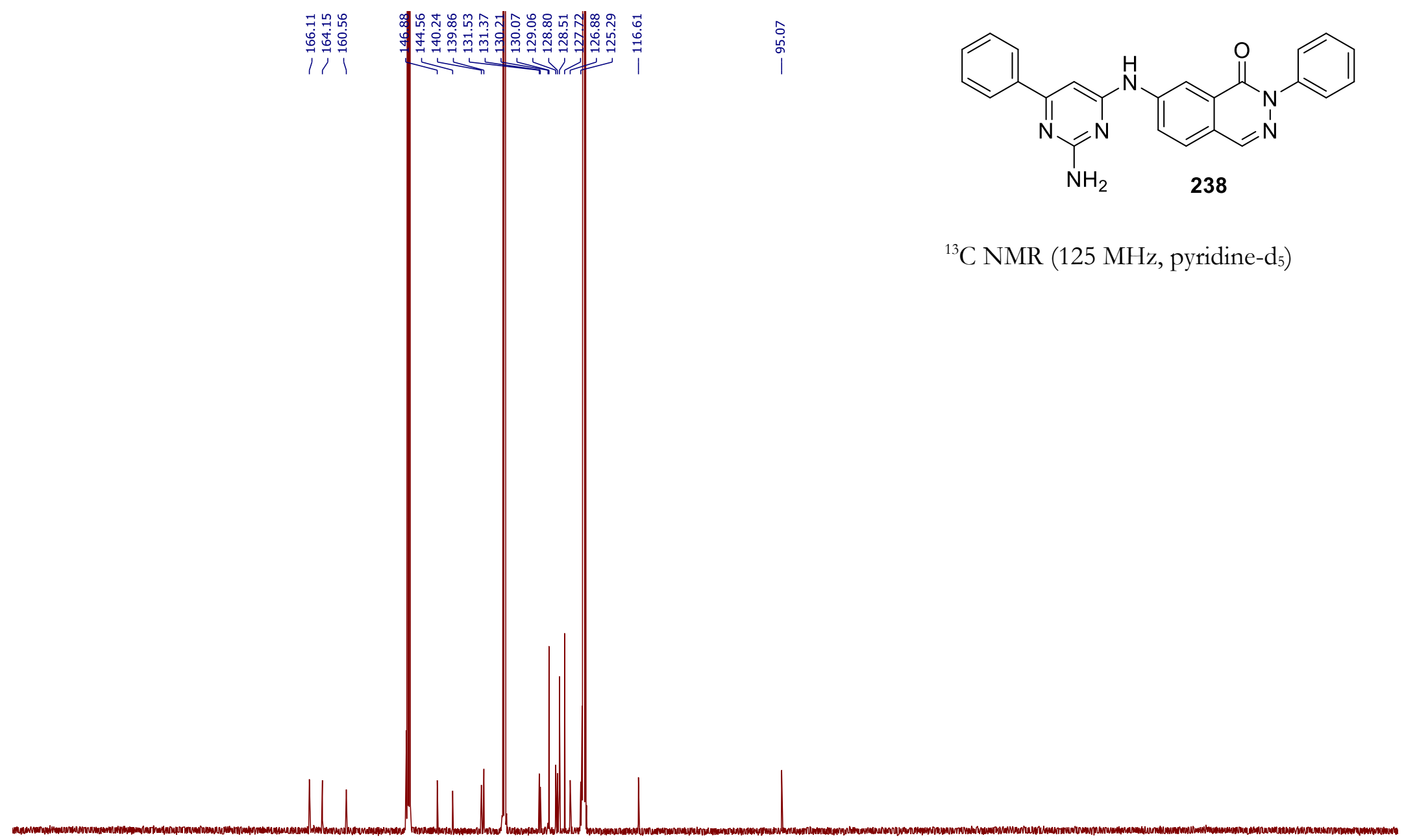

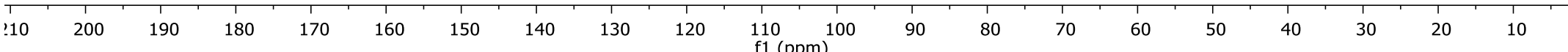




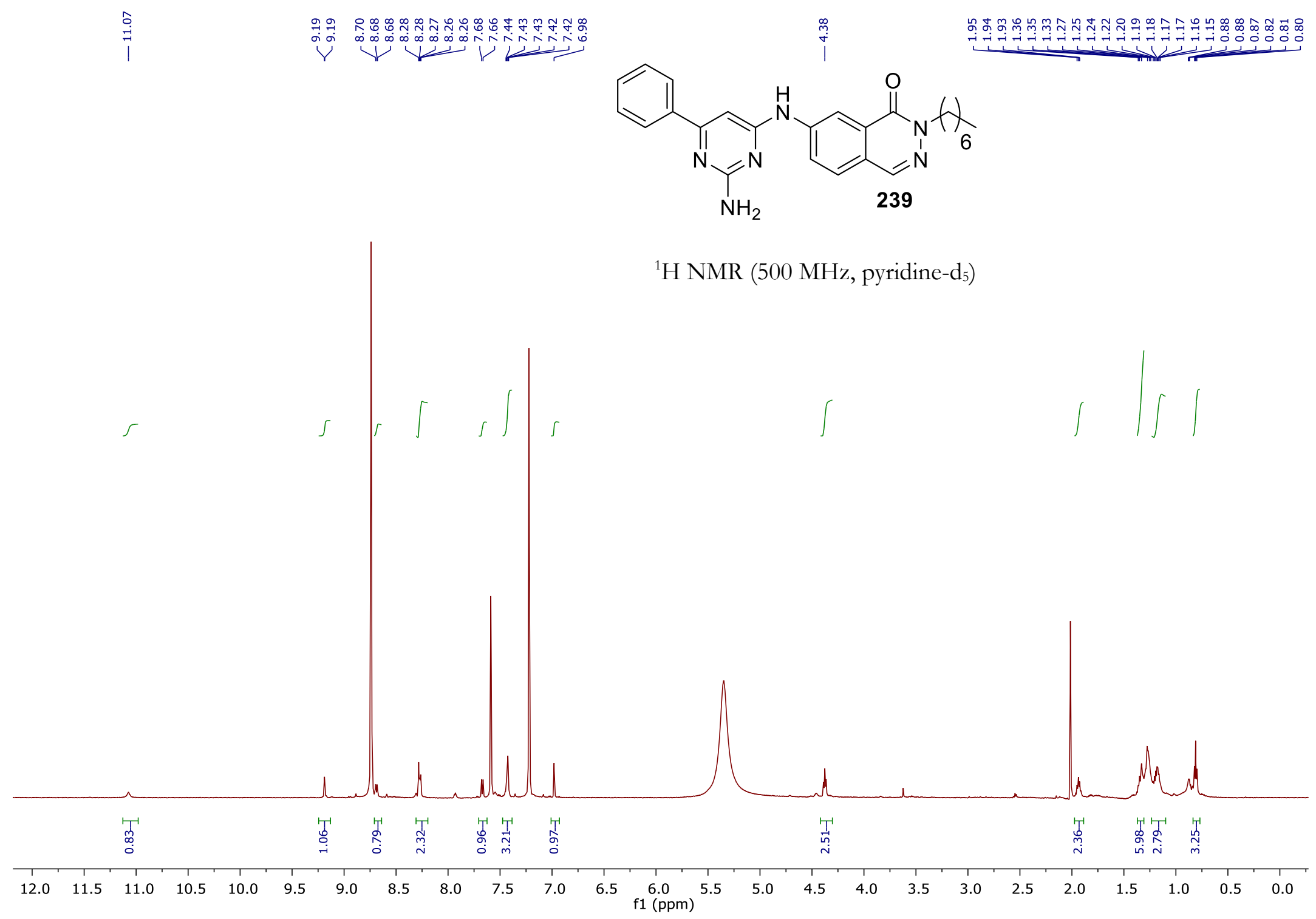




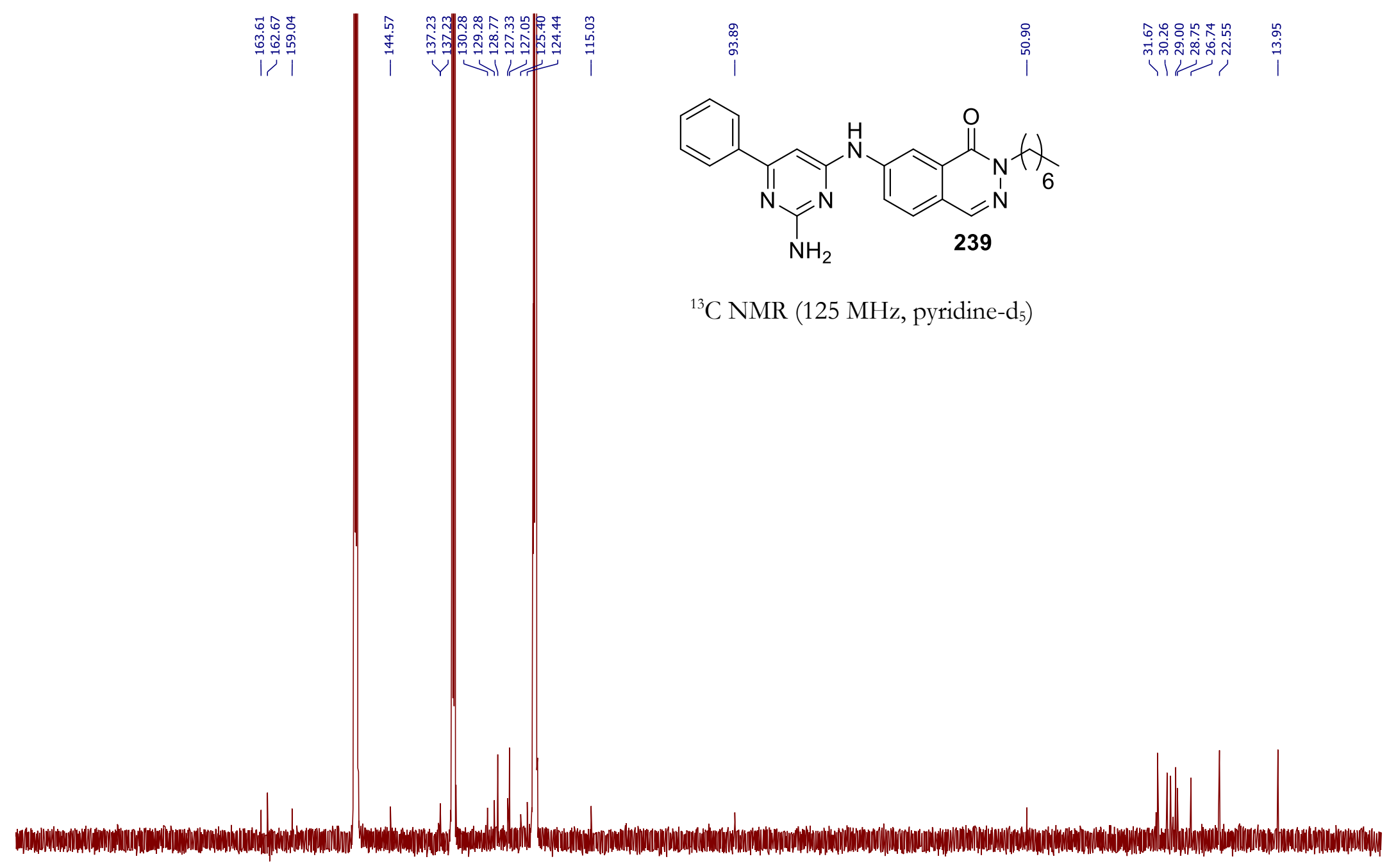

$\begin{array}{llll}190 & 180 & 170 & 160\end{array}$

$150 \quad 140$

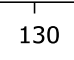

120

10100

100
$\mathrm{f} 1(\mathrm{ppm})$$\quad 90 \quad 80$

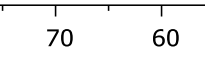




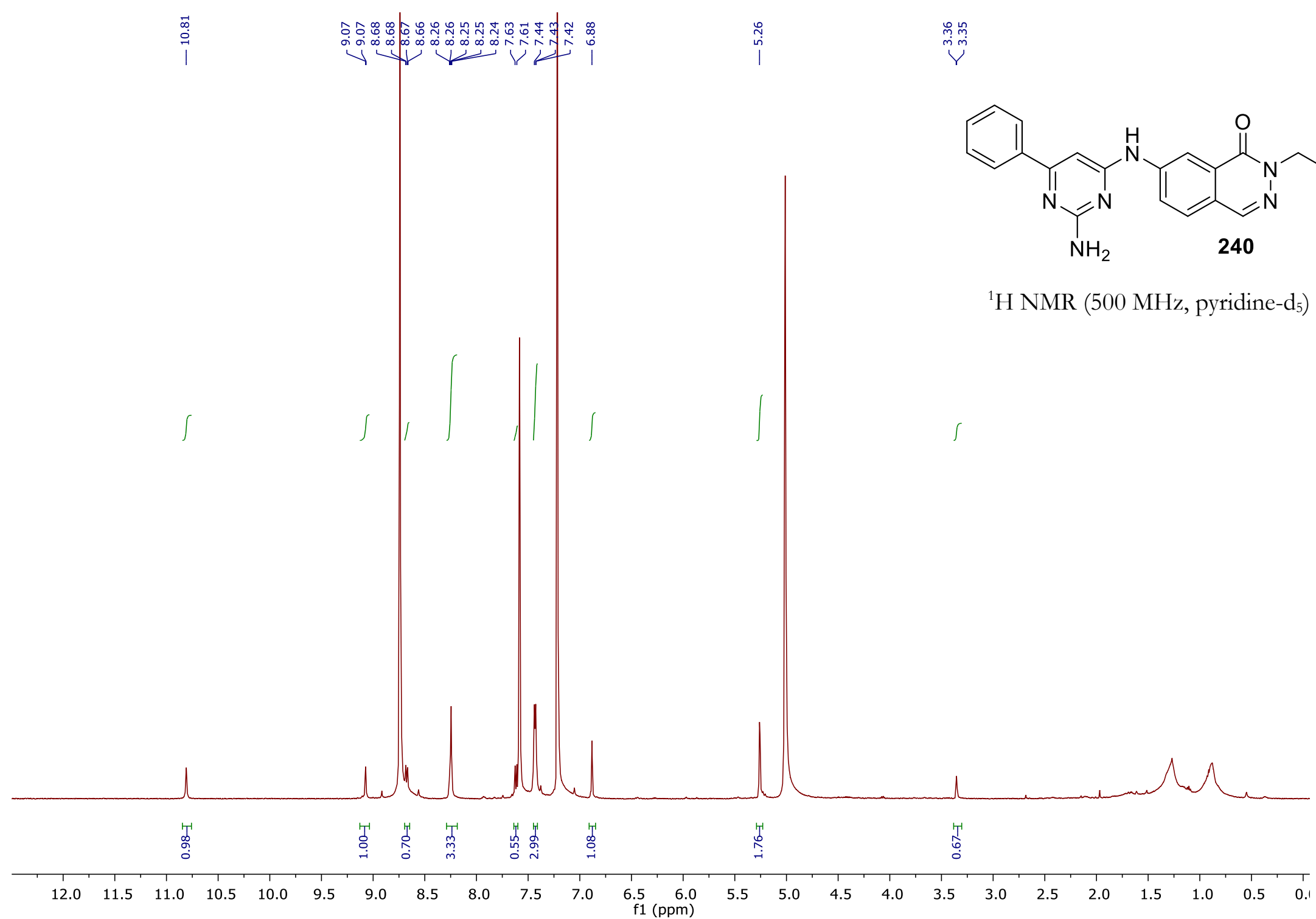




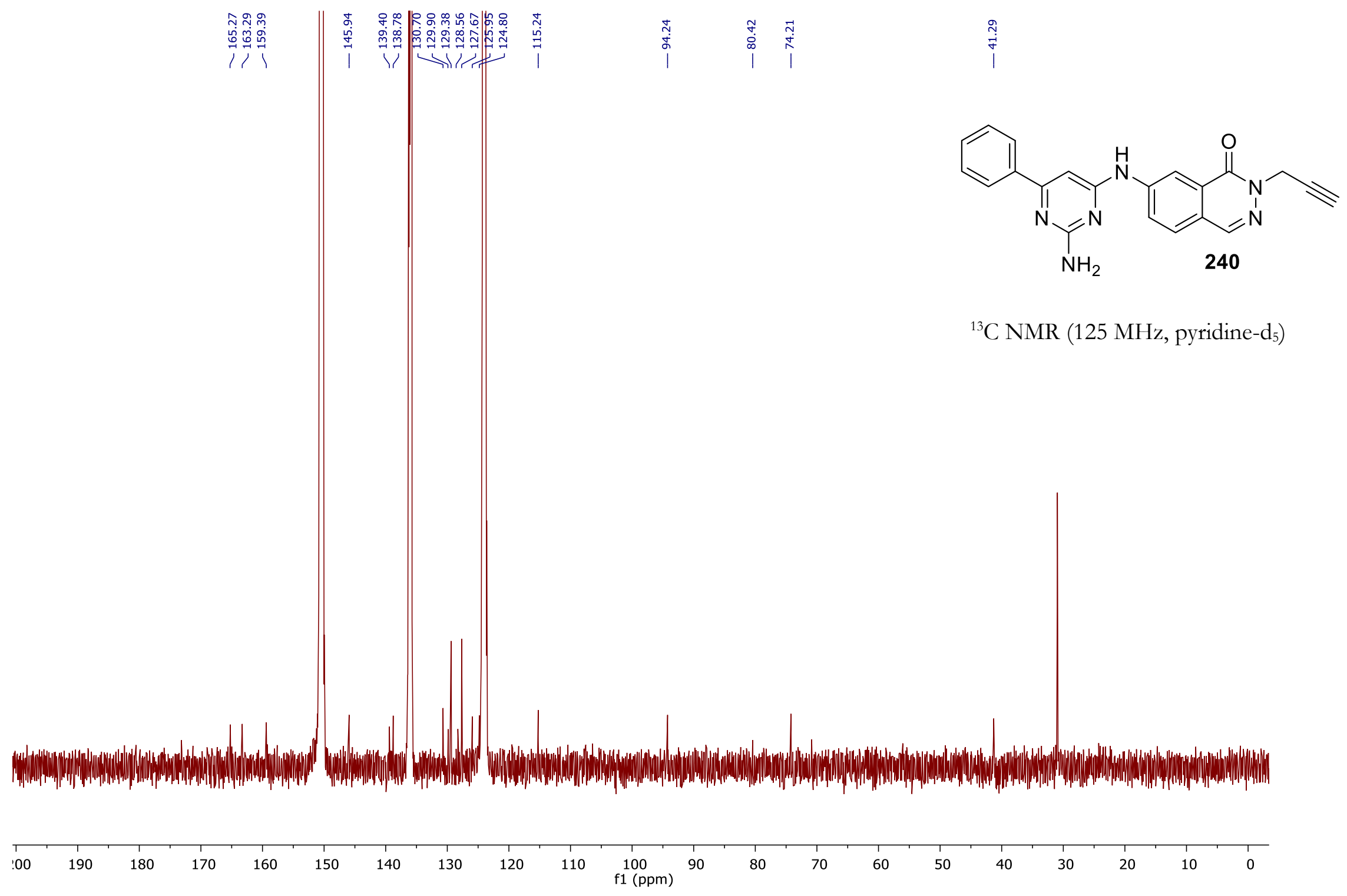




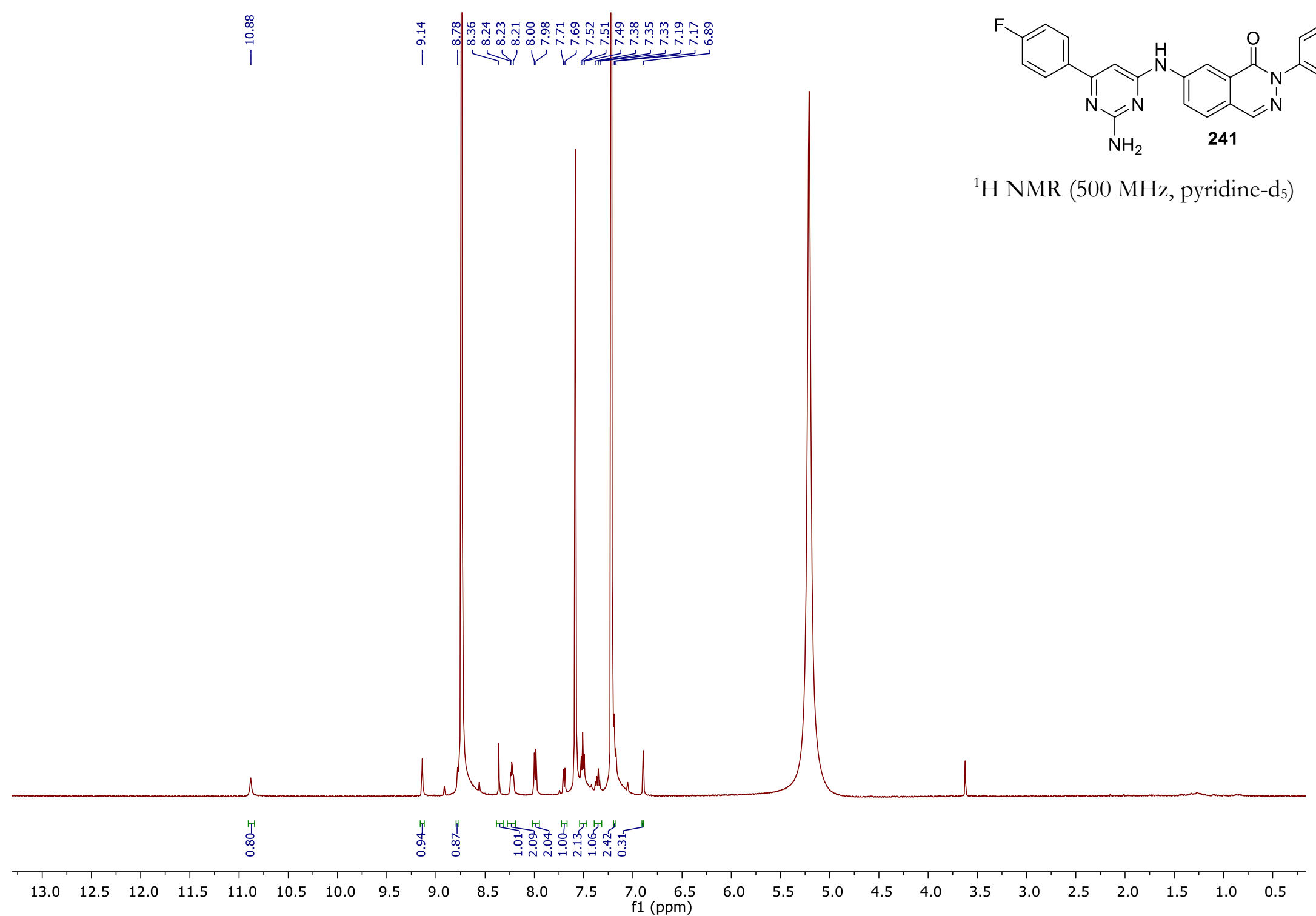




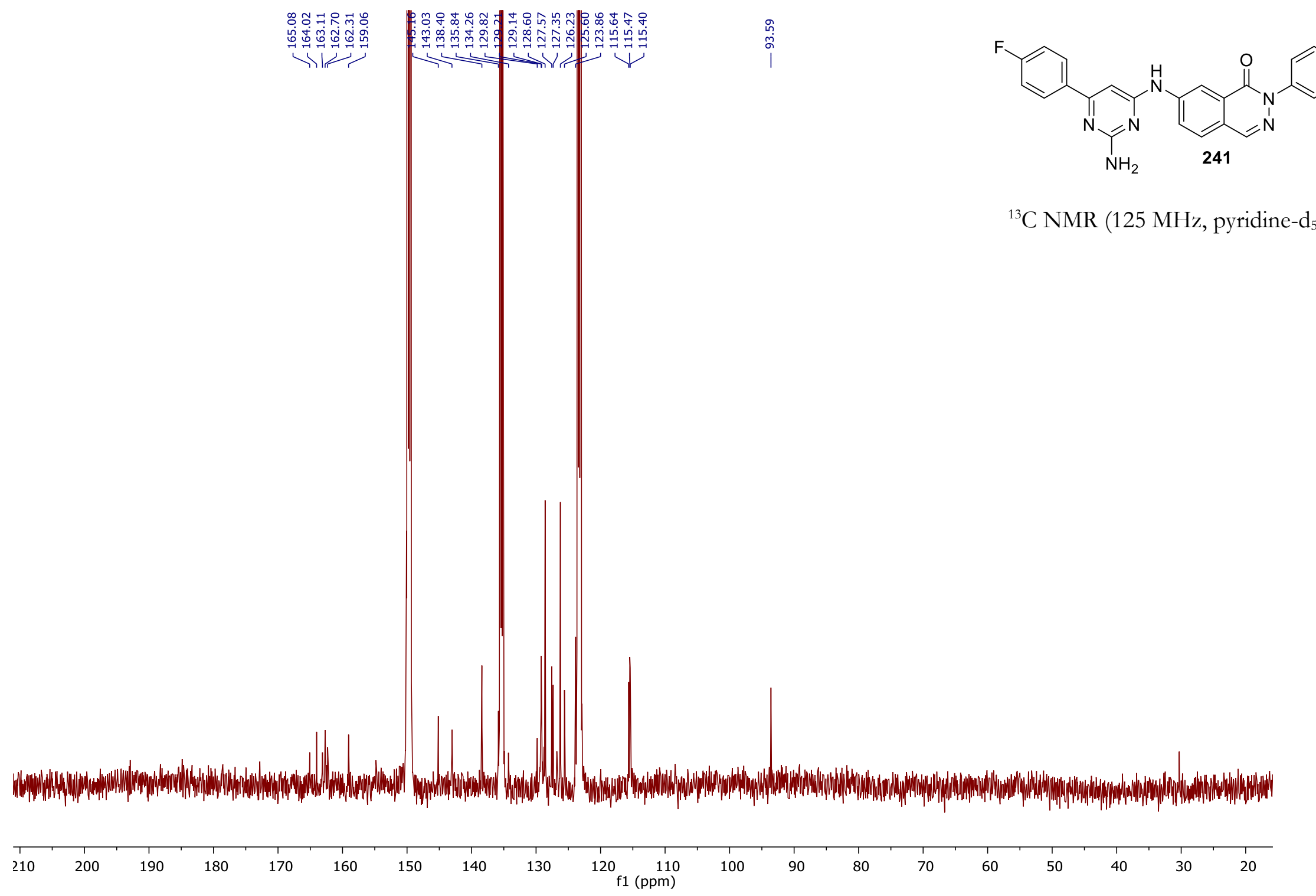




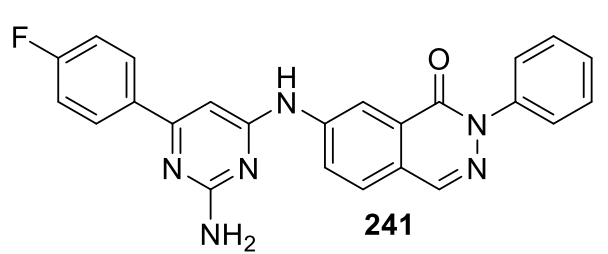

${ }^{19}$ F NMR (282 MHz, pyridine- $\left.\mathrm{d}_{5}\right)$

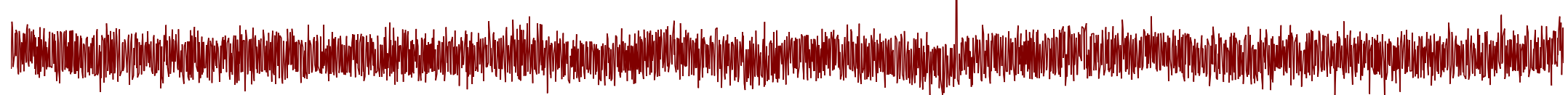

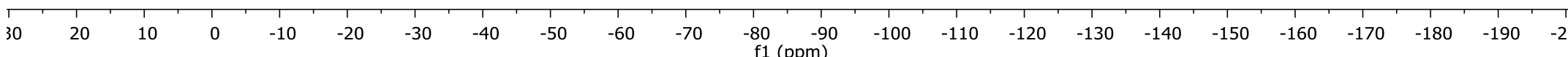




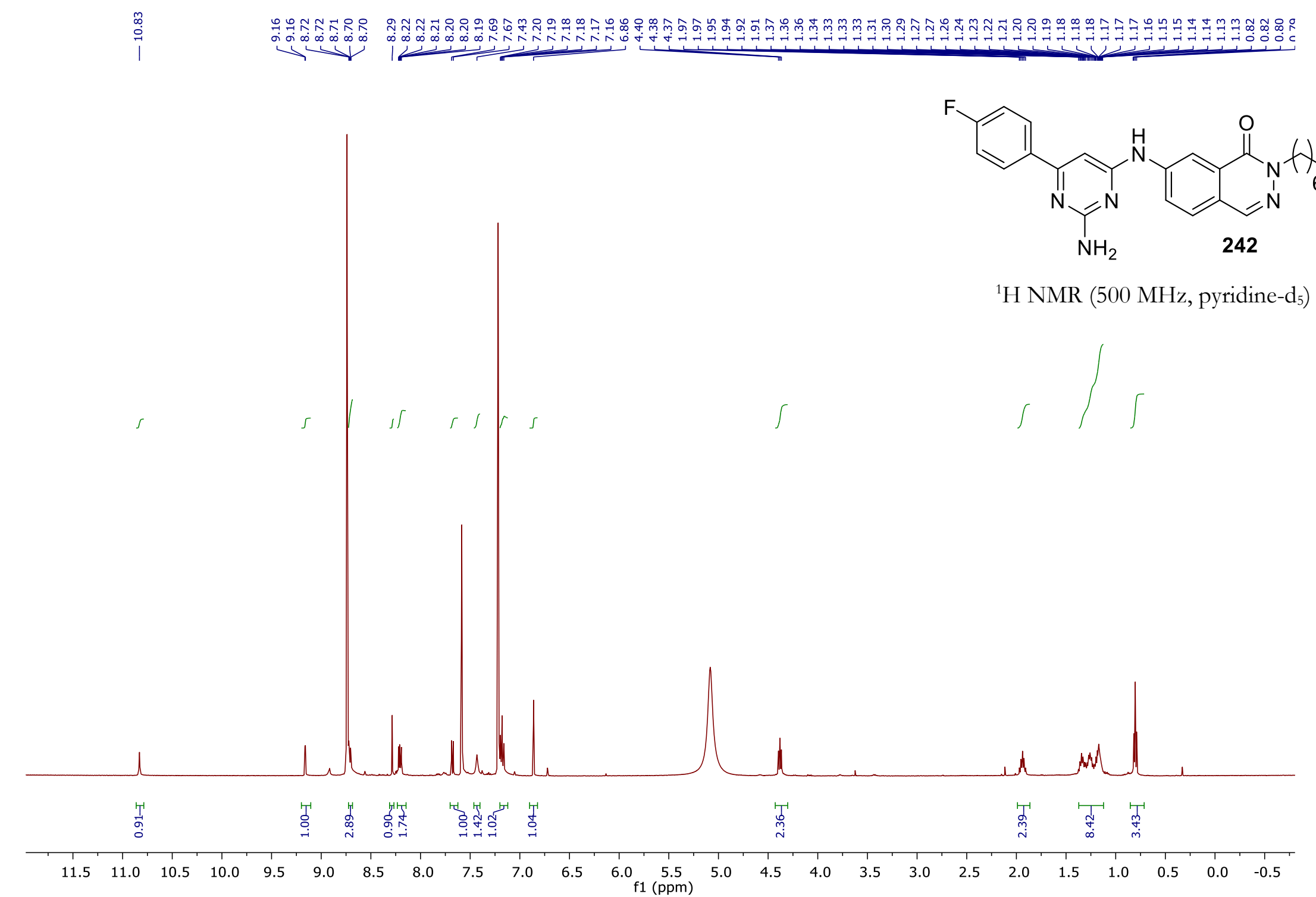



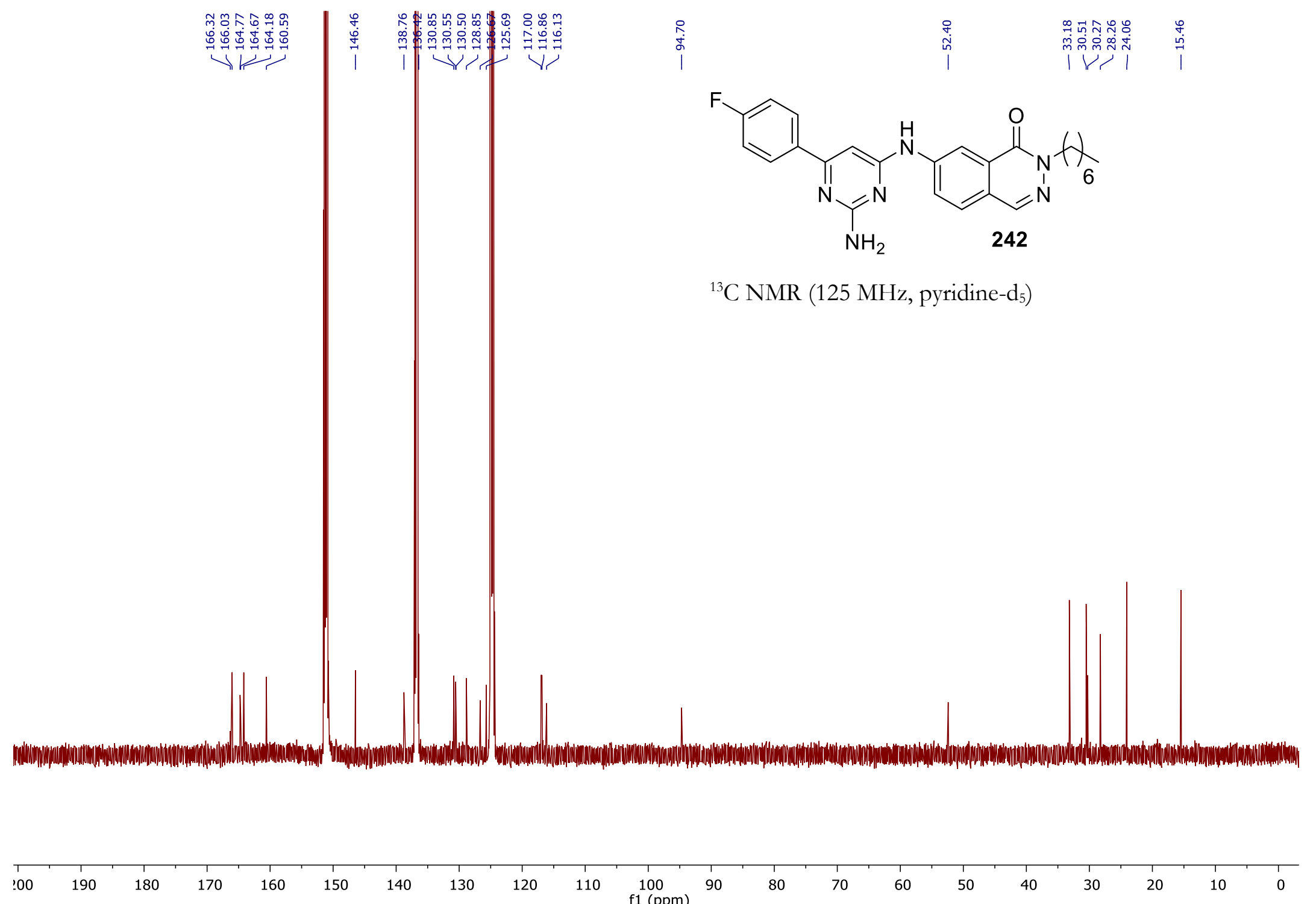


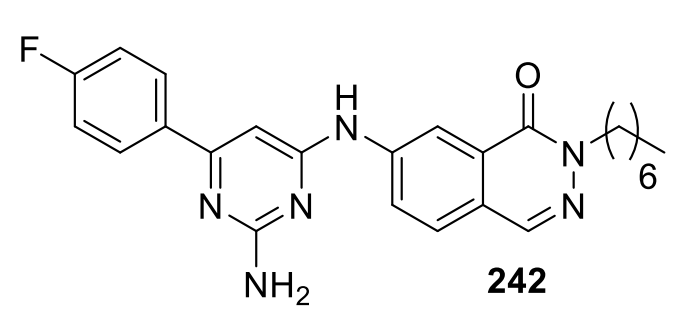

${ }^{19} \mathrm{~F}$ NMR (282 MHz, pyridine- $\mathrm{d}_{5}$ )

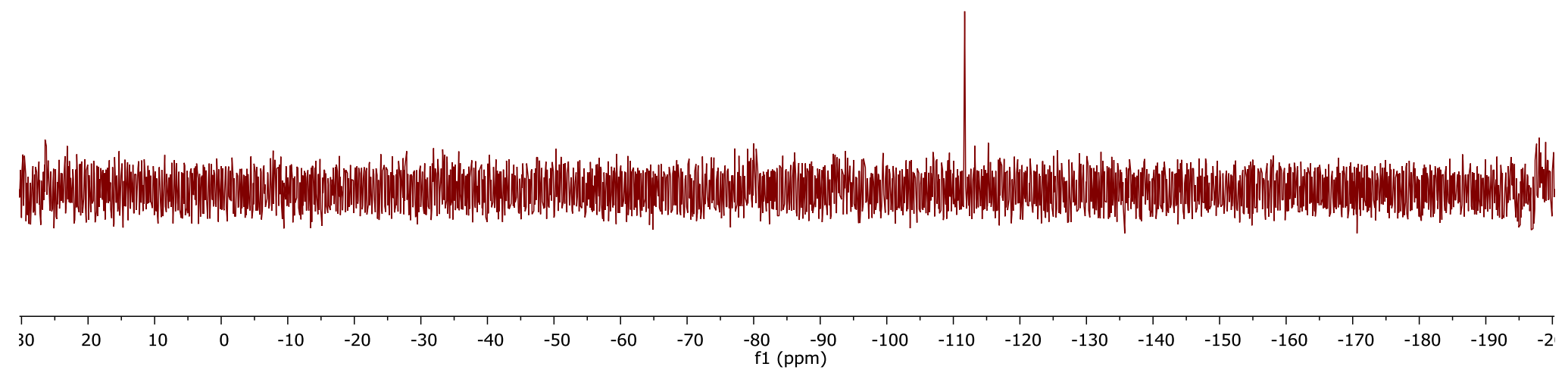




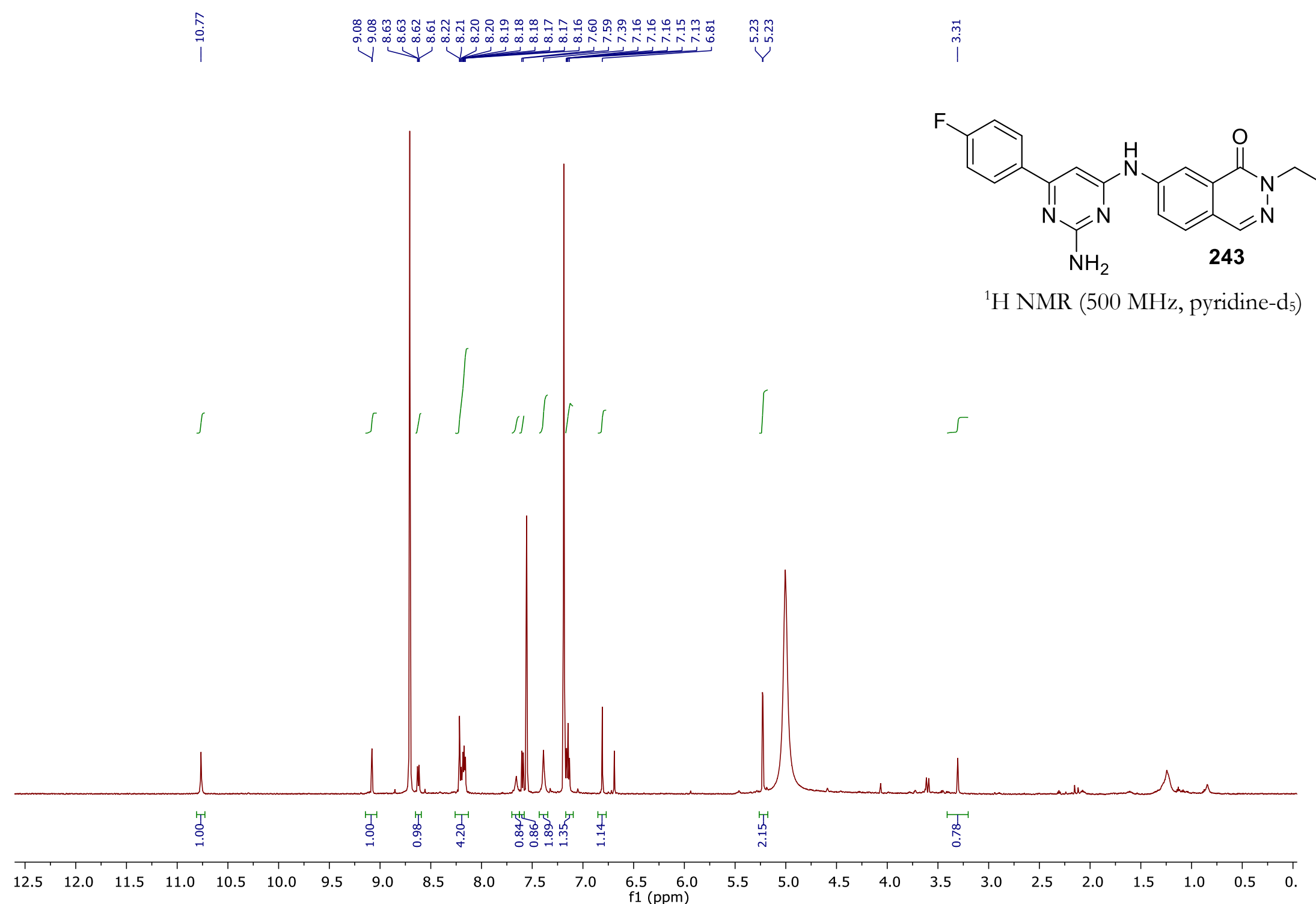




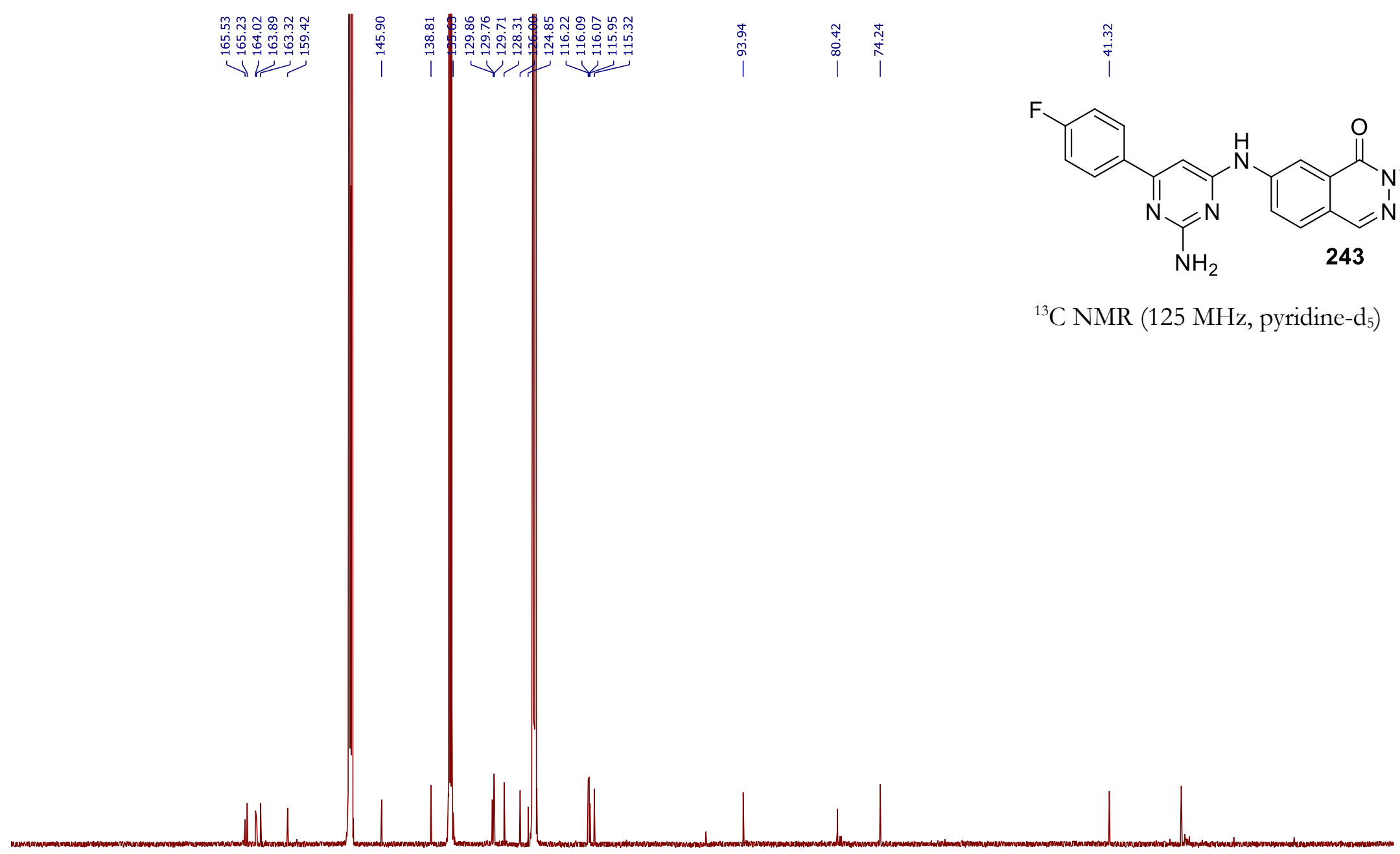

190

170

160

150

$40 \quad 130$

120

$110 \quad 100$

90

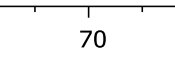

60 


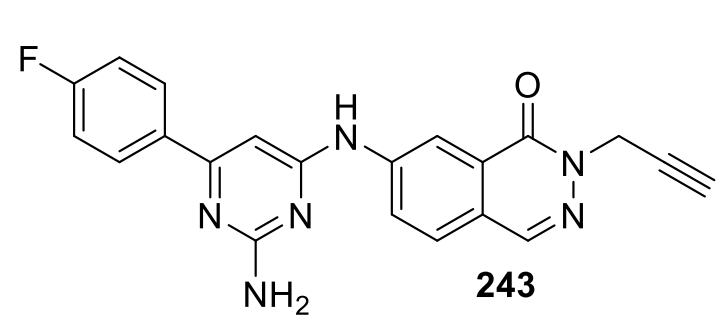

$\underset{\substack{+\stackrel{+}{i}}}{i}$

${ }^{19}$ F NMR (282 MHz, pyridine- $\left.\mathrm{d}_{5}\right)$

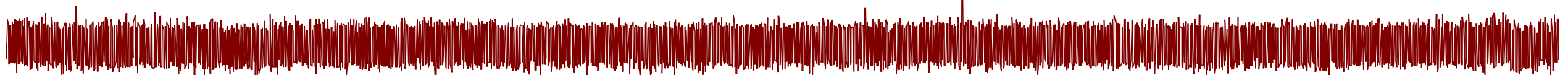

\begin{tabular}{llllllllllllllllllllllllllllll}
\hline & 20 & 10 & 0 & -10 & -20 & -30 & -40 & -50 & -60 & -70 & -80 & -90 & -100 & -110 & -120 & -130 & -140 & -150 & -160 & -170 & -180 & -190 & -2
\end{tabular} 
